\title{
Quantitative Analysis of Shallow Earthquake Sequences and Regional Earthquake Behavior: \\ Implications for Earthquake Forecasting
}

by

Katrina Maureen Jacobs

\begin{abstract}
A thesis
Submitted to the Victoria University of Wellington

In fulfillment of the requirements for the degree of

Doctor of Philosophy
\end{abstract}

Victoria University of Wellington

2013 



\section{Abstract}

This study is a quantitative investigation and characterization of earthquake sequences in the Central Volcanic Region (CVR) of New Zealand, and several regions in New Zealand and Southern California. We introduce CURATE, a new declustering algorithm that uses rate as the primary indicator of an earthquake sequence, and we show it has appreciable utility for analyzing seismicity. The algorithm is applied to the CVR and other regions around New Zealand. These regions are also compared with the Southern California earthquake catalogue. There is a variety of behavior within these regions, with areas that experience larger mainshock-aftershock (MS-AS) sequences having distinctly different general sequence parameters than those of more swarm dominated regions. The analysis of the declustered catalog shows that Lake Taupo and at least three other North Island regions have correlated variations in rate over periods of $\sim 5$ years. These increases in rate are not due to individual large sequences, but are instead caused by a general increase in earthquake and sequence occurrence. The most obvious increase in rate across the four North Island subsets follows the 1995-1996 magmatic eruption at Ruapehu volcano. The fact that these increases are geographically widespread and occur over years at a time suggests that the variations may reflect changes in the subduction system or a broad tectonic process.

We examine basic sequence parameters of swarms and MS-AS sequences to provide better information for earthquake forecasting models. Like MS-AS sequences, swarm sequences contain a large amount of decay (decreasing rate) throughout their duration. We have tested this decay and found that $89 \%$ of MS-AS sequences and 55\% of swarm sequences are better fit with an Omori's law decay than 
a linear rate. This result will be important to future efforts to forecast lower magnitude ranges or swarm prone areas like the CVR.

To look at what types of process may drive individual sequences and may be associated with the rate changes, we examined a series of swarms that occurred to the South of Lake Taupo in 2009. We relocated these earthquakes using doubledifference method, hypoDD, to obtain more accurate relative locations and depths. These swarms occur in an area about $20 \times 20 \mathrm{~km}$. They do not show systematic migration between sequences. The last swarm in the series is located in the most resistive area of the Tokaanu geothermal region and had two $\mathrm{M}=4.4$ earthquakes within just four hours of each other. The earthquakes in this swarm have an accelerating rate of occurrence leading up to the first $\mathrm{M}=4.4$ earthquakes, which migrate upward in depth. The locations of earthquakes following the $M=4.4$ event expand away from it at a rate consistent with fluid diffusion.

Our statistical investigation of triggering due to large global $(\mathrm{M} \geq 7)$ and regional earthquakes $(M \geq 6)$ concludes that more detailed (waveform level) investigation of individual sequences will be necessary to conclusively identify triggering, but sequence catalogs may be useful in identifying potential targets for those investigations. We also analyzed the probability that a series of swarms in the central Southern Alps were triggered by the 2009 Dusky Sound Mw $=7.8$ and the 2010 Darfield $\mathrm{Mw}=7.1$ earthquake. There is less than a one-percent chance that the observed sequences occurred randomly in time. The triggered swarms do not show a significant difference to the swarms occurring in that region at other times in the 1.5year catalog. Waveform cross-correlation was performed on this central Southern Alps earthquake catalog by a fellow $\mathrm{PhD}$ student Carolin Boese, and reveals that individual swarms are often composed of a single waveform family or multiple 
waveform families in addition to earthquakes that did not show waveform similarities. The existence of earthquakes that do not share waveform similarity in the same swarm ( $2.5 \mathrm{~km}$ radius) as a waveform family indicates that similar waveform groups may be unique in their location, but do not necessarily necessitate a unique trigger or driver. In addition to these triggered swarms in the Southern Alps we have also identified two swarms that are potentially triggered by slow-slip earthquakes along the Hikurangi margin in 2009 and 2010. The sequence catalogs generated by the CURATE method may be an ideal tool for searching for earthquake sequences triggered by slow-slip. 


\section{Acknowledgements}

In addition to my own work, this thesis represents the work of many people. I would like to thank my supervisors Euan Smith and Martha Savage for formulating the project which became the basis of my thesis. You have both been incredibly generous with your time, encouragement, and support.

This work would not have been possible without funding from the EQC and the continuous efforts of the GeoNet project to maintain the New Zealand earthquake catalog. Thanks to Jiancang Zhuang for doing ETAS modeling and for discussions that strengthened our publication (Chapter 2) significantly. I am also grateful to Tony Hurst for discussions about swarms that have helped to developed and direct some of my analyses.

Thank you to my friends, especially Anya, Rosie, Dee, Wiebke, and Jo who have encouraged me by getting me out of the office for regular intake of coffee and fresh air. I also owe much gratitude to my various officemates who have kept the atmosphere light hearted and ready for discussion; with special thanks to Sapi and Adrian who have been stuck with me the longest. Thank you to the New Zealand School of Music Orchestra for making me welcome and giving me a place to clear my head.

Finally I'd like to thank my family for answering the phone as often as I called. Your support is priceless and it has gotten me through this work. 


\section{Contents}

Abstract......

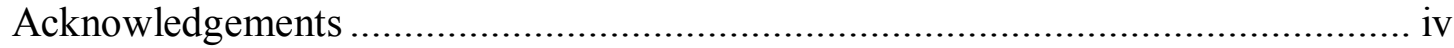

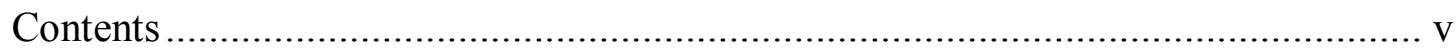

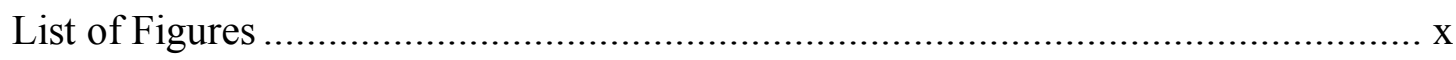

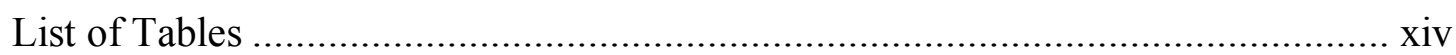

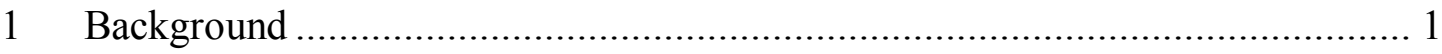

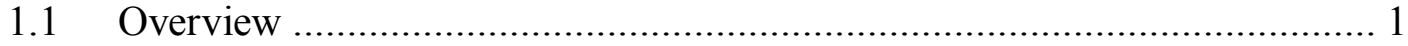

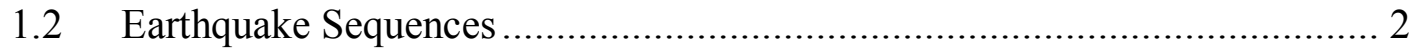

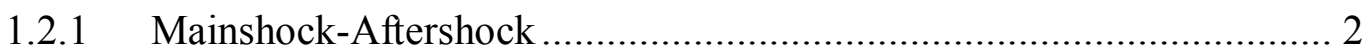

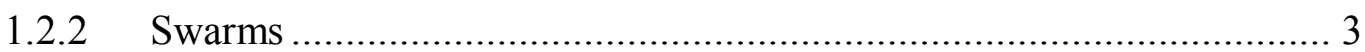

1.2.3 Physical processes and crossover between types............................. 6

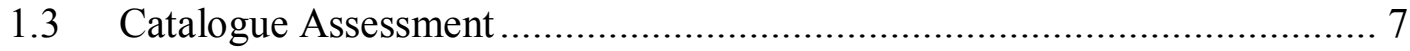

1.3.1 Magnitude Distribution ............................................................. 7

1.3.2 Magnitude of Completeness …...................................................... 8

1.3.3 Background Rate.......................................................................... 11

1.3.4 Sequence Identification and declustering .......................................12

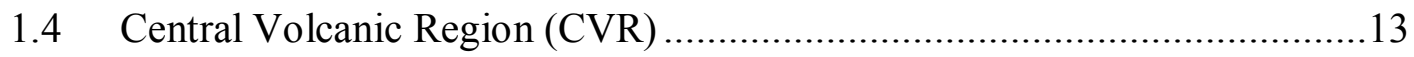

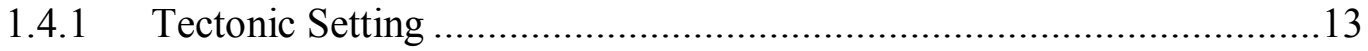

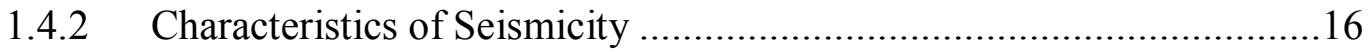

1.4.2.1 Sequence Characteristics .......................................................... 17

1.4.2.2 Association with Volcanic Activity..............................................20

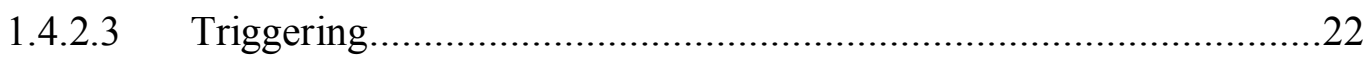

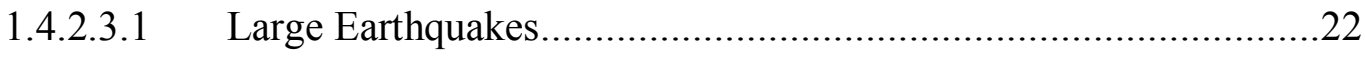

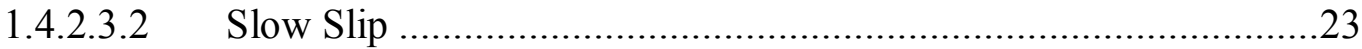

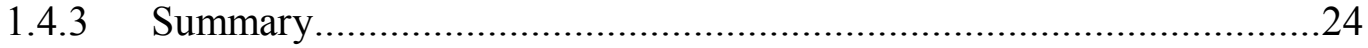

2 Cumulative Rate Analysis (CURATE): A Clustering Algorithm for Swarm Dominated Catalogs .....................................................................................25

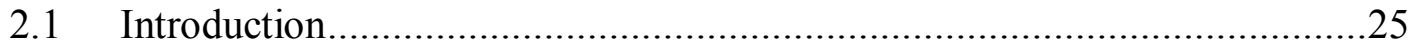

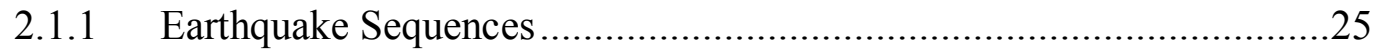

2.1.2 Motivation for a New Method .......................................................26

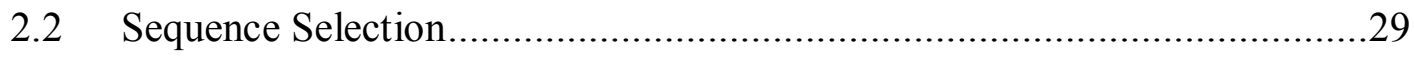

2.2.1 Introduction to Sequence Selection...............................................29

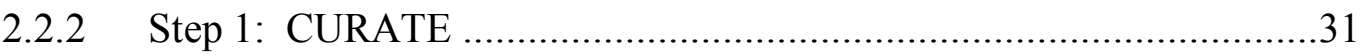




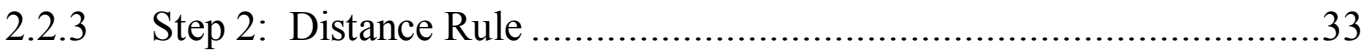

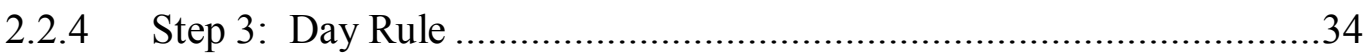

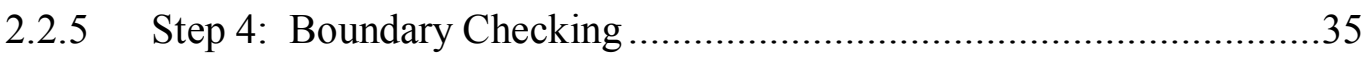

2.3 Application of the Method: Central Volcanic Region, New Zealand ...........36

2.3.1 The Setting of the CVR, New Zealand ............................................36

2.3.2 Earthquake Data and Completeness...................................................37

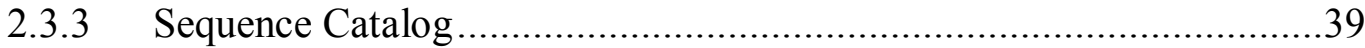

2.4 Comparison With Other Methods ...........................................................43

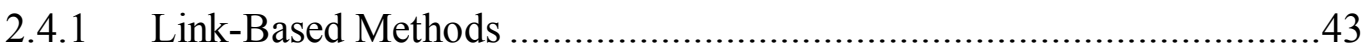

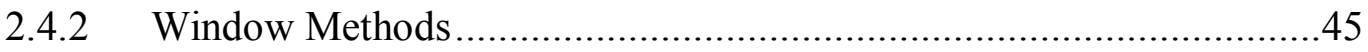

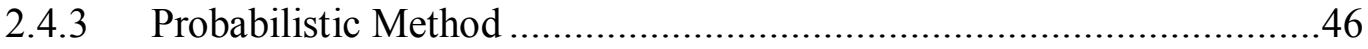

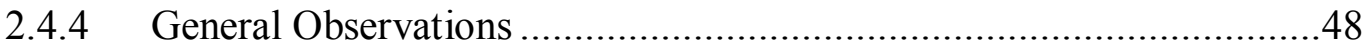

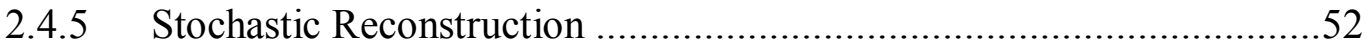

2.4.6 Further Comparison with Link-Based Methods ..................................55

2.5 Testing Sequence Selection Parameters .................................................60

2.5.1 Comparison with Previous Foreshock Results .................................60

2.5.2 Temporal Distribution of the Declustered Catalog ............................61

2.6 Discussion: Utility of Sequence Catalogs ..............................................68

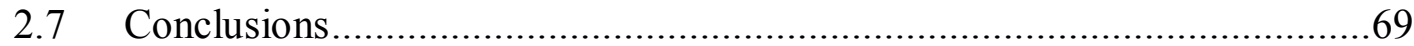

3 Declustered Catalogs and Regional Behavior ................................................ 71

3.1 Comment on clustering/declustering algorithms and Poisson behavior .......71

3.1.1 Dispersion and Size of the Declustered Catalogs ..............................72

3.1.2 Synthetic Poisson Catalog ...............................................................73

3.1.3 CURATE of the Declustered Catalog ..............................................78

3.2 Southern California: Testing the influence of Mcut ..................................79

3.2.1 Southern California, Complete Catalog: ..........................................79

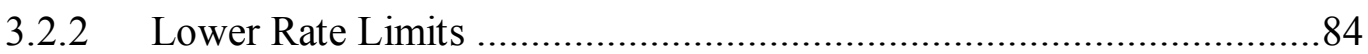

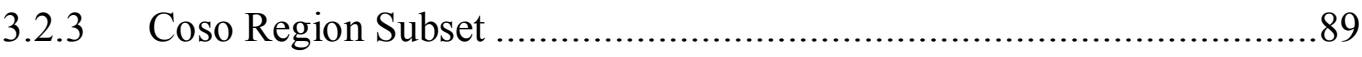

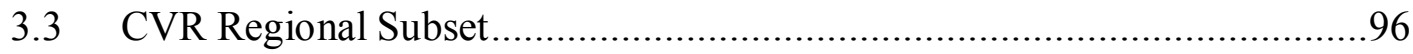

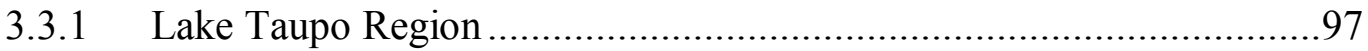

3.4 New Zealand Aftershock Regions ........................................................ 101

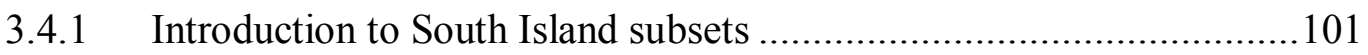

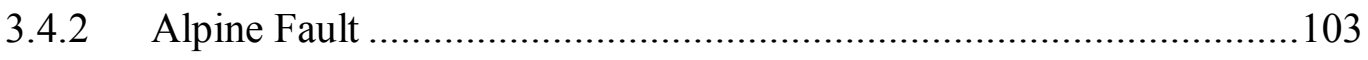


3.4.3 Fiordland (steeply dipping subduction) ........................................110

3.4.4 Northwest Nelson.......................................................................113

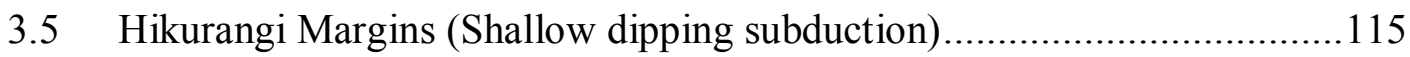

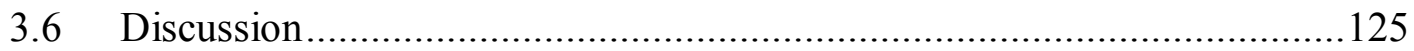

3.6.1 Clustering of Sequences in time .................................................125

3.6.2 Search for rate changes in the broader CVR catalog ........................126

3.6.3 Previous studies of rate changes ................................................. 130

3.6.4 Rate changes in Hikurangi...............................................................132

3.6.5 Possible Causes of Observed Variations in Rate ...............................133

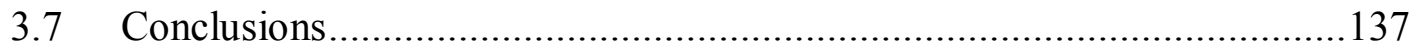

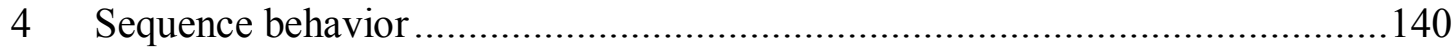

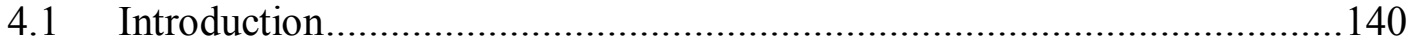

4.2 . Basic Sequence Parameters ................................................................. 144

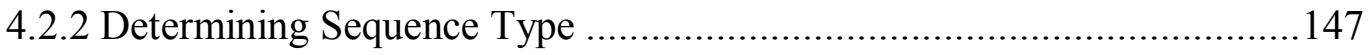

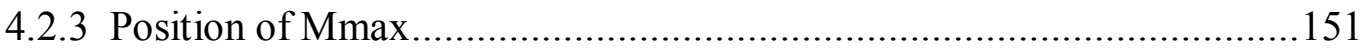

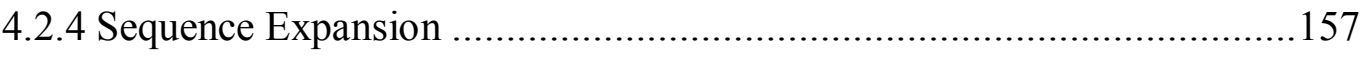

4.2.5 Correlation of Parameters................................................................. 161

4.2.6 Earthquake Rates (Acceleration and Decay) ......................................173

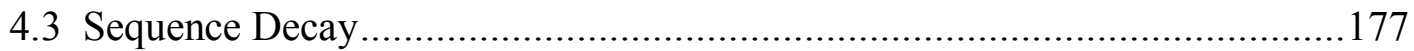

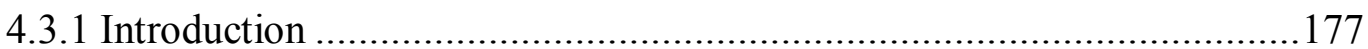

4.3.2 Omori’s Law Modeling .....................................................................179

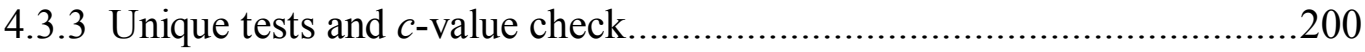

4.3.4 Sequence Parameters.......................................................................203

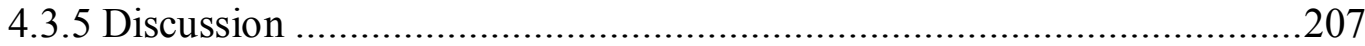

4.4 Rate Patterns in Individual Sequences .........................................................208

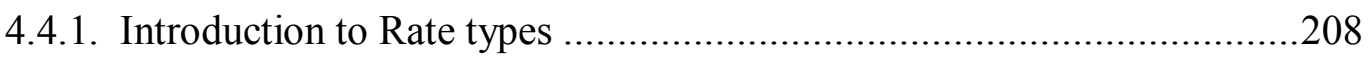

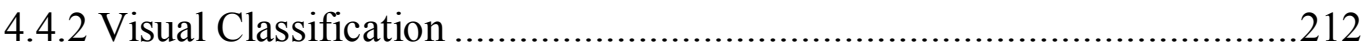

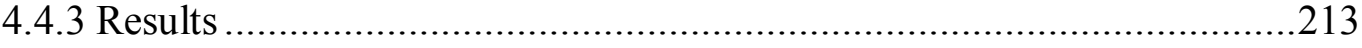

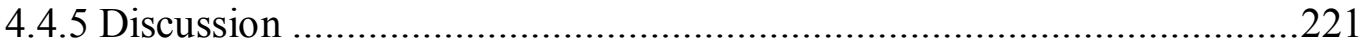

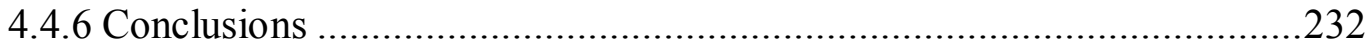

4.5 Sequence Example: Tokaanu Sequences 2009 ........................................233

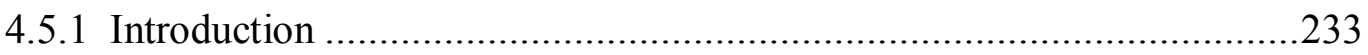

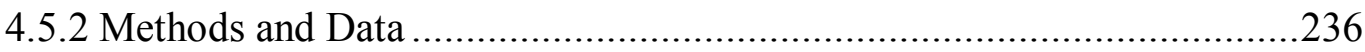


4.5.3 Results

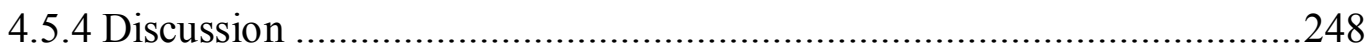

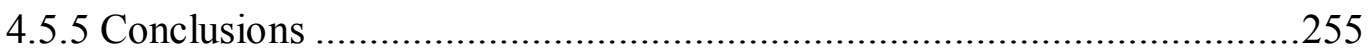

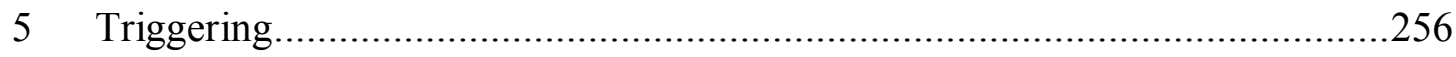

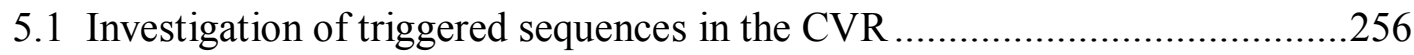

5.1.2 Trigger Test Parameters ......................................................................256

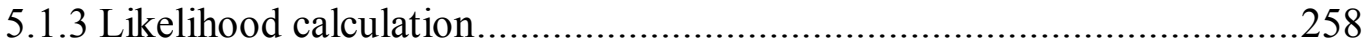

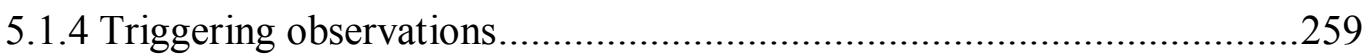

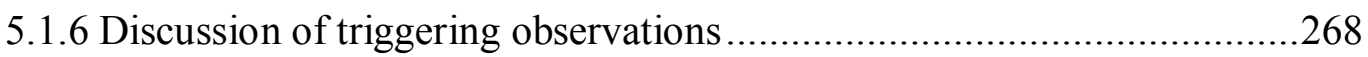

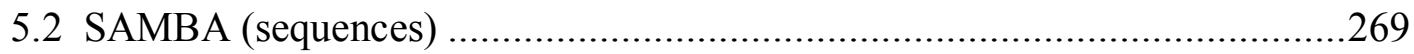

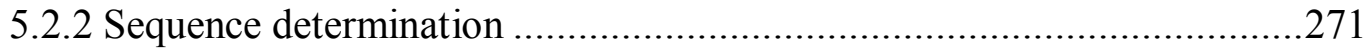

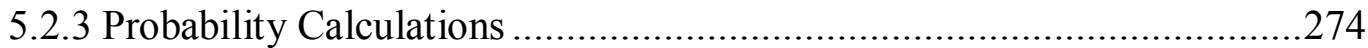

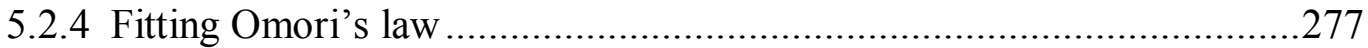

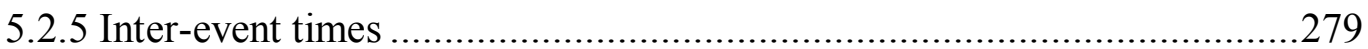

5.2.6 Triggering investigation in the long term catalogue ............................28

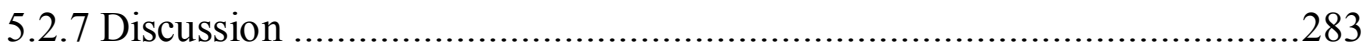

5.3 Investigation of potential triggering by slow slip events ..........................286

5.3.1 Previous seismic and slow slip observations in New Zealand ................286

5.3.2 Relationship to Seismicity (past observations)....................................28

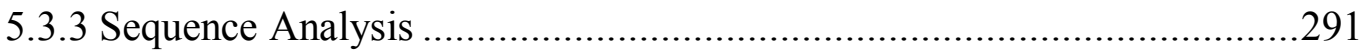

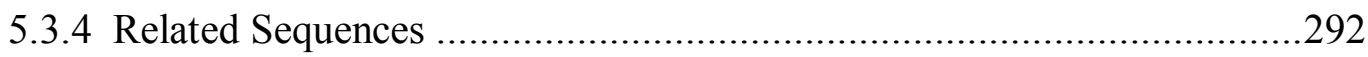

5.3.5 Discussion: Possibilities for seismic detection of slow slip events ........302

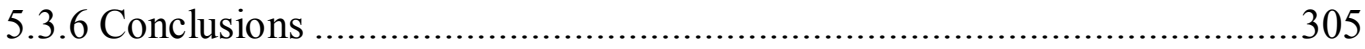

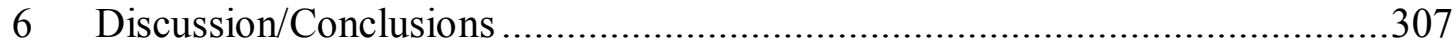

6.1 How can we best identify sequences in an earthquake catalogue that contains

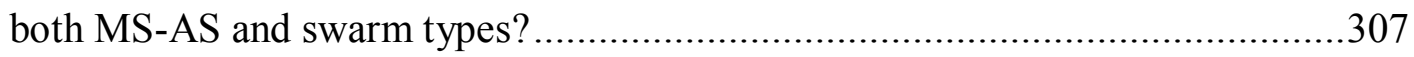

6.2 What kind of sequences occur in the CVR, and how does this compare to other regions of New Zealand and sequences in Southern California?..........................308

6.3 How much decay is observed in swarm sequences and can decay, where

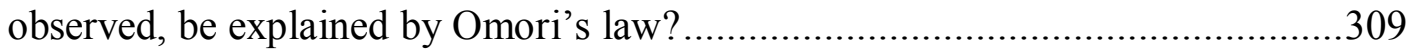

6.4 Are there patterns in sequence occurrence, and does sequence occurrence relate

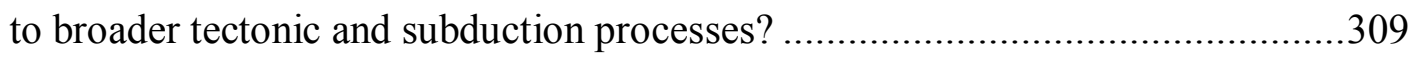

6.4.1: Identified Patterns: Omori and others ................................................309 
In addition to measuring the amount of decay, and comparing sequence decay

6.4.2 Are sequences related to broader tectonic and subduction processes? ....311 6.5 Can statistics using complete catalogues identify potential triggering? .........311 6.6 Does triggering occur in New Zealand? If so, what are its causes?

6.7 Conclusions: So, what can complete catalogues at high Mc tell us about

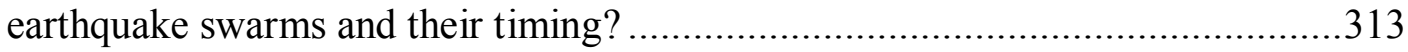

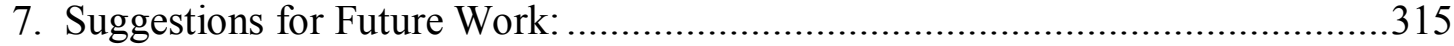

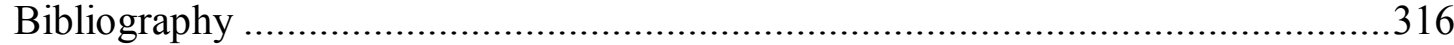

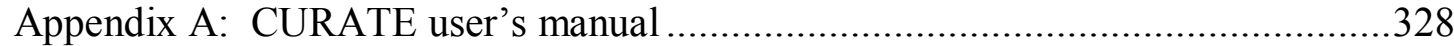

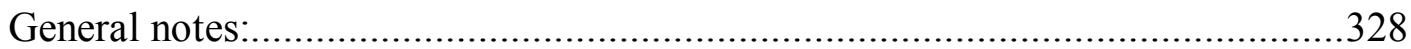

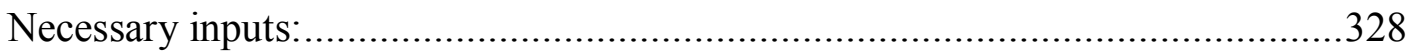

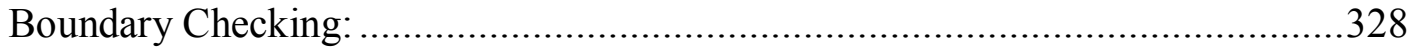

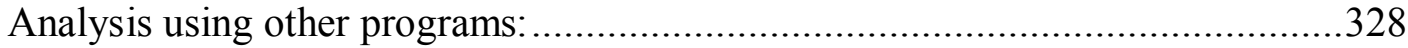

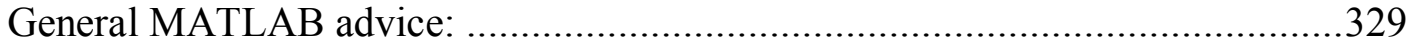

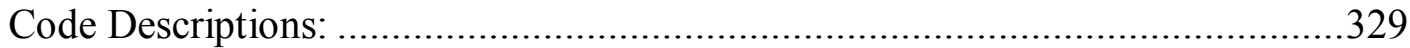

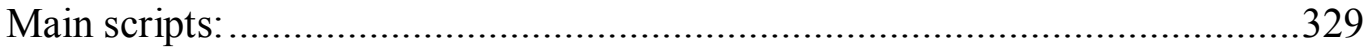

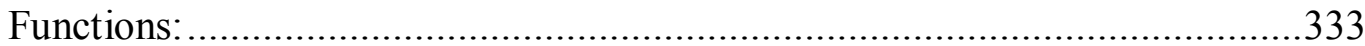

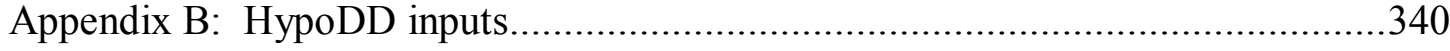

Appendix C: Earthquakes used in triggering analyses .......................................342 


\section{List of Figures}

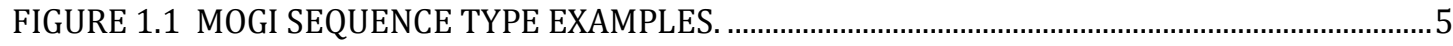

FIGURE 1.2 TWO MAGNITUDE OF COMPLETENESS DETERMINATION METHODS...........................11

FIGURE 1.3 MAP OF THE NORTH ISLAND OF NEW ZEALAND WITH ACTIVE FAULTS.....................15

FIGURE 1.4 MAP OF EARTHQUAKES COLORED WITH DEPTH........................................................ 16

FIGURE 2.1 FLOW CHART OUTLINING THE STEPS THE CURATE METHOD USES TO IDENTIFY SEQUENCES (AND AN ACCOMPANYING DECLUSTERED CATALOG........................................... 30

FIGURE 2.2 PLOTS OF THE CURATE AND CUMULATIVE NUMBER OF EARTHQUAKES WITH

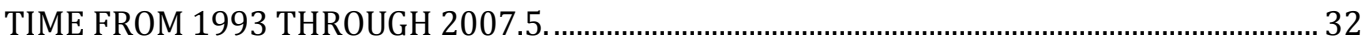

FIGURE 2.3 LOCATION MAP OF THE CENTRAL VOLCANIC REGION WITH GEONET DATA FOR ALL SHALLOW (<40 KM) SEISMICITY M > 2.45 FROM 2005 ................................................... 38

FIGURE 2.4 PLOT USED TO DETERMINE THE MAGNITUDE OF COMPLETENESS IN THE METHOD

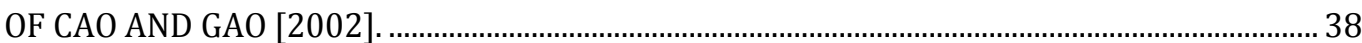

FIGURE 2.5 SEQUENCE ABUNDANCE AND DISTRIBUTION................................................................... 41

FIGURE 2.6 CUMULATIVE NUMBER OF EARTHQUAKES IN THE DECLUSTERED CATALOGS WITH TIME FOR FIVE DIFFERENT CLUSTERING TECHNIQUES, SOME WITH MULTIPLE PARAMETER VALUES.

FIGURE 2.7 DURATION HISTOGRAMS FOR CLUSTERS DEFINED BY FIVE DIFFERENT CLUSTERING METHODS, SOME WITH MULTIPLE PARAMETER VALUES. 50

FIGURE 2.8 CUMULATIVE NUMBER OF EVENTS WITH TIME FOR THE REAL AND TEN STOCHASTICALLY RECONSTRUCTED CATALOGS

FIGURE 2.9 MAP AND MAGNITUDE COMPARISONS FOR TIME PERIODS AROUND TWO LARGE SEQUENCES NEAR HAROHARO.

FIGURE 2.10 POISSON FITS FOR DECLUSTERED CATALOGS.........................................................................

FIGURE 3.1 DISPERSION VALUES PLOTTED WITH SIZE OF THE DECLUSTERED CATALOG......... 72

FIGURE 3.2 CUMULATIVE NUMBER OF EARTHQUAKES FOR SYNTHETIC SEISMICITY CATALOG

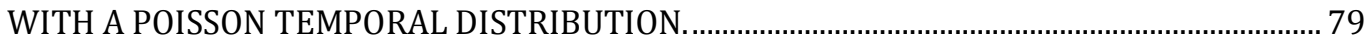

FIGURE 3.3 LOCATION MAP FOR SOUTHERN CALIFORNIA AND COSO GEOTHERMAL AREA (BLUE RECTANGLE). 80

FIGURE 3.4 TWO FIGURES RELATING TO THE EFFECTS OF LARGE EARTHQUAKES ON THE OVERALL RATE OF EVENTS. . .83

FIGURE 3.5 YEARLY EARTHQUAKE TOTALS FROM 100 INITIAL TIMES CHOSEN RANDOMLY FOR THE SOUTHERN CALIFORNIA DATASET. . .85

FIGURE 3.6 CUMULATIVE NUMBER OF EARTHQUAKES WITH TIME FOR THE ORIGINAL AND VARIOUS DECLUSTERED CATALOGS FOR THE SOUTHERN CALIFORNIA DATASET..............8 88

FIGURE 3.7 DISPERSION VALUES FOR THE DECLUSTERED CATALOG OF THE SOUTHERN CALIFORNIA DATASET.

FIGURE 3.8 CURATE PLOTS FOR THE COSO SUBSET AT VARIOUS MCUT VALUES. ......................... 90

FIGURE 3.9 COMPARISON OF THE ORIGINAL AND DECLUSTERED CATALOGS FOR DATA FROM THE COSO GEOTHERMAL REGIONAL SUBSET.

FIGURE 3.10 COMPARISON OF THE DECLUSTERED CATALOGS CREATED BY THE CURATE AND GARDNER AND KNOPOFF CLUSTERING METHODS

FIGURE 3.11 LOCATION MAP FOR SUBSET POLYGONS IN THE NORTH ISLAND, NEW ZEALAND.

FIGURE 3.12 COMPARISON OF THE ORIGINAL AND DECLUSTERED CATALOGS FOR THE LAKE TAUPO REGION. 100

FIGURE 3.13 LOCATION MAP FOR FOUR POLYGONS IN THE SOUTH ISLAND OF NEW ZEALAND.

FIGURE 3.14 CURATE PLOTS FOR FOUR AREAS DOMINATED BY MAINSHOCK-AFTERSHOCK SEQUENCES. 
FIGURE 3.15 YEARLY EARTHQUAKE TOTAL FROM 100 RANDOMLY CHOSEN START TIMES FOR THE FOUR REGIONS IN THE SOUTH ISLAND

FIGURE 3.16 COMPARISON OF THE ORIGINAL AND DECLUSTERED CATALOGS FOR THE ALPINE FAULT DATASET.

FIGURE 3.17 COMPARISON OF THE ORIGINAL AND DECLUSTERED CATALOGS FOR THE SOUTHERN ALPINE FAULT DATASET.

FIGURE 3.18 COMPARISON OF THE ORIGINAL AND DECLUSTERED CATALOGS FOR THE FIORDLAND DATASET.

FIGURE 3.19 COMPARISON OF THE ORIGINAL AND DECLUSTERED CATALOGS FOR THE NELSON DATASET.

FIGURE 3.20 LOCATION MAP FOR THREE POLYGONS IN THE HIKURANGI MARGIN OF NEW ZEALAND

FIGURE 3.21 YEARLY EARTHQUAKE TOTALS FROM 100 START-TIMES CHOSEN RANDOMLY FOR THE THREE HIKURANGI MARGIN SUBSETS.

FIGURE 3.22 COMPARISONS OF THE ORIGINAL AND DECLUSTERED CATALOGS FOR THE HIKURANGI-EAST CAPE DATASET.

FIGURE 3.23 COMPARISON OF THE ORIGINAL AND DECLUSTERED CATALOGS FOR THE HIKURANGI-GISBORNE DATASET.

FIGURE 3.24 COMPARISON OF THE ORIGINAL AND DECLUSTERED CATALOGS FOR THE HIKURANGI-CASTLE POINT DATASET.

FIGURE 3.25 POISSON FRACTION AND DISPERSION FOR THE THREE HIKURANGI DATASETS.

FIGURE 3.26 YEARLY EARTHQUAKE TOTAL FROM 100 START TIMES CHOSEN RANDOMLY FOR THE ENTIRE CVR CATALOG DISCUSSED IN CHAPTER TWO AND FOR THE LAKE TAUPO REGIONAL SUBSET

FIGURE 3.27 CURATE PLOTS FOR THE FOUR REGIONAL SUBSETS SHOWN IN FIGURE 3.11....130

FIGURE 3.28 CUMULATIVE NUMBER OF EARTHQUAKES PLOTTED WITH MONTHLY EXTRACTION AND INJECTION DATA FROM THE COSO GEOTHERMAL REGION.

FIGURE 3.29 VARIANCE/MEAN (DISPERSION) WITH SIZE OF THE DECLUSTERED CATALOG FOR VARIOUS SELECTION PARAMETERS, AND MAGNITUDE CUTOFFS.

FIGURE 4.1 COMPARISONS OF THE NUMBER OF SEQUENCES THAT FIT DIFFERENT DEFINITIONS OF A MAINSHOCK-AFTERSHOCK SEQUENCE.

FIGURE 4.2 STACK PLOTS OF THE NUMBER OF MS-AS SEQUENCES IDENTIFIED BY EACH REGION FOR VARIOUS DEFINITIONS OF A MAINSHOCK-AFTERSHOCK SEQUENCE...........150

FIGURE 4.3 CUMULATIVE DISTRIBUTION OF THE POSITION OF MMAX WITH NORMALIZED DURATION FOR ALL SEQUENCES, AND THE SEQUENCE TYPES. 152

FIGURE 4.4 CUMULATIVE DISTRIBUTION OF THE TIMING OF ALL EARTHQUAKES IN DIFFERENT SEQUENCE TYPES. 153

FIGURE 4.5 CUMULATIVE DISTRIBUTION OF THE POSITION OF MMAX IN DURATION FOR VARYING DEFINITIONS OF MAINSHOCK-AFTERSHOCK SEQUENCES (MS-AS)....................154

FIGURE 4.6 CUMULATIVE DISTRIBUTION OF THE POSITION OF MMAX WITH SEQUENCE TYPE FOR EACH REGION.

FIGURE 4.7 CUMULATIVE DISTRIBUTION OF MDIFF FOR ALL SEQUENCES IN FIVE DIFFERENT REGIONS.

FIGURE 4.8 PLOTS OF THE AMOUNT OF TIME BEFORE AND AFTER MMAX FOR ALL SEQUENCES, AND SEQUENCES BY TYPE.

FIGURE 4.9 SEQUENCE EXPANSION PLOTS. .

FIGURE 4.10 EXPANSION PLOTS BY REGION. 160

FIGURE 4.11 CORRELATION MATRIX FOR THE ABSOLUTE VALUE OF SIGNIFICANT CORRELATIONS OF 33 PARAMETERS FOR THE TWO SEQUENCE TYPES. 164 
FIGURE 4.12 CUMULATIVE DISTRIBUTION OF THE TIME BETWEEN THE LARGEST AND SECOND LARGEST MAGNITUDE EARTHQUAKES.

FIGURE 4.13 AMOUNT OF DECAY OBSERVED FOR SEQUENCES AS A FRACTION OF THE DURATION.

FIGURE 4.14 CUMULATIVE DISTRIBUTION PLOTS OF THE AMOUNT OF A SEQUENCE (BY DURATION) THAT IS TAKEN UP BY DECAY AND ACCELERATION FOR EACH OF OUR FIVE REGIONS (A-E)

FIGURE 4.15 ILLUSTRATING THE CALCULATIONS CONNECTED TO MAXIMUM LIKELIHOOD AND CREATION OF SYNTHETIC DISTRIBUTIONS FROM AN OMORI'S LAW DECAY MODEL.

FIGURE 4.16 EXAMPLE OF THE OMORI'S LAW DECAY MODEL FITS OF C AND K VALUES...........182 FIGURE 4.17 CUMULATIVE DISTRIBUTIONS OF THE MAXIMUM MISFIT FROM AN OMORI'S LAW DECAY MODEL OF ALL SEQUENCES THAT PASS THE MAXIMUM MISFIT FOR ANY OF THE 6 TESTS 184

FIGURE 4.18 EXAMPLE OF A LINEAR COMPARISON TEST.. 188

FIGURE 4.19 COMPARISON OF THE TWO GOODNESS OF FIT TESTS FOR OMORI'S LAW DECAY PARAMETERS MODELS. 188

FIGURE 4.20 ANOTHER EXAMPLE OF TWO GOODNESS OF FIT TESTS FOR OMORI'S LAW DECAY PARAMETERS MODELS. .190

FIGURE 4.21 EXAMPLES OF THREE TYPICAL MS-AS FITS WITH THE LINEAR-COMPARISON TEST.

FIGURE 4.22 LINEAR-COMPARISON TESTS FOR THREE LARGE SWARMS IN THE CVR...............194 FIGURE 4.23 IMPROVEMENT FACTOR (RESIDUAL RATIO) OF THE LINEAR FIT FOR ALL SIX TESTS WITH SEQUENCE TYPE

FIGURE 4.24 RESIDUAL RATIOS WITH SEQUENCE TYPE.

FIGURE 4.25 RESIDUAL RATIOS WITH SEQUENCE TYPE FOR EACH OF THE SIX TESTS. .............199

FIGURE 4.26 SIX OF EIGHT MS-AS SEQUENCES THAT DON'T PASS THE LINEAR-COMPARISON FOR ANY OF THE SIX TESTS.

FIGURE 4.27 SIX SWARMS > 50 EARTHQUAKES THAT FAIL THE LINEAR COMPARISON FOR ALL SIX OMORI'S LAW TESTS.

FIGURE 4.28 CUMULATIVE DISTRIBUTIONS OF FIVE BASIC SEQUENCE PARAMETERS FOR SEQUENCES THAT PASS AND FAIL THE LINEAR COMPARISON TEST BY SWARM TYPE...205

FIGURE 4.29 ARCHETYPES OF FOUR DIFFERENT RATE-PATTERNS FOUND IN AVERAGE INTEREVENT TIME PLOTS.

FIGURE 4.30 DETAILED VIEW OF THE FIRST PART OF THE AVERAGE INTER-EVENT TIME PLOT SHOWN IN FIGURE $4.29 \mathrm{~A}$.

FIGURE 4.31 HISTOGRAMS OF THE NUMBER OF SEQUENCES THAT BELONG TO EACH RATETYPE CATEGORY.

FIGURE 4.32 SPATIAL LOCATIONS OF RATE-TYPES FOR SEQUENCES OF 20 OR MORE EARTHQUAKES.

FIGURE 4.33 EXAMPLES OF THE FIVE RATE-TYPE CATEGORIES OF AVERAGE INTER-EVENT TIMES.

FIGURE 4.34 CUMULATIVE DISTRIBUTION FUNCTION (CDF) PLOTS OF RATE-TYPES WITH FOUR PARAMETERS.

FIGURE 4.35 SYNTHETIC AVERAGE INTER-EVENT TIME FOR OMORI'S LAW DECAY. NINE SEQUENCES ARE SHOWN WITH VARYING K AND C VALUES.

FIGURE 4.36 AVERAGE INTER-EVENT TIMES FOR TWO DIFFERENT L-SHAPED SEQUENCES..224 FIGURE 4.37 MAP OF MATATA SEQUENCES COLORED WITH DAYS THROUGH THE SEQUENCES.

FIGURE 4.38 AVERAGE INTER-EVENT TIME PLOT FOR THE TWO MATATA SEQUENCES SHOWN IN FIGURE 4.37 A) 2005, B) 2007. 
FIGURE 4.39 MAP AND VP/VS RATIOS FOR FOUR STATIONS DURING TWO EARTHQUAKE SEQUENCES IN THE OKATINA CALDERA.............................................................................2.

FIGURE 4.40 AVERAGE INTER-EVENT TIME PLOT FOR THE THREE HAROHARO SEQUENCES SHOWN IN FIGURE 4.39

FIGURE 4.41 MAP OF EARTHQUAKE EPICENTERS FROM MARCH $1^{\text {ST }}$ THROUGH SEPTEMBER $1^{\text {ST }}$ 2009.

FIGURE 4.42 LOCATION MAP OF STATIONS USED TO PICK PHASE ARRIVALS FOR RELOCATION. BLACK STARS SHOW THE LOCATION OF TWO M $=4.4$ EARTHQUAKES.

FIGURE 4.43 COMPARISONS BETWEEN THE ORIGINAL CATALOG LOCATIONS AND DOUBLEDIFFERENCE RELOCATIONS.

FIGURE 4.44 A COMPARISON OF THE LOCATIONS FOR TWO SETS OF P-PHASE ONLY EARTHQUAKE RELOCATIONS.

FIGURE 4.45 EARTHQUAKE RELOCATIONS (USING P-PHASE ONLY) IN 3D.. .244

FIGURE 4.46 PLOT OF THE ARRIVAL TIME OF THE P- VS THE S- PHASES RELATIVE TO THE

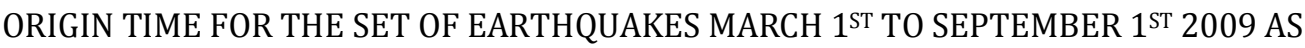
MEASURED ON ALL TWELVE STATIONS.

FIGURE 4.47 MEAN VP/VS VALUES BY STATION FOR ALL TAUPO EARTHQUAKES (BLACK)....247

FIGURE 4.48 DEPTH AND DISTANCE WITH TIME FOR TOKAANU SWARM 4.

FIGURE 4.49 DEPTH WITH TIME COMPARISONS OF P-PHASE ONLY, AND P- AND S- PHASE RELOCATION RESULTS.

FIGURE 4.50 EARTHQUAKE RELOCATIONS OF ALL TAUPO EARTHQUAKES USING BOTH P AND S PHASES FOR RELOCATION.

FIGURE 4.51 AVERAGE INTER-EVENT TIME VERSUS NORMALIZED DURATION OF SWARM 4.

FIGURE 5.1 MAP OF LARGE GLOBAL EARTHQUAKES FROM THE USGS CATALOG (M > 7.0) BETWEEN JANUARY1993 AND JULY 2007.

FIGURE 5.2 MAP OF LARGE NEW ZEALAND EARTHQUAKES ( $M$ > 6.0) FROM JANUARY 1993 TO JULY 2007

FIGURE 5.3 CUMULATIVE DISTRIBUTION FUNCTIONS (CDF) OF PARAMETERS FOR LARGE EARTHQUAKES INCLUDING POTENTIALLY TRIGGERING EVENTS. ..........................................267

FIGURE 5.4 LOCATION MAP FOR THE SAMBA DEPLOYMENT.......................................................2270

FIGURE 5.5 SEISMICITY WITH TIME DETAILS FOR THE EARTHQUAKES IN THE SAMBA NETWORK.

FIGURE 5.6 EARTHQUAKE RATE FITTING FOR THE SAMBA CATALOG FOLLOWING THE DUSKY SOUND EARTHQUAKE. 278

FIGURE 5.7 SYNTHETIC AND OBSERVED AVERAGE INTER-EVENT TIMES FOR SEQUENCES IN THE SAMBA NETWORK. .280

FIGURE 5.8 SEARCH FOR TRIGGERED SEQUENCES IN THE CENTRAL SOUTHERN ALPS REGION

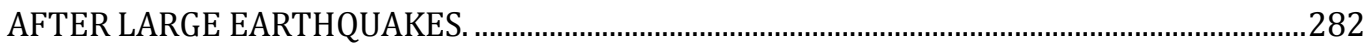

FIGURE 5.9 MAP OF MAIN SLOW-SLIP REGIONS AND RELATED LOCATIONS...............................288

FIGURE 5.10 MAPS OF SLOW SLIP EVENTS (SSE) AND RELATED SEISMICITY IN DECEMBER 2009 AND JUNE AND JULY 2011.........................................................................................29

FIGURE 5.11 SEISMICITY AND SLIP CONTOURS ASSOCIATED WITH GISBORNE 2004 AND 2010 SSES.

FIGURE 5.12 PLOT OF SEISMICITY M > 4.0 FROM JANUARY 2008 THROUGH DECEMBER 2010.

FIGURE 5.13 RATE WITH TIME FOR THREE SEQUENCES ASSOCIATED WITH SLOW SLIP..........301 


\section{List of Tables}

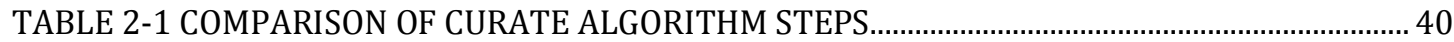

TABLE 2-2 CHANGES MADE BY DIFFERENT PROCESSING STEPS........................................................... 42

TABLE 2-3 STATISTICS OF KEY SEQUENCE PARAMETERS................................................................ 42

TABLE 2-4 NUMBER OF EARTHQUAKES IN CATALOGS WITH VARYING DAY AND DISTANCE

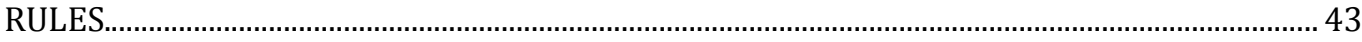

TABLE 2-5 PARAMETERS OF DIFFERENT DECLUSTERING METHODS (DURATIONS IN DAYS, AND AREAS IN KMำ.

TABLE 2-6 RESULTS OF POISSON TESTING FOR DIFFERENT SLC AND CURATE DECLUSTERED CATALOGS.

TABLE 3-1 DETAILS OF DECLUSTERED CATALOGS OF SYNTHETIC POISSON TEMPORAL EARTHQUAKE CATALOGS FOR VARIOUS CLUSTERING TECHNIQUES. ………………............76

TABLE 3-2 POISSON TESTING OF THE SCA CATALOG................................................................................ 81

TABLE 3-3 POISSON TESTING OF THE SCA CATALOG WITH MEDIAN AND MINIMUM RATES ... 86

TABLE 3-4 POISSON TESTING USING MINIMUM RATE THRESHOLD FOR VARIOUS TIME PERIODS OF THE SCA CATALOG 87

TABLE 3-5 POISSON TESTING OF THE COSO SUBSET DECLUSTERED CATALOG USING THE CURATE METHOD WITH A MINIMUM RATE.

TABLE 3-6 POISSON TESTING OF THE COSO SUBSET DECLUSTERED CATALOG FROM THE GARDNER AND KNOPOFF METHOD

TABLE 3-7 POISSON TESTING OF THE LAKE TAUPO SUBSET DECLUSTERED CATALOG USING THE CURATE METHOD.

TABLE 3-8 ALPINE FAULT POISSON TESTING FOR DIFFERENT POLYGONS AND TIME PERIODS.

TABLE 3-9 SUMMARY FOR COMPARISON OF ALPINE FAULT POLYGONS FOR DIFFERENT TIME PERIODS.

TABLE 3-10 POISSON TESTING OF THE FIORDLAND SUBSET USING THE CURATE METHOD..112

TABLE 3-11 POISSON TESTING OF THE NELSON SUBSET USING THE CURATE METHOD..........114

TABLE 3-12 POISSON TESTING OF SUBSETS IN THE HIKURANGI MARGIN USING THE CURATE METHOD WITH MEAN RATE.

TABLE 3-13 POISSON TESTING OF DIFFERENT TEMPORAL SUBSETS FOR THE GISBORNE REGION USING THE CURATE METHOD WITH MEAN RATE.

TABLE 4-1 BASIC PARAMETERS OF ALL CATALOGS USED TO INVESTIGATE SEQUENCES........142

TABLE 4-2 TABLE OF BASIC SEQUENCE PARAMETERS BY REGION. ..............................................144

TABLE 4-3 TABLE OF BASIC SEQUENCE PARAMETERS FOR ALL SEQUENCE, AND BY SEQUENCE TYPE.

TABLE 4-4 COMPARISON OF THE MEDIAN AND MAXIMUM DISTANCE RATIOS FOR ALL REGIONS

TABLE 4-5A CORRELATION OF KEY PARAMETER PAIRS FOR SEQUENCE TYPES IN ALL REGIONS.

TABLE 4-6 THE NUMBER OF SEQUENCES (BY TYPE) THAT PASS THE DIFFERENT OMORI'S LAW DECAY TESTS.

TABLE 4-7 CONFIDENCE BOUNDS AND ABSOLUTE VALUE OF THE MAXIMUM MISFIT FOR SEQUENCES PASSING AND FAILING ALL OMORI'S LAW DECAY TESTS.................................186

TABLE 4-8 THE NUMBER OF SEQUENCES (BY TYPE) THAT PASS THE LINEAR COMPARISON DECAY TEST.

TABLE 4-9 RESIDUAL RATIOS OF OMORI'S LAW DECAY AND LINEAR FITS TO THE DATA FROM EACH TEST.

TABLE 4-10 NUMBER OF SEQUENCES THAT PASS OMORI'S DECAY FOR THREE DIFFERENT TESTS. 
TABLE 4-11 PARAMETER VARIATIONS FOR SEQUENCES THAT PASS AND FAIL THE OMORI'S LAW DECAY TESTS

TABLE 4-12 NUMBER OF SEQUENCES THAT PASS AND FAIL THE LINEAR COMPARISON TEST

BY REGION.

.206

TABLE 4-13 SEQUENCE PARAMETERS OF DISCUSSED INTER-EVENT TIME EXAMPLES........................228

TABLE 4-14 PARAMETERS OF THE FOUR MAIN SWARMS IN 2009................................................237

TABLE 4-15 VELOCITY MODEL USED FOR RELOCATION...................................................................240

TABLE 5-1 RESULTS OF LIKELIHOOD TESTS LOOKING FOR POTENTIAL SEQUENCE

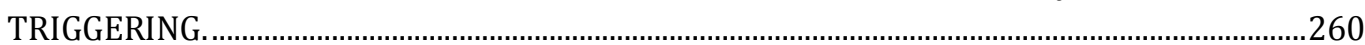

TABLE 5-2 LIKELIHOOD OF SEQUENCES RANDOMLY ALIGNING WITH A LIST OF TIMES THE LENGTH OF THE POTENTIAL TRIGGERS FOR THE GLOBAL AND NEW ZEALAND LISTS THAT YIELD THE LARGEST NUMBER OF POTENTIALLY TRIGGERED SEQUENCES (TABLE

5.1).

TABLE 5-3 SEQUENCE CATALOG FOR THE SAMBA AND DFDP10 ARRAYS (CENTRAL ALPINE FAULT). 



\section{Background}

\subsection{Overview}

In this study we investigate shallow earthquakes sequences with a focus on earthquake swarms, which are common in the Central Volcanic Region (CVR) of New Zealand. We focus on this region, as it has been a persistent source of swarms since records have been kept. The historically recorded activity would have a much bigger impact if it were to occur today as populations have increased in many areas. Additionally, because the area is volcanic, the occurrence of earthquake swarms may give insight to volcanic, geothermal, and tectonic activity.

Earthquake swarms do not have a quantitative definition and so studies of swarms rely on systematic reporting or individual characterization of each sequence. To establish a firm basis for systematically identifying earthquake sequences, we have developed a new Cumulative Rate (CURATE) declustering algorithm that uses rate as the primary way to identify whether an earthquake sequence is occurring (Chapter 2). This new method allows for a better characterization of swarm sequences than other common declustering methods. The sequence catalog produced by using this method allows us to objectively and systematically compare seismicity over large periods of time, and spatial regions that may vary significantly in their behavior. This chapter is a reprint of a paper we published in JGR [Jacobs et al., 2013].

In addition to analyzing seismicity in the CVR as a whole, we also examine regional subsets through the North Island to try to gain additional insights into regional sub-processes and to how broad-scale processes manifest in different regions (Chapter 3). Chapters 3 and 4 contain analysis of sequence catalogs created by the 
CURATE method applied throughout New Zealand and in Southern California. Chapter 3 focuses on the application of the CURATE method in the presence of rate variations with time caused by large aftershock sequences and other sources.

Chapter 4 uses the data and sequence catalogues created in Chapter 3 to quantify earthquake sequence types and characteristics. This analysis includes quantification of the amount of observed sequence decay. We also conducted an average rate analysis within sequences is also used to analyze sequence behavior (section 4.4). The rate analysis reveals several distinct rate-patterns that differ from Omori's law decay. We have also undertaken a study of a set of individual swarm sequences near Tokaanu, at the South end of Lake Taupo, to test what kind of processes could be linked to the broader patterns and processes observed through the collective swarm analysis (section 4.5). Finally Chapter 5 will explore whether large earthquakes and slow slip influence the timing of sequence occurrence and also provides brief examples of how sequence catalogs created using the CURATE method can be utilized.

\subsection{Earthquake Sequences}

\subsubsection{Mainshock-Aftershock}

Mainshock sequences are much more studied, and better understood than earthquake swarms. Classically a mainshock-aftershock sequence is one in which the largest magnitude event occurs first, or early in the case of foreshocks [Mogi, 1963], and according to Bath's law there is also an expected magnitude difference between the two largest shocks of 1.2 magnitude units; however that value is an average and there is a wide range of globally observed magnitude differences for mainshockaftershock sequences [Bath, 1965; Felzer et al., 2002; Felzer et al., 2004]. 
Mainshock-aftershock sequences are also known to have a power law decay rate which is described by a modified Omori's law (eq. 1.1).

$$
n(t)=\frac{K}{(t+c)^{p}}
$$

Where $n$ is the number of earthquakes that occur per unit time at a time $t . K$ is proportional to the aftershock productivity (number of subsequent earthquakes for a given mainshock), $p$ is a decay parameter close to one, and $c$ is a small amount of time to avoid the singularity at $\mathrm{t}=0$ [Utsu et al., 1995]. The $c$-value typically has a small value on the order of minutes; whether or not it has a physical meaning is still under debate [Enescu et al., 2009] and will be discussed further in section 4.3. Recent work by Christophersen and Smith [2008] confirms that foreshock sequences behave like mainshock-aftershock sequences which happen to have a smaller first event [Felzer et al., 2004]. We will consider sequences with foreshocks to be mainshockaftershock type sequences as long as they also have a characteristic magnitude separation (Bath's law) between the largest and second largest earthquakes and these sequences will generally be included when we use the terms 'mainshock-aftershock' or 'aftershock sequence'.

\subsubsection{Swarms}

The most common way to define an earthquake swarm is as a group of spatiotemporally related earthquakes that lack a mainshock. The rate, duration, and spatial extent of earthquake swarms varies so widely that there are no strict definitions to identify what constitutes a swarm, let alone any equations which forecast their spatial extent or the number of earthquakes. Few studies endeavor to define what they characterize as a "swarm" and others seek to use separate terminology (including 
bursts) to emphasize the time relation. One of the most widely cited definitions of an earthquake swarm is that of Mogi [1963], who describes three types of earthquake sequences (Fig. 1.1 A). Mogi generally defines an earthquake swarm as a group of earthquakes that are closely clustered in time and space and have no predominant earthquake or 'mainshock' [Mogi, 1963]. 'Close' in time is generally determined as an increase from the original or background rate and 'close' in space is not defined, but is generally within tens of kilometres or less. The schematic rate diagram he presents (Fig 1.1a) is often seen in studies that differentiate earthquake swarms from other sequences [Benoit and McNutt, 1996; Scholz, 2002]. In his textbook, Scholz [2002] goes on to define swarms as "sequences that often start and end gradually and in which no single earthquake dominates in size." Though the absence of a mainshock often provides a more uniform rate with time when compared to mainshock-aftershock sequences, Mogi's original formulation does not require earthquake swarms to have gradual changes in rate and a gradual decrease at the end of a swarm is in contrast to the swarm example provided by Mogi (Fig 1.1b). The example case study histograms (Fig. 1.1b) are rarely cited and not provided in literature that reproduces the schematic diagram (Fig. 1.1a). Note that the earthquake swarm (type 3) has an increasing rate, but appears to end abruptly towards the maximum event rate without a gradual decline. This shows that even within the broad definition of earthquake swarms there are variations. 


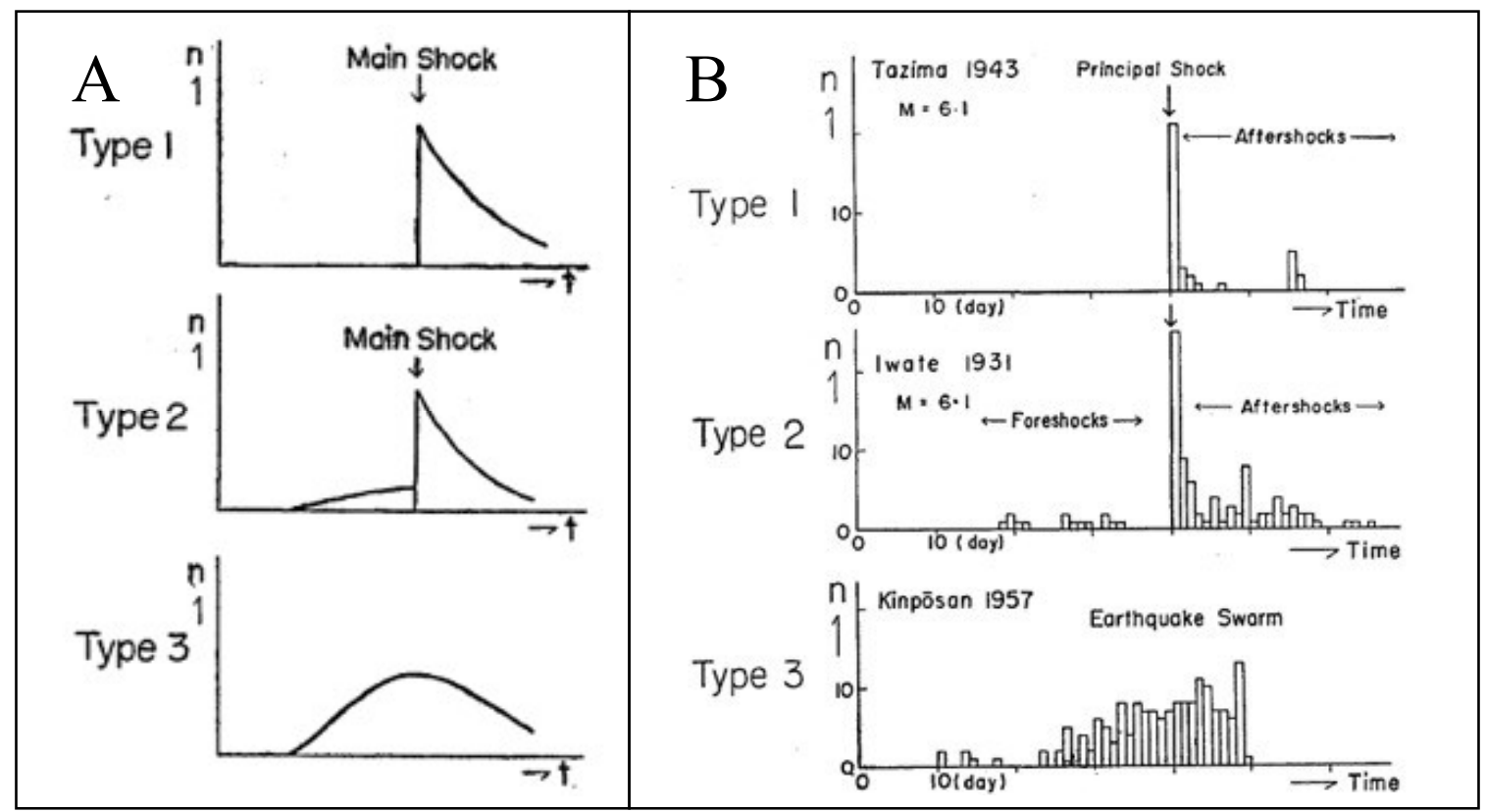

Figure 1.1 Mogi sequence type examples.

A) Schematic rate with time histograms for three sequence types, Mainshock, foreshock-mainshock-aftershock, and swarm types. B) Rate histograms from earthquake sequences in Japan that exemplify each of the three sequence types. Taken from Mogi [1963].

One of the most extensive studies of earthquake swarms is the Global Volcanic Earthquake Swarm Database of volcanic earthquake swarms that occurred between 1979 and 1989 compiled by Benoit and McNutt [1996]. The database contains more than 600 earthquake swarms, and was an attempt to characterize swarm behavior associated with volcanic activity. The most frequently reported parameter for the swarms in their study was duration, and the second most frequently reported parameter, maximum magnitude, was reported in less than 30-percent of the entries. This highlights the main problem that they encountered in characterizing swarm activity, which is the lack of systematic reporting of basic earthquake sequence parameters. This lack of reporting may stem directly from the lack of a clear definition by which one can interpret observed activity. To this end we intend that the use of our strict algorithm will provide a practical definition. 
Swarms (by various definitions) are observed worldwide, often near active volcanoes or in areas of recent volcanism [Benoit and McNutt, 1996; Eiby, 1966; Sherburn, 1992b]. Due to the common occurrence of swarms at volcanoes they are often interpreted in terms of magma movement, but even at volcanoes swarms may represent more benign processes related to fluid intrusion or changes in stress without the presence of magma [Hainzl and Fischer, 2002b; Hill, 1977; Roman and Cashman, 2006].

\subsubsection{Physical processes and crossover between types}

Earthquake swarms can be triggered by large earthquakes $(M \geq 7)$ up to $3660+$ km away [Eberhart-Phillips et al., 2003; Hill et al., 1993; Moran et al., 2004; West et al., 2005]. Studies after the Denali Fault earthquake in 2002 have revealed that geothermal and volcanic areas are particularly susceptible to this triggering [EberhartPhillips et al., 2003]. While certain local physical conditions are likely to be necessary for this triggering to occur, it is important to consider that triggers outside the local area of occurrence may affect the timing of earthquakes and sequences.

Brodsky [2006] has shown that some triggered swarm events following distant large earthquakes may simply be explained as aftershocks of events that are triggered during the passage of the wave-train. However, in section 5.2 we show an example in the Southern Alps of New Zealand that demonstrates that despite a lack of waveform triggered events there are subsequent swarms observed that are not easily explained by the typical time occurrence of swarms in that area. This point will be further investigated in Section 4.3 where we compare the temporal rate decay of mainshock sequences to other (swarm) type sequences.

This similarity in triggered earthquake swarms and MS-AS sequences highlights an important point: that there are no clear lines between sequence types. Our analysis 
shows that increased rate is the main commonality between all types of sequences and this rate may be the key factor to understanding the underlying physical processes. We think of earthquake sequences as having two essential parts, a sequence trigger, and a sequence driver. For instance in a classic MS-AS sequence the trigger may be a critical stress build up and frictional failure on a fault and the driver may be a redistribution of stresses in the area on and around the fault ruptured in the mainshock. In swarms, the sequence triggers and drivers are less clear. Some swarms do exhibit decay and all earthquakes have some ability to produce subsequent earthquakes. In section 4.3 we quantify the amount of swarm decay that is observed and test whether it can be described by Omori's law. Swarms appear to have a variety of driving mechanisms and hence are poorly modeled by aftershock decay models that include spatial faulting assumptions and Omori-like power law decay (Section 2.4.5). For this study we call any group of spatially and temporally related earthquakes ranging from MS-AS sequences to earthquake swarms an earthquake sequence. Mainshockaftershock sequences (including those with foreshocks) will be distinguished from earthquake swarms by the early occurrence of the largest magnitude earthquake (Mmax) and a characteristic magnitude separation (Bath's law) between the two largest earthquakes.

\subsection{Catalogue Assessment}

\subsubsection{Magnitude Distribution}

There are two main concepts that are thought to describe earthquake magnitude behavior. The first is the seismic b-value discovered Ishimoto and Ida [1939], and put in its classic form by Gutenberg and Richter [1944] (Eq. 1.2),

$$
\log _{10} N=a-b M
$$


where $N$ is the number of earthquakes magnitude $\geq M, a$ and $b$ are constants.

Generally the formula relates the rate of occurrence of large magnitude events to the rate of small magnitude events. Tectonic areas often have a b-value near one over long time scales [Frohlich and Davis, 1993]. Volcanic areas tend to have higher bvalues (larger ratio of small/large magnitudes) [McNutt, 2005; Scholz, 2002]. The bvalue has also been shown to vary temporally and spatially although care should be taken as changes in this parameter are very sensitive to the catalog completeness and changes to the completeness [Woessner and Wiemer, 2005]. Variations are thought to be due to four main factors: material heterogeneity [Mogi, 1962], stress [Scholz, 1968], changes in pore pressure [Wyss, 1973], and thermal gradient [Warren and Latham, 1970].

Bath's law is the other major law associated with magnitude distributions. Unlike the b-value, it only pertains to individual sequences and governs the expected average magnitude separation between the two largest events in a sequence. The parameter is known to vary widely [Felzer et al., 2004]. The magnitude threshold used to define mainshocks and the catalog itself can also affect the range in these values as shown by Console et al. [Console et al., 2003]. Thus, the observed variation may stem from our incomplete knowledge of the processes underlying specific sequences or from how the sequences are determined.

\subsubsection{Magnitude of Completeness}

The Magnitude of Completeness (Mc) is the magnitude at which all earthquakes of a certain magnitude and higher are located consistently (e.g. [Woessner and Wiemer, 2005]). Earthquakes below this magnitude may appear in the catalog and be located during routine location processes, but analysis shows that a percentage of the expected population of those events is missing. If all earthquakes in 
the catalog are used instead of $M \geq M c$, studies of earthquake properties, especially the number of events, may be skewed. The Mc is an attribute of the earthquake catalogue, not of the earthquakes themselves and it can be influenced by station spacing, attenuation of the area under investigation, and even anthropogenic and other noise sources. The Mc has also been shown to vary locally following large earthquakes (e.g. [Kagan and Houston, 2005]).

Whatever factors contribute to the magnitude of completeness in a given catalog, it is of the utmost importance for statistical and comparative studies. Husen and Hardbeck [2010] summarize this, writing, "Statistical seismology strives to quantify, test, and understand the spatial-temporal behavior of earthquakes. However, the spatial-temporal properties of earthquakes cannot be studied directly; one must instead study the spatial-temporal properties of earthquake catalogs. The applicability of any particular statistical result to the real Earth depends in part on the extent to which the utilized catalog accurately represents the real earthquake properties. Therefore, when undertaking a statistical study using an earthquake catalog, it is vital to understand the original purpose and limitations of the catalog and how these limitations may affect the study's results."

Methods for the determination of Mc and its error analysis have been extensively studied (e.g. Woessner and Wiemer [2005]). Our current study focuses on what we can learn about swarm behavior using such a complete catalog (in an area with a high magnitude of completeness $\sim \geq 2.0$ ). This completeness may also be a factor in studies that consider aftershock probabilities and magnitude scaling laws such as Bath's law [Vere-Jones and Zhuang, 2008]. Completeness determinations used in this study are detailed briefly below. For a complete list and comparison of methods see Woessner and Wiemer [2005]. 
The Maximum Curvature method (MAXC) [Wiemer and Wyss, 2000] looks for the sharpest change in slope (maximum of the first derivative of a b-value plot). In a standard b-value plot the cumulative number of earthquakes with a magnitude $M$ and greater are plotted as a function of those magnitudes. We expect this cumulative number to form a linear trend, the slope of which is the b-value (Fig 1.2a). At some low magnitude, the cumulative number deviates from the linear trend and starts to roll or level off. The maximum curvature method identifies the maximum change in slope over this cumulative trend and identifies that as the magnitude of completeness [Rydelek and Sacks, 1989]. While this method can underestimate the Mc, Woessner and Wiemer [2005] show that the Mc estimate of maximum curvature is more stable with a decreasing number of data than other methods, and suggest that this method is suitable for small datasets.

The other Mc determination method used in our study is the Mc by b-value stability (MBS) method of Cao and Gao [2002], which takes advantage of the idea that the b-value should be constant over a range of magnitudes above the true completeness magnitude. The graphical method calculates a b-value using only $\mathrm{M} \geq$ $\mathrm{X}$ and the accompanying errors. These $\mathrm{b}$-values are plotted with error bars against the minimum magnitude used in each calculation (Fig 1.2b) and then a line is drawn from high to low magnitudes to determine how well lower magnitudes are consistent with those determined with large magnitudes alone. The point at which low magnitudes deviate (outside of errors) is determined to be the magnitude of completeness. Woessner and Wiemer [2005] show that this method gives consistently higher Mc than other methods, and while it is not as stable as other methods for small numbers of data, it is useful for regional catalogs. We have determined an Mc value of 2.45 for the CVR using the Cao and Gao [2002] method. Note that while this is significantly 
higher than the value using the maximum curvature method, it does correspond to a significant roll off in cumulative events (triangles in Fig. 1.2 A).
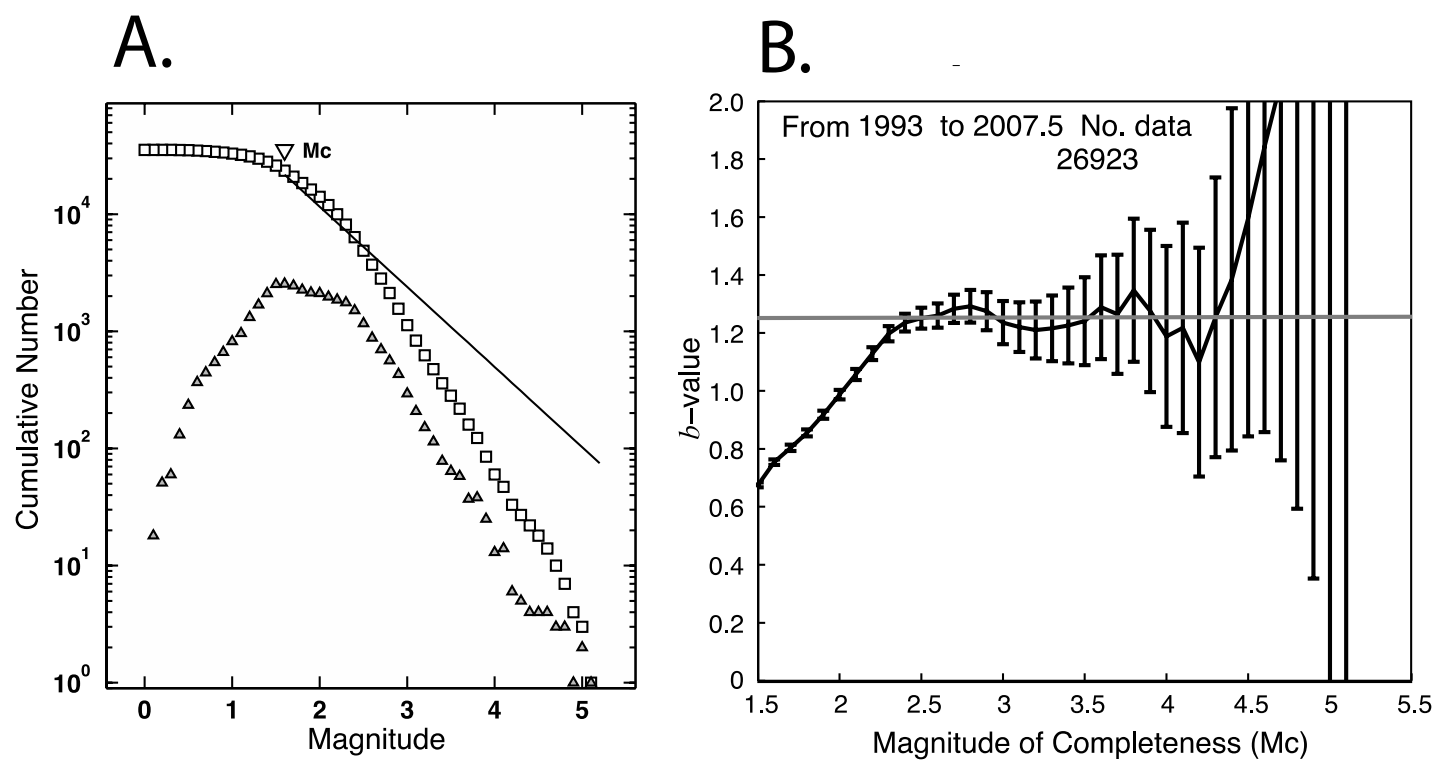

Figure 1.2 Two Magnitude of completeness determination methods.

A) Maximum Curvature determination with b-value; Wiemer and Wyss [2000]. B) Mc by b-value stability method of $\mathrm{Ca}$ and Gao [2002]. Both methods use 26923 earthquakes $\mathrm{M} \geq 1.45$ from the GeoNet earthquake catalog in the Central Volcanic Region (CVR) of New Zealand from 1993-2007.5.

\subsubsection{Background Rate}

After ensuring that we are working with a complete catalog we can then begin to assess the substance of the catalog. The rate of earthquakes observed worldwide is thought to be composed of two main parts, a rate of independent events (background) and a number of earthquakes that are causally linked to those in the independent part (e.g. [Ogata, 1988; Zhuang et al., 2002]). The background rate of an earthquake catalog is believed to follow a Poisson time pattern (with a known average rate and independent of the time since the last event). In fact clustering algorithms are often tested to check that the set of main events determined has a Poisson temporal behavior in time (e.g. [Gardner and Knopoff, 1974]). The other part of the catalog is temporally clustered from the causal relationship between specific earthquakes. We 
do not know precisely why any individual earthquake occurs and so it is impossible to determine absolutely which earthquakes belong to the background process and which are caused by other earthquakes in the catalog.

Thus, background is often taken to be the set of mainshocks that is determined from clustering and declustering algorithms. This assumption is also central to the Epidemic-type aftershock sequence (ETAS) model. The ETAS model is a point process model that consists of a background process $(\mu)$ plus a modified Omori law for temporal decay with a magnitude dependent abundance defined as,

$$
\kappa(M)=A e^{\alpha\left(M-M_{c}\right)}
$$

where $\alpha$ is the efficiency of an earthquake to produce aftershocks, $A$ is the productivity, and $\mathrm{Mc}$ is the cutoff magnitude of the earthquake catalog [Ogata, 1988]. The parameter $\mu$ is often referred to as the background rate but is more accurately the rate of independent events (after [Zhuang et al., 2002]); those that are not explained by preceding seismicity. This distinction becomes especially important in the case of earthquake swarms (section 2.4.5) because they represent a clustering of independent events that are not caused (as aftershocks) by preceding earthquakes, and so disrupt the presumed Poisson rate. Chapter 3 investigates how background rates change between regions and over time.

\subsubsection{Sequence Identification and declustering}

The main purpose of any declustering algorithm is to separate the independent and dependent parts of the earthquake catalog. Declustering can be performed either to analyze the background behavior of earthquakes, or to analyze the earthquake sequences (clusters). Chapter two details the most common clustering algorithm types: Window Method [Gardner and Knopoff, 1974], Reasenberg declustering 
[Reasenberg, 1985], Single-Link-Clustering [Frohlich and Davis, 1990], and Probabilistic methods [Zhuang et al., 2002].

\subsection{Central Volcanic Region (CVR)}

\subsubsection{Tectonic Setting}

The North Island of New Zealand is situated on the Australian plate with subduction of the Pacific plate occurring along the East Coast in the Hikurangi subduction zone. This subduction dominates the seismic and tectonic features we observe in the North Island. A volcanic arc is present, coincident with a large region of backarc spreading [Stern et al., 2006] known as the Central Volcanic Region (CVR). The eastern portion of this zone is delineated by the volcanically active region over the last $2 \mathrm{Ma}$ [Wilson et al., 1995]. This zone contains a majority of the present day seismic and volcanic activity and is referred to separately as the Taupo Volcanic Zone (TVZ).

There have been at least 34 caldera forming eruptions, the most active period of which began around 300,000 years ago [Houghton et al., 1995]. Wilson et al. [1995] noted that the CVR has a similar eruptive volume output to that observed at Yellowstone volcano (Wyoming, USA), but has a contrasting style with smaller single eruption volumes. They attribute this to the rapid extension rates (7-20 mm.yr) that make the CVR crust hotter and thinner than the Yellowstone hotspot [Wilson et al., 1995].

In conjunction with this volcanic activity the CVR is a region of high heat flow that is manifest with seventeen distinct geothermal systems with a total heat flow of 4200+500MW [Bibby et al., 1995]. The number of geothermal systems implies that they are persistent features for at least as long as 200,000 years. Most of this 
output is concentrated in the Eastern TVZ with an apparent anti-correlation of geothermal fields and surface fault traces [Bibby et al., 1995]. The heat flow is partially driven by the extension (between 5-20 mm/yr), however, conductive heating from the zone of shallow brittle-ductile transition can account for only up to $60 \%$ of the observed heat output, implying that mantle input (dike intrusions etc.) must account for the remainder [Bibby et al., 1995].

The fault belt associated with extension in the CVR is called the Taupo Fault Belt (TFB) [Villamor and Berryman, 2001]. It is densest in the central and northern regions (Figure 1.3). There are two proposals to explain the change from north to south. The first is that the opening of the CVR is the product of rotation that has the highest extension rates to the North and decreases gradually to the South [Beanland and Haines, 1998; Wallace et al., 2004]. Alternatively, based on the overall shape of the CVR and gravity mapping, Villamor and Berryman [2006] proposed a step like opening of the CVR with faults propagating southwards in steps associated with increases in rhyolitic volcanism. These two alternate models both predict similar seismicity on a decadal scale and we do not attempt to contribute towards selection of a better model with the present study. 


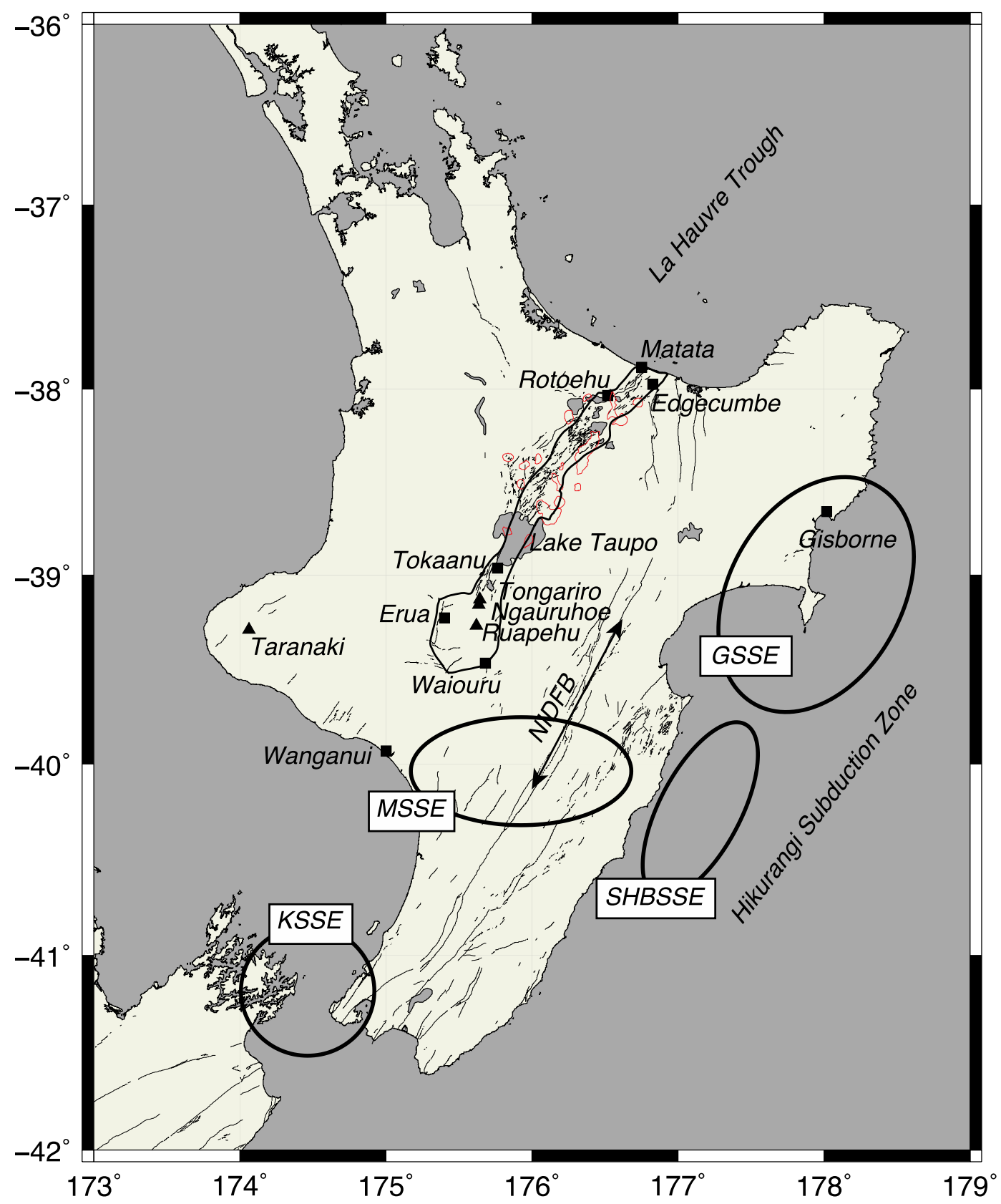

Figure 1.3 Map of the North Island of New Zealand with active faults. Active faults shown are from the Active Faults Database of New Zealand (http://www.gns.cri.nz/Home/Products/Database/Active-Faults-Database-of-NewZealand). Black squares show the locations of towns mentioned in the text, black triangles show the location of volcanoes, and red outlines show the polygons of geothermal areas. The modern Taupo Fault Belt (TFB) as defined by Villamor and Berryman [2006] is outlined in black, and the North Island Dextral Fault Belt (NIDFB) is also labeled. Ellipses show four dominant areas of slow-slip: Gisborne (GSSE), Manawatu (MSSE), Southern Hawke's Bay (SHBSSE), and Kapiti (KSSE). 


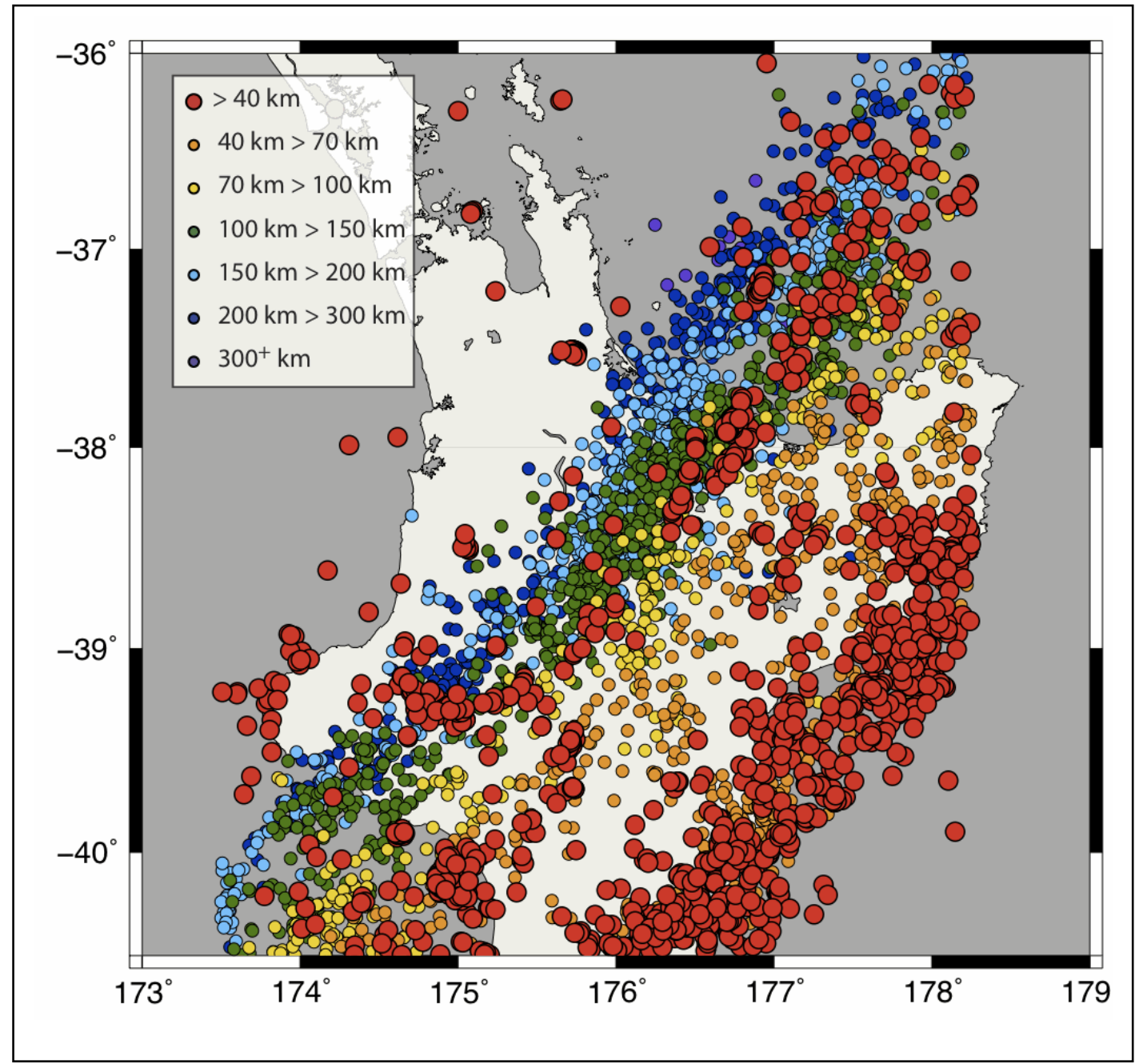

Figure 1.4 Map of earthquakes colored with depth.

Red are shallow earthquakes $(\leq 40 \mathrm{~km})$ depth) and progressive colors show depths up to $400 \mathrm{~km}$.

\subsubsection{Characteristics of Seismicity}

All of these tectonic and volcanic processes are manifest in the observed seismicity in the North Island. The main seismicity in the North Island is dominated by the plate boundary (Fig. 1.4). Shallow seismicity also occurs in the TFB, typical of backarc systems. 


\subsubsection{Sequence Characteristics}

There are many documented sequences in the North Island, especially in the TVZ [Bryan et al., 1999; Eiby, 1966; Gibowicz, 1973b; Hurst et al., 2008; Sherburn, 1992b; Smith and Webb, 1986]. Sherburn [1992b] was one of the first authors to make observations about earthquake sequences after the network in the CVR was improved. He concluded that despite the short duration of most sequences, their temporal distribution is complex. He also proposed that sequences in the CVR represent a continuum of behavior between MS-AS and swarms [Sherburn, 1992b].

Bryan et al. [1999] conducted analysis of seismicity recorded by a temporary deployment of 87 seismometers (23 broadband) in the first five-months of 1995 . They compared their results to the observations from the permanent seismic network and found that areas that appeared quiet in the years preceding the temporary deployment also appeared quiet during the deployment, suggesting true quiescence rather than detection or catalog completeness problems [Bryan et al., 1999].

Some of the earliest documented swarms occurred around Lake Taupo (caldera) [Eiby, 1966]. Activity was recorded around the area in 1922, were felt over a wide area and had reports of surface faulting [Eiby, 1966]. The location and size of the events are unknown although Smith and Webb [1986] proposed magnitudes around $5.5-6.0$ for the largest events. The first well documented swarm in the Lake Taupo area occurred on the northwestern edge of the lake over 59 days during 1964 and 1965 and included at least 1127 earthquakes $\geq$ M 2.7 [Gibowicz, 1973b]. In February 1983 a small swarm occurred in a similar location to the 1964-65 activity and another swarm later that year occurred beneath the lake itself [Webb et al., 1986]. A series of events that is a likely swarm also occurred with an $M=5.4$ earthquake in 1984, but work on that sequence has not been published. 1987 again saw the 
occurrence of swarm-like seismicity accompanied by $\mathrm{M} \sim 4$ earthquakes, this time to the southwest of Lake Taupo [Sherburn, 1992a]. These sequences and our understanding of their significance will be explored in section 4.5 , which investigates a recent 2009 earthquake swarm South of Lake Taupo, near the known TokaanuWaihi geothermal area (section 4.5). The historical record of earthquake swarms in this region gives us a good context in which to interpret the likelihood of magmatic/volcanic involvement in the 2009 activity.

The Matata area has experienced both frequent earthquake swarms and large earthquakes [Hurst et al., 2008; Richardson, 1989; Smith and Oppenheimer, 1989]. In 1977 an $\mathrm{M}_{\mathrm{L}}>5.4$ earthquake occurred that included more than 20 foreshocks and numerous $\mathrm{M}>4$ aftershocks [Richardson, 1989]. The foreshocks and aftershocks had distinct spatial clustering, a feature that has accompanied all subsequent aftershocks and swarms in the region [Hurst et al., 2008; Mouslopoulou and Hristopulos, 2011; Smith and Oppenheimer, 1989]. The $1987 \mathrm{M}_{\mathrm{L}}=6.3$ Edgecumbe earthquake was accompanied by a large foreshock sequence with around 130 events $>$ M 3.0 over 10 days that had an accelerating rate and migrated spatially towards the eventual mainshock [Smith and Oppenheimer, 1989]. This seemed to be possible evidence to support Evison's [Evison, 1982; Evison, 1977] precursory swarm hypothesis that swarms were signs of eventual mainshocks and served in some way to build the stress of a region. The testing of this hypothesis in New Zealand along with the precursory quarm [Evison and Rhoades, 1993; 1997; 1999b] has not been validated. Evison and Rhoades [1999a] later pointed out that this may only be true in a subset of tectonic settings (mainly shallow subduction) and the phenomena may manifest differently in other settings. Despite the negative result of their theory, the Matata area has been the subject of intense intermittent swarm activity from 2004-2007 [Hurst et al., 2008; 
Mouslopoulou and Hristopulos, 2011]. This swarm activity has not been as intense (in rate or energy) as the foreshock activity leading to the Edgecumbe earthquake, but does leave the question about whether swarms in this area may be a reflection of the loading process that builds before large earthquakes. A paper published by Mouslopoulou and Hristopulos [2011], with relocations by Stephen Bannister, show that the pattern of seismicity there is complex and does not give clear evidence for migration of stress or fluid in any recognizable way. There are likely to be more large earthquakes in the future and we will have to wait and see whether the activity mimics the cycle leading up to the Edgecumbe earthquake. Geological investigations, including trenching, immediately after the 1987 Edgcumbe earthquake exposed evidence of a previous rupture on the Edgcumbe Fault around 800 years B.P., narrowly preceding the deposition of the Kaharoa ash and a possible second event just above the Taupo Pumice Alluvium (c 1850 years B.P.) [Beanland et al., 1989]. Beanland et al. [1989] used this to suggests a recurrence time between 800 and 1000 years for rupture along the Edgecumbe Fault. This suggests that recently observed swarms will not lead to another rupture along the Edgecumbe Fault, but as there are many faults in the region a relationship to future larger earthquakes should not be ruled out.

The Matata 2005 swarm was one of three sequences studied by Hurst et al. [2008] in the northern TVZ. They conclude that the Rotoehu (2004) sequence may be due to fluid processes similar to the well-studied Vogtland-Boehmia swarm [Hainzl and Fischer, 2002a; Hainzl, 2004; Hainzl and Ogata, 2005; Hurst et al., 2008]. The Haroharo (1998) swarm appears to occur in a broader context of increased seismicity across the TVZ during that period and is discussed further in section 3.6 and 4.4.5. The diversity among these three earthquake swarms shows that like global 
observations, swarms in the TVZ vary widely in size, duration, and rate characteristics [Gibowicz, 1973b; Sherburn, 1992b]. Kanamori [1981] observed, "In general, seismicity patterns vary substantially from event to event, even though some of the fundamental physical processes leading to an earthquake may be common to all events." Identifying, describing, and quantifying the characteristics and differences of sequences is the key to understanding their physical mechanisms and their broader implications for hazards and tectonic processes; this lies at the heart of this study.

\subsubsection{Association with Volcanic Activity}

While seismicity is concentrated in the TFB and around caldera centers, andesitic volcanoes in New Zealand have not been observed to be a large source of seismicity [Bryan and Sherburn, 1999; Hurst and McGinty, 1999; Sherburn, 1992a]. Mount Ruapehu had its most recent magmatic eruption in 1995-1996 with an approximate eruptive volume of $0.05 \mathrm{~km}^{3}$ [Nakagawa et al., 1999]. While that eruption was accompanied by seismicity, it did not have detectable precursory seismicity and does not have a high background rate of events. Some activity was observed $\sim 20 \mathrm{~km}$ to the west of Ruapehu prior to the eruption, but its character was not distinctive enough to forecast impending activity [Hurst and McGinty, 1999]. In addition to magmatic eruptions, Mount Ruapehu also experiences phreatic eruptions from the crater lake that have no seismic signature and are referred to as "blue-sky" eruptions (e.g [Kilgour et al., 2010]). The most recent phreatic eruption occurred in 2007.

While little precursory seismicity has been observed directly under Mount Ruapehu, two nearby areas, Ruarimu Fault/Erua, and Waiouru, experience frequent earthquake activity. The Waiouru area was first noted for its frequent seismicity by Reyners [1980], and was studied in more detail by Hayes et al. [2004]. They found 
that the Waiouru area is not likely to be of volcanic origin itself, but did have increased activity following the Ruapehu 1995-1996 eruptions, showing a sensitivity to volcanic stresses [Hayes et al., 2004]. The Erua region is also thought to be a potential indicator of changes at Ruapehu, despite displaying somewhat typical MSAS behavior [Hurst and McGinty, 1999]. A recent study of seismicity around the phreatic eruptions in 2006 and 2007 found that shear-wave splitting and other parameters may have changed around the times of the phreatic eruptions [Keats et al., 2011]. These recent changes further support the Erua region reacting to volcanic stresses despite being $20 \mathrm{~km}$ west of Ruapehu.

For nearly 7 years, since 2005, Ngauruhoe volcano has exhibited repeating low frequency earthquakes [Jolly et al., 2012]. Despite the frequent association of low frequency events with fluid and magma movement, no eruption has occurred at Ngauruhoe during this time. The neighboring Tongariro volcano had a small eruption in August 2012 and had a minor, but noticeable, increase in seismicity for a few weeks prior to the eruption. Another small eruption occurred in November 2012, but no change or buildup in seismicity occurred prior to that eruption.

While we, thankfully, have yet to observe a major rhyolitic eruption in New Zealand, Sherburn and Nairn (2004) have explored the potential geophysical precursors that could precede such an event. In their five-year scenario Sherburn and Nairn [2004] anticipate being able to observe a seismic build up on the order of a few months prior to another caldera forming eruption. Sequences in the TVZ vary widely in size, and it is important to track them in order to be able to assess their relative position and strength through time. The prevalence of non-eruptive sequences heightens the importance of systematically cataloging sequences in order to be able to identify sequences and patterns in sequence occurrence as anomalous. These patterns 
may be associated with eruption cycles or tectonic processes that occur over longer time scales than the duration of individual sequences.

\subsubsection{Triggering}

\subsection{Large Earthquakes}

In addition to triggering by nearby phenomena such as volcanic and geothermal activity and other earthquakes, earthquakes may be triggered by large regional and global earthquakes. Triggering of small earthquakes became a widely accepted seismic phenomenon in the wake of widespread observations of triggering following the Landers 1992 earthquake [Hill et al., 1993]. Areas that experience such triggering do not experience it in every large global or large regional event. The factors believed to affect the likelihood of triggering include: directivity, stress or other current local state, distance, peak ground acceleration and velocity (PGA/PGV) (e.g. [Gomberg et al., 2001; Prejean et al., 2004]). Accordingly, any investigation into what initiates and drives earthquake sequences in the CVR, or anywhere, must consider the possibility of triggering by distant (global or regional) earthquake sources (Chapter 5).

Observations of earthquake triggering in New Zealand have not been published until recently (Boese [2012], and section 5.2). Triggered deep tremor was identified by Fry et al. [2011] after the Maule Chile $M=8.8$ earthquake. The size of typical triggered events is often $\mathrm{M}<1$ (e.g. [Prejean et al., 2004]). We conducted an investigation into New Zealand-wide triggering to determine whether it is possible to detect triggering with the present catalog completeness (Section 5.1). We also show the results of a study using a much lower Mc in the South Island of New Zealand with 
Boese looking at triggered earthquakes in a swarm prone area just east of the Alpine Fault after large regional earthquakes $\mathrm{M} \sim 7$ in the South Island (Section 5.2).

\subsection{Slow Slip}

Slow slip has now also been documented along the Hikurangi Margin and across the lower North Island since 2002 (e.g. [Douglas et al., 2005]), with some studies suggesting the possibility of its presence from at least 1994 (e.g. [Robinson, 2003]). New Zealand is uniquely situated relative to its subduction margin and so stations cover a much larger profile across the subduction margin than is possible in other locations, where the trench lies much further offshore. The major locations of slow slip observations are shown in figure 1.3. In the Hikurangi margin slow slip is not always accompanied by observable tremor, and instead may trigger small seismic events in the surrounding area [Delahaye et al., 2009; Ide, 2012]. This has not been studied systematically, but at least two studies have shown increased seismicity directly associated with slow slip [Delahaye et al., 2009; Wallace et al., 2012]. Two other sequences may also be associated with stress changes due to slow slip events [Reyners and Bannister, 2007; Robinson, 2003]. This accompanying seismic activity takes up a very small portion $(\sim 1 \%)$ of the total slip calculated for these slow-slip events [Reyners and Bannister, 2007; Wallace et al., 2012]. The accompanying seismicity could be due to stress changes, or the release of fluids [Llenos et al., 2009; Okutani and Ide, 2011; Reyners and Bannister, 2007; Wallace et al., 2012]. Reyners and Bannister [2007] suggest that systematic investigation of earthquake swarms may lead to better understanding of slow slip and the coupling on the plate interface. We believe that the CURATE method developed here is ideal for such studies. Possible triggering due to slow slip is explored in section 5.3. 


\subsubsection{Summary}

This rich dataset of earthquakes and sequences in the CVR is ideal to investigate our primary questions:

- How can we best identify sequences in an earthquake catalogue that contains both MS-AS and swarm types?

- What kind of sequences occur in the CVR, and how does this compare to other regions of NZ and sequences worldwide?

- What can complete catalogues at high Mc tell us about earthquake swarms and their timing?

- Are there patterns in sequence occurrence, and does this relate to broader tectonic and subduction processes?

- How much decay is observed in swarm sequences and can decay, where observed, be explained by Omori's law?

- Can statistics using complete catalogues identify potential triggering?

- Does triggering occur in the CVR? If so, what are its causes?

Answering these questions will lead to a better understanding of sequences in general and the hazards they pose, and the clues they hold about broader tectonic processes. 


\section{Cumulative Rate Analysis (CURATE): A Clustering Algorithm for Swarm Dominated Catalogs}

\subsection{Introduction}

This chapter contains a paper published in JGR that contains the bulk of our method development and initial testing [Jacobs et al., 2013]. It is reproduced as originally published.

\subsubsection{Earthquake Sequences}

Earthquakes often occur in groups, clustering in time and space. This temporal clustering is defined as an increase in rate above a given background rate, where the background is the typical rate of earthquakes observed in the area of interest. These clusters have often been divided into two categories: mainshock-aftershock sequences and earthquake swarms [Mogi, 1963; Scholz, 2002]. For this study we define an earthquake sequence to be any group of earthquakes with a rate above an average rate that are also related in space; this will include both categories. An increase in rate is the common element between both types of earthquake sequences. Classically a mainshock-aftershock sequence is one in which the largest magnitude event occurs first, or early in the sequence if there are foreshocks. According to Bath's law there is also an expected magnitude difference between the two largest shocks of 1.2 magnitude units [Bath, 1965]. However that value is an average and many authors have shown there is a wide range of observed magnitude differences for mainshockaftershock sequences globally [e.g. Felzer et al., 2002; Helmstetter and Sornette, 2003].

Recent work by Christophersen and Smith [2008] and Zhuang et al. [2008] confirms that foreshock sequences behave like mainshock-aftershock sequences which happen to have a smaller first event [Felzer et al., 2004]. We will thus include foreshock sequences when we refer to 'mainshock-aftershock' sequences. Equations 
and models (e.g. Omori's law [Utsu et al., 1995] and ETAS [Ogata, 1998]) can now be used to calculate the expected number of earthquakes (aftershocks) once a large mainshock occurs, and the physical cause of those aftershocks seems to be relatively clear [Scholz, 2002]. The same is not true for earthquake swarms in the sense that no parallel laws of abundance and decay exist for swarm sequences and there is no way to forecast them. There is not even a generally accepted quantitative definition of an earthquake swarm, although most authors would agree with a qualitative definition similar to Mogi’s, [1963], that earthquake swarms are groups of earthquakes which are closely clustered in time and space (though the duration may be years) and which have no predominant earthquake or 'mainshock'. Because the term "swarm" is used to classify sequences which simply have the absence of a distinct mainshock, they encompass a variety of spatial and temporal patterns. This implies that the largest earthquake is not the main physical trigger for the subsequent sequence and that magnitude dependent clustering techniques (e.g. [Gardner and Knopoff, 1974; Reasenberg, 1985]) may not be as effective in identifying swarm sequences.

Most authors who have studied earthquake sequences in volcanic regions note the variation in earthquake rate, duration, and magnitudes [Eiby, 1966; Gibowicz, 1973b; Hurst et al., 2008; McNutt, 2005; Sherburn, 1992b; Toda et al., 2002]. Benoit and McNutt [1996] report that swarm sequences have a tendency to be recorded with durations that are related to fixed periods of observation (e.g. daily, weekly). These observations emphasize the need for a general model to detect and quantify the observed behavior.

\subsubsection{Motivation for a New Method}

Clustering methods allow objective quantitative comparisons of sequences within the same catalog and between different geographic regions. The resulting 
sequence catalogs (catalogs of clusters identified by clustering methods) can be used to set a benchmark to measure the relative size of future sequences and thus lend themselves as aides to hazard assessment and physical interpretation. While the comparison of sequences within any one method is objective, because many methods exist to identify clusters and remove them from an earthquake catalog, the choice of which method to use is subjective (see vanStiphout et al. [2012] from the CORSSA project [Zechar et al., 2011] for a review of methods). Standard clustering techniques often focus on the removal of sequences rather than the sequences themselves and rely on aftershock behavior and relationships to previous seismicity for cluster assignment. These assumptions may not apply to regions or over magnitude ranges with swarm seismicity, for which the laws of temporal and spatial scaling are not known. Thus, despite the large number of available methods, standard clustering methods are not used in studies of swarm sequences with many authors instead defining arbitrary spatial and rate boundaries to define individual sequences [e.g. Vidale and Shearer, 2006]. Therefore, we have developed a new cumulative rate method, which we call "CURATE" to characterize sequences of earthquakes that could include both mainshock-aftershock and swarm sequences.

To demonstrate the differences between our method and other declustering and clustering methods we have applied several of the standard methods to our dataset. The three types of methods analyzed here are window methods [GardnerKnopoff, 1974], link based methods (Single-Link Clustering, [Frohlich and Davis, 1990]; and Reasenberg, [1985]) and Stochastic Declustering [Zhuang, 2002]. Summaries of the four specific methods are presented in section 4.

All four of the standard methods presented here assume a single process or suite of processes that depend on earthquake-earthquake interaction and are roughly 
governed by some combination of Omori's law time decay (aftershock) or a fault interaction zone between successive earthquakes. Single-link clustering makes the least physical assumptions, but still relies on the idea that earthquakes are directly caused by preceding seismicity.

Swarms are understood [Scholz, 2002] to be distinct from the decay processes of aftershock sequences. Brodsky [2006] identified some late triggered swarm earthquakes as aftershocks of events that occurred during the passage of surface waves. These triggered swarms were well fit by an Omori's law decay. It remains to be seen whether non-triggered swarm sequence decay, where observed, can be described by Omori's law and if so whether the parameters fall within the range of values observed in true aftershock sequences.

The CURATE method uses seismicity rate as the main link between earthquakes. Using rate allows us to eliminate the assumption that earthquakes close in time and space are caused by each other and allows us to make the more general statement that an increased rate of earthquakes is evidence that they likely share a common physical trigger. In some cases that physical trigger will be another earthquake, but in the case of many swarms it could be a different physical impetus that may be completely separate from the preceding seismicity (e.g. increased porefluid pressure over a region (e.g. [Hainzl, 2004]). Separating the definition of increased activity from a direct distance relationship between events will enable us to select and vary a distance parameter based on the region of interest and the anticipated scale of activity. It will also allow quantitative analysis of swarms with multiple bursts (potentially multiple inputs) and the variation of duration, number of events, and energy output between such sub-swarms. The method is easy to apply and can be used at a variety of temporal and spatial scales. We anticipate it will be most useful 
at regional (not global) and local scales in magnitude ranges that include some swarm activity (likely $\mathrm{M}_{\max }<6.5$ ).

\subsection{Sequence Selection}

\subsubsection{Introduction to Sequence Selection}

In order to use any clustering technique, including the CURATE method, it is first necessary to start with a complete earthquake catalog (i.e. any catalog where the lower magnitude limit $\left[M_{\text {cut }}\right]$ is greater than or equal to the magnitude of completeness, $M_{c}$, [e.g. Wiemer and Wyss, 2000]). Unlike typical clustering methods the CURATE method starts by placing a portion of the earthquake catalog (that with rates below the mean rate) into an initial declustered catalog. We essentially make a subset of the catalog that contains earthquakes that occur at a rate above a certain rate threshold and search for spatial links between those earthquakes. Most earthquakes occurring above this rate will not be background earthquakes and so the probability of including background earthquakes does not increase dramatically when the spatial search window is extended marginally. The remaining steps of the method assign all earthquakes occurring at rates above the mean rate into individual clusters and may place additional earthquakes into the declustered catalog. Figure 1 shows a detailed flow chart through the steps of the method that are described in the following sections. The free parameters of the method are the spatial and temporal boundaries that define the earthquake catalog (this defines the mean earthquake rate), a distance search rule (which limits the area size of potential sequences), and a time window (used to allow for lulls in activity above $M_{c}$ where there may be ongoing activity at a magnitude below $M_{c}$ ). 


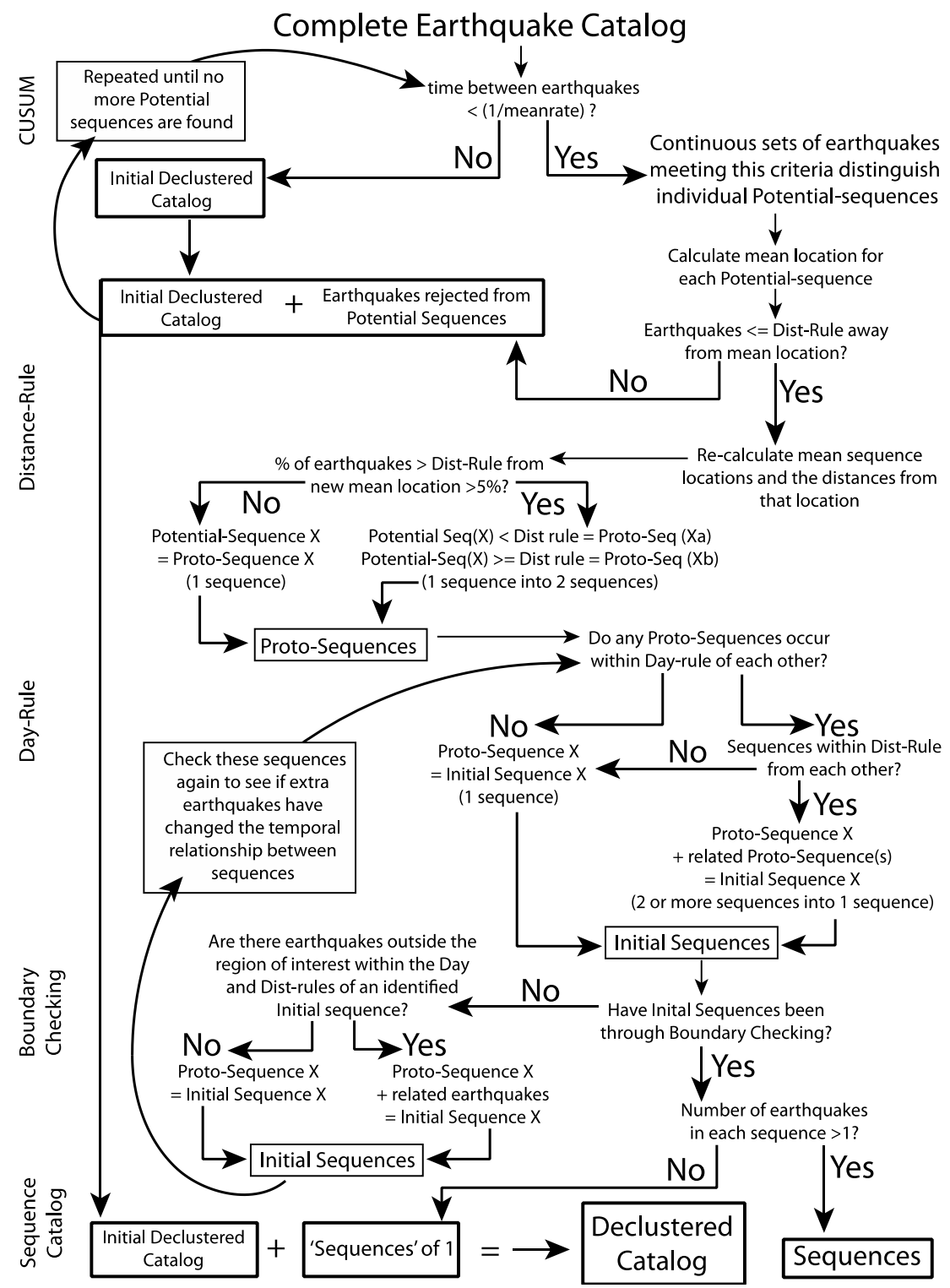

Figure 2.1 Flow chart outlining the steps the CURATE method uses to identify sequences (and an accompanying declustered catalog.

Curved paths represent steps where processed data is rerun through an earlier step one or more times. 


\subsubsection{Step 1: CURATE}

The first step we take to identify earthquake sequences is to check for a temporal relationship between earthquakes. We use an application of the CUSUM [Page, 1954; Tam, 2009] method to characterize the rate. At the time of each earthquake in the catalog, the CUSUM uses the average daily number of earthquakes for the entire period to estimate the expected cumulative number of earthquakes from the beginning of the catalog up to that point in time. The expected cumulative total is subtracted from the real (observed) cumulative total for each earthquake in the catalog (see figure 2.2). This produces a comparison to the average rate that is analogous to a reduced travel-time curve. CUSUM methods are often used to detect subtle changes in rate from a background rate that also has fluctuations [Page, 1954; Tam, 2009]. The average or mean rate used in this method will always be higher than the true background rate because it is calculated from the raw earthquake catalog that includes sequences. The resulting sequences produced by this method contain earthquakes that occur at the highest rates and are, we propose, most likely to related. The use of the mean makes the CURATE method more sensitive to the actual temporal distribution of earthquakes than methods that only consider the individual times between earthquakes.

Equations 2.1 and 2.2 describe the CUSUM calculation carried out for each day in the catalog, where $D a$ is the daily average number of earthquakes, $t_{\mathrm{s}}$ is the time of the first earthquake in the catalog, $t_{i}$ is the time (in decimal days from the beginning of the catalog) of an earthquake in the catalog, $t_{\mathrm{f}}$ is the time of the last earthquake in the catalog, and $E o b_{t}$ is the number of earthquakes observed between time $t_{\mathrm{s}}$ and $t$.

$$
D a=E o b_{t_{f}} /\left(t_{f}-t_{s}\right)
$$




$$
\text { CURATE }=E_{\text {Eob }}-D a\left(t_{i}-t_{s}\right) \quad t_{s} \leq t_{i} \leq t_{f}
$$

This calculation identifies all earthquakes for which the time from the previous earthquake is less than $1 /$ (mean rate), i.e., upward slopes in Fig. 2.2. These events are marked as parts of potential sequences with individual sequences being defined by continuous periods of increase. These periods of increased activity generally look like vertical lines on the graph because of the short time span (hours to days) over which they occur. This approach identifies all types of sequences. If desired, mainshock sequences can be differentiated by other criteria later. This first step creates a list of temporally related earthquakes which we refer to as "potential sequences."

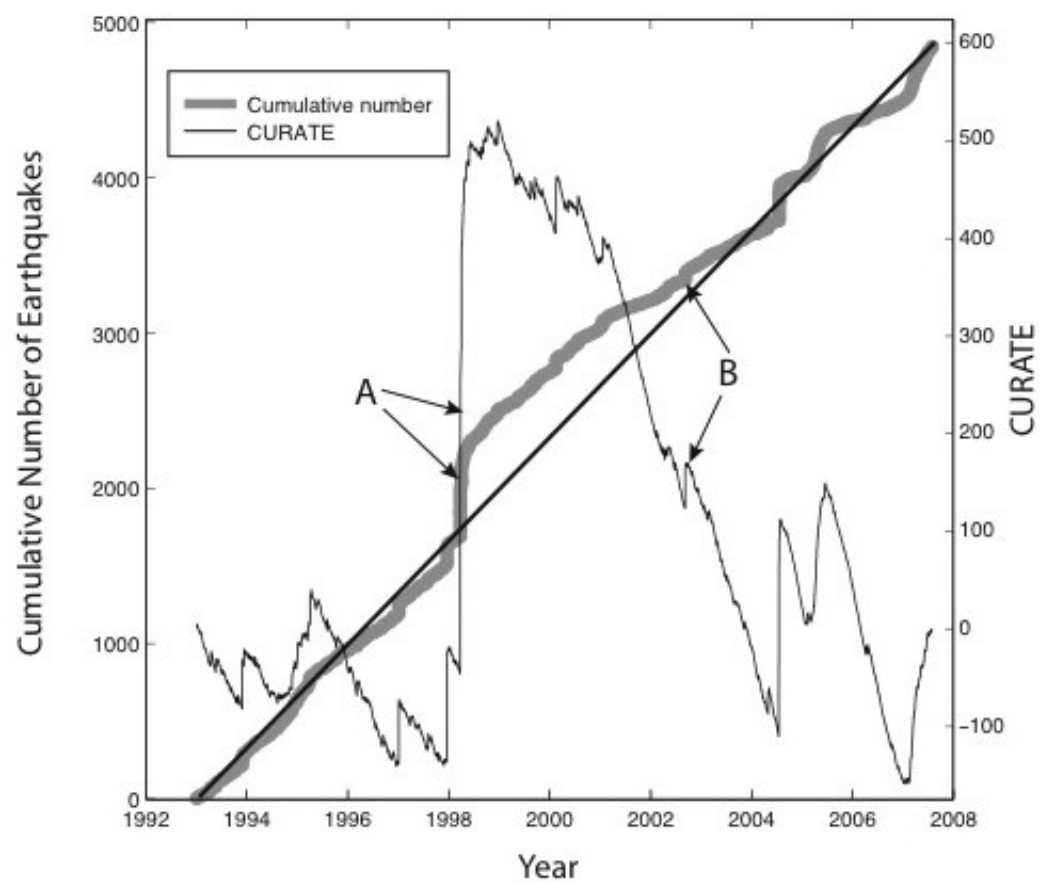

Figure 2.2 Plots of the CURATE and cumulative number of earthquakes with time from 1993 through 2007.5.

Any upward movement (Positive slope, but not necessarily greater than zero value) is an indication of above average seismicity rate. For example, A) points to the upward line related to the 1998 Haroharo earthquake swarm (380 earthquakes) and B) points out the upward line related to a mainshock-aftershock sequence of a magnitude 4.85 earthquake in 2002 ( 50 earthquakes). The bold black line shows a mean cumulative rate trend for reference. 


\subsubsection{Step 2: Distance Rule}

Once potential sequences have been identified we apply a distance rule to check that these groups of earthquakes are spatially related. This step is akin to the distance element of a windowing method but it will not sweep in as much seismicity because the time element is not a window, and part of the seismicity (i.e., the seismicity on days of low rate) has already been eliminated from inclusion in the spatial window. The distance rule is chosen by the user, and essentially functions as the scale on which we expect, or wish to search for, possible physical mechanisms underlying the sequences. The initial CURATE calculation will select all earthquakes in our study area during periods of elevated activity regardless of the geographic location. Thus the potential sequences may include distant earthquakes that are part of the background activity away from the sequence location, or simultaneous sequences occurring in separate locations. To ensure earthquakes in each sequence are spatially related we first calculate a mean location for each potential sequence, and the distance of each earthquake from the mean location. If the distance from the mean is greater than the distance-rule, the earthquake is eliminated from the sequence.

After events have undergone the distance selection, the mean sequence location is recalculated with the remaining earthquakes. Due to this re-calculation it is possible that some sequences will have earthquakes which are now at distances greater than the distance-rule from the mean location (although all remaining earthquakes in the sequence will be within the distance-rule of the original mean location). To ensure that this step does not create a 'cookie-cutter' effect and include only parts of a secondary sequence that may fall along the search boundary, any sequence which has more than 5 percent of the re-calculated distances greater than the distance rule is split into two sequences, with earthquakes less than the distance-rule 
forming one sequence and earthquakes above that limit in another. This creates an upper limit $95 \%$ on our distance-rule and eliminates some ambiguity on the spread of distances between earthquakes in a sequence. These two sequences are not rechecked against the distance rule because they already belong to a subset of events within the distance rule of some point.

To account for cases where a smaller, distant sequence is occurring simultaneously with a large sequence (that dominates the mean sequence location), earthquakes that are initially rejected from a potential sequence are returned to the catalog of earthquakes that have not yet been assigned to any sequence. This catalog subset is then searched again for earthquakes related in time (using the previously calculated value of $D a$ from equation 1) iteratively, including application of the distance rule to any found sequences, until no more sequences are found among the rejected earthquakes. Groups of earthquakes that are produced after the application of the distance-rule are called "proto-sequences." Earthquakes not in a proto-sequence are considered to be the background seismicity and are equivalent to a declustered catalog (see section 4.4 for a comparison of this background seismicity to that from standard declustering algorithms).

\subsubsection{Step 3: Day Rule}

The third step of the CURATE method, which combines related protosequences, is akin to a link based system in that it allows for expansion of the sequence spatial dimensions outside of the initial circular search area through the concatenation of sequences. To determine the expected cumulative number of earthquakes at a particular time, the CURATE method multiplies the time (in decimal days) of the catalog by the daily average number of earthquakes. So to be selected as 
part of a cluster the inter-event time between earthquakes must be less than $1 /($ mean rate). This may produce sequences that are in the same location whose start and end dates are just a day or two apart. Depending on $M_{\text {cut }}$ (chosen minimum magnitude of the catalog $\geq M_{c}$ ) this may or may not reflect separate causal processes. To address this potential problem, we have introduced an allowance of a certain number of days between sequences or a 'day rule'. If activity continues in a similar location (defined by the distance-rule) within the time defined by the day rule, then the activity is assumed to be related. If no other proto-sequences exist within the day and distance rules the sequence will remain unchanged. Sequences that are found to be related are then concatenated into a single sequence with new parameters (duration/mean location/etc). This concatenation can expand the total sequence area from the original distance-rule limits. Note that this step does not re-introduce background earthquakes which were identified in step 2 (earthquakes which do not exceed the CURATE). It simply concatenates existing proto-sequences. The products of Step 3 are our "Initial Sequences."

\subsubsection{Step 4: Boundary Checking}

The final step is designed to ensure that sequences in our catalog are not arbitrarily truncated at the catalog border. This is necessary for our current study because the northern (offshore) boundary of the CVR is not defined tectonically or by a natural decrease of activity, but instead by our ability to measure the activity. Due to the arbitrary nature of that boundary we do a search for earthquakes (not new sequences) beyond the border that are related by the distance and day rules to an existing initial sequence. Because earthquakes may be added which change the initial sequence start and end, the results are run through step 3 once more to check for 
related sequences. If any sequences of only one event are identified during the method they are added to the declustered catalog and any sequence of two or more earthquakes make up our final sequence catalog, and groups of earthquakes that have been through this final step are simply called "sequences." Step 4 may be unnecessary in studies where the boundaries are drawn solely on the basis of seismic activity, but should be performed in area where boundaries are based on arbitrary parameters related to network or catalog variability. See Wang et al. [2010a] for more about the importance of boundary testing in clustering methods.

\subsection{Application of the Method: Central Volcanic Region, New Zealand}

\subsubsection{The Setting of the CVR, New Zealand}

As an example application we use seismicity in the Central Volcanic Region (CVR) in the North Island, New Zealand. The CVR is interpreted as a backarc extensional basin that represents a continuation of the southern end of the Lau-Havre trough [Stern et al., 2006; Wallace et al., 2004]. The Hikurangi trough lies off the east coast of the North Island where the Pacific plate subducts beneath the Australian plate. The eastern portion of the CVR has high heat flow and volcanism [Bibby et al., 1995; Wilson et al., 1995], and is often referred to separately as the Taupo Volcanic Zone (TVZ). Most of the earthquakes observed in the CVR occur in the TVZ. We chose this catalog because it is an active swarm region and swarms there have been observed and documented as early as 1922 [Bryan et al., 1999; Eiby, 1966; Garrick and Gibowicz, 1983; Gibowicz, 1973a; Hayes et al., 2004; Hurst and McGinty, 1999; Hurst et al., 2008; Sherburn, 1992b]. While the chosen region is dominated by swarms, we note it also has experienced large mainshock-aftershock sequences, of which the largest event recorded in the last fifty years was the 
Edgecumbe 1987, $\mathrm{M}=6.3$ [Smith and Oppenheimer, 1989]. Many other regions worldwide also experience some degree of swarm activity in addition to mainshockaftershock activity [Scholz, 2002; Vidale and Shearer, 2006].

\subsubsection{Earthquake Data and Completeness}

Our earthquake data come from the New Zealand GeoNet catalog of located earthquakes from 1993 through July 2007 within a triangular boundary around the Central Volcanic Region, CVR (vertices: -39.7 175.25; -37.65 178;-37.65 175.25) and a depth of $40 \mathrm{~km}$ or less (Fig. 2.3). The northern boundary, unlike the other boundaries, has been defined on the basis of completeness and not as a delineation of change in earthquake activity. A spatial test of Mc shows that north of -37.25 values of $\mathrm{Mc}$ increase to $\mathrm{Mc}=3.0$ and continue to increase northward (offshore) due to a land based seismic station network. The stochastic reconstruction presented in section 4.5 requires data outside the target region to establish the background rate and history of the catalog so we chose a northern limit lower by $0.4 \mathrm{~S}$ to accommodate the need for completeness beyond the boundaries of the polygon. Using the method of Cao and Gao, which uses successive magnitudes to determine a stable $b$-value and establish Mc, with a northern boundary of -37.65 (Fig. 2.4) we find a magnitude of completeness (Mc) of 2.45. Mc has improved (decreased) with time in general and is also lower in some limited geographic regions, but we have chosen to work with a single Mc to make comparisons in time and space. 


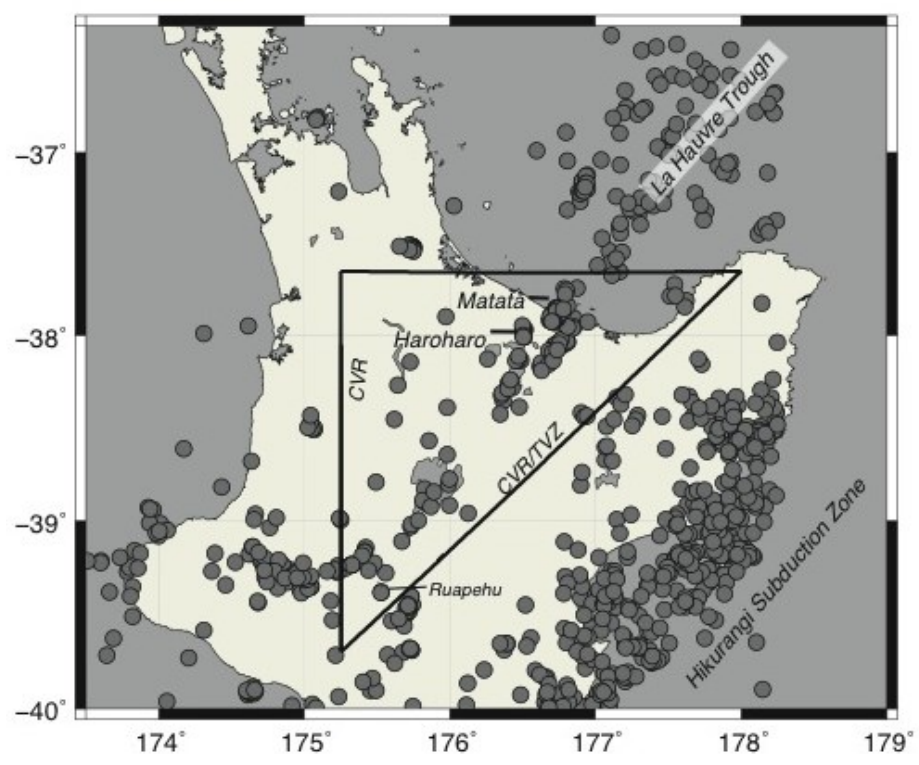

Figure 2.3 Location map of the Central Volcanic Region with GeoNet data for all shallow $(<40 \mathrm{~km})$ seismicity $\mathrm{M} \geq 2.45$ from 2005 .

Note that Southeast of the CVR/TVZ is an area of shallow subduction related earthquakes.

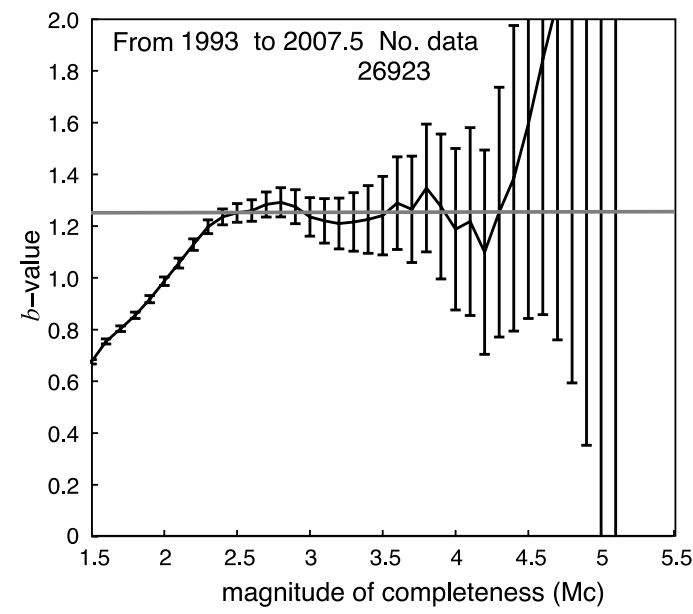

Figure 2.4 Plot used to determine the magnitude of completeness in the method of Cao and Gao 「20021.

The horizontal line is shown to help trace the stable range of $M c$ and the corresponding b-value (1.24). 


\subsubsection{Sequence Catalog}

Here we present the results of a single sequence catalog using a minimum number of events in a sequence $\left(\mathrm{N}_{\min }\right)=4$, distance-rule $=20 \mathrm{~km}$, and a day-rule $=3.5$ days. The CURATE plot for this catalog is shown in figure 2.2. Like all clustering algorithms, the CURATE method identifies clusters as small as two earthquakes; however the smallest clusters in any method are most likely to be affected by selection rules specific to the individual method. We chose to reduce the possible bias of small clusters in the sequence parameters by using a larger value $\left(\mathrm{N}_{\min }=4\right)$ for the sequence analysis. These parameters have been chosen on the basis of previously described swarm activity in the CVR. Large swarms have been documented by Hurst et al [2008], Sherburn [1992] and others. We leave the distance rule relatively large to encompass such activity and to see if any precursory activity is seen on these scales.

Using these parameters the CURATE method defines 163 sequences in the CVR comprising 2583 earthquakes (out of 4845), with individual sequences containing between $\mathrm{N}_{\min }=4$ and 380 events (fig. 2.5). Table 2.1 shows the number of sequences affected by each processing step. Only six earthquakes are added in the final processing step (combination of two sequences of three earthquakes.) Table 2.2 gives the range of changes made to individual sequences in each of the later steps of the method. We exclude steps one and two from the table as earthquakes kept and rejected at these stages depend more on the earthquake catalog itself than the method. Note that we do not allow sequences to exist entirely above the northern boundary so the Northern boundary step cannot add sequences, and very few sequences are affected by the final boundary check. The mean, median, and standard deviation of Table 2 step $4 \mathrm{~b}$ are the same, indicating that just 2 sequences have been joined 
together by the final application of the Day-rule. The median time added to the sequence duration is less than half of the Day-Rule (1.2 vs. 3.5 days), which suggests that the selection parameter is not at a critical value: it is not being used to its maximum extent and is therefore reasonable. While the median number of earthquakes added is small, the maximum is large. This likely indicates that a large sequence was continuing with events below $M_{c}$ (as suggested by the change in sequence size in Table 2.1). Table 2.3 gives the sequence statistics including the number of earthquakes, duration, $\mathrm{M}_{\max }$ (maximum magnitude), and area (an elliptical area enclosing all earthquakes in the sequence). The values indicate that the majority of identified sequences are small and over quickly. The low mean and median values for the area (relative to the possible area of the search radius $\sim 1256 \mathrm{~km}^{2}$ ) indicate that the large search radius for sequence earthquakes is not inflating sequence areas (Table 2.3). The three largest sequences in the catalog have all been identified by previous authors as likely swarm sequences [Hurst et al., 2008].

Table 2-1 Comparison of CURATE Algorithm Steps Number of Sequences and Earthquakes for Different Sequence Selection Steps

\begin{tabular}{lrrr}
\hline & proto sequences & initial sequences & sequences \\
\# sequences & 177 & 163 & 163 \\
\# earthquakes & 2373 & 2577 & 2583 \\
Max \# eqs in a sequence & 336 & 380 & 380 \\
\# seqs above 10 & 47 & 43 & 43 \\
\#seqs above 100 & 3 & 6 & 6 \\
\hline
\end{tabular}




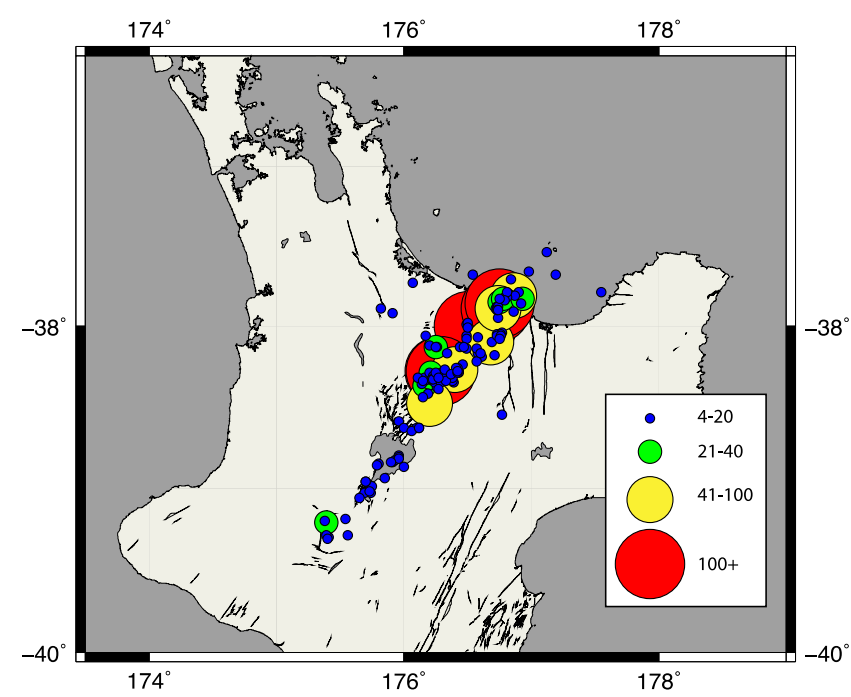

Figure 2.5 Sequence abundance and distribution. All sequences are plotted at their mean locations and scaled and colored by the number of earthquakes they contain.

Distances rules from 10-50 km and day rules from 3-7 were also tested. The largest increase in the number of earthquakes included in sequences between any two distance rules occurred between 10 and $20 \mathrm{~km}$ (Table 2.4). The $10 \mathrm{~km}$ rule was too small to encompass all activity. The $20 \mathrm{~km}$ rule appeared to be the smallest distance rule capable of describing sequences in ways that matched published descriptions of that activity. We do not attempt to prove a "best" set of parameters. The sequence catalogs are not sensitive to the day and distance rule parameters (for further discussion see section 5.2). We will show in section 5.1 that we can reproduce foreshock probabilities previously calculated for this region. This gives us further confidence that the parameters presented here are reasonable. 
Table 2-2 Changes made by different processing steps

\begin{tabular}{rrrr}
\hline & Day-Rule & $\begin{array}{c}\text { Northern } \\
\text { Boundary }\end{array}$ & North Day-Rule \\
\hline \#seqs added & & & \\
\hline min & 2 & 0 & 2 \\
max & 7 & 0 & 2 \\
mean & 2.47 & 0 & 2.00 \\
std & 1.05 & 0 & 0.00 \\
median & 2 & 0 & 2 \\
\hline \#eqs added & & & 3 \\
min & 1 & 3 & 3 \\
max & 87 & 3 & 3.00 \\
mean & 7.78 & 3.00 & 0.00 \\
std & 16.03 & 0.00 & 3 \\
median & 2 & 3 & 3.16 \\
\hline $\mathbf{t}$ (days) & \multicolumn{3}{c}{} \\
\hline min & 1.1791531 & 0 & 3.16 \\
max & 41.35 & 0.00 & 3.16 \\
mean & 5.45 & 0.00 & 0.00 \\
std & 7.48 & 0.00 & 3.16 \\
\hline median & 1.1791531 & 0 & \\
\hline$\Delta$ Moment & & & $1.35 \mathrm{E}+14$ \\
min & $1.89 \mathrm{E}+13$ & $1.40 \mathrm{E}+15$ & $1.35 \mathrm{E}+14$ \\
max & $3.92 \mathrm{E}+16$ & $1.40 \mathrm{E}+15$ & $1.35 \mathrm{E}+14$ \\
mean & $2.42 \mathrm{E}+15$ & $1.40 \mathrm{E}+15$ & $0.00 \mathrm{E}+00$ \\
std & $7.16 \mathrm{E}+15$ & $0.00 \mathrm{E}+00$ & $1.35 \mathrm{E}+14$ \\
\hline median & $1.89 \mathrm{E}+13$ & 0 & \\
\hline & & &
\end{tabular}

Table 2-3 Statistics of Key Sequence Parameters

\begin{tabular}{|c|c|c|c|c|c|}
\hline & $\min$ & $\max$ & mean & $\begin{array}{l}\text { Standard } \\
\text { deviation }\end{array}$ & median \\
\hline Duration(days) & $<1$ & 56.95 & 3.46 & 7.22 & 0.00 \\
\hline \# Earthquakes & 4 & 380 & 16 & 39 & 4 \\
\hline $\mathrm{M}_{\max }$ & 2.53 & 5.11 & 3.28 & 0.52 & 2.76 \\
\hline $\operatorname{Area}\left(\mathrm{km}^{2}\right)$ & $<1$ & 930.87 & 94.62 & 142.72 & 34.24 \\
\hline
\end{tabular}


Table 2-4 Number of earthquakes in Catalogs with Varying Day and Distance Rules. Values in Bold Text Correspond to Catalogs Displayed in Figure 6 and Listed in Table 5.

\begin{tabular}{|c|c|c|c|c|c|c|}
\hline Days & 3 & 3.5 & 4 & 5 & 7 & $\mathrm{~km}+/ \mathrm{eq}^{+}$ \\
\hline \multicolumn{7}{|c|}{ Radius(km) } \\
\hline 10 & 2364 & 2380 & 2396 & 2433 & 2497 & \\
\hline 20 & 2566 & 2583 & 2608 & 2664 & 2765 & 4.27 \\
\hline 30 & 2711 & 2754 & 2798 & 2874 & 2998 & 8.5 \\
\hline 40 & 2866 & 2914 & 2971 & 3085 & 3194 & 12.45 \\
\hline 50 & 3050 & 3129 & 3201 & 3904 & 4040 & 9.52 \\
\hline
\end{tabular}

\subsection{Comparison With Other Methods}

\subsubsection{Link-Based Methods}

The single-link cluster (SLC) analysis of Frohlich and Davis [1990] was run using MATLAB scripts we generated. The SLC method defines a single space-time parameter as: $d_{\mathrm{ST}}=\sqrt{\left(d^{2}+C^{2} \tau^{2}\right)}$, where $\tau$ is the time between each pair of earthquakes, $d$ is the corresponding distance between those earthquakes, and $C$ (often given a value of $1 \mathrm{~km} /$ day) is a constant to convert the time to an equivalent space/time distance. This single space-time distance $\left(d_{\mathrm{ST}}\right)$ is used to link earthquakes. Thus earthquakes closer in time can be further apart in space for the same $d_{\mathrm{ST}}$.

A value of $d_{\mathrm{ST}}$ is calculated for each earthquake pair in the catalog and each earthquake is then linked to the earthquake with the minimum $d_{\mathrm{ST}}$ value. This creates a set of trees. These trees are then linked by the shortest distance between any two trees until all earthquakes in the catalog are linked. Then, a limiting value $D_{\mathrm{ST}}$ is chosen and links greater than this value are severed to create distinct clusters. Davis and Frohlich [1991] give an equation for determining the best $D_{\mathrm{ST}}$ value to use to make clusters. The equation depends on the median link length of the catalog when all earthquakes are joined, and can be applied for median link lengths between 8 and 
$300 \mathrm{~km}$. The close temporal proximity of lower magnitude earthquakes dramatically lowers the median link length even at high $C$ values. The calculated median link lengths for the CVR catalog at $M_{\mathrm{c}}=2.45$ were 2.07-4.89 for $C$ values between 0.0510; these are well below $8 \mathrm{~km}$. This is likely to create a generic problem with interpreting results for local catalogs or at low cutoff magnitudes. Davis and Frohlich [1991] suggest that smaller $D_{\mathrm{ST}}$ values should be used in regions with higher background rates. This means that the maximum separation between clustered events is partially dependent on the background rate of seismicity. Because the temporal and spatial distances are treated equally, their errors are also treated equally. For catalogs with low $M_{\mathrm{c}}$, the value of $D_{\mathrm{ST}}$ becomes very close to the location errors, hence some of the decisions of whether to link earthquakes will be influenced by location error and will not be consistent if different times or regions of the catalog have different location errors (this is a separate issue from variations in $M_{\mathrm{c}}$ ). Note that variation of the $D_{\mathrm{ST}}$ and $C$ parameters can create a declustered catalog of almost any size. We present results at three different $C / D_{\mathrm{ST}}$ combinations to show the variation in the duration, and the size of clusters with parameter choice. A discussion on the effect of these parameter choices is presented in section 5.2.

The other common link-based method is Reasenberg [1985], which we implemented using ZMAP codes by A. Allmann [Wiemer, 2001]. The Reasenberg method defines a maximum space and time "interaction zone" to look for earthquakes which may be related to each earthquake in the catalog. This distinguishes it from the SLC method because one earthquake can create multiple links to other earthquakes. Both the spatial and temporal zones assume typical mainshock-aftershock fault behavior. The time of the interaction zone is based on the maximum time (according to an expectation value derived from Omori's law decay) to observe the next 
earthquake in the sequence. For simplification this time takes on a maximum value of one day for earthquakes not already in a cluster and a maximum of ten days for events already associated with a cluster. The spatial interaction zone is based on Kanamori and Anderson [1975] fault source dimension model multiplied by a scaling factor 'Q' (generally equal to 10). Like the SLC method, this distance assumption limits the area of the defined sequence by assuming each earthquake is triggered by another earthquake in the catalog.

\subsubsection{Window Methods}

The Gardner and Knopoff [1974] method is a forward looking window-based technique that focuses on the creation of the declustered catalog. The method sets magnitude dependent space-time windows within which to remove earthquakes from the catalogs as clusters. We use the normal Gardner-Knopoff windows, which are conservative and err on the side of removing more earthquakes from the catalog with e.g. (31 km/ 22 days) for $M=3.5$, and (100 km/ 790 days) for $M=6.5$. We ran the Gardner-Knopoff [1974] method using ZMAP codes by J. Woessner [Wiemer, 2001]. Gardner and Knopoff windows specifically for New Zealand were proposed by Savage and Rupp [2000]. Their windows are systematically larger (more conservative) than the normal parameters and we chose to have smaller clusters by using the smaller, original windows. Thus the results presented here will return a larger declustered catalog. The forward looking nature of the algorithm separates some clear mainshock-aftershock sequences into two sequences due to other earthquakes occurring beforehand. In such cases the clusters themselves are not physically meaningful (arbitrary separation of a single sequence), but the desired declustered outcome is unchanged. The windowing technique also takes out large 
amounts of seismicity due to the time window involved. As we show below with stochastic reconstructions of the catalog, the decay time is probably largely overestimated for swarm activity (Section 4.5).

\subsubsection{Probabilistic Method}

The final method we use for comparison is the stochastic declustering technique of Zhuang et al. [2002]. Stochastic declustering assigns a probability that each earthquake is a background event or an offspring of a previous earthquake in the catalog. Earthquakes are then put into clusters based on these probabilities. The method estimates the background intensity $[\mu(x, y)]$ by first estimating the total spatial intensity (variable kernel method) and modeling the branching structure (assigned using modeled epidemic-type aftershock sequence [ETAS] model parameters for the specific earthquake catalog to be analyzed as inputs into a productivity function with Omori's law temporal decay). The ETAS model is a point process model which consists of a background process plus a modified Omori law for temporal decay with a magnitude dependent abundance defined as

$$
\kappa(M)=A e^{\alpha\left(M-M_{c}\right)}
$$

Where $\alpha$ is the efficiency of an earthquake to produce aftershocks, $A$ is the productivity, and $M_{\mathrm{c}}$ is the cutoff magnitude of the earthquake catalog [Ogata, 1998].

That is to say, each time an event, namely $\left(t_{i}, x_{i}, y_{i}, M_{i}\right)$, occurs, it triggers a nonstationary Poisson process with a rate $\kappa\left(M_{i}\right) g\left(t-t_{i}\right) f\left(x-x_{i}\right)$, where $g$ represents a probability density function (p.d.f.) corresponding to the Omori law, and $f$ is the p.d.f. for the locations of the direct triggered events. Here we refer to Zhuang et al. [2008] for details. The ETAS parameters modeled for the CVR $(\alpha=0.74, A=0.66)$ 
are relatively low for productivity values, but are consistent with previous models which included the CVR [Zhuang et al., 2002]. However, this CVR $\alpha$-value is much less than the $\alpha$-value for the whole New Zealand region in Zhuang et al. [2008], which is 1.92 for all shallow events (depth $<40 \mathrm{~km}$ ) for M4.3+ and 1.75 of M4.0+, implying that the difference in triggering productivity between events of different magnitudes in CVR is not as significant as in other parts of NZ. Other possible reasons for the low $\alpha$ value are discussed in Section 2.4.5.

The probability that an event belongs to the class of triggered events can be estimated as the proportion of the contribution of the triggering by all the previous events to the total occurrence rate. Once probabilities of being an offspring event $\left(\mathrm{p}_{j}\right)$ have been constructed for each earthquake, background earthquakes can be (nonuniquely) identified from offspring events stochastically by generating a set of uniform random numbers $U$ within the set $\left[\begin{array}{ll}0 & 1\end{array}\right]$ and assigning each earthquake $(j)$ as background if $\mathrm{U}_{\mathrm{j}}<1-\mathrm{p}_{j}$, and all others as offspring. Similarly, to create specific clusters, the ancestor for any offspring events can be found by taking the earliest earthquake (smallest value of $I$ ) such that $\mathrm{U}_{\mathrm{j}}<\left(1-\mathrm{p}_{j}\right)+\sum_{i=1}^{I} p_{i, j}$ where the probability that $j$ is an offspring of event $i, \mathrm{p}_{i, j}$, is dependent on the magnitude, time, and spatial position of earthquakes $i$ and $j$. See Zhuang et al. [2002, 2004] for a full description of the method. Due to the stochastic nature of the simulations, each declustering run is different. Zhuang et al. [2002] observed that most earthquakes $(70-80 \%)$ show clear probabilities $\left(\mathrm{p}_{\mathrm{j}}<0.1, \mathrm{p}_{\mathrm{j}}>0.9\right)$ of being either a background or a clustered event. Thus the exact number of earthquakes (and hence the area and duration) of individual sequences will vary but the overall number of sequences and their relative size is similar between simulations. 


\subsubsection{General Observations}

In the following section the CURATE sequence catalog has been set to $\mathrm{N}_{\min }=2$ to include the possibility of clusters as small as two events, which occur in other methods. Figure 2.6 shows the range of declustered catalogs (seismicity identified as background) for the different methods. The CURATE declustered catalog falls within normal limits of the standard methods presented. The Reasenberg [1985] and SingleLink-Clustering $C / D_{\mathrm{ST}}=10 / 3$ [Davis and Frohlich, 1991] are not as smooth as the other curves, indicating they may not be identifying all clustering activity (see figure 2.6). At the other end of the range Stochastic Declustering, Gardner and Knopoff, and SLC $\left(C / D_{\mathrm{ST}}=10 / 30\right)$ have assigned the most events to sequences and have the smallest declustered catalogs. Table 2.5 gives a comparison of the area, duration, and parameters of the largest identified sequence for all five methods and for varying selection parameters of the CURATE method. The assumptions about earthquakeearthquake interaction and causation tend to give the Reasenberg catalog more short clusters, but where larger clusters develop, the durations are larger than observed in the CURATE catalog. The Gardner-Knopoff and Stochastic Declustering methods give longer durations and larger areas than the CURATE and link-based methods. Figure 2.7 shows duration histograms that more clearly illustrate the differences between the sequences defined by the different methods. The CURATE and link based methods assign most sequences durations of one day or less and have very few sequences lasting longer than a week. More conservative techniques (GardnerKnopoff, Stochastic Declustering, and large $C / D_{\mathrm{ST}}$ value Single-Link-Clustering) define sequence catalogs with a broader range of durations, with durations up to years long. 


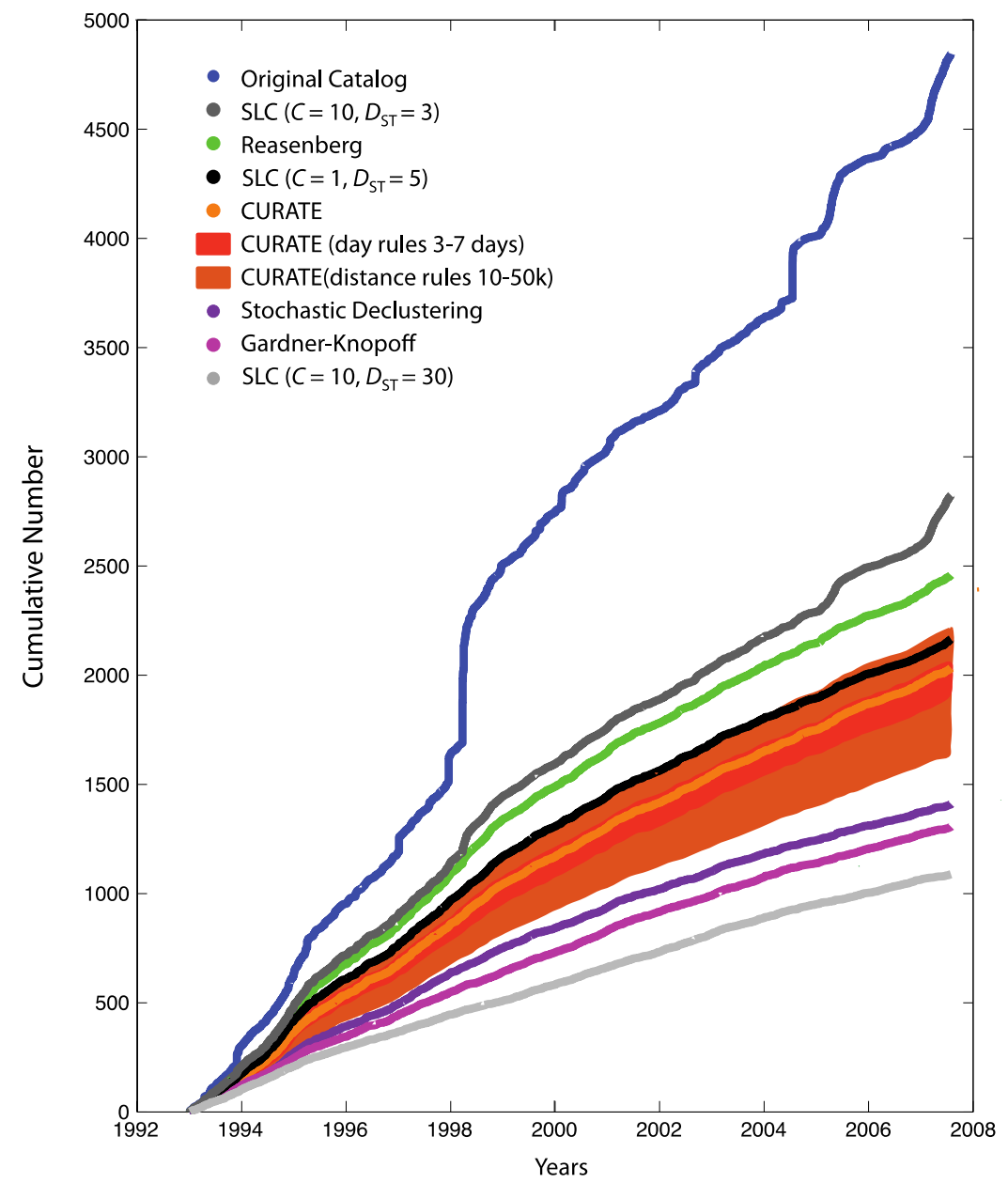

Figure 2.6 Cumulative number of earthquakes in the declustered catalogs with time for five different clustering techniques, some with multiple parameter values. 

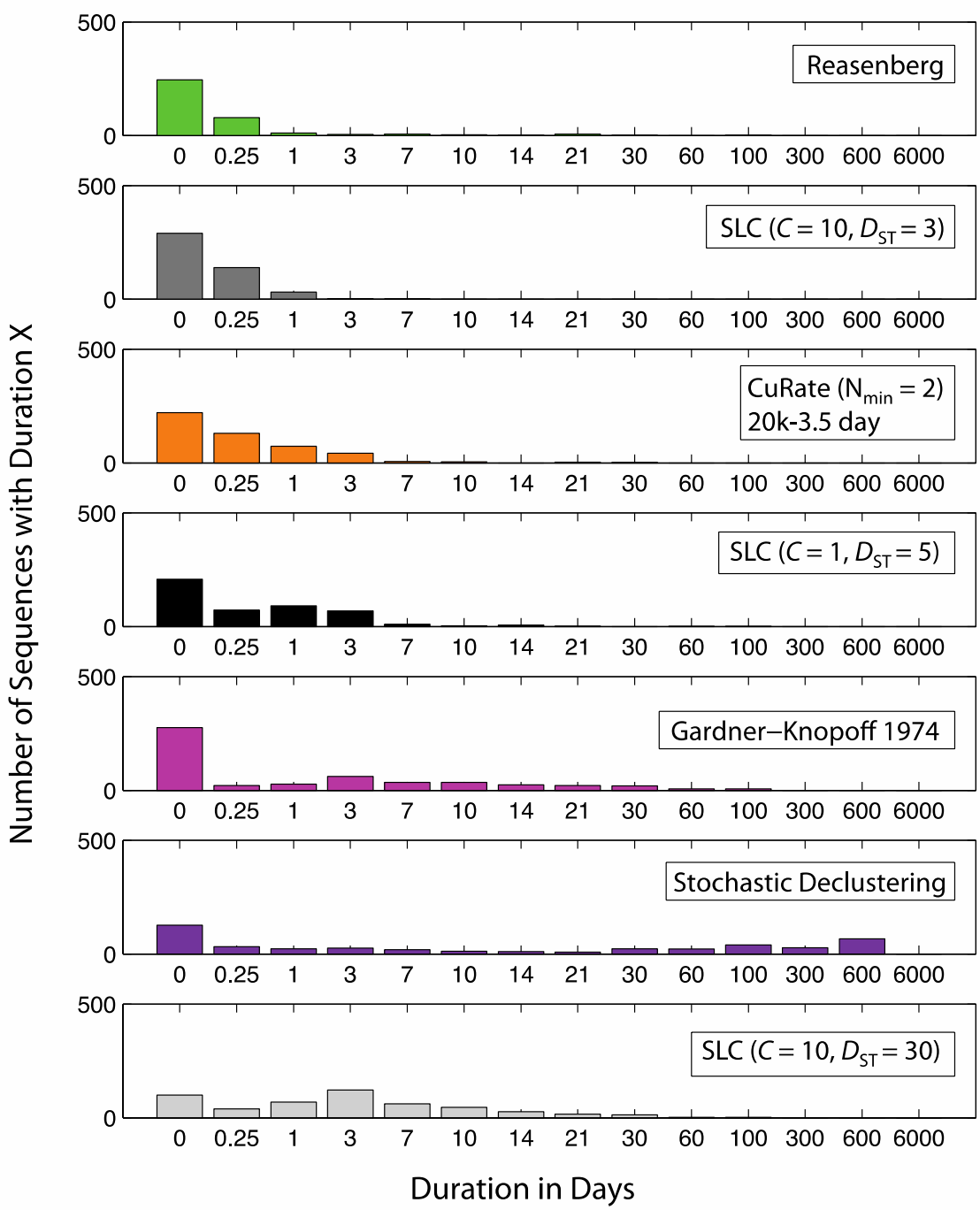

Figure 2.7 Duration histograms for clusters defined by five different clustering methods, some with multiple parameter values. 
Table 2-5 Parameters of Different Declustering Methods (Durations in Days, and Areas in $\mathrm{km}^{2}$ )

\begin{tabular}{|c|c|c|c|c|c|c|c|c|c|c|c|c|c|}
\hline & $\begin{array}{c}\# \\
\text { seqs }\end{array}$ & $\begin{array}{c}\# \\
\text { seqs } \\
4+ \\
\end{array}$ & $\begin{array}{c}\# \\
\text { seqs } \\
10+ \\
\end{array}$ & $\begin{array}{c}\text { Irgst } \\
\text { seq } \\
\# \\
\end{array}$ & $\begin{array}{c}\text { Irgst } \\
\text { seq } \\
M_{\max } \\
\end{array}$ & $\begin{array}{l}\text { lrgst seq } \\
\text { duration }\end{array}$ & $\begin{array}{l}\text { lrgst seq } \\
\quad \text { area }\end{array}$ & $\begin{array}{l}\text { Area } \\
\text { mean }\end{array}$ & $\begin{array}{c}\text { Area } \\
\text { median }\end{array}$ & $\begin{array}{c}\text { Area } \\
\text { max }\end{array}$ & $\begin{array}{c}\text { Duration } \\
\text { mean }\end{array}$ & $\begin{array}{c}\text { Duration } \\
\text { median }\end{array}$ & $\begin{array}{c}\text { Duration } \\
\max \end{array}$ \\
\hline \multicolumn{14}{|l|}{ Method } \\
\hline Reasenberg & 359 & 99 & 29 & 359 & 4.68 & 8.6 & 331 & 17 & 3.8 & 331 & 2 & 0.1 & 158.7 \\
\hline $\operatorname{SLC}\left(C=1, D_{\mathrm{ST}}=5\right)$ & 466 & 141 & 37 & 370 & 4.68 & 29.1 & 407 & 17.7 & 4.7 & 407 & 2.1 & 0.4 & 108.2 \\
\hline $\operatorname{SLC}\left(C=10, D_{\mathrm{ST}}=3\right)$ & 463 & 124 & 26 & 293 & 4.68 & 3 & 202 & 8 & 2.3 & 202 & 0.34 & 0.12 & 8.07 \\
\hline $\operatorname{SLC}\left(C=10, D_{\mathrm{ST}}=30\right)$ & 495 & 218 & 68 & 596 & 4.75 & 97.1 & 3290 & 429.9 & 134.1 & 5942 & 7.4 & 4.1 & 203.4 \\
\hline Gardner-Knopoff & 531 & 200 & 61 & 604 & 4.75 & 120.6 & 2354 & 169 & 49.1 & 2354 & 7.7 & 0.1 & 162 \\
\hline Stochastic -Declustering & 438 & 154 & 49 & 647 & 4.68 & 2410 & 2821 & 106 & 8.8 & 6568 & 306 & 8.5 & 4570 \\
\hline CURATE $\left(\mathrm{N}_{\min }=4\right)$ & 482 & 163 & 43 & 380 & 4.68 & 29.1 & 932 & 71.4 & 17.4 & 932 & 1.5 & 0.3 & 57 \\
\hline CURATE 20_3 & 488 & 166 & 43 & 341 & 4.68 & 11.56 & 931.62 & 70.8 & 17.36 & 931.62 & 1.32 & 0.34 & 56.95 \\
\hline CURATE 20_3.5 & 482 & 163 & 43 & 380 & 4.68 & 29.12 & 931.62 & 71.39 & 17.36 & 931.62 & 1.45 & 0.35 & 56.95 \\
\hline CURATE 20_4 & 476 & 163 & 43 & 380 & 4.68 & 29.12 & 931.62 & 79.36 & 17.14 & 931.62 & 1.62 & 0.38 & 56.95 \\
\hline CURATE 20_5 & 455 & 162 & 43 & 380 & 4.68 & 29.12 & 947.16 & 85.95 & 18.3 & 947.16 & 2.02 & 0.42 & 114.37 \\
\hline CURATE 20_7 & 420 & 157 & 44 & 380 & 4.68 & 29.12 & 945.99 & 102.32 & 25.74 & 945.99 & 3.01 & 0.47 & 159.56 \\
\hline CURATE 10_3.5 & 456 & 141 & 40 & 371 & 4.68 & 29.12 & 509.1 & 30.18 & 8.55 & 509.1 & 1.29 & 0.2 & 56.95 \\
\hline CURATE 15_3.5 & 464 & 154 & 44 & 377 & 4.68 & 29.12 & 685.07 & 43.22 & 12.91 & 685.07 & 1.39 & 0.25 & 56.95 \\
\hline CURATE 20_3.5 & 482 & 163 & 43 & 380 & 4.68 & 29.12 & 931.62 & 71.39 & 17.36 & 931.62 & 1.45 & 0.35 & 56.95 \\
\hline CURATE 25_3.5 & 489 & 172 & 48 & 513 & 4.68 & 31.25 & 1974.16 & 132.7 & 26.96 & 1974.16 & 1.58 & 0.41 & 56.95 \\
\hline CURATE 30_3.5 & 495 & 179 & 54 & 519 & 4.68 & 31.25 & 2471.66 & 181.3 & 32.58 & 2471.66 & 1.64 & 0.43 & 56.95 \\
\hline CURATE $40 \_3.5$ & 504 & 192 & 54 & 522 & 4.68 & 31.25 & 7526.54 & 386.24 & 65.03 & 7526.54 & 1.79 & 0.54 & 60.37 \\
\hline CURATE 50_3.5 & 492 & 210 & 61 & 525 & 4.68 & 31.25 & 7686.64 & 667.43 & 135.66 & 7686.64 & 2.05 & 0.65 & 74.26 \\
\hline
\end{tabular}




\subsubsection{Stochastic Reconstruction}

We present the results of a reconstructed synthetic catalog to fully compare the ability of an aftershock based model to describe the seismicity in the CVR. If aftershock models and temporal decay assumptions are appropriate, then a synthetic catalog generated using ETAS parameters should match the general characteristics of the observed seismicity. A simplified description of the reconstruction method is included here. See Zhuang et al [2004] for a complete description of stochastic reconstruction. Stochastic reconstruction utilizes the same principles as stochastic declustering. It is based on the idea that if you can model the background process and know the aftershock productivity, time, and spatial functions associated with that productivity then you should be able to stochastically create a synthetic earthquake catalog. The technique introduced by Zhuang et al. [2004] uses ETAS parameters as input to the productivity function of a branching structure with Omori's law temporal decay. Once background events are determined, a Gaussian deviation is added to the locations of those background events. The times of those background events are kept, but the locations are randomly reordered and a magnitude is assigned to each background time by re-sampling the magnitudes of all events in the target catalog. To account for boundary effects a larger space/time window around the target catalog is used to carry out the simulation. These background events are then allowed to produce offspring according to the same formulations used in stochastic declustering with an ETAS branching processes, with their temporal occurrence relating to Omori's law and their spatial distribution governed by a long-range inverse power decay. The reconstruction method allows for direct comparisons between the model assumptions and the real catalog data. Here the polygon 
$\left(-37^{\circ}, 175^{\circ} ;-39.9^{\circ}, 175^{\circ} ;-39.9^{\circ}, 175.25^{\circ} ;-37.25^{\circ}, 178.25^{\circ},-37^{\circ}, 178.25^{\circ}\right)$ is used to account for boundary effects, and the catalog from 1987 through 1992 is used to inform the background process history. We have then extracted all reconstructed events within the target boundaries:

$\left(-39.7^{\circ}, 175.25^{\circ} ;-37.65^{\circ}, 178^{\circ} ;-37.65^{\circ}, 175.25^{\circ}\right)$ and times $(1993-2007.5)$ of the catalog analyzed earlier. Ten simulations were run and returned between 5104 and 5364 earthquakes. A cumulative time plot of the reconstructed catalogs and the observed catalog is shown in figure 2.8 with insets showing the calculated CURATE for the real catalog, and one of the reconstructed catalogs (5231 events). A clear deficiency of temporal clustering is seen in the reconstructed catalog. 


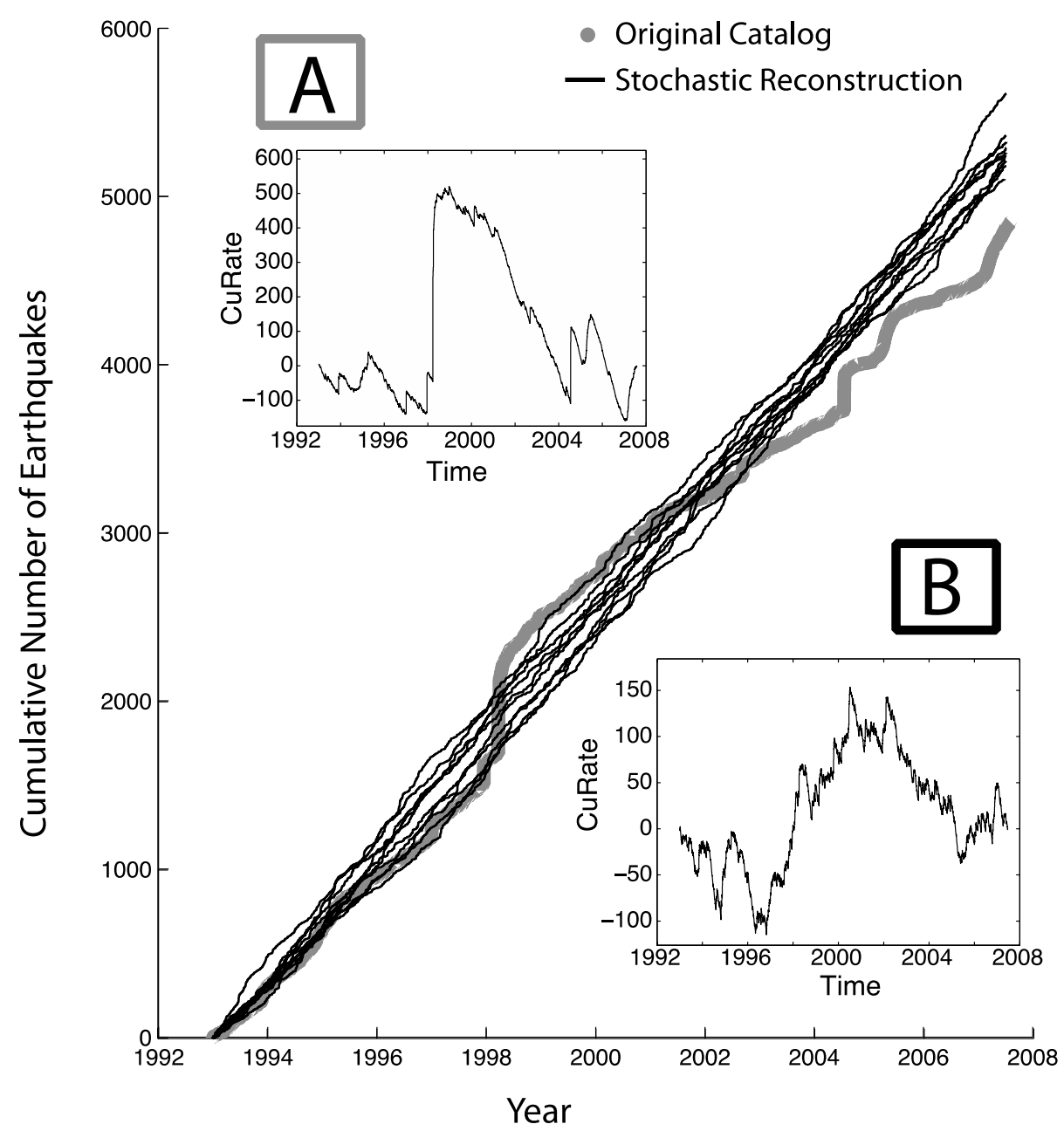

Figure 2.8 Cumulative number of events with time for the real and ten stochastically reconstructed catalogs.

Note the relatively uniform slope of the reconstructed catalogs with very weak temporal clustering. Insets A and B show the CURATE plots for the real catalog and one of the stochastic reconstructions.

This lack of clustering may result from an underestimate of $\alpha$. A number of studies on smaller regions have shown that misfits of ETAS models may sometimes be corrected by using a background function $[\mu(\mathrm{x}, \mathrm{y})]$ that is temporally non-stationary (Hainzl and Ogata [2005], Lombardi et al. [2006], Lomardi et al. [2010], Lohman \& McGuire [2007], and Llenos \& McGuire [2009]). Our results show that this problem is also observable on a catalog wide scale over long time periods. Note that while the parameter $[\mu(\mathrm{x}, \mathrm{y})]$ is often referred to as the background rate in a time varying context it is much more accurately the rate of independent events, and not the background rate 
in a traditional sense. These previous studies shown that the number of events with time (and temporal clustering) can be reproduced by allowing $[\mu(\mathrm{x}, \mathrm{y})]$ to vary with time. However, an increase in the rate of independent events would also alter the results of stochastic declustering and limit the ability to identify individual swarm clusters, with more individual clusters likely during periods with increased rates of independent events. The inability of temporally stationary $[\mu(\mathrm{x}, \mathrm{y})]$ to characterize our dataset also suggests that catalog-scale analysis methods that use a single set of aftershock decay parameters (time invariant ETAS, Reasenberg, Gardner-Knopoff) are unlikely to be useful in assessing the likelihood of sequences of a given size.

\subsubsection{Further Comparison with Link-Based Methods}

Most of the declustered catalogs produced by the two link based methods are larger, but similar in number to the declustered catalog of the CURATE method (fig. 2.6). The SLC method with parameters of $C=10$ and $D_{\mathrm{ST}}=3$ is in this range; however the cumulative declustered catalog (fig. 2.6) is not as smooth and figure 2.6 and table 2.5 demonstrate that those parameters limit almost all sequence durations to less than 3 days. Other possible SLC catalogs $\left(C / D_{\mathrm{ST}}\right)$ combinations are considered in section 5.2. The Reasenberg and SLC method with parameters of $C=1$ and $D_{\mathrm{ST}}=$ 5 are the closest matches to our CURATE method in terms of the largest sequence and the total number of sequences (table 2.5), as well as the duration distributions (fig. 2.7), so we have looked at those two sequence catalogs in more detail. As noted above, the CURATE sequence catalog is shown at $\mathrm{N}_{\text {min }}=2$. Despite the different approaches of these two methods and the CURATE method, a few sequences are identical in all three sequence catalogs. These are all sequences which occur over short time scales and are extremely close in space (day/km range). Figures 2.9a and 
$2.9 \mathrm{~b}$ show a direct comparison of methods for time periods around two large sequences near Haroharo in the Okataina caldera complex (fig 2.3). The larger 1998 sequence (2.9b) is described by Hurst et al. [2008] as the 'Haroharo sequence'. 

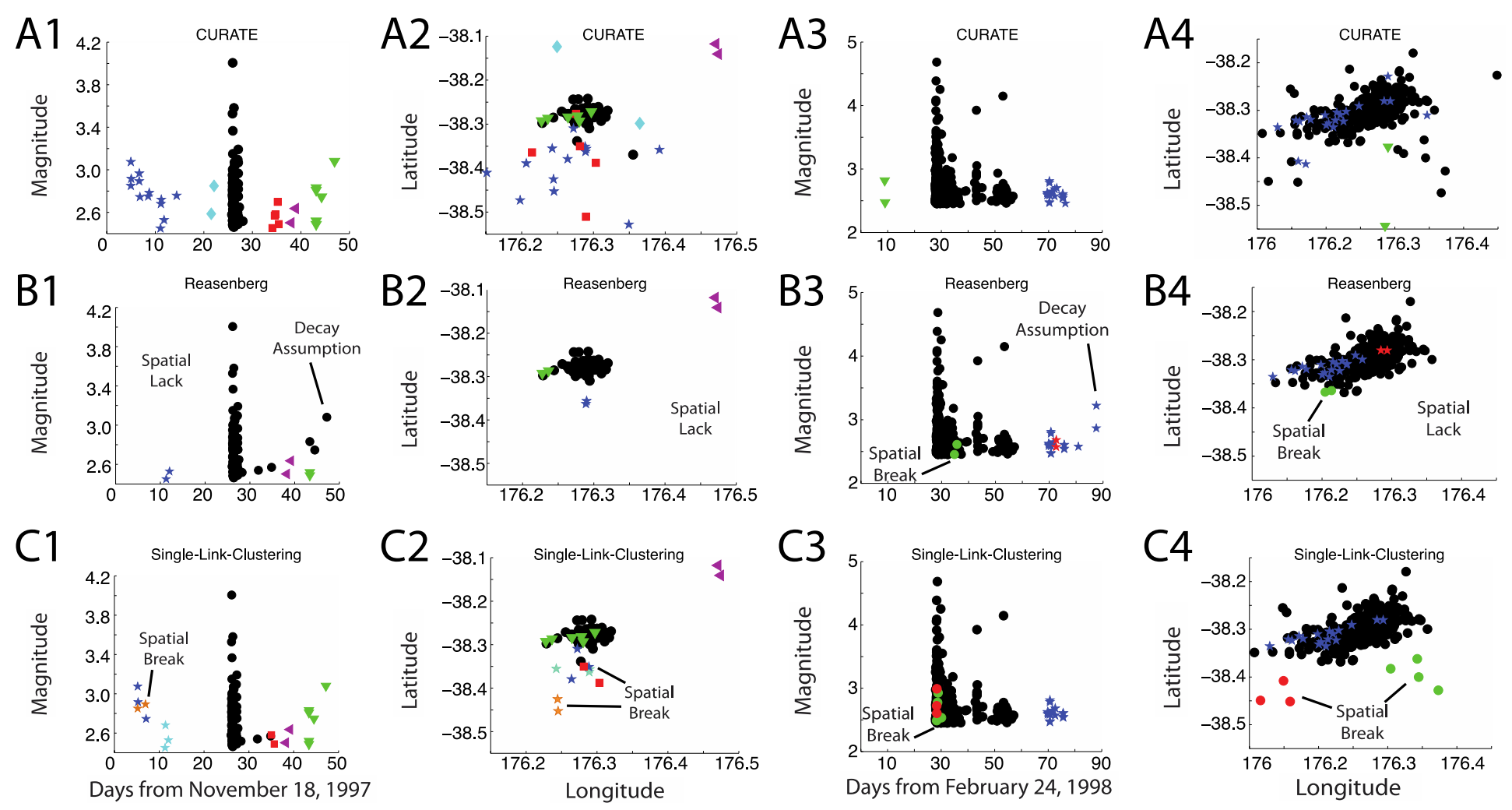

Figure 2.9 Map and magnitude comparisons for time periods around two large sequences near Haroharo.

1-2) November 1997, 3-4) February 1998. Column 1 is a magnitude with time plot for each of three clustering methods: A) CURATE, B) Reasenberg, C) SLC (C=1, DST=5). Columns 2 and 4 are map views of the same sequences shown in columns 1 and 3 . The colors and shapes in A are each distinct sequences identified by that method. Shapes in plots B and C represent sequences which contain earthquakes that overlap with the sequences identified by the CURATE method (A); and colors in B and C still identify distinct sequences identified by these methods. The largest sequence in each time period is represented by black circles. Colors and shapes do not translate between 2.9a (1-2) and 2.9b (3-4). See the text for further explanation. 
There are three types of differences seen in figure 2.9. The first is the difference caused by assigning time decay/physical causality to preceding seismicity. The Reasenberg method is the only method of these three that explicitly assumes temporal decay in keeping with aftershocks and Omori's law. This can be seen in figure 2.9a (B1) by the assignment (black dots) of 5 more earthquakes to the main sequence in the 20 days following the bulk of the activity. This affect is also apparent in $2.9 \mathrm{~b}(\mathrm{~B} 1)$ where the blue star sequence that starts around day 70 continues after day 80 with three more earthquake not identified by the CURATE (A1) or the SLC (C1) methods.

The second major difference apparent in figure 2.9 is a lack of events arising from the way spatially diffuse increases in seismicity and migration of events are dealt with. The CURATE searches an entire area over the time period of interest whereas the other two link-based methods assume that all earthquakes are caused by other earthquakes in the catalog, thus inter-earthquake distances must be within a certain maximum length. This spatial lack of events is most evident in the Reasenberg method (2.9a-b [B2]) with fewer events overall and a lack of events away from the densest part of clusters. Not including these more distant or sparse events in the sequence catalog is one of the reasons why the Reasenberg declustered catalog (Fig. 2.6) has more events than any other method presented here. While this effect is less evident in the SLC method $(2.9 \mathrm{a}-\mathrm{b}, \mathrm{C})$ it is worth noting that in both $2.9 \mathrm{a}$ (blue diamonds) and $2.9 \mathrm{~b}$ (A1, green triangles) there is a sequence of two distant events preceding the main sequence that are not identified by either Reasenberg or SLC.

Finally, some sequences that the CURATE method identifies as a single sequence are separated into two or more sequences on the basis of their lack of spatial proximity (spatial break). This division is often not based on the area over which the 
sequence is occurring but arises from the nature of link-based methods that necessarily link successive earthquakes. In figure $2.9 \mathrm{a}$ a single sequence of thirteen events (blue stars) is identified in the CURATE method (A). The same sequence appears as eight events (stars) in three separate sequences in the SLC (C) method. The effect is also seen in figure $2.9 \mathrm{~b}$. Because we do not have a priori knowledge of the area over which to expect swarm sequences, it is desirable to look for precursors to large scale activity over the same spatial area as the anticipated activity. The spatial break is most evident in the SLC method because each earthquake only shares a single link; however, figure $2.9 \mathrm{~b}$ (B2) shows that the Reasenberg method also contains traces of this effect. The Reasenberg method (B) does not include two events (green circles) in the larger main sequence (black circles) even though the events look spatially proximal to that activity. This effect can be caused if there is migration with time of the main activity while decay continues in the original regions. Thus it is appears that the CURATE method's independent treatment of distance may better capture the decay of sequences than other link-based methods. In addition to possible decay, the CURATE method allows for larger sequence areas that are independent of the spatial succession of events. In contrast to the link-based methods the Stochastic Declustering combines both large sequences in $2.9 \mathrm{a}$ and $2.9 \mathrm{~b}$ into a single sequence lasting over 5 years. 


\subsection{Testing Sequence Selection Parameters}

Next we analyze the robustness of the selection parameters presented here for CURATE. We also propose a method for determining reasonable resulting sequence properties.

\subsubsection{Comparison with Previous Foreshock Results}

To test the consistency of our sequence definitions and the resulting sequences, we have attempted to replicate results of a recent study of foreshocks in the Taupo Volcanic Zone (TVZ) conducted by Tormann et al. [2008]. They define a foreshock as 'an earthquake that is followed by an event of equal or larger magnitude within 5 days and $50 \mathrm{~km}$ from the initial event'. They used a catalog of earthquakes with magnitudes $\geq 4.0$ from 1964-2007 (they also determined that their findings are independent of the time period chosen). Tormann et al. [2008] used a magnitude and time dependent window to remove aftershocks based on the Gardner and Knopoff [1974] method modified for New Zealand by Savage and Rupp [2000]. We have compared our sequences to their findings by estimating the empirical probability of foreshocks occurring in our sequence catalog. We assume that any foreshockmainshock pair will be contained in a single sequence. We do not allow for a foreshock to be an earthquake outside of the sequences. A foreshock sequence then will be any sequence with a maximum magnitude $M \geq 4.0$ event with at least one smaller $M \geq 4.0$ event preceding it within 5 days and $50 \mathrm{~km}$ in the same sequence. Multiple foreshocks will not be taken into account. The number of possible foreshock sequences will be composed of sequences with at least one $M \geq 4.0$ event and any $\mathrm{M}$ $\geq 4.0$ earthquakes that are not part of any sequence (declustered catalog). The $M \geq$ 4.0 events in the declustered catalog are not in a sequence and therefore are not considered foreshocks in this analysis, so the foreshock probability can only reach 1 if 
there are no $M \geq 4.0$ earthquakes in the declustered catalog. The boundaries of our catalog are larger than those used by Tormann et al. [2008] so first we limited our search to earthquakes and sequences that are within the TVZ boundaries $\left(-37.00^{\circ}\right.$, $\left.175.85^{\circ} ;-39.29^{\circ}, 175.55^{\circ} ;-37.50^{\circ}, 177.40^{\circ}\right)$. Then we identified sixteen sequences within those boundaries that contained at least one $M \geq 4.0$ event. The number of M $\geq 4.0$ earthquakes (in the same area) in the declustered catalog was six. Using the constraints above there were four sequences with at least one foreshock. This gives us an empirical probability of $\sim 18.2 \%(4 / 22)$, which is within the $24.2 \% \pm 7.7 \%$ range of Tormann et al. [2008].

\subsubsection{Temporal Distribution of the Declustered Catalog}

It has long been suggested that the background rate of seismicity is random in time and should follow a Poisson distribution (e.g. [Gardner and Knopoff, 1974; Ogata, 1988; Vere-Jones, 1970]). At this stage we do not offer a method for optimizing the sequence selection parameters (catalog boundaries, distance-rule, dayrule) but we suggest that a Poisson test of the declustered catalog can be used to test whether a given set of selection parameters are appropriate. This will not give a unique solution of parameters, but will have the ability to identify unreasonable selection parameters and catalogs whose mean rate is unrepresentative of a background rate. This approach may be developed to find optimized parameters by identifying the best fit to a Poisson distribution.

Luen and Stark [2012] recently suggested that some declustering methods may remove too much seismicity to achieve a Poisson result. If too many earthquakes are removed from the catalog, the number of aftershocks expected for each earthquake will be overestimated and the rate of mainshocks will be underestimated. It is therefore ideal to leave in as much seismicity as possible. 
We have undertaken two different methods to test the declustered catalogs presented in Section 2.4.4 for a temporal Poisson distribution. The first method utilizes the $\chi^{2}$ test as used in Gardner and Knopoff [1974]. This consists of binning the seismicity into 10 day time windows and counting the number of earthquakes that occur in each bin. To test the influence of the choice of bin we have incremented the starting bin by the minimum inter-event time in the declustered catalog ( 0.05 days) for start times between 0 and 10 days. This gives us 210 different bin starts. For each possible bin start the seismicity is tabulated over 10-day windows and the mean is used to calculate a Poisson distribution of those window counts. If the position of the bins does not affect the results we will only get binary answers of either 0 (completely non-Poisson) or 100\% (completely Poisson). Another property of a Poisson distribution is that its mean should be equal to its variance [Dixon and Massey, 1968]. Thus the variance divided by the mean (dispersion) should be insignificantly different from one. The second test simply calculates the dispersion for each set of bins. According to Vere-Jones [1970], over-dispersion $(\geq 1)$ indicates some degree of clustering, and under-dispersion $(\leq 1)$ indicates more regularity than a Poisson distribution.

A surprisingly low percent of catalogs returned a Poisson result when using the entire time period (fig. 2.10 A2). It looks possible from the declustered catalog rates (fig. 2.6) that there is a significant change in rate before and after 1998. The variation in the Poisson fraction (fig. 2.10) also shows that the position of the bins does have an effect on whether the result passes the $\chi^{2}$ test. 
A: $1993-2007.5$

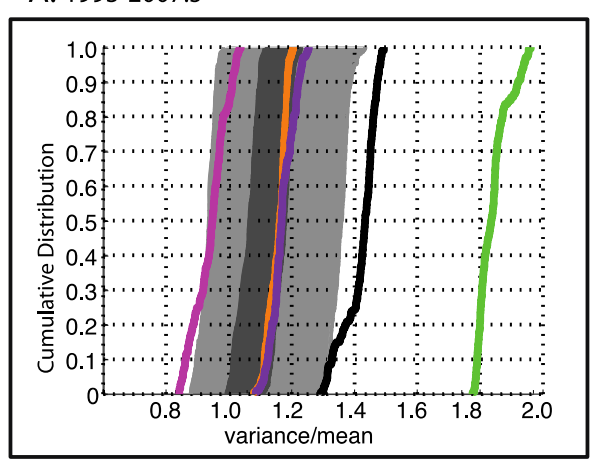

B: $1993-1997$

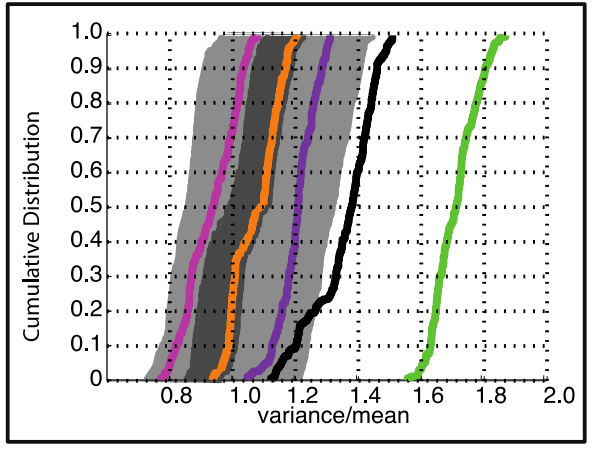

C: $1998-2007.5$

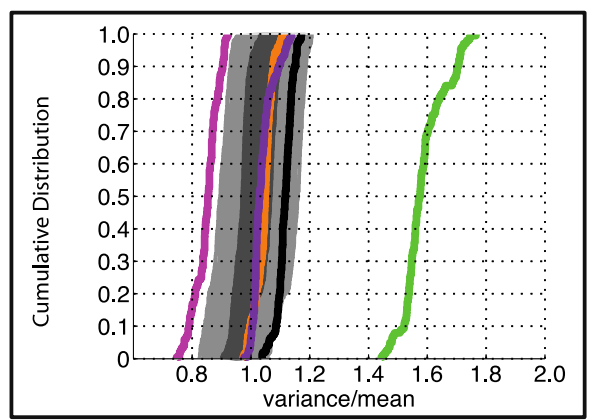

Gardner-Knopoff (GK)

CURATE (CR)

CURATE (day rules 3-7 days)

CURATE(distance rules 10-50k)

Stochastic Declustering (SD)

- $\operatorname{SLC}\left(C=1, D_{\mathrm{ST}}=5\right)(\mathrm{SLC})$

Reasenberg (RB)

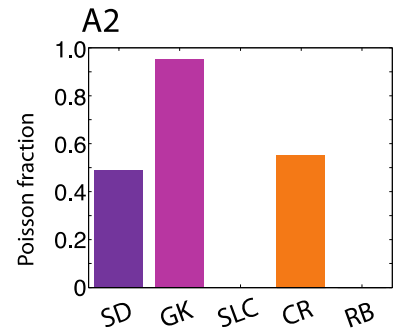

$S D \quad G K \quad S L C \quad C R \quad R B$
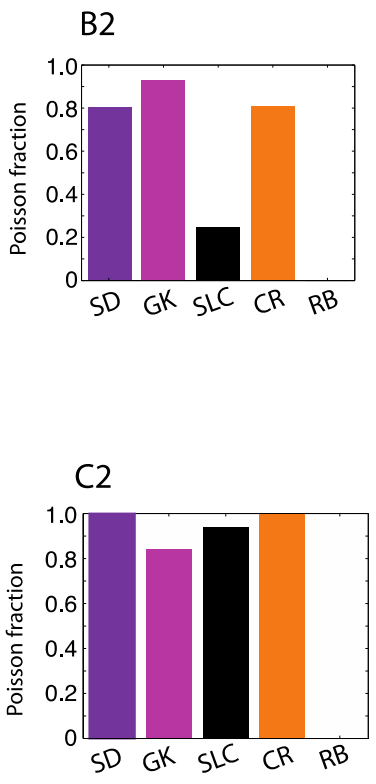

Figure 2.10 Poisson fits for declustered catalogs.

A-C cumulative distribution of values of variance/mean (dispersion) for 210 different binning configurations of 10 day windows for the three time periods marked (e.g. A: 1993-2007.5), colored by method in the key. Poisson distribution should have a dispersion of one. A2-C2 show the relative fraction of those 210 configurations that return a $\chi^{2}$ value consistent with a Poisson distribution. Methods are ordered by the number of earthquakes in the declustered catalog from smallest to largest. The lack of a colored bar indicates no configurations returned a Poisson result. 
The Reasenberg catalog returns the highest dispersion values and never returns a Poisson result for any time period (fig. 2.10). It is unsurprising that the Reasenberg declustered catalog is not Poisson given that it has the largest declustered catalog. The maps in figure 2.9a (low amounts of clustered seismicity in B2 compared to A2 and C2) also show how much temporally clustered seismicity is left in the declustered catalog. Van Stiphout et al. [2012] ran several declustering algorithms on the ANSS catalog in the California CSEP testing region and found that varying the parameters of the Reasenberg and Gardner and Knopoff methods has little impact on the $\chi^{2}$ test results. While their conclusions suggest that other parameter choices for the Reasenberg method would not influence our results, we did run several tests of different $\mathrm{p}, \mathrm{Q}$, and $\tau$ values to ensure that we had not chosen an unfairly large catalog. The smallest declustered catalog produced from the range of parameters tested was with $\mathrm{p} / \mathrm{Q} / \tau$ values of $.95 / 100 / 10$ which gave a declustered catalog of 1944 earthquakes. This value of $\mathrm{Q}$ is beyond those typically tested, yet even with this catalog the method gives a median dispersion value of 1.45 and still does not return any Poisson results. This matches the conclusions of Van Stiphout et al. [2012] and other authors indicating that the Reasenberg method rarely returns a Poisson declustered catalog and implies that there is something in the method itself, not just the large size of this declustered catalog that leads to high dispersion value.

Our results for the Gardner and Knopoff method also match those found by Van Stiphout et al. [2012] with that method returning a high percentage of Poisson results. However by tracking the dispersion values directly we find that the GardnerKnopoff method is consistently under-dispersed. It is tempting to think that this is due to an overabundance of long time intervals (low number of events in the declustered catalog), but the stochastic declustering method (branching-ratio .7198) 
also has a relatively small declustered catalog and returns dispersion values which are either near one or slightly over-dispersed. The reason for this is unclear and warrants further investigation. The CURATE method has dispersion values that are closer to one than Gardner and Knopoff except in the early time period.

The SLC method does not perform well in the overall catalog or in the early time period (1993-1997). We ran the two Poisson tests on six different $C / D_{\mathrm{ST}}$ pairs which gave definitions of the largest sequence that were similar to the CURATE method. Three of the declustered catalogs $\left(C / D_{\mathrm{ST}}=: 1 / 10,3 / 10,10 / 15\right)$ have fewer events than the CURATE declustered catalog, and three $\left(C / D_{\mathrm{ST}}=: 1 / 5,0.25 / 3\right.$, $0.05 / 3$ ) have more events. The results of the tests and the size of the declustered catalogs are given in Table 2.6 where the median value is given to represent the dispersion test. The catalog presented for earlier comparison, $C / D_{\mathrm{ST}}=1 / 5$, is highlighted with a black box in Table 2.6. As expected based on the catalog sizes, the three largest declustered catalogs give the largest dispersion values. The dispersion values of catalogs with larger $D_{\mathrm{ST}}$ values do not strictly increase with increasing catalog size. Increases in $C$ for the same $\mathrm{D}_{\mathrm{ST}}$ cause a corresponding increase in dispersions, whereas increases in $D_{\mathrm{ST}}$ for the same $C$ value lead to a decrease in dispersion. The poor performance of the small $D_{\mathrm{ST}}$ catalogs in the early time period may indicate that the increased rate of seismicity in the early time period was due to relatively dispersed seismicity. Table 6 also shows that the CURATE method (highlighted in gray) performs reasonably well over a range selection parameters. For the whole time period (1993-2007.5) and the early time period (1993-1997) all the SLC catalogs are over-dispersed and return higher dispersion values than the CURATE. 
The range of CURATE selection parameters shows that several possible selection parameters return better Poisson results than our initial selection parameters. We have elected to use the smaller (20k) Distance-rule to match previous descriptions of large seismicity. The day rule analysis shows that we may be able to improve our results by using slightly higher day-rules of 4 or 5 days. While these day rules give slightly better values, note that the size of the declustered catalogs vary less than $3.0 \%$ from that reported for the $20 \mathrm{k} / 3.5$ day rules. Tables like this paired with information about the scale of activity in a dataset will allow users of the CURATE method to find parameter ranges that are appropriate. Small distance rules $\leq 5 \mathrm{~km}$ may be appropriate for small studies of micro-seismicity, but care should be taken when the distance rule approaches the size of the location errors. 
Table 2-6 Results of Poisson Testing for Different SLC and CURATE Declustered Catalogs..

The SLC and CURATE Catalogs Considered Throughout the Text are Highlighted in Bold Throughout the Row.

\begin{tabular}{|c|c|c|c|c|c|c|c|c|c|}
\hline \multirow[b]{2}{*}{ Method } & \multirow[b]{2}{*}{$\begin{array}{l}\text { C/Dist- } \\
\text { Rule }\end{array}$} & \multirow[b]{2}{*}{$\begin{array}{c}\text { DST }_{\text {ST }} / \text { Day- } \\
\text { Rule }\end{array}$} & \multirow[b]{2}{*}{$\begin{array}{c}\text { Catalog } \\
\text { Size }\end{array}$} & \multicolumn{2}{|c|}{$1993-2007.5$} & \multicolumn{2}{|c|}{ 1993-1997 } & \multicolumn{2}{|c|}{$1998-2007.5$} \\
\hline & & & & Dispersion & $\begin{array}{c}\% \\
\text { Poisson }\end{array}$ & Dispersion & $\begin{array}{c}\% \\
\text { Poisson }\end{array}$ & Dispersion & $\begin{array}{c}\% \\
\text { Poisson }\end{array}$ \\
\hline SLC & 1 & 10 & 1708 & 1.21 & 0.1841 & 1.17 & $97.51 \%$ & 0.99 & $99.50 \%$ \\
\hline SLC & 10 & 15 & 1799 & 1.18 & $32.84 \%$ & 1.12 & $79.60 \%$ & 0.97 & $94.53 \%$ \\
\hline SLC & 3 & 10 & 1871 & 1.26 & $12.94 \%$ & 1.19 & $75.12 \%$ & 1 & $94.03 \%$ \\
\hline CURATE & 20 & 3.5 & 2033 & 1.16 & $54.73 \%$ & 1.09 & $80.60 \%$ & 1.05 & $100 \%$ \\
\hline SLC & 1 & 5 & 2165 & 1.43 & $0.00 \%$ & 1.37 & $32.84 \%$ & 1.14 & $78.61 \%$ \\
\hline SLC & 0.05 & 3 & 2181 & 1.69 & $0.00 \%$ & 1.6 & $1.00 \%$ & 1.37 & $30.85 \%$ \\
\hline SLC & 0.25 & 3 & 2365 & 1.72 & $0.00 \%$ & 1.66 & $0.00 \%$ & 1.41 & $13.93 \%$ \\
\hline CURATE & 10 & 3.5 & 2218 & 1.36 & $0.00 \%$ & 1.33 & $31.34 \%$ & 1.16 & $87.06 \%$ \\
\hline CURATE & 20 & 3.5 & 2033 & 1.16 & $54.73 \%$ & 1.09 & $80.60 \%$ & 1.05 & $100 \%$ \\
\hline CURATE & 30 & 3.5 & 1874 & 1.08 & $94.53 \%$ & 1 & $98.51 \%$ & 0.98 & $100 \%$ \\
\hline CURATE & 40 & 3.5 & 1748 & 0.99 & $100 \%$ & 0.89 & $93.53 \%$ & 0.93 & $100 \%$ \\
\hline CURATE & 50 & 3.5 & 1648 & 0.94 & $100 \%$ & 0.85 & $87.56 \%$ & 0.9 & $100 \%$ \\
\hline CURATE & 20 & 3 & 2049 & 1.18 & $44.78 \%$ & 1.11 & $76.62 \%$ & 1.07 & $100 \%$ \\
\hline CURATE & 20 & 3.5 & 2033 & 1.16 & $54.73 \%$ & 1.09 & $80.60 \%$ & 1.05 & $100 \%$ \\
\hline CURATE & 20 & 4 & 2013 & 1.14 & $68.66 \%$ & 1.06 & $82.59 \%$ & 1.05 & $100 \%$ \\
\hline CURATE & 20 & 5 & 1979 & 1.1 & $90.05 \%$ & 1 & $95.52 \%$ & 1.02 & $100 \%$ \\
\hline CURATE & 20 & 7 & 1917 & 1.07 & $93.03 \%$ & 0.98 & $94.03 \%$ & 0.97 & $99.50 \%$ \\
\hline
\end{tabular}




\subsection{Discussion: Utility of Sequence Catalogs}

The map view of the sequence catalog (fig. 2.5) provides us with a first-order look at sequence activity in the study region. One initial observation is the apparent increased likelihood for larger sequences to occur in the northern part of our study region (fig. 2.5). This is unsurprising given the larger area and much denser distribution of faults in the northern part of the TVZ ([Villamor and Berryman, 2006]. The largest sequence south of Lake Taupo is a sequence to the west of Ruapehu volcano (fig. 2.3), preceding its 1995 eruption [Hurst and McGinty, 1999]. While Hurst and McGinty (1999) refer to this activity as 'swarms' we note that the largest of these sequences picked out by our method has many characteristics of a mainshockaftershock sequence. The largest event is $\mathrm{M}=4.8$ which occurs as the $9^{\text {th }}$ out of 37 earthquakes, has a magnitude separation of 0.86 (high for the TVZ), and it also exhibits temporal decay after the occurrence of the largest event. All of these characteristics seem to indicate it may be a foreshock-mainshock-aftershock sequence. Its most unusual feature is its proximity in time to the later eruptive activity at Ruapehu. Other distant (10-30 km) sequences preceding eruptions have been documented [Fisher et al., 2009; Umakoshi et al., 2001]. In all cases links to the subsequent eruptive/magmatic activity are not conclusive; however all reported cases show the preceding seismicity to be anomalous compared to previously recorded decades of seismicity. Hurst and McGinty [1999] suggested that a broader region around volcanoes be monitored for pre-eruptive seismicity. We propose that sequences catalogs created with the CURATE method are an ideal way to facilitate such observations. Even without probabilistic modeling, sequence catalogs can be used as a first order tool to identify anomalous behavior. 


\subsection{Conclusions}

We have developed CURATE, a new clustering method that is more general than standard clustering techniques in that no specific causality is assumed. The use of earthquake rate as the primary indicator of activity allows us to vary the distance search parameter more independently than other methods. This independent treatment of distance will enable better identification of sparse increases in activity and lead to the better categorization of decay of sequences. It will allow us to investigate the spatial scale of any potential precursory activity.

Applying the method to a dataset from the Central Volcanic Region of New Zealand we identified small earthquake sequences preceding two large swarms in the Haroharo region. If other large swarm sequences are found to have small precursors it could have implications for hazard assessments and investigations of underlying physical processes. Anomalous earthquake sequences can readily be identified using sequence catalogs.

A stochastic reconstruction, based on ETAS parameters, indicates that methods with a single set of temporal decay assumptions will put background earthquakes into clusters more frequently in areas or magnitude ranges with swarm activity. The lack of temporal clustering in the reconstructed catalogs replicates on a large spatial and temporal scale results for small spatio-temporal datasets (e.g. Hainzl and Ogata [2005], Lombardi et al. [2006] ) that have shown that ETAS models with temporally stationary background rates cannot replicate swarms well.

Despite the novelty of the CURATE method, it produces a declustered catalog which is consistent with other clustering algorithms. We propose Poisson testing of the declustered catalog to check for a sufficient choice of selection parameters. 
Testing of the declustered catalog presented here also shows that the CURATE method will add to the suite of clustering tools already available. The use of sequence catalogs generated by the CURATE method in seismically active areas will promote the ability to identify the timing and scale of anomalous behavior and provide useful parameters for incorporating earthquake swarm information into earthquake forecasts and hazard assessments. 


\section{Declustered Catalogs and Regional Behavior}

In this chapter we take a closer look at the declustered catalog itself and what can be gained by examining rates in the raw earthquake catalog. This investigation started largely as a way to check that the different regions we were investigating were getting good clustering results (as discussed in chapter 2). We were surprised how difficult it was to achieve Poisson declustered catalogs with our method. This chapter develops a more general way to process a variety of earthquake catalogs with the CURATE method, and discusses the departure from Poisson distributions in terms of differences in regional earthquake behavior and the temporal stability of the background rate.

\subsection{Comment on clustering/declustering algorithms and Poisson behavior}

This section includes a few brief studies that were conducted largely in response to reviewer comments on the published paper that constitutes Chapter 2. The first two subsections here focus on testing the performance of different clustering algorithms, and the final section introduces the CURATE of the declustered catalog. The declustered CURATE will be used as one way to compare real declustered catalogs to ideal Poisson distributions. 


\subsubsection{Dispersion and Size of the Declustered Catalogs}

First we present another comparison of the CURATE method to other clustering techniques using Poisson temporal distributions. We have already established in Chapter 2 that the CURATE method gives larger declustered catalogs than other methods while still achieving a Poisson temporal distribution. Figure 3.1 clarifies this statement by showing that the catalogs derived from various selection parameters of the CURATE method (orange) sit on a lower trend than other methods, indicating that the CURATE returns larger declustered catalogs for similar Poisson values. Note in the plot of dispersion values that smaller declustered catalogs correspond to larger selection parameters, which place more events in clusters. The lower trend indicates that we have satisfied the appeal by Luen and Stark [2012] for a method that recovers Poisson catalogs with a larger fraction of the seismicity in the declustered catalog.

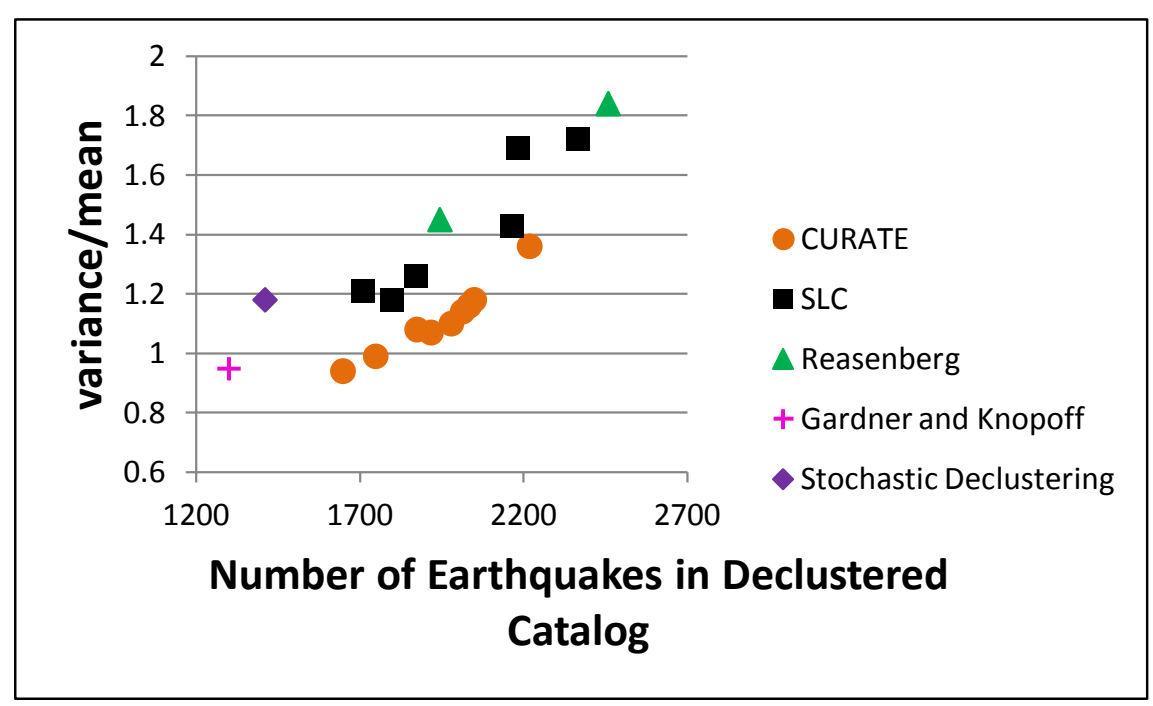

Figure 3.1 Dispersion values plotted with size of the declustered catalog. Poisson behavior corresponds to dispersion $=1$. 


\subsubsection{Synthetic Poisson Catalog}

Another way to test how much excess seismicity is being removed by a given method is to see how it performs on a (synthetic) catalog that has a Poisson temporal distribution. Ideally, if the data are not clustered no seismicity should be removed. However, the basic assumption of all clustering algorithms is that clustering does exist, so some seismicity will be removed in all cases.

The CURATE method can identify single earthquakes as being anomalous in terms of rate. This contrasts with the SLC method most strongly, because it uses short space-time distances to identify earthquake pairs (another way of inherently implying the causality between earthquakes) instead of being based on individual events. We do not consider single events as being clusters or sequences thus; "sequences" of one event are returned to the declustered catalog at the end of all other processing steps. To test the sizes of the final declustered catalogs, we made a Poisson distribution of times by choosing a window length (10 days, as used for the declustering testing) and getting a random Poisson distribution with the same mean rate as the catalog for the number of events that would be in each time bin (representative of the bins over the whole catalog $(\sim 530$ in this case $=14 * 365 / 10)$. Note that this creates slightly different catalog lengths for each simulation. To get to a stage where we had a time for each synthetic event, instead of a number of events/10 days, we then generated a list of random number between 0-1 with a length equal to the number of earthquakes. These numbers were used to assign a placement to events within the 10-day window (i.e. $0.9=$ occurring on day 9). Because there are a large number of earthquakes in the catalog, there are few if any bins that expect zero events (some catalogs were without any). This becomes problematic for the Reasenberg method as discussed later. The addition of this timescale means the distribution is not exactly Poisson, but it is a reasonable 
approximation. For the purposes of testing the CURATE method without day and distance rules we ran 1000 such simulations. Using just the initial CURATE calculation without a distance or day rule (assuming zero distance and not applying a day rule) produces a declustered catalog with $36.81 \%$ of the total earthquakes. That value is approximately equal to the $37 \%$ probability for an exponentially distributed waiting time to be greater than the mean. When sequences of only one earthquake are also put into the declustered catalog the percentage goes up to $45.34 \%$. Standard declustering procedures also typically put one earthquake back into the declustered catalog from each sequence (either the first or largest earthquake) to represent the triggering capability of the background. This practice is referred to as 'mainshockreplacement'. The percentages reported so far do not include mainshock replacement: if mainshocks are also added back in then the percentage goes up to $60.03 \%$. While it may be ideal for $100 \%$ of the catalog to be in the declustered catalog as came from a Poisson distribution, there are no existing clustering algorithms we are aware of that have a possible output of no clustering. The number of sequences of one is entirely dependent on the temporal distribution of earthquakes. Thus, for the Poisson simulations the median of the percent of events in the initial sequences that are sequences of only one event is about $13.6 \%$ as opposed to $8 \%$ in the real CVR catalog. The results for this test are listed in table 3.1.

We chose to run several possible catalogs through the other methods and through the whole CURATE method (with day rule). To keep the problem simple we set all locations to a single latitude and longitude to simulate a temporal-only catalog. Note that the Reasenberg and Gardner and Knopoff methods are more difficult to test because they are also magnitude dependent. Thus we coupled the Poisson temporal distribution with three possible magnitude distributions: a random 
magnitude drawn from the real magnitudes of the presented CVR catalog $(\mathrm{CH} .2)$, a fixed magnitude at $\mathrm{M}_{\min }$ (2.5) and a larger fixed magnitude $\mathrm{M}=3.0$ (Table 3.1). 
Table 3-1 Details of declustered catalogs of synthetic Poisson temporal earthquake catalogs for various clustering techniques.

'w10' indicates a window length of 10 days.

\begin{tabular}{|c|c|c|c|c|c|c|}
\hline & $\begin{array}{l}\text { \# eqs in } \\
\text { catalog }\end{array}$ & $\begin{array}{l}\# \text { eqs in a } \\
\text { sequence }\end{array}$ & $\begin{array}{l}\text { \% not } \\
\text { in seq }\end{array}$ & $\begin{array}{l}\text { \# not in } \\
\text { clus }\end{array}$ & $\begin{array}{l}\# \text { seqs } \\
\text { (MS) }\end{array}$ & $\begin{array}{l}\text { \% declus with } \\
\text { MS replace }\end{array}$ \\
\hline \multicolumn{7}{|c|}{ SLC with fixed $C=1($ window $=10)$} \\
\hline \multicolumn{7}{|l|}{ DST } \\
\hline 5 & 4861 & 4860 & $0.02 \%$ & 1 & 48 & $1.01 \%$ \\
\hline 3 & 4861 & 4843 & $0.37 \%$ & 18 & 297 & $6.48 \%$ \\
\hline 1 & 4861 & 4048 & $16.72 \%$ & 813 & 1160 & $40.59 \%$ \\
\hline 0.5 & 4861 & 2841 & $41.56 \%$ & 2020 & 1100 & $64.18 \%$ \\
\hline \multicolumn{7}{|l|}{ Reasenberg } \\
\hline \multicolumn{7}{|l|}{ Rand Mag } \\
\hline w10 & 4907 & 4906 & $0.02 \%$ & 1 & 2 & $0.06 \%$ \\
\hline \multicolumn{7}{|l|}{ Fixed $M=2.5$} \\
\hline w10 & 4892 & 4144 & $15.29 \%$ & 748 & 1161 & $39.02 \%$ \\
\hline w20 & 4851 & 4058 & $16.35 \%$ & 793 & 1145 & $39.95 \%$ \\
\hline w30 & 4812 & 4026 & $16.33 \%$ & 786 & 1156 & $40.36 \%$ \\
\hline \multicolumn{7}{|l|}{ Fixed $M=3.0$} \\
\hline w10 & 4892 & 4144 & $15.29 \%$ & 748 & 1161 & $39.02 \%$ \\
\hline $\mathrm{w} 20$ & 4851 & 4058 & $16.35 \%$ & 793 & 1145 & $39.95 \%$ \\
\hline w30 & 4812 & 4026 & $16.33 \%$ & 786 & 1156 & $40.36 \%$ \\
\hline \multicolumn{7}{|l|}{ Gardner and Knopoff } \\
\hline \multicolumn{7}{|l|}{ Rand Mag } \\
\hline w10 & 4907 & 4907 & $0.00 \%$ & 0 & 83 & $1.69 \%$ \\
\hline \multicolumn{7}{|l|}{ Fixed $M=2.5$} \\
\hline w10 & 4892 & 4891 & $0.02 \%$ & 1 & 705 & $14.43 \%$ \\
\hline w20 & 4814 & 4814 & $0.00 \%$ & 0 & 700 & $14.54 \%$ \\
\hline w30 & 4812 & 4809 & $0.06 \%$ & 3 & 712 & $14.86 \%$ \\
\hline \multicolumn{7}{|l|}{ Fixed $M=3.0$} \\
\hline w10 & 4892 & 4892 & $0.00 \%$ & 0 & 409 & $8.36 \%$ \\
\hline \multicolumn{7}{|l|}{ CURATE } \\
\hline \multicolumn{7}{|l|}{ w/o day-rule } \\
\hline w10* & 4856 & 2653 & $45.36 \%$ & 2203 & 713 & $60.05 \%$ \\
\hline $\mathrm{w} 20 *$ & 4859 & 2658 & $45.34 \%$ & 2201 & 714 & $59.99 \%$ \\
\hline w30* & 4860 & 2654 & $45.41 \%$ & 2206 & 713.5 & $60.07 \%$ \\
\hline day-rule 2 & 4861 & 2809 & $42.21 \%$ & 2052 & 656 & $55.71 \%$ \\
\hline day-rule 3 & 4861 & 2956 & $39.19 \%$ & 1905 & 502 & $49.52 \%$ \\
\hline day-rule 3.5 & 4861 & 2998 & $38.33 \%$ & 1863 & 413 & $46.82 \%$ \\
\hline day-rule 4 & 4861 & 3032 & $37.63 \%$ & 1829 & 355 & $44.93 \%$ \\
\hline day-rule 5 & 4861 & 3059 & $37.07 \%$ & 1802 & 248 & $42.17 \%$ \\
\hline
\end{tabular}


Before running the SLC method through the code we anticipated that it would perform similarly to the CURATE method because without the distance element the link calculation boils down to a time link. So DST in this test, if $\mathrm{C}=1$, is simply a maximum time between individual events. We left $\mathrm{C}=1$ for all tests to try to make the relationship to the average time as clear as possible. The average rate of earthquakes in the real (and simulated Poisson catalogs) is $\sim 1.08$ days between earthquakes. So we might expect that a DST=1 would give similar results to the CURATE method, with all earthquakes within one day of each other included in sequences. However the Table 3.1 shows that at a value of DST=1 yields a smaller declustered catalog (both with and without mainshock replacement) than the CURATE. By defining the link instead of the earthquake that has occurred before an average or expected recurrence time, there is an implicit assumption, albeit less obvious than for Reasenberg, Gardner and Knopoff, or other methods, that earthquake occurrence is caused by preceding seismicity. Thus it is possible that in some instances where the time between earthquakes is shorter than the average time, the SLC method that ends up linking two earthquakes where the CURATE method may only identify one of those earthquakes as unusual in time.

Another possible test of the CURATE method is to apply the day and distance rules or run the full code. The distance rule will keep all events if they all share a common location, but the day rule may act to concatenate sequences (including adding sequences of 1 event to sequences with more events, thus preventing its addition to the declustered catalog). So when the day rule is applied both the number of earthquakes that are not part of a cluster and the overall number of clusters decreases. These decrease by relatively similar amounts, resulting in a decreased declustered catalog. While all clustering methods will choose some clusters even if 
the initial temporal distribution is Poisson, because the CURATE uses the mean-time, it is sensitive to the temporal distribution of events and performs well in this test. Thus the CURATE method takes out more than is necessary from a Poisson temporal distribution, but in most cases would still produce a declustered catalog similar or larger to that produced by other methods.

\subsubsection{CURATE of the Declustered Catalog}

Having created synthetic catalogs with Poisson temporal distributions we can show what CURATE applied to such a catalog would look like. Figure 3.2 shows that the cumulative rate of the synthetic Poisson catalog is nearly linear and thus the CURATE has a very shaggy appearance as most deviations from the mean are very small. Most deviations are of similar size with no outstanding periods of increase. This looks very similar to the synthetic ETAS catalogs produced earlier in Chapter 2 (Fig. 2.8). This is the kind of plot we should anticipate in our declustered catalogs, and we will use CURATE plots of the declustered catalogs as another indicator of whether they have Poisson behavior. 


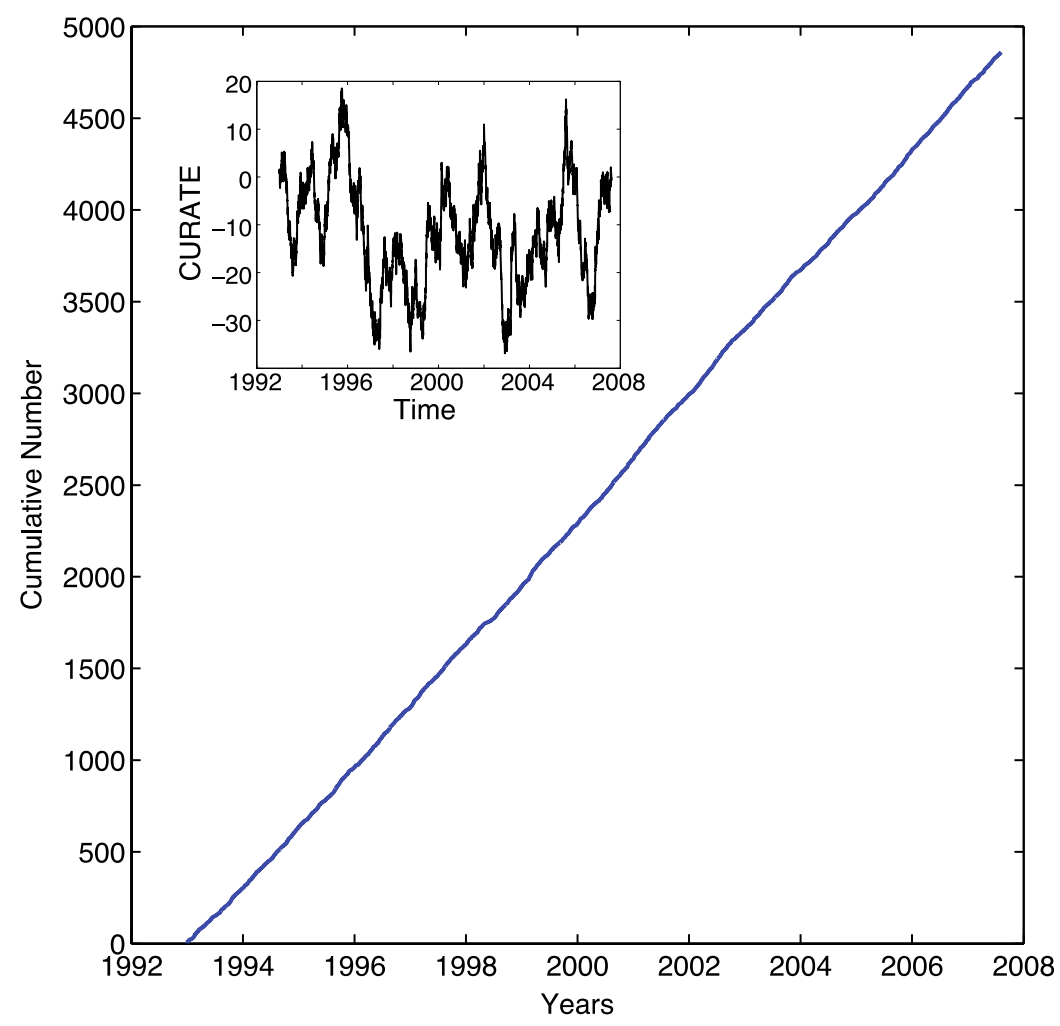

Figure 3.2

Cumulative number of earthquakes for synthetic seismicity catalog with a Poisson temporal distribution. Inset shows the CURATE of the same catalog.

\subsection{Southern California: Testing the influence of Mcut}

\subsubsection{Southern California, Complete Catalog:}

Southern California has been instrumented since 1932 [Hutton 2010] and has produced a large earthquake catalog. The present network there operated by the Southern California Earthquake Data Center has over 400 stations [Hauksson et al., 2012; Hutton et al., 2010]. For details on the history of instrumentation, routine processing procedures, and Mc and b-value data see Hutton et al. [2010].

We applied the CURATE method to the Southern California waveform relocated earthquake catalog of Hauksson et al., [2012]. Hauksson et al. [2012] use waveform cross-correlation and other techniques [Lin et al., 2007] as well as a 3-D velocity model (where possible) to relocate the Southern California Seismic Network (SCSN) catalog. These are the only data we use from Southern California, but to identify its geographic location we will refer to it as the SCA catalog although some 
authors refer to the specific relocated catalog as the HYS catalog [Hauksson et al., 2012]. We compare sequences and swarms from this area to those found in the CVR.

To make the sequence catalog comparable, we used a magnitude cutoff of $\mathrm{Mc}=2.45$, well above the reported Mc for time periods 1981-2008 of 1.8 [Hutton et al., 2010]. The catalog at that magnitude cutoff contains 44174 earthquakes (Fig. 3.3). There are thirteen $\mathrm{M} \geq 6$, and three $\mathrm{M} \geq 7(\mathrm{Mw}=7.3,1992$ Landers; $\mathrm{Mw}=7.1,1999$ Hector Mine; and the $\mathrm{Mw}=7.2$ El Mayor-Cucapah) earthquakes during this time period.

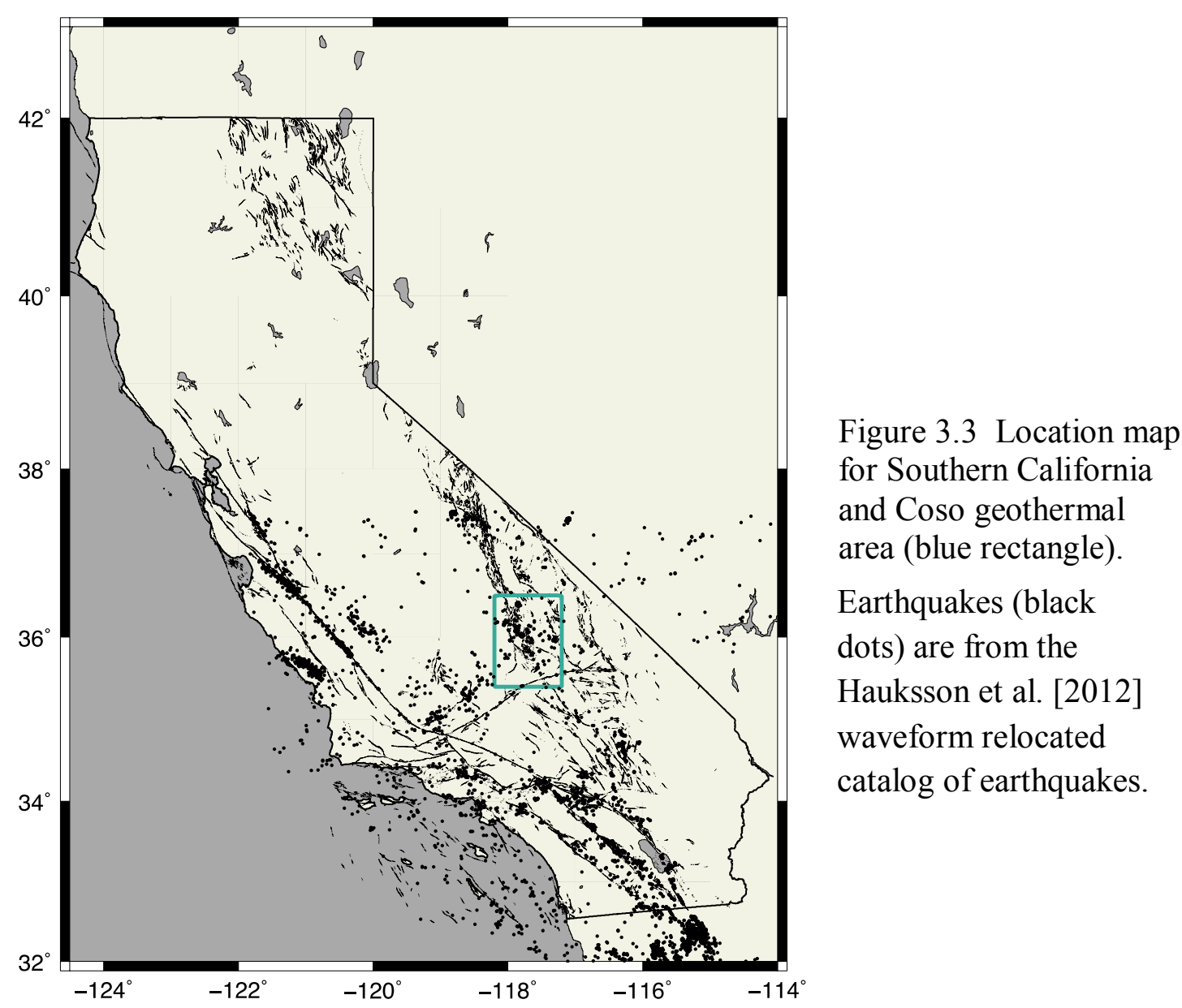

To make sequence catalogs for this dataset we used a range of selection parameters from 15-25 $\mathrm{km}$ and 3-7 days. After creating the various sequence catalogs we tested the declustered catalogs to find the best selection parameters. Unfortunately, the chi-squared tests gave no Poisson results. As an example, for 
selection parameters of $20 \mathrm{~km}$ and 3 days the declustered catalog contains 23588

earthquakes (53.4\%) and did not return any Poisson results from the chi-squared tests.

The dispersion values are also considerably higher than those produced for the CVR declustered catalog (Table 3.2 and Table 2.6). The declustered catalog is composed of two primary elements: the earthquakes that are not part of any sequence, and a representative earthquake from each identified sequence (mainshock replacement). These pieces are almost always tested as a single entity, but throughout this chapter we will show both the declustered catalog without mainshock-replacement (WoMSR) and the whole declustered catalog (with mainshock-replacement, referred to as 'All Declus' in the tables throughout the chapter).

Table 3-2 Poisson testing of the SCA catalog. 'WoMsr' is the declustered catalog without-mainshock replacement and 'All Declus' is the whole declustered catalog (with mainshock-replacement). Degrees of Freedom has been abbreviated as DOF.

\begin{tabular}{|c|c|c|c|c|c|c|}
\hline & & & $\%$ Poisson & Variance/ Mean & \# eqs & DOF \\
\hline & & & \multicolumn{4}{|c|}{ 1981-2011 mean rate (1448.8) } \\
\hline \multirow[t]{6}{*}{20} & 3 & WoMsr & $0.00 \%$ & 3.46 & 22222 & 23 \\
\hline & 3 & All Declus & $0.00 \%$ & 4.17 & 24011 & 23 \\
\hline & 4 & WoMsr & $0.00 \%$ & 3.36 & 22064 & 23 \\
\hline & 4 & All Declus & $0.00 \%$ & 3.97 & 23777 & 24 \\
\hline & 5 & WoMsr & $0.00 \%$ & 3.30 & 21930 & 23 \\
\hline & 5 & All Declus & $0.00 \%$ & 3.84 & 23588 & 22 \\
\hline \multirow[t]{6}{*}{25} & 3 & WoMsr & $0.00 \%$ & 3.09 & 21786 & 22 \\
\hline & 3 & All Declus & $0.00 \%$ & 3.74 & 23588 & 22 \\
\hline & 4 & WoMsr & $0.00 \%$ & 3.02 & 21623 & 22 \\
\hline & 4 & All Declus & $0.00 \%$ & 3.58 & 23345 & 23 \\
\hline & 5 & WoMsr & $0.00 \%$ & 2.98 & 21478 & 22 \\
\hline & 5 & All Declus & $0.00 \%$ & 3.47 & 23154 & 23 \\
\hline \multirow[t]{6}{*}{30} & 3 & WoMsr & $0.00 \%$ & 2.90 & 21472 & 22 \\
\hline & 3 & All Declus & $0.00 \%$ & 3.47 & 23258 & 23 \\
\hline & 4 & WoMsr & $0.00 \%$ & 2.83 & 21304 & 22 \\
\hline & 4 & All Declus & $0.00 \%$ & 3.32 & 23021 & 23 \\
\hline & 5 & WoMsr & $0.00 \%$ & 2.80 & 21154 & 22 \\
\hline & 5 & All Declus & $0.00 \%$ & 3.23 & 22833 & 23 \\
\hline
\end{tabular}


The large dispersion values 2.8-4.2 indicate that there is still a large degree of clustering in the declustered catalog. The most likely cause of this is that large earthquakes and their aftershocks skew the mean rate towards high values, leading to an inefficient identification of clustering as few time periods exceed these high rates. This is an effect of the CURATE being a relative method. Relative to the clustering caused by large earthquakes in the catalog, the other clustering pales in comparison. Essentially, there is a breakdown of the assumption of the method that the mean rate is a reasonable estimate of the background rate. We also note that some authors use a negative-binomial distribution to model seismicity rates to avoid the need for declustering (e.g. [Jackson and Kagan, 1999; Kagan and Jackson, 2000]). The Poisson distribution is generally thought to fit the declustered catalog better, but the negative-binomial distribution has a larger variance and may be easier to fit in some instances [Kagan and Jackson, 2000].

The CURATE plot shows the dominant effects of these large earthquakes on the rate (Fig. 3.4A). The large earthquakes, and their associated aftershocks, clearly dominate the number of events (sharp increases) with the trend for times outside large aftershock sequences continuously decreasing (relative to the skewed mean rate). The rate during large aftershock sequences is clearly elevated by the large events and is not representative of time periods or geographic locations without large events. To show how the mean rate is skewed by large events we show a calculation of the percent of earthquakes with various inter-event times for the SCA and CVR catalogs (Fig. 3.4B). There is a clear difference in slope in the cumulative number for the two catalogs. The large number of very small inter-event times (time between earthquakes) skews the mean rate of the SCA catalog to a small value. The 
dominance of aftershock sequences results in less earthquakes being identified as part of sequences, leading to larger declustered catalogs that may still contain clustering.

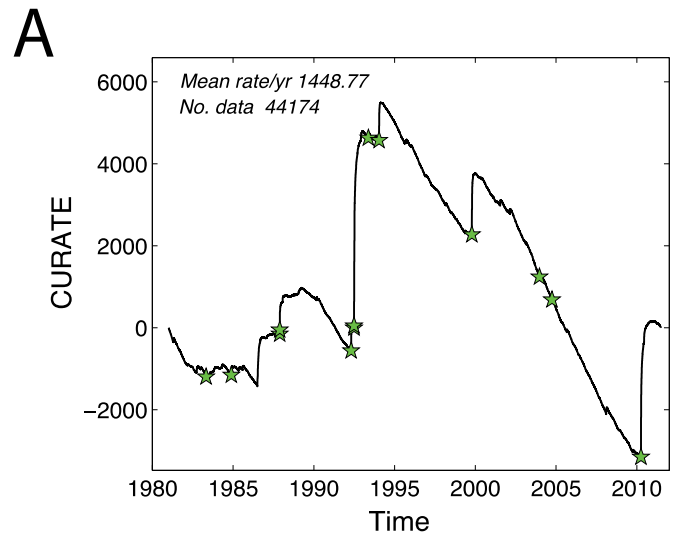

B

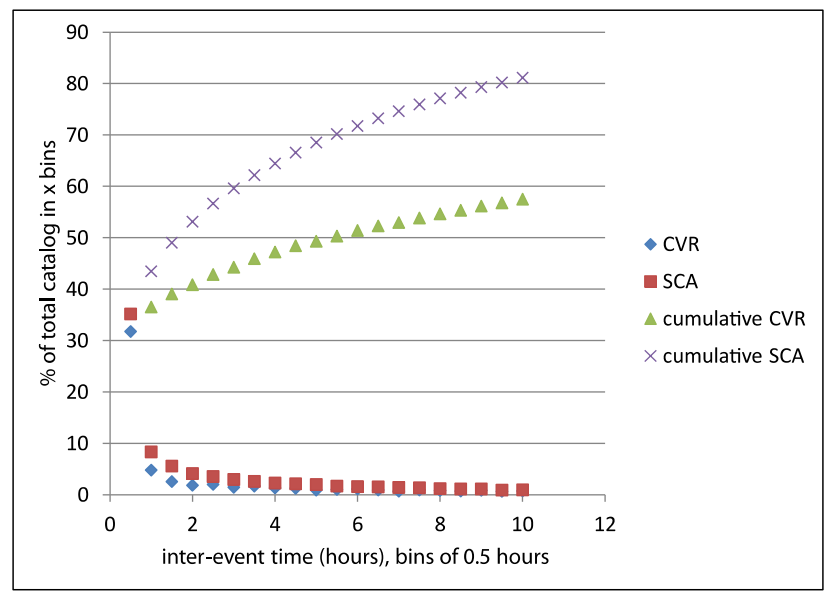

Figure 3.4 Two figures relating to the effects of large earthquakes on the overall rate of events.

A) CURATE of the Southern California catalog. Green stars represent the time of $\mathrm{M}$ $\geq 6.0$ earthquakes. B) Histogram of the percent of an earthquake catalog that falls into various interevent time bins as well as the cumulative percentage for both the CVR and SCA catalogs.

This problem is found with the CURATE method, and to some extent the ETAS method, but not in other clustering methods because most methods do not take into account a background rate, and put earthquakes into clusters on the basis of whether they fit a certain time/distance profile. The attempt of the CURATE method to initially reject a certain amount of the earthquake catalog from possible inclusion into sequences makes it unfortunately very sensitive to such rate changes. The CURATE method is similar to the ETAS method (Section 2.4.5) in this respect. Over 
certain time periods or regions with similar behavior a set of ETAS parameters (decay, background, mag dependence) can be obtained that can accurately model seismicity. However, if background rate or other parameters change across time or space the ETAS model is forced to use values that satisfy the total number of earthquakes, but may fail to reflect the behavior of the real catalog.

\subsubsection{Lower Rate Limits}

We hypothesize that there is still a stable background rate in regions affected by large aftershock sequences, but that it is overshadowed when we use a simple mean rate. To try to find an appropriate rate limit (lower than the mean rate) to model this stable background rate, we created random year-long segments of the catalog. First we chose 100 random decimal year values and then counted the number of earthquakes in the catalog during a one year period from those dates. This creates a kind of random re-sampling of the possible data. After calculating the 100 yearly rates, we then find, and exclude any of those periods that include $M \geq 6$ earthquakes. Finally we calculate the median and the minimum of the 100 random time samples that do not have any large earthquakes in the yearly observation. This may still include parts of the aftershock sequence of (preceding) large earthquakes. Both the minimum and the median values are tested because we want to lower the rate sufficiently to remove the clustering, but to avoid putting earthquakes into clusters unnecessarily. These random yearly rates do show significant variation with time (Fig. 3.5). Because it appears that the earlier part of the catalog is more variable we additionally used the catalog after 1994 (starting 1995) with both the median and minimum rate. 


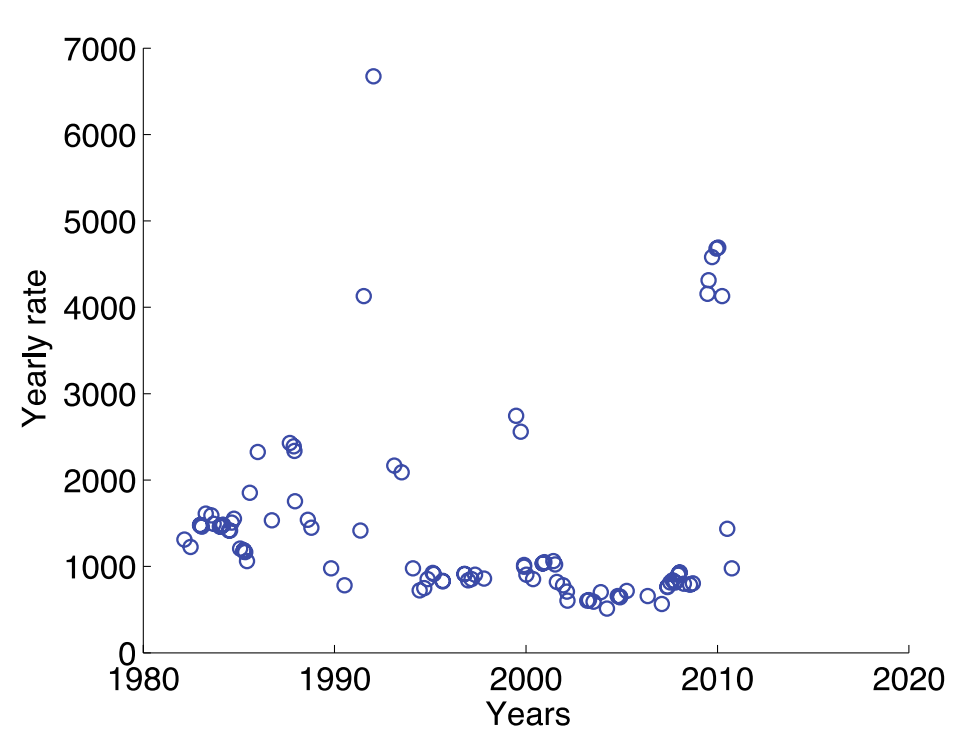

Figure 3.5 Yearly earthquake totals from 100 initial times chosen randomly for the Southern California dataset.

See text for discussion on the variations in rate.

The catalog was run through the CURATE method again with the median and minimum rates using the same range of selection parameters (Table 3.3). The median rate (768) is nearly half of the original mean rate (1448.8) used initially. Despite the reduction of nearly fifty-percent, the number of earthquakes in the declustered catalogs has been reduced by only about fifteen-percent (Fig. 3.6). The dispersion values are lower (1.84-2.65), but none of the selection parameter combinations return any Poisson results for the chi-squared tests. The minimum-rate, for the shortened time period, is thirty-percent lower again than the median rate and it reduces the number of earthquakes in the declustered catalog by more than half (compared to the mean-rate). This significantly improves the dispersion values, and returns Poisson fractions for the declustered catalog WoMsr of up to $76 \%$ Poisson, but less than $20 \%$ for the whole declustered catalog (Table 3.3). Limiting the time period and using the lowest rate that can be justified still has not produced completely Poisson declustered catalogs. 
To further reduce the rate variations we split the shorter time period still further, into an early (1995-2001) and late (2002-2011) time period. This reduction produces nearly perfect Poisson results in the early time period (Table 3.4). The late time period, however, shows a different behavior. The dispersion values of the later time period are close to one (1.07-1.65), but the lowest dispersion values (1.07-1.26) all come from the declustered catalog WoMsr. The whole declustered catalog dispersion values (1.31-1.65) are still above one and give no Poisson results in the chi-squared tests (Table 3.4, Fig. 3.7). The good chi-squared results for the WoMsr catalog indicates that reducing the rate and time period further will not eliminate this problem, and we will address this issue in greater depth in the discussion.

Table 3-3 Poisson testing of the SCA catalog with median and minimum rates

\begin{tabular}{|c|c|c|c|c|c|c|c|c|c|c|}
\hline & & & & & & & & & \\
\hline & & & $\begin{array}{c}\% \\
\text { Poisson }\end{array}$ & $\begin{array}{c}\text { Variance/ } \\
\text { Mean }\end{array}$ & \# eqs & DOF & $\begin{array}{c}\% \\
\text { Poisson }\end{array}$ & $\begin{array}{c}\text { Variance/ } \\
\text { Mean }\end{array}$ & $\begin{array}{l}\# \\
\text { eqs }\end{array}$ & DOF \\
\hline & & & \multicolumn{4}{|c|}{$1981-2011$ median rate (768) } & \multicolumn{4}{|c|}{$1995-2011$ min rate (551) } \\
\hline \multirow[t]{6}{*}{20} & 3 & WoMsr & $0.00 \%$ & 2.08 & 19398 & 21 & $30.85 \%$ & 1.18 & 7974 & 17 \\
\hline & 3 & All Declus & $0.00 \%$ & 2.65 & 21561 & 22 & $0.00 \%$ & 1.50 & 9052 & 18 \\
\hline & 4 & WoMsr & $0.00 \%$ & 1.99 & 19173 & 20 & $45.77 \%$ & 1.15 & 7888 & 17 \\
\hline & 4 & All Declus & $0.00 \%$ & 2.50 & 21257 & 22 & $0.00 \%$ & 1.43 & 8931 & 17 \\
\hline & 5 & WoMsr & $0.00 \%$ & 1.94 & 19004 & 20 & $54.73 \%$ & 1.13 & 7824 & 17 \\
\hline & 5 & All Declus & $0.00 \%$ & 2.42 & 21041 & 22 & $0.00 \%$ & 1.38 & 8839 & 18 \\
\hline \multirow[t]{6}{*}{25} & 3 & WoMsr & $0.00 \%$ & 1.93 & 18987 & 21 & $41.79 \%$ & 1.10 & 7826 & 17 \\
\hline & 3 & All Declus & $0.00 \%$ & 2.47 & 21192 & 22 & $0.00 \%$ & 1.36 & 8892 & 18 \\
\hline & 4 & WoMsr & $0.00 \%$ & 1.85 & 18750 & 21 & $51.74 \%$ & 1.07 & 7729 & 17 \\
\hline & 4 & All Declus & $0.00 \%$ & 2.33 & 20873 & 21 & $0.00 \%$ & 1.30 & 8769 & 18 \\
\hline & 5 & WoMsr & $0.00 \%$ & 1.79 & 18545 & 21 & $57.21 \%$ & 1.05 & 7658 & 17 \\
\hline & 5 & All Declus & $0.00 \%$ & 2.23 & 20615 & 22 & $1.00 \%$ & 1.26 & 8667 & 18 \\
\hline \multirow[t]{6}{*}{30} & 3 & WoMsr & $0.00 \%$ & 1.84 & 18676 & 21 & $65.17 \%$ & 1.06 & 7670 & 17 \\
\hline & 3 & All Declus & $0.00 \%$ & 2.33 & 20901 & 21 & $0.00 \%$ & 1.30 & 8768 & 18 \\
\hline & 4 & WoMsr & $0.00 \%$ & 1.77 & 18427 & 21 & $75.62 \%$ & 1.03 & 7552 & 16 \\
\hline & 4 & All Declus & $0.00 \%$ & 2.21 & 20560 & 22 & $1.49 \%$ & 1.24 & 8625 & 18 \\
\hline & 5 & WoMsr & $0.00 \%$ & 1.71 & 18204 & 20 & $76.12 \%$ & 1.00 & 7476 & 16 \\
\hline & 5 & All Declus & $0.00 \%$ & 2.11 & 20278 & 22 & $19.40 \%$ & 1.20 & 8523 & 17 \\
\hline
\end{tabular}


Table 3-4 Poisson testing using minimum rate threshold for various time periods of the SCA catalog

\begin{tabular}{|c|c|c|c|c|c|c|c|c|c|c|c|c|c|c|}
\hline & & & $\begin{array}{c}\% \\
\text { Poisson }\end{array}$ & $\begin{array}{l}\text { Variance/ } \\
\text { Mean }\end{array}$ & $\begin{array}{c}\# \\
\text { eqs }\end{array}$ & DOF & $\begin{array}{c}\% \\
\text { Poisson }\end{array}$ & $\begin{array}{c}\text { Variance/ } \\
\text { Mean }\end{array}$ & $\begin{array}{l}\# \\
\text { eqs }\end{array}$ & DOF & $\begin{array}{c}\% \\
\text { Poisson }\end{array}$ & $\begin{array}{c}\text { Variance/ } \\
\text { Mean }\end{array}$ & $\begin{array}{c}\# \\
\text { eqs }\end{array}$ & DOF \\
\hline & & & \multicolumn{4}{|c|}{$1995-2011$ min rate } & \multicolumn{4}{|c|}{$1995-2001 \mathrm{~min}$ rate } & \multicolumn{4}{|c|}{ 2002-2011 min rate } \\
\hline \multirow[t]{6}{*}{20} & 3 & WoMsr & $30.85 \%$ & 1.18 & 7974 & 17 & $98.51 \%$ & 0.97 & 7974 & 15 & $100 \%$ & 1.26 & 7974 & 14 \\
\hline & 3 & All Declus & $0.00 \%$ & 1.50 & 9052 & 18 & $87.56 \%$ & 1.22 & 9052 & 15 & $0 \%$ & 1.65 & 9052 & 15 \\
\hline & 4 & WoMsr & $45.77 \%$ & 1.15 & 7888 & 17 & $93.03 \%$ & 0.95 & 7888 & 15 & $100 \%$ & 1.23 & 7888 & 15 \\
\hline & 4 & All Declus & $0.00 \%$ & 1.43 & 8931 & 17 & $92.04 \%$ & 1.17 & 8931 & 16 & $0 \%$ & 1.55 & 8931 & 15 \\
\hline & 5 & WoMsr & $54.73 \%$ & 1.13 & 7824 & 17 & $96.52 \%$ & 0.94 & 7824 & 14 & $100 \%$ & 1.21 & 7824 & 15 \\
\hline & 5 & All Declus & $0.00 \%$ & 1.38 & 8839 & 18 & $97.01 \%$ & 1.15 & 8839 & 16 & $0 \%$ & 1.49 & 8839 & 16 \\
\hline \multirow[t]{6}{*}{25} & 3 & WoMsr & $41.79 \%$ & 1.10 & 7826 & 17 & $92.04 \%$ & 0.90 & 7826 & 14 & $100 \%$ & 1.17 & 7826 & 15 \\
\hline & 3 & All Declus & $0.00 \%$ & 1.36 & 8892 & 18 & $95.52 \%$ & 1.13 & 8892 & 16 & $0 \%$ & 1.49 & 8892 & 16 \\
\hline & 4 & WoMsr & $51.74 \%$ & 1.07 & 7729 & 17 & $92.54 \%$ & 0.88 & 7729 & 14 & $100 \%$ & 1.14 & 7729 & 15 \\
\hline & 4 & All Declus & $0.00 \%$ & 1.30 & 8769 & 18 & $99.50 \%$ & 1.07 & 8769 & 15 & $0 \%$ & 1.42 & 8769 & 16 \\
\hline & 5 & WoMsr & $57.21 \%$ & 1.05 & 7658 & 17 & $93.03 \%$ & 0.88 & 7658 & 14 & $100 \%$ & 1.12 & 7658 & 15 \\
\hline & 5 & All Declus & $1.00 \%$ & 1.26 & 8667 & 18 & $97.51 \%$ & 1.06 & 8667 & 15 & $0 \%$ & 1.36 & 8667 & 16 \\
\hline \multirow[t]{6}{*}{30} & 3 & WoMsr & $65.17 \%$ & 1.06 & 7670 & 17 & $95.02 \%$ & 0.87 & 7670 & 14 & $100 \%$ & 1.14 & 7670 & 15 \\
\hline & 3 & All Declus & $0.00 \%$ & 1.30 & 8768 & 18 & $97.51 \%$ & 1.08 & 8768 & 15 & $0 \%$ & 1.42 & 8768 & 16 \\
\hline & 4 & WoMsr & $75.62 \%$ & 1.03 & 7552 & 16 & $95.02 \%$ & 0.85 & 7552 & 15 & $100 \%$ & 1.10 & 7552 & 15 \\
\hline & 4 & All Declus & $1.49 \%$ & 1.24 & 8625 & 18 & $98.01 \%$ & 1.03 & 8625 & 15 & $0 \%$ & 1.34 & 8625 & 15 \\
\hline & 5 & WoMsr & $76.12 \%$ & 1.00 & 7476 & 16 & $93.53 \%$ & 0.84 & 7476 & 14 & $100 \%$ & 1.07 & 7476 & 14 \\
\hline & 5 & All Declus & $19.40 \%$ & 1.20 & 8523 & 17 & $98.51 \%$ & 1.00 & 8523 & 15 & $0 \%$ & 1.31 & 8523 & 15 \\
\hline
\end{tabular}




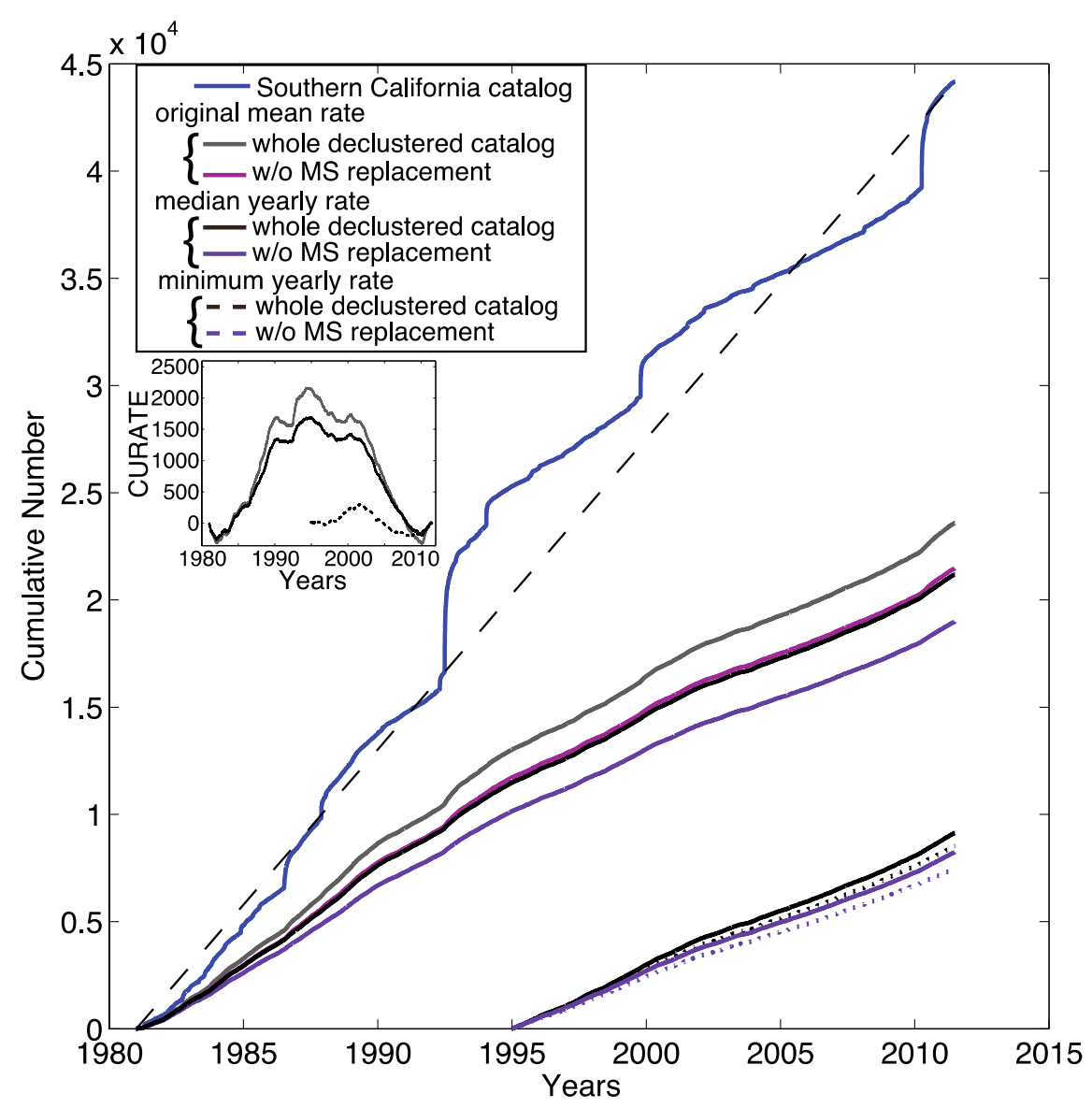

Figure 3.6 Cumulative number of earthquakes with time for the original and various declustered catalogs for the Southern California dataset.

The original catalog is shown in blue, the whole declustered catalogs in gray and black, and the declustered catalogs without-mainshock replacement are plotted in pink and purple. Dashed line approximates the mean rate of the original catalog. Gray and pink lines correspond to declustered catalogs obtained using the mean-rate. Black and purple lines represent declustered catalogs obtained using the median-rate. Inset shows the CURATE of the whole declustered catalog for mean (gray) and median rate (black). Median (black and purple, solid) and minimum rates (black and purple, dashed) are also shown for the period 1995-2012. 

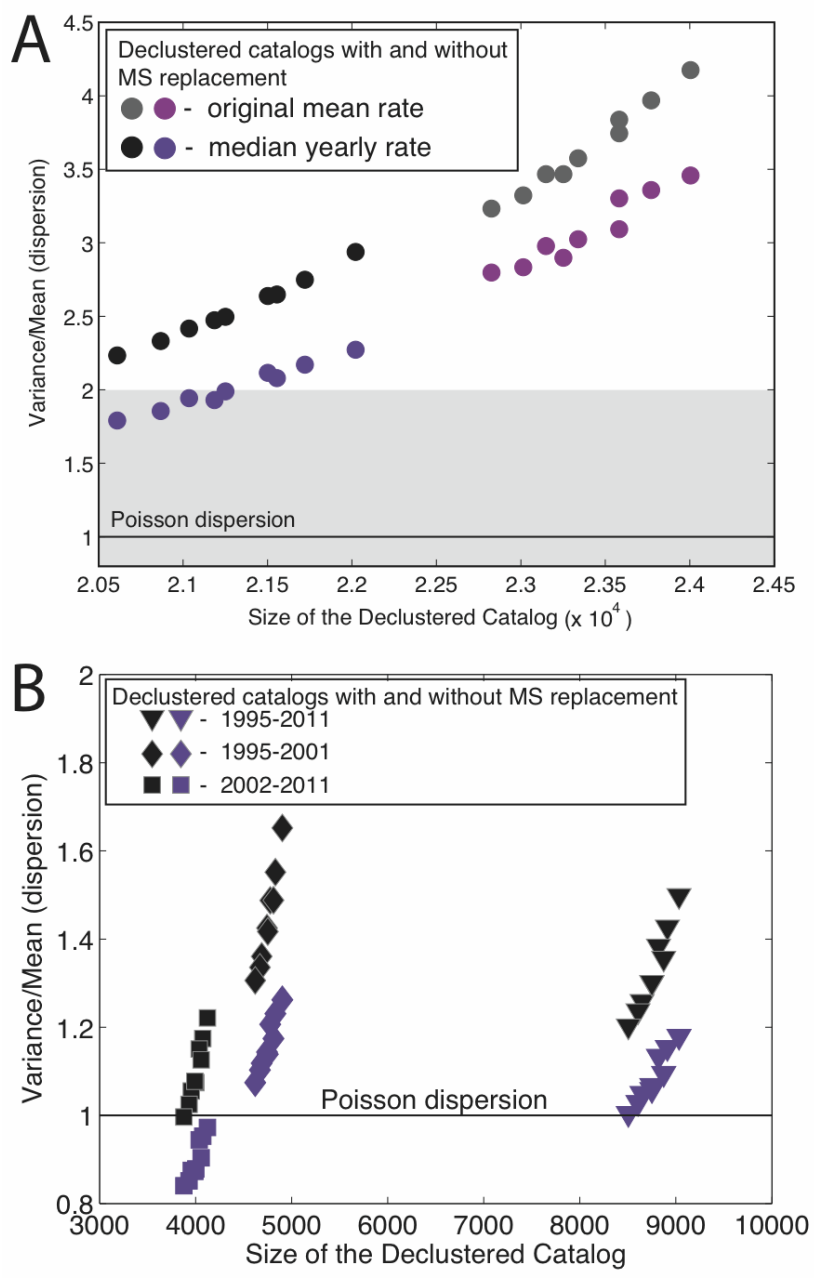

Figure 3.7 Dispersion values for the declustered catalog of the Southern California dataset. Declustered catalogs obtained using the mean-rate are shown in gray (with mainshock replacement) and pink (without mainshock-replacement). Declustered catalogs obtained using the median rate are shown in black (with mainshock-replacement) and purple (without mainshock replacement). A) Comparison of mean and median rate techniques B) Minimum rate technique over 3 different time periods (see legend for shapes of each time period).

\subsubsection{Coso Region Subset}

We also applied the CURATE method on a smaller subset of SCA around the Coso geothermal region (boundaries: 35.4:36.5, -117.2:-118 Figure 3.3). The seismic setting and the number of earthquakes are comparable to the TVZ (TVZ, 4845; Coso, $3045>=2.45$ ). The Coso region surrounding the geothermal area is a boundary between a transpressive regime to the East and an extensional regime to the West 
[Bhattacharyya and Lees, 2002]. Like the TVZ, the seismicity there is mostly small magnitude $(\mathrm{M}<3)$ earthquakes that often occur in tectonic and geothermally driven swarm sequences [Bhattacharyya and Lees, 2002]. The lower Mc and the relocated catalog [Hauksson et al., 2012] will also allow us to more easily look at Mc and spatial effects.

Given the findings of changing dispersion values with changing Mcut in the previous section (Fig. 3.7), we want to ensure that the CURATE itself is not sensitive to a selected value of Mcut $\geq$ Mc. The low Mc of 1.45 in the Coso region makes the area a good target for such a test. Figure 3.8 shows the CURATE plots for three different Mcut values. The shape of each plot is nearly identical, including points of upturn, despite the different scaling due to Mcut variation. This gives us confidence that we can identify the same sequences at a variety of magnitude cutoffs and that the sequence catalogs produced by the CURATE method are not dependent on the Mc of the catalog.
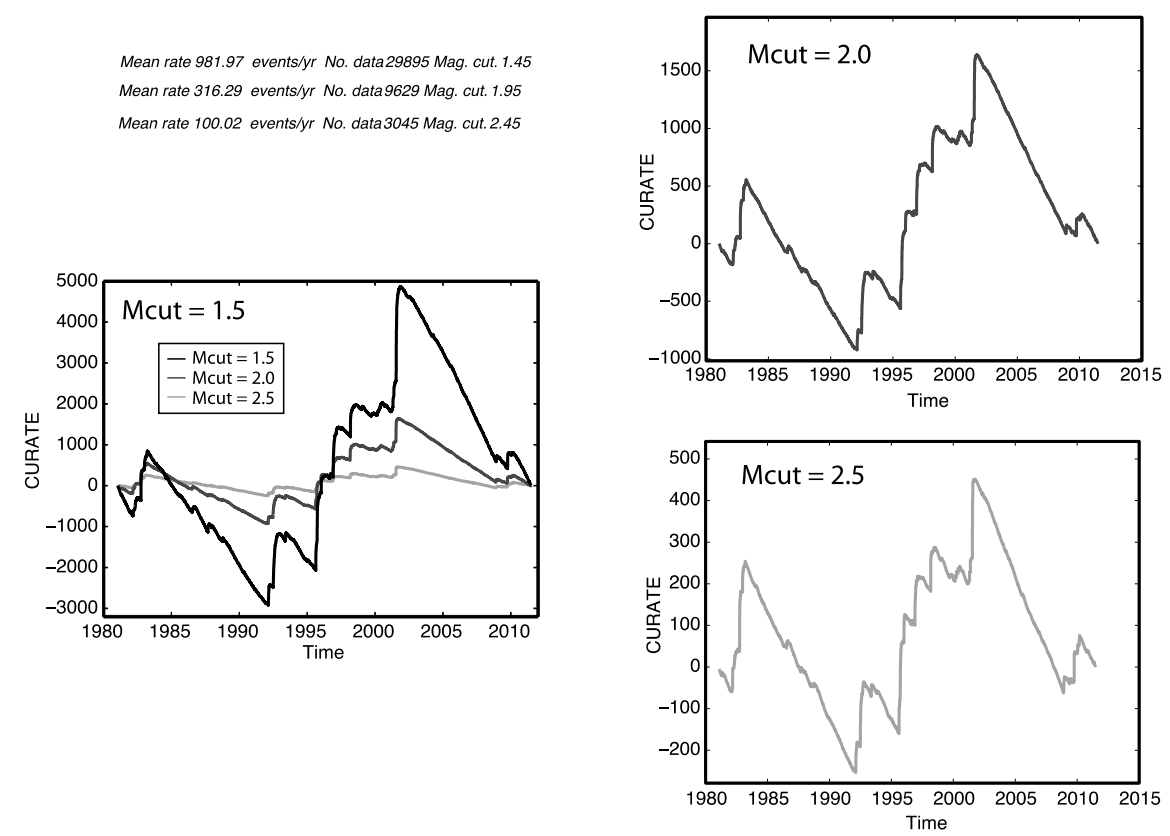

Figure 3.8 CURATE plots for the Coso subset at various Mcut values. 
To compare this area to the CVR we ran the CURATE method on the Coso Catalog at an Mcut of 2.45 and a range of selection parameters from 10-25 km and 25 days (Table 3.5, Fig. 3.9). The $10 \mathrm{~km}$ distance-rule returns the worst chi-squared results. Most day rules for distance rules of 15-25 km give good dispersion values and Poisson results in the chi-squared test for the declustered catalog WoMSR. However, the results for the whole declustered catalog are poor with no chi-squared test values above $11 \%$ for any of the tested selection parameters. The good results of the WoMSR catalog indicate that the mean rate is sufficient to identify clustering and that a median or minimum rate is unnecessary. 


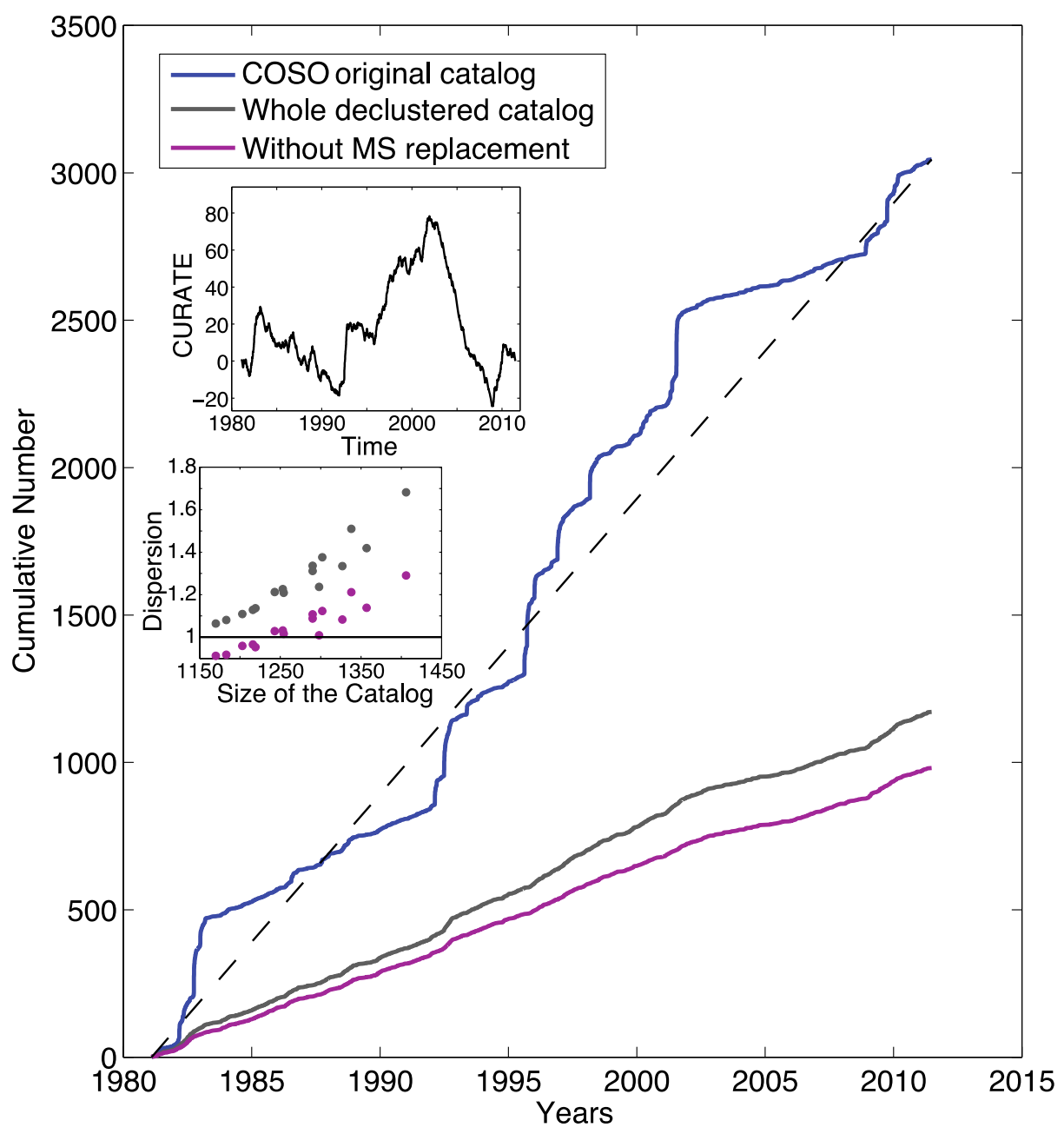

Figure 3.9 Comparison of the original and declustered catalogs for data from the Coso geothermal regional subset.

Cumulative number of earthquakes with time for the original, declustered, and declustered-without-mainshock replacement (WoMSR) catalogs. Insets show the CURATE of the declustered catalog with mainshock replacement and dispersion with size of the declustered catalogs for the range of tested selection parameters. The dashed line approximates the mean rate of the original catalog. 
Table 3-5 Poisson testing of the Coso subset declustered catalog using the CURATE method with a minimum rate.

Distance-rule is given in kilometeres and Degrees of Freedom has been abbreviated DOF.

\begin{tabular}{|c|c|c|c|c|c|c|}
\hline \multirow{2}{*}{$\begin{array}{l}\text { Dist- } \\
\text { rule }\end{array}$} & \multirow{2}{*}{$\begin{array}{l}\text { Day- } \\
\text { rule }\end{array}$} & \multirow{2}{*}{$\begin{array}{c}\text { Catalog } \\
\text { Type }\end{array}$} & \% Poisson & $\begin{array}{c}\text { Variance/ } \\
\text { Mean }\end{array}$ & \# eqs & DOF \\
\hline & & & \multicolumn{4}{|c|}{$1981-2011 \mathrm{~min}$ rate } \\
\hline \multirow[t]{8}{*}{10} & 2 & WoMsr & $0.00 \%$ & 1.29 & 1202 & 5 \\
\hline & 2 & All Declus & $0.00 \%$ & 1.68 & 1407 & 5 \\
\hline & 3 & WoMsr & $7.96 \%$ & 1.21 & 1150 & 4 \\
\hline & 3 & All Declus & $0.00 \%$ & 1.51 & 1339 & 5 \\
\hline & 4 & WoMsr & $54.23 \%$ & 1.12 & 1121 & 4 \\
\hline & 4 & All Declus & $0.00 \%$ & 1.38 & 1303 & 5 \\
\hline & 5 & WoMsr & $76.12 \%$ & 1.11 & 1113 & 4 \\
\hline & 5 & All Declus & $0.00 \%$ & 1.34 & 1291 & 5 \\
\hline \multirow[t]{8}{*}{15} & 2 & WoMsr & $40.80 \%$ & 1.14 & 1150 & 4 \\
\hline & 2 & All Declus & $0.00 \%$ & 1.42 & 1358 & 5 \\
\hline & 3 & WoMsr & $88.56 \%$ & 1.09 & 1097 & 4 \\
\hline & 3 & All Declus & $0.00 \%$ & 1.31 & 1291 & 5 \\
\hline & 4 & WoMsr & $93.03 \%$ & 1.03 & 1071 & 4 \\
\hline & 4 & All Declus & $0.00 \%$ & 1.23 & 1254 & 5 \\
\hline & 5 & WoMsr & $97.51 \%$ & 1.03 & 1067 & 4 \\
\hline & 5 & All Declus & $0.00 \%$ & 1.21 & 1244 & 5 \\
\hline \multirow[t]{8}{*}{20} & 2 & WoMsr & $89.05 \%$ & 1.08 & 1113 & 4 \\
\hline & 2 & All Declus & $0.00 \%$ & 1.33 & 1328 & 5 \\
\hline & 3 & WoMsr & $99.00 \%$ & 1.02 & 1053 & 4 \\
\hline & 3 & All Declus & $0.00 \%$ & 1.21 & 1255 & 5 \\
\hline & 4 & WoMsr & $94.03 \%$ & 0.97 & 1024 & 4 \\
\hline & 4 & All Declus & $0.50 \%$ & 1.13 & 1217 & 5 \\
\hline & 5 & WoMsr & $93.03 \%$ & 0.96 & 1019 & 4 \\
\hline & 5 & All Declus & $1.00 \%$ & 1.11 & 1204 & 5 \\
\hline \multirow[t]{8}{*}{25} & 2 & WoMsr & $100 \%$ & 1.01 & 1077 & 4 \\
\hline & 2 & All Declus & $0.00 \%$ & 1.24 & 1299 & 5 \\
\hline & 3 & WoMsr & $100 \%$ & 0.95 & 1012 & 4 \\
\hline & 3 & All Declus & $4.48 \%$ & 1.14 & 1220 & 5 \\
\hline & 4 & WoMsr & $89.55 \%$ & 0.92 & 985 & 4 \\
\hline & 4 & All Declus & $5.47 \%$ & 1.08 & 1184 & 5 \\
\hline & 5 & WoMsr & $81.09 \%$ & 0.91 & 981 & 4 \\
\hline & 5 & All Declus & $10.95 \%$ & 1.06 & 1171 & 4 \\
\hline
\end{tabular}


To test whether the lack of Poisson results for the whole declustered catalog might be a product of the CURATE method itself, we also applied the Gardner and Knopoff [1974] declustering algorithm in MATLAB. The overall size of the Gardner and Knopoff declustered catalog is 706 earthquakes, just slightly more than half the size of that returned by the CURATE method. This difference in size between the two methods is comparable to that observed in the CVR catalog (Chapter 2). The Gardner and Knopoff declustered catalog and its individual components are shown in figure 3.10A. In the COSO catalog there are four earthquakes in the $\mathrm{M}=5-6$ range between 1995 and 1998 [Bhattacharyya et al., 1999]. The Gardner and Knopoff method's large windows removes too many earthquakes over this time and so, too little seismicity is in the declustered catalog, which creates a rate deficiency (Fig. 3.10A) that precludes it from returning Poisson values. Initially it seems that the GK method fails because it takes out too much seismicity. However a closer look at the cumulative number plots (Fig. 3.10B) shows that there is still a large degree of clustering in the declustered catalog. To see whether this time period alone caused the non-Poisson results, we ran the catalog in two separate time periods (1981-1995.5, and 1997-2011). The earlier time period is better behaved (more Poisson) for some window lengths, but the later time period still returns non-Poisson results at any window size (Table 3.6). In our tests of other catalogs Gardner and Knopoff was the method most likely to return Poisson results (Chapter 2, Figure 2.10) because of the small size of the declustered catalog. The long time windows of the Gardner and Knopoff methods partially protect it from seeing sequence clustering in time. While it is taking out large amounts of seismicity in the COSO catalog as well, it still does not return a well behaved declustered catalog. 


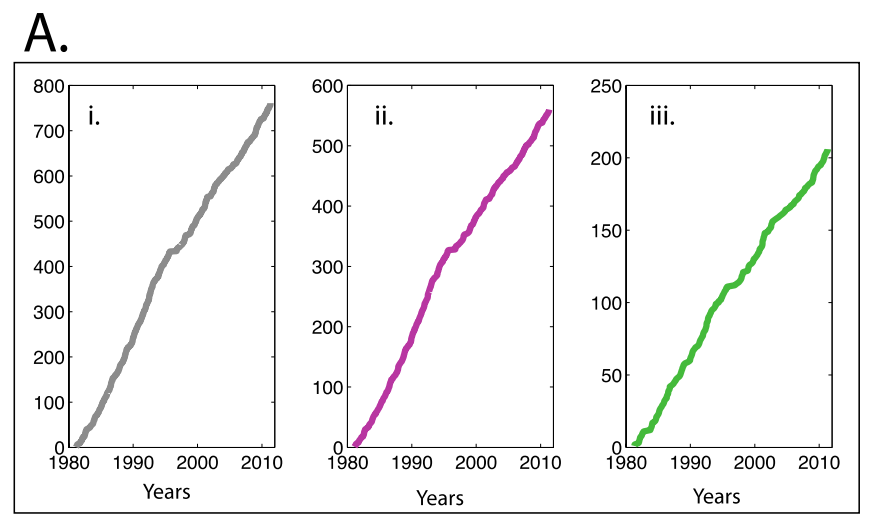

B.

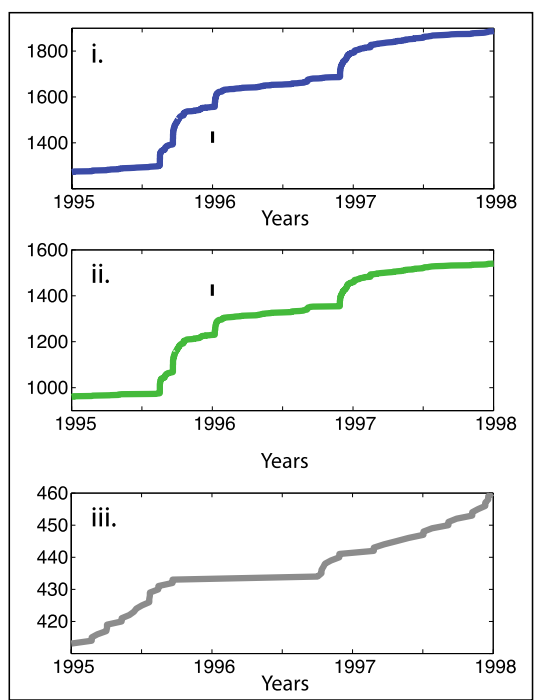

Figure 3.10 Comparison of the declustered catalogs created by the CURATE and Gardner and Knopoff clustering methods.

Figure 3.10. A) Declustered catalogs of the Coso subset from the Gardner and Knopoff method. i) Whole declustered catalog, ii) declustered catalog without MS replacement, iii) MS only. B). temporal subset of the same Coso subset showing the large proportion of seismicity in sequences and declustered catalog. i) the original Coso subset, ii) the earthquakes in sequences, iii) the whole declustered catalog. The small black bars in i) and ii) show the vertical scale of iii). 
Table 3-6 Poisson Testing of the Coso subset declustered catalog from the Gardner and Knopoff method

\begin{tabular}{|c|c|c|c|c|c|c|}
\hline & $\begin{array}{l}\text { window } \\
\text { length }\end{array}$ & & $\begin{array}{l}\text { Poisson } \\
\text { Fraction }\end{array}$ & $\begin{array}{l}\text { Variance } \\
\text { /mean }\end{array}$ & $\begin{array}{c}\% \text { bins } \\
\text { with zero } \\
\text { count }\end{array}$ & DOF \\
\hline \multirow[t]{6}{*}{ 1981-2011 } & 10 & WoMsr & $2.49 \%$ & 0.90 & $58.45 \%$ & 2 \\
\hline & & All Declus & $0.00 \%$ & 1.34 & $58.45 \%$ & 3 \\
\hline & 20 & WoMsr & $96.76 \%$ & 0.93 & $35.56 \%$ & 3 \\
\hline & & All Declus & $0.00 \%$ & 1.33 & $35.56 \%$ & 4 \\
\hline & 50 & WoMsr & $91.21 \%$ & 1.10 & $11.76 \%$ & 5 \\
\hline & & All Declus & $0.00 \%$ & 1.54 & $11.76 \%$ & 7 \\
\hline \multirow[t]{6}{*}{ 1981-1995 } & 10 & WoMsr & $14.43 \%$ & 0.89 & $51.80 \%$ & 2 \\
\hline & & All Declus & $0.00 \%$ & 1.29 & $51.80 \%$ & 2 \\
\hline & 20 & WoMsr & $64.84 \%$ & 0.84 & $26.89 \%$ & 3 \\
\hline & & All Declus & $51.37 \%$ & 1.17 & $26.89 \%$ & 4 \\
\hline & 50 & WoMsr & $99.70 \%$ & 0.90 & $5.71 \%$ & 5 \\
\hline & & All Declus & $97.70 \%$ & 1.22 & $5.71 \%$ & 6 \\
\hline \multirow[t]{6}{*}{$1997-2011$} & 10 & WoMsr & $0.00 \%$ & 0.83 & $62.21 \%$ & 1 \\
\hline & & All Declus & $0.00 \%$ & 1.33 & $62.21 \%$ & 2 \\
\hline & 20 & WoMsr & $100 \%$ & 0.90 & $40.68 \%$ & 2 \\
\hline & & All Declus & $0.00 \%$ & 1.43 & $40.68 \%$ & 3 \\
\hline & 50 & WoMsr & $100 \%$ & 1.00 & $12.26 \%$ & 4 \\
\hline & & All Declus & $0.00 \%$ & 1.60 & $12.26 \%$ & 5 \\
\hline
\end{tabular}

\subsection{CVR Regional Subset}

Another effect of the relative nature of the CURATE method, is that when regions with varying sequence behavior are included, only the strongest activity will be highlighted. Taking smaller subsets decreases the mean rate and so includes more earthquakes in each sequence. Smaller scale geographic investigations are most likely to be useful in identifying possible volcanic precursory activity. While such activity may be identifiable on broader scales (Raurimu area, Chapter 2) it should be studied on smaller scales as well in case the activity has unusual timing but small magnitude. This section examines how regional subsets within and surrounding the CVR behave. The subsets discussed in this section are shown in figure 3.11 . 


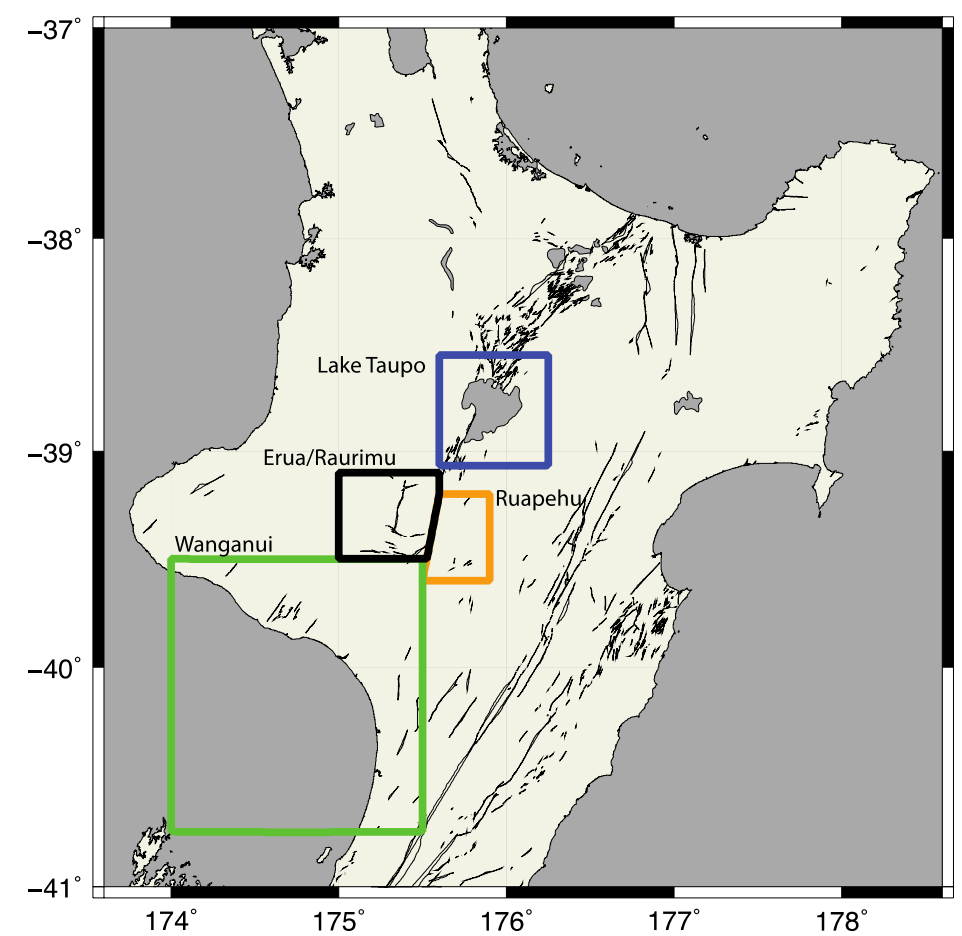

Figure 3.11 Location map for subset polygons in the North Island, New Zealand.

\subsubsection{Lake Taupo Region}

The Lake Taupo area has frequent swarm activity, although more recent sequences have been too small to see at the CVR-wide scale of chapter 2. There have been historic swarms of magnitude 4 and 5 earthquakes in the 1960s [Bryan et al., 1999; Eiby, 1966; Otway and Sherburn, 1994; Sherburn, 1992b; Smith et al., 2007].

There have also been large amounts of deformation in the region that has been documented with and without accompanying seismic activity. To investigate this region more closely we processed it with distance rules of 10,15 , and $20 \mathrm{~km}$ and dayrules between 3 and 5 days. The time period from 1993-2007.5 gives no Poisson results for the whole declustered catalog, but the piece without mainshock replacement is Poisson. The Poisson results of the declustered catalog without mainshock-replacement imply that the sequences are clustering in time. If the 
sequences cluster in time that also suggests that whatever drives or triggers the sequences also changes over these times. The change in rates is easily seen with the cumulative number plots (Fig. 3.12A) that show the mean rate is unrepresentative of any time through the catalog. This suggests that the mean is an average of multiple behaviors rather than an estimate of the whole. The poor results do not appear to be caused by sudden changes in rate due to isolated events, but rather longer changes in the broad trend of activity. We tested several time periods each with a start date of 1998, and end years of 2001, 2002, 2003, and 2007 (Table 3.7). The 1998-2003 time period yielded the most Poisson results. In the cumulative number plot of this time period (Fig 3.12B), the rate deviates from the mean rate but it also returns to that rate between periods of increase. Over this period of time both the declustered catalog with and without mainshock replacement return Poisson results. 
Table 3-7 Poisson Testing of the Lake Taupo subset declustered catalog using the CURATE method

\begin{tabular}{|c|c|c|c|c|c|c|c|}
\hline & $\begin{array}{l}\text { Dist } \\
\text { rule }\end{array}$ & $\begin{array}{l}\text { Day } \\
\text { rule }\end{array}$ & & $\begin{array}{l}\text { Poisson } \\
\text { Fraction }\end{array}$ & $\begin{array}{c}\text { Variance/ } \\
\text { Mean }\end{array}$ & $\begin{array}{l}\% \text { bins with } \\
\text { zero count }\end{array}$ & DOF \\
\hline 1993- & 10 & 3 & WoMsr & $20.40 \%$ & 1.18 & $65.23 \%$ & 2 \\
\hline \multirow[t]{17}{*}{2007.5} & & 3 & All Declus & $0.00 \%$ & 1.29 & $61.62 \%$ & 2 \\
\hline & & 4 & WoMsr & $14.93 \%$ & 1.18 & $65.92 \%$ & 2 \\
\hline & & 4 & All Declus & $0.00 \%$ & 1.27 & $61.62 \%$ & 2 \\
\hline & & 5 & WoMsr & $37.81 \%$ & 1.17 & $66.27 \%$ & 2 \\
\hline & & 5 & All Declus & $0.00 \%$ & 1.21 & $61.79 \%$ & 2 \\
\hline & 15 & 3 & WoMsr & $96.02 \%$ & 1.08 & $66.44 \%$ & 2 \\
\hline & & 3 & All Declus & $0.00 \%$ & 1.17 & $61.79 \%$ & 2 \\
\hline & & 4 & WoMsr & $88.06 \%$ & 1.07 & $67.64 \%$ & 1 \\
\hline & & 4 & All Declus & $0.00 \%$ & 1.13 & $61.79 \%$ & 2 \\
\hline & & 5 & WoMsr & $98.01 \%$ & 1.04 & $67.99 \%$ & 1 \\
\hline & & 5 & All Declus & $44.28 \%$ & 1.06 & $62.13 \%$ & 2 \\
\hline & 20 & 3 & WoMsr & $99.00 \%$ & 1.04 & $66.44 \%$ & 2 \\
\hline & & 3 & All Declus & $0.00 \%$ & 1.13 & $61.79 \%$ & 2 \\
\hline & & 4 & WoMsr & $100 \%$ & 1.04 & $68.33 \%$ & 1 \\
\hline & & 4 & All Declus & $14.93 \%$ & 1.08 & $62.13 \%$ & 2 \\
\hline & & 5 & WoMsr & $100 \%$ & 1.02 & $68.85 \%$ & 1 \\
\hline & & 5 & All Declus & $25.87 \%$ & 1.03 & $62.65 \%$ & 2 \\
\hline 1998- & 10 & 3 & WoMsr & $100 \%$ & 0.96 & $53.92 \%$ & 1 \\
\hline \multirow[t]{17}{*}{2003} & & 3 & All Declus & $41.29 \%$ & 1.08 & $49.31 \%$ & 2 \\
\hline & & 4 & WoMsr & $100 \%$ & 0.96 & $55.76 \%$ & 1 \\
\hline & & 4 & All Declus & $84.58 \%$ & 1.02 & $49.77 \%$ & 2 \\
\hline & & 5 & WoMsr & $100 \%$ & 0.93 & $56.22 \%$ & 1 \\
\hline & & 5 & All Declus & $94.53 \%$ & 0.97 & $49.77 \%$ & 2 \\
\hline & 15 & 3 & WoMsr & $100 \%$ & 0.92 & $54.84 \%$ & 1 \\
\hline & & 3 & All Declus & $48.26 \%$ & 1.04 & $49.31 \%$ & 2 \\
\hline & & 4 & WoMsr & $100 \%$ & 0.90 & $57.14 \%$ & 1 \\
\hline & & 4 & All Declus & $83.08 \%$ & 0.97 & $49.77 \%$ & 2 \\
\hline & & 5 & WoMsr & $98.01 \%$ & 0.88 & $58.06 \%$ & 1 \\
\hline & & 5 & All Declus & $99.00 \%$ & 0.91 & $49.77 \%$ & 2 \\
\hline & 20 & 3 & WoMsr & $100 \%$ & 0.91 & $55.76 \%$ & 1 \\
\hline & & 3 & All Declus & $69.65 \%$ & 0.98 & $49.31 \%$ & 2 \\
\hline & & 4 & WoMsr & $100 \%$ & 0.88 & $58.53 \%$ & 1 \\
\hline & & 4 & All Declus & $75.62 \%$ & 0.90 & $49.77 \%$ & 2 \\
\hline & & 5 & WoMsr & $100 \%$ & 0.87 & $59.45 \%$ & 1 \\
\hline & & 5 & All Declus & $99.50 \%$ & 0.86 & $49.77 \%$ & 2 \\
\hline
\end{tabular}



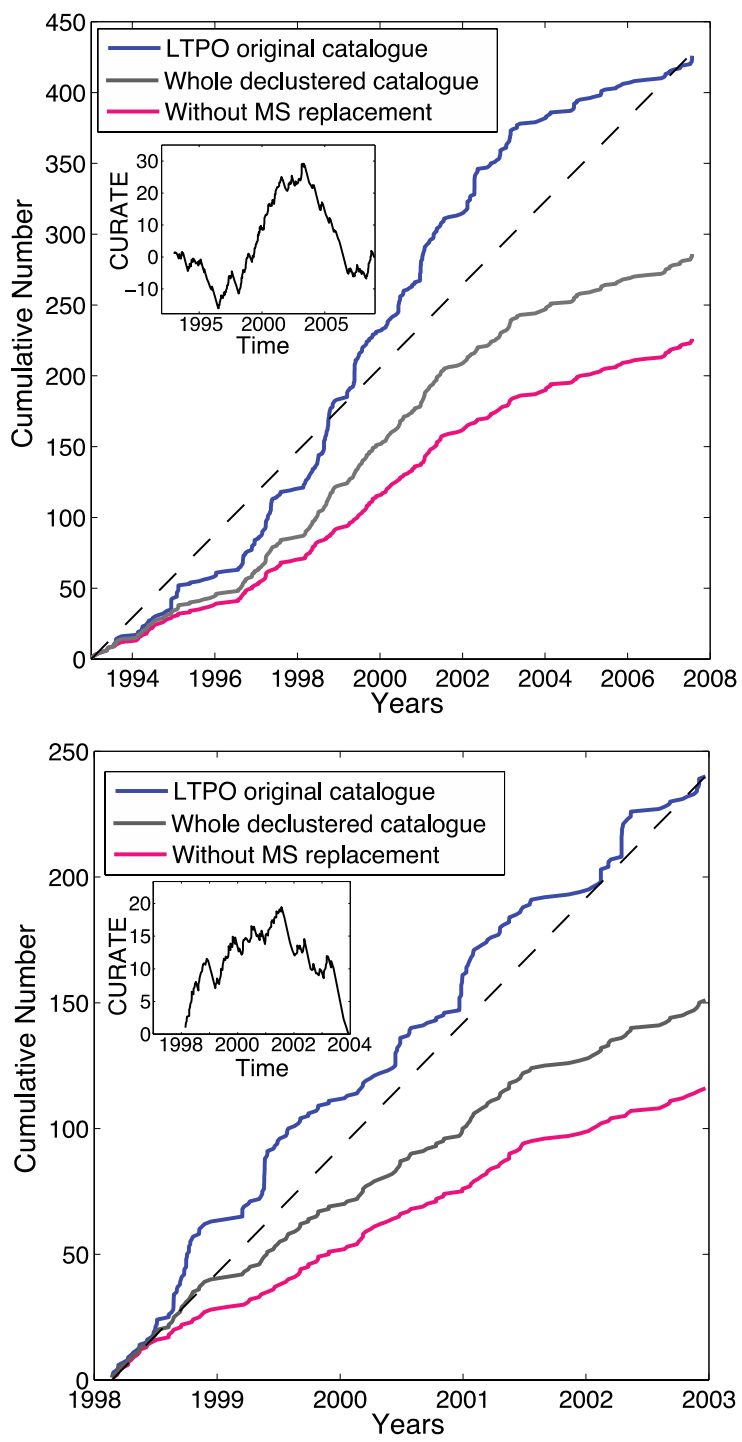

Figure 3.12 Comparison of the original and declustered catalogs for the Lake Taupo region.

Cumulative number of earthquakes with time for the original, declustered, and declustered-without-mainshock replacement catalogs. A) Entire catalog time period 1993-2007.5, B) temporal subset 1998-2003. Dashed line approximates the mean rate of the original catalog. Inset shows the CURATE of the declustered catalog with mainshock replacement. 


\subsection{New Zealand Aftershock Regions}

\subsubsection{Introduction to South Island subsets}

One way to test whether the Lake Taupo sequence clustering is produced by something specific to the region is to test other regions at the same period of time. We wanted to test regions in the South Island of New Zealand because they are more likely to be dominated by mainshock-aftershock sequences like the SCA catalog. The difference in tectonic setting and their distance from the CVR should clarify whether the observed rate changes in the Lake Taupo subset are unique to the region (and tectonic setting). We have chosen four subsets in the South Island (Fig. 3.13). The earthquake map (Fig 3.13) shows that the seismicity along the Alpine Fault is not continuous. We were unsure whether we should treat the entire length of the Alpine Fault as a single subset, or to break it up into a northern and southern portion according to the seismicity. Both the full polygon and the two sub-regions (Alpine Fault, and Southern Alpine Fault) were tested independently to see which gave the best declustered catalogs. The results of the sub-polygons are better than the overall polygon, and they are presented separately hereafter with the testing of two possibilities given below. The CURATES (Fig. 3.14) and random yearly rates (Fig. 3.15) suggest that at least three of these subsets have outstanding large events that influence the rate, and, like SCA, will need an alternative rate method to identify other sequences. 


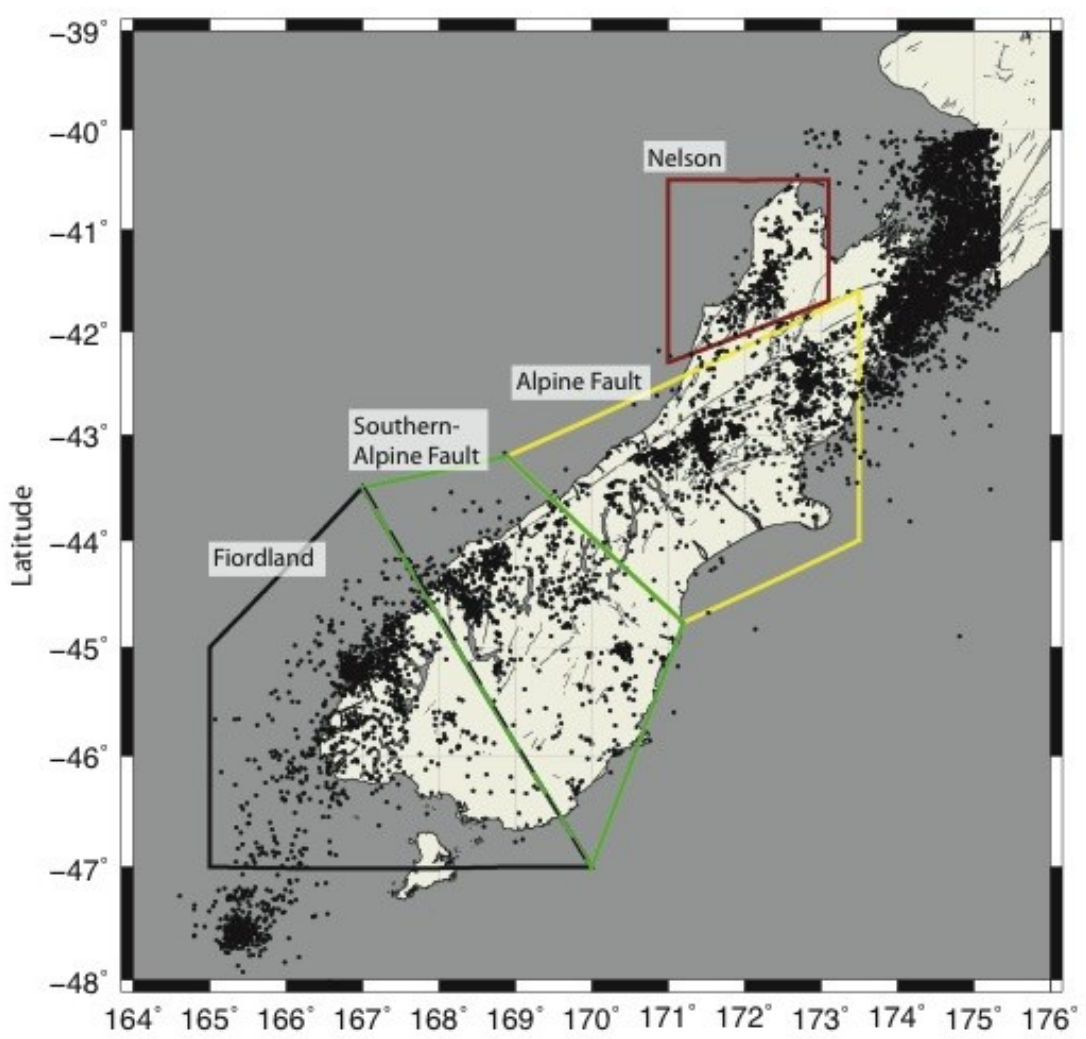

Figure 3.13 Location map for four polygons in the South Island of New Zealand. See text for an explanation of the choice of polygon.
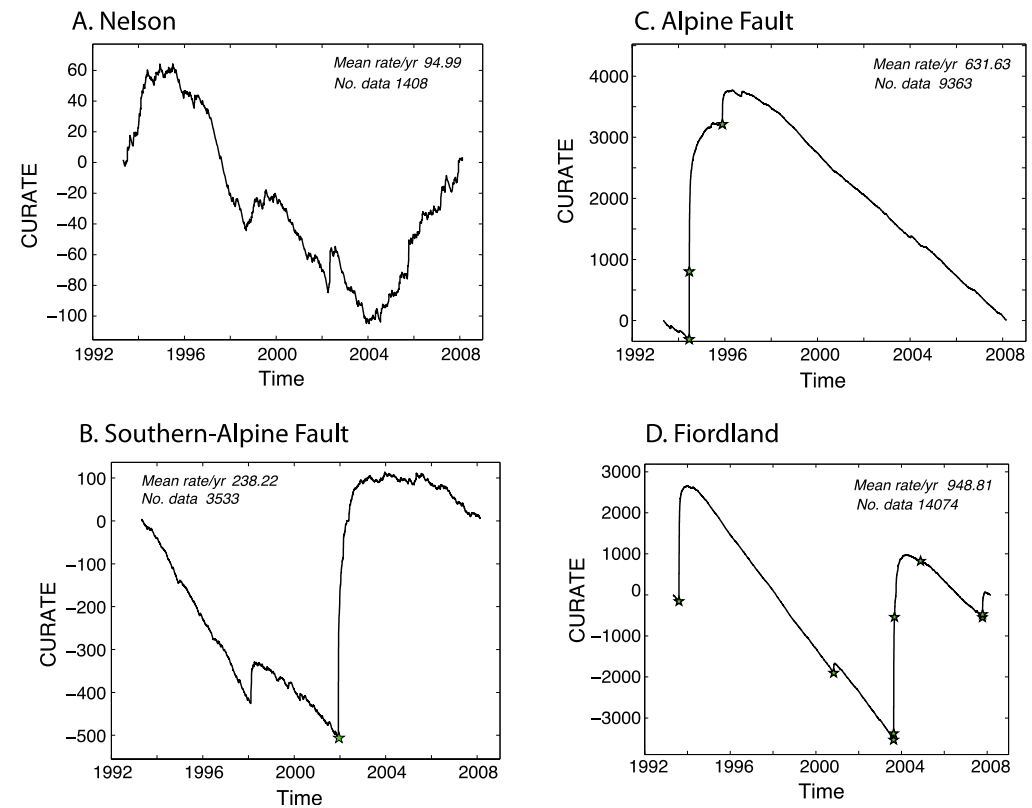

Figure 3.14 CURATE plots for four areas dominated by mainshock-aftershock sequences.

Green stars are plotted at the time of earthquakes $M \geq 6$. 

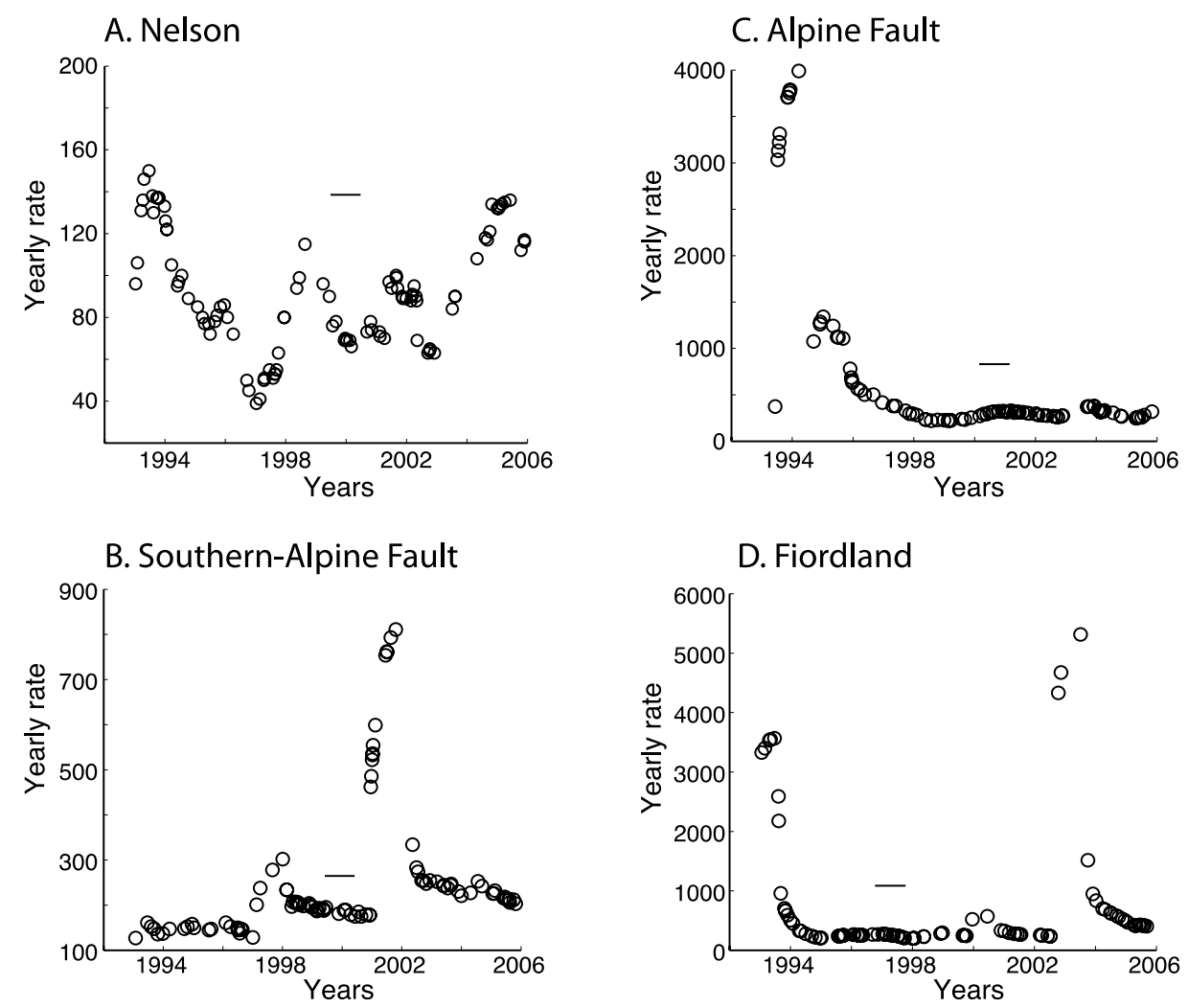

Figure 3.15 Yearly earthquake total from 100 randomly chosen start times for the four regions in the South Island.

Note the differences in yearly-rate scale. Horizontal lines in A-D represent a length of one year.

\subsubsection{Alpine Fault}

First we apply this method to the Alpine Fault polygons (Fig. 3.13, yellow and green polygons). As anticipated, there are some times when the yearly rate is very high, but more importantly there are also long periods of time in which the rate is relatively stable (Fig. 3.14, B-C). It is this stable rate that we want to use as our reference rate to identify sequences. The random start-time sampling method will produce slightly different answers for any given run, but should not change significantly. Here we report the results from a single run of 100 random start times. The original mean rate is 631.6 earthquakes/year, and the median excluding periods with large earthquakes $(M \geq 6.0)$ is less than half of that value, 311.5 earthquakes/year. 
We used the median rate as the rate threshold to identify sequences and ran the data through the CURATE algorithm with the new lower rate. The resulting declustered catalog is smaller and smoother than the one obtained using the mean rate (Figure 3.16, inset). It is also easy to see how unrepresentative the mean rate (thin dashed line) is of the background rate. While the median rate method produces an improvement in dispersion values, none of the selection parameter combinations give good results in the chi-squared test (Table 3.8). Unlike in the swarm-dominated regions, it does not appear that the misfit arises from a clustering of sequences because the mean-rate declustered catalog without mainshock replacement (Fig. 3.16, pink line) also gives poor results. The declustered CURATE plots (Fig. 3.16, inset) also show that there is still a deviation in rate in the years following the 1994 Arthur's Pass $(\mathrm{M}=6.7)$ earthquake [Abercrombie et al., 2000].

Table 3.9 gives a summary of the Poisson testing in Table 3.8 by showing minimum, maximum, mean, and median values for the Poisson fraction and dispersion. In general the separate polygons outperform the combined Alpine Fault polygon. The late time period seems to be less sensitive to the division. It consistently returns more Poisson results in the chi-squared tests, but is slightly underdispersed for all polygons. Similarly in the late time period there is less difference between the median and minimum rates. In the early time period the minimum rate gives better results, especially for the Alpine Fault (northern) polygon that contains the 1994 Arthur's Pass earthquake. The Southern Alpine fault polygon contains fewer earthquakes in general, and is less affected by the difference between the declustered catalogs created with the mean and median rates. This can be seen clearly in the difference between cumulative rates of both regions (Fig. 3.16 and Fig 3.17) where the declustered catalog for the Southern Alpine Fault using the median-rate 
(black) is not smaller than the declustered catalog WoMsr for the mean-rate (pink). In the Northern Alpine Fault polygon the declustered catalog using the median-rate (black) has fewer earthquakes than the mean-rate declustered catalogs both with (gray) and without mainshock-replacement (pink).

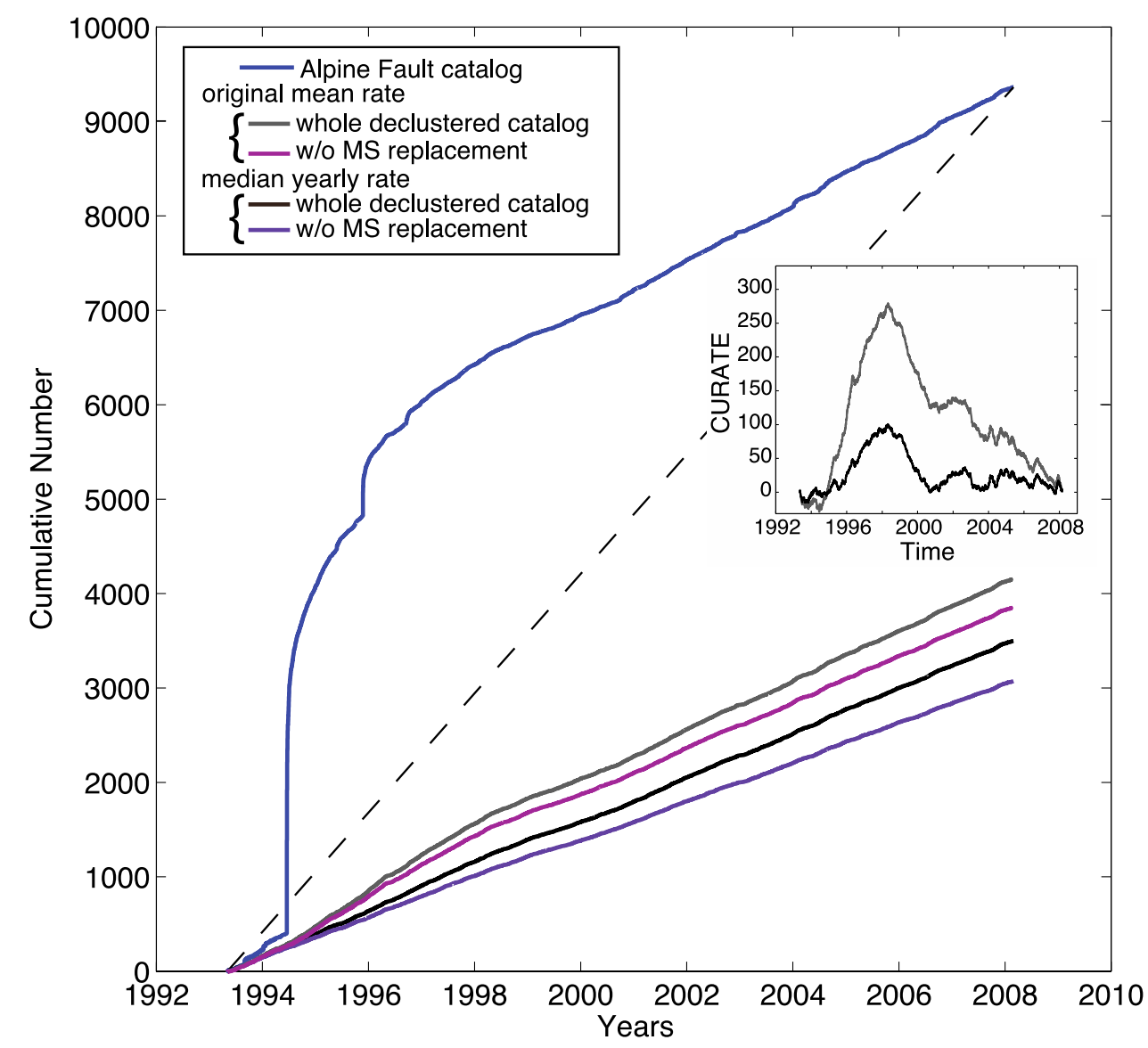

Figure 3.16 Comparison of the original and declustered catalogs for the Alpine Fault dataset.

Cumulative number of earthquakes with time for the original (blue), declustered (gray and black), and declustered-without-mainshock replacement (pink and purple) catalogs for the Alpine Fault dataset. Dashed line approximates the mean rate of the original catalog. Gray and pink lines correspond to declustered catalogs obtained using the mean-rate. Black and purple lines represent declustered catalogs obtained using the median-rate. Inset shows the CURATE of the whole declustered catalog for mean (gray) and median rate (black). 


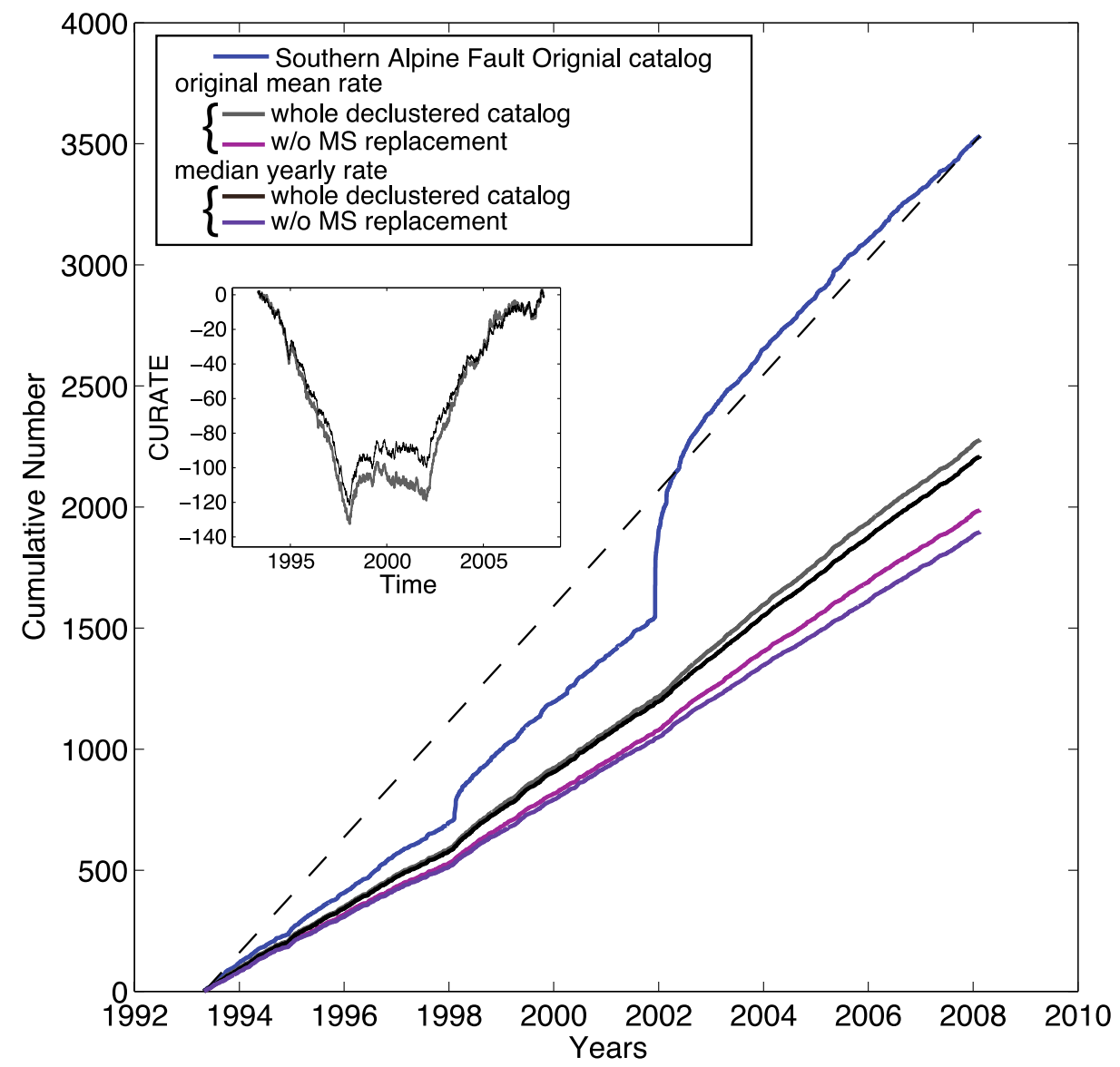

Figure 3.17 Comparison of the original and declustered catalogs for the Southern Alpine Fault dataset.

Cumulative number of earthquakes with time for the original (blue), declustered (gray and black), and declustered-without-mainshock replacement (pink and purple) catalogs for the Southern Alpine Fault dataset. Dashed line approximates the mean rate of the original catalog. Gray and pink lines correspond to declustered catalogs obtained using the mean-rate. Black and purple lines represent declustered catalogs obtained using the median-rate. Inset shows the CURATE of the whole declustered catalog for mean (gray) and median rate (black). 
Table 3-8 Alpine Fault Poisson Testing for different polygons and time periods.

Poisson distributions should have a Variance/Mean (dispersion) value of 1.

\begin{tabular}{|c|c|c|c|c|c|c|c|c|c|c|c|c|c|c|c|c|c|}
\hline & $\begin{array}{c}\% \\
\text { Poisson }\end{array}$ & $\begin{array}{l}\text { Variance } \\
\text { / Mean }\end{array}$ & $\begin{array}{c}\# \\
\text { eqs }\end{array}$ & DOF & $\begin{array}{c}\% \\
\text { Poisson }\end{array}$ & $\begin{array}{l}\text { Variance } \\
\text { / Mean }\end{array}$ & $\begin{array}{c}\# \\
\text { eqs }\end{array}$ & DOF & $\begin{array}{c}\% \\
\text { Poisson }\end{array}$ & $\begin{array}{l}\text { Variance } \\
\text { / Mean }\end{array}$ & $\begin{array}{c}\# \\
\text { eqs }\end{array}$ & DOF & $\begin{array}{c}\% \\
\text { Poisson }\end{array}$ & $\begin{array}{l}\text { Variance } \\
\text { / Mean }\end{array}$ & $\begin{array}{c}\# \\
\text { eqs }\end{array}$ & DOF \\
\hline & & \multicolumn{4}{|c|}{ 1993-1999 median rate } & \multicolumn{4}{|c|}{$1993-1999$ min rate } & \multicolumn{4}{|c|}{ 2000-2007.5 median rate } & \multicolumn{4}{|c|}{ 2000-2007.5 min rate } \\
\hline \multicolumn{18}{|c|}{ Southern Alpine Fault } \\
\hline & 3 WoMsr & $29.4 \%$ & 0.95 & 805 & 7 & $59.7 \%$ & 0.98 & 784 & 7 & $87.6 \%$ & 0.85 & 1275 & 8 & $84.1 \%$ & 0.83 & 1200 & 9 \\
\hline & 3 All Declus & $15.4 \%$ & 1.02 & 918 & 8 & $22.9 \%$ & 1.01 & 903 & 7 & $97.5 \%$ & 0.90 & 1462 & 9 & $92.5 \%$ & 0.86 & 1406 & 9 \\
\hline & 5 WoMsr & $33.3 \%$ & 0.93 & 787 & 7 & $80.6 \%$ & 0.96 & 763 & 7 & $77.1 \%$ & 0.83 & 1250 & 8 & $63.7 \%$ & 0.80 & 1164 & 9 \\
\hline & 5 All Declus & $22.9 \%$ & 0.97 & 901 & 7 & $28.9 \%$ & 0.96 & 882 & 7 & $96.5 \%$ & 0.87 & 1427 & 9 & $79.6 \%$ & 0.81 & 1359 & 8 \\
\hline & 3 WoMsr & $59.2 \%$ & 0.89 & 727 & 6 & $75.1 \%$ & 0.89 & 692 & 6 & $29.4 \%$ & 0.73 & 1149 & 8 & $22.4 \%$ & 0.71 & 1082 & 8 \\
\hline & 3 All Declus & $29.4 \%$ & 0.93 & 866 & 7 & $29.9 \%$ & 0.91 & 842 & 7 & $72.1 \%$ & 0.78 & 1362 & 8 & $46.3 \%$ & 0.74 & 1309 & 8 \\
\hline & 5 WoMsr & $77.6 \%$ & 0.88 & 703 & 6 & $91.0 \%$ & 0.87 & 663 & 6 & $17.4 \%$ & 0.70 & 1108 & 8 & $2.0 \%$ & 0.67 & 1023 & 7 \\
\hline & 5 All Declus & $30.3 \%$ & 0.89 & 844 & 7 & $26.4 \%$ & 0.86 & 813 & 7 & $26.4 \%$ & 0.72 & 1306 & 8 & $3.5 \%$ & 0.66 & 1240 & 7 \\
\hline \multicolumn{18}{|c|}{ Alpine Fault (North) } \\
\hline \multirow[t]{4}{*}{20} & 3 WoMsr & $10.0 \%$ & 1.22 & 1726 & 10 & $14.4 \%$ & 1.08 & 1453 & 10 & $78.1 \%$ & 0.81 & 1809 & 9 & $96.5 \%$ & 0.84 & 1755 & 10 \\
\hline & 3 All Declus & $0.5 \%$ & 1.46 & 1957 & 11 & $24.9 \%$ & 1.22 & 1735 & 11 & $96.0 \%$ & 0.90 & 2010 & 10 & $99.5 \%$ & 0.90 & 1975 & 10 \\
\hline & 5 WoMsr & $20.4 \%$ & 1.21 & 1692 & 10 & $26.4 \%$ & 1.03 & 1407 & 10 & $83.6 \%$ & 0.80 & 1787 & 10 & $84.6 \%$ & 0.79 & 1720 & 10 \\
\hline & 5 All Declus & $0.0 \%$ & 1.35 & 1883 & 11 & $79.1 \%$ & 1.13 & 1639 & 10 & $97.0 \%$ & 0.89 & 1994 & 10 & $95.0 \%$ & 0.87 & 1948 & 10 \\
\hline \multirow[t]{4}{*}{30} & 3 WoMsr & $85.1 \%$ & 1.13 & 1558 & 9 & $82.1 \%$ & 0.98 & 1268 & 8 & $78.1 \%$ & 0.78 & 1718 & 10 & $98.5 \%$ & 0.81 & 1639 & 10 \\
\hline & 3 All Declus & $9.0 \%$ & 1.28 & 1773 & 11 & $88.6 \%$ & 1.11 & 1540 & 9 & $92.5 \%$ & 0.84 & 1944 & 10 & $98.5 \%$ & 0.84 & 1895 & 10 \\
\hline & 5 WoMsr & $84.6 \%$ & 1.11 & 1520 & 9 & $92.0 \%$ & 0.95 & 1227 & 8 & $81.1 \%$ & 0.76 & 1677 & 9 & $96.0 \%$ & 0.77 & 1597 & 9 \\
\hline & 5 All Declus & $35.3 \%$ & 1.19 & 1698 & 10 & $1.5 \%$ & 1.01 & 1448 & 10 & $94.0 \%$ & 0.82 & 1909 & 10 & $95.0 \%$ & 0.81 & 1849 & 10 \\
\hline
\end{tabular}




\begin{tabular}{|c|c|c|c|c|c|c|c|c|c|c|c|c|c|c|c|c|}
\hline \multicolumn{17}{|l|}{ Table 3.8 cont. } \\
\hline & $\begin{array}{c}\% \\
\text { Poisson }\end{array}$ & $\begin{array}{l}\text { Variance } \\
\text { / Mean }\end{array}$ & $\begin{array}{c}\# \\
\text { eqs }\end{array}$ & DOF & $\begin{array}{c}\% \\
\text { Poisson }\end{array}$ & $\begin{array}{l}\text { Variance } \\
\text { / Mean }\end{array}$ & $\begin{array}{c}\# \\
\text { eqs }\end{array}$ & DOF & $\begin{array}{c}\% \\
\text { Poisson }\end{array}$ & $\begin{array}{l}\text { Variance } \\
\text { / Mean }\end{array}$ & $\begin{array}{c}\# \\
\text { eqs }\end{array}$ & DOF & $\begin{array}{c}\% \\
\text { Poisson }\end{array}$ & $\begin{array}{l}\text { Variance } \\
\text { / Mean }\end{array}$ & $\begin{array}{c}\# \\
\text { eqs }\end{array}$ & DOF \\
\hline & \multicolumn{4}{|c|}{ 1993-1999 median rate } & \multicolumn{4}{|c|}{$1993-1999 \mathrm{~min}$ rate } & \multicolumn{4}{|c|}{ 2000-2007.5 median rate } & \multicolumn{4}{|c|}{ 2000-2007.5 min rate } \\
\hline \multicolumn{17}{|c|}{ Combined Alpine Fault } \\
\hline 203 WoMsr & $0.0 \%$ & 1.54 & 2908 & 13 & $70.6 \%$ & 1.24 & 2409 & 12 & $95.0 \%$ & 0.91 & 3305 & 13 & $97.0 \%$ & 0.90 & 3187 & 14 \\
\hline 3 All Declus & $0.0 \%$ & 1.76 & 3179 & 14 & $47.8 \%$ & 1.41 & 2779 & 13 & $94.0 \%$ & 1.01 & 3642 & 15 & $97.0 \%$ & 0.98 & 3559 & 14 \\
\hline 5 WoMsr & $4.5 \%$ & 1.48 & 2852 & 13 & $0.5 \%$ & 1.19 & 2320 & 12 & $87.1 \%$ & 0.89 & 3248 & 13 & $78.6 \%$ & 0.86 & 3127 & 13 \\
\hline 5 All Declus & $1.5 \%$ & 1.64 & 3096 & 13 & $2.5 \%$ & 1.30 & 2656 & 13 & $95.0 \%$ & 0.97 & 3581 & 14 & $91.0 \%$ & 0.93 & 3494 & 14 \\
\hline 303 WoMsr & $69.7 \%$ & 1.47 & 2723 & 12 & $35.8 \%$ & 1.21 & 2170 & 12 & $81.1 \%$ & 0.84 & 3159 & 13 & $64.2 \%$ & 0.80 & 3027 & 12 \\
\hline 3 All Declus & $0.0 \%$ & 1.65 & 2989 & 14 & $47.8 \%$ & 1.33 & 2551 & 13 & $93.5 \%$ & 0.91 & 3532 & 14 & $93.0 \%$ & 0.87 & 3442 & 14 \\
\hline 5 WoMsr & $11.9 \%$ & 1.43 & 2661 & 13 & $33.3 \%$ & 1.17 & 2101 & 11 & $70.1 \%$ & 0.81 & 3078 & 13 & $65.2 \%$ & 0.79 & 2943 & 12 \\
\hline 5 All Declus & $7.5 \%$ & 1.54 & 2897 & 13 & $52.7 \%$ & 1.25 & 2436 & 12 & $88.1 \%$ & 0.87 & 3451 & 13 & $83.1 \%$ & 0.84 & 3352 & 13 \\
\hline
\end{tabular}


Table 3-9 Summary for comparison of Alpine Fault polygons for different time periods.

\begin{tabular}{|c|c|c|c|c|c|c|c|c|c|c|}
\hline & & \multicolumn{2}{|c|}{ minimum } & \multicolumn{2}{|c|}{ maximum } & \multicolumn{2}{|c|}{ mean } & \multicolumn{2}{|c|}{ median } & \multirow{2}{*}{$\begin{array}{c}\text { mean } \\
\text { \# } \\
\text { earthquakes }\end{array}$} \\
\hline & & $\begin{array}{c}\% \\
\text { Poisson }\end{array}$ & $\begin{array}{c}\text { Variance } \\
\text { /mean }\end{array}$ & $\begin{array}{c}\% \\
\text { Poisson }\end{array}$ & $\begin{array}{l}\text { Variance } \\
\text { /mean }\end{array}$ & $\begin{array}{c}\% \\
\text { Poisson }\end{array}$ & $\begin{array}{c}\text { Variance } \\
\text { /mean }\end{array}$ & $\begin{array}{c}\% \\
\text { Poisson }\end{array}$ & $\begin{array}{c}\text { Variance } \\
\text { /mean }\end{array}$ & \\
\hline \multicolumn{11}{|l|}{ 1993-1999 median rate } \\
\hline WoMsr & SALP & $18.91 \%$ & 0.87 & $77.61 \%$ & 0.99 & $43.33 \%$ & 0.92 & $40.80 \%$ & 0.92 & 773.08 \\
\hline & Alpine Fault & $0.00 \%$ & 1.10 & $85.07 \%$ & 1.32 & $38.14 \%$ & 1.19 & $33.08 \%$ & 1.18 & 1669.58 \\
\hline & All Alpine Fault & $0.00 \%$ & 1.43 & $69.65 \%$ & 1.68 & $22.14 \%$ & 1.52 & $8.21 \%$ & 1.49 & 2829.50 \\
\hline All Declus & SALP & $2.99 \%$ & 0.89 & $30.35 \%$ & 1.07 & $19.03 \%$ & 0.97 & $21.39 \%$ & 0.98 & 894.42 \\
\hline & Alpine Fault & $0.00 \%$ & 1.19 & $35.32 \%$ & 1.63 & $5.56 \%$ & 1.37 & $0.00 \%$ & 1.35 & 1876.58 \\
\hline & All Alpine Fault & $0.00 \%$ & 1.54 & $7.46 \%$ & 1.95 & $1.16 \%$ & 1.70 & $0.00 \%$ & 1.66 & 3087.75 \\
\hline \multicolumn{11}{|l|}{ 1993-1999 min rate } \\
\hline WoMsr & SALP & $40.30 \%$ & 0.87 & $91.04 \%$ & 1.03 & $69.11 \%$ & 0.94 & $70.15 \%$ & 0.94 & 746.17 \\
\hline & Alpine Fault & $14.43 \%$ & 0.95 & $93.53 \%$ & 1.16 & $55.14 \%$ & 1.03 & $57.21 \%$ & 1.02 & 1383.17 \\
\hline & All Alpine Fault & $0.50 \%$ & 1.15 & $85.07 \%$ & 1.29 & $28.86 \%$ & 1.21 & $22.14 \%$ & 1.20 & 2311.58 \\
\hline All Declus & SALP & $10.95 \%$ & 0.86 & $30.35 \%$ & 1.07 & $24.50 \%$ & 0.96 & $27.36 \%$ & 0.96 & 874.92 \\
\hline & Alpine Fault & $0.00 \%$ & 1.01 & $88.56 \%$ & 1.41 & $37.89 \%$ & 1.16 & $37.31 \%$ & 1.13 & 1638.92 \\
\hline & All Alpine Fault & $2.49 \%$ & 1.25 & $52.74 \%$ & 1.49 & $26.91 \%$ & 1.34 & $20.65 \%$ & 1.34 & 2665.50 \\
\hline \multicolumn{11}{|l|}{ 2000-2007.5 median rate } \\
\hline WoMsr & SALP & $17.41 \%$ & 0.70 & $91.04 \%$ & 0.88 & $66.50 \%$ & 0.81 & $75.12 \%$ & 0.82 & 1229.58 \\
\hline & Alpine Fault & $65.67 \%$ & 0.76 & $90.55 \%$ & 0.81 & $76.53 \%$ & 0.79 & $77.36 \%$ & 0.79 & 1770.25 \\
\hline & All Alpine Fault & $70.15 \%$ & 0.81 & $97.01 \%$ & 0.96 & $86.69 \%$ & 0.88 & $88.06 \%$ & 0.88 & 3239.17 \\
\hline All Declus & SALP & $26.37 \%$ & 0.72 & $100.00 \%$ & 0.96 & $83.96 \%$ & 0.86 & $97.01 \%$ & 0.87 & 1415.42 \\
\hline & Alpine Fault & $91.54 \%$ & 0.82 & $99.00 \%$ & 0.92 & $95.85 \%$ & 0.87 & $96.02 \%$ & 0.88 & 1981.33 \\
\hline & All Alpine Fault & $88.06 \%$ & 0.87 & $99.00 \%$ & 1.07 & $95.32 \%$ & 0.97 & $95.52 \%$ & 0.96 & 3584.67 \\
\hline \multicolumn{11}{|l|}{ 2000-2007.5 min rate } \\
\hline WoMsr & SALP & $1.99 \%$ & 0.67 & $90.55 \%$ & 0.88 & $55.27 \%$ & 0.78 & $59.70 \%$ & 0.79 & 1148.58 \\
\hline & Alpine Fault & $79.60 \%$ & 0.77 & $98.51 \%$ & 0.84 & $90.42 \%$ & 0.80 & $89.55 \%$ & 0.80 & 1706.25 \\
\hline & All Alpine Fault & $64.18 \%$ & 0.79 & $99.00 \%$ & 0.94 & $81.18 \%$ & 0.85 & $81.34 \%$ & 0.85 & 3118.08 \\
\hline All Declus & SALP & $3.48 \%$ & 0.66 & $99.00 \%$ & 0.93 & $69.20 \%$ & 0.80 & $80.85 \%$ & 0.81 & 1353.58 \\
\hline & Alpine Fault & $92.54 \%$ & 0.81 & $99.50 \%$ & 0.92 & $97.10 \%$ & 0.86 & $97.76 \%$ & 0.87 & 1937.83 \\
\hline & All Alpine Fault & $83.08 \%$ & 0.84 & $97.01 \%$ & 1.04 & $93.49 \%$ & 0.93 & $94.28 \%$ & 0.92 & 3498.17 \\
\hline
\end{tabular}




\subsubsection{Fiordland (steeply dipping subduction)}

Next we applied the median rate method to the Fiordland subduction zone polygon in the South Island. This area has also experienced large earthquakes and the CURATE plot (fig 3.14D) shows signs that the aftershock sequences of these large events dominate the overall rate. The yearly rate plot (fig 3.15) shows that this is indeed the case. There is a relatively stable rate of earthquakes that is only elevated around the times of large earthquakes. The mean rate of earthquakes is 948.8 earthquakes per year, and the median excluding periods with large earthquakes $(\mathrm{M} \geq$ 6.0 ) is less than a third of that value, 264 earthquakes per year. The mean rate does remove a large portion of the seismicity, but there are clear increases following the times of large earthquakes, indicating that a portion of the aftershocks has been left in the declustered catalog (Fig. 3.18). The median rate method has a smaller declustered catalog overall, and has also largely eliminated the rate increases seen in the mean rate catalog. This is also clear in the CURATE of the declustered catalogs. Similarly to the Alpine Fault polygon, there are deviations in the declustered CURATE that are common to both the mean and median rates, further suggesting that the background rate potentially has real deviations with time. Also similar to the Alpine Fault polygon, the Fiordland area shows reasonable dispersion values for some sets of selection parameters but returns relatively few good results in the chi-squared tests (Table 3.10). The larger declustered catalogs seem to have better chi-squared results for the declustered catalog without mainshock replacement, however this is coupled with a drop in the Poisson results of the overall catalog, which may indicate that more, smaller sequences have been identified. 


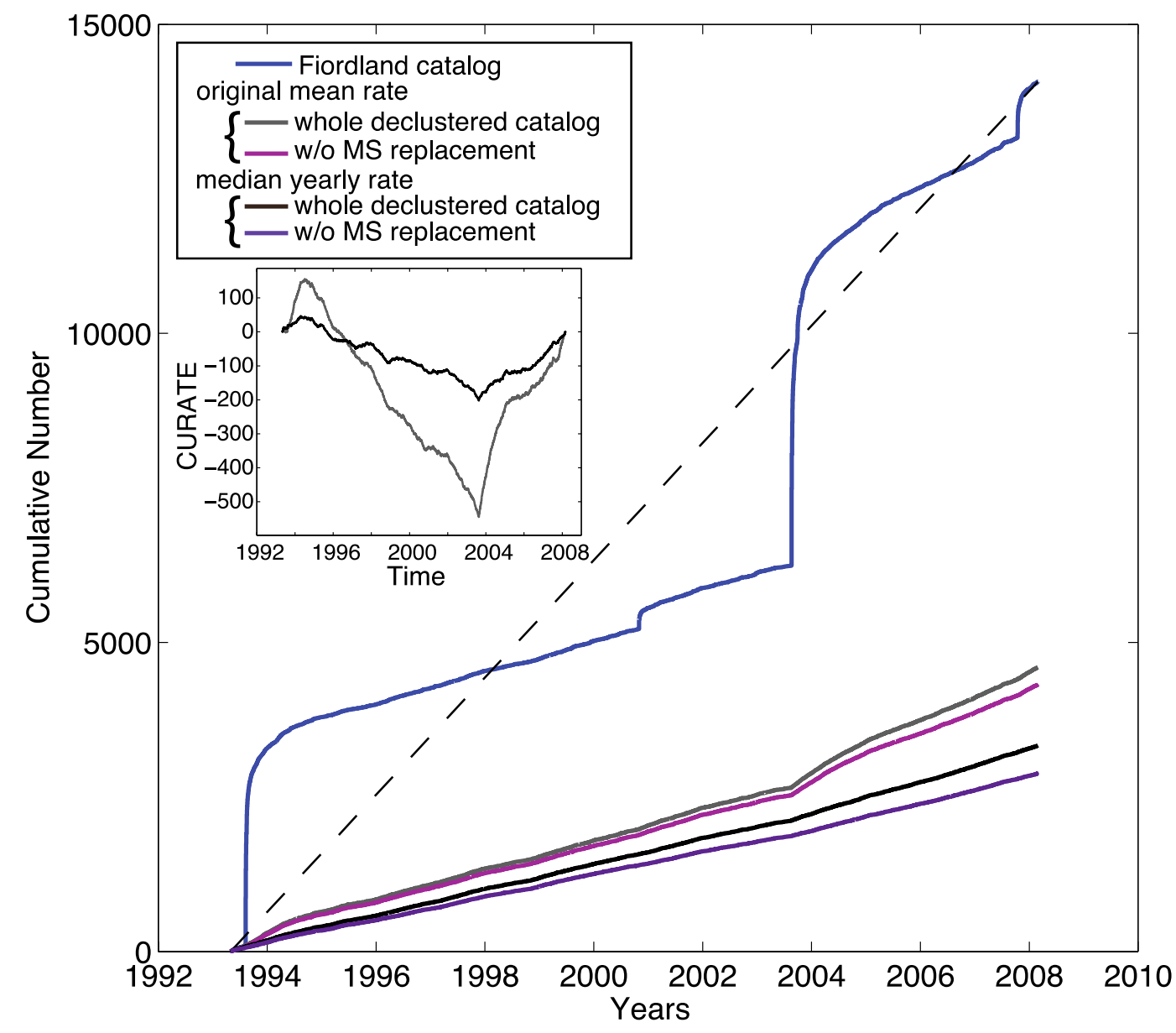

Figure 3.18 Comparison of the original and declustered catalogs for the Fiordland dataset. Cumulative number of earthquakes with time for the original (blue), declustered (gray and black), and declustered-without-mainshock replacement (pink and purple) catalogs. Dashed line approximates the mean rate of the original catalog. Gray and pink lines correspond to declustered catalogs obtained using the mean-rate. Black and purple lines represent declustered catalogs obtained using the median-rate. Inset shows the CURATE of the whole declustered catalog for mean (gray) and median rate (black). 
Table 3-10 Poisson Testing of the Fiordland subset using the CURATE method. Results from both the mean and the median rate methods are shown.

\begin{tabular}{|c|c|c|c|c|c|c|c|c|c|}
\hline $\begin{array}{l}\text { distance } \\
\text { rule }\end{array}$ & $\begin{array}{l}\text { day } \\
\text { rule }\end{array}$ & $\begin{array}{c}\% \\
\text { Poisson }\end{array}$ & $\begin{array}{c}\text { Variance/ } \\
\text { Mean }\end{array}$ & $\begin{array}{c}\text { \% bins } \\
\text { with } \\
\text { zero } \\
\text { count }\end{array}$ & DOF & $\begin{array}{c}\% \\
\text { Poisson }\end{array}$ & $\begin{array}{l}\text { Variance/ } \\
\text { Mean }\end{array}$ & $\begin{array}{l}\text { \% bins } \\
\text { with } \\
\text { zero } \\
\text { count }\end{array}$ & DOF \\
\hline Fiordland & & \multicolumn{4}{|c|}{ mean rate $(948.8)$} & \multicolumn{4}{|c|}{ median rate (264) } \\
\hline 15 & 3 WoMsr & $0.00 \%$ & 2.79 & $2.17 \%$ & 12 & $6.47 \%$ & 1.23 & $2.17 \%$ & 11 \\
\hline & 3 All Declus & $0.00 \%$ & 3.51 & $2.17 \%$ & 13 & $0.00 \%$ & 1.75 & $2.17 \%$ & 11 \\
\hline & 4 WoMsr & $0.00 \%$ & 2.75 & $2.17 \%$ & 12 & $13.93 \%$ & 1.20 & $2.17 \%$ & 11 \\
\hline & 4 All Declus & $0.00 \%$ & 3.34 & $2.17 \%$ & 13 & $0.00 \%$ & 1.64 & $2.17 \%$ & 11 \\
\hline & 5 WoMsr & $0.00 \%$ & 2.68 & $2.17 \%$ & 13 & $46.77 \%$ & 1.15 & $2.17 \%$ & 11 \\
\hline & 5 All Declus & $0.00 \%$ & 3.18 & $2.17 \%$ & 13 & $0.00 \%$ & 1.54 & $2.17 \%$ & 11 \\
\hline 20 & 3 WoMsr & $0.00 \%$ & 2.31 & $2.17 \%$ & 13 & $81.09 \%$ & 1.04 & $2.17 \%$ & 10 \\
\hline & 3 All Declus & $0.00 \%$ & 2.84 & $2.17 \%$ & 13 & $0.00 \%$ & 1.37 & $2.17 \%$ & 11 \\
\hline & 4 WoMsr & $0.00 \%$ & 2.24 & $2.17 \%$ & 13 & $91.04 \%$ & 1.00 & $2.17 \%$ & 10 \\
\hline & 4 All Declus & $0.00 \%$ & 2.65 & $2.17 \%$ & 12 & $0.00 \%$ & 1.28 & $2.17 \%$ & 11 \\
\hline & 5 WoMsr & $0.00 \%$ & 2.22 & $2.17 \%$ & 12 & $93.03 \%$ & 0.98 & $2.17 \%$ & 10 \\
\hline & 5 All Declus & $0.00 \%$ & 2.57 & $2.17 \%$ & 12 & $1.49 \%$ & 1.22 & $2.17 \%$ & 11 \\
\hline 25 & 3 WoMsr & $0.00 \%$ & 2.05 & $2.17 \%$ & 12 & $40.80 \%$ & 0.88 & $2.17 \%$ & 9 \\
\hline & 3 All Declus & $0.00 \%$ & 2.46 & $2.17 \%$ & 12 & $8.96 \%$ & 1.15 & $2.17 \%$ & 11 \\
\hline & 4 WoMsr & $0.00 \%$ & 2.00 & $2.17 \%$ & 12 & $30.85 \%$ & 0.85 & $2.17 \%$ & 9 \\
\hline & 4 All Declus & $0.00 \%$ & 2.32 & $2.17 \%$ & 13 & $24.38 \%$ & 1.05 & $2.17 \%$ & 11 \\
\hline & 5 WoMsr & $0.00 \%$ & 1.99 & $2.17 \%$ & 12 & $26.37 \%$ & 0.85 & $2.17 \%$ & $\underline{9}$ \\
\hline & 5 All Declus & $0.00 \%$ & 2.26 & $2.17 \%$ & 13 & $31.34 \%$ & 1.02 & $2.17 \%$ & 10 \\
\hline 30 & 3 WoMsr & $0.00 \%$ & 1.84 & $2.17 \%$ & 12 & $13.93 \%$ & 0.85 & $2.17 \%$ & 9 \\
\hline & 3 All Declus & $0.00 \%$ & 2.16 & $2.17 \%$ & 13 & $24.88 \%$ & 1.04 & $2.17 \%$ & 11 \\
\hline & 4 WoMsr & $0.00 \%$ & 1.79 & $2.17 \%$ & 12 & $1.49 \%$ & 0.81 & $2.17 \%$ & 9 \\
\hline & 4 All Declus & $0.00 \%$ & 2.05 & $2.17 \%$ & 13 & $22.89 \%$ & 0.94 & $2.17 \%$ & 10 \\
\hline & 5 WoMsr & $0.00 \%$ & 1.79 & $2.17 \%$ & 12 & $6.47 \%$ & 0.81 & $2.17 \%$ & $\underline{9}$ \\
\hline & 5 All Declus & $0.00 \%$ & 1.98 & $2.17 \%$ & 12 & $24.88 \%$ & 0.93 & $2.17 \%$ & 10 \\
\hline
\end{tabular}




\subsubsection{Northwest Nelson}

Unlike the other South Island polygons, the Nelson region is not dominated by a small number of large earthquakes (Figs. 3.14 and 3.15 A). The overall rate is relatively stable. Applying the CURATE method with the standard mean-rate technique gives a reasonable declustered catalog (Table 3.11, Fig. 3.19). Because the mean-rate seems sufficient to identify sequences in this region, we did not apply other rates, or divide the catalog into temporal subsets.

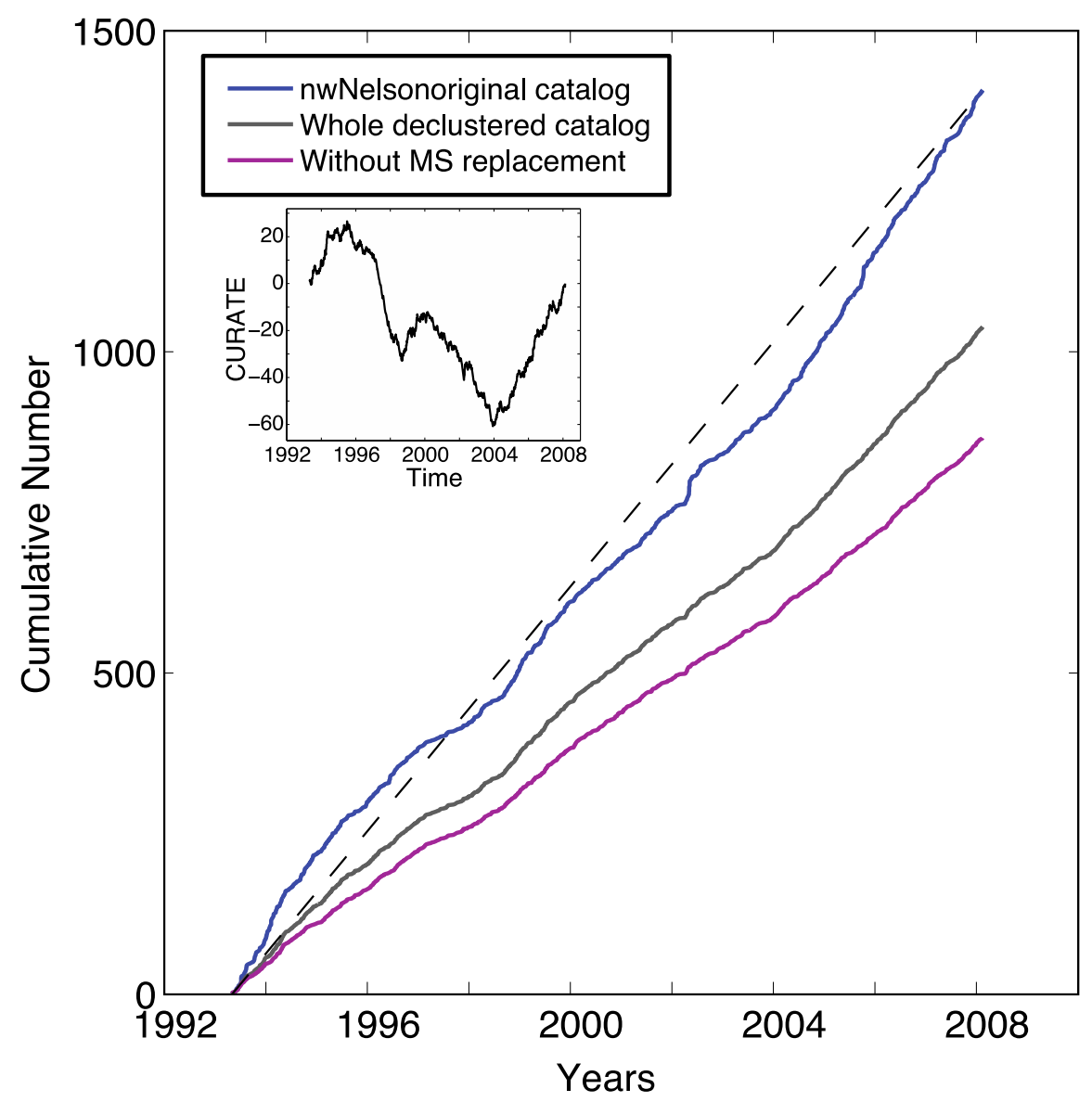

Figure 3.19 Comparison of the original and declustered catalogs for the Nelson dataset.

Cumulative number of earthquakes with time for the original (blue), declustered (gray), and declustered-without-mainshock replacement (pink) catalogs. Dashed line approximates the mean rate of the original catalog. Gray and pink lines correspond to declustered catalogs obtained using the mean-rate. Inset shows the CURATE of the whole declustered catalog for mean. 
Table 3-11 Poisson Testing of the Nelson subset using the CURATE method.

\begin{tabular}{|c|c|c|c|c|c|}
\hline & & $\begin{array}{c}\% \\
\text { Poisson } \\
\end{array}$ & $\begin{array}{c}\text { Variance } \\
\text { / Mean }\end{array}$ & \begin{tabular}{|c|}
$\#$ \\
eqs \\
\end{tabular} & DOF \\
\hline & & \multicolumn{4}{|c|}{ 1993-2007.5 mean rate } \\
\hline \multirow[t]{6}{*}{10} & 3 WoMsr & $99.00 \%$ & 1.01 & 1075 & 6 \\
\hline & 3 All Declus & $42.29 \%$ & 1.09 & 1189 & 6 \\
\hline & 4 WoMsr & $99.50 \%$ & 0.97 & 1052 & 6 \\
\hline & 4 All Declus & $68.16 \%$ & 1.06 & 1174 & 6 \\
\hline & 5 WoMsr & $97.51 \%$ & 0.96 & 1037 & 6 \\
\hline & 5 All Declus & $72.14 \%$ & 1.04 & 1165 & 6 \\
\hline \multirow[t]{6}{*}{15} & 3 WoMsr & $92.54 \%$ & 0.95 & 1024 & 5 \\
\hline & 3 All Declus & $82.09 \%$ & 1.02 & 1154 & 6 \\
\hline & 4 WoMsr & $93.53 \%$ & 0.91 & 983 & 5 \\
\hline & 4 All Declus & $85.07 \%$ & 0.98 & 1129 & 6 \\
\hline & 5 WoMsr & $87.06 \%$ & 0.90 & 974 & 5 \\
\hline & 5 All Declus & $84.58 \%$ & 0.97 & 1122 & 6 \\
\hline \multirow[t]{6}{*}{20} & 3 WoMsr & $81.09 \%$ & 0.91 & 987 & 5 \\
\hline & 3 All Declus & $94.03 \%$ & 0.99 & 1131 & 6 \\
\hline & 4 WoMsr & $49.75 \%$ & 0.84 & 932 & 5 \\
\hline & 4 All Declus & $85.57 \%$ & 0.93 & 1095 & 6 \\
\hline & 5 WoMsr & $30.85 \%$ & 0.82 & 921 & 5 \\
\hline & 5 All Declus & $60.70 \%$ & 0.91 & 1085 & 6 \\
\hline \multirow[t]{6}{*}{25} & 3 WoMsr & $59.70 \%$ & 0.86 & 936 & 5 \\
\hline & 3 All Declus & $73.63 \%$ & 0.93 & 1093 & 6 \\
\hline & 4 WoMsr & $18.91 \%$ & 0.81 & 880 & 5 \\
\hline & 4 All Declus & $53.23 \%$ & 0.88 & 1054 & 6 \\
\hline & 5 WoMsr & $7.96 \%$ & 0.77 & 866 & 5 \\
\hline & 5 All Declus & $46.27 \%$ & 0.84 & 1039 & 5 \\
\hline
\end{tabular}




\subsection{Hikurangi Margins (Shallow dipping subduction)}

We have also applied the CURATE method to the shallow parts of the

Hikurangi subduction margin. The area is broken up into three polygons on the basis of the location and behavior of known slow slip earthquakes (e.g. [Beavan et al., 2007; McCaffrey et al., 2008; Wallace and Beavan, 2010; Wallace et al., 2012]). We have titled the three subsets from north to south Hikurangi-East Cape, HikurangiGisborne, and Hikurangi-Castle Point (Fig 3.20). While the subduction zone continues to the South, most identified slow-slip events along the East Coast lie within these three polygons. These subsets are another way to explore the behavior of the CURATE method and the declustered catalog. For processing these subsets we use the same GeoNet earthquake catalog that was downloaded for the CVR and we processed the data at the same magnitude and depth cut offs of $\mathrm{M} \geq 2.45$, and $\mathrm{d} \leq$ $40 \mathrm{~km}$.

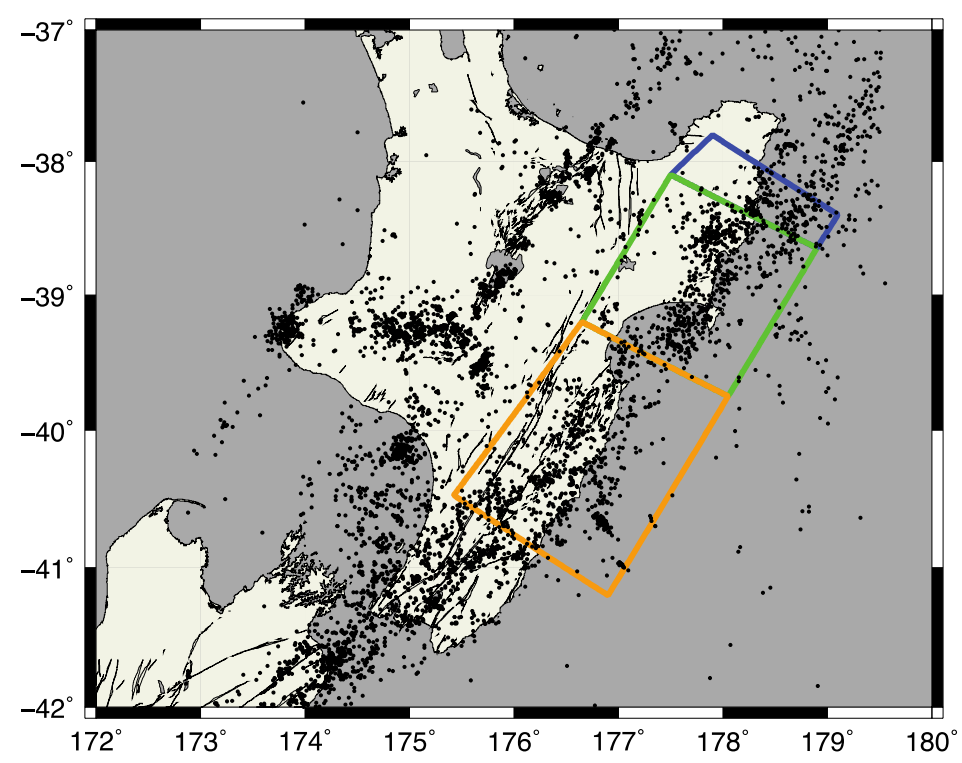

Figure 3.20 Location map for three polygons in the Hikurangi Margin of New Zealand. See text for an explanation of the choice of polygon. 
There is some variation in the yearly rates (Fig 3.21) in the Hikurangi subsets, but the scale of the variations in all subsets is much smaller than the catalogs dominated by large earthquakes and aftershocks (Figs. 3.5 and 3.15). The Castle Point set shows a nearly regular variation, and is overall more stable than the other two subsets. We ran both mean and median yearly rates for all three of these subsets. The resulting cumulative number plots of the declustered catalogs are shown in Figures 3.22-3.24. The Castle Point subset is much more linear than the other Hikurangi subsets, and the mean rate is a much better fit to the original catalog. The nearly linear shape of the original catalog is similar to the Nelson subset on the South Island. Although the Castle Point subset looks better than the others, it too shows some periods of variation, and it also displays a more active period from 1993 through mid-1995. The East Cape and Castle Point subsets have well behaved declustered catalogs for some selection parameters using both the mean and median rates (Table 3.12, Fig. 3.25). Note that because it is well behaved in some of the mean-rate catalogs we prefer to use that method to have more events in the declustered catalog and so only parameters for the mean-rate runs are given in Table 3.12. The dispersion and Poisson fraction results overlap for the mean and median rate methods (Fig. 3.25), indicating that similar results are achieved for both methods. The East Cape subset shows better Poisson results for the declustered catalog without mainshock replacement than with replacement. This may again indicate some kind of clustering of sequences in time. The Gisborne subset is not well behaved. Despite the overlap in the two methods only the largest selection parameters (30 km 3-5 days) return some Poisson results in the chi-squared test (Table 3.12). 
Table 3-12 Poisson Testing of subsets in the Hikurangi margin using the CURATE method with mean rate.

\begin{tabular}{|c|c|c|c|c|c|c|c|c|c|c|c|c|c|}
\hline & & $\begin{array}{c}\% \\
\text { Poisson }\end{array}$ & $\begin{array}{l}\text { Variance } \\
\text { / Mean }\end{array}$ & $\begin{array}{c}\# \\
\text { eqs }\end{array}$ & DOF & $\begin{array}{c}\% \\
\text { Poisson }\end{array}$ & $\begin{array}{l}\text { Variance } \\
\text { / Mean }\end{array}$ & $\begin{array}{c}\# \\
\text { eqs }\end{array}$ & DOF & $\begin{array}{c}\% \\
\text { Poisson }\end{array}$ & $\begin{array}{c}\text { Variance } \\
\text { / Mean }\end{array}$ & $\begin{array}{c}\# \\
\text { eqs }\end{array}$ & DOF \\
\hline & & \multicolumn{4}{|c|}{ East Cape } & \multicolumn{4}{|c|}{ Gisborne } & \multicolumn{4}{|c|}{ Castle Point } \\
\hline \multirow[t]{6}{*}{10} & 3 WoMsr & $0.00 \%$ & 1.33 & 825 & 5 & $0.00 \%$ & 2.22 & 2677 & 10 & $21.89 \%$ & 1.23 & 4406 & 14 \\
\hline & 3 All Declus & $0.00 \%$ & 1.77 & 976 & 6 & $0.00 \%$ & 2.88 & 3011 & 11 & $0.00 \%$ & 1.44 & 4817 & 14 \\
\hline & 4 WoMsr & $1.49 \%$ & 1.21 & 796 & 5 & $0.00 \%$ & 2.10 & 2640 & 10 & $28.86 \%$ & 1.22 & 4374 & 14 \\
\hline & 4 All Declus & $0.00 \%$ & 1.58 & 948 & 6 & $0.00 \%$ & 2.74 & 2974 & 11 & $0.00 \%$ & 1.41 & 4787 & 14 \\
\hline & 5 WoMsr & $13.43 \%$ & 1.12 & 775 & 5 & $0.00 \%$ & 2.05 & 2620 & 10 & $42.29 \%$ & 1.20 & 4349 & 13 \\
\hline & 5 All Declus & $0.00 \%$ & 1.50 & 932 & 5 & $0.00 \%$ & 2.67 & 2955 & 11 & $0.00 \%$ & 1.39 & 4766 & 14 \\
\hline \multirow[t]{6}{*}{15} & 3 WoMsr & $54.23 \%$ & 1.12 & 745 & 5 & $0.00 \%$ & 1.77 & 2442 & 10 & $93.03 \%$ & 1.08 & 4128 & 13 \\
\hline & 3 All Declus & $0.00 \%$ & 1.41 & 898 & 5 & $0.00 \%$ & 2.29 & 2814 & 10 & $2.99 \%$ & 1.27 & 4614 & 13 \\
\hline & 4 WoMsr & $97.51 \%$ & 1.03 & 707 & 5 & $0.00 \%$ & 1.67 & 2401 & 10 & $91.54 \%$ & 1.08 & 4077 & 13 \\
\hline & 4 All Declus & $0.00 \%$ & 1.28 & 865 & 5 & $0.00 \%$ & 2.16 & 2768 & 10 & $19.90 \%$ & 1.24 & 4564 & 13 \\
\hline & 5 WoMsr & $98.51 \%$ & 1.02 & 694 & 5 & $0.00 \%$ & 1.64 & 2376 & 10 & $96.52 \%$ & 1.05 & 4042 & 12 \\
\hline & 5 All Declus & $0.00 \%$ & 1.23 & 849 & 5 & $0.00 \%$ & 2.10 & 2738 & 10 & $51.74 \%$ & 1.20 & 4529 & 13 \\
\hline \multirow[t]{6}{*}{20} & 3 WoMsr & $98.51 \%$ & 1.02 & 687 & 5 & $0.00 \%$ & 1.46 & 2230 & 10 & $99.00 \%$ & 0.94 & 3847 & 12 \\
\hline & 3 All Declus & $0.00 \%$ & 1.19 & 846 & 5 & $0.00 \%$ & 1.85 & 2625 & 10 & $90.05 \%$ & 1.10 & 4402 & 14 \\
\hline & 4 WoMsr & $96.02 \%$ & 0.96 & 645 & 4 & $0.00 \%$ & 1.40 & 2191 & 10 & $98.01 \%$ & 0.93 & 3792 & 12 \\
\hline & 4 All Declus & $15.92 \%$ & 1.08 & 807 & 5 & $0.00 \%$ & 1.74 & 2570 & 10 & $93.03 \%$ & 1.07 & 4335 & 13 \\
\hline & 5 WoMsr & $90.05 \%$ & 0.92 & 632 & 4 & $0.00 \%$ & 1.37 & 2167 & 9 & $97.51 \%$ & 0.91 & 3754 & 12 \\
\hline & 5 All Declus & $24.88 \%$ & 1.04 & 791 & 5 & $0.00 \%$ & 1.67 & 2532 & 9 & $96.02 \%$ & 1.03 & 4282 & 13 \\
\hline \multirow[t]{6}{*}{25} & 3 WoMsr & $80.60 \%$ & 0.93 & 646 & 4 & $0.00 \%$ & 1.32 & 2084 & 9 & $94.53 \%$ & 0.83 & 3637 & 11 \\
\hline & 3 All Declus & $16.42 \%$ & 1.06 & 805 & 5 & $0.00 \%$ & 1.65 & 2498 & 9 & $92.04 \%$ & 0.97 & 4220 & 13 \\
\hline & 4 WoMsr & $76.62 \%$ & 0.88 & 611 & 4 & $0.00 \%$ & 1.25 & 2045 & 9 & $89.55 \%$ & 0.82 & 3568 & 12 \\
\hline & 4 All Declus & $27.36 \%$ & 1.00 & 767 & 5 & $0.00 \%$ & 1.54 & 2438 & 10 & $88.56 \%$ & 0.94 & 4138 & 13 \\
\hline & 5 WoMsr & $57.21 \%$ & 0.85 & 599 & 4 & $1.00 \%$ & 1.21 & 2013 & 9 & $69.15 \%$ & 0.79 & 3517 & 12 \\
\hline & 5 All Declus & $23.38 \%$ & 0.96 & 751 & 5 & $0.00 \%$ & 1.47 & 2387 & 10 & $89.05 \%$ & 0.89 & 4069 & 12 \\
\hline
\end{tabular}


Hikurangi - East Cape

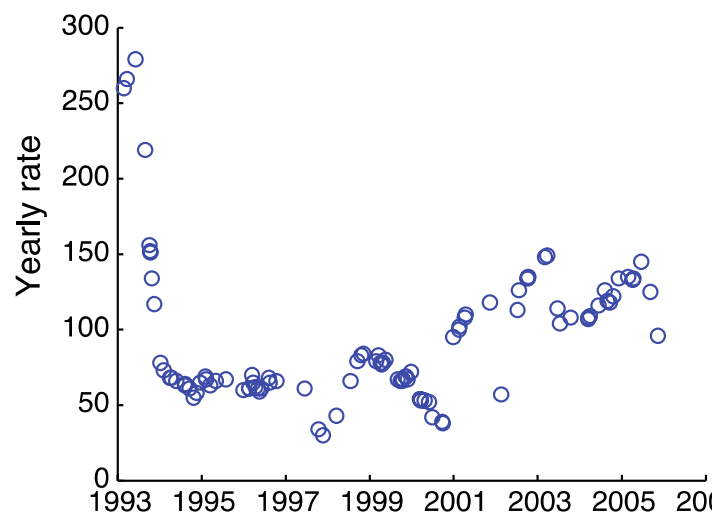

Hikurangi - Gisborne

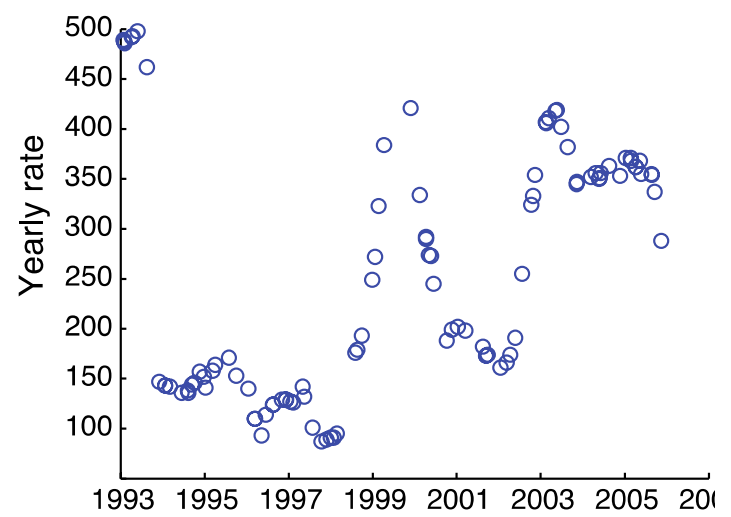

Hikurangi - Castle Point

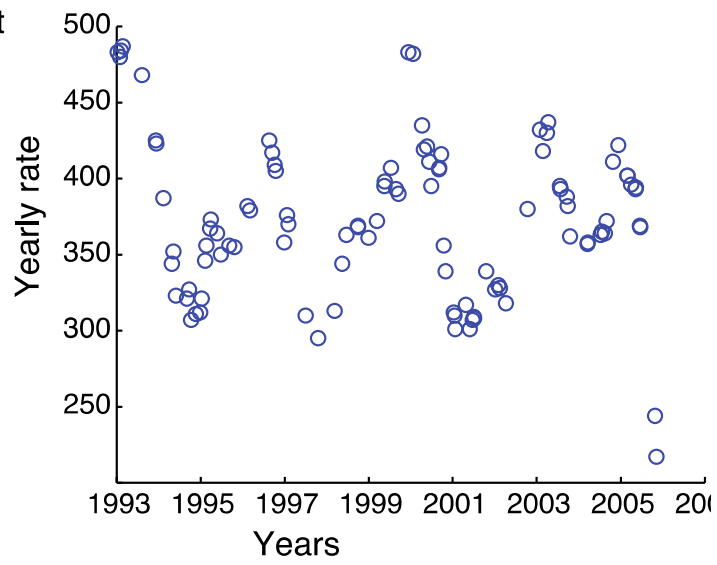

Figure 3.21 Yearly earthquake totals from 100 start-times chosen randomly for the three Hikurangi margin subsets. 


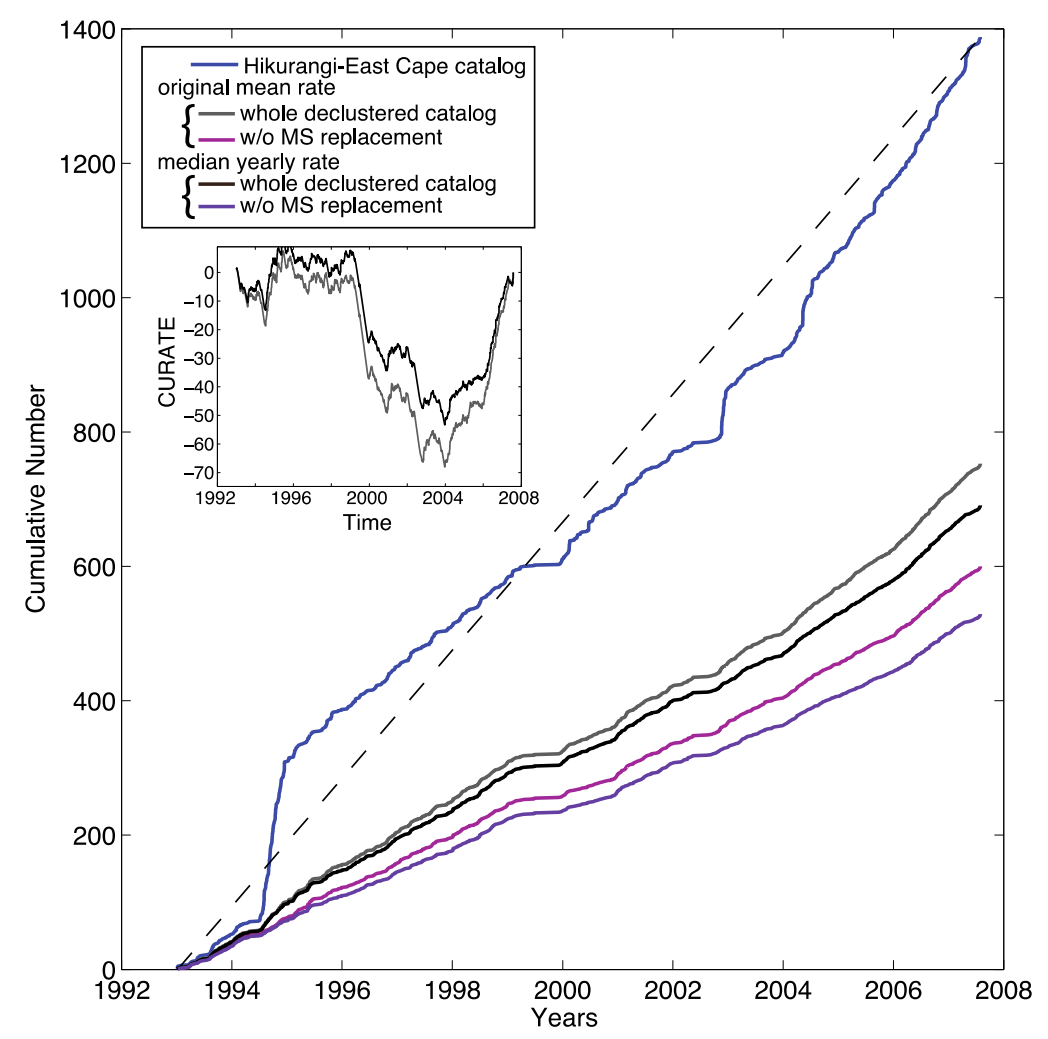

Figure 3.22 Comparisons of the original and declustered catalogs for the Hikurangi-East Cape dataset.

Cumulative number of earthquakes with time for the original (blue), declustered (gray and black), and declustered-without-mainshock replacement (pink and purple) catalogs. Dashed line approximates the mean rate of the original catalog. Gray and pink lines correspond to declustered catalogs obtained using the mean-rate. Black and purple lines represent declustered catalogs obtained using the median-rate. Inset shows the CURATE of the whole declustered catalog for mean (gray) and median rate (black). 


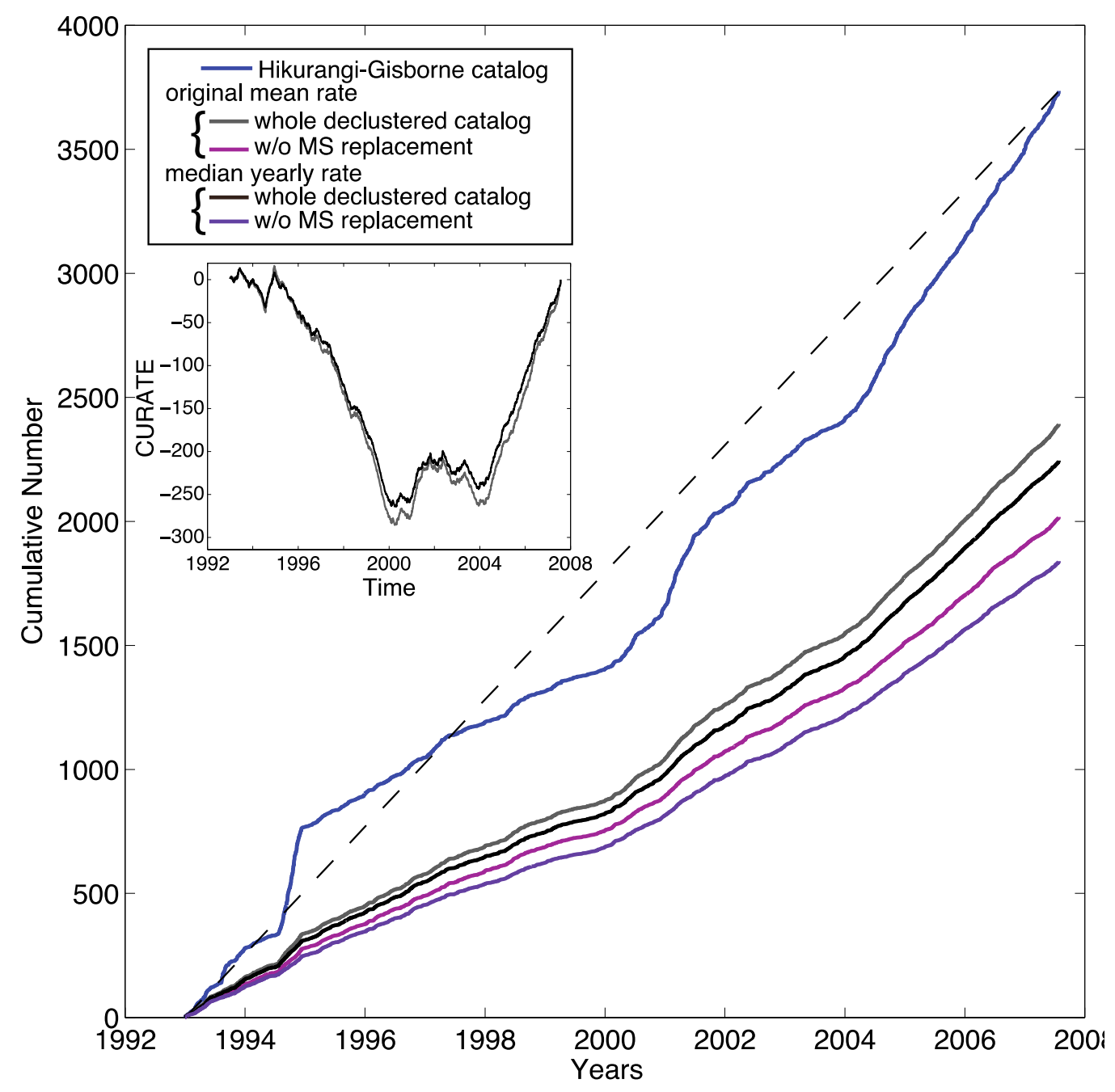

Figure 3.23 Comparison of the original and declustered catalogs for the HikurangiGisborne dataset.

Cumulative number of earthquakes with time for the original (blue), declustered (gray and black), and declustered-without-mainshock replacement (pink and purple) catalogs. Dashed line approximates the mean rate of the original catalog. Gray and pink lines correspond to declustered catalogs obtained using the mean-rate. Black and purple lines represent declustered catalogs obtained using the median-rate. Inset shows the CURATE of the whole declustered catalog for mean (gray) and median rate (black). 


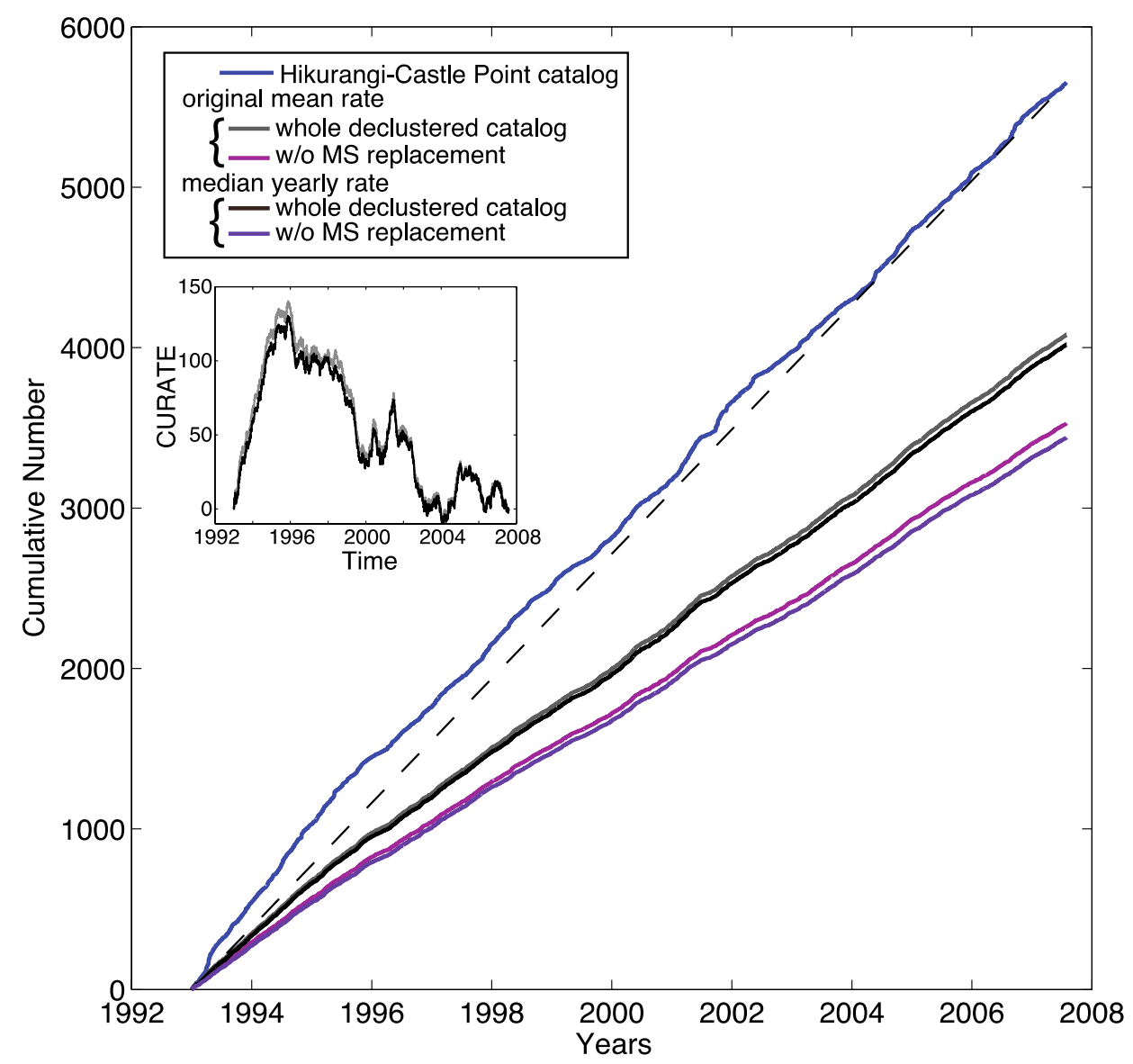

Figure 3.24 Comparison of the original and declustered catalogs for the HikurangiCastle Point dataset.

Cumulative number of earthquakes with time for the original (blue), declustered (gray and black), and declustered-without-mainshock replacement (pink and purple) catalogs. Dashed line approximates the mean rate of the original catalog. Gray and pink lines correspond to declustered catalogs obtained using the mean-rate. Black and purple lines represent declustered catalogs obtained using the median-rate. Inset shows the CURATE of the whole declustered catalog for mean (gray) and median rate (black). 
Hikurangi - East Cape
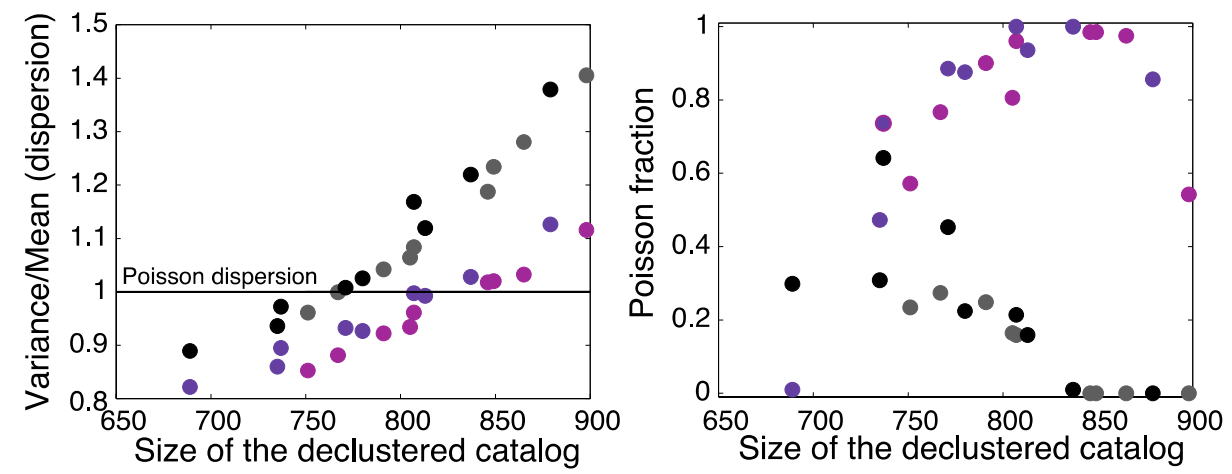

Hikurangi - Gisborne
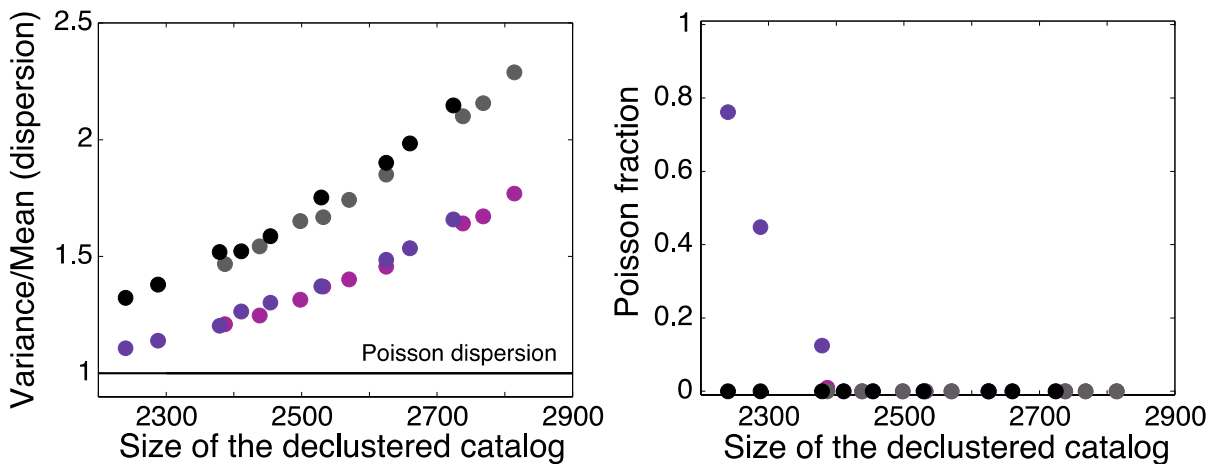

Hikurangi - Castle Point
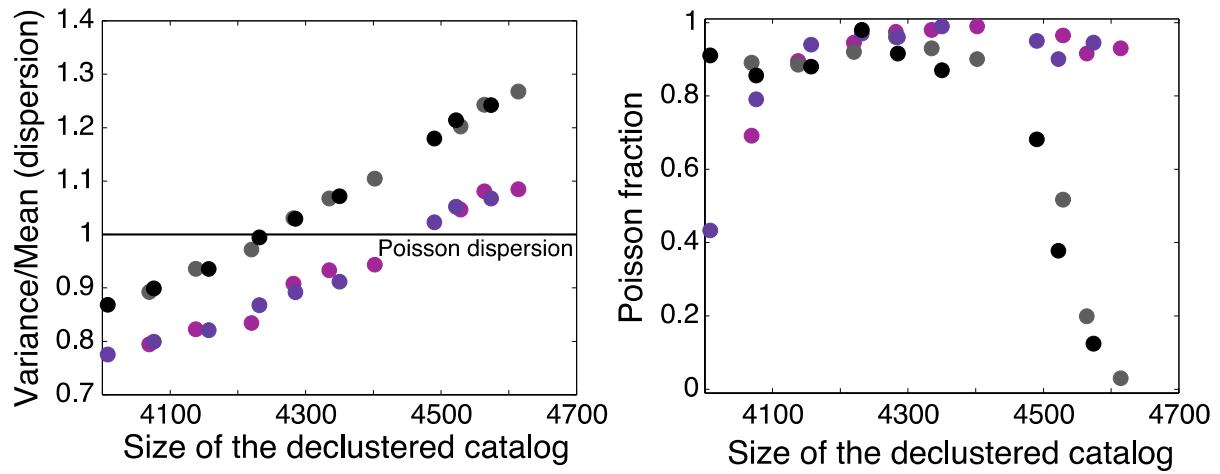

Figure 3.25 Poisson fraction and Dispersion for the three Hikurangi datasets.

Both quantities are calculated for the declustered catalogs with and without mainshock-replacement using both the mean (gray/pink) and median (black/purple) yearly rates. 
To see whether this could be explained by temporal variation we broke the Gisborne catalog into a number of different temporal subsets. Because the initial tests showed little difference between the mean and median rates we only ran the mean rate method on the temporal subsets. We did not try all possible temporal variations, but we found three subsets (1993-1998, 2000-2003, 2004-2007.5) that produce reasonable Poisson results and dispersions (Table 3.13). There is not a sharp change, but the behavior appears to change sometime between 1999 and 2000, with another change around the beginning of 2004 (Fig. 3.23). The early period has a rate of 219 earthquakes/year, and during the time period 2000-2003 the rate increases to 251 earthquakes/year. Because there is so little seismicity in 1999 the period from 19992003 has a lower average rate than 1993-1998 (219 earthquake/year) if it is included in the middle time period. The later time period has an even higher rate of 371 earthquakes/year. Once these time periods were identified it also appears that there are hints of parallel behavior in the other two Hikurangi subsets, but to an extent that is within the expected variability of the catalog. 
Table 3-13 Poisson Testing of different temporal subsets for the Gisborne region using the CURATE method with mean rate.

\begin{tabular}{|c|c|c|c|c|c|c|c|c|c|c|c|c|c|c|c|c|}
\hline & & $\begin{array}{c}\% \\
\text { Poisson }\end{array}$ & $\begin{array}{l}\text { Variance } \\
\text { /Mean }\end{array}$ & \# eqs & DOF & $\begin{array}{c}\% \\
\text { Poisson }\end{array}$ & $\begin{array}{l}\text { Variance } \\
\text { /Mean }\end{array}$ & \# eqs & DOF & $\begin{array}{c}\% \\
\text { Poisson }\end{array}$ & $\begin{array}{l}\text { Variance } \\
\text { /Mean }\end{array}$ & \# eqs & DOF & $\begin{array}{c}\% \\
\text { Poisson }\end{array}$ & $\begin{array}{l}\text { Variance } \\
\text { /Mean }\end{array}$ & \# eqs \\
\hline & & \multicolumn{4}{|c|}{ Gisborne 1993-1998 } & \multicolumn{4}{|c|}{ Gisborne 1999-2003 } & \multicolumn{4}{|c|}{ Gisborne $2000-2003$} & \multicolumn{3}{|c|}{ Gisborne 2004-2007.! } \\
\hline \multirow[t]{6}{*}{15} & 3 WoMsr & $0.00 \%$ & 1.72 & 784 & 8 & $0.00 \%$ & 1.86 & 720 & 7 & $1.49 \%$ & 1.64 & 684 & 8 & $89.55 \%$ & 0.80 & $\overline{941}$ \\
\hline & 3 All Declus & $0.00 \%$ & 2.62 & 916 & 8 & $0.00 \%$ & 2.26 & 835 & 8 & $0.00 \%$ & 2.08 & 779 & 8 & $81.59 \%$ & 0.86 & 1057 \\
\hline & 4 WoMsr & $0.00 \%$ & 1.55 & 767 & 8 & $0.00 \%$ & 1.70 & 701 & 7 & $9.45 \%$ & 1.50 & 664 & 8 & $91.04 \%$ & 0.78 & 933 \\
\hline & 4 All Declus & $0.00 \%$ & 2.38 & 897 & 8 & $0.00 \%$ & 2.07 & 814 & 8 & $0.00 \%$ & 1.93 & 760 & 8 & $81.09 \%$ & 0.84 & 1048 \\
\hline & 5 WoMsr & $0.00 \%$ & 1.48 & 759 & 8 & $0.00 \%$ & 1.65 & 689 & 7 & $16.42 \%$ & 1.47 & 658 & 8 & $90.05 \%$ & 0.77 & 921 \\
\hline & 5 All Declus & $0.00 \%$ & 2.20 & 883 & 7 & $0.00 \%$ & 2.03 & 804 & 8 & $0.00 \%$ & 1.91 & 753 & 9 & $87.56 \%$ & 0.81 & 1037 \\
\hline \multirow[t]{6}{*}{20} & 3 WoMsr & $36.82 \%$ & 1.39 & 718 & 7 & $0.00 \%$ & 1.44 & 663 & 7 & $51.24 \%$ & 1.26 & 627 & 7 & $76.12 \%$ & 0.76 & 884 \\
\hline & 3 All Declus & $0.00 \%$ & 1.93 & 847 & 7 & $0.00 \%$ & 1.90 & 790 & 8 & $0.00 \%$ & 1.71 & 731 & 8 & $87.56 \%$ & 0.78 & 1008 \\
\hline & 4 WoMsr & $55.22 \%$ & 1.30 & 707 & 7 & $0.00 \%$ & 1.36 & 650 & 8 & $72.64 \%$ & 1.19 & 613 & 7 & $83.08 \%$ & 0.76 & 866 \\
\hline & 4 All Declus & $0.00 \%$ & 1.70 & 824 & 8 & $0.00 \%$ & 1.71 & 767 & 8 & $0.00 \%$ & 1.57 & 710 & 8 & $87.06 \%$ & 0.77 & 990 \\
\hline & 5 WoMsr & $65.17 \%$ & 1.27 & 704 & 7 & $5.97 \%$ & 1.30 & 635 & 7 & $85.07 \%$ & 1.17 & 605 & 7 & $77.61 \%$ & 0.74 & 852 \\
\hline & 5 All Declus & $0.00 \%$ & 1.66 & 819 & 8 & $0.00 \%$ & 1.62 & 751 & 7 & $0.50 \%$ & 1.51 & 699 & 8 & $72.64 \%$ & 0.73 & 973 \\
\hline \multirow[t]{6}{*}{25} & 3 WoMsr & $80.10 \%$ & 1.23 & 670 & 7 & $51.24 \%$ & 1.30 & 622 & 7 & $78.61 \%$ & 1.17 & 599 & 7 & $62.19 \%$ & 0.70 & 828 \\
\hline & 3 All Declus & $1.00 \%$ & 1.55 & 795 & 8 & $0.00 \%$ & 1.73 & 759 & 7 & $0.00 \%$ & 1.57 & 709 & 8 & $61.69 \%$ & 0.71 & 964 \\
\hline & 4 WoMsr & $98.01 \%$ & 1.16 & 658 & 7 & $82.09 \%$ & 1.18 & 603 & 7 & $92.54 \%$ & 1.08 & 583 & 7 & $73.63 \%$ & 0.71 & 809 \\
\hline & 4 All Declus & $4.98 \%$ & 1.41 & 775 & 8 & $0.00 \%$ & 1.48 & 725 & 7 & $7.96 \%$ & 1.42 & 685 & 8 & $56.22 \%$ & 0.70 & 941 \\
\hline & 5 WoMsr & $99.50 \%$ & 1.11 & 650 & 7 & $89.55 \%$ & 1.15 & 596 & 7 & $98.01 \%$ & 1.05 & 574 & 7 & $57.21 \%$ & 0.69 & 797 \\
\hline & 5 All Declus & $19.40 \%$ & 1.31 & 762 & 8 & $0.00 \%$ & 1.42 & 710 & 7 & $21.89 \%$ & 1.35 & 670 & 8 & $49.75 \%$ & 0.69 & 923 \\
\hline \multirow[t]{6}{*}{30} & 3 WoMsr & $100.00 \%$ & 1.05 & 640 & 7 & $85.57 \%$ & 1.17 & 592 & 7 & $98.51 \%$ & 1.04 & 565 & 7 & $86.57 \%$ & 0.73 & 776 \\
\hline & 3 All Declus & $60.20 \%$ & 1.26 & 760 & 8 & $0.00 \%$ & 1.52 & 730 & 7 & $13.93 \%$ & 1.37 & 677 & 8 & $73.63 \%$ & 0.72 & 924 \\
\hline & 4 WoMsr & $100.00 \%$ & 0.99 & 630 & 6 & $95.02 \%$ & 1.12 & 579 & 7 & $98.51 \%$ & 1.00 & 553 & 6 & $83.58 \%$ & 0.70 & 753 \\
\hline & 4 All Declus & $73.13 \%$ & 1.20 & 743 & 7 & $0.50 \%$ & 1.33 & 702 & 7 & $49.25 \%$ & 1.24 & 654 & 7 & $73.13 \%$ & 0.71 & 898 \\
\hline & 5 WoMsr & $100.00 \%$ & 0.97 & 621 & 6 & $99.00 \%$ & 1.08 & 568 & 7 & $97.01 \%$ & 0.97 & 541 & 6 & $65.17 \%$ & 0.70 & 739 \\
\hline & 5 All Declus & $88.56 \%$ & 1.10 & 727 & 7 & $14.43 \%$ & 1.28 & 685 & 7 & $70.65 \%$ & 1.18 & 637 & 7 & $52.74 \%$ & 0.68 & 877 \\
\hline
\end{tabular}




\subsection{Discussion}

\subsubsection{Clustering of sequences in time}

By analyzing seismicity in Southern California and throughout New Zealand, we have observed a wide variety of behavior in both the original and declustered catalogs. For the entire SCA catalog, and the aftershock areas in New Zealand, the mean rate was insufficient to adequately identify sequence activity. Due to the dominance of large earthquakes in these catalogs there is often clustering of sequences (mainshocks) that prevents the whole declustered catalog from returning Poisson values. In swarm areas we have also recovered catalogs where, although the whole declustered catalog is non-Poisson, the catalog WoMSR is Poisson. The Poisson results of the declustered catalog WoMSR indicates that sequences cluster in time. There are no large earthquakes in some of the subsets (e.g. Lake Taupo) that display the non-Poisson declustered catalog, which suggests that large earthquakes alone do not cause the changes in clustering with time. While there are some selection parameters $(10 \mathrm{~km})$ that seem too small to suit the data, it does not appear that there is an obvious variation with selection parameters as we might expect if the problem were due in part to incorrectly identifying sequences as being smaller than their actual size. In the swarm areas, when small temporal subsets are taken they produce Poisson results in the whole declustered catalog. This leads us to the interesting possibility that, while background earthquakes may follow a Poisson distribution in time, sequences themselves may not.

In interpreting the yearly rate plots it is important to realize that short periods that appear to have a high rate may be showing sampling bias. Trends that last more than one year may be actual changes in rate. 


\subsubsection{Search for rate changes in the broader CVR catalog}

In light of the results in this chapter, we also went back and tested the entire CVR catalog presented in the previous chapter. We find some similarities to the regional results. The catalog without mainshock replacement gives more Poisson results in all window lengths (tested 10, 20, and 50 days). However, in the larger CVR catalog, it is less clear than in the Lake Taupo subset, when the rate changes occur in time. The more subdued signature of the rate changes may be due to a mixture of broad-scale and more localized influences. Another difference between the CVR-wide case and the Lake Taupo subset is that the Gardner and Knopoff method does not fail to give good Poisson results for the entire period in the CVR. However, the stochastic declustering and SLC methods give better results when the time period is broken into an early and late period. (Note CURATE is the only method that could potentially need to be re-run over smaller temporal subsets; other methods should return identical sequences over different time periods if boundaries between time periods are accounted for.) Because the Gardner and Knopoff declustered catalogs are so small, the potential variations in rate are also smaller. Thus the long time windows of the Gardner and Knopoff methods partially protect it from seeing sequence clustering in time.

To see how spatially extensive potential rate-changes with time are we plotted an additional three subsets in the North Island: Waiouru, Ruahpehu (including Erua/Raurimu fault), and Wanganui regions (Fig. 3.11). The Waiouru and Raurimu Fault (Erua) earthquake clusters, to the East and West of Ruapehu respectively, have both been cited as potential areas that show stress changes associated with volcanic activity at Ruapehu [Hayes et al., 2004; Hurst and McGinty, 1999; Keats et al., 2011]. While there is only one major eruptive episode (two eruptions) during the recorded 
earthquake catalog, it is worth comparing these two locations to illustrate the possible or expected behavior of future activity. Figure 3.27 shows the CURATE plots for all four subset areas. Both the Ruapehu and Waiouru regions have their biggest CURATE increases in 1997, following eruptive activity. However figure 3.27 shows that there was spatial-temporal clustering at Ruapehu preceding the eruptive activity while that at Waiouru follows the main eruptions. This may be specific to the direction or location of magmatic intrusion during the 1995-1996 eruptions. It might also indicate that the Waiouru zone is unlikely to be as useful for monitoring as the Raurimu fault area. It is interesting to note that the Wanganui region also has an increase in activity following the eruptions. There is also an increase in the Lake Taupo area during 1998-2003. Although this period is after the eruption, the declustered catalogs are more Poisson if 1997 and periods directly after the eruption are excluded, suggesting the process may not be directly linked to the eruptive activity or that complicating factors immediately following the eruption do not allow us to detect the trend. Although the increased rate in the Wanganui and Lake Taupo catalogs continues in time after activity settles down in the Waiouru and Ruapehu/Raurimu regions, the fact that all subsets show some kind of increase suggests that there may be broad scale changes in the subduction zone which cause widespread activity. Given that the increased seismicity is observed from 1996-2003, depending on the region, these changes may be related to stress re-adjustment following the eruptions, or to the same processes that influence the timing of eruptions. Keats et al. [2011] examined changes in shear-wave splitting and b-values from 1999-2010 in the Erua/Raurimu area. They found several periods of stable parameters separated by shorter transition periods. While the temporal changes of the Lake Taupo subset around 1998-2003 (Fig. 3.12, 3.26, 3.27) and the increases in the 
CURATES of Ruapehu/Raurimu (black) and Waiouru (orange) shown in Figure 3.27 do not have identical timing, this only tells us that these transition periods or changes are not a dominant feature of the seismicity rate. A more detailed analysis is needed to identify rate changes associated with the changes observed by Keats et al. [2011]. 

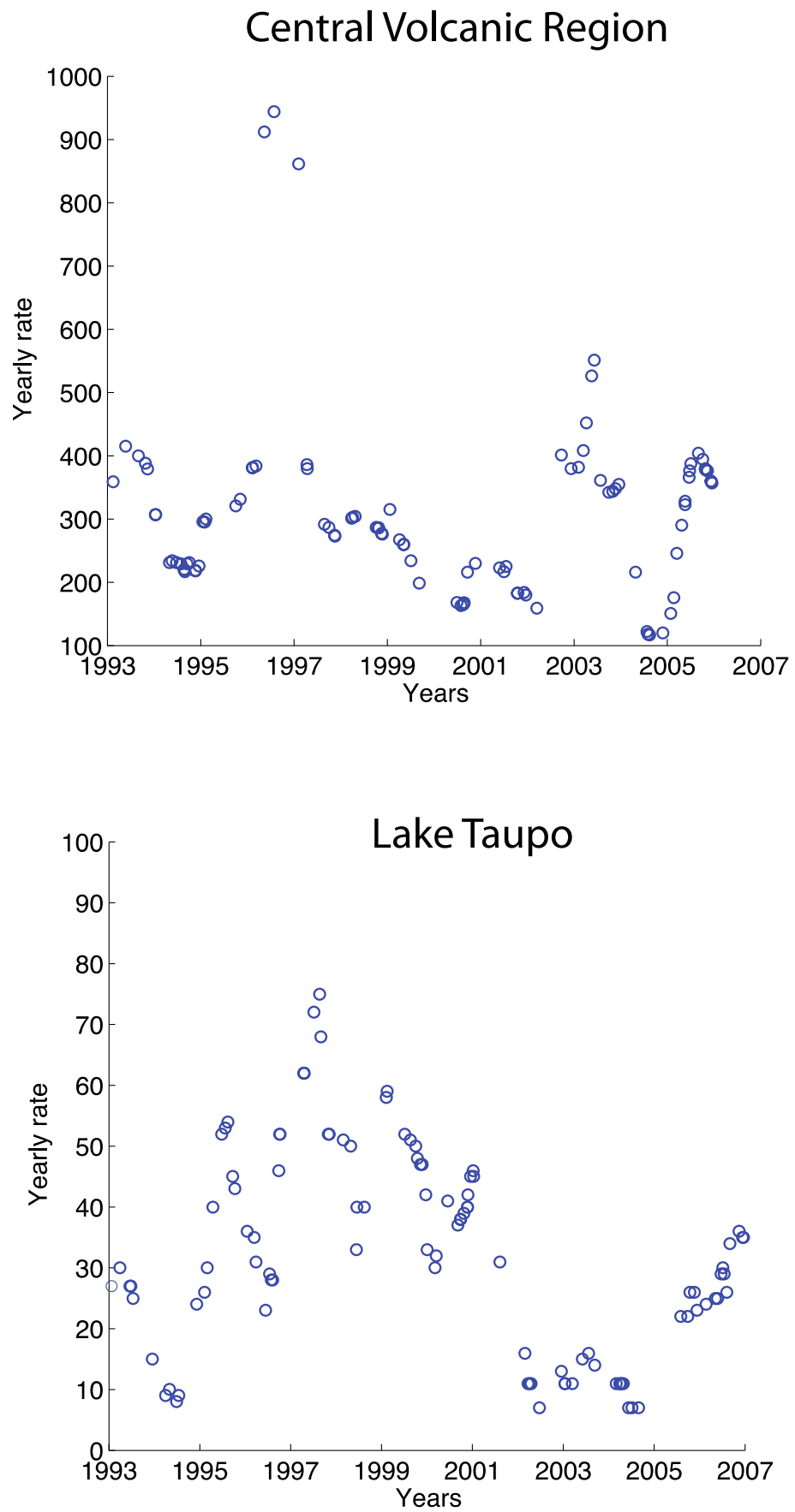

Figure 3.26 Yearly earthquake total from 100 start times chosen randomly for the entire CVR catalog discussed in Chapter two and for the Lake Taupo regional subset. 


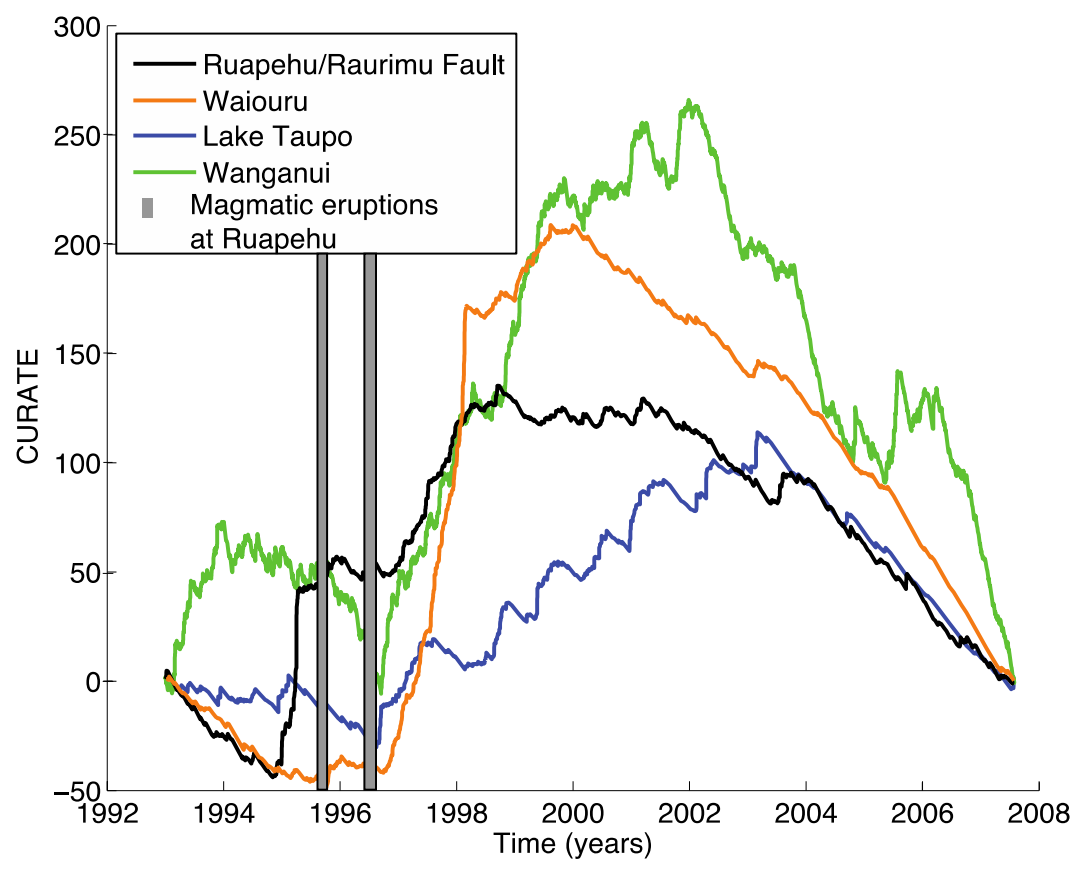

Figure 3.27 CURATE plots for the four regional subsets shown in Figure 3.11. Gray vertical bars represent the timing of magmatic eruptions at Ruapehu volcano in 1995 and 1996.

\subsubsection{Previous studies of rate changes}

\subsubsection{New Zealand}

Robinson [1979] found that there was clustering in the moment release of the New Zealand earthquake catalog. He looked at earthquakes with $M \geq 4.3$ in the Main Seismic Region of New Zealand, excluding the TVZ, and found a relatively stable moment release from 1950 up to the 1968 Inangahua earthquake. Robinson anticipated that if the observed increases seen in the catalog up to the time of his publication were indeed a pattern, that another such increase may be observed around the year 2000. Our observed increase in sequence occurrence is not directly comparable to the moment-release study since the sequence identification only involves numbers of earthquakes and does not necessarily correlate to moment release. It is also somewhat unlikely that the clustering of sequences could be seen 
through increases in moment that include $M>6$ earthquakes. Our present study conflicts with this finding in two ways. First, we see variations across a shorter period than described by Robinson. Secondly, as a minor point, we see some initial evidence in figure 3.27 that the distinction between volcanic zones and non-volcanic zones may vanish for broad-scale seismicity rate changes. Robinson [1979] also noted a potential cycle of about 9 years in earthquake rates. The cycle in earthquake rates may make a more fruitful comparison than the study of moment, but it is unclear how to compare it directly since our major changes are observed at lower magnitudes and in regions Robinson did not examine.

\subsubsection{Southern California}

The SCA catalog is perhaps the most interesting of those studied as it covers the longest period of time and is often the catalog used to test the question of whether the declustered catalogs are Poisson (e.g. [Gardner and Knopoff, 1974]). In the yearly rate plot (Fig. 3.5), the time period from 1985, until the Lander's earthquake in 1992 appears extremely variable. However this is due in part to the sparse random start times over this time. There is a clear decrease in rate in the 2-3 years preceding the 1992 Lander's mainshock. Wiemer and Wyss [1994] also investigated rate changes in the SCA catalog. Their study was much more detailed and found two independent volumes to the north and south of the aftershock region that experienced a $75 \%$ drop in seismic activity starting in 1988, and 1989 respectively. Further work is needed to see whether the changes Wiemer and Wyss [1994] observed might be attributable to more broad-scale changes, because they do not find a decrease preceding the Joshua Tree $1992 \mathrm{M}=6.1$ earthquake. In addition to sharp increases in rate associated with the 1999 Hector Mine and the 2010 El-Mayor Cucapah earthquakes, there are also smaller changes after Landers. The first is a gradual 
increase from 1994-2001, and then the second pattern seems to repeat this. After dropping back down in 2002 the rate gradual increases again through 2009. The SCA catalog is also twelve years longer than the NZ region catalog and so may simply be revealing more changes that can be observed over longer time scales.

\subsubsection{Rate changes in Hikurangi}

Out of the three Hikurangi subsets, only the Gisborne subset exhibits enough variability to return poor Poisson results in the overall catalog. Gisborne only returns Poisson results if it is subdivided into at least three time periods (Table 3.13, Fig. 3.23). The middle time period starts around 1999 and continues through 2003. This overlaps with the change seen in the Lake Taupo subset (Fig. 3.12) from 1998-2003. If the same process caused both changes in rates, it would suggest that the change acted over a large area. The last time period, starting in 2004, has a rate increase not seen at Lake Taupo. It may be associated with an upgrade of the GeoNet nationwide network that included the installation of new seismometers in the North Island [Petersen et al., 2011]. Although the change in rate may be attributable to a better network, there is also a change in behavior from 1999 to 2004 that is inexplicable. In both the East-cape and Gisborne subsets, the early time period, until around 1999 is relatively smooth, i.e. with stable rate (Fig. 3.22 and 3.23). Then, until about 2004, the rate is much more variable and the cumulative number curve is quite bumpy with some times at very low rates (flat curve), and other times with sharp increases. From around the beginning of 2004 the rate again becomes more smooth and stable, although the cumulative trend tends to curve upwards. So, while the underlying rate may change due to changes in the network, the rate variations cannot be explained in a similar way. 
The plots (Figs. 3.22-3.24) also show that the mean rate does a reasonable job compared to the median rate for the Hikurangi subsets. Unlike in most aftershock regions, the declustered catalogs run with the median rate (black and purple) sit within the mean-rate declustered catalogs (gray, with mainshock-replacement; pink without mainshock-replacement). Despite the mean rate appearing to be relatively representative of the background rate for the Hikurangi regions, they still show temporal variations in rate. The variability in these swarm regions suggests that large earthquakes are not the only source influencing rate changes in earthquake catalogs. In Chapter 5.3 we will investigate the potential relationship of increased seismicity and aseismic or slow slip.

\subsubsection{Possible Causes of Observed Variations in Rate}

\subsubsection{Geothermal Production}

Clustering of sequences was first observed in the Coso subset of the SCA catalog (Ch. 3.2.3). The Coso geothermal field stands out from other parts of SCA because it is an area undergoing active geothermal production [Bhattacharyya and Lees, 2002] (and references therein) and because it has experienced well documented triggering after both the Landers [Hill et al., 1993] and Denali Fault earthquakes [Prejean et al., 2004]. This raises the possibility that either distant triggering or geothermal activity has contributed to the observed clustering of sequences in time. We have investigated both of these possibilities by plotting the cumulative number of earthquakes in the Coso catalog with data from extraction (fig 3.28a) and injection (fig 3.28b). The data is from the California Department of Conservation, Division of Oil, Gas and Geothermal Resources. These figures also show the time of all local M $\geq 5.0$ earthquakes and global $\mathrm{M} \geq 7.0$ earthquakes known to have caused long range triggering. The rate of earthquakes does not appear to be clearly related to withdrawal 
or injection rates at the COSO geothermal plant. In particular, the onset of operations, c. 1987 and the peak in extraction/injection in c. 1990 were not accompanied by rate changes. There is a possibility that the increase in seismicity is a delayed reaction to the exploitation, but the time lag and the reason why the rates would return to preexploitation levels despite ongoing operations is unclear. Although these simple plots cannot completely rule out that field operations affect the seismicity, the observation of rate changes happening around Lake Taupo, possibly CVR-wide, and along the Hikurangi margin suggests a different cause as these other catalogs only contain small portions of active geothermal areas and have not been shown to experience triggering (triggering is discussed further in the chapter 5).
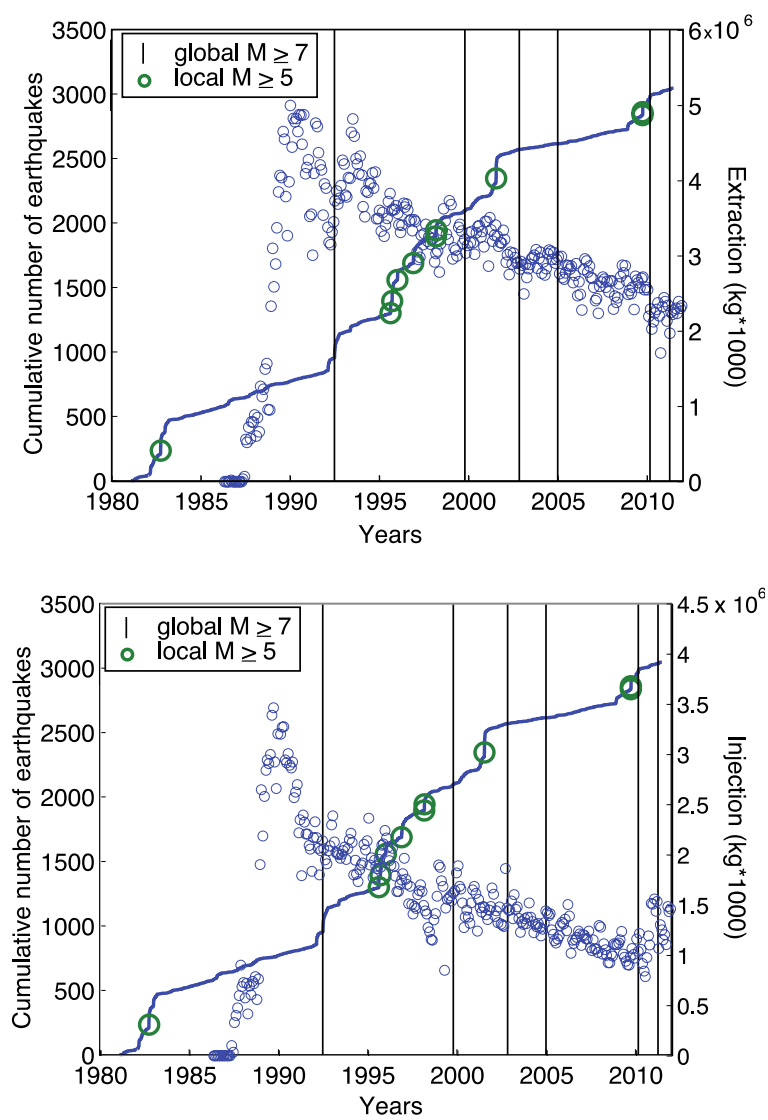

Figure 3.28 Cumulative number of earthquakes plotted with monthly extraction and injection data from the Coso geothermal region.

The cumulative earthquake rate is shown as a blue line, extraction and injection data are shown as open blue circles. The times of significant global earthquakes and large local magnitude earthquakes are also shown (vertical lines, green circles respectively). Injection and extraction data are from the California Department of Conservation, Division of Oil, Gas and Geothermal Resources. 


\subsubsection{Earthquake Triggering}

Figure 3.28 shows that, although the earthquakes were similar in size, there is much more seismicity (number of events) after Landers (1992) than after the Hector Mine earthquake (1999). The larger size of the 1992 activity is due to the widespread triggering observed following the Landers earthquake [Hill et al., 1993]. We see no reason why the CURATE algorithm (or other clustering methods) would not recover triggered seismicity. The timescale of persistently increased activity is much longer than documented triggering sequences, which are typically on the order of hours to tens of days [Brodsky, 2006; Husen et al., 2004]. The durations reported for triggered sequences are for the most obvious increases in earthquake occurrence directly following the triggering event, but background seismicity rates may experience changes that lasts for longer time periods [Toda et al., 2011; Wyss and Wiemer, 2000].

\subsubsection{Background distribution}

Wang et al. [2010b] have presented tests for stationarity of the California (both north and south) earthquake catalog at magnitudes $M \geq 4.0$. They note that some variation is expected for random processes even if they are 'stationary' and that the background distribution (e.g. Poisson or Negative Binomial) is important in characterizing how much variability to expect [Wang et al., 2010b]. Kagan and Jackson $[1991 ; 2004]$ have used a negative-binomial distribution to model the entire earthquake catalog to avoid the need for declustering. If the background distribution of earthquakes is also better fit by a negative-binomial, this would be another possible reason for some of the analyzed catalogs having greater variability than expected for the Poisson distribution. Similarly, Jackson [1996] has also argued that because there are variations in random processes, that a change in seismicity rate does not necessarily imply changes to the processes that cause the earthquakes. 


\subsubsection{Magnitude Range and Mc}

Shearer [2012] recently used small mainshocks, $M=2.5-5.5$, to test the Southern California Catalog for self-similarity of aftershock and foreshock rates with magnitude of the mainshock. He found that for low magnitude thresholds the concept of self-similarity breaks down (and also does not support Bath's Law) [Shearer, 2012]. The findings presented in this chapter seem to support the idea that lower magnitudes catalogs show different behavior than we expected at larger magnitudes and higher Mc. We offer one new possibility for the observed change at low magnitudes: that the changes in rate with time and swarm interactions exist at all magnitude levels, but are subsequently damped to an undetectable level at higher magnitudes due to the lower number of earthquakes. To show this possibility we plotted the dispersion values against size of the declustered catalog for Mcut of 2.45, 2.95, and 3.45 using the CURATE method with a mean rate and a range of selection parameters (15-30 km and 3-5 days), and the Gardner and Knopoff [1974] method for Mcut of 2.45 and 2.95 (Fig. 3.29). Gardner and Knopoff's original study used data from 1932-1971 for $M \geq 3.8$ and 1952-1971 for $M \geq 2.8$. It is clear in the figure that difference in cutoff magnitude can make large changes in the character of the catalog. Increasing the magnitude cutoff by 0.5-1 magnitude units decreases the dispersion values by more than fifty percent. Essentially the effect of clustering is magnified at lower magnitude thresholds due to the log relationship in the number of earthquakes with magnitude. Given the sensitivity to magnitude changes, we think that the difference between large and small Mcut catalogs is likely due to a combination of this magnification at small magnitudes and the likelihood that small magnitude ranges may include more swarm (non-earthquake-earthquake triggering) behavior. 


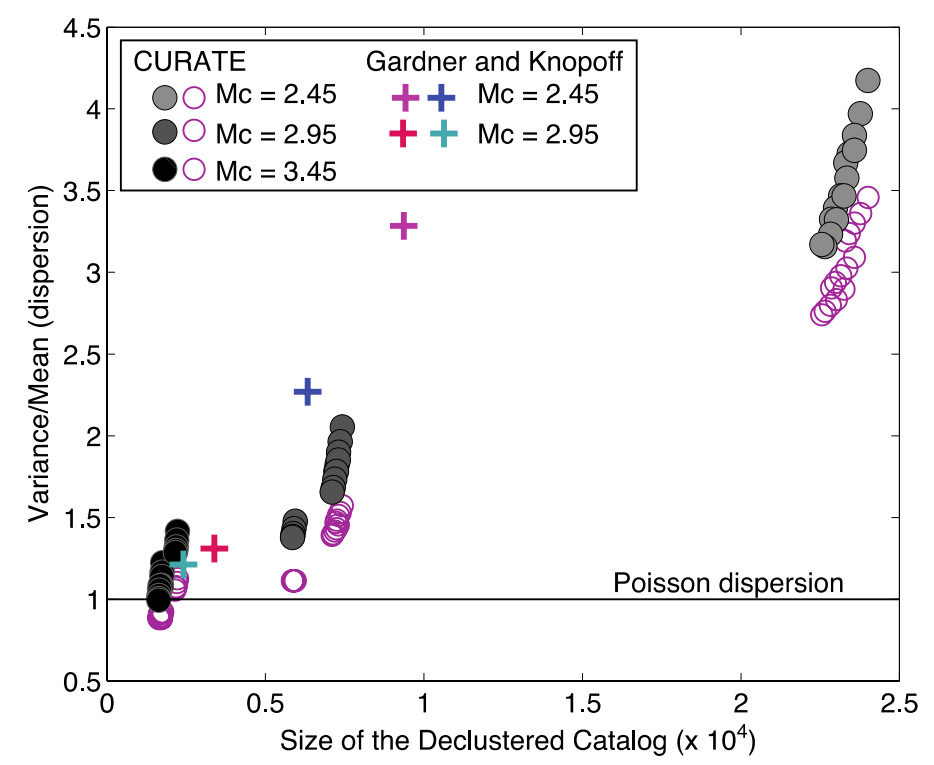

Figure 3.29 Variance/mean (dispersion) with size of the declustered catalog for various selection parameters, and magnitude cutoffs.

Data are from the Hauksson et. al (2012) waveform relocated Southern California catalog of earthquakes using the CURATE and Gardner and Knopoff methods. For each entry in the legend the first symbol corresponds to the whole declustered catalog, and the second symbol represents the declustered catalog without mainshockreplacement.

\subsection{Conclusions}

Is the catalog of earthquakes in Southern California, with aftershocks

removed, Poissonian? No, and neither are many other catalogs at low magnitude cutoffs over long time periods (decades). The Poisson testing that started as a simple test of methods has developed in this chapter to provide some interesting insights into earthquake catalogs and earthquake behavior. The catalogs and subsets in this chapter show a wide range of behaviors with time. We have explained some of the nonPoisson results as an inadequacy of using the mean rate in the initial CURATE method. This has been addressed through the introduction of the use of median and minimum rates that excludes times of exceptionally high rates. There may be more sophisticated approaches, but this method appears to be sufficient for periods with stable background rates. 
The background rate is not always stable for long time periods. In Coso, Lake Taupo, the entire CVR, and Hikurangi margin, which may be manifest as the clustering of sequences in time (with a stable background rate). We have also shown that over subsets of time ( $\sim 5$ years) the background rate does exhibit the expected Poisson behavior.

The CURATE is the one of the few declustering method inherently sensitive to the temporal distribution of earthquakes. Despite the variation in dispersion with Mcut, the CURATE shape does not vary with Mcut and does not affect sequence identification as long as Mcut $>$ Mc. The Gardner and Knopoff method is also affected by low magnitude cut-off values and does not return good dispersion values at Mcut $=2.45$ for the SCA catalog.

We cannot rule out that the observed variability arises (in part) from our method (or more generally of any method used). The fact that the longer-duration SCA catalog contains more varied rates and amounts of clustering supports the idea that the background rate changes with time. Further investigation is necessary to verify that these variations exist at low Mcut, but are damped in higher magnitude ranges. Traditional assumptions about Poisson rates of earthquakes without time variation may be incorrect and we may have to build new models in order to understand the earthquake cycle.

Some driving force is necessary to create the background rate, whether that rate is stable or not. The results of the study presented here and other recent, smallerscale, ETAS studies [Hainzl and Ogata, 2005; Lombardi et al., 2010] show that the background rate may indeed change with time. Documenting and understanding these changes may lead us towards uncovering the relationship between this driving force 
and the rate. If the rate is generally variable then this will have implications for both forecast and hazard models. 


\section{Sequence behavior}

\subsection{Introduction}

In this chapter we use the previous processing and testing of different earthquake regions and earthquake catalogs from Chapter 3 to produce a large sequence catalog that we can use to test the general characteristics of sequences. There is a tradeoff between the information we can gain about temporal and magnitude characteristics of the catalog, and the spatial features of sequences and individual catalogs. The spatial development of individual sequences (and relocated catalogs) can be extremely useful in identifying specific physical mechanisms occurring during a specific sequence. We have favored the collective behavior of swarms to provide the maximum data to work with, but this makes it impractical to re-locate all the earthquakes in this study. However, to examine the spatial evolution of sequences we have relocated a small subset of the more recent GeoNet catalog, to examine the possible underlying processes for a set of sequences at the southern end of Lake Taupo in 2009.

The details (catalog name, time period, selection parameters, and various aspects) of the resulting catalogs are shown in Table 4.1. Throughout the rest of the chapter we will present results for five individual regions [CVR, Hikurangi, Alpine Fault, Fiordland, and Southern California (SCA)], as well as the combined sequence catalog of all regions. The regional code for each catalog is listed in Table 4.1. In the interest of brevity we do not report the results of each individual catalog, (i.e., only report values for Hikurangi region, not Hikurangi-East Cape specifically. As in Chapter 3, we characterize regions that require a lower rate than the mean be used as the background rate in the CURATE calculation to be mainshock-aftershock (MS-AS) 
dominated regions (Alpine Fault, Fiordland, and SCA). The overall make-up of sequence types is explored further in the next sections. 
Table 4-1 Basic Parameters of All Catalogs used to Investigate Sequences.

Reg. is the regional code assignment for geographically groupings. \#eqs is the number of earthquakes in the total catalog. Rate, Dist, and day rule columns are the rate method and distance and day rules used to run the CURATE method for that particular catalog. \#seqs $10+$ indicates the number of sequences with at least 10 earthquakes. The 'Lrgst Seq' columns give the parameters of the largest sequence (by number of earthquakes) identified in each catalog. Duration and Area columns report the mean and median values of these parameters for all sequences of at least 10 earthquakes.

\begin{tabular}{|c|c|c|c|c|c|c|c|c|c|c|c|c|c|c|c|}
\hline Catalog & Time & Reg. & $\begin{array}{c}\# \\
\text { eqs }\end{array}$ & Rate & $\begin{array}{l}\text { Dist } \\
\text { rule } \\
(\mathrm{km})\end{array}$ & $\begin{array}{l}\text { Day } \\
\text { rule }\end{array}$ & $\begin{array}{c}\text { \#seqs } \\
10+\end{array}$ & $\begin{array}{c}\text { Lrgst } \\
\text { seq } \\
\text { \#eqs }\end{array}$ & $\begin{array}{l}\text { Lrgst } \\
\text { Seq } \\
\text { Mmax }\end{array}$ & $\begin{array}{c}\text { Lrgst } \\
\text { Seq } \\
\text { Dur }\end{array}$ & $\begin{array}{c}\text { Lrgst } \\
\text { Seq } \\
\text { Area }\end{array}$ & $\begin{array}{c}\text { Dur } \\
\text { Mean } \\
\text { (days) }\end{array}$ & $\begin{array}{c}\text { Dur } \\
\text { Med. } \\
\text { (days) }\end{array}$ & $\begin{array}{c}\text { Area } \\
\text { Mean } \\
(\mathrm{km} 2)\end{array}$ & $\begin{array}{l}\text { Area } \\
\text { Med. } \\
(\mathrm{km} 2)\end{array}$ \\
\hline CVR & $\begin{array}{l}1993- \\
2007.5\end{array}$ & 1 & 1866 & mean & 20 & 3 & 37 & 380 & 4.7 & 29.1 & 1184 & 9.3 & 5.2 & 249 & 158 \\
\hline $\begin{array}{l}\text { Hikurangi East } \\
\text { Cape }\end{array}$ & $\begin{array}{l}1993- \\
2007.5\end{array}$ & 2 & 254 & mean & 15 & 4 & 14 & 43 & 4.4 & 27.1 & 789 & 10.1 & 7.1 & 431 & 413 \\
\hline $\begin{array}{l}\text { Hikurangi } \\
\text { Gisborne }\end{array}$ & $\begin{array}{l}1993- \\
1998 \\
\end{array}$ & 2 & 387 & mean & 30 & 5 & 8 & 130 & 4.1 & 88.2 & 16484 & 33.3 & 30.2 & 4629 & 3152 \\
\hline $\begin{array}{l}\text { Hikurangi } \\
\text { Gisborne }\end{array}$ & $\begin{array}{l}1999- \\
2003\end{array}$ & 2 & 168 & mean & 30 & 5 & 9 & 35 & 3.6 & 26.6 & 2454 & 13.5 & 12.2 & 1945 & 1968 \\
\hline $\begin{array}{l}\text { Hikurangi } \\
\text { Gisborne }\end{array}$ & $\begin{array}{l}2004- \\
2007.5\end{array}$ & 2 & 23 & mean & 20 & 3 & 2 & 12 & 3.9 & 4.5 & 574 & 5.3 & 5.3 & 688 & 688 \\
\hline $\begin{array}{l}\text { Hikurangi } \\
\text { Castle Point }\end{array}$ & $\begin{array}{l}1993- \\
2007.5\end{array}$ & 2 & 330 & mean & 20 & 4 & 20 & 26 & 5.6 & 9.6 & 243 & 6.5 & 5.9 & 599 & 675 \\
\hline $\begin{array}{l}\text { Hikurangi East } \\
\text { Cape }\end{array}$ & $\begin{array}{l}2008.5- \\
2011.7 \\
\end{array}$ & 2 & 12 & mean & 20 & 4 & 1 & 12 & 3.2 & 4.9 & 1176 & 4.9 & 4.9 & 1176 & 1176 \\
\hline $\begin{array}{l}\text { Hikurangi } \\
\text { Gisborne }\end{array}$ & $\begin{array}{l}2008.5- \\
2011.7\end{array}$ & 2 & 363 & mean & 20 & 4 & 9 & 169 & 5.0 & 15.0 & 612 & 9.8 & 9.0 & 741 & 612 \\
\hline
\end{tabular}


Table 4.1 continued

\begin{tabular}{|c|c|c|c|c|c|c|c|c|c|c|c|c|c|c|c|}
\hline Catalog & Time & Reg. & $\begin{array}{l}\# \\
\text { eqs }\end{array}$ & Rate & $\begin{array}{l}\text { Dist } \\
\text { rule } \\
(\mathrm{km}) \\
\end{array}$ & $\begin{array}{l}\text { Day } \\
\text { rule }\end{array}$ & $\begin{array}{c}\text { \#seqs } \\
10+\end{array}$ & $\begin{array}{c}\text { Lrgst } \\
\text { seq } \\
\text { \#eqs } \\
\end{array}$ & $\begin{array}{l}\text { Lrgst } \\
\text { Seq } \\
\text { Mmax }\end{array}$ & $\begin{array}{l}\text { Lrgst } \\
\text { Seq } \\
\text { Dur }\end{array}$ & $\begin{array}{l}\text { Lrgst } \\
\text { Seq } \\
\text { Area }\end{array}$ & $\begin{array}{l}\text { Dur } \\
\text { Mean } \\
\text { (days) }\end{array}$ & $\begin{array}{c}\text { Dur } \\
\text { Median } \\
\text { (days) }\end{array}$ & $\begin{array}{l}\text { Area } \\
\text { Mean } \\
(\mathrm{km} 2)\end{array}$ & $\begin{array}{c}\text { Area } \\
\text { Median } \\
(\mathrm{km} 2) \\
\end{array}$ \\
\hline $\begin{array}{l}\text { Hikurangi } \\
\text { Castle Point }\end{array}$ & $\begin{array}{l}2008.5- \\
2011.7 \\
\end{array}$ & 2 & 99 & mean & 20 & 4 & 3 & 46 & 5.9 & 7.5 & 468 & 8.9 & 7.5 & 627 & 481 \\
\hline $\begin{array}{l}\text { Hikurangi } \\
\text { Wairarapa }\end{array}$ & $\begin{array}{l}1993- \\
2007.5\end{array}$ & 2 & 247 & mean & 15 & 3 & 8 & 100 & 5.6 & 18.7 & 180 & 6.9 & 5.2 & 99 & 52 \\
\hline $\begin{array}{l}\text { Northwest } \\
\text { Nelson }\end{array}$ & $\begin{array}{l}1993- \\
2007.5\end{array}$ & 3 & 22 & mean & 20 & 3 & 1 & 22 & 5.5 & 5.8 & 84 & 5.8 & 5.8 & 84 & 84 \\
\hline Alpine Fault & $\begin{array}{l}1993- \\
1999 \\
\end{array}$ & 3 & 4973 & $\min$ & 30 & 4 & 22 & 3764 & 6.7 & 306.5 & 7529 & 34.1 & 14.3 & 1770 & 1236 \\
\hline Alpine Fault & $\begin{array}{l}2000- \\
2007.5\end{array}$ & 3 & 56 & med. & 15 & 3 & 3 & 28 & 4.4 & 6.2 & 20 & 3.9 & 3.1 & 47 & 52 \\
\hline $\begin{array}{l}\text { S. Alpine } \\
\text { Fault }\end{array}$ & $\begin{array}{l}1993- \\
1999 \\
\end{array}$ & 3 & 101 & $\min$ & 30 & 5 & 3 & 50 & 5.1 & 18.5 & 211 & 15.5 & 15.4 & 1786 & 1449 \\
\hline $\begin{array}{l}\text { S. Alpine } \\
\text { Fault }\end{array}$ & $\begin{array}{l}2000- \\
2007.5\end{array}$ & 3 & 585 & med. & 20 & 3 & 9 & 425 & 6.2 & 70.2 & 1149 & 16.1 & 11.1 & 555 & 405 \\
\hline Fiordland & $\begin{array}{l}1993- \\
2007.5\end{array}$ & 4 & 9422 & med. & 20 & 4 & 46 & 4126 & 7.0 & 283.1 & 6031 & 25.4 & 12.5 & 1354 & 1141 \\
\hline SCA & $\begin{array}{l}\text { 1995- } \\
2011\end{array}$ & 5 & 8512 & $\min$ & 30 & 5 & 102 & 1908 & 7.2 & 158.2 & 9894 & 13.4 & 7.4 & 763 & 174 \\
\hline
\end{tabular}




\subsection{Basic Sequence Parameters}

To begin our study of sequence characteristics we report the values of five basic

parameters (Duration, Number of earthquakes, Largest magnitude [Mmax],

magnitude difference between the two largest magnitudes [Mdiff], and Area) for each

of our five regions (Table 4.2). A more detailed analysis of the relationship between

individual parameters follows in section 4.5.

Table 4-2 Table of Basic Sequence Parameters by Region.

CVR stands for the Central Volcanic Region, and SCA for Southern California, and (AFD) after a region indicates aftershock-dominated region. Mmax is the largest magnitude earthquake. Mdiff is the magnitude difference between the largest and second largest earthquake.

\begin{tabular}{|c|c|c|c|c|c|c|}
\hline & & Min & Max & Mean & StDv & Median \\
\hline \multirow[t]{5}{*}{ CVR } & Duration (days) & 0.08 & 56.95 & 9.26 & 13.40 & 5.16 \\
\hline & \# Earthquakes & 11.00 & 380.00 & 50.43 & 72.57 & 21.00 \\
\hline & Mmax & 2.81 & 5.11 & 3.73 & 0.64 & 3.75 \\
\hline & Mdiff & 0.00 & 1.04 & 0.25 & 0.29 & 0.11 \\
\hline & Area $(\mathrm{km} 2)$ & 6.83 & 1183.84 & 248.93 & 244.85 & 158.13 \\
\hline \multirow[t]{5}{*}{ Hikurangi } & Duration (days) & 1.03 & 88.19 & 11.42 & 13.04 & 7.92 \\
\hline & \# Earthquakes & 11.00 & 169.00 & 25.45 & 26.62 & 15.00 \\
\hline & Mmax & 2.89 & 5.90 & 3.93 & 0.75 & 3.74 \\
\hline & Mdiff & 0.01 & 2.12 & 0.37 & 0.45 & 0.22 \\
\hline & Area $(\mathrm{km} 2)$ & 22.51 & 16484.21 & 1141.13 & 2124.40 & 593.10 \\
\hline \multirow[t]{5}{*}{ Alpine Fault (AFD) } & Duration (days) & 0.59 & 306.53 & 25.25 & 50.70 & 12.82 \\
\hline & \# Earthquakes & 11.00 & 3764.00 & 150.97 & 612.06 & 17.50 \\
\hline & Mmax & 3.03 & 6.67 & 4.45 & 1.00 & 4.25 \\
\hline & Mdiff & 0.00 & 1.84 & 0.48 & 0.50 & 0.27 \\
\hline & Area $(\mathrm{km} 2)$ & 19.99 & 7528.51 & 1303.38 & 1542.64 & 868.50 \\
\hline \multirow[t]{5}{*}{ Fiordland (AFD) } & Duration (days) & 0.47 & 283.10 & 25.45 & 48.23 & 12.50 \\
\hline & \# Earthquakes & 11.00 & 4126.00 & 204.83 & 722.53 & 18.00 \\
\hline & Mmax & 3.00 & 6.99 & 4.56 & 0.95 & 4.44 \\
\hline & Mdiff & 0.02 & 1.75 & 0.49 & 0.38 & 0.39 \\
\hline & Area $(\mathrm{km} 2)$ & 109.08 & 6030.86 & 1353.72 & 1075.10 & 1140.57 \\
\hline \multirow[t]{5}{*}{$\overline{S C A}($ AFD) } & Duration (days) & 0.13 & 158.23 & 13.38 & 21.01 & 7.42 \\
\hline & \# Earthquakes & 11.00 & 1908.00 & 83.45 & 281.69 & 20.50 \\
\hline & Mmax & 2.94 & 7.20 & 4.44 & 0.83 & 4.37 \\
\hline & Mdiff & 0.00 & 2.03 & 0.52 & 0.43 & 0.40 \\
\hline & Area $(\mathrm{km} 2)$ & 0.72 & 9894.48 & 763.46 & 1498.90 & 174.37 \\
\hline
\end{tabular}


Table 4-3 Table of Basic Sequence Parameters for all Sequence, and by Sequence Type.

Mmax is the largest magnitude earthquake. Mdiff is the magnitude difference between the largest and second largest earthquake.

\begin{tabular}{|l|l|c|c|c|c|c|}
\hline & & Min & Max & Mean & StDv & Median \\
\hline All Sequneces & Duration (days) & 0.08 & 306.53 & 15.76 & 30.89 & 8.11 \\
& \# Earthquakes & 11.00 & 4126.00 & 93.80 & 404.90 & 19.00 \\
\hline & Mmax & 2.81 & 7.20 & 4.23 & 0.87 & 4.12 \\
\hline & Mdiff & 0.00 & 2.12 & 0.44 & 0.43 & 0.29 \\
\hline & Area (km2) & 0.72 & 16484.21 & 953.96 & 1576.08 & 472.38 \\
\hline MS-AS & Duration (days) & 0.47 & 306.53 & 26.09 & 56.18 & 7.20 \\
\hline & \# Earthquakes & 11.00 & 4126.00 & 250.63 & 758.66 & 27.00 \\
\hline & Mmax & 3.26 & 7.20 & 5.16 & 0.83 & 4.93 \\
\hline & Mdiff & 0.29 & 2.12 & 1.01 & 0.39 & 0.91 \\
\hline & Area (km2) & 1.56 & 9894.48 & 1174.96 & 1962.20 & 425.01 \\
\hline & Duration (days) & 0.08 & 88.19 & 12.40 & 12.88 & 8.87 \\
\hline & \# Earthquakes & 11.00 & 1537.00 & 40.73 & 113.22 & 17.00 \\
\hline & Mmax & 2.81 & 5.83 & 3.95 & 0.66 & 3.87 \\
\hline & Mdiff & 0.00 & 1.38 & 0.26 & 0.24 & 0.21 \\
\hline
\end{tabular}

\subsubsection{Duration}

CVR durations are the shortest of all regions, and are similar to the durations observed by Benoit and McNutt's [1996] Global Volcanic Earthquake Swarm Database that gave geometric-mean and median values of 7 and 5.5 days respectively for a global sample of volcanic earthquake swarms. The swarm database was updated by Jacobs and McNutt [2006] and the mean and the median values increased to 8 and 7.3 days. The Alpine Fault and Fiordland regions have mean values that are double those of other regions, and they also have larger median values. Vidale and Shearer [2006] did not measure duration as such, but instead calculated median time lag from the first event for 71 seismicity bursts in SCA. They found the mean and median of this time lag to be 6.2 and 4.5 for their small seismicity bursts (concentrated within a $2 \mathrm{~km}$ radius). SCA in this study has similar mean and median duration values to the Hikurangi region, lower than the other aftershock-dominated regions. In the SCA case we think these smaller duration values are probably due to a higher background value and thus a quicker return to background despite potentially ongoing activity. 
SCA has the smallest maximum duration of all the aftershock-dominated regions, which also suggests that these calculated durations are affected by background levels.

\subsubsection{Number of Earthquakes}

To get our initial sequences to study sequence behavior, we required the sequences from each catalog to be more than 10 earthquakes, thus the minimum number is the same for all regions. The median number is also close for all regions. The aftershock-dominated regions all have maximum numbers of events in the thousands. Both the CVR and Hikurangi regions have maximum numbers of earthquakes an order of magnitude lower (Table 4.2). The smaller maximum number of earthquakes in SCA may be due to the smaller duration (Table 4.2) and higher background rate, as discussed above in 4.2.1.1. There is also a possibility that the number is limited by a lack of completeness directly following the largest magnitude earthquakes

\subsubsection{Mmax and Mdiff}

Mmax is larger in mainshock dominated regions (Alpine Fault, Fiordland, and SCA). Mdiff is also larger for the aftershock-dominated regions, but Mdiff in Hikurangi is similar to the aftershock-dominated regions. The CVR is the only region that stands out with significantly smaller Mdiff despite similar median values of Mmax as Hikurangi.

\subsubsection{Area}

Area is our least well determined parameter. The time and magnitude of the earthquakes are easy parameters to collect from a catalog. Area on the other hand can be measured in a variety of ways. We have chosen to measure area as the smallest elliptical area that encompasses all earthquake locations. This will inevitably overestimate the areas. And the amount of the calculated area that is genuinely active 
over the course of the sequence may be much less than total area. For this reason we take the correlations with area more roughly than we interpret other, more precise, parameters. The median area of all regions is less than $\sim 1256 \mathrm{~km}^{2}$, which is equivalent to a search area with a radius of $20 \mathrm{~km}$ (Tble. 4.2). Nearly one third of earthquake catalogs use search radii of $30 \mathrm{~km}$ (Table 4.1, dist-rule), yet they still have median areas less than an equivalent $20 \mathrm{~km}$ search radius. The low values of median area are further evidence that the distance-rule search parameter in the CURATE method is robust.

Hikurangi has diffuse seismicity. That manifests as the largest area of a single sequence observed in all catalogs. SCA region has many small sequences with mean and median areas smaller than those for other aftershock-dominated regions.

\subsubsection{Determining Sequence Type}

We also want to measure how parameters vary with sequence types and whether this can give any insights into regional behavior. We chose to distinguish mainshock-aftershock (MS-AS) sequences and swarms by setting a criteria to define MS-AS sequences and considering the rest of the sequences to be swarms. To define MS-AS sequences we use two parameters, an event-window (Mmax in the first Xpercent of earthquakes) and a magntidue difference (Mdiff) between the largest (Mmax) and second largest magnitude earthquakes. This form of definition is convenient for looking at sequence catalogs, but any definition reliant on the total number of earthquakes or duration will not be able to be applied as a sequence occurs in real time. However, this problem is currently not a limitation as we are also unable to unequivocally identify the largest earthquake until the sequence has finished. 
In Chapter 2 for the CVR we used an Mdiff value of $\geq 0.5$ magnitude units, and an event window of the first $30 \%$ of earthquakes (by number). This definition is suitable for the CVR, which has lower Mdiff than other regions (Table 4.2) and more swarm-like MS-AS sequences (Sherburn 1992). With our larger dataset we are able to investigate the effect of variations of this sequence-type definition.

First we varied the Mdiff from 0.1-1.5 magnitude units and calculated how many MS-AS sequences were identified at different event-window ranges from 530\% (Fig. 4.1). Even at the lowest Mdiff (0.1) less than half(128) of the total sequences (297) are classified as MS-AS sequences at the largest event window of $30 \%$. The number of MS-AS sequences identified for different definition decreases relatively smoothly from Mdiff $0.1-0.7$, and there is a marked change in behavior at higher Mdiff values (Fig. 4.1). The event-window size becomes less important at high Midff $(\geq 1.0)$. This is the first indication that there may not be a clear best definition or discriminant between sequence types. We also looked at the same variations in definition for our five regional subsets (Fig. 4.2). The Hikurangi region has a low number of sequence defined as MS-AS, but those that occur appear to have larger Mdiff values (shown by consistent size bars across Mdiff from left to right). At the smallest event-window the decrease with increasing Mdiff is flatter, indicating that sequences with a very early Mmax are more likely to have a higher Mdiff. These two figures do not show an obvious best definition to classify sequence types and so we move on to investigating the temporal position of Mmax to further test the effects of the definition of MS-AS sequences. 


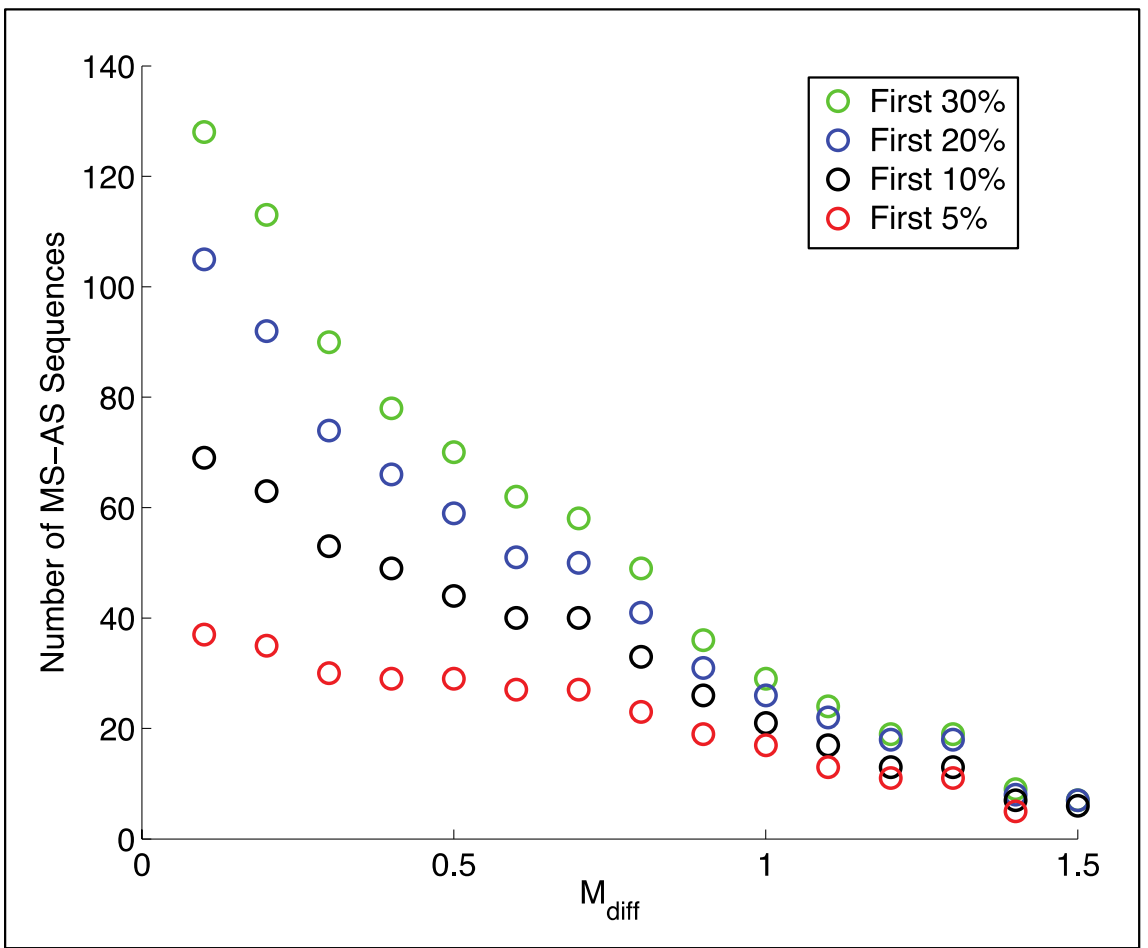

Figure 4.1 Comparisons of the number of sequences that fit different definitions of a mainshock-aftershock sequence.

Number of mainshock-aftershock sequences identified for different combinations of the two limiting parameters (Mmax in the first X-percent of events, and magnitude difference (Mdiff) between the largest and second largest earthquakes) for all regions. 
CVR $\square$ Hikurangi $\square$ Alpine Fault $\square$ Fiordland $\square$ SCA
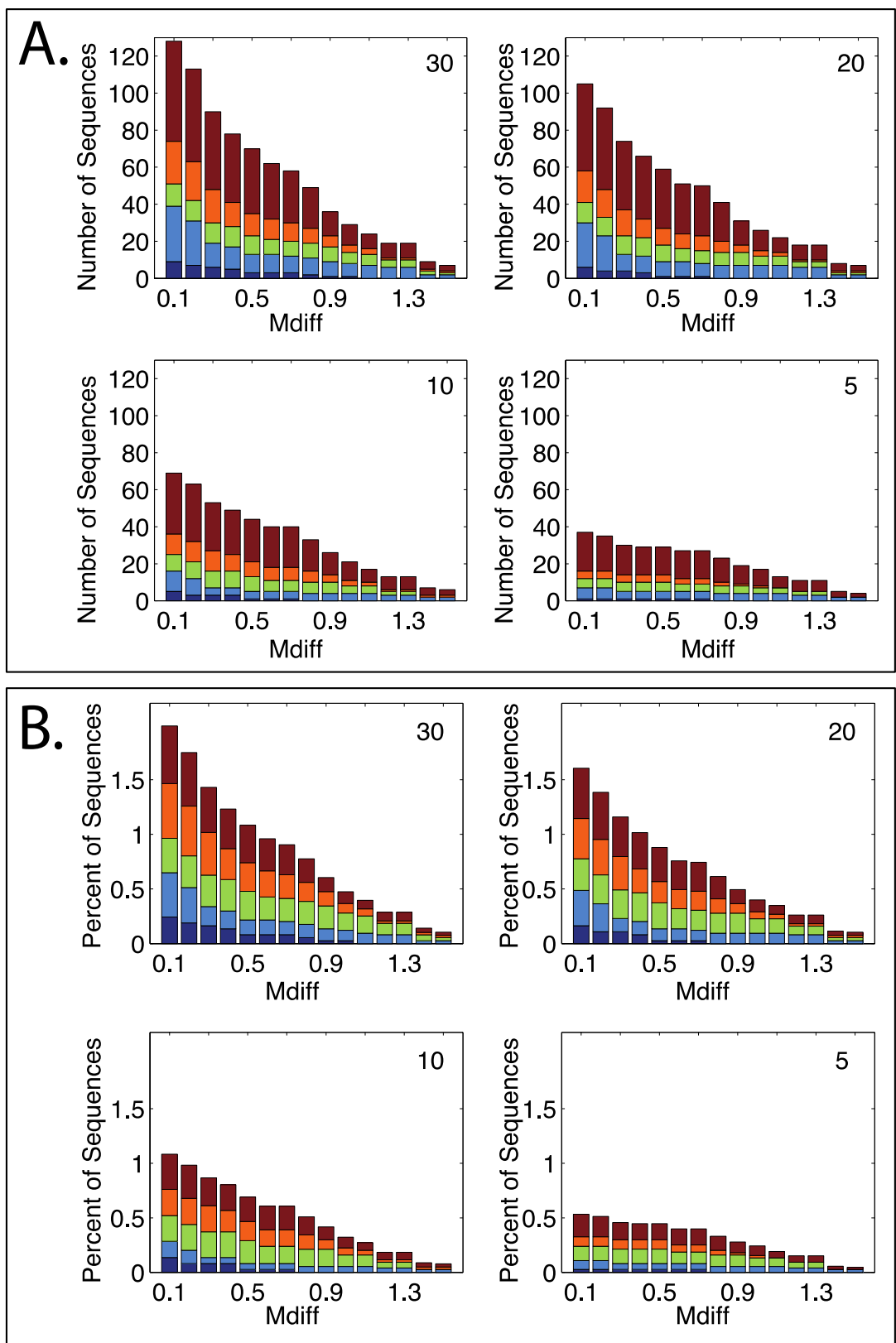

Figure 4.2 Stack plots of the number of MS-AS sequences identified by each region for various definitions of a mainshock-aftershock sequence.

The number in the top-right hand corner of each plot indicates the first X-percent of earthquakes in which Mmax is allowed to occur, the x-axis corresponds to the Mdiff requirement for the definition. A) Number of sequences. B) Fraction of the sequences for a given region. Note the $y$-axis values may exceed 1 collectively, but no individual region will have a value greater than 1 . The colors represent each region (see legend at the top of the figure). 


\subsubsection{Position of Mmax}

The position (in time) of Mmax is a key sequence parameter of interest for hazard forecasting, and also for how we define sequence types. It is likely to be near the beginning of a sequence (by time and number) for MS-AS sequence but we do not have a way to quantitatively estimate when it will occur in swarm sequences. To look at the potential distribution of this parameter with sequence type we use the initial definition of MS-AS sequences used in our investigations of the CVR (Mdiff $\geq 0.5$, event-window 30\%). Figure 4.3 shows a cumulative distribution for the position of Mmax as a function of normalized duration for all sequences and for sequences by type. The duration is normalized for all sequence by setting the first earthquake time equal to zero and the last equal to 1 . Note that while the number of earthquakes is related to the duration, we fix the mainshock event window and not the duration window in our definition of MS-AS sequences (ie. we consider $30 \%$ of the number of events, and not by time). Because the definition is relies on the number of events, we are free to compare where Mmax falls as a function of duration between sequence types. There are three times more swarm-type (dashed line) sequences than MS-AS (black line) sequences $(224 / 73)$ and it is clear that the swarm-type sequences dominate the shape of the overall curve (gray line). The two sequence types have distinctly different distributions. The distribution of Mmax with duration in swarm sequences is far more uniform than in MS-AS sequences. Although in swarms, the occurrence of Mmax is evenly distributed with time, there is still an increased probability of occurrence in the first 10 and $40 \%$ of a sequence by duration (Fig. 4.3). Forcing one earthquake to occur at the beginning and the end increases the number of earthquakes at these times, and thus the probability of an earthquake (including Mmax) occurring in those bins. We can show that for both MS-AS and swarm 
sequences the position of Mmax is related to the probability of earthquakes occurring at a given point in the duration (Fig. 4.4). Swarm earthquakes occur at a steady rate throughout the sequence, and so it should be unsurprising that the probability of Mmax is also spread more uniformly throughout the sequence. The CDF of number of earthquakes for MS-AS (4.4A) may also be skewed by aftershock sequences of large mainshocks, which have very large numbers of events following Mmax.

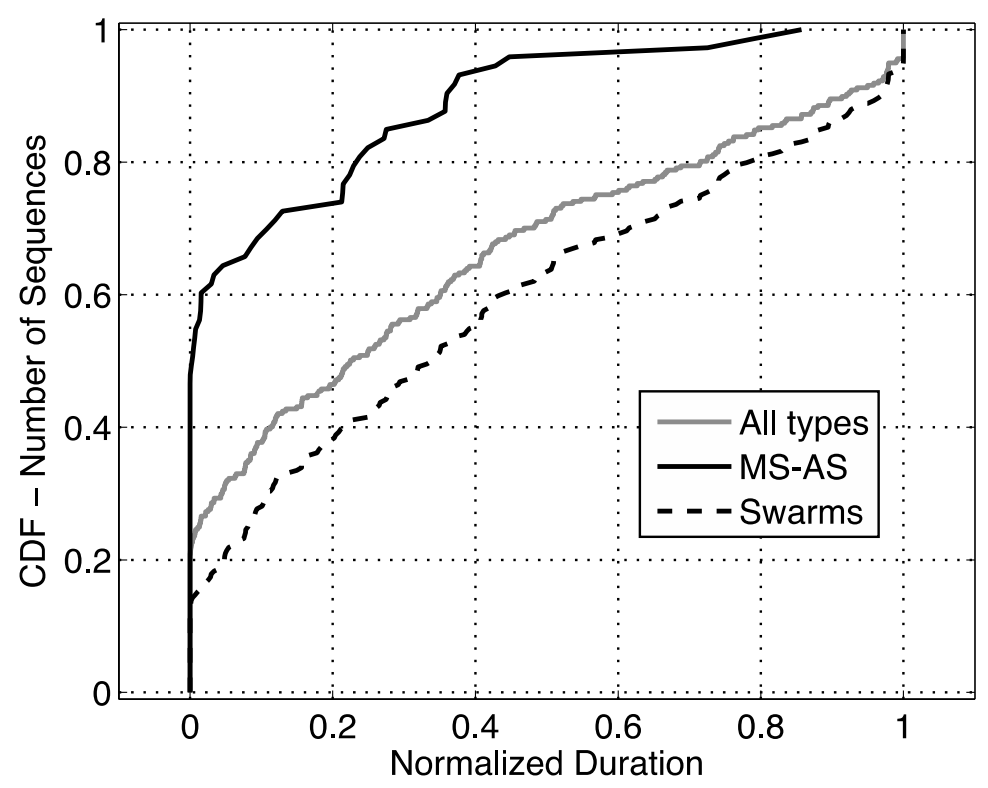

Figure 4.3 Cumulative distribution of the Position of Mmax with normalized duration for all sequences, and the sequence types. 

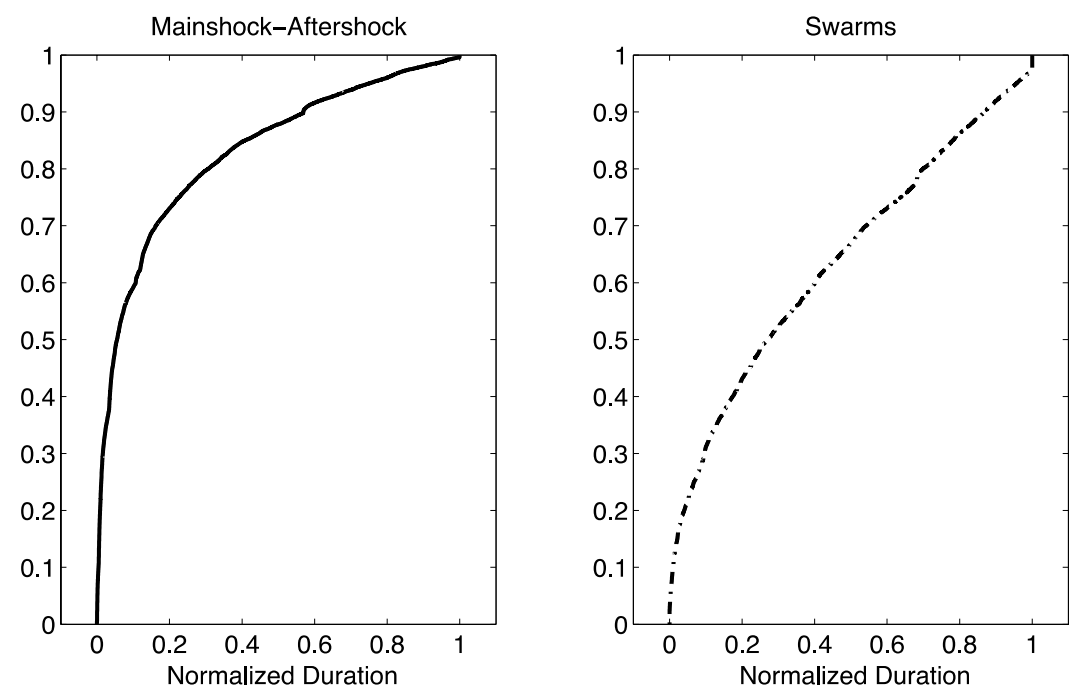

Figure 4.4 Cumulative distribution of the timing of all earthquakes in different sequence types.

To test whether the shape of the Position of Mmax curves is sensitive to our definition of aftershock sequences we plot the curves using a subset of the parameter range used in Figures 4.1 and 4.2 (Mdiff: 0.1-1.5, \%Number 10-30\%). From the earlier investigation, there are less than 40 sequences that have Mmax in the first 5\% of events, regardless of Mdiff requirements. We found that although the number of sequences obviously varies with different definitions of aftershock sequences, the overall shape of the Position of Mmax in duration curves is similar at almost all Mdiff variations (Fig. 4.5). The most different looking Mdiff in 4.5 A and B is 0.1 , which is likely due to a poor distinction between the sequence types. At the $10 \%$ number requirement even that distinction almost disappears (4.5C). Because the shape is stable we chose to continue to use the definition used for the CVR in Chapter 2 to identify MS-AS sequences (Mdiff $\geq 0.5$, in the first $30 \%$ by number). 
A: In the first $30 \%$

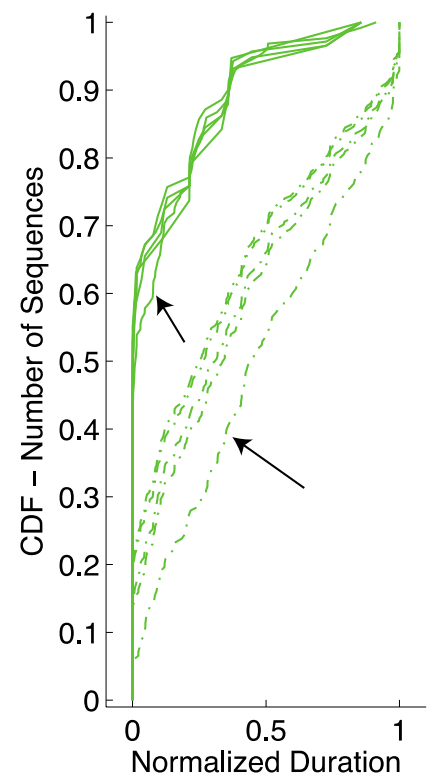

B: In the first $20 \%$

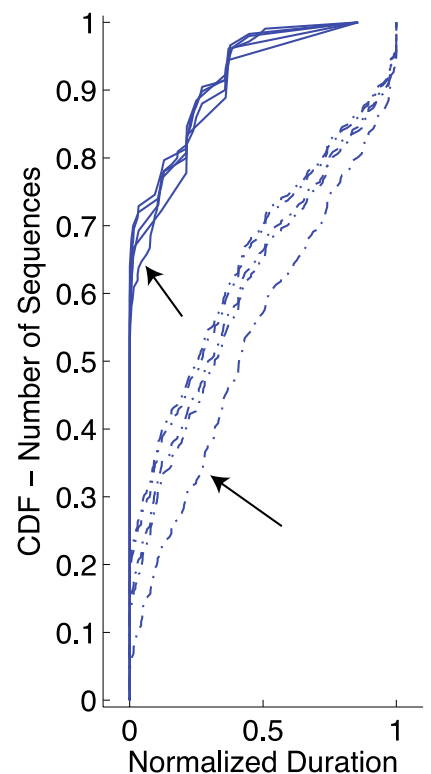

C: In the first $10 \%$

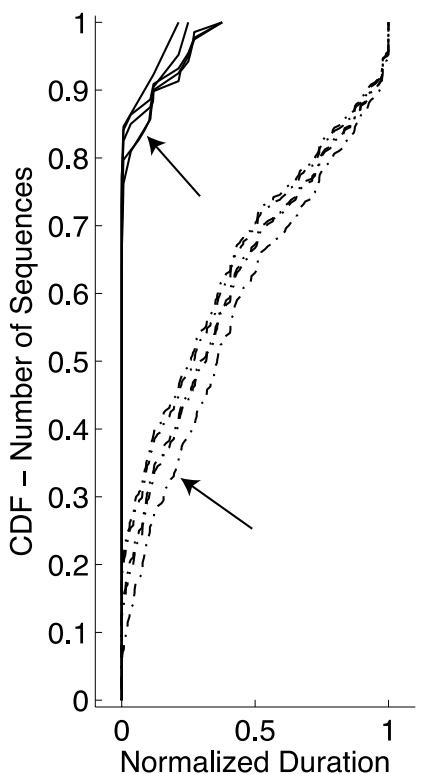

Figure 4.5 Cumulative distribution of the Position of Mmax in duration for varying definitions of mainshock-aftershock sequences (MS-AS).

Solid lines indicate those that are categorized as MS-AS and dashed lines indicate swarm type sequences under a particular definition. A-C show three different variations of the requirement that the largest magnitude earthquake must occur in the first X-\% of earthquakes. The different lines in each set of plots represent different Mdiff parameters $(0.1,0.5,0.7,1.0,1.2)$. Arrows point to Mdiff $=0.1$. See text for further discussion on defining MS-AS sequences.
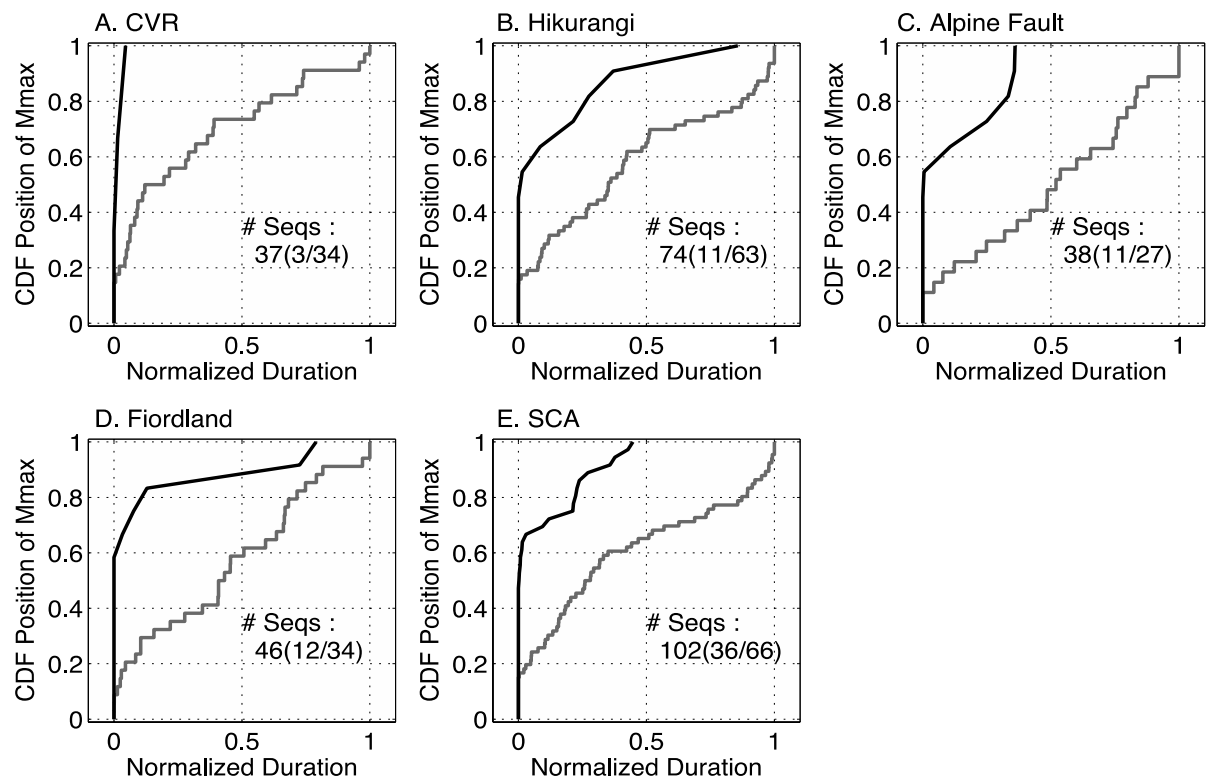

Figure 4.6 Cumulative distribution of the position of Mmax with sequence type for each region.

Solid black lines are mainshock-aftershock sequences and the lighter gray lines are for swarm sequences. Number printed on each plot show the total number of sequences represented along with the ratio (number of MS-AS/and swarm type). 
We also wanted to know whether this duration distribution was universal across the five regions. The regions do not all have a robust number of sequences for both types, but we have plotted them all (Fig. 4.6) for comparison. The CVR (4.6A) is different from other regions. The number of MS-AS sequences is too low to draw conclusions for the CVR, but the swarm distribution appears to have one of the highest probabilities in the first $10 \%$ of the duration compared to other regional swarm distributions. We think this may be due to sequences that behave like MS-AS sequence, but have Mdiff lower than our threshold of 0.5. Hikurangi and Fiordland (4.6 B and D) both have some mainshocks that occur relatively late in the sequence. The Alpine Fault and SCA MS-AS sequences all have Mmax that occurring within the first half of the sequence by duration. The Alpine fault and Fiordland regions have the most linear distribution of the position of Mmax for swarm sequences, and hence most uniform probability.

To look more closely at the potential relationship to Mdiff we plotted the cumulative distributions of Mdiff for each region (Fig. 4.7). Fiordland and SCA have the most similar cumulative distributions of Mdiff. Table 4.2 showed that the median Mdiff value for Hikurangi and the Alpine Fault regions is similar, but figure 4.7 shows that the Alpine Fault region has more larger Mdiff values (likely aftershock sequences). While the CVR is the most distinctive region in both figure 4.6 and 4.7 , other regions do not show a correlation between the timing of Mmax and their overall distribution of Mdiff. 


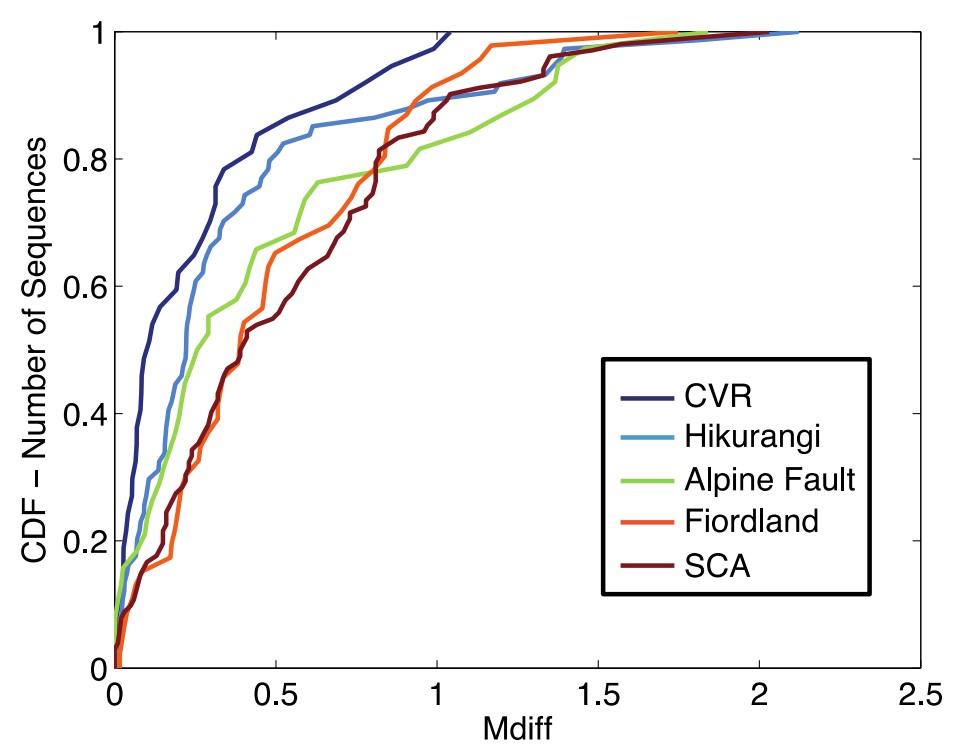

Figure 4.7 Cumulative distribution of Mdiff for all sequences in five different regions. See table 4.2 for minimum, mean, median, and maximum values for all of the distributions.

Another obvious potential correlation for the timing of Mmax is the duration of the sequences. Figure 4.8 shows distributions of duration in absolute time [A and B] and as a percentage of duration [C and D] that occurs before and after Mmax. Mainshock-aftershock sequences all have their largest magnitude earthquake in less than 20 days from the start of the sequence, but more than $10 \%$ of them continue for longer than 20 days following Mmax (Fig. 4.8 MS-AS A and B). Swarm sequences on the other hand have shorter durations in general and $95 \%$ of them have less than 20 days before and after Mmax (Fig. 4.8 Swarm A and B). Plots C and D in Figure 4.8 show the percentages of duration described by the time length in $\mathrm{A}$ and $\mathrm{B}$, where $\%$ of duration occurring before Mmax is equivalent to the Position of Mmax in Duration and $\%$ after Mmax is simply the inverse of percent-time before. Because of the requirement that Mmax occur early in MS-AS sequences a large percentage of them have the bulk of their duration following Mmax. Conversely, swarm sequences have 
a relatively uniform distribution, although it appears there is a slight tendency to have more of the sequence after Mmax than MS-AS.

The main thing we can see in this investigation of the timing of Mmax is that regardless of sequence type, Mmax is more likely to occur in the first half of a sequence than in the second half. This is more dramatic with mainshock-aftershock sequences, but is apparent for swarms too.
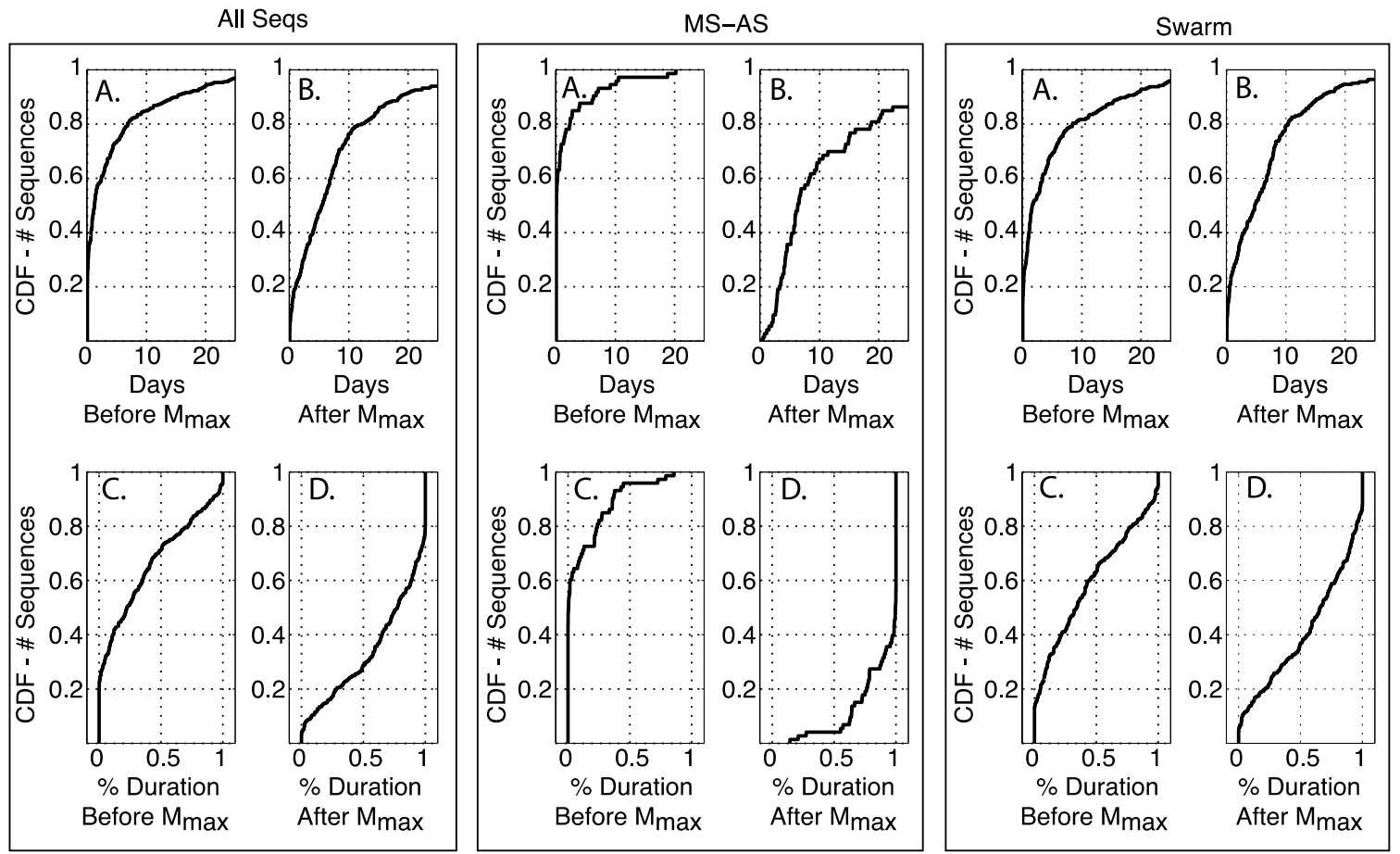

Figure 4.8 Plots of the amount of time before and after Mmax for all sequences, and sequences by type.

A) and B) are the distributions of the number of days before Mmax, plotted from 0-25 days. C) and D) are the percent of duration that occurs before and after Mmax (these are inverses of each other, whereas $\mathrm{A}$ and $\mathrm{B}$ are independent).

\subsubsection{Sequence Expansion}

Another potential way to distinguish MS-AS from swarm types sequence is by the amount of spatial expansion that occurs during the sequence. Vidale and Shearer [2006] suggested a way to quantify the spatial expansion using two calculated 
parameters related to the spatial position and magnitude of the first earthquake in the sequence. They define a distance ratio between two halves of the sequence by taking the median distance from the first event and every event in the first half of the sequence and the median distance from the first event to every event in the second half of the sequence. This ratio should be near 1 for MS-AS sequences, which are not expected to have much spatial expansion with time. Swarm sequences on the other hand may be caused by fluid diffusion and could show substantial expansion from the first event. The second parameter is a magnitude difference calculated by taking the difference between the magnitude of the first event and the largest magnitude in the rest of the sequence. Note that this is different from the value 'Mdiff' that we used earlier to defined MS-AS sequences and will only be the same when the first earthquake is either Mmax or the second largest earthquake in the sequence. Because we have used a magnitude difference in our criteria to define MS-AS sequences, 0.5 is the smallest magnitude difference possible in MS-AS sequences. A positive magnitude difference indicates that Mmax is the first earthquake. The Vidale and Shearer [2006] are unclear on how they chose the sequence by halves, and so we calculated it using both duration and number of earthquakes to split the sequence into halves (Fig. 4.9). The zoom in $4.9 \mathrm{C}$ and $\mathrm{D}$ shows that there is not a systematic difference in distance ratio (expansion) between the two sequence types. Regional plots (Fig 4.10) show a similar effect, with magnitude difference by definition different for the sequence types, but with no discernable difference in distance ratio. 


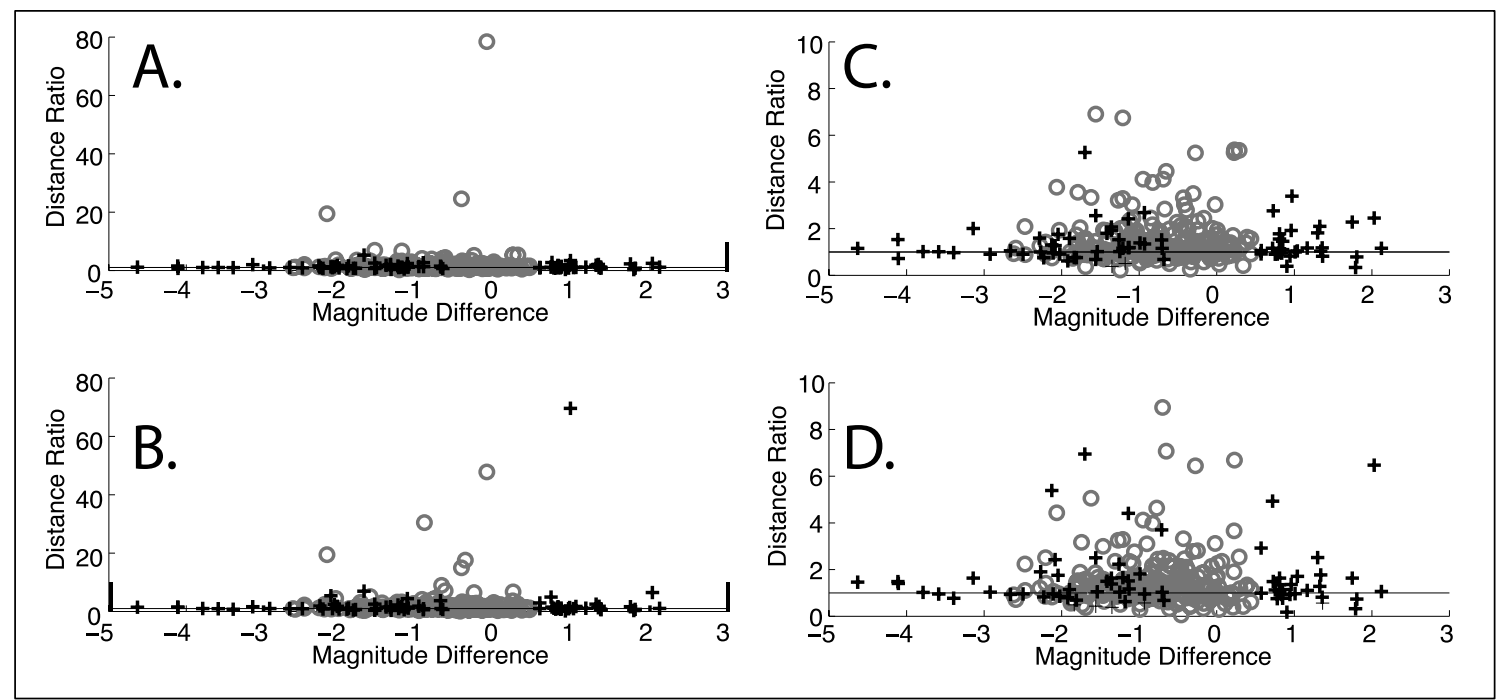

Figure 4.9 Sequence Expansion plots.

The distance-ratio is the ratio between the second and first halves of the sequence of the median distance of all earthquakes from the first event. A) Halves of the sequences are determined by number of earthquakes. B) Halves of the sequence are determined by duration. C) and D) are the same as A) and B), but with a smaller yscale (indicated by the vertical black bar at a Magnitude Difference of 3 in A and B). Values near one indicate little spatial change with time, and values away from one indicate expansion $(>1)$ or contraction $(<1)$. Plus symbols are mainshock-aftershock (MS-AS) sequences and open circles are swarm sequences. The absence of MS-AS sequences around zero is due to the definition that MS-AS sequences must have a magnitude difference of at least 0.5 .

Table 4-4 Comparison of the Median and Maximum Distance Ratios for All Regions (Sequence midpoint determined by number of earthquakes)

\begin{tabular}{lrrrrrr} 
& $\begin{array}{c}\text { Median } \\
\text { All }\end{array}$ & $\begin{array}{c}\text { Median } \\
\text { MS-AS }\end{array}$ & $\begin{array}{c}\text { Median } \\
\text { Swarms }\end{array}$ & $\begin{array}{c}\text { Max } \\
\text { All }\end{array}$ & $\begin{array}{c}\text { Max } \\
\text { MS-AS }\end{array}$ & $\begin{array}{c}\text { Max } \\
\text { Swarms }\end{array}$ \\
\hline All Seqs & 1.17 & 1.15 & 1.20 & 78.45 & 5.27 & 78.45 \\
CVR & 1.01 & 1.19 & 1.00 & 2.27 & 1.93 & 2.27 \\
Hikurangi & 1.15 & 1.16 & 1.14 & 6.74 & 2.69 & 6.74 \\
Alpine & & & & & & \\
Fault & 1.04 & 0.97 & 1.17 & 3.50 & 2.01 & 3.50 \\
Fiordland & 1.28 & 1.13 & 1.37 & 4.11 & 2.28 & 4.11 \\
SCA & 1.29 & 1.16 & 1.36 & 78.45 & 5.27 & 78.45
\end{tabular}



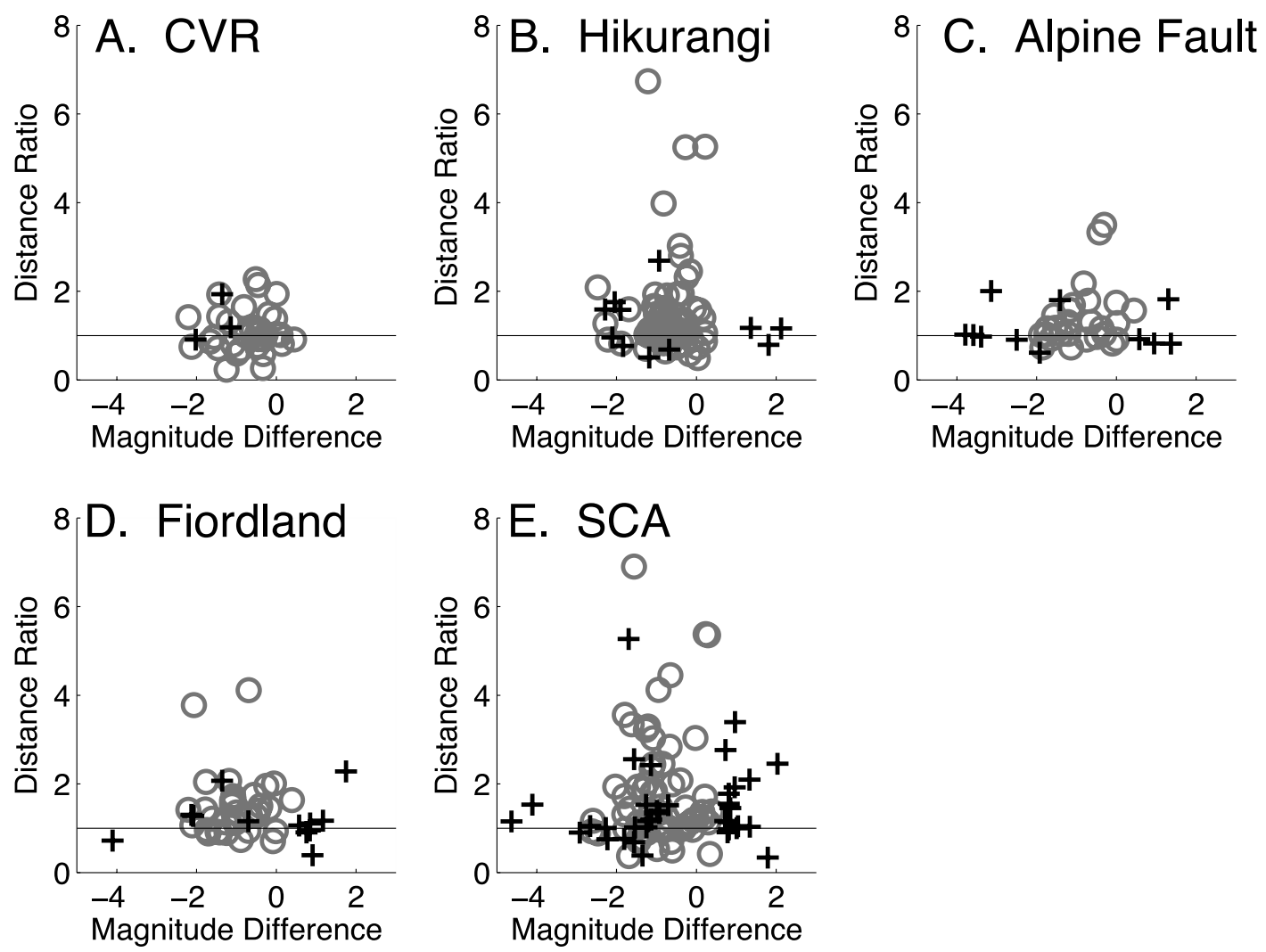

Figure 4.10 Expansion plots by region.

The distance-ratio is the ratio between the second and first halves of the sequence (by number of earthquakes) of the median distance of all earthquakes from the first event. Values near one indicate little spatial change with time, and values away from one indicate expansion $(>1)$ or contraction $(<1)$. Plus symbols are mainshock-aftershock sequences and open circles are swarm sequences. Note there are some values that are too large to display in the figure. See table 4.3 for details.

The obvious difference with the study of Vidale and Shearer [2006] is that their sequences all have positive magnitude differences, while our mainshock aftershock sequences also have negative magnitude differences (4.9 and 4.10). Negative magnitude differences are not observed in the Vidale and Shearer study because they require abrupt rate increases in order to consider the seismicity burst, excluding bursts that have more than 3 events within the $2 \mathrm{~km}$ inner sequence area during the 28 days prior to an abrupt rate increase [Vidale and Shearer, 2006]. Their exclusion of sequences with events prior to the occurrence of a certain event rate excludes some foreshock sequences and so will increase the probability of Mmax 
being the first event. The Vidale and Shearer bursts are also limited to have very small sequence areas (concentrated within a $2 \mathrm{~km}$ radius). They state that they have avoided larger sequences because it is dubious whether larger sequences will show similar behavior. While there is some tendency for MS-AS to occur near one, the trend is not clear and our results seem to indicate that there was reason to doubt whether such behavior was observable in larger sequences.

There does not appear to be a correlation between greatest expansion (Table 4.4) and area (Table 4.2). The Hikurangi and SCA regions have the largest expansion values, but they have intermediate area values compared to the other three regions. While the figures do not show an obvious correlation, we test this parameter in the next section. Aftershock-dominated regions do show smaller expansions for MS-AS sequences than for swarm sequences, but the expected relationship does not hold for Hikurangi and the CVR (Fig. 4.10).

\subsubsection{Correlation of Parameters}

\subsubsection{Parameters and general correlations}

To see how the basic parameters were related and whether additional, less obvious, parameters could help constrain sequence type, we gathered a list of parameters for each sequence and correlated them all to test for significant links. From our initial basic list of five parameters we added an additional 28 parameters for potential correlation. Many of them are related and they are all listed below.

1: Number of earthquakes

2: Duration 
3: $\operatorname{Mmax}$

4: Area

5: total Moment

6: total Moment-Moment Mmax

7: Average Moment/earthquake

8: Mdiff (difference between the two largest magnitudes)

9: Difference between the magnitdues of the $1^{\text {st }}-2^{\text {nd }}$ and $2^{\text {nd }}-3^{\text {rd }}$ largest earthquakes,

10: Start date

11: time/earthquake

12) $\mathrm{M} \geq 3.0,13) \# \mathrm{M} \geq 3.5,14) \# \mathrm{M} \geq 4.0,15) \# \mathrm{M} \geq 4.5$

16-17: time before/after Mmax

18-19: \% time before/after Mmax

20-23: \# of earthquakes of magnitudes given in 12-15, divided by duration

24-27: \# of earthquakes of magnitudes given in $12-15$, divided by the total number of earthquakes

28-29: \# earthquakes before/after Mmax

30-31: \%earthquakes before/after Mmax

32: time between the first and second largest magnitude earthquakes (diff Mdiff)

33: Expansion (after Vidale and Shearer [2006])

The values of all these parameters were determined for all sequences and then run through the 'corrcoef' function in MATLAB. This function calculates the correlation based on the covariance between the parameters. Corrcoef also tests the hypothesis of no correlation and returns a p-value indicating how likely it is to get a correlation as high as the one reported when the true correlation is zero. We use this matrix of pvalues to limit the correlation matrix to values with a significance level of at least .95 . Non-significant correlations are given values of zero. The absolute value of the 
significant correlations are plotted as matrices in figure 4.11. Note that the use of the p-value has removed the auto-correlation along the diagonal. It is hard to get much information from these plots without labeling all 33 parameters, but we show it here to make a few simple observations. The most obvious is that swarm sequences seem to have more significant correlations, but where correlations exist for both sequence types MS-AS sequences often have higher correlations. The arrows in fig 4.11 point to Mmax (expect high correlations) and start date (expect no correlations) as benchmarks to compare with other correlations. Note that for most parameters, there is a non-significant correlation with the start date. For the few parameters where there is some correlation, this low correlation value should be considered a zero value.

Blocks of color are due to related parameters, such as ' $\mathrm{E}$ ', the number of $\mathrm{M}=3.0,3.5$, 4.0, 4.5; these numbers will be related even if the relationship is that none exist for the sequence. Low numbers of $\mathrm{M}=3$ imply even lower numbers of $\mathrm{M}=4.0$ (etc.) according to Gutenberg-Richter relationship. We also note that figure 4.11 only has 32 rows/columns because the expansion (Distance ratio) has no significant correlations.

Using figure 4.11 we chose a subset of 25 parameter combinations for investigation and comparisons by region in Table $4.5 \mathrm{a}$ and $4.5 \mathrm{~b}$. The columns are given a letter label corresponding to figure 4.11 and a number in order of columns for ease of reference. The first two columns (A1 and B2) are the summed values of all correlations for Mmax, for which we would expect good correlations, and Start-Date, for which we would expect low if any correlations. These are designed to be a sort of benchmark to test that we are getting real correlations for other parameters. 

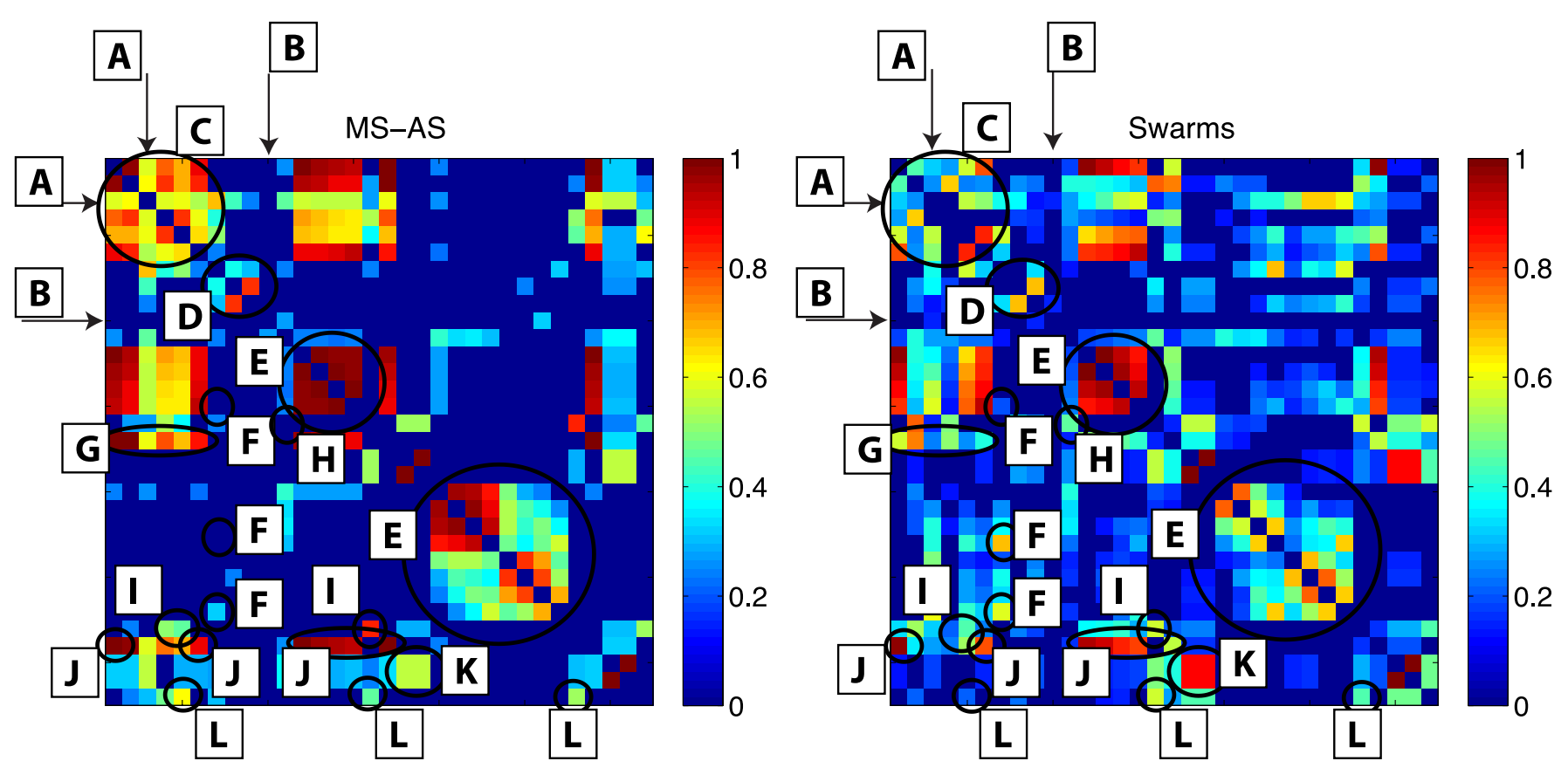

Figure 4.11 Correlation matrix for the absolute value of significant correlations of 33 parameters for the two sequence types.

Each square cell represents the significant correlation value between two parameters. The plot is mirrored along the diagonal and all labels are kept below the diagonal so parts obscured can be seen in the upper portion of each plot. Labels A-K correspond to columns in table 4.4 with explanations given in the text. Repeated labels indicate belonging to the same parameter or related parameter set. A) Arrows indicate the row and column associated with the parameter Mmax. B) Arrows indicate the row and column associated with Start date. C) cross-correlations of parameters listed in Table 4.2. D) average moment, Mdiff, and diff Mdiff, E) correlation related to the number of earthquakes at specific magnitudes. F) Average Moment, and number of $\mathrm{M} \geq 4.5$ earthquakes. G) time after Mmax with key sequence parameters . H) Rate-time before Mmax I) Number of earthquakes before Mmax with Area and Moment. J) Number of earthquakes after Mmax with total number of earthquakes, Moment-Mmax, number and time after Mmax. K) percent duration before/after Mmax with the percent of earthquakes before/after Mmax. L) Time between Mdiff with total Moment, time and number of earthquakes before Mmax. See table 4.4 and the text for further explanation. 
Table 4-5a Correlation of Key Parameter Pairs for Sequence Types in All Regions.

Numbers represent correlation values 0-1 for different parameter combinations and regions. Zero values indicate non-significant correlation.

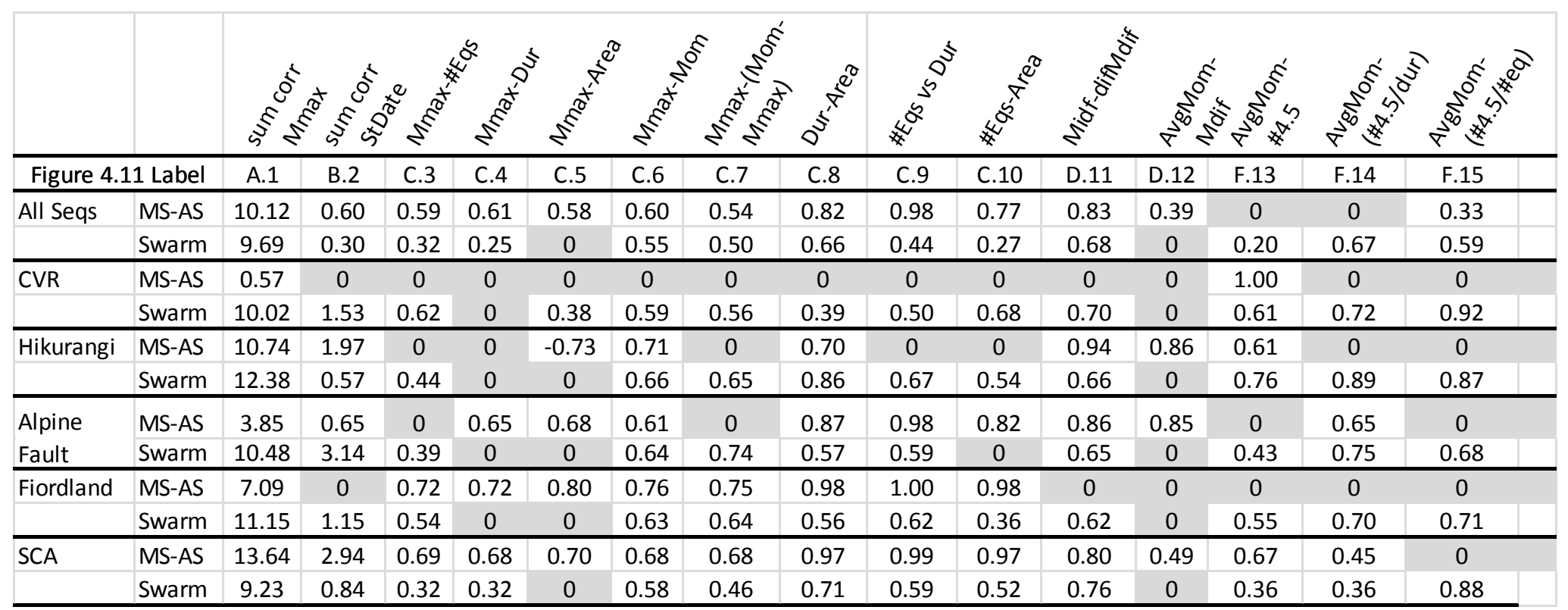


Table 4.5b Correlation of Key Parameter Pairs for Sequence Types in All Regions. Numbers represent the correlation values 0-1 for different parameter combinations and regions. Zero values indicate non-significant correlation.

\begin{tabular}{|c|c|c|c|c|c|c|c|c|c|c|c|c|c|}
\hline & & $0^{55^{50}}$ & $0^{2}$ & 5 & 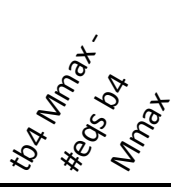 & $\vec{x}^{e^{2}}$ & $2^{2}$ & 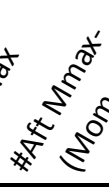 & $c^{2} \underbrace{0}_{0}$ & $\underbrace{\infty}_{0}$ & 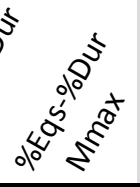 & 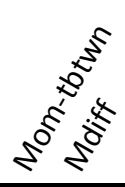 & $\frac{x^{2}}{x^{2}}$ \\
\hline \multicolumn{2}{|c|}{ Figure 4.11 Label } & G.16 & G.17 & H.18 & 1.19 & 1.20 & 1.21 & J.22 & J.23 & J.24 & K.25 & L. 26 & L. 27 \\
\hline \multirow[t]{2}{*}{ All Seqs } & MS-AS & 0 & 0 & 0 & 0.84 & 0.51 & 0.47 & 0.88 & 1.00 & 0.98 & 0.55 & 0.62 & 0.46 \\
\hline & Swarm & 0.40 & 0.77 & 0.46 & 0.57 & 0.21 & 0.44 & 0.78 & 0.98 & 0.36 & 0.86 & -0.21 & -0.56 \\
\hline \multirow[t]{2}{*}{ CVR } & MS-AS & 0 & 0 & 0 & 0 & 0 & 0 & 0 & 0 & 0 & 0 & 0 & 0 \\
\hline & Swarm & 0.42 & 0.78 & 0.37 & 0.56 & 0.69 & 0 & 0.69 & 0.96 & 0 & 0.85 & 0 & -0.79 \\
\hline \multirow[t]{2}{*}{ Hikurangi } & MS-AS & 0 & 0 & 0.83 & 0.63 & 0.75 & 0 & 0.76 & 1.00 & 0 & 0 & 0 & -0.65 \\
\hline & Swarm & 0.40 & 0.83 & 0.37 & 0.71 & 0.41 & 0 & 0 & 0.90 & 0.58 & 0.89 & 0 & -0.53 \\
\hline Alpine & MS-AS & 0 & 0 & 0 & 0.93 & 0 & 0 & 0.99 & 1.00 & 0.98 & 0.82 & 0 & 0 \\
\hline Fault & Swarm & 0.67 & 0.82 & 0.60 & 0.70 & 0 & 0 & 0 & 0.77 & 0 & 0.85 & 0 & -0.57 \\
\hline \multirow[t]{2}{*}{ Fiordland } & MS-AS & 0 & 0 & 0 & 0.93 & 0 & 0 & 0.88 & 1.00 & 1.00 & 0 & 0 & 0 \\
\hline & Swarm & 0 & 0.85 & 0 & 0.67 & 0 & 0.85 & 0.57 & 0.97 & 0.57 & 0.88 & -0.61 & -0.67 \\
\hline \multirow[t]{2}{*}{ SCA } & MS-AS & 0 & 0 & 0 & 0.89 & 0.78 & 0.82 & 0.99 & 1.00 & 0.99 & 0.54 & 0.75 & 0.83 \\
\hline & Swarm & 0.35 & 0.59 & 0.65 & 0.63 & 0.36 & 0 & 0.92 & 1.00 & 0.56 & 0.84 & 0 & -0.48 \\
\hline
\end{tabular}




\subsubsection{Correlations with Mmax}

Mmax is a parameter that we expect to have more significant correlations with MS-AS sequences than swarms. The duration (C4), the area (C5), and the moment (C6) all show the expected positive correlation with Mmax for MS-AS sequences; larger Mmax gives larger and longer sequences. Mmax and the area (C5) are negatively correlated in the Hikurangi region. This could be due to the large areas identified for some sequences there. We think it is likely an artifact of our area identification and not a real property of that region. For MS-AS sequences, the number of earthquakes (C3) is less well correlated to Mmax for the CVR, Hikurangi, and Alpine Fault regions. Swarm sequences in these three regions do have correlations between Mmax and the number. For SCA and Fiordland the correlations are higher, and similar to those observed with duration, area, and moment (C4-6).

Swarm sequences also show a correlation with Mmax and the Moment (C6). Moment is calculated from magnitudes and it is inescapable that these parameters will be tied whether Mmax controls other sequence parameters or not. To see whether Mmax influenced the moment of the rest of the sequence we also show the correlation of Mmax with the Moment-Moment(Mmax) (C7). Mmax and the moment of the remaining sequence correlates well for swarm sequences in all regions. It also correlates for MS-AS sequences in Fiordland and SCA, where Mmax correlates with the number of earthquakes (C3).

\subsubsection{Correlations with Duration}

The number of earthquakes and the duration (C9) are more strongly linked than the number of earthquakes and $\operatorname{Mmax}(\mathrm{C} 3)$. For MS-AS sequences in the aftershock dominated regions the duration also varies with Mmax (C4), but not as strongly as the number (C9). For MS-AS sequences the correlations for the number 
of earthquakes after Mmax (J24) is identical to the correlations for the total number of earthquakes. For swarm sequences the correlations are higher for the total number of earthquakes (C9) versus the number after Mmax (J24). The lower correlation for swarm sequences should be expected because the rate may be more constant than MSAS sequences and because Mmax is more likely to happen later than in MS-AS sequences (by definition), the number after Mmax should be less correlated.

\subsubsection{Correlations with Area}

The area correlates better with number of earthquakes (C10) than Mmax (C5). This is expected for swarm sequences, but is also evidently true for MS-AS sequences in the three aftershock dominated regions. Swarms in fact show no correlation with Mmax and the area. This is another example of why it is important to have the ability to search for sequences independently of magnitude.

\subsubsection{Correlations with Rate}

In general we expect and observe swarms to correlate better with rate [any measure of earthquakes per time whether individual rate parameters (e.g. 11, 24-27 in parameter list), or comparisons between two parameters (e.g. I.19, time before Mmax - Number of earthquakes before Mmax) than MS-AS sequences due to less overall change in the rate through the course of a sequence. The time before Mmax and the rate (H18) show no correlation for MS-AS sequences overall, and by region only for

Hikurangi. Swarm sequences show a correlation between these parameters (H18) for all regions except Fiordland. The correlation may not be surprising, but the positive value of it is. The positive correlation implies that the higher the rate of earthquakes in swarm sequences the longer the time it will be before Mmax occurs. For forecasting this would imply that the higher the rate of earthquakes, the less sure we 
should be that Mmax has already occurred. This is also connected to a correlation (for swarms) between duration and rate (G16), as well as duration and the time before Mmax (G17). The correlation implies that the longer time before Mmax implies a longer duration overall.

The two other rate parameters we have included in the table are the number of earthquakes before Mmax -time before Mmax (I19) and the percentage version of those parameters, the percent of earthquakes before Mmax to the percent of the duration before Mmax (K25). The number of earthquakes and the time before Mmax (I19) are strongly correlated for both sequence types, with a slightly stronger correlation for MS-AS sequences. Both sequence types show a positive correlation indicating that the more time that goes by, the more earthquakes occur. The percentages of these parameters (K25) tell a different story however with less or no correlation for MS-AS sequences and stronger correlations than for absolute values (I19) for swarm sequences. This is likely to be due to the fact that Mmax has a control on both the duration and the number for MS-AS sequences. It is also unsurprising that the percentages are not correlated given that the rate (H18) does not correlate to the time before Mmax for MS-AS sequences either.

\subsubsection{Moment (average and Mom-Mmax)}

We expect that average moment will be a parameter that correlates better with other parameters for swarm sequences than for aftershock sequences in general. On figure 4.11 however, $\mathrm{D}$ shows that there is a small correlation for Average Moment and Mdiff (D12) for MS-AS and not for swarm sequences. The breakdown by region in Table 4.5a shows that this correlation does not hold in Fiordland and is much stronger in Hikurangi and the Alpine Fault than for SCA. The overall correlation for all regions is low, and where it does correlate we presume that it correlates with the 
average Moment because large magnitude differences push the remaining magnitudes towards Mc and are thus likely to have less events. A decrease in the number of events could cause the average Moment to increase.

For swarm sequences we find good correlations with average moment and the number of $\mathrm{M} \geq 4.5$ earthquakes (F13). Where a correlation also exists for the number of $\mathrm{M} \geq \mathrm{M} 4.5$ as a percent of the total number of earthquakes (F15), this has a higher correlation for swarms than MS-AS. The other way that the average moment correlates is the number $\mathrm{M} \geq \mathrm{M} 4.5$ divided by the duration (F14). The percent by duration (F14) has a higher overall correlation for all swarm sequences, but the correlation region to region varies, with the CVR and SCA regions having better correlation with the percent by number (F15) and the remaining regions having similar or slightly better correlations for percent by duration (F14). The difference in correlation between these two parameters may be interpreted as a measure of the variation in rate for each region. Where rates are similar between sequences we would expect these correlations to be similar, where they are different (or where we have not done well in distinguishing between sequence types) then the correlations will vary.

\subsubsection{Time between the two largest earthquakes (L26 L27)}

One of the most interesting parameters observed to have correlations in figure 4.11 is the time between Mmax and the second largest magnitude (M2) earthquake with Moment and time before Mmax (L26-27). We term this time between the two largest magnitude earthquakes 'tMdiff'. Examination by region in Table 4.5b (L26) shows that the correlation with Moment is probably an artifact arising from a few sequences in specific regions. On the other hand swarms seem consistently to have negative correlations with tMdiff and the time before Mmax (L27). Positive 
correlation of these parameters indicates that the more time a sequence has before Mmax occurs the more time there will be between the largest two earthquakes. Negative correlation implies that the longer time before Mmax would lead to shorter times between the largest two earthquakes. A correlation between the tMdiff and any parameter is exciting as it is important for hazards and risk assessment. It is important to note here that even if the correlation is valid for MS-AS sequences, it may not apply directly to large earthquakes, as our definition of sequences may put large, lateaftershocks into separate sequences.

Because the correlation (L27) seems most strong in swarm sequences we wanted to investigate tMdiff as a parameter with sequence type. One of the first things we suspected was that swarm sequences are more likely to have a negative tMdiff due to the higher probability of Mmax occurring later in the sequence (Figs. 4.3-4.6). Figure 4.12 (A) shows that this is the case and only a single MS-AS sequence (1/73) has the second largest earthquake before Mmax, while nearly half $(104 / 224)$ of all swarm sequences have the second largest earthquake before Mmax. The most obvious thing from this plot is that the majority of tMdiff are $\leq$ one day. There is a higher likelihood for MS-AS sequences $(\sim 80 \%)$ to have tMdiff $\leq$ one day, but $\sim 60 \%$ of swarms also observe the two largest earthquakes within one day. We also plotted tMdiff by region to match the regional probabilities in Table 4.5b. Figure 4.12 B shows that the MS-AS sequences in SCA have longer tMdiff values than other regions. The Hikurangi region is the only one with the second largest earthquake preceding Mmax, which explains its negative correlation. For swarm sequences Hikurangi also has the widest spread of tMdiff. This spread of timing is somewhat surprising since the Hikurangi region has lower mean and median durations than the three aftershock dominated regions. All regions display some swarms with negative 
tMdiff values (second largest earthquake preceding Mmax). The other surprising feature of the swarm timing plot (4.12B) is that where the times occur prior to Mmax (negative) they are longer than the times observed following Mmax. The larger values of negative tMdiff relative to positive tMdiff are surprising given that Fig 4.6 showed swarm sequences have a tendency to be skewed with more of the duration following Mmax than preceding it. This reinforces our earlier conclusion that Mmax is more likely to occur in the first half of the sequence (by duration) regardless of sequence type.
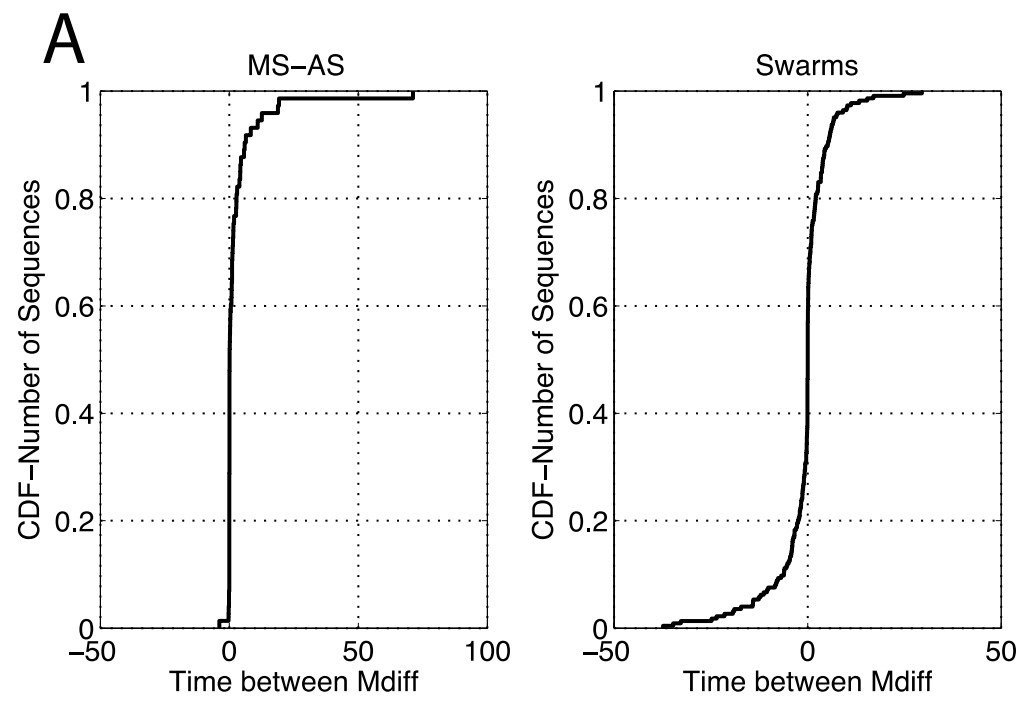

\section{B}
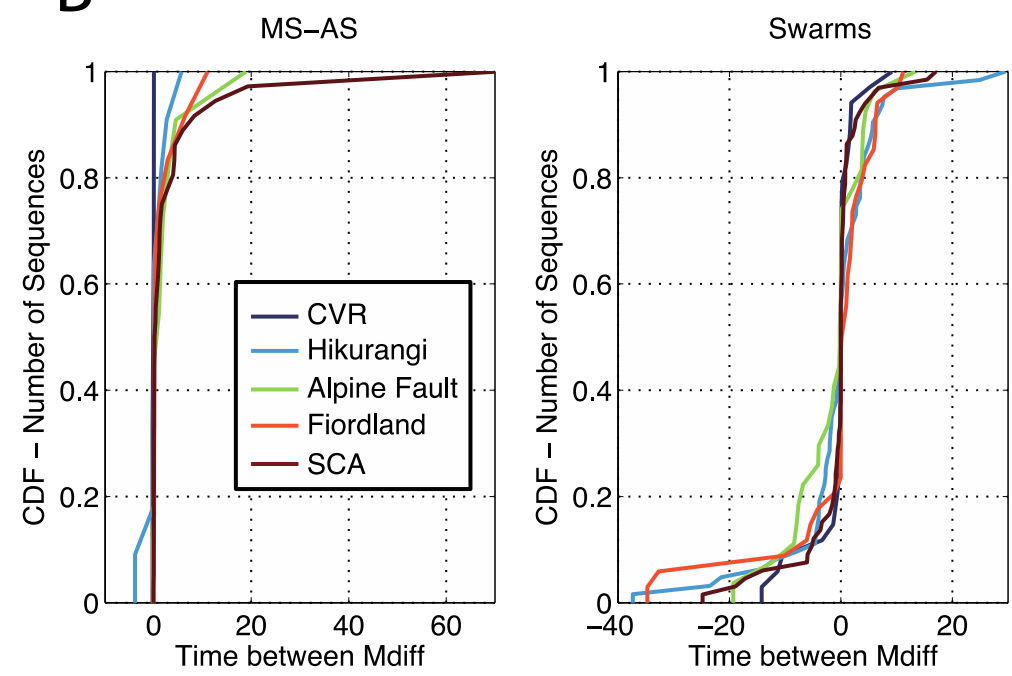

Figure 4.12 Cumulative distribution of the time between the largest and second largest magnitude earthquakes.
A) for sequence types.
B) for sequence types in five different regions. 
If we separate the tMdiff into positive and negative (instead of by sequence type) we find that the population with negative tMdiff have a high correlation of -0.91 and the positive tMdiff population has a lower, but still significant correlation of 0.29. The reason for the opposite sign of correlation is not intuitive as it indicates that the longer the time between the two largest earthquakes the shorter the total time before Mmax. The longer the two earthquakes have between them the shorter the total time before Mmax indicates possible triggering between events. Whereas when tMdiff is small, and Mmax and M2 are very close in swarm sequences, the time before Mmax gets longer, indicating a driving mechanism other than the current or preceding seismicity. The positive correlation for the positive tMdiff population (M2 earlier than Mmax) is likewise unclear in its origins. The longer a sequence goes before observing Mmax, the longer it will continue, regardless of sequence type. While we do not know the exact cause of these correlations, it warrants further, future investigation as it has important implications for hazards.

\subsubsection{Earthquake Rates (Acceleration and Decay)}

To begin investigating how sequences develop in time, we calculate the amount of acceleration and decay occurring in sequences for both absolute and average inter-event times. We calculate whether each earthquake time in a sequence represents acceleration or decay by simply taking the time difference between successive inter-event times. Times which are shorter than the previous time are accelerations and times that are longer are categorized as decay. Fig 4.13 shows that all sequences are dominated by decay. In $4.13 \mathrm{~B}$ we can see that $20 \%$ of MS-AS sequences have $80 \%$ or less of their duration described by decay, implying that $80 \%$ of MS-AS sequences have more than $80 \%$ of their duration described by decay. On the other hand, twice as many (40\%) of swarm sequences have $80 \%$ of their duration 
explained by decay. Although the swarms have less decay, $60 \%$ of swarms still have $80 \%$ or more of decay through their duration. Thus, all sequences experience a large amount of decay. Absolute inter-event times vary little with sequence type although MS-AS curve does sit slightly above swarm sequences in 4.13-A, showing more decay (reflexive). Figure 4.13-B shows substantial difference with sequence type. We naturally expect more decay than acceleration as decay is by definition a longer time interval than an acceleration. While swarms are more likely to have greater proportions of acceleration, they still experience large amounts of decay. Figure 4.14 shows that the amount of relative acceleration and decay from average inter-event times by region. All MS-AS sequence have more decay than acceleration, with slightly different slopes for different regions. Most regions show differences at the median value, but Hikurangi and SCA are the most similar between types (also most expansion, Fig. 4.10 and Tbl. 4.4). The observation that all sequences contain large portions of decay is the subject of investigation in the next two sections, which focus on temporal patterns of acceleration and decay, and the nature of the observed decay. 
A. Absolute Inter-event times
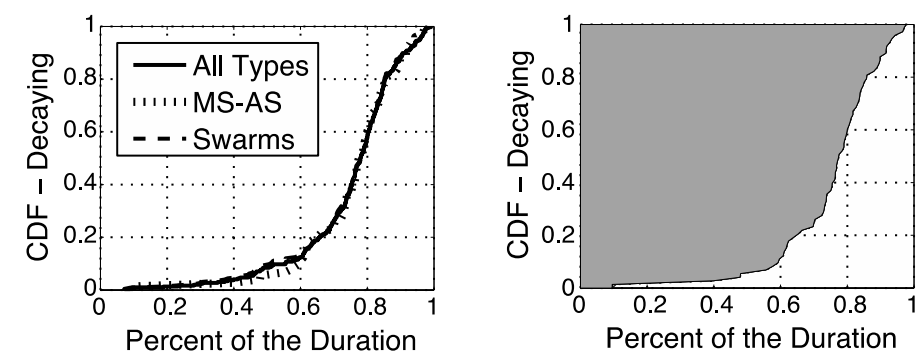

B. Average Inter-event times
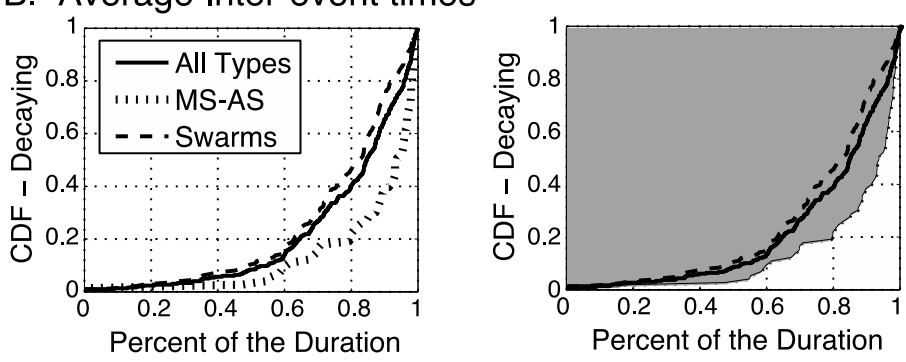

Figure 4.13 Amount of Decay observed for sequences as a fraction of the duration. Acceleration and decay are determined by taking the difference in successive interevent times. Negative differences indicate acceleration, and positive differences correspond to decay. The negative and positive time periods are summed respectively leading to a total amount of acceleration and decay for each sequence. As the amount of acceleration and decay will be inverses of each other, only plots of decay are shown. A) Absolute Inter-event times. B) Average Inter-Event times. The solid line shows all sequence types, the thick dashed line shows swarm sequences, and the thin dashed line shows mainshock aftershock type sequences. For both A and B the lefthand panel shows the CDF plots for the amount of decay by sequence type and the right-hand plot shades the amount that decay explains for MS-AS sequences to more obviously show the dominance of decay. 
A. CVR

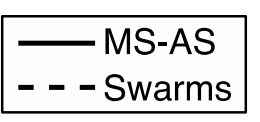

B. Hikurangi
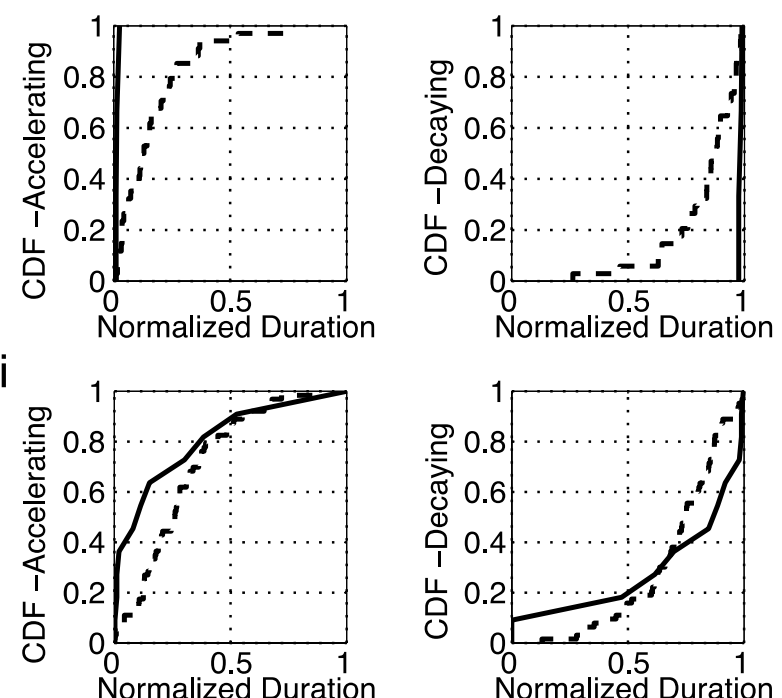

C. Alpine Fault

Normalized Duration

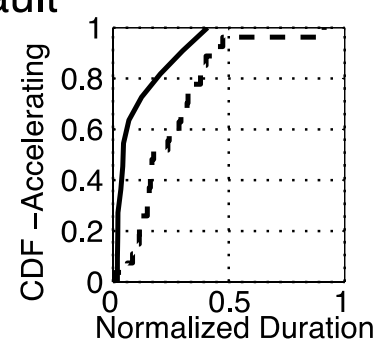

D. Fiordland

E. SCA
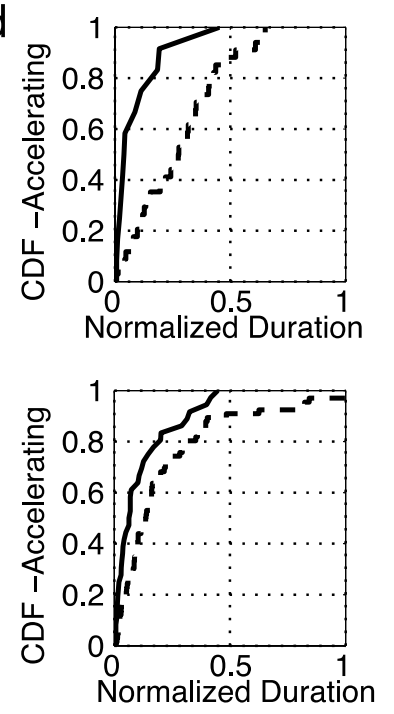
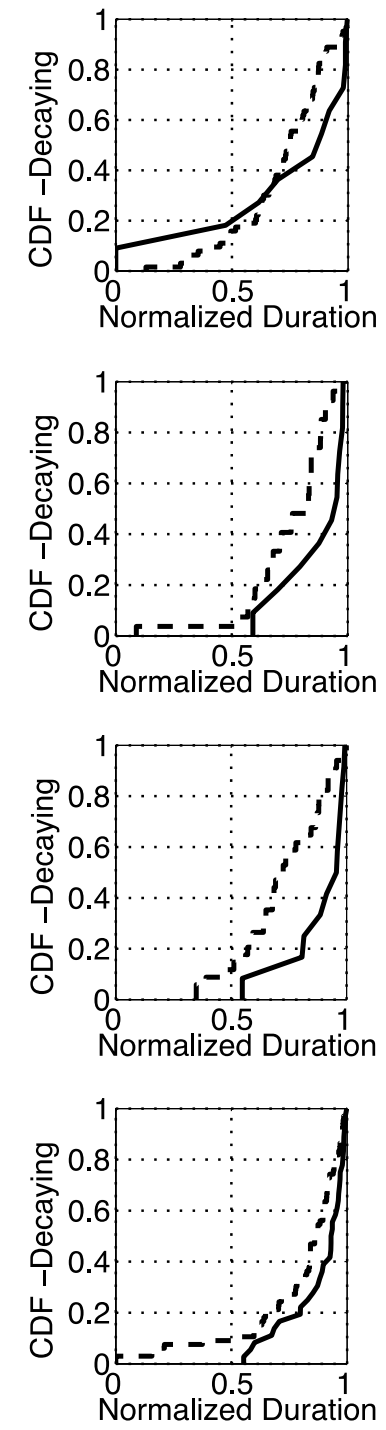

Figure 4.14 Cumulative distribution plots of the amount of a sequence (by duration) that is taken up by decay and acceleration for each of our five regions (A-E).

The curves in the left and right hand plots are inverses of each other. Dotted lines indicate the population of swarm type sequence, and solid lines represent mainshockaftershock type sequences. 


\subsection{Sequence Decay}

\subsubsection{Introduction}

Given the large amount of decay observed in all sequence types, we wanted to test whether an Omori's law decay (Eq. 4.1) could explain decay observed in swarm type sequences. The rate of aftershocks is known to decay according to Omori's law, which in its cumulative form, with the p-value set to 1 , is given by:

$$
\mathrm{N}(\mathrm{t})=K^{\star} \log (1+(\mathrm{t} / \mathrm{c}))
$$

Where $K$ is a constant productivity factor, $c$ is a constant time delay, and $t$ is the time of each earthquake relative to the time of the first event. Our main goal is to see whether the temporal development of the sequences can be described by Omori's law. We may not have enough information to fit three separate parameters $(c, K, p)$, so we have left $p$ fixed to the reasonable value of 1 (e.g. [Utsu et al., 1995]). ETAS models and some earthquake forecasting tools incorporate some sort of Omori decay with time. We have shown that at magnitude thresholds less that $M=3.0$ sequence catalogs are composed of $>60 \%$ swarms. If we can quantify the degree to which Omori's law decay can explain decay in swarm sequences we may be able to anticipate how well such models will forecast swarm dominated catalogs. A better understanding of swarm decay processes may also enable us to augment existing seismicity models to create more realistic earthquake clustering.

We do not expect swarm sequences to follow Omori's law for their entire duration, and so we have chosen a variety of potential temporal breakpoints within a sequence to test different parts of sequences that may fit Omori's law. The list below details the breakpoint, or starting point, for each test.

Test 1: First Earthquake (whole sequence) 
Test 2: Mmax

Test 3: minimum absolute inter-event time

(shortest time between two earthquakes)

Test 4: minimum average inter-event time

(smallest average time between earthquakes)

Test 5: minimum average inter-event time following Mmax

Test 6: last Mdiff within 0.5 of Mmax (Swarms only, by definition)

All tests model Omori's law parameters using earthquake times from the breakpoint until the end of the sequence. The fitted parameters will change between tests because the fraction of a sequence included will change for each test. The only requirement to test a sequence is that it has at least 10 earthquakes from the breakpoint to the end of the sequence. The total sequence size has been limited to a minimum of ten earthquakes, so all sequences will go through Test 1 . We anticipate that most MS-AS sequences should be well fit by Omori's law, either from the first event or from Mmax (tests 1 and 2). Swarms are unlikely to perform well over the whole sequence (test 1), but may be well fit for some of the other tests.

After fitting the Omori's law parameters for each breakpoint in all sequences, we use two different methods to evaluate the goodness of fit. The first method calculates the amount of variation expected based on the number of earthquakes used in the fitting and the second method compares the Omori fit to a linear fit for the seismicity. The fitting of the Omori's law parameters and the two methods to evaluate the goodness of fit are detailed in the next section. 


\subsubsection{Omori's Law Modeling}

\subsubsection{Fitting Omori's Law}

To fit Omori's law for each test sequence, we get times relative to breakpoints, with the first earthquake at the breakpoint equal to time zero and other earthquakes times reflecting the time since the first (breakpoint) event. We use those times to determine the best possible $c$ value through maximum likelihood (code written by Euan Smith and adapted for this purpose). The code assumes a $p$-value of 1, and tests a range of $c$-value parameters between input values of $c \min (0.1)$ and $\operatorname{cmax}(25)$ in increments $(c i)$ of 0.1 between the input bounds. If the minimum $c$-value is found to have the maximum likelihood then the bounds are adjusted and retested with the new $c \min =c i / 10, c \max =c i^{*} 2$, and $c i=c i / 100$. Typical $c$-values are small $(<1)$ when the catalog is complete, but completeness can vary directly following large earthquakes (e.g. [Kagan and Houston, 2005; Utsu et al., 1995]). We have allowed a large cmax value (longer than median durations for both sequence types). Therefore, reaching the maximum $c$-value will indicate a poor fit.

Once the maximum-likelihood value is found we use the best fitting c-value to calculate a corresponding $K$-value. This is done by fixing the end of the sequence and finding a $K$ that, with the best-fitting $c$-value, will give the correct observed total number of earthquakes (equation 4.2). Note that this is $K$ for the Omori's law cumulative number with time formula (Eq. 4.1).

$$
\mathrm{K}=1 / \log (1+\mathrm{tmax} / \text { Bestc })
$$

where tmax is the time of the last earthquake, and Bestc is the c-value determined from maximum likelihood fitting. 
We also determined a 95\% confidence bound for the maximum likelihood cvalue. Using Equation 4.3 [Williams, 2001; p. 230] any c-values with a corresponding negative $\mathrm{Z}$ value are within the $95 \%$ confidence region (Figure 4.15A) where $\log L$ is the $\log$-likelihood and MLmax is the maximum likelihood.

$$
Z=\operatorname{abs}(\log L-M L \max )-(1.96)
$$

Models using these $95 \%$ boundary $c$-values often have smaller deviations from the best model than the observed misfit between the data and the best-fit model.

\subsubsection{Maximum Misfit Testing}

The maximum misfit test determines whether the misfit between the data and the modeled Omori's law can be attributed to the sample size. To calculate the misfit expected from the sample size alone, we take the number of earthquakes used to fit the Omori parameters for each sequence and we create the same number of random yvalues (between 0-1). These random y-values are then projected onto the model to obtain times from the beginning of the sequence (Figure $4.15 \mathrm{~B}$ ). Once we have the synthetic times, we take the cumulative distribution of the times and measure a maximum offset from the model. Because these values have been taken directly from the model, any observed misfit can be attributed to the sample size. This is done 1000 times to give 1000 maximum offset measurements. These 1000 measurements are then used to make a cumulative distribution of potential maximum offsets, which gives us a bound for which $95 \%$ of these random misfits fall below. We then compare the observed maximum misfit value between the Omori model and the data to the $95 \%$ misfit-bound.

If the maximum misfit between the Omori model and the data is less than the $95 \%$ misfit-bound it is possible that the observed misfit is simply due to a small 
sample size. If, however, the real misfit is greater than the $95 \%$ random misfit it cannot be explained by sample size. An example of this testing is shown in figure 4.16 on a linear and log scale. In this example data vary more than the minimum and maximum c-value models (black) but are well within the 95\% sample size boundary (gray).

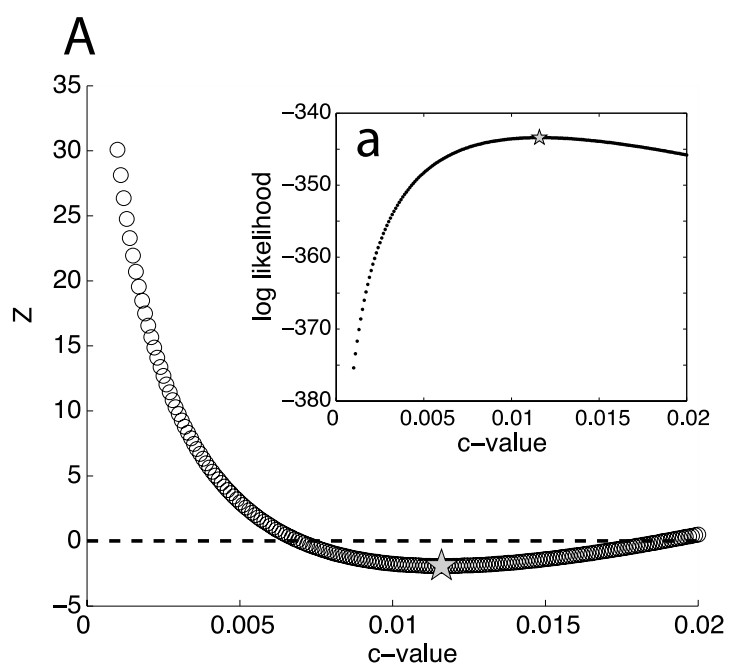

\section{B}

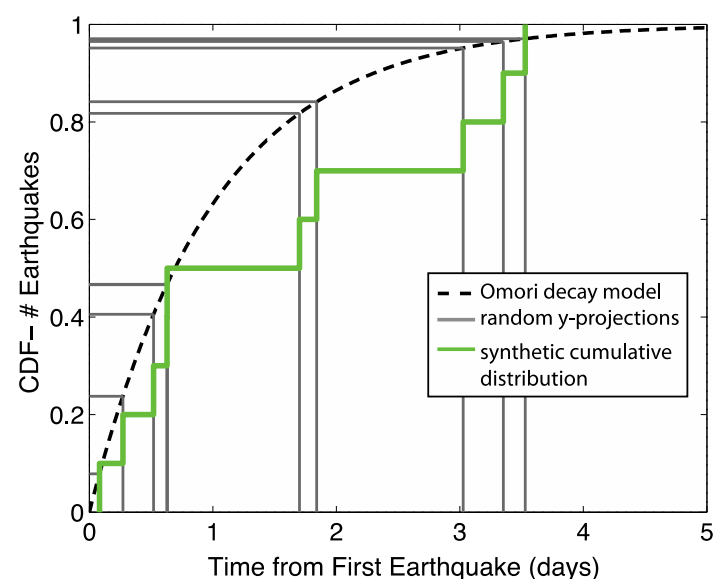

Figure 4.15 Illustrating the calculations connected to maximum likelihood and creation of synthetic distributions from an Omori's law decay model.

A) Plots showing an example of how the maximum likelihood c-value (a) and 95\% confidence bounds are calculated. See the text and Equation 4.3. B) Figure of random sampling from the model. A set of random numbers (with size equal to the number of earthquakes) is generated and used as as y-values (gray lines) in the model (dashed black line) to get a set of x-times that are used to make a cumulative distribution (not shown). The deviation of this cumulative distribution will reflect the amount of misfit that can be attributed to the sample size. 

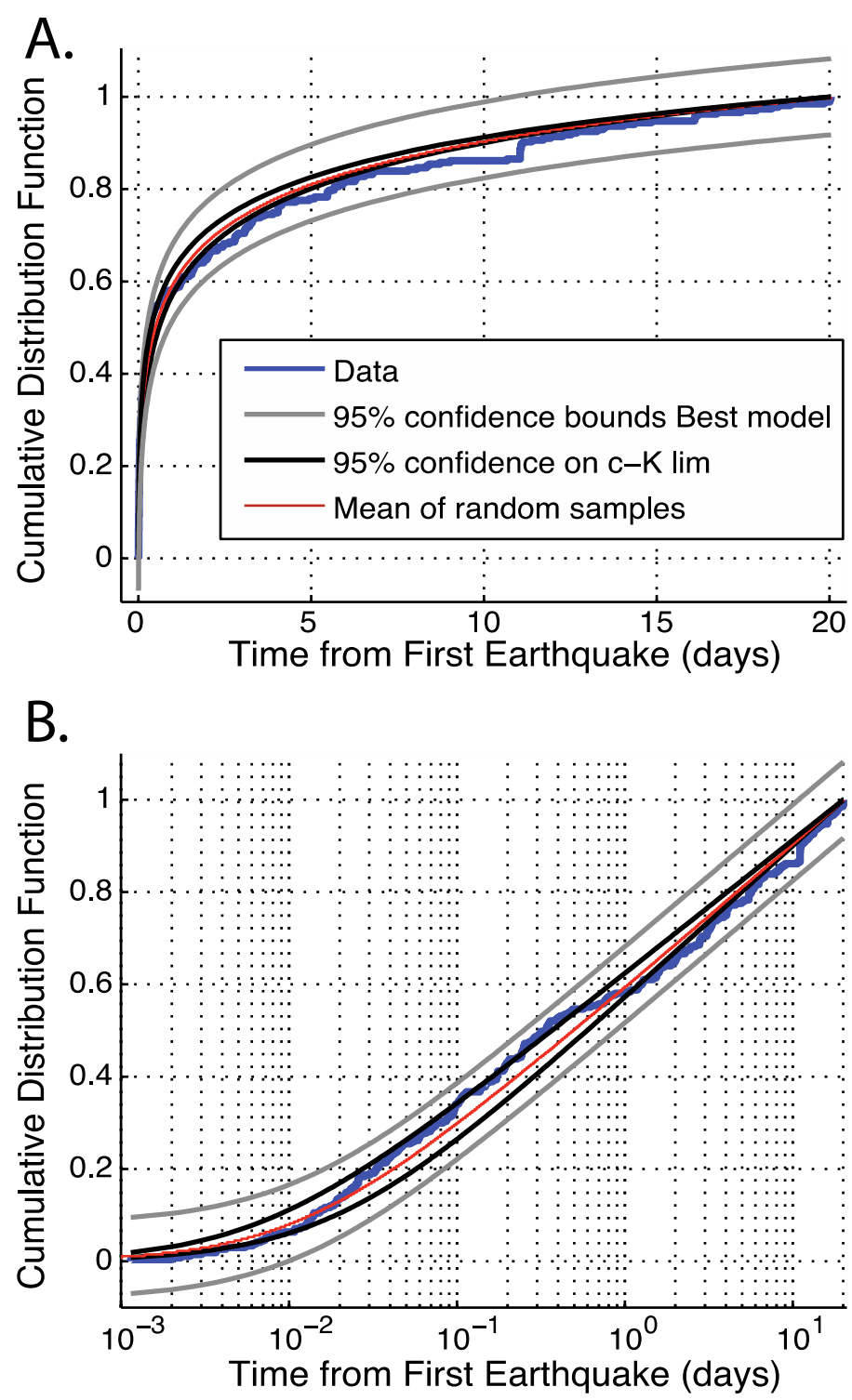

$C$ values $=0.007: 0.012: 0.019$

$\mathrm{K}$ values $=0.126: 0.134: 0.143$

$95 \%$ confidence from random sampling $=0.0825$

Maximum deviation of real data $=-0.0559$

Figure 4.16 Example of the Omori's law decay model fits of $\mathrm{c}$ and $\mathrm{K}$ values.

The mean of the sampled models (red) is shown with $95 \%$ confidence boundaries (black) as well as the $95 \%$ confidence interval based on the sample size (gray). The data (blue) is from a MS-AS sequence of 268 earthquakes in the Fiordland region with $M m a x=6.22$, and Mdiff $=0.76$ starting in November 2000 .

Table 4.6 shows the number of sequences that were tested and passed for both MS-AS and swarm sequences for all six tests. The results are somewhat surprising 
with a higher percentage of swarms than MS-AS passing all six tests. There is some overlap in percentage between individual tests. The percent of unique sequences that pass at least one test is $84 \%(62 / 73)$ for MS-AS and nearly $89 \%(199 / 224)$ for swarm sequences.

Despite the high percentage of the swarms passing the maximum misfit test, the c-values for swarms in most of the tests in Table 4.6 have relatively large values, indicating that even the passing swarms may have poor fits. Test 2, earthquakes from Mmax, is the only test that gives reasonable c-values. On the other hand for MS-AS sequences only two tests, 1 and 5, show inflated c-values (Table 4.6). In general the median passing c-value is much lower for MS-AS than for swarm sequences. One possible reason for the discrepancy is that swarms tend to have lower numbers of earthquakes and so may have larger confidence bounds, resulting in 'pass' values that may not have real meaning.

Figure 4.17-A shows the cumulative distributions of the maximum misfit values for sequences passing any of the six tests. Swarm sequences show a larger misfit, but this may simply be a product of smaller sequence sizes. Figure 4.17B compares between misfit for the whole sequence (test 1) and other tests. Swarms, and MS-AS sequences are similar overall, but MS-AS sequences have a smaller range of misfits. There is a clear variation between bounds for sequences that pass and fail (Table 4.7), suggesting a possible dependence. The maximum median data misfit is larger for swarm sequences on most tests, but because the misfit may depend on sample size it is difficult to determine whether this is reasonable. For these reasons we conclude that the maximum misfit test is insufficient to determine whether sequences experience Omori's law decay. 
A.

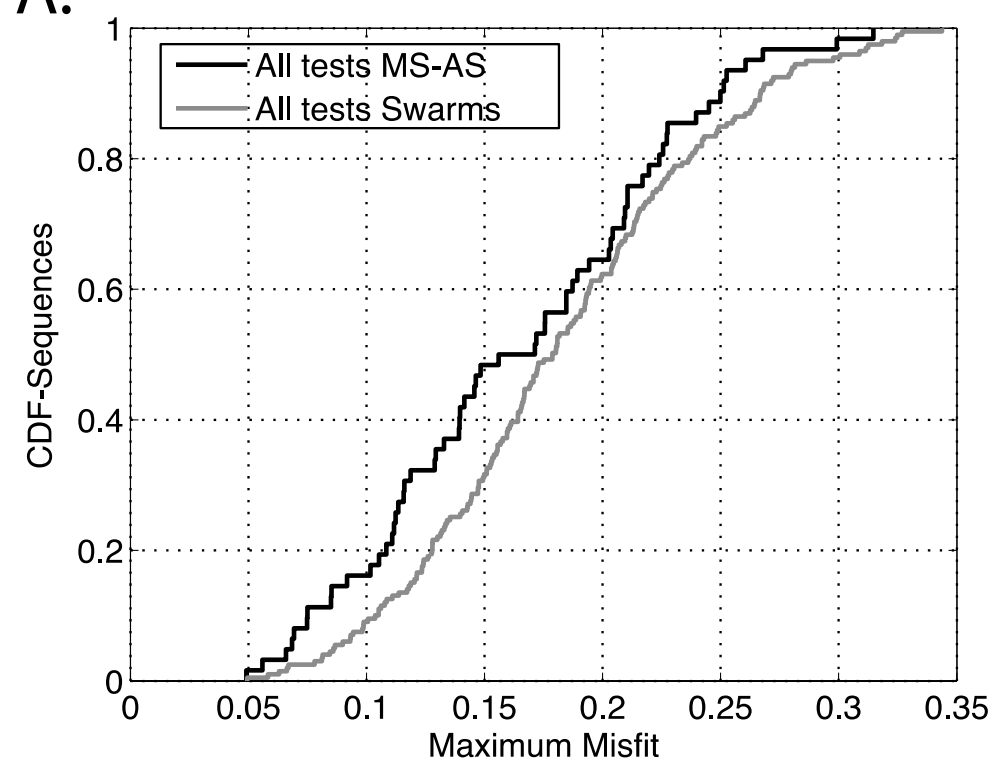

B.

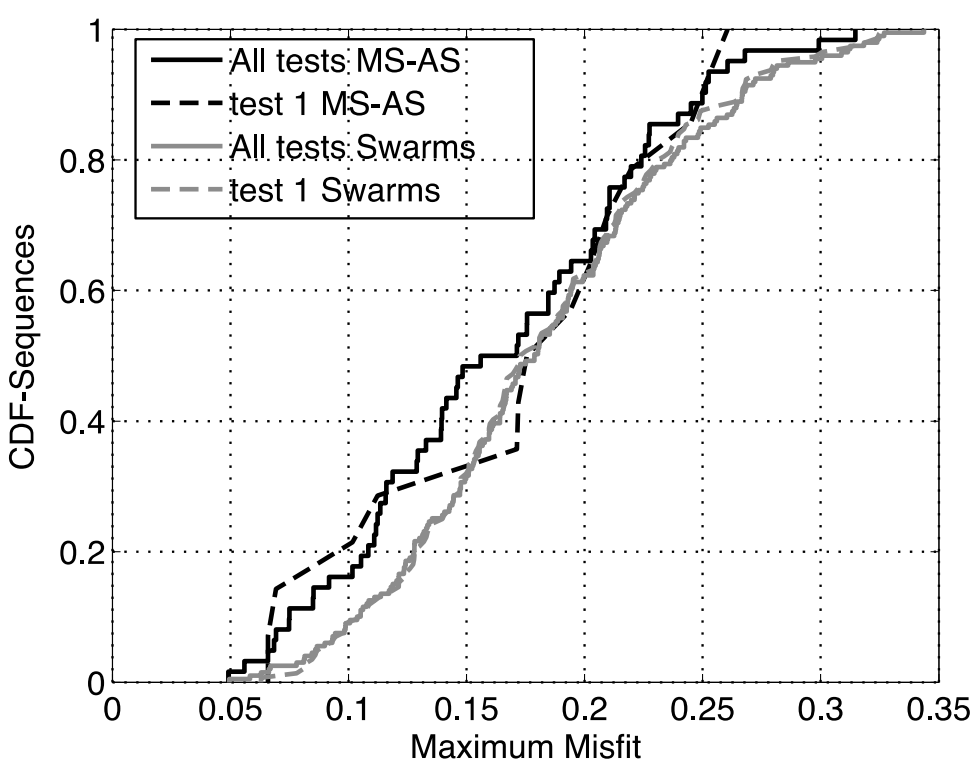

Figure 4.17 Cumulative distributions of the maximum Misfit from an Omori's law decay model of all sequences that pass the maximum misfit for any of the 6 tests.

A) By sequence type. Black indicates MS-AS, and gray represents swarm sequences.

B) By sequence type for those that pass any test (solid lines) and those that pass test 1, the whole sequence (dashed). Note that the solid and dashed lines overlap for swarm sequences. See Table 4.6 for further details. 
Table 4-6 The Number of Sequences (by type) that pass the different Omori's Law Decay Tests.

The test column indicates the starting point of the test. All tests go to the end of the sequence. The number tested corresponds to the number of sequences that have more than 10 earthquakes between the starting point and the end of the sequence.

\begin{tabular}{|c|c|c|c|c|c|c|c|c|c|c|}
\hline & & $x^{s^{e^{\alpha}}} x^{e^{x^{e^{d}}}}$ & $p^{p^{s^{c}}}$ & $000^{p^{\delta^{s^{e^{\partial}}}}}$ & $n^{e^{j^{2}}}$ & $r^{e^{j^{2}}}$ & 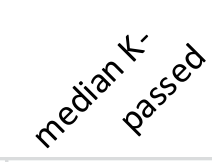 & 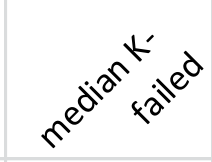 & 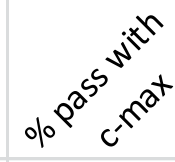 & $000^{00^{n}} c^{n^{2}}$ \\
\hline \multirow[t]{6}{*}{ MS-AS } & Test & & & & & & & & & \\
\hline & 1. Whole Sequence & 73 & 43 & $58.90 \%$ & 0.21 & 6.23 & 9.08 & 71.97 & $13.95 \%$ & $33.33 \%$ \\
\hline & 2. From Mmax & 69 & 51 & $73.91 \%$ & 0.05 & 0.16 & 6.41 & 41.39 & $9.80 \%$ & $11.11 \%$ \\
\hline & 3. Min abs iEtime & 29 & 17 & $58.62 \%$ & 0.29 & 1.53 & 6.65 & 119.12 & $17.65 \%$ & $16.67 \%$ \\
\hline & 4. Min avg iEtime & 54 & 38 & $70.37 \%$ & 0.38 & 1.16 & 11.59 & 124.26 & $15.79 \%$ & $37.50 \%$ \\
\hline & $\begin{array}{l}\text { 5. Min avg iEtime } \\
\text { after Mmax }\end{array}$ & 7 & 4 & $57.14 \%$ & 2.30 & 24.99 & 18.21 & 140.95 & $0.00 \%$ & $66.67 \%$ \\
\hline \multirow[t]{7}{*}{ Swarms } & Test & & & & & & & & & \\
\hline & 1. Whole Sequence & 224 & 146 & $65.18 \%$ & 21.19 & 24.99 & 26.87 & 62.76 & $49.32 \%$ & $62.82 \%$ \\
\hline & 2. From $M \max$ & 129 & 104 & $80.62 \%$ & 0.62 & 1.88 & 9.19 & 55.53 & $24.04 \%$ & $44.00 \%$ \\
\hline & 3. Min abs iEtime & 111 & 77 & $69.37 \%$ & 12.65 & 24.99 & 27.53 & 54.81 & $42.86 \%$ & $55.88 \%$ \\
\hline & 4. Min avg iEtime & 135 & 93 & $68.89 \%$ & 16.91 & 24.99 & 27.39 & 54.30 & $48.39 \%$ & $61.90 \%$ \\
\hline & $\begin{array}{l}\text { 5. Min avg iEtime } \\
\text { after Mmax }\end{array}$ & 84 & 53 & $63.10 \%$ & 24.99 & 24.99 & 31.76 & 71.65 & $52.83 \%$ & $77.42 \%$ \\
\hline & $\begin{array}{l}\text { 6. from last } M \\
w / \text { in } 0.5 \mathrm{Mmax}\end{array}$ & 40 & 29 & $72.50 \%$ & 0.23 & 2.76 & 8.59 & 61.23 & $20.69 \%$ & $45.45 \%$ \\
\hline
\end{tabular}


Table 4-7 Confidence Bounds and Absolute Value of the Maximum Misfit for Sequences Passing and Failing All Omori's Law Decay Tests. The $95 \%$ confidence bound is related to the number of earthquakes used to determine $c$ and $K$ values for the Omori's Law models.

\begin{tabular}{|c|c|c|c|c|c|c|c|c|c|}
\hline & & 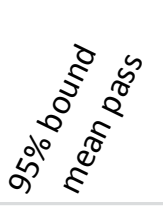 & 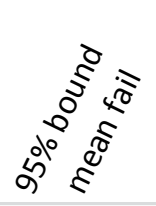 & 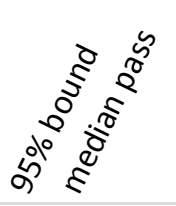 & 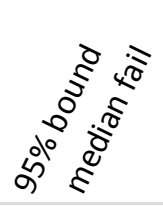 & 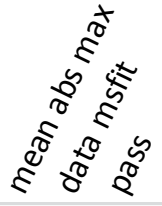 & 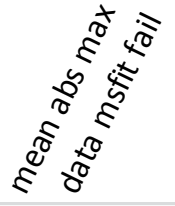 & 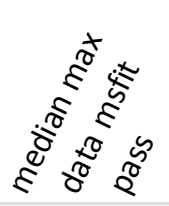 & 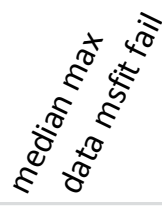 \\
\hline \multirow[t]{7}{*}{ MS-AS } & Test & & & & & & & & \\
\hline & 1. Whole Sequence & 0.262 & 0.175 & 0.278 & 0.194 & 0.167 & 0.301 & -0.075 & 0.257 \\
\hline & 2. From Mmax & 0.258 & 0.135 & 0.280 & 0.161 & 0.154 & 0.202 & -0.075 & 0.090 \\
\hline & 3. Min abs iEtime & 0.299 & 0.119 & 0.313 & 0.107 & 0.178 & 0.224 & -0.115 & -0.140 \\
\hline & 4. Min avg iEtime & 0.240 & 0.144 & 0.260 & 0.141 & 0.153 & 0.258 & -0.083 & 0.188 \\
\hline & $\begin{array}{l}\text { 5. Min avg iEtime } \\
\text { after Mmax }\end{array}$ & 0.275 & 0.193 & 0.291 & 0.224 & 0.207 & 0.435 & 0.031 & 0.374 \\
\hline & & 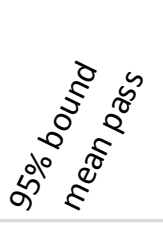 & 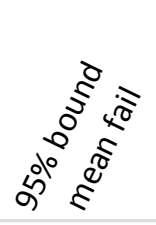 & 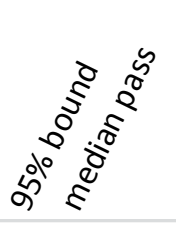 & 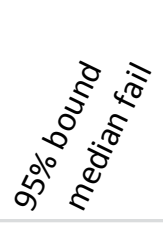 & 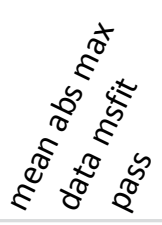 & 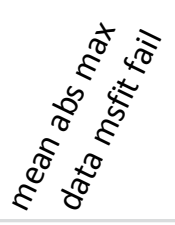 & 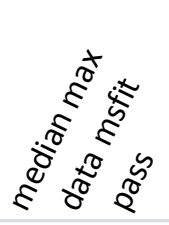 & 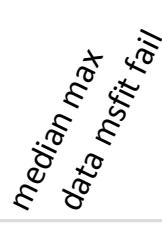 \\
\hline \multirow[t]{6}{*}{ Swarms } & Test & & & & & & & & \\
\hline & 1. Whole Sequence & 0.290 & 0.224 & 0.305 & 0.222 & 0.182 & 0.357 & 0.008 & 0.249 \\
\hline & 2. From Mmax & 0.279 & 0.210 & 0.292 & 0.201 & 0.170 & 0.269 & -0.093 & 0.210 \\
\hline & 3. Min abs iEtime & 0.289 & 0.206 & 0.300 & 0.204 & 0.183 & 0.320 & -0.111 & 0.257 \\
\hline & 4. Min avg iEtime & 0.280 & 0.193 & 0.299 & 0.198 & 0.180 & 0.307 & -0.078 & 0.235 \\
\hline & $\begin{array}{l}\text { 5. Min avg iEtime } \\
\text { after Mmax }\end{array}$ & 0.271 & 0.192 & 0.272 & 0.203 & 0.186 & 0.328 & -0.080 & 0.270 \\
\hline
\end{tabular}




\subsubsection{Linear Comparison Test}

To refine our testing we introduced a comparison to a linear time fit. Such fits have been used previously in the literature (e.g. Brodsky [2006]). For this test we calculate the cumulative residuals of the Omori's law model for each sequence (or sequence piece) and the cumulative residuals to a simple linear model that starts at zero time and finishes at the end time of the sequence, tmax, with the total number of earthquakes (Figure 4.18). The cumulative residuals will vary for individual sequences and with the number of earthquakes, so we take the ratio of the cumulative residuals to the minimum cumulative residual. Values of 1 indicate that a particular fit has the minimum cumulative residual, and the corresponding ratio of the other fit gives an 'improvement factor' from the fit of the other method. This was run for all the tests and sequences as above for the maximum misfit testing. For this test each sequence gets a 'pass' if the Omori's law fit has the minimum cumulative residual. The percent of each sequence type that passes this test is closer to what we had initially anticipated for the sequence types (Table 4.8, Fig. 4.19). For the linear comparison test, up to $91.3 \%$ of MS-AS sequences pass, and a slightly lower $80 \%$ of swarm sequences pass for any given breakpoint test. The c-values are also much more reasonable, with the median c-value of passing swarms having much smaller values than those observed for the maximum -misfit test (Table 4.6)). The smaller cvalues indicate that the linear comparison test is able to identify sequences that have a poor Omori's law fit. 


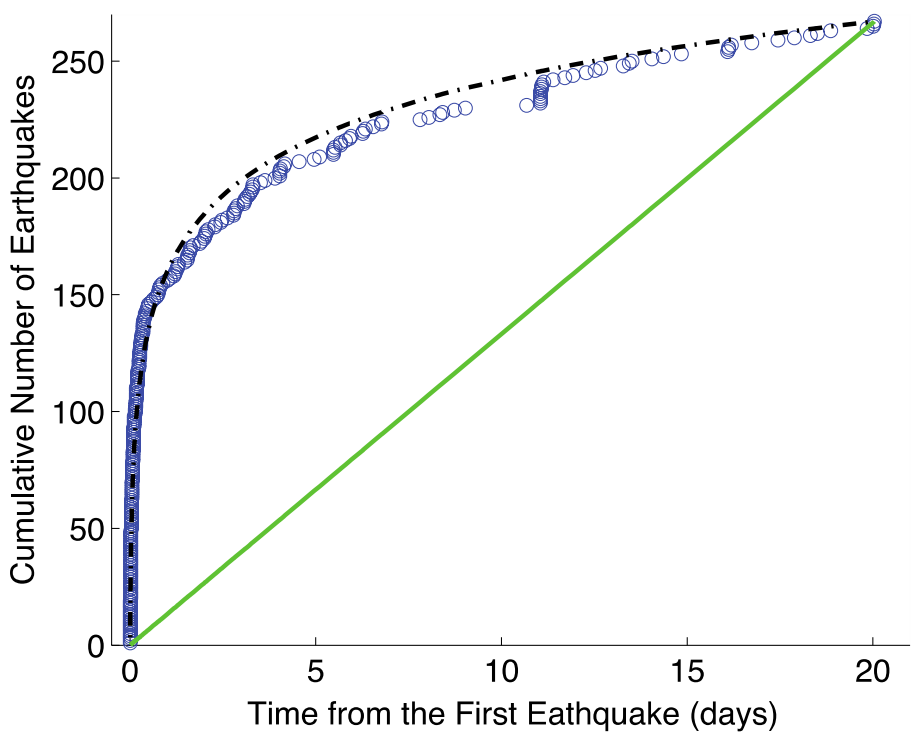

(blue).

A. Max Misfit: Test 1

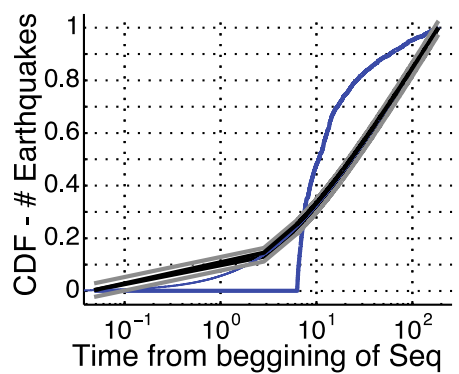

B. Linear Comp: Test 1

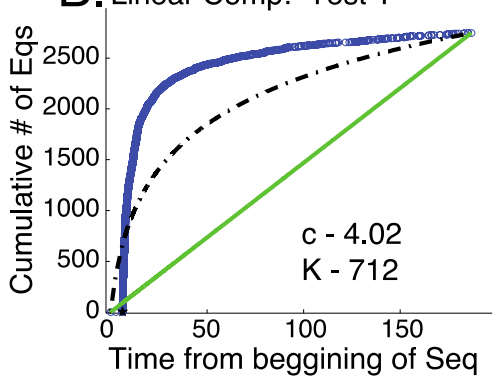

C. Max Misfit: Test 2

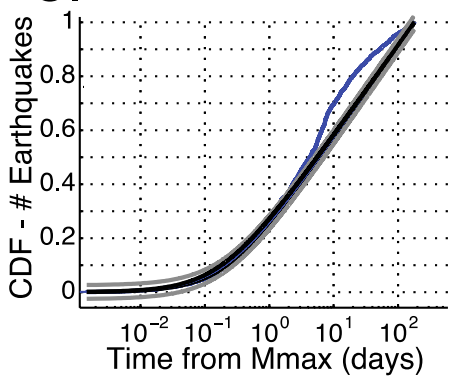

D. Linear Comp: Test 2

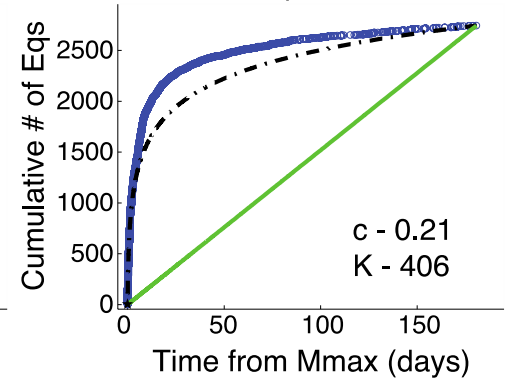

Figure 4.18 Example of a linear comparison test.

Data (blue) is from the same Fiordland MS-AS sequence in figure 4.16. Dashed black line shows the bestfitting Omori's law model and the green line shows a linear seismicity rate model The test compares the cumulative residuals between these two models and the data

Figure 4.19 Comparison of the two goodness of fit tests for Omori's law decay parameters models.

The whole sequence is used in $\mathrm{A}$ and $\mathrm{B}$, and the sequence from Mmax is used in C and $\mathrm{D}$. Data are from an $\mathrm{Mmax}=6.7$ sequence in Fiordland starting in early August 1993. Real data are shown in blue in all plots. The maximum misfit method (as shown in

Fig. 4.16) is shown for A) test 1 on a $\log$ scale, and C) test 2 , also on a log scale. To pass the maximum misfit test the data (blue) must fall inside the gray lines. This sequence fails the maximum misfit for both tests 1 and 2 (A and $\mathrm{C}$ ). The linear comparison goodness of fit (as shown in Fig. 4.18) for B) test 1 and D) test 2. The modeled Omori's law decay is shown as a black dashed line with the green line for comparison. To pass the linear comparison test the sum of the residuals for the Omori fit (dashed) must be smaller than the sum of the residual for the linear rate (green). This sequence passes the linear comparison fit test for both test 1 and 2 (B and D). Note with the improved fit of D over B the c-value has decreased substantially. 
Figure 4.19 and 4.20 show examples of two large MS-AS sequences that failed our original maximum misfit tests, but pass the linear comparison tests. In figure 4.20 we show results for a Fiordland sequence with Mmax=6.7, and an Mdiff of 1.07 that has three identified foreshocks prior to Mmax. The foreshocks, as expected, appear to throw off the Omori model (A). The linear fit comparison shows that an Omori's law type decay is more suitable than a linear model. For test 2 (4.19 C and D) the Omori model is significantly improved, but a large overshoot still occurs towards the end of the sequence. The linear comparison from Mmax shows a much more stable fit with the improved Omori model parameters. Another advantage of the linear comparison is the ability to deal with late large aftershocks without using compound Omori decay models. In figure 4.20 the $2010 \mathrm{M}=7.2$ Cucapah earthquake in Southern California is shown. This sequence has 31 foreshocks and a large late aftershock. Like the Fiordland sequence it fails the maximum misfit test for tests 1 and 2. It fails the linear comparison for test 1 because the foreshocks disturb the fit and no true maximum is reached in the model determination $(\mathrm{c}=c \max )$. The Cucapah sequence passes the linear-comparison tests for the sequence starting from Mmax as the Omori model is improved. Despite the large aftershock, the sequence can still be much better modeled with Omori's decay than a linear fit. 


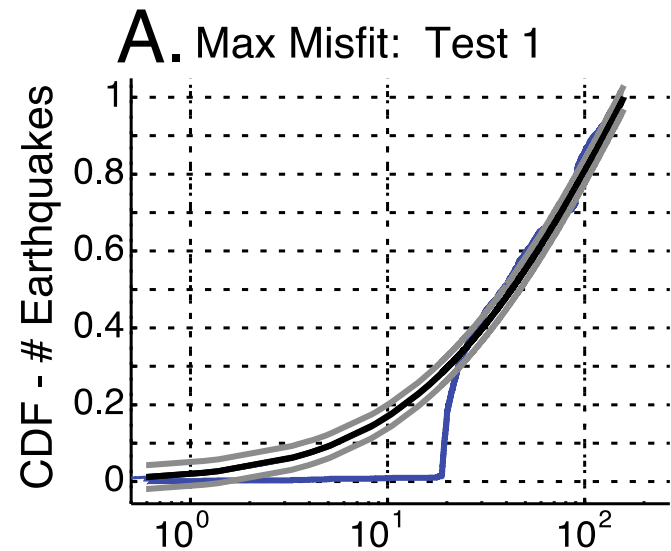

Time from beggining of Seq

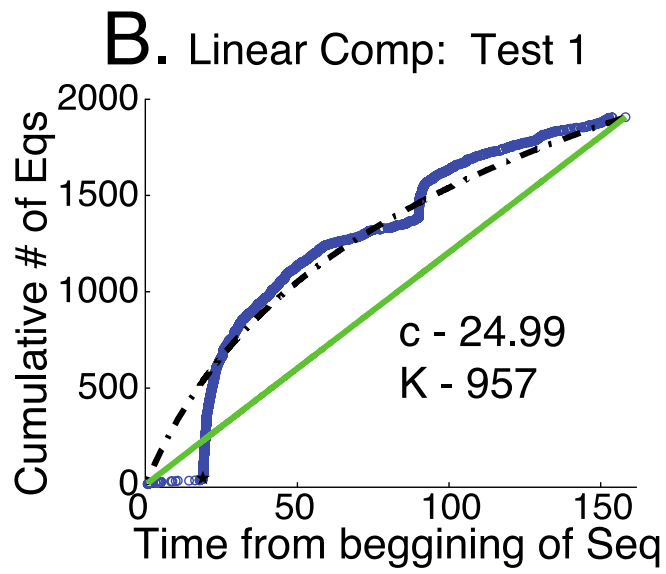

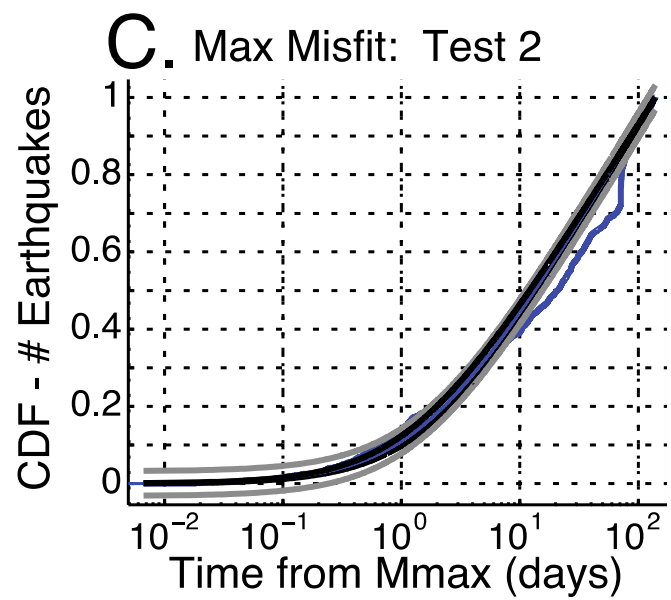

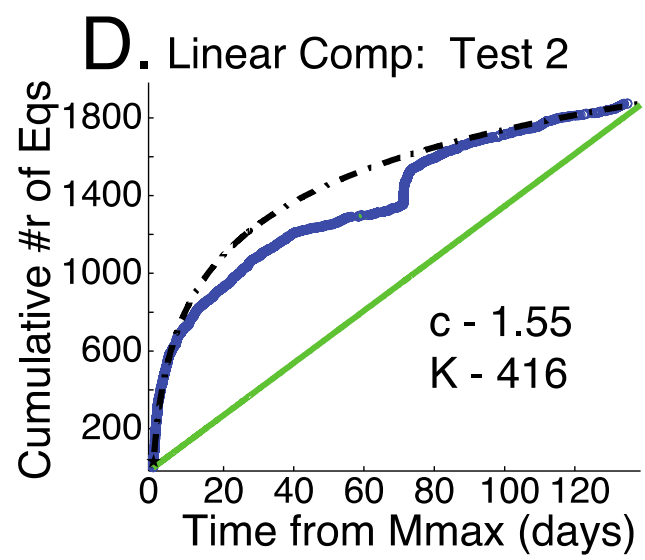

Figure 4.20 Another example of two goodness of fit tests for Omori's law decay parameters models.

The whole sequence is used in $\mathrm{A}$ and $\mathrm{B}$ and the sequence from Mmax is used in $\mathrm{C}$ and D. The sequence shown is the $M \max =7.2$ Cucapaha 2010 earthquake in Southern California. Real data are shown in blue in all plots. The maximum misfit method (as shown in Fig. 4.16) is shown for A) test 1 on a log scale, and C) are test 2, also on a $\log$ scale. To pass the maximum misfit test the data (blue) must fall inside the gray lines. This sequence fails the maximum misfit for both tests 1 and 2 (A and C). The linear comparison goodness of fit (as shown in Fig. 4.18) for B) test 1 and D) test 2. The modeled Omori's law decay is shown as a black dashed line with the green line for comparison. To pass the linear comparison test the sum of the residuals for the Omori fit must be smaller than the sum of the residual for the linear rate. Parameters $\mathrm{c}$ and K are given for each Omori's law fit. See the text for an explanation of these parameters and the fitting procedure. This sequence fails the linear comparison fit test for test 1 (B), but passes the linear comparison for test 2 (D). Note the more realistic c-value in test 2 (D). 
Table 4-8 The Number of Sequences (by type) that Pass the Linear Comparison Decay Test.

The test column indicates the starting point of the test. All tests go to the end of the sequence. The number tested corresponds to the number of sequences that have at least 10 earthquakes between the starting point and the end of the sequence.

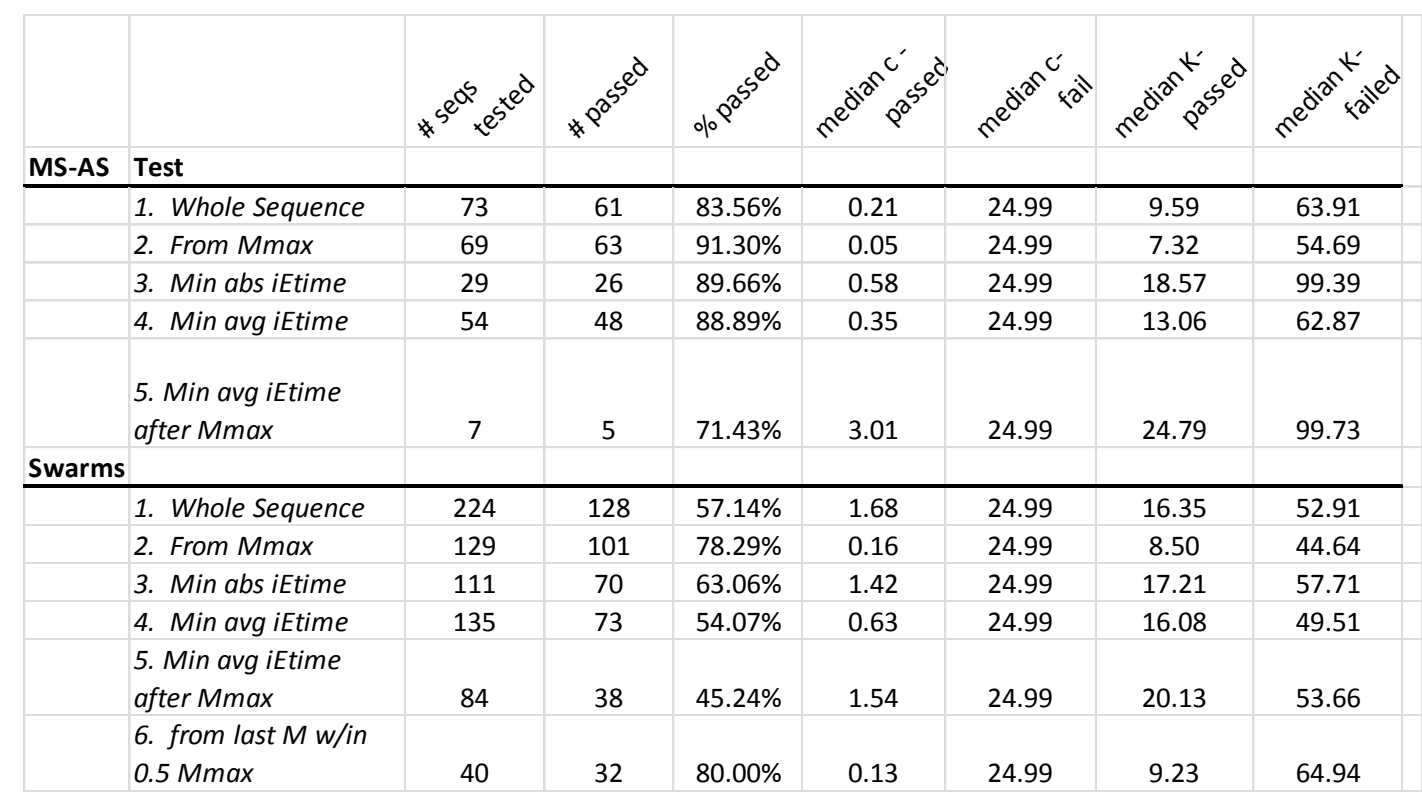



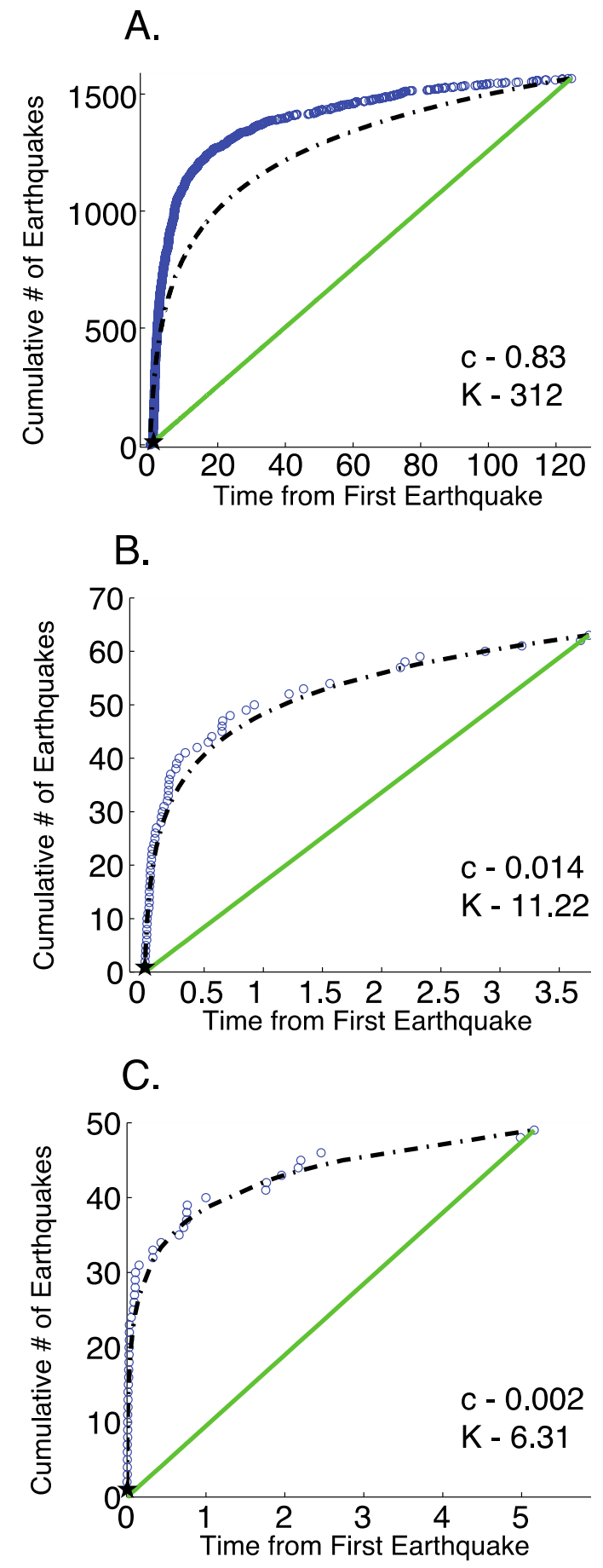

Figure 4.21 Examples of three typical MS-AS fits with the linear-comparison test.

Parameters c and K are given for each Omori's law fit. See the text for an explanation of these parameters and the fitting procedure. A) Hector Mine $\mathrm{M}=7.1$ earthquake (c=0.85, K=312). B) SCA Mmax=5.36 in August 1995 ( $c=0.0137, \mathrm{~K}=11.22) . \mathrm{C}$ ) CVR Mmax=4.85 in September 2002. $(\mathrm{c}=.002, \mathrm{~K}=6.31)$. 
Figure 4.21 shows three additional MS-AS sequences. A) is the Hector Mine $\mathrm{M}=7.1$, another large Mmax that does poorly with the maximum misfit testing. The linear-comparison shows that it is more productive than we have modeled but that it roughly fits the expected decay pattern. The second and third panels are two smaller MS-AS sequences from SCA (B) and the CVR (C). These slightly smaller Mmax values $(5.36,4.85)$ are much better fits with our simple Omori model. Both of the smaller sequences passed the maximum misfit test. We also looked at the difference between the whole sequence fits (test 1) and the fits from Mmax (test 2) for our three largest swarm sequences in the CVR (Fig. 4.22). All three are reasonably well modeled by Omori's law decay, with residual ratios of at least 2 for test 1 . Haroharo sequence (A) does not pass the maximum misfit test despite having residual ratios of 3.3, and 6.78 for tests 1 and 2. Only the Matata sequence (C) passes the maximum misfit test for the whole sequence although the c-value is high. Rotoehu (B) passes the maximum misfit test from Mmax and is described as behaving similarly to a mainshock aftershock sequence by Hurst et al. [2008]. While the Matata sequence passes the maximum misfit tests, the Rotoehu sequence has the best improvement factor from Mmax with a value of 19.55, nearly three times the value for Haroharo and Matata (Fig. 4.22). 
A. Haroharo (1998)

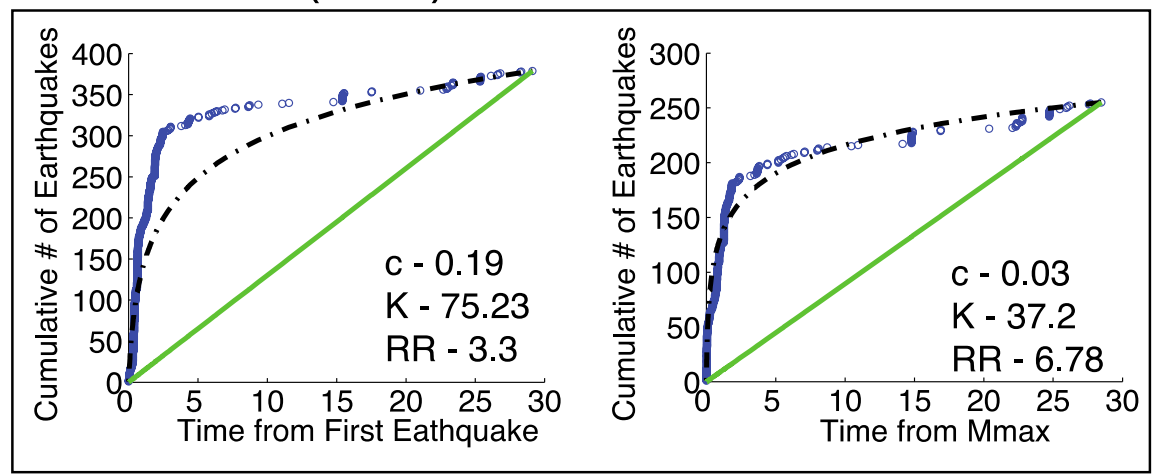

B. Rotoehu (2004)

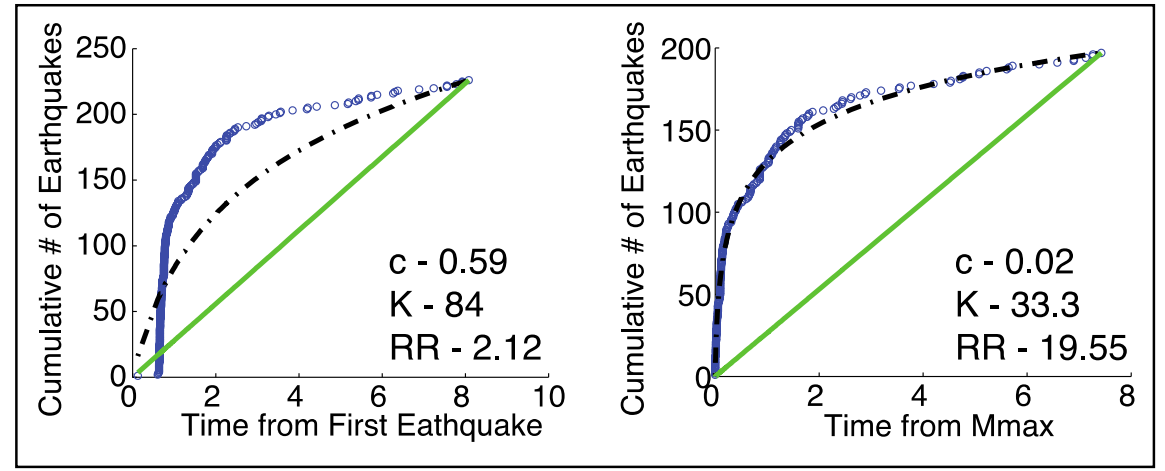

\section{Matata (2005)}

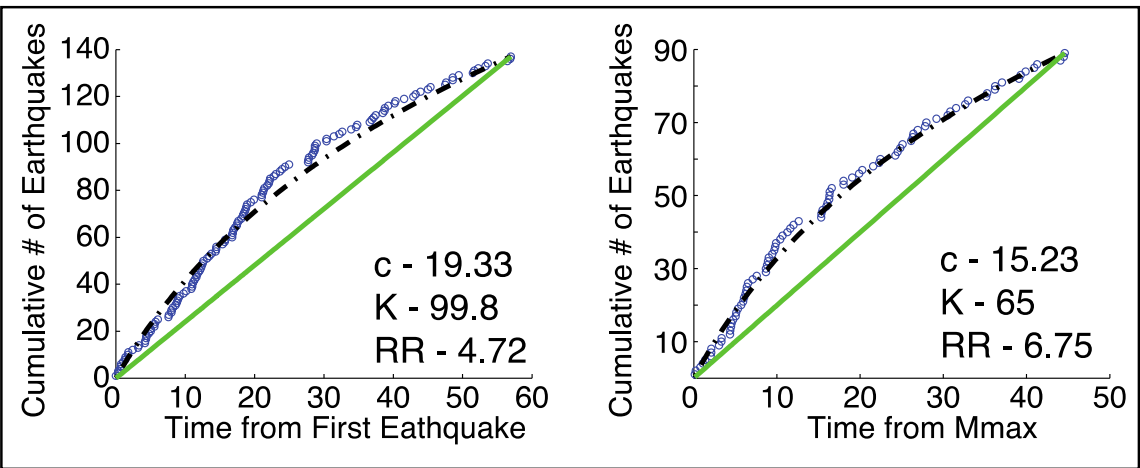

Figure 4.22 Linear-comparison tests for three large swarms in the CVR.

The first column is test 1 (whole sequence) and the second column is for test 2 (from Mmax). A) Haroharo, $M \max =4.68$. B) Rotoehu, $M \max =5.10$. C) Matata, $\mathrm{Mmax}=3.86$. The Omori model $\mathrm{c}$ and $\mathrm{K}$ parameters are shown for each model as well as the residual ratio from the linear fit comparison. 
We can also use the residual ratios to compare the relative improvement between MS-AS and swarm sequences, and we also refer to this residual ratio as an improvement factor. The residual ratio of the linear model should be large for sequences that are well fit by an Omori's law decay. This comparison can also give us ideas of which of the six tests are capturing real decay. We found that MS-AS sequences have a much larger residual ratio (improvement-factor) than swarm sequences (Figure 4.23, Table 4.9). The portion of a sequence after Mmax has the biggest improvement factor for both MS-AS sequence and swarms. Because MS-AS sequences are constrained to have Mmax early in the sequence this does not have a large impact on the percent of earthquakes in the test. Swarm sequences, however, have a larger distribution of the timing of Mmax within the sequence and the later occurrences of Mmax reduces the total percent of earthquake used in the Omori's law fit down to $77 \%$ in test 2 . The next best test for MS-AS sequences is the portion of the sequence from the minimum average inter-event time (test 4). This improvement comes at a cost in the percent of the sequence that is described by the decay. Test 4 uses $83 \%$ of the total earthquakes on average. Despite the potential reduction in the number of earthquakes used, all MS-AS sequences use a mean of $92.7 \%$ of all events to calculate the Omori's fit. Swarms also have reasonably large percentages used in all tests. The lowest percent, $54 \%$, is in test 6 (the last earthquake within 0.5 of Mmax). Test 4 is the only test for which swarms have a larger mean-percentage of earthquakes used in the test than aftershocks. The higher percentage indicates that swarms have their minimum average inter-event time earlier in time than MS-AS sequences. This is surprising since we think of MS-AS sequences decaying from the outset, which would suggest an average inter-event time at the beginning of the sequence. 


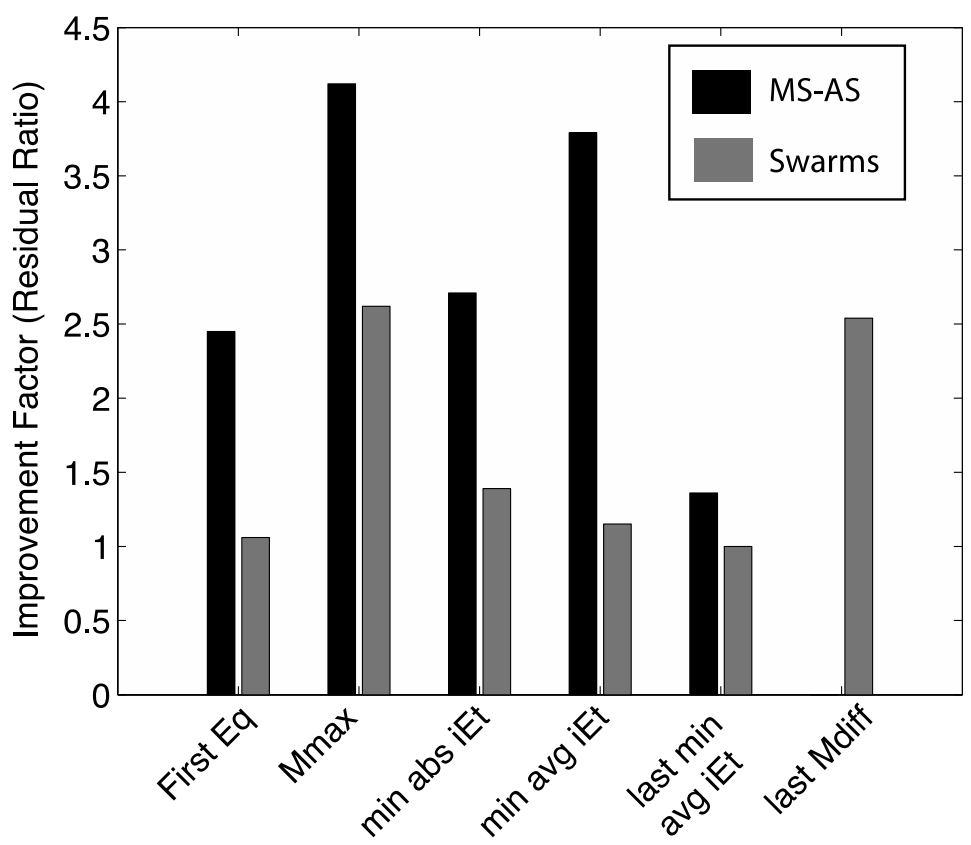

Figure 4.23 Improvement Factor (Residual ratio) of the Linear fit for all six tests with sequence type.

Mainshock-aftershock (black) and Swarm (gray). Note that MS-AS by definition cannot have any earthquakes within 0.5 of Mmax, so only swarm sequences are tested in test 6 (last Mdiff).

Table 4-9 Residual Ratios of Omori's Law Decay and Linear Fits to the Data from Each Test.

The ratio is made by dividing the each cumulative residuals for the Omori and Linear fits by whichever cumulative residual has the lowest value. Values of 1 represent cases where that fit has the minimum, or best, cumulative residual. Small differences from 1 represent small differences between the goodness of the two fits.

\begin{tabular}{|c|c|c|c|c|c|c|c|c|c|c|c|c|}
\hline \multirow[b]{2}{*}{ Test } & \multirow[t]{2}{*}{ 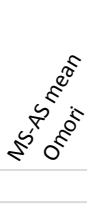 } & \multicolumn{2}{|c|}{ 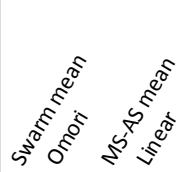 } & \multirow[t]{2}{*}{ 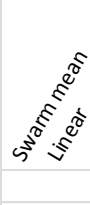 } & \multirow[t]{2}{*}{ 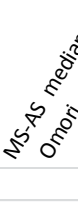 } & \multirow[t]{2}{*}{ है } & \multicolumn{2}{|c|}{ 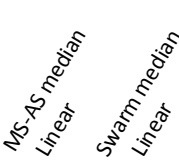 } & \multirow[t]{2}{*}{ 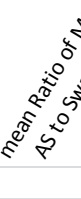 } & \multicolumn{2}{|c|}{ 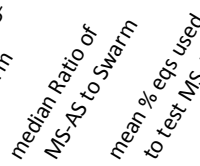 } & \multirow[t]{2}{*}{$\underbrace{4}$} \\
\hline & & & & & & & & & & & & \\
\hline 1. Whole Sequenct & 1.03 & 1.15 & 3.95 & 2.01 & 1.00 & 1.00 & 2.45 & 1.06 & 1.96 & 2.30 & $100 \%$ & $100 \%$ \\
\hline 2. From $M \max$ & 1.02 & 1.06 & 5.28 & 3.53 & 1.00 & 1.00 & 4.12 & 2.62 & 1.50 & 1.58 & $94.50 \%$ & $76.63 \%$ \\
\hline 3. Min abs iEtime & 1.00 & 1.14 & 2.93 & 1.84 & 1.00 & 1.00 & 2.71 & 1.39 & 1.59 & 1.94 & $91.25 \%$ & $87.55 \%$ \\
\hline 4. Min avg iEtime & 1.02 & 1.21 & 4.75 & 2.27 & 1.00 & 1.00 & 3.79 & 1.15 & 2.09 & 3.30 & $82.88 \%$ & $87.57 \%$ \\
\hline $\begin{array}{l}\text { 5. Min avg iEtime } \\
\text { after Mmax }\end{array}$ & 1.03 & 1.23 & 1.66 & 1.82 & 1.00 & 1.02 & 1.36 & 1.00 & 0.91 & 1.36 & $94.61 \%$ & $93.30 \%$ \\
\hline $\begin{array}{l}\text { 6. from last } M \\
\text { w/in } 0.5 \mathrm{Mmax}\end{array}$ & & 1.02 & & 3.00 & & 1.00 & & 2.54 & & & 0 & $53.93 \%$ \\
\hline
\end{tabular}


Figure 4.24 shows the improvement factor for each sequence type. MS-AS sequences have more sequences that pass (residual ratio $=1$ ) and have larger improvement factors. If we look at the improvement factors by test (Fig. 4.25), we see from the range of values along the $\mathrm{x}$-axis, that Test 2 (from Mmax) shows the largest residual ratios (biggest improvement) for both sequence types. Test 6 (only swarms) has a similar shape to the residual ratio curve as this test is meant to mimic the Mmax test (Test 2). Swarms have a lower improvement factor distribution for all tests. 

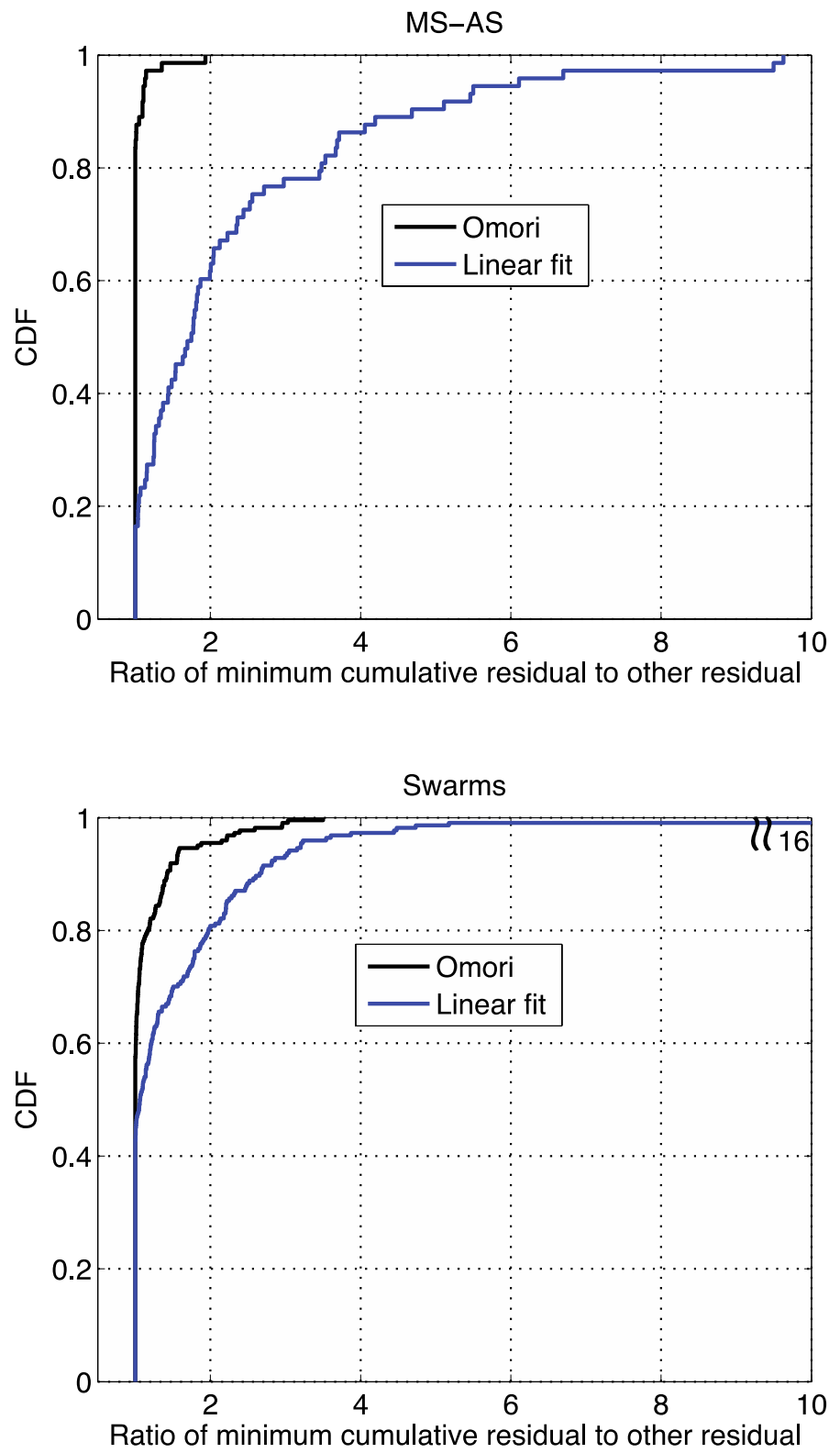

Figure 4.24 Residual Ratios with sequence type.

For sequences where the Omori's law decay model gives a lower cumulative residual than a linear fit the Omori fit (black) will have a value of one. The residual ratio of the Linear fit (blue) is a measure of how much better the Omori model is than the linear model. 
A. Test 1

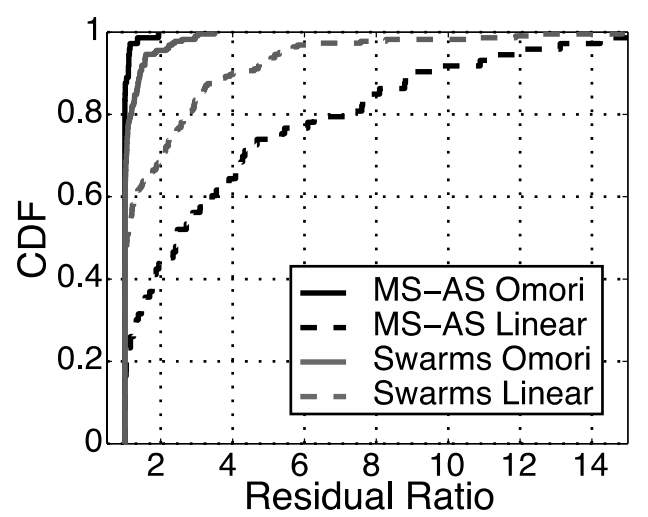

C. Test 3

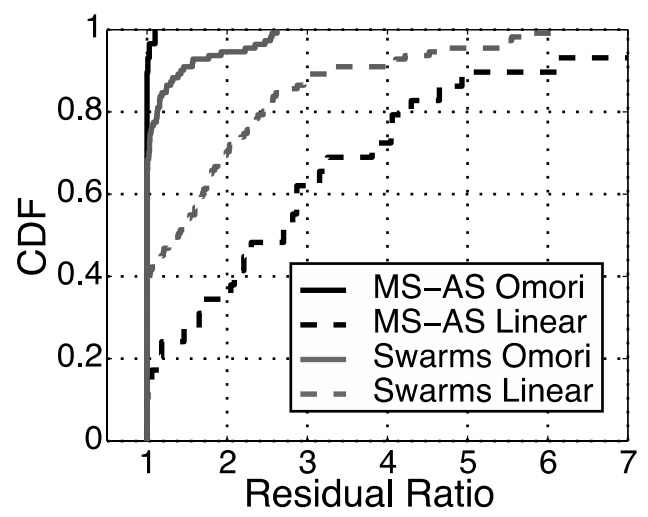

E. Test 5

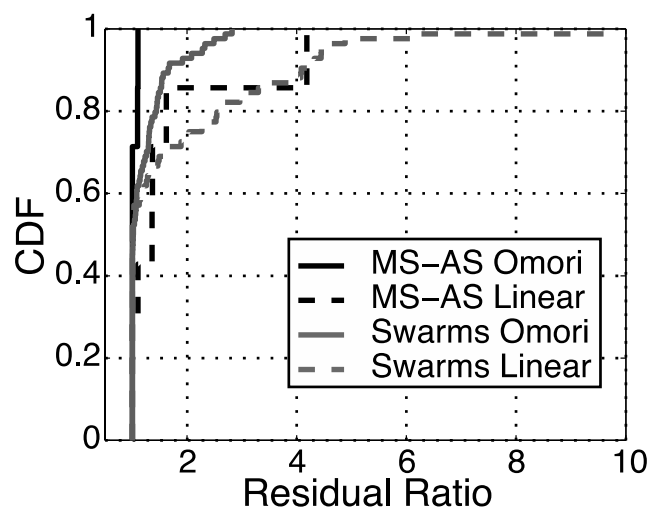

B. Test 2

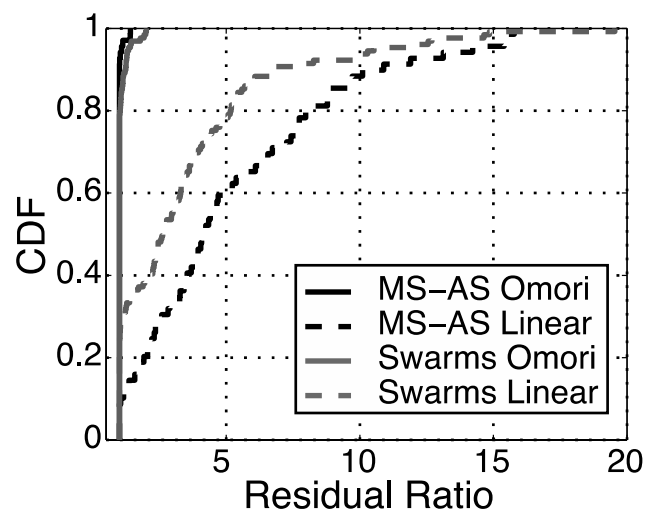

D. Test 4

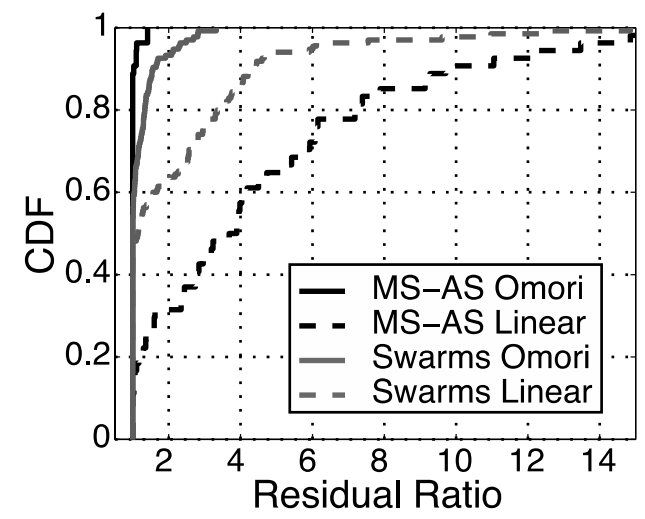

F. Test 6

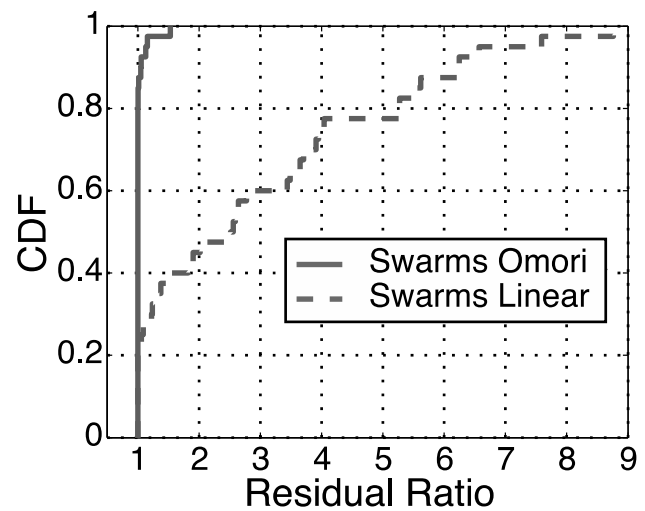

Figure 4.25 Residual Ratios with sequence type for each of the six tests.

For sequences where the Omori's law decay model gives a lower cumulative residual than a linear fit the Omori fit (solid) will have a value of one. The residual ratio of the Linear fit (dashed) is a measure of how much better the Omori model is than the linear model. MS-AS sequences are shown in black and swarms are shown in gray. 


\subsubsection{Unique tests and $c$-value check}

Next we selected a single test fit for each sequence. Because many sequences fit more than one of the tests we used two criteria to select the best test fit. First we looked for the test that explained the largest number of earthquakes in each sequence; we term this largest fit as the 'best fit'. Our preference is to be able to say what percent of each sequence can be explained as decay. If we were more interested in the most appropriate $c$ and $K$ values for each region, then we could have weighted our preference towards higher residual ratios. When we completed this step we saw that we only needed to use the first three tests. Sequences that passed tests 4 , 5 , and 6 were all successful in passing tests 1,2, or 3 and had a larger number of earthquakes in one of the earlier tests. Once we had established the best test for each sequence we then checked for unreasonable $c$-values ( $c=\operatorname{cmax}$, or $c>$ sequence duration). The linear-comparison seemed to have differentiated between most good and bad $c$-values (lower $c$-values for swarms than maximum misfit test), but we wanted to be sure the Omori's law decay models were sensible. There were 11 MS-AS and 57 swarms sequences that returned unreasonable $c$-values for the best test. We then looked to see whether any of these sequences passed another test that gave more reasonable cvalues. Seven of the eleven MS-AS sequences and ten of the swarm sequences had more reasonable values in another test. For the sequences without reasonable cvalues we changed their status to 'failed', and continued our testing. Table 4.10 shows the number of swarms in each of the three unique tests with good and bad cvalues and the mean percent of earthquakes that each test used. More swarm sequences are eliminated in this step and this increases the difference in the percent of sequences that pass between the sequence types to almost $34 \%$. 
MS-AS sequences that fail the Omori modeling have bad c-values (either $c=c \max$, or $c>$ duration) and the Omori fits are very close to the linear models (Figure 4.26). None of the sequences are well fit, and none look as though a different selection point within the sequence would give a better model. At least four of the six sequences $(\mathrm{B}, \mathrm{C}, \mathrm{D}, \mathrm{F})$ show an internal structure that suggests they may be able to be fit by multiple decay models. Figure 4.27 shows the six largest swarms that fail the Omori decay tests. The Omori models are more distinct from the linear models than in figure 4.26 despite still having c-values equal to cmax. The sequences themselves have a temporal pattern that does not show evidence of decay. The exception to this is $4.27 \mathrm{C}$ that looks as though it might have Omori decay late in the sequence. The fact that sequence $\mathrm{C}$ did not pass any tests indicates that the visual change in behavior does not occur at any of our tested breakpoints. This sequence is in the Hikurangi region and will be discussed further in Chapter 5.
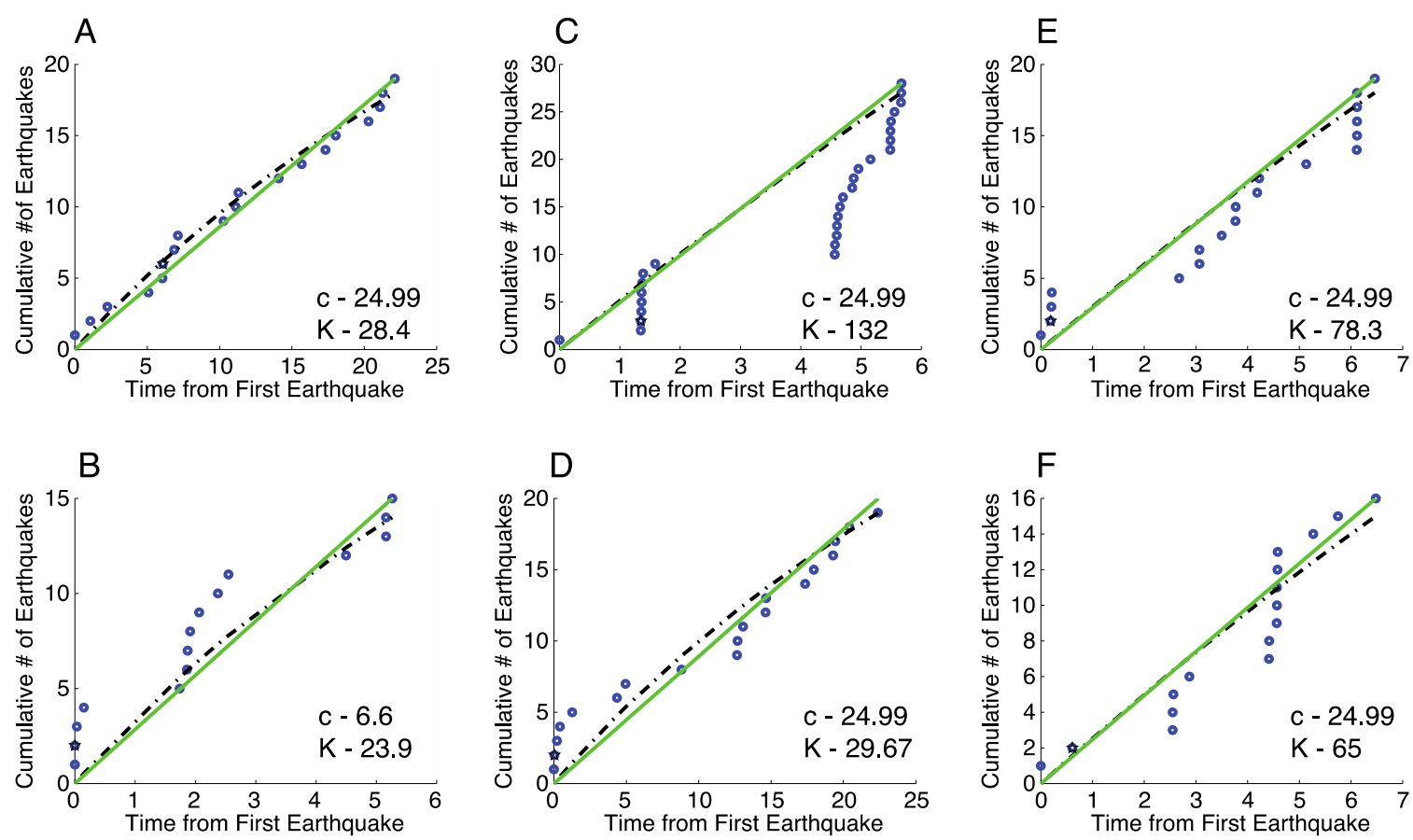

Figure 4.26 Six of eight MS-AS sequences that don't pass the linear-comparison for any of the six tests.

All of them have bad c-values that are either equal to cmax (A, C, D, E, F), or longer than the sequence duration (B). Data are shown in blue. Dashed black line shows the best-fitting Omori's law model and the green line shows a linear seismicity rate model. 

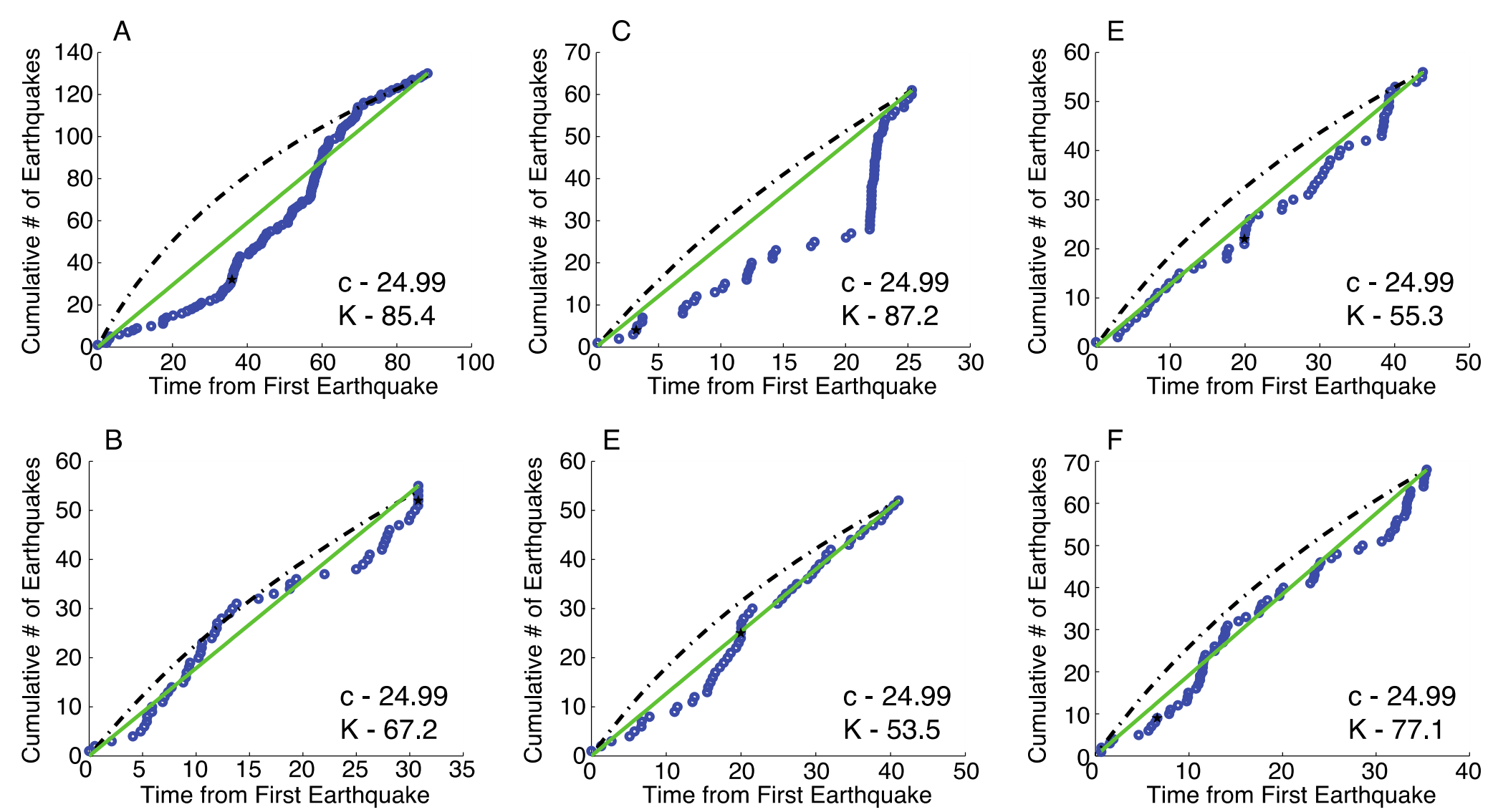

Figure 4.27 Six swarms $\geq 50$ earthquakes that fail the linear comparison for all six Omori's law tests.

All six sequence have a c-value equal to cmax. Data are shown in blue. Dashed black line shows the best-fitting Omori's law model and the green line shows a linear seismicity rate model. 
Table 4-10 Number of sequences that pass Omori's Decay for Three Different Tests. All indicates sequences that the cumulative misfit to Omri's law was less than the cumulative misfit to a linear fit. The 'good-c' columns are the number of sequences after unreasonable c-values are removed. The last two columns show the mean percentage of earthquakes used to get an Omori fit for each test.

\begin{tabular}{|c|c|c|c|c|c|c|c|c|}
\hline Test & $\frac{p^{S^{5}}}{s^{s^{s}}}$ & $5^{s^{5}} 5^{5^{5}}$ & $s_{1}^{s_{0}}$ & $5^{2} 5^{5}$ & 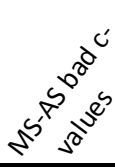 & 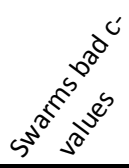 & 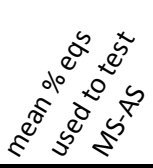 & 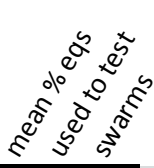 \\
\hline 1. Whole Sequence & 54 & 122 & 52 & 88 & 2 & 34 & 1 & 1 \\
\hline 2. From Mmax & 11 & 22 & 11 & 19 & 0 & 3 & $87.64 \%$ & $63.25 \%$ \\
\hline 3. Min abs iEtime & 4 & 27 & 2 & 17 & 2 & 10 & $83.05 \%$ & $85.57 \%$ \\
\hline total & 69 & 171 & 65 & 124 & 4 & 47 & & \\
\hline$\%$ passing & $94.52 \%$ & $76.34 \%$ & $89.04 \%$ & $55.36 \%$ & & & & \\
\hline $\begin{array}{l}\% \text { Difference } \\
\text { between Seq-types }\end{array}$ & $18.18 \%$ & & $33.68 \%$ & & & & & \\
\hline
\end{tabular}

\subsubsection{Sequence Parameters}

Figure 4.28 shows cumulative distributions of five basic parameters for sequence types passing and failing the Omori's law - linear comparison test. Swarm sequences that pass the Omori's law decay tests have slightly shorter durations (A), a larger number of earthquakes (B), slightly smaller area (C), and larger Mmax (D). The distributions of Mdiff are extremely similar and Table 4.11 shows that if anything the sequences that pass have a slightly smaller Mdiff than those that fail. However, the Mdiff values for pass and failing swarms are small and are similar. MS-AS sequences on the other hand show that the sequences that fail have smaller Mdiff values than the overall distribution. Mmax is also smaller for the failing MS-AS sequences. Because of the small number of MS-AS sequences that fail (8), we cannot say much about the distribution of passing versus failing, but we can say that all failing MS-AS sequences have short durations and a relatively small number of earthquakes (B).

We have also evaluated the sequences that pass and fail by region (Table 4.12). The Hikurangi and SCA regions are the only two with MS-AS sequences that 
fail. Both of these regions still have at least a $70 \%$ success rate for MS-AS sequences. All regions have swarm sequences that fail. The Alpine Fault region has the worst pass ratio with only one-third of swarms passing the linear-comparison test. The other four regions have at least a 50\% success rate for swarms. All regions have a higher pass rate for MS-AS than swarm sequences. The differences in success rate between the sequence type ranges from 20-40 percent. 

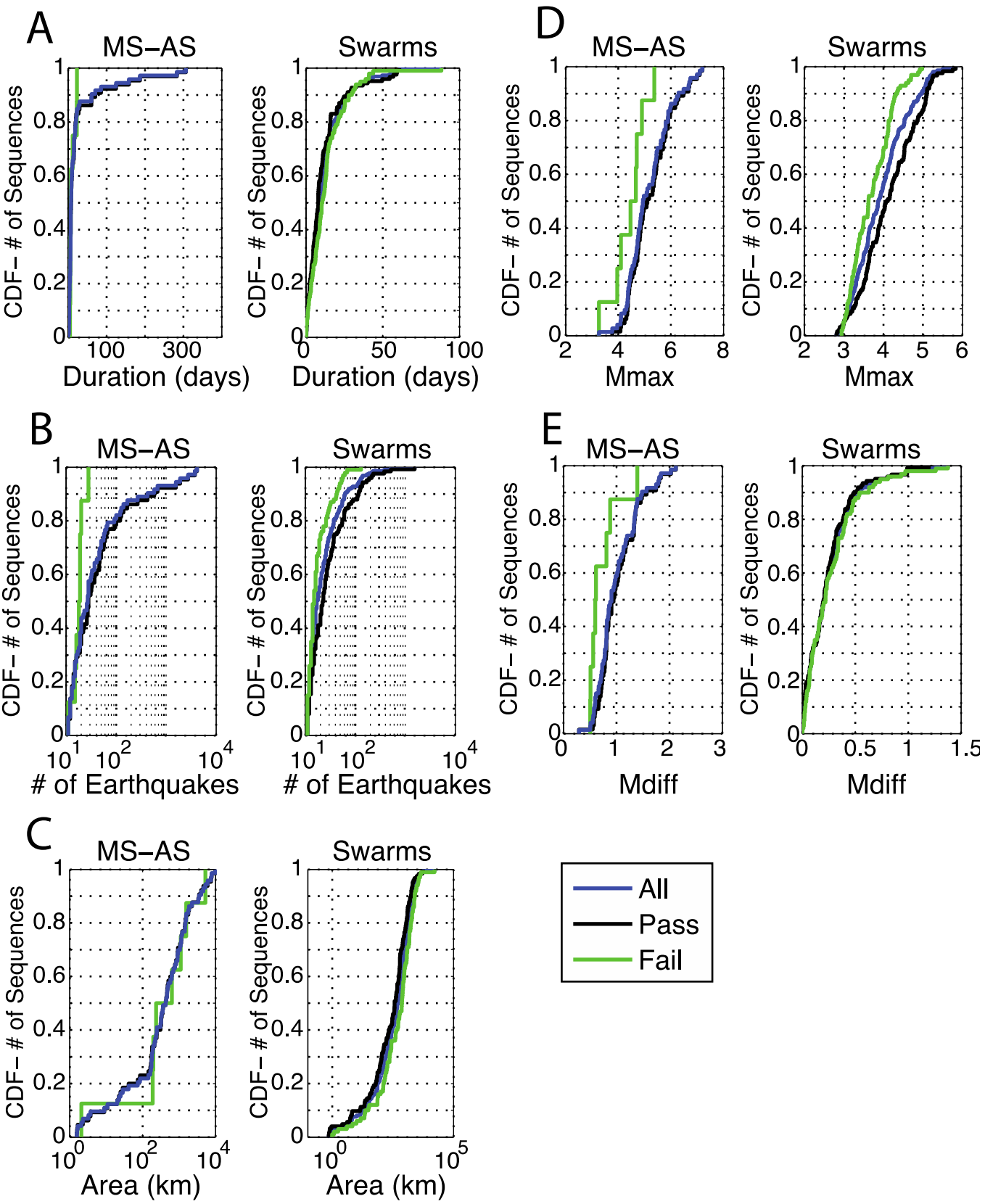

Figure 4.28 Cumulative distributions of five basic sequence parameters for sequences that pass and fail the linear comparison test by swarm type.

The total distribution for any sequence type is in blue, those that pass are shown in black, and the green line indicates sequences that fail the linear comparison test. A) Duration. B) Number of Earthquakes. C) Area. D) Mmax. E) Mdiff. 
Table 4-11 Parameter Variations for Sequences that Pass and Fail the Omori's Law Decay Tests.

\begin{tabular}{|c|c|c|c|c|c|c|c|c|}
\hline & $r^{e^{2}} r^{p^{s^{s}}}$ & $n^{e^{\gamma^{2}}} e^{\rho^{\delta^{s}}}$ & $r^{e^{d^{\gamma^{r}}}} e^{\gamma^{s^{5}}}$ & $r^{e^{j^{2}}} e^{\partial^{s^{5}}}$ & $r^{e^{2}} r^{x \theta^{\prime \prime}}$ & 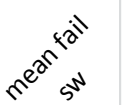 & $\varepsilon^{e^{j 0}} \varepsilon^{x}$ & $s_{e^{9}}^{a_{0}}$ \\
\hline Dur & 28.00 & 11.63 & 7.39 & 7.93 & 10.55 & 13.36 & 6.47 & 10.47 \\
\hline Number & 279.23 & 56.60 & 28.00 & 22.50 & 18.25 & 21.05 & 18.50 & 15.00 \\
\hline Mmax & 5.25 & 4.14 & 5.11 & 4.09 & 4.43 & 3.70 & 4.57 & 3.62 \\
\hline Mdiff & 1.04 & 0.24 & 0.95 & 0.20 & 0.73 & 0.27 & 0.61 & 0.22 \\
\hline Area & 1177.93 & 669.81 & 425.01 & 368.51 & 1150.83 & 1144.98 & 434.44 & 737.62 \\
\hline
\end{tabular}

Table 4-12 Number of Sequences that Pass and Fail the Linear Comparison Test by Region.

\begin{tabular}{|c|c|c|c|c|c|c|}
\hline & $s^{5}$ & $N^{s} p^{s} \partial^{s}$ & $N^{5}{ }^{5}$ & $x^{50^{5^{5}}}$ & $s^{s^{5}} \rho^{\delta^{5}}$ & 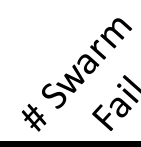 \\
\hline CVR & 3 & 3 & 0 & 34 & 22 & 12 \\
\hline Hikurangi & 11 & 8 & 3 & 63 & 32 & 31 \\
\hline Alpine Fault (AFD) & 11 & 11 & 0 & 27 & 9 & 18 \\
\hline Fiordland (AFD) & 12 & 12 & 0 & 34 & 21 & 13 \\
\hline SCA (AFD) & 36 & 31 & 5 & 66 & 40 & 26 \\
\hline
\end{tabular}

The distributions of basic parameters by region largely follow the same trends as observed in the overall distribution for sequence types. The eight failing sequences belong to only the Hikurangi and SCA regions so, while we make observations about the difference between the passing and failing MS-AS sequences, the conclusions are preliminary and a larger dataset is needed to confirm these findings. The most obvious trends are that for both sequence types the number of earthquakes in sequences that exhibit Omori's law decay is greater than or equal to the number of earthquakes observed in failing sequences. Similarly the Mmax values of passing sequences are greater than those that fail for both sequence types. Best fit MS-AS sequences have larger Midff values than those that fail. Smaller Mdiff values in MSAS sequences that fail the Omori's law fit tests could indicate that these sequences are 
not true aftershock sequences. The small Mdiff values of failing MS-AS sequences may also be due to large aftershocks with their own substantial aftershock sequences that are not well fit by a single Omori's law decay. For swarms on the other hand, only the CVR and Hikurangi regions have larger Mdiff values for passing sequences. In the aftershock dominated regions the Mdiff values of passing swarms sequences are less than those of sequences that fail. The Mdiff values are small for both passing and failing swarms and the difference is smaller $(<0.2)$ than the difference between passing and failing MS-AS sequences ( $>0.2)$. Note this is the difference between Mdiff values of passing and failing sequences of all types and it should not be affected directly by our requirement that MS-AS sequences have an Mdiff $\geq 0.5$.

\subsubsection{Discussion}

Using a maximum likelihood estimate of Omori's law $c$-values, a $p$-value $=1$, in conjunction with a comparison to an average rate (linear-fit) we have been able to observe decay in both MS-AS and swarm type sequences. MS-AS sequences have more observed decay with both a higher percentage of sequences in which the whole sequence passes $(71 \%)$, for any of the tests $(89 \%)$ and a larger percentage of the total earthquakes in those sequences used in the Omori models. The fact that not all of our MS-AS sequences fit Omori's law may indicate that our loose Mdiff criteria has arbitrarily included some swarm sequences that happen to have larger Mdiff values and early occurrence of Mmax. Swarms show less decay with only 39\% of whole sequences passing, and $55 \%$ of sequences passing any of the tests. All sequences that pass the linear-comparison test have larger number of earthquakes, and larger Mmax values than the respective failing sequences for that type. Mdiff may be a distinguishing parameter between sequences that pass and fail for MS-AS, but is not an important factor for swarms. This modeling has given us an indication of how 
accurate forecast models based on Omori's law will be in swarm areas. The parameter differences between sequences that are and are not well fit by Omori's law will also be important for future forecasting and hazard analysis efforts in swarm regions.

\subsection{Rate Patterns in Individual Sequences}

\subsubsection{Introduction to Rate types}

\subsubsection{Motivation}

In terms of hazard analysis and earthquake forecasting, the primary advantage mainshock-aftershock (MS-AS) sequences have over swarms is that they have a predictable rate that is governed by Omori's law. On the other hand, no universal patterns have been found to describe the temporal development of swarms. Omori's law does not result in spatial information, but it can be paired with the GutenbergRichter formula to estimate the number of earthquakes expected at different magnitudes with time. This information is an important aspect of hazard analysis following large earthquakes. While earthquake swarms in general have smaller maximum magnitudes (Chapter 4.2), they tend to have more earthquakes in the sequence that are at that maximum level, and swarms with maximum magnitudes $\mathrm{M} \geq$ 4 have occurred in New Zealand previously and up to $M \geq 5$ and 6 globally (e.g. [Dewey et al., 2007; Toda et al., 2002]). The majority ( 70\%) of observed sequences are swarms (Chapter 4.2) so it is extremely important to make progress on classifying these sequences and identifying potential physical mechanisms. Sherburn [Sherburn, 1992b] observed that there were a variety of patterns occurring in a handful of swarm sequences that occurred between 1982-1987. He described the number of events with time in terms of a mixture of foreshock, mainshock behavior, or as lacking a specific 
rate structure [Sherburn, 1992b]. This is a tantalizing suggestion that rate patterns do exist in swarm sequences. Any information that could help analyze the probability that an earthquake swarm will continue will be very useful when large swarms occur in the future. Likewise the constraint of a rate pattern suggests a consistent physical mechanism driving such a pattern. A constraint on the physical mechanism would have an even greater impact on hazard analysis and on determining whether volcanic and magmatic processes are at work in a given sequence. The use of the CURATE algorithm to systematically and objectively identify swarm sequences gives us a new opportunity to thoroughly investigate whether we can observe patterns in seismicity rate, besides Omori's law, that may lead to better understanding of swarm sequences.

\subsubsection{Inter-Event times and Basic Patterns}

To carry out our investigations of rate development we decided to use the time between each earthquake (inter-event times). We find it preferable to talk about interevent times rather than rate as times are the actual observed data. Inter-event times are often turned into rates, but rate information can be misleading because there can be rates of hundreds of earthquakes per day during sequences that contain less than 100 events. Furthermore rate information varies depending on the timescale that different studies report (e.g. events/day, or events/hour). Therefore we have chosen to analyze the inter-event times in their more natural state. Previous studies (e.g. [Hainzl and Fischer, 2002a; Hurst et al., 2008]) have also investigated inter-event times within sequences, and have focused on their distribution over entire sequences. In general, we have found that that there are not sufficient numbers of earthquakes to perform such analyses on individual sequences due to the relatively high Mc (2.5) in the CVR. Most of the observed swarms have less than 100 earthquakes. As an alternative to the distribution of inter-event times, we chose to plot the average inter- 
event time as it evolved with time through each sequence. This analysis has revealed what we think represent several distinct patterns in the average-inter event time plots (Fig 4.29). We will call these rate-patterns and rate-types instead of sequence-types to distinguish them from the terms used earlier to differentiate between MS-AS and swarm sequences. All of the plots presented here have been normalized by their duration so that patterns are more obvious. The first rate-pattern is associated with constantly increasing inter-event times, or a decreasing rate (Fig. 4.29 A). We term this rate-type as decay and will analyze its relationship to Omori's law later. The three remaining types all have elements of acceleration (decreasing inter-event times) that cannot be explained by aftershock decay. The second rate-pattern (Fig. 4.29 B) we term, $L$-shaped for its distinct acceleration followed by decay. The third pattern is dominated by acceleration (Fig. 4.29 C), and thus we simply term this pattern accelerating. The final steady rate-pattern often exhibits an initial decay over a few events and then have the bulk of earthquakes happen at a constant rate and end without the observation of much decay (Fig. 4.29 D). The steady rate-pattern was borne out of the observation that two large sequences in the Matata area in the CVR had rather similar rate patterns despite different spatial development and clustering (see 4.4.4.1 for further discussion of these sequences). The emergence of several apparently distinct rate patterns is surprising given that there are no widely accepted rate patterns for swarms. Individual sequences vary from these archetypes, and the study presented here investigates the robustness of the patterns and whether they do represent distinct patterns. 

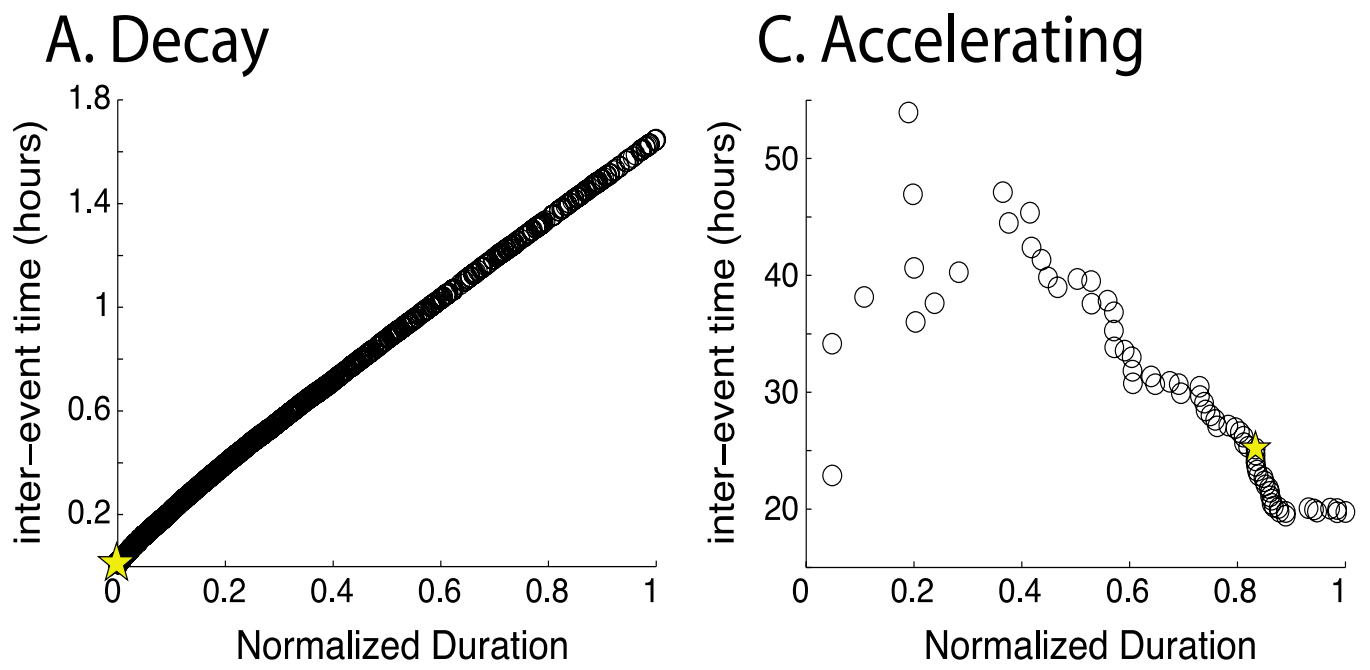

\section{B. L-shaped}
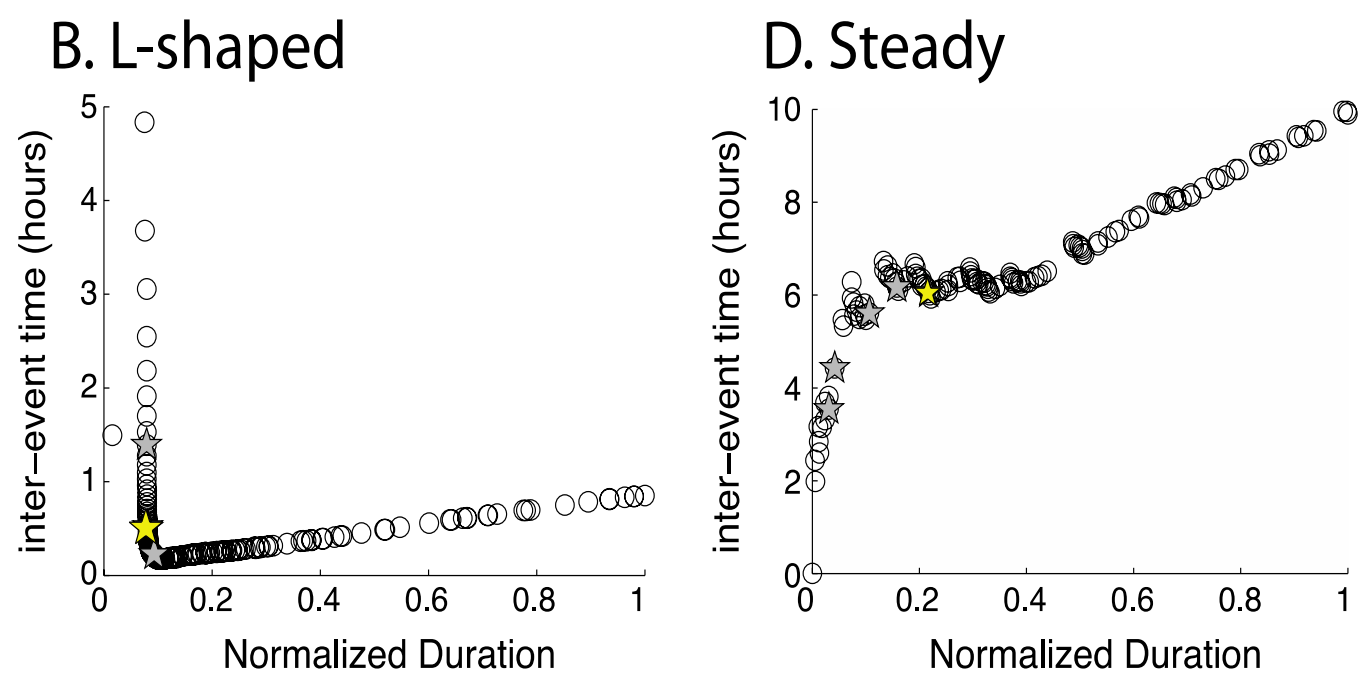

Figure 4.29 Archetypes of four different rate-patterns found in average inter-event time plots.

Note that a decrease in inter-event times corresponds to an increase in rate. The ratetypes refer to the activity during the bulk of the events. A) Decay, B) L-shaped (acceleration followed by decay), C) Accelerating, D) Steady. See text for further explanation of types. Yellow stars represent Mmax and gray stars show all earthquakes within 0.5 magnitude units of Mmax. 


\subsubsection{Visual Classification}

To categorize each sequence as a member of a specific rate-pattern, we plotted the rate-patterns and then visually assessed which rate-pattern best fit each sequence. Without a quantitative way to define these patterns we limited our investigations to sequences with at least 20 earthquakes to reduce the possibility of mis-identification. This criterion left us with 145 sequences (45 MS-AS, 100 swarms). The rate-type categorization also included a fifth category for sequences that did not clearly fit into any of the four rate-patterns described above (Fig. 4.29). The accelerating sequences were probably assessed most liberally and if most events in the sequences showed acceleration we assigned them to this category.

We also wanted to use these initial classifications to identify possible quantitative ways of determining the rate-types, but variations, even within the most robust looking rate-type examples (Fig. 4.30), have prevented us from completing such an analysis. These variations make objective analysis difficult. In the final section of the discussion we briefly discuss the attempts made at objective classification and possible ways forward.

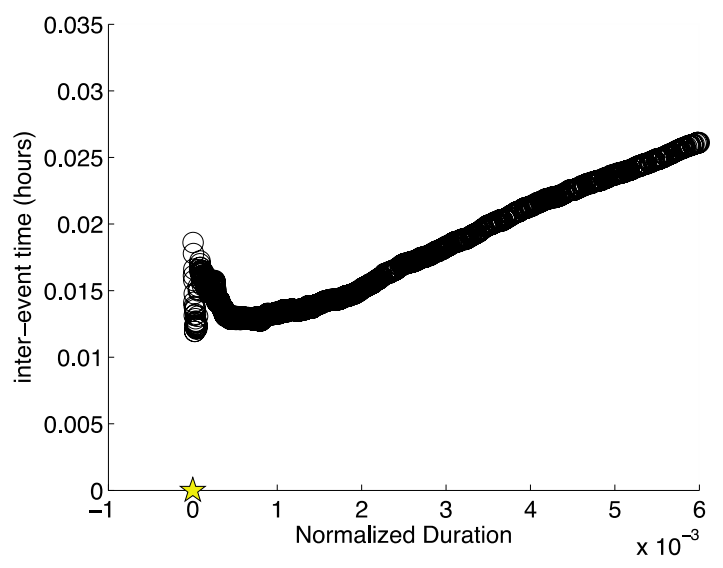

Figure 4.30 Detailed view of the first part of the average interevent time plot shown in Figure 4.29 A.

Shortly after the mainshock (yellow star) there is a complex rate variability which may indicate nondetection or a genuine rate development. 


\subsubsection{Results}

\subsubsection{Distribution of Rate Types}

Our visual classification of sequences rates resulted in a spread across all ratetypes with at least $10 \%$ of the total sequences in each type. Figure 4.31 -A shows a histogram of rate-types by sequence type. Note that MS-AS sequences, as expected, largely occupy the decay category, and also have approximately another one-third of sequences in the L-shaped category, while very few MS-AS fall into the accelerating, steady, or other. The five geographic regions discussed in chapters 3 and 4 contain a mix of all five rate-types $(4.31 \mathrm{~B})$. All regions, except the Alpine Fault region, have the highest percent of decay (1) sequences. The highest percent for the Alpine Fault region is $L$-shaped (2) rate-pattern. This is discussed further in section 4.4.4.2. Hikurangi has a higher percentage of accelerating sequences than any other region. Steady sequences are most common in the CVR and Fiordland. All regions have $25 \%$ or fewer sequences that fall into the other category. 

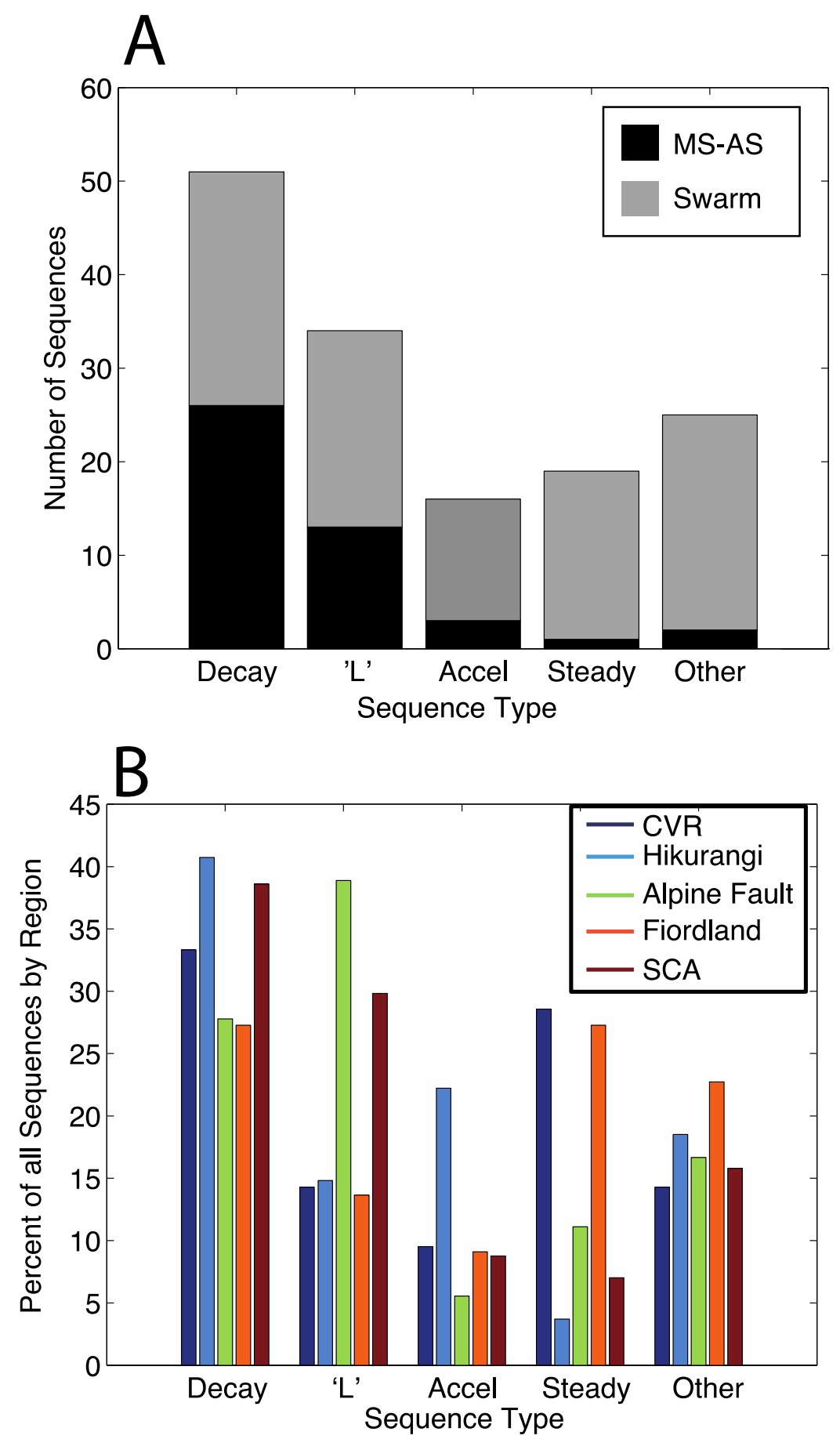

Figure 4.31 Histograms of the number of sequences that belong to each rate-type category.

A) By sequence type. B) By geographic region. All sequences contain at least 20 earthquakes. 


\subsubsection{Spatial Distribution}

Because there are only 145 sequences in total, many of the sequences occur in an isolated spatial location. For areas that do have multiple sequences, a variety of rate-patterns are observed (Fig. 4.32). This is compatible with previous observations throughout New Zealand that a variety of sequence types often occur in overlapping regions (e.g. [Bryan et al., 1999; Eiby, 1966; Evison, 1977; Sherburn, 1992b]). As seen in the distribution, all rate-types are seen in each area. Figure 4.33 shows further examples of rate-type sequences from different regions. 
A.

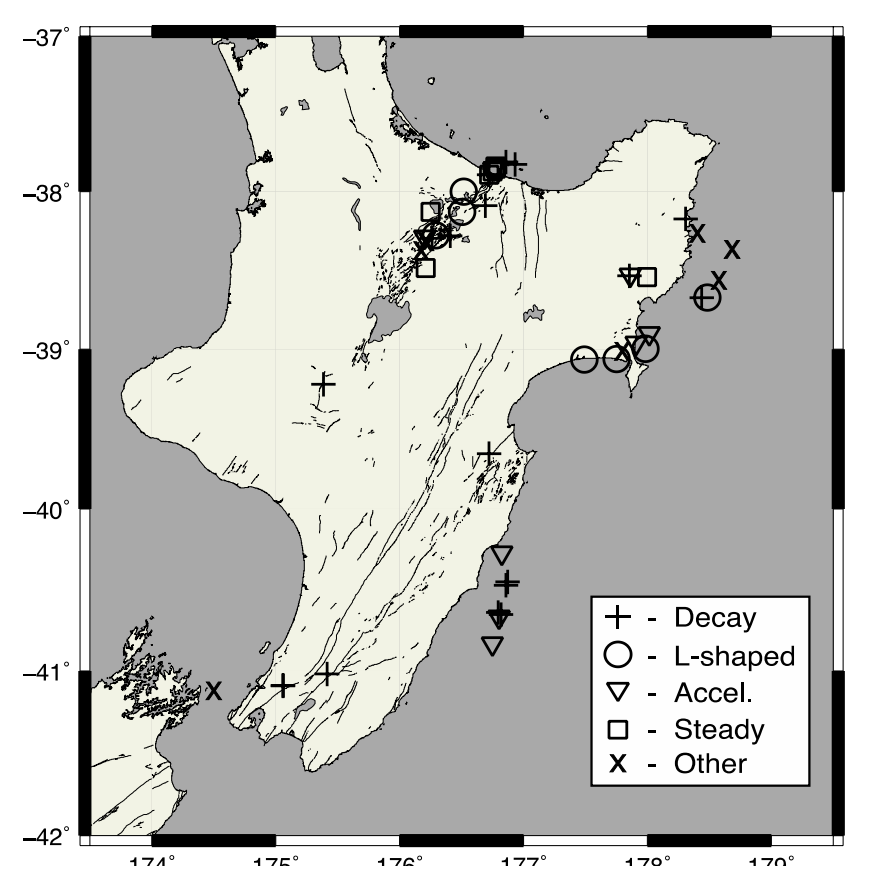

B.

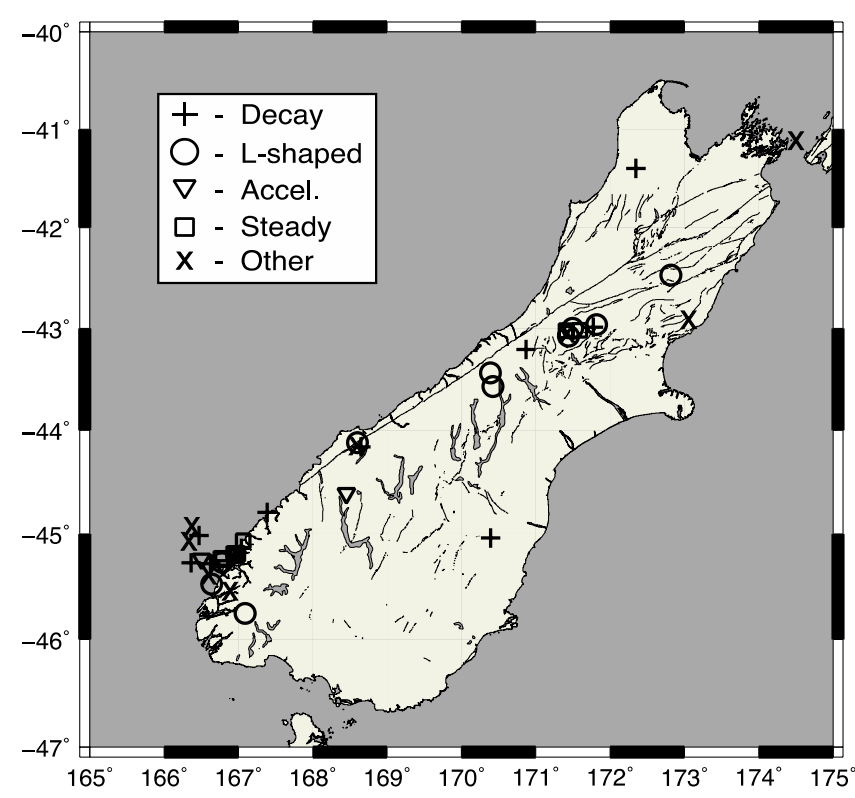

Figure 4.32 Spatial locations of rate-types for sequences of 20 or more earthquakes.

A) North Island. B) South Island. Shapes indicate rate-type: (+)-Decay, (O) - L-shaped, (triangle) - Accelerating, ( $\square)-$ Steady, (X) - other. 


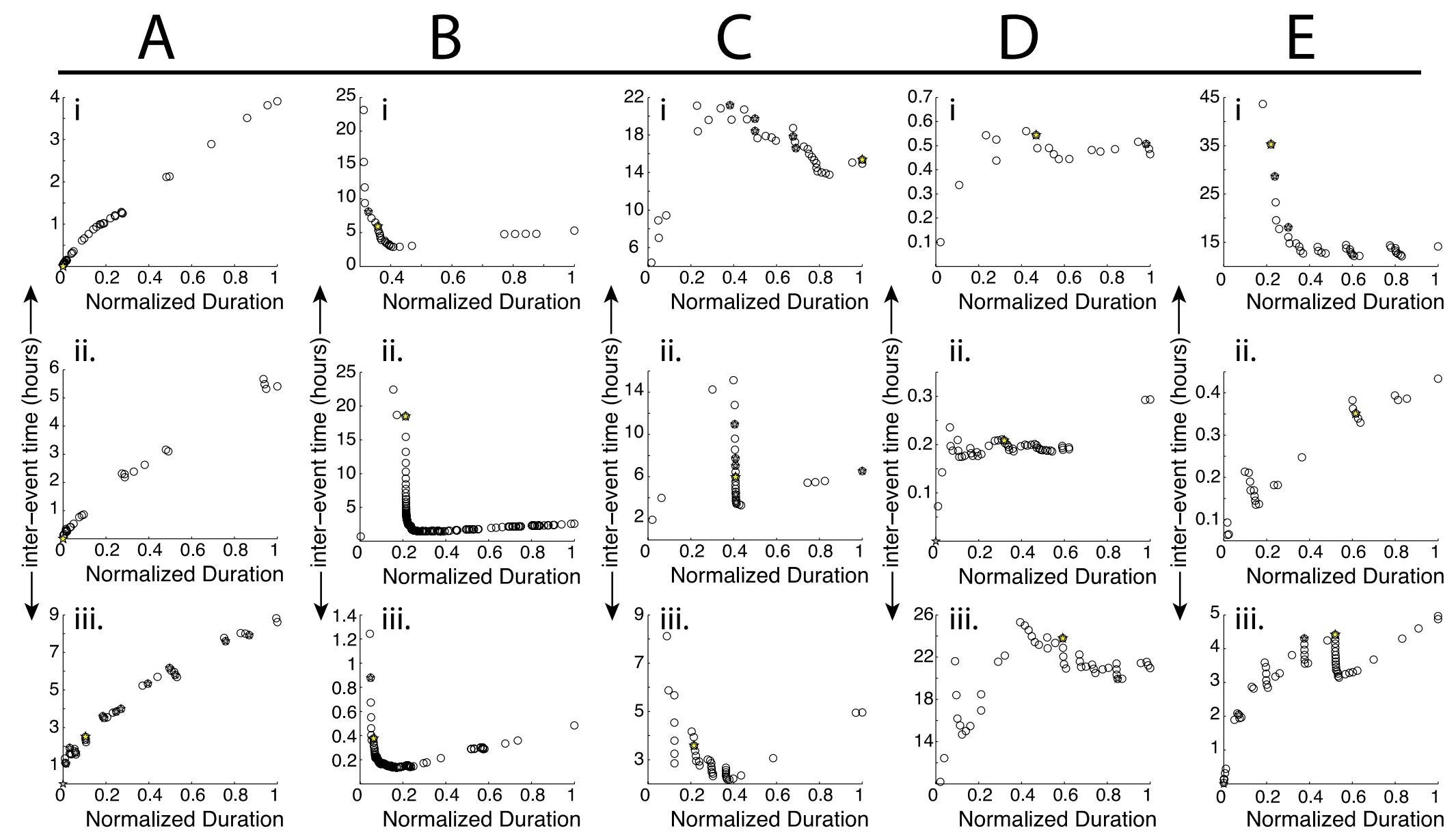

Figure 4.33 Examples of the five rate-type categories of average inter-event times.

A) Decay, B) L-shaped, C) Accelerating, D) Steady, E) Other. Each rate-type has three examples taken from a different region (i-iii). 


\subsubsection{Parameters of rate-types}

To test whether rate-types contained sequences with distinct characteristics, we plotted the distributions of Duration, Mmax, the number of earthquakes in a sequence, and the maximum inter-event time (Fig. 4.34). For the most part the different rate-types have similar distributions. The outstanding rate type in duration (4.34 A) is the steady sequences, which have longer durations in general, though not the longest durations. The steady sequences also have smaller Mmax values than the other sequence types (4.34 B). Accelerating sequences also tend to have smaller Mmax values, although their maximum Mmax value is $>5.5$. The distribution of decay and $L$-shaped sequences are very similar for both duration and especially for Maximum magnitude. The potential crossover between these two rate-patterns is discussed in the following sections. The L-shaped and decay sequences are the largest sequences in terms of the number of events in each sequence (Fig 4.34C). The smallest number of earthquakes occurs in the other rate-type ( Fig 4.34C). The y-axis of the inter-event time plots has a lot of variation and so we also looked at the maximum average inter-event time (slowest average rate observed within a sequence). The decay type sequences tend to have faster average rates, and have the lowest maximum average inter-event times (Fig 4.34 D). Despite the overall large duration and maximum inter-event times, there are short sequences in the steady rate-pattern (Fig 4.34-A and D). The maximum inter-event time (Fig. $4.34 \mathrm{D})$ is the only parameter in which the decay type shows a significant difference from L-shaped ratetype. Corresponding to their longer durations, the steady sequences largely are skewed towards higher maximum inter-event times, though, like duration distributions, they do not have the largest maximum values. 

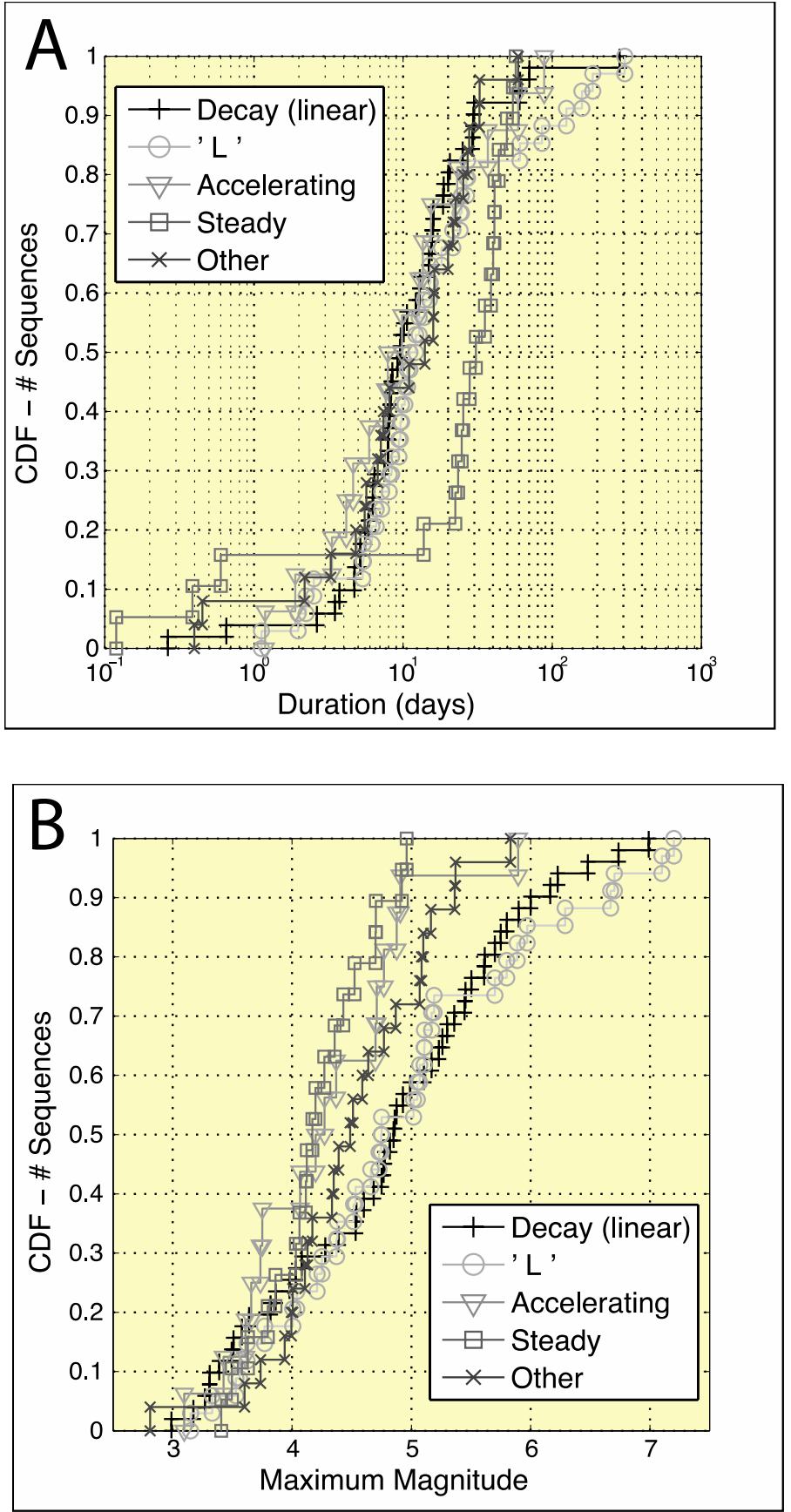

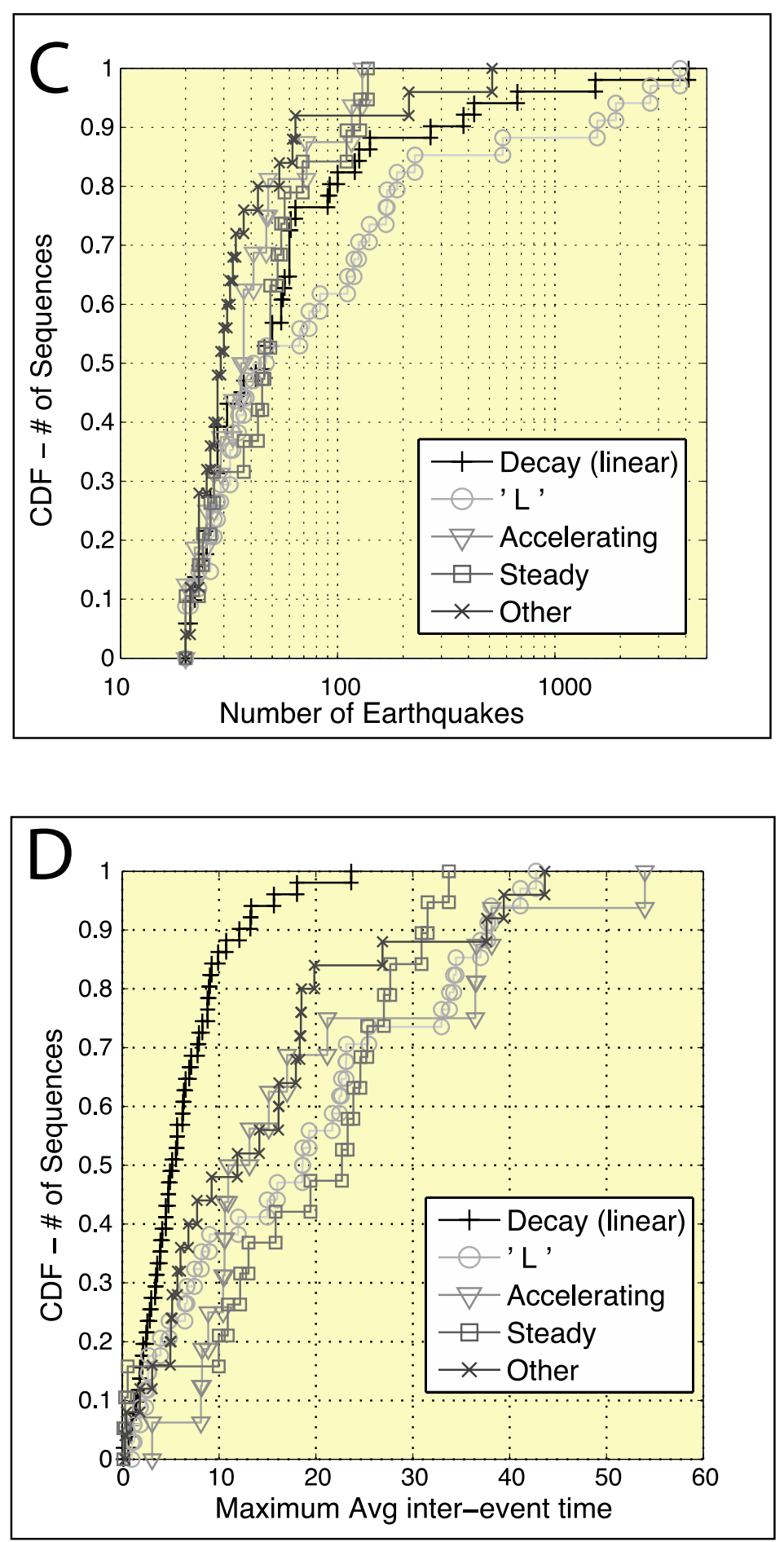

Figure 4.34 Cumulative Distribution Function (CDF) plots of rate-types with four parameters.

A) Duration, B) Mmax, C), Number of earthquakes in the sequence, D) Maximum average inter-event time. Symbols along each line match the rate-type symbols used in figure 4.32 . 


\subsubsection{Discussion}

4.4.5.1 Synthetic Omori's law sequence rates

The observation of a purely decaying rate-type that contains the majority of identified MS-AS sequences suggests that this is related to Omori's law and aftershock decay. To show this explicitly we created synthetic Omori's law temporal distributions and calculated the average inter-event times from these ideal synthetic sequences (Fig. 4.35). The range of c (.05-1.7) and $\mathrm{K}(7-17)$ values is chosen from the median values of the sequences that passed Omori's law tests in section 4.3 (Tbl. 4.6 and 4.8). As anticipated the synthetic Omori's law sequences have nearly linear inter-event time patterns. This is what we expected, but it also emphasizes an important point: a single Omori's law decay cannot explain any amount of acceleration. This is one reason why Omori's law fits are often given as cumulative number $[\mathrm{N}(\mathrm{t})]$ instead of instantaneous $[\mathrm{n}(\mathrm{t})]$, because the rate may temporarily increase due to secondary aftershocks or other effects. Secondly, MS-AS sequences are not all perfectly explained by Omori's law. Thus, the variations observed in other rate-patterns cannot be used as sole evidence to disregard their integrity as a pattern. 


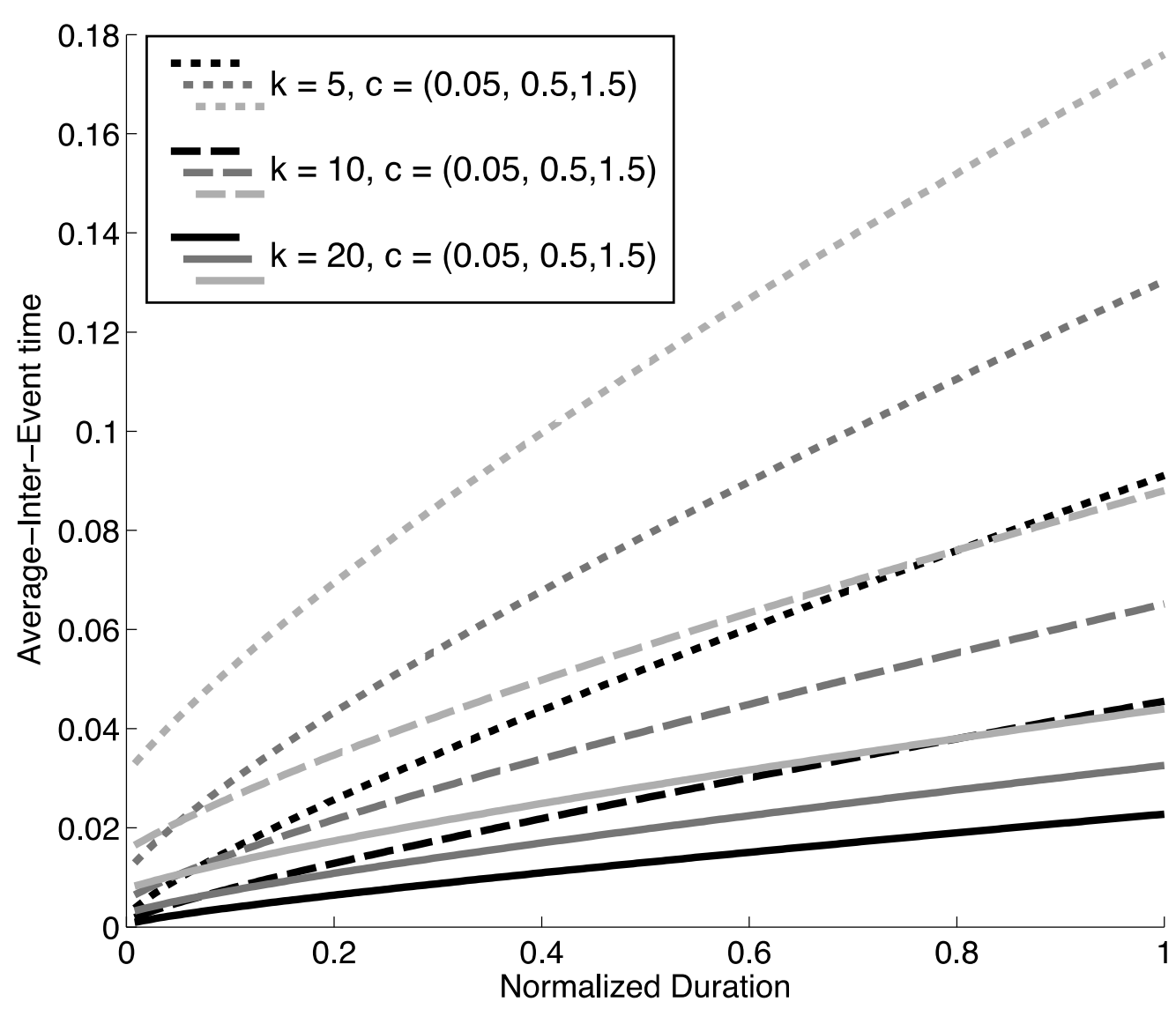

Figure 4.35 Synthetic average inter-event time for Omori's law decay. Nine sequences are shown with varying $\mathrm{k}$ and $\mathrm{c}$ values.

\subsubsection{Types of L-shaped}

Several observations necessitate investigating whether $L$-shaped sequences may be a special form of decay sequences. Overall the $L$-shaped sequences have similar parameter distributions to decay type sequences (4.34). The one difference is in maximum average inter-event time (4.34 D). A variation in Mc following a large mainshock may create an $L$-shaped pattern because rates would initially look lower and then accelerate as the completeness returned to a lower value. Foreshocks could also potentially create this pattern; a few sparse events preceding the mainshock would lead to a dramatic acceleration of rates following the mainshock. If foreshocks 
did cause this pattern we would anticipate observing Mmax towards the top of the $L$ pattern. We observe this phenomenon in several known MS-AS sequences in the Alpine Fault region. Both Arthur's Pass and Cass earthquakes show this behavior (Fig 4.36 B, 4.33 Bii). However, in many of the L-shaped patterns Mmax occurs towards the bend (decay initiation) in the pattern (4.29B, 4.33B). A large portion of swarms also exhibit this pattern, and the mainshock is not always seen at the start of the acceleration (4.36 A). The Rotoehu sequence was one of three sequences studies by Hurst et al. [Hurst et al., 2008] who described it as an intermediate sequence between a MS-AS and a swarm sequence. They propose that due to the similarity between the Rotoehu sequence and the Vogtland-Bohemia swarm that an injection of fluids may have initiated this sequence [Hurst et al., 2008]. Two further examples of L-shaped rate-pattern in other catalogs that are not controlled by aftershock processes are shown in sections 4.5 and 5.2. While we observe some MS-AS sequences that appear to have an $L$-shaped distribution as an artifact of foreshocks or sequence selection, there are also swarm-type sequences that appear to exhibit the $L$-shape as a real pattern. The $L$-shaped pattern is also consistent with observations by Sherburn [1992b], who noted that some foreshock-type swarm sequences had very extended foreshocks sequence. That kind of extended foreshock sequence is likely to be associated with an L-shaped rate. 


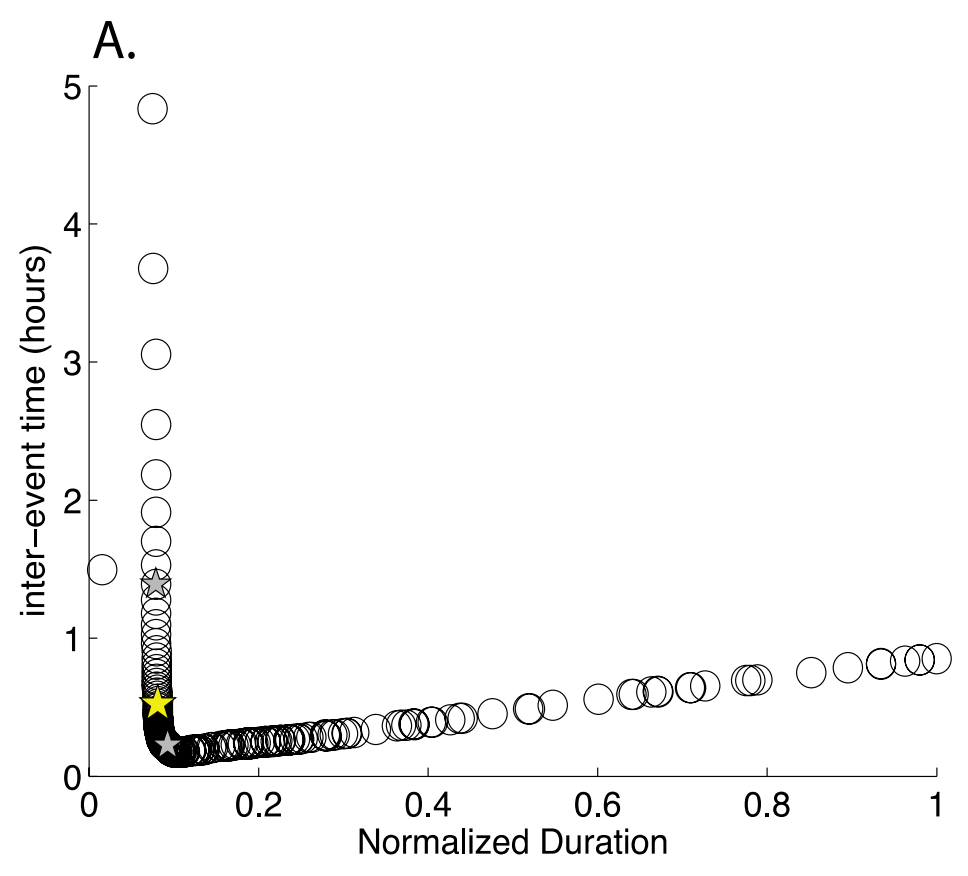

B.

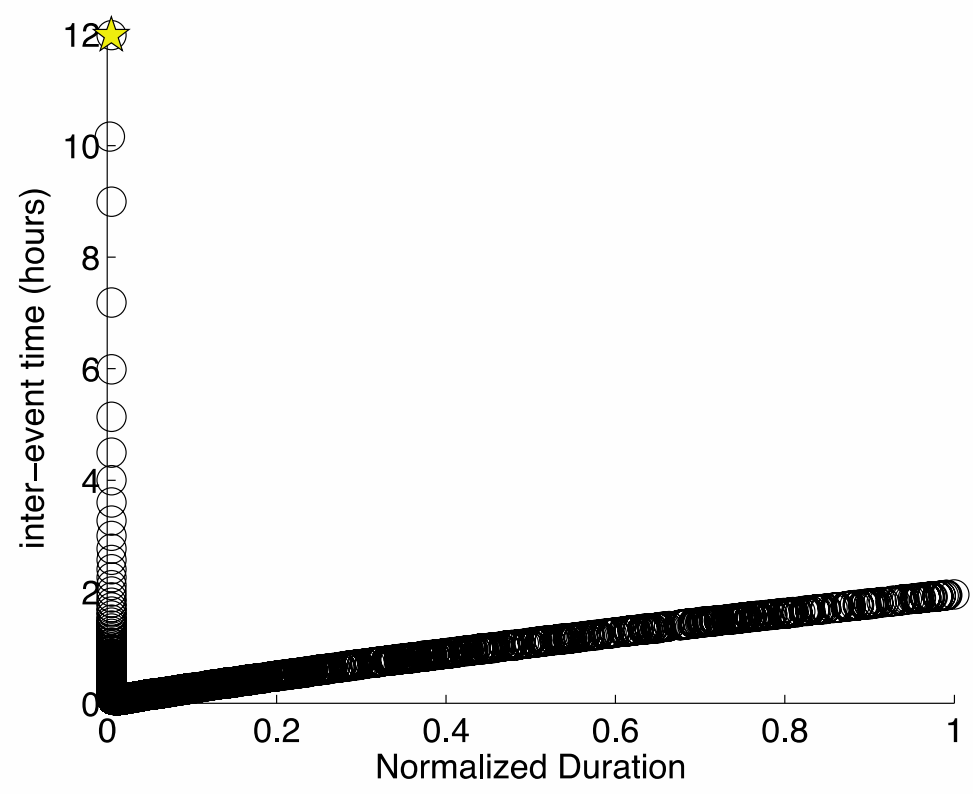

Figure 4.36 Average inter-event times for two different L-shaped sequences. A) Rotoehu sequence, $M_{L} \max =5.1$. Note this sequence is also shown in figure 4.29B. B) Arthur's Pass $\mathrm{Mw}=6.7$ earthquake 1994 . 


\subsubsection{Evidence for repeating processes}

In this section we show two examples that further support the idea that the observed rate-patterns may be evidence for repeating processes. Sequences that are both spatially and temporally proximal have a greater likelihood of sharing a common underlying process. Basic Parameters of all the swarms discussed in this section are given in Table 4.13 .

\subsection{Matata}

The first example is made from two sequences from the Matata area. Matata is an active area with both large foreshock-mainshock-aftershock and swarm sequences (see Ch. 1 for background information). Because the region experienced large foreshock sequences prior to the Edgecumbe $\mathrm{M}=6.7$ earthquake [Smith and Oppenheimer, 1989], swarms in the area are monitored closely. In 2005 and 2007 the Matata area had prolonged swarms of a few hundred events over several months (Fig. 4.37). The later sequence had more events and higher event rates (Tbl 4.13). The two sequences do not repeat in a spatial sense, but both contain similar strong clustering, without interpretable migration. The event rates for both these sequences are smaller than the event rates of the Edgecumbe foreshock sequence. The 2005 sequence was studied and classified as a swarm by Hurst et al. [2008]. Despite the differences between the 2005 and 2007 Matata sequences, the rate patterns of these sequences (which inspired the steady-pattern) are similar (Fig. 4.38). The 2007 sequences had more fluctuation at the beginning but settled down to a steady rate as the bulk of the earthquakes occurred. Hurst et al. [2008] described the rate as, "quasi-symmetrical development over time", which fits well with our observations for both 2005 and 2007. They also suggest that the steady nature of the seismicity rate could be due to a slow influx of fluids (as opposed to injection as postulated for Rotoehu) coupled with the ideas of Yamashita [1999] that pore spaces can be opened by fault movement, 
lowering the fluid pressure and hence lowering the rate of immediate aftershocks

[Hurst et al., 2008]. Therefore the steady rate-pattern may be associated with changes in porosity (leading to a change in fluid movement without sudden injection or introduction of fluids), or slow fluid fluxes.

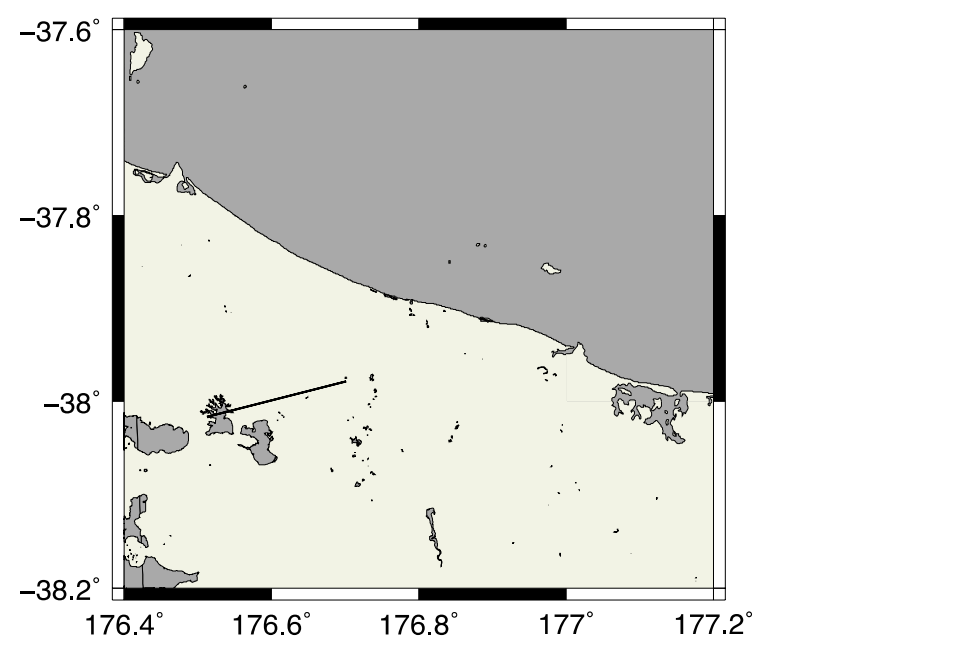

Figure 4.37 Map of Matata sequences colored with days through the sequences.

A) Largest single sequence of 2005 B) Largest single sequence of 2007. Black star shows the locations on the $1987 \mathrm{M}=6.3$

Edgecumbe earthquake.

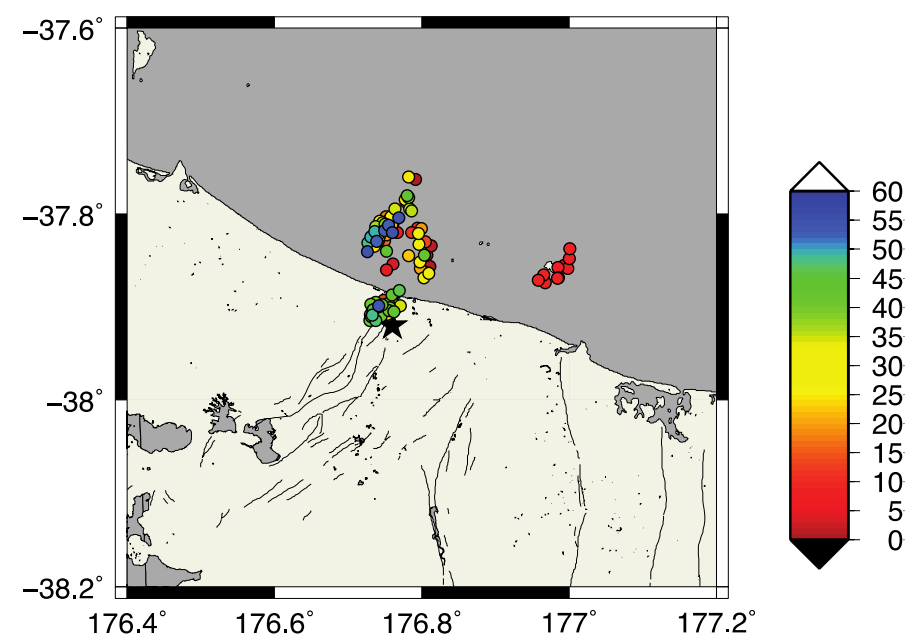



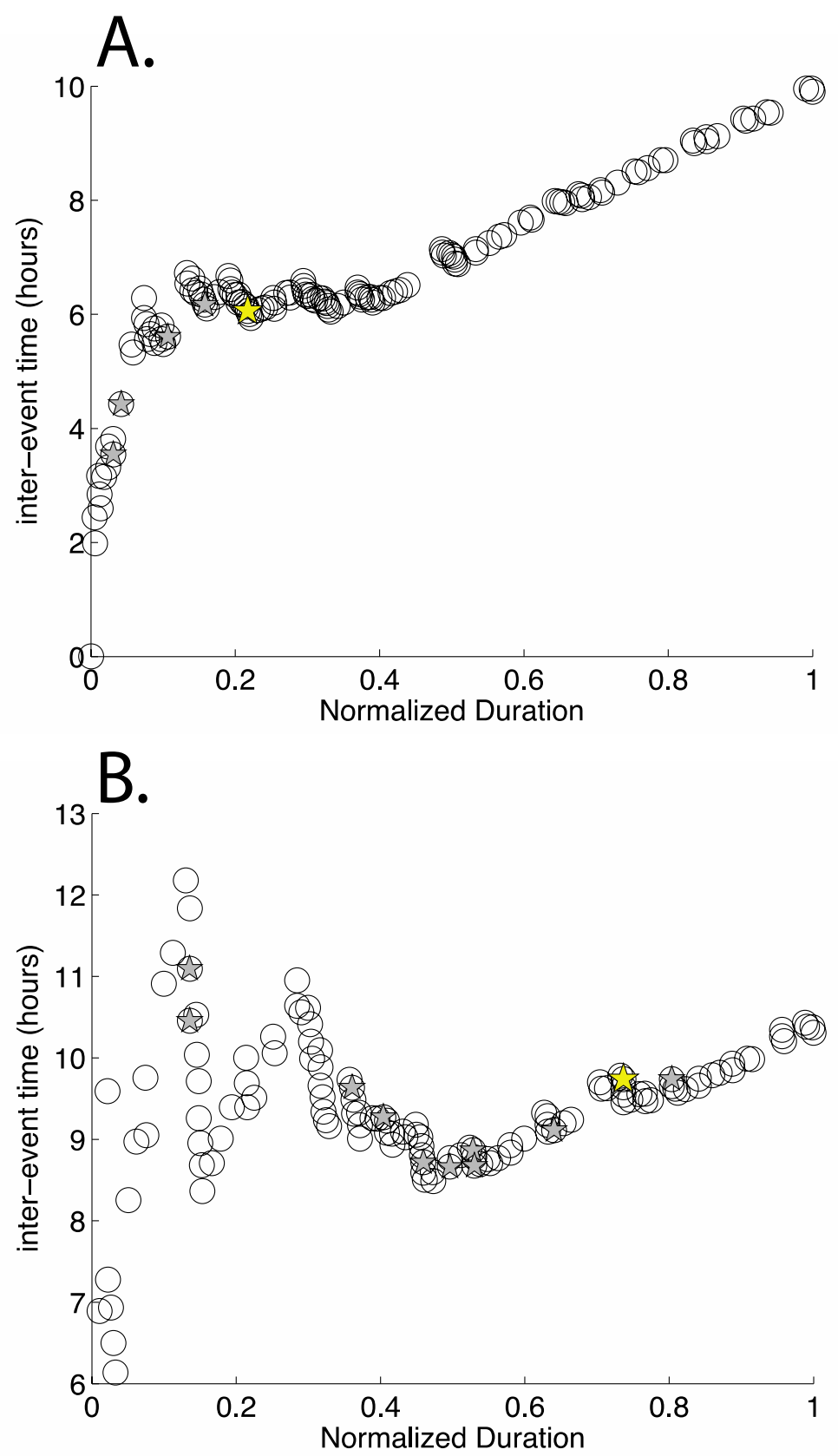

Figure 4.38 Average inter-event time plot for the two Matata sequences shown in Figure 4.37 A) 2005, B) 2007.

Note that the 2005 sequence (A) is also shown in figure 4.29D. 
Table 4-13 Sequence Parameters of discussed inter-event time examples.

All parameters are taken from the sequence definitions in the CURATE sequence catalog analyzed earlier in chapter 4.

\begin{tabular}{lccccc}
\hline Location & \# eqs & Start Date & $\begin{array}{c}\text { Position of } \\
\text { Mmax }\end{array}$ & Mmax & Duration \\
\hline Matata & 138 & April 1st 2005. & 49 & 3.86 & 56.95 \\
Matata & 127 & Feb. 16th 2007. & 99 & 4.03 & 54.55 \\
Haroharo & 111 & Dec. 17th 1997. & 9 & 4.01 & 2.24 \\
Haroharo & 380 & March 24th 1998. & 125 & 4.68 & 29.12 \\
Haroharo & 125 & April 1st 1998. & 33 & 4.75 & 13.89
\end{tabular}

\subsection{Haroharo}

The Haroharo sequence is another one of the three sequences analyzed by

Hurst et al. [2008]. In 1998, two large sequences occurred around the Okataina caldera. The two sequences occurred on opposite sides of Lake Tarawera (Fig. 4.39 E), and seismicity was not detected beneath the lake, despite the two sequences overlapping in time. The southern, larger sequences began March $23^{\text {rd }} 1998$ and the northern one, began April $1^{\text {st }} 1998$ during the decay portion of the southern sequence (Tbl. 4.13). Another southern sequence occurred prior to these two simultaneous swarms in December 1997 in the same region as the Southern sequence shown in figure 4.48-E. These all have an L-shaped rate pattern (4.40). Hurst et. al. [2008] interpreted the northern sequence in this pair, similarly to the Rotoehu sequence, as an intermediate sequence between MS-AS and swarm type. No extra stations were deployed during the occurrence of this swarm and so Hurst et al. [2008] do not draw many further conclusions about this sequence specifically. We calculated $\mathrm{Vp} / \mathrm{Vs}$ 
ratios using available GeoNet catalog picks (4.39 A-D). The Vp/Vs ratio is calculated by taking dividing the arrival time of the S-phase by the arrival time of the P-phase. We then used a 30 point moving average (that moves by one event each calculation) to look for changes with time. For more details about Vp/Vs ratios see section 4.5.3. Two stations (4.39, A-HARZ, C-TAZ) show brief significant increases during the swarms. Increase in $\mathrm{Vp} / \mathrm{Vs}$, if caused by a decrease in the S-velocity, may indicate the involvement of fluids $[\mathrm{Nur}, 1972]$. At the time of these swarms the network was almost exclusively 1-component vertical seismometers. The Vp/Vs information and the noted similarity between this swarm and the Rotoehu swarm, both point towards fluid involvement in these three Haroharo swarms. Unlike the steady rate-pattern, the $L$-shaped pattern is associated with faster seismicity rates (lower maximum average inter-event times in Fig 4.34D). If fluids do drive these sequences it may be due to an injection of fluid or more rapid process than implicated by steady rate patterns. 

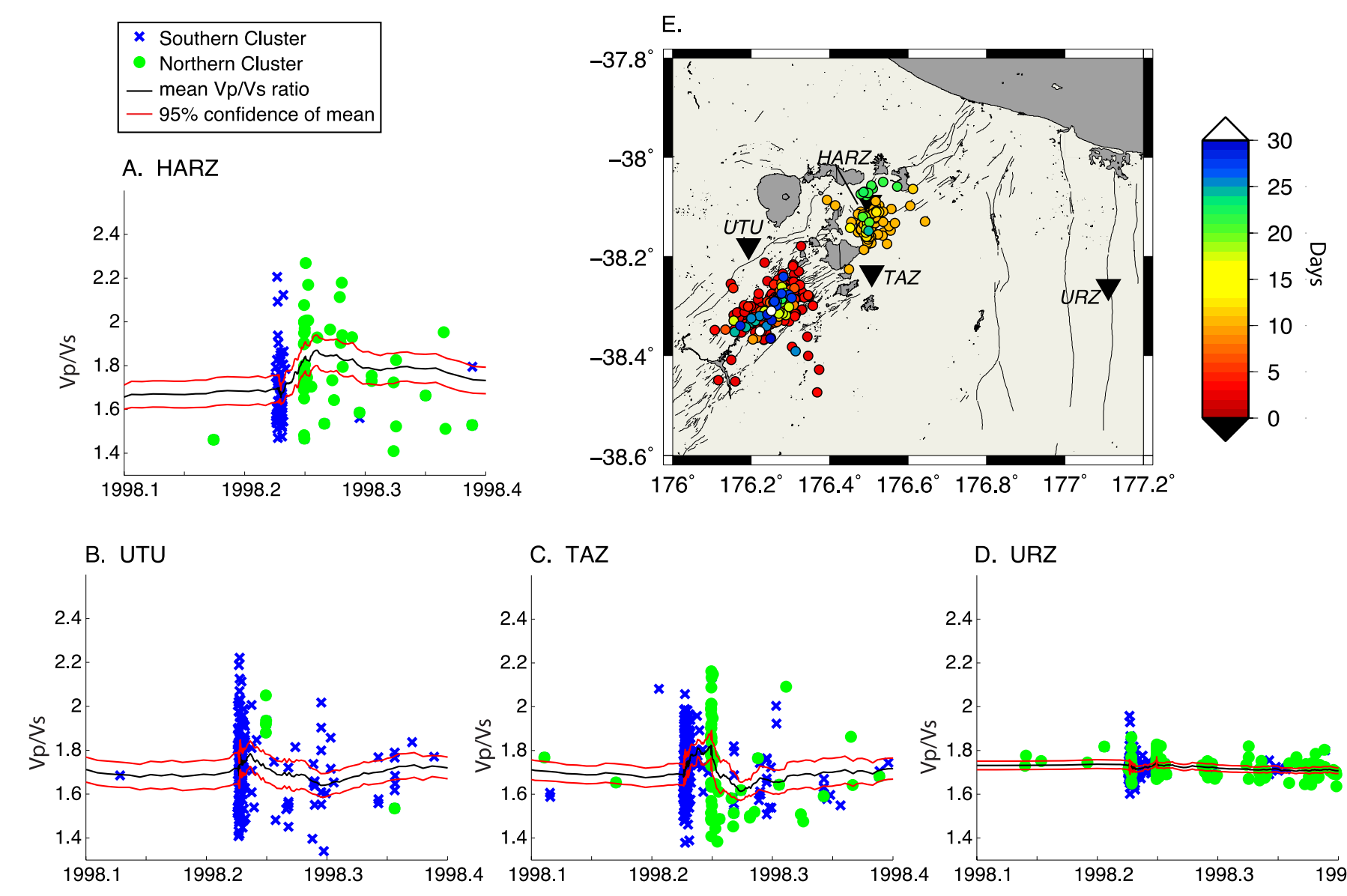

C. TAZ

D. URZ
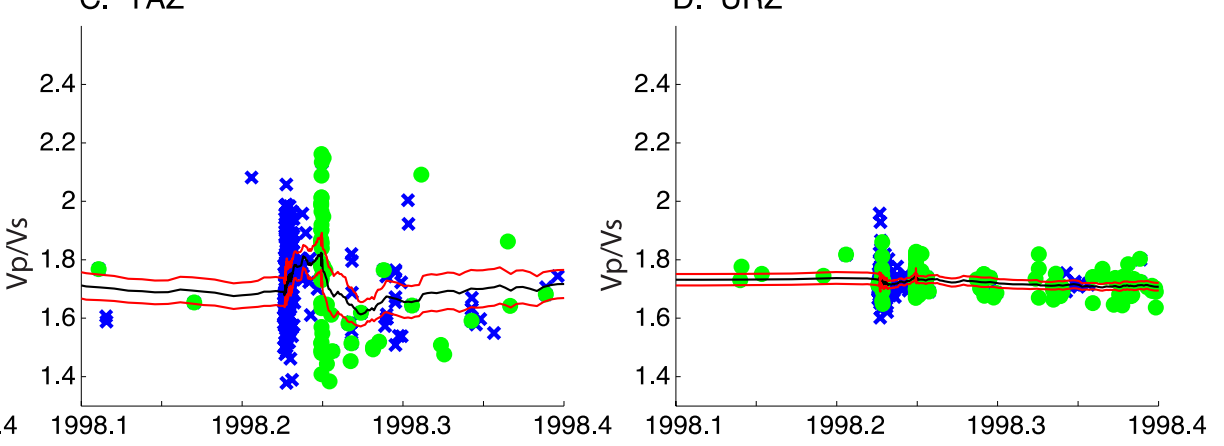

Figure 4.39 Map and Vp/Vs ratios for four stations during two earthquake sequences in the Okatina caldera.

Earthquakes on the map are colored by days since March $23^{\text {rd }} 1998$. Blue on Vp/Vs diagrams (A-D) represent earthquakes occurring in the southern cluster, and green events occur in the northern cluster. The black lines represent a 30 point moving average, with a $95 \%$ confidence bound shown as red lines. 

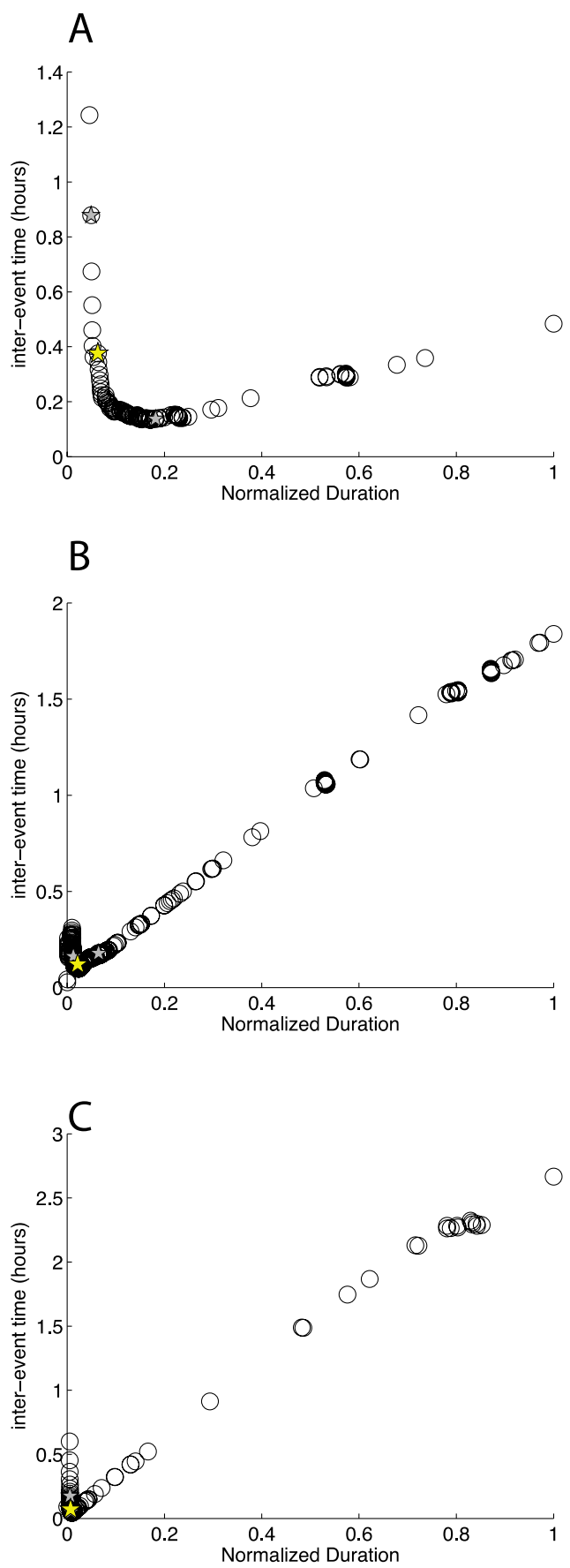

Figure 4.40 Average inter-event time plot for the three Haroharo sequences shown in Figure 4.39

A) Decmber $17^{\text {th }} 1997$ (southern) B) March $23^{\text {rd }} 1998$ (southern), and C) April $1^{\text {st }}$ 1998 (northern). 


\subsubsection{Comments on the 'other' rate type}

For all parameters the 'other' rate-pattern category has intermediate values. This may suggest that this category is made up of sequences that belong to another rate-type, but are difficult to identify, or are made of multiple rate-types in a single sequence (Fig. 4.33E). The threshold size of at least 20 earthquakes also may not be large enough to detect patterns consistently. The other rate-type does have the smallest sequence sizes in general (Fig. 4.34-C). Larger sequences may also have more clear patterns as individual events have less influence on the average through the course of the sequence.

\subsubsection{Conclusions}

Using sequence catalogs created by the CURATE method we have been able to analyze the rate patterns of a large number of sequences. We have identified four distinct rate-patterns using average inter-event times for 145 sequences $(\mathrm{N} \geq 20)$. Swarm sequences are evenly distributed among the four rate-patterns, and in the other category. The decay pattern is well modeled by Omori's law models and MS-AS sequences fall largely into this rate-type. In general, steady sequences have longer durations and, smaller values of Mmax. They are exemplified by two sequences in the Matata area in 2005 and 2007. The Matata swarms do not show consistent spatial development and may be caused by slow fluid flow or changes in porosity. The $L$ shaped rate-pattern is a mix of MS-AS sequences with foreshocks and swarms with a large amount of accelerating seismicity. Three similar large sequences in the Okataina caldera complex from 1997-1998 display this rate-pattern and observed increases in $\mathrm{Vp} / \mathrm{Vs}$ ratios suggest that fluids were involved in these sequences.

The identification of these patterns suggests that we may be able to incorporate swarm sequences directly into forecasting models in the future. The 
existence of multiple patterns gives further weight to the common proposition that there are a variety of physical processes that trigger and drive earthquake swarms. All regions contain some sequences in each of the four rate-patterns, as well as sequences that fall into the other category. We are confident that these are real patterns that will help advance our understanding of the mechanisms underlying swarm sequences.

\subsection{Sequence Example: Tokaanu Sequences 2009}

\subsubsection{Introduction}

This section describes our investigation of a series of sequences at the southern end of Lake Taupo during 2009. We discovered these events in the sequence catalog we created to look at earthquakes leading up to the eruption at Tongariro in August 2012. The two largest sequences in the pre-eruptive sequence catalog were near the south end of Lake Taupo in 2009 with start dates only about one week apart. We investigated this to see if this classification of activity was an artifact of the CURATE method or whether the division into two sequences was warranted. These sequences also stood out because one of them contained two $\mathrm{M}_{\mathrm{L}}=4.4$ earthquakes within a few hours. These two sequences turned out to be part of a series of four sequences during 2009. These sequences also allow us to add a more detailed perspective to our overall investigation of swarm sequences and provide an opportunity to investigate the relationship between shallow seismicity, geothermal, and possible volcanic activity.

\subsubsection{Background}

Lake Taupo sits within a large rhyolitic caldera and it is one of the most productive known rhyolitic systems [Wilson et al., 1995]. Seismicity and deformation were documented around Lake Taupo as early as 1922 [Eiby, 1966]. The first well documented swarm in the Lake Taupo area occurred over 59 days during 1964 and 
1965 and included at least 1127 earthquakes $\geq$ M 2.7 [Gibowicz, 1973b]. A series of swarms occurred at various locations around the lake during the 1980s [Otway and Sherburn, 1994; Webb et al., 1986]. The 1983 swarms are thought to be related to subsidence that was detected through lake leveling that began around the same time [Otway and Sherburn, 1994; Smith et al., 2007]. Subsidence continued, with exponential decay, through 1998, however other swarms were not tied to specific changes in the deformation signal [Otway and Sherburn, 1994]. The lake leveling changes have been modeled as dewatering of a magma body at $>8 \mathrm{~km}$ beneath Lake Taupo [Smith et al., 2007]. The historical record of earthquake swarms in this region gives us a good context in which to interpret the likelihood of magmatic involvement in the 2009 activity.

\subsubsection{Taupo 2009 activity}

To examine the two sequences in June 2009 more completely we looked for other sequences around the same time and found swarms to the north and south of this activity in April and May respectively. Throughout this section we will refer to the swarms in the order in which they occurred (Table 4.14 gives the parameters of each

swarm). Swarm 1 is the northernmost swarm, which occurred in April, swarm 2 is the southernmost swarm in May, swarm 3 is directly north of Lake Rotoaira and south of Tihia in mid-June, and swarm 4 is just north of swarm 3 in late June (Fig. 4.41). Spatially it looks as if swarms 3 and 4 could be continuous, and start dates a week apart also made us question whether this was a single sequence. On looking closer at the spatio-temporal occurrence we could see that despite the spatial proximity, swarm 4 starts in the north and then migrates back towards the area occupied by swarm 3. Swarm 4 contains two magnitude 4.4 earthquakes about four hours apart and swarm 3 has a maximum magnitude $<3.0$. The GeoNet catalogue locations have poor depth 
control and often depths are relegated to a boundary in the velocity model. In order to get improved depth control and further investigate this series of swarms we chose to relocate the earthquakes.

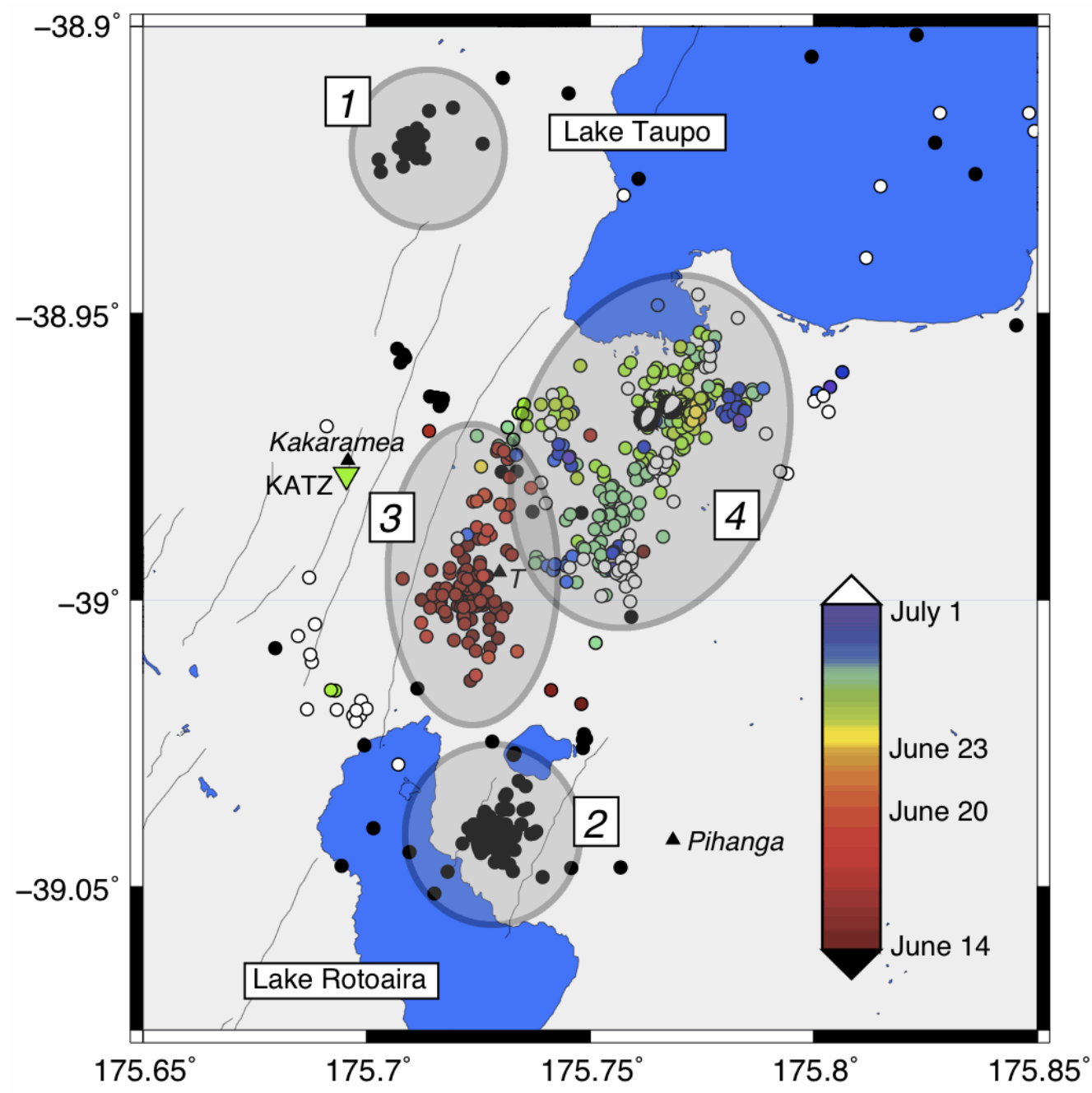

Figure 4.41 Map of earthquake epicenters from March $1^{\text {st }}$ through September $1^{\text {st }} 2009$. Swarms 3 and 4 in June and July are colored with time. Despite the overlapping spatial areas, there is not a clear spatial migration from one swarm to the next. Stations KATZ is shown as a green inverted triangle. Three small andesitic cones are shown with black triangles (Kakaramea Pihanga and Tihia, abbreviated with T). Transparent polygons highlight the four main swarms discussed in the text (numbered 1-4). The focal mechanisms for the two $M=4.4$ earthquakes (swarm 4) are shown. See table 4.5.1 for details of the four swarms. 


\subsubsection{Methods and Data}

\subsubsection{Sequence Analysis}

To look into earthquake activity preceding the Tongariro 2012 eruption at Te Maari craters, we analyzed the GeoNet catalog from 2008-2012. At the time of our analysis (conducted 13/8/2012) the GeoNet catalog had patchy completeness (not all locations finalized) and we have experienced large changes between reviewed and finalized catalogs. For this reason we only used earthquakes with a finalized status. This restriction eliminated all earthquakes in 2012, and so was not very useful for analyzing seismicity prior to the Te Maari eruption. Using finalized events within a $15 \mathrm{~km}$ radius of Tongariro, we first determined a magnitude of completeness $(\mathrm{Mc})$ for the dataset of 1.8. We used earthquakes with $M \geq 1.75$ to run through the CURATE method and create a sequence catalog for this dataset. The sequence catalog with a day-rule of 3 days and distance-rule of $15 \mathrm{~km}$ contained thirty-two sequences with at least 10 earthquakes. Swarms 3 (134 earthquakes) and 4 (278 earthquakes) in June 2009 were by far the largest two sequences in the catalog. The third largest sequence in the catalog contains 80 earthquakes and a Mmax value of 3.14. The only swarm with a comparable Mmax to sequence 4 is a sequence in September 2008 that also occurred within the swarm 4 area (Fig. 4.41) and it had only 34 earthquakes. Our interest was piqued by the fact that the largest two sequences were so close together in time and space, but we should note that using longer day rules swarms 3 and 4 are concatenated in some sequence catalogs. 
Table 4-14 Parameters of the Four Main Swarms in 2009.

Day-rule $=3$ days; distance-rule $=15 \mathrm{~km} ; \mathrm{Mc}=1.8$

\begin{tabular}{|c|c|c|c|c|c|c|}
\hline & $\begin{array}{l}\text { start } \\
\text { date }\end{array}$ & $\begin{array}{l}\text { CURATE } \\
\# \text { of eqs }\end{array}$ & $\begin{array}{l}\text { CURATE } \\
\text { duration } \\
\text { (days) }\end{array}$ & Mmax & $\begin{array}{c}\text { mean } \\
\text { latitude }\end{array}$ & $\begin{array}{c}\text { mean } \\
\text { longitude }\end{array}$ \\
\hline & April & & & & & \\
\hline Swarm 1 & $\begin{array}{l}20 . \\
\text { May }\end{array}$ & 22 & 2.67 & 2.90 & -38.92 & 175.72 \\
\hline Swarm 2 & $\begin{array}{l}30 . \\
\text { June }\end{array}$ & 67 & 2.67 & 3.83 & -39.04 & 175.70 \\
\hline Swarm 3 & $\begin{array}{l}14 . \\
\text { June }\end{array}$ & 134 & 6.26 & 2.91 & -39.00 & 175.72 \\
\hline Swarm 4 & 23. & 278 & 11.64 & 4.42 & -38.97 & 175.76 \\
\hline
\end{tabular}

\subsubsection{Data selection for phase picking and relocation}

To get more accurate phase arrivals for use in a relocation algorithm we identified a set of approximately 600 earthquakes $M \geq 1.8$ between April $1^{\text {st }}$ and September $1^{\text {st }} 2009$ and downloaded waveform data from GeoNet for 12 stations (Fig. 4.42). The time period was chosen to encompass the two sequences during June and July that we were most interested in, and to give us a long enough time period to test for potential changes in $\mathrm{Vp} / \mathrm{Vs}$ ratios with time. Not many catalogue S-picks are available so manual picking was required to compile comparable station plots for $\mathrm{Vp} / \mathrm{Vs}$ and to ensure that the stations had enough common events for comparisons in time. The twelve stations were chosen by their proximity to swarms 3 and 4 . Additionally station BKZ was added to try to improve the azimuthal coverage to the southeast. P and S phases were identified for all earthquakes where possible. 
The ratios of $\mathrm{P}$ - to $\mathrm{S}$-velocties $(\mathrm{Vp} / \mathrm{Vs})$ along the raypath are calculated by dividing the time from the origin to the $\mathrm{S}$-arrival by the time to the $\mathrm{P}$-arrival at each station [Nur, 1972]. A diagram that plots the arrival time of the P-phase against the arrival time of the S-phase is also compiled to calculate an approximate Vp/Vs ratio for the region (similar to a Wadati [1933] plot).

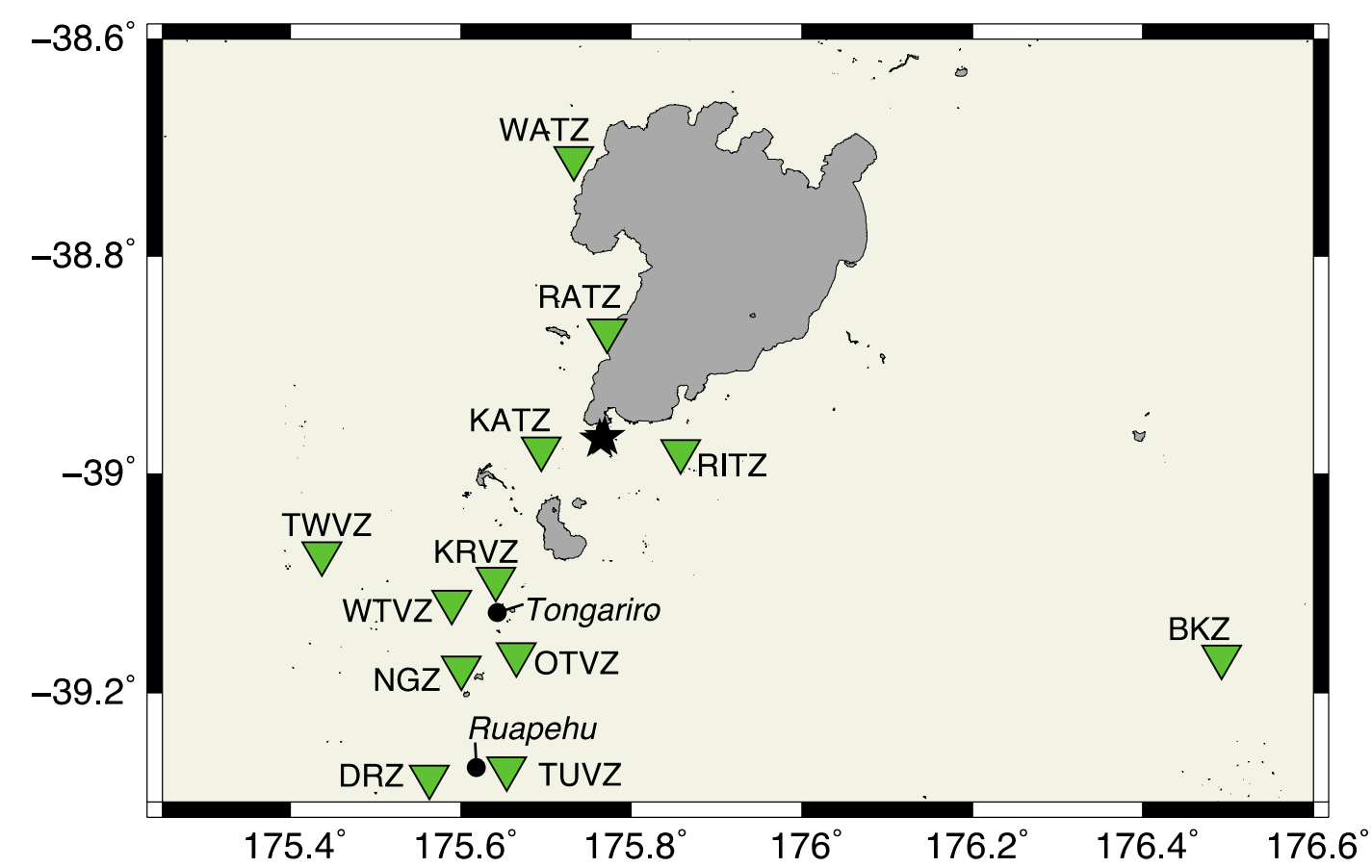

Figure 4.42 Location map of stations used to pick phase arrivals for relocation. Black stars show the location of two $\mathrm{M}=4.4$ earthquakes. 


\subsubsection{Parameter inputs for Double-Difference relocation}

Double-difference relocation has become a common technique to produce high accuracy relative locations. The technique utilizes the fact that for earthquakes that are relatively close in space compared to event-station distances and velocity heterogeneity scales, the arrival times differences can be assumed to come from the difference between their locations rather than from potential path differences [Waldhauser and Ellsworth, 2000]. The term double-difference comes from using the difference between observed and calculated arrival time differences between event pairs.

We chose to relocate the 2009 Taupo earthquakes with the hypoDD method of Waldhaser and Ellsworth [2000]. Because we thought fluids might be involved in the swarms, in addition to running hypoDD with both $\mathrm{P}$ and $\mathrm{S}$ phases, we also ran relocations with $\mathrm{P}$-phases only to avoid potential changes in the S-wave velocities with time. HypoDD uses two different methods to solve the least squared inversion. For larger datasets ( $>\sim 300$ earthquakes) hypoDD uses the LSQR algorithm of Paige and Saunders [1982], which utilizes a conjugate gradient algorithm to solve the damped least-squares problem. However, the LSQR method does not provide accurate error estimates of the relocated earthquakes [Waldhauser and Ellsworth, 2000]. Therefore the alternate, Single-Value-Decomposition (SVD), method must be used on a subset of the data to obtain error estimates for the relocated events.

For the velocity model input into hypoDD we used the 1-D velocity model determined by Sherbern et. al. [2003] for the TVZ (Tbl. 4.15). They used this 1-D model to further investigate 3-D Vp/Vs changes in the northern TVZ. Similarity of the tectonic settings suggests that this is a good model for the Tokaanu region. Sherburn et. al. [2003] report that the model is not well constrained below $15 \mathrm{~km}$, but 
this should not affect the current study as all initial depths of the earthquakes considered here are less than $14 \mathrm{~km}$ and more than $90 \%$ of initial depths are less than $10 \mathrm{~km}$.

Table 4-15 Velocity model used for relocation.

Taken from Sherburn et. al. [2003]

$\begin{array}{cc}\text { Top depth of layer } & \mathrm{Vp}(\mathrm{km} / \mathrm{s}) \\ 0 & 3.2 \\ 2 & 4.1 \\ 4 & 5 \\ 6 & 5.78 \\ 10 & 5.87 \\ 20 & 7.33 \\ 40 & 7.88 \\ 100 & 8.08\end{array}$

Our main interest in these swarms, in addition to their spatial locations, is their progression in time. To keep as much temporal information as possible we wanted to retain most of the events through the relocation process. Our input parameters are all designed to achieve this goal. Events can still be eliminated during relocation if they have bad picks (residual errors will be too high), or become too shallow (air-quakes). We wanted to know the location of all events relative to one another and so we did not perform any clustering prior to relocation. Appendix $\mathrm{C}$ contains a complete description of all input parameters and scripts for running hypoDD. 


\subsubsection{Results}

Once the phases had been picked for all the data we ran hypoDD and we were able to get better locations without much adjustment to the iteration parameters (Fig. 4.41, map of P-phase only relocations and 4.43, comparison of catalog locations with both P-only and $\mathrm{P}$ and $\mathrm{S}$ relocations). The relocated events also allow us to observe the depth relationship that was not apparent in the catalog locations (Fig. 4.43). One immediate observation is that the depth of the last swarm (4) is shallower than the three previous swarms.

To obtain accurate error estimates we ran a subset of earthquakes through hypoDD using the SVD analysis. The SVD method cannot handle more than a few hundred events (depending on the number of stations and phases used) and so we selected 200 of the earthquakes in swarm 4 , including the two $M=4.4$ earthquakes. This subset was rerun with similar weighting through the iterations, but only five iterations were performed in each set (see appendix for more details). The mean horizontal errors are 30 and $32 \mathrm{~m}$, and the mean vertical errors are $45.5 \mathrm{~m}$. These small errors allow us to interpret the depth differences with confidence.

Station DRZ was found to have the highest RMS residuals during the relocations. To test what effect this had on the absolute locations we repeated one run using only the other 11 stations. This had a relatively small impact on the overall centroid of events, moving it by about $400 \mathrm{~m}$ in both horizontal directions and only 100 meters in depth (Fig. 4.44). The change appears to be a shift to the northwest

(Fig 4.44), suggesting it is a change in the absolute rather than the relative locations. The small magnitude of the change indicates that the locations are robust. 
A.

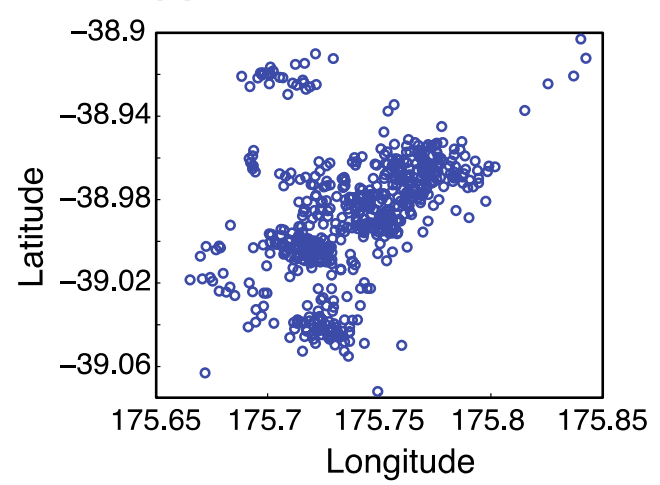

D.

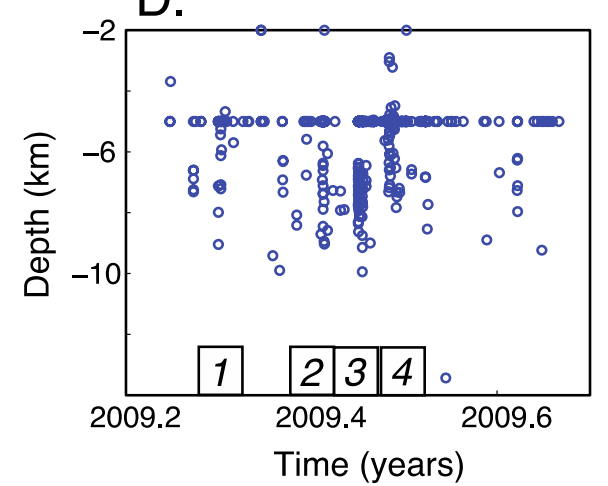

B.
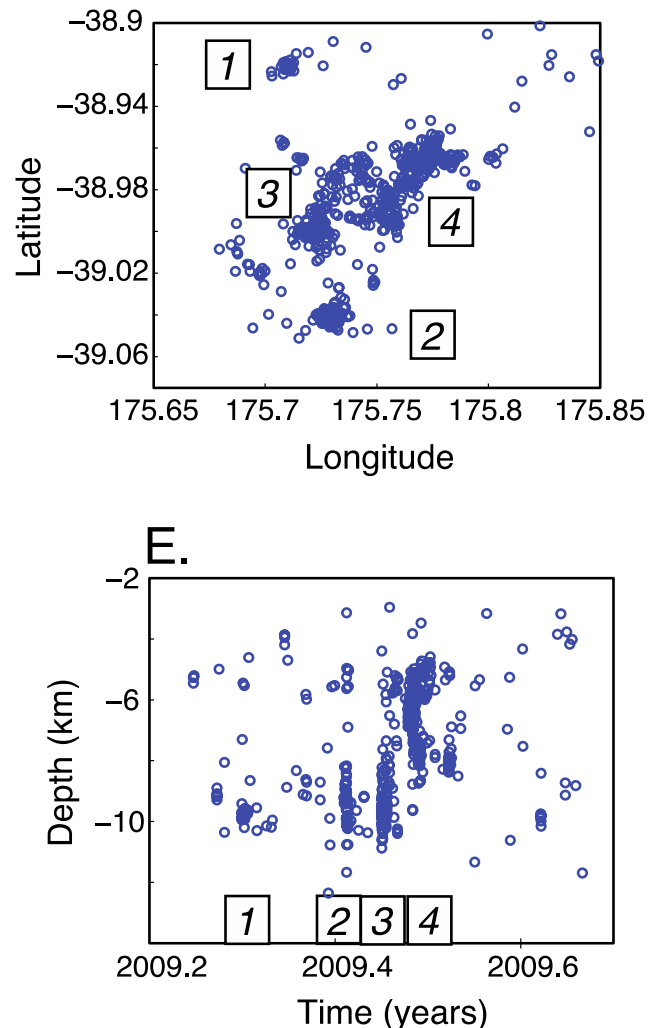

C.

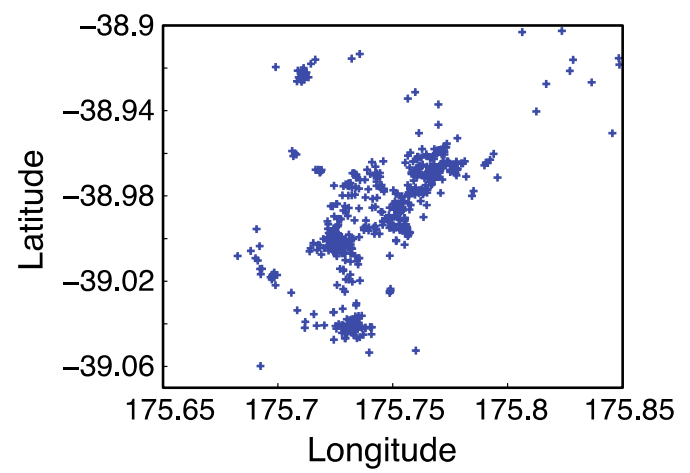

F.

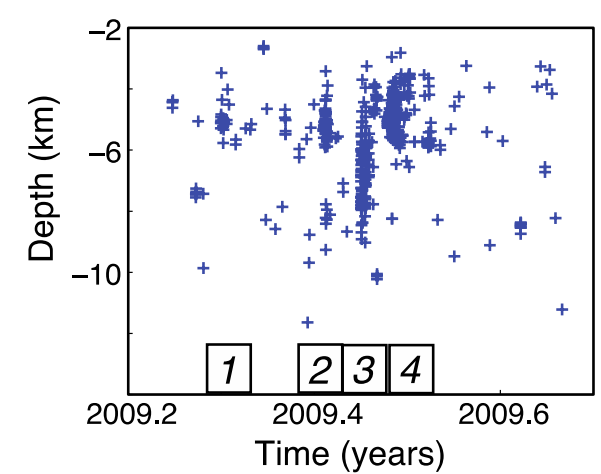

Figure 4.43 Comparisons between the original catalog locations and double-difference relocations.

Plots showing the original (A and D) and double-difference relocated earthquake locations for relocations performed with P-phases only (B and E), and for relocations performed with $\mathrm{P}$ and $\mathrm{S}$ phases $(\mathrm{C}$ and $\mathrm{F})$. A.-C. are map views, D-F show depth with time. See the text for details on the relocation procedures. The four swarms discussed in the text are roughly marked with numbers in $\mathrm{B}$, and the times of the swarms are shown along the bottom axis of D-F. 


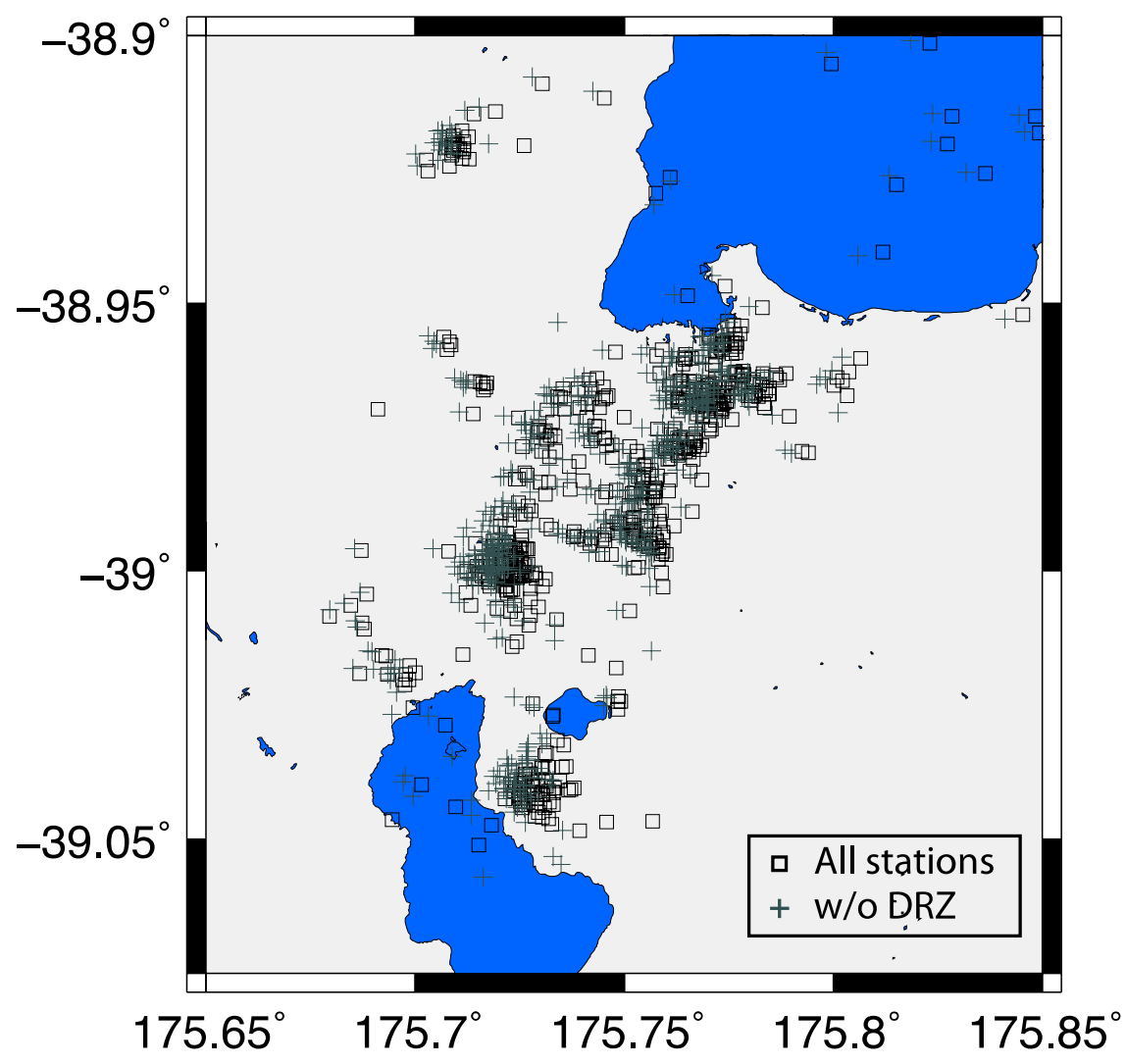

Figure 4.44 A comparison of the locations for two sets of P-phase only earthquake relocations.

The squares represent a set of relocations using all stations and the plus signs show a relocation calculated with the same input parameters, but without station DRZ.

The absolute depths vary by several kilometers between the SVD locations and the LSQR runs. The average depth difference is $1 \mathrm{~km}$ using all stations between the two methods and the LSQR run without station DRZ has deeper overall depths and thus a larger, $2.3 \mathrm{~km}$ average difference in depths from the LSQR and SVD methods that include all stations. It could be that the earlier more distant swarms in April (north) and May (south) help to constrain the depths, which could explain the difference in the SVD locations, which only contains events from the central clusters. We think the relative depth structure is well constrained, because the depths of these sequences match previous geophysical interpretations as discussed in the following 
section. We also note that one thing common to all relocations is the set of events that occur just below the hypocenter of the first $\mathrm{M}=4.4$ earthquake directly prior to its occurrence (yellow circles Fig. 4.45). This apparent depth migration will also be examined further in the next section.

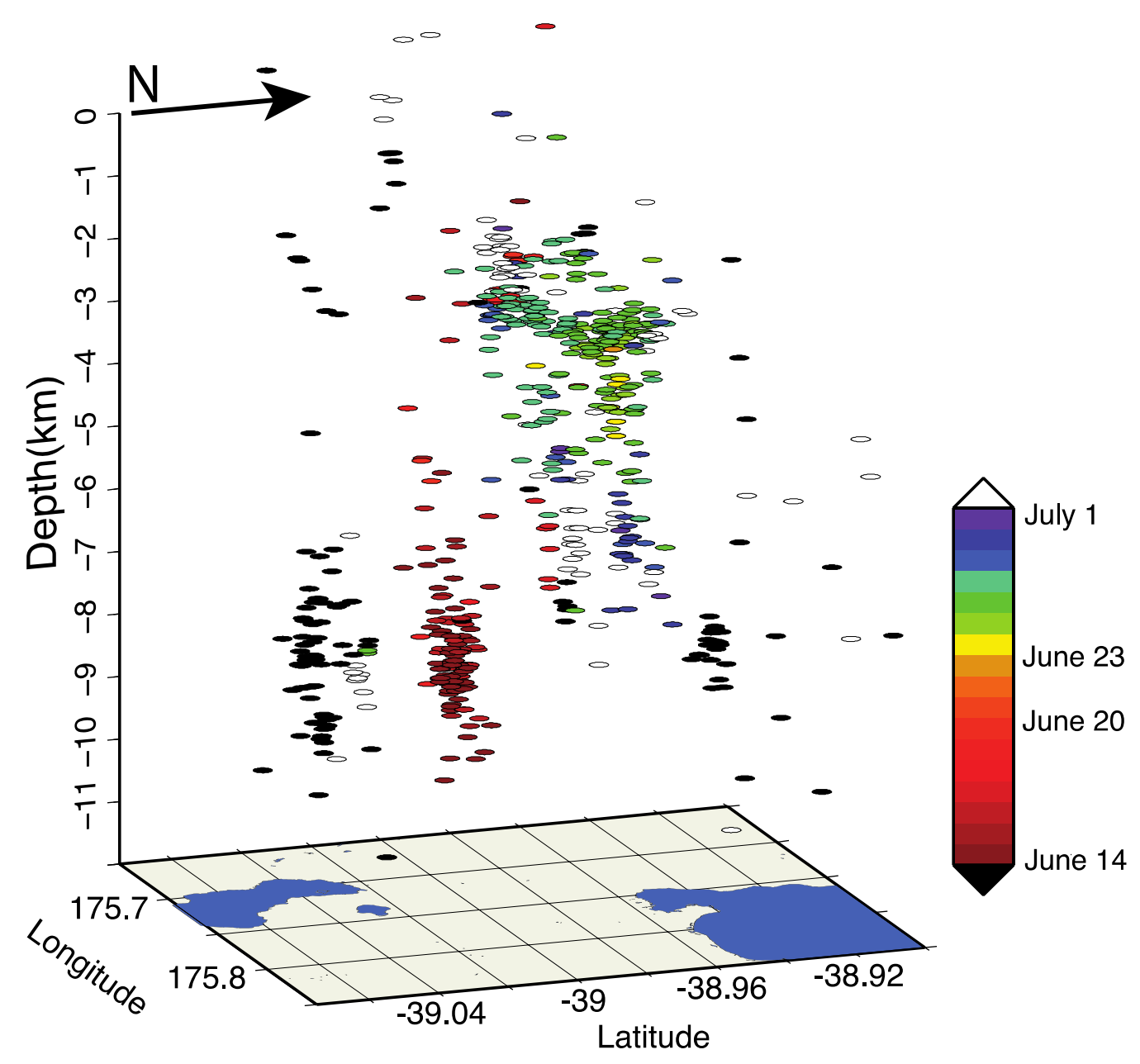

Figure 4.45 Earthquake relocations (using P-phase only) in 3D.

Colors are the same as figure 4.43 and represent the period June $14^{\text {th }}-\mathrm{July} 1^{\text {st }} 2009$. Black earthquakes occur prior to June $14^{\text {th }}$, and white earthquakes occur after July $1^{\text {st }}$. 
The $\mathrm{Vp} / \mathrm{Vs}$ ratio from all events is 1.70 taken from the slope of a best fit line through a plot of Tp vs Ts, where Tp and Ts are the arrival times of the P- and Sphases from the origin time (Fig 4.46). The value of 1.70 is similar to the value of 1.73 estimated by Sherburn et. al. [2003] for the northern TVZ. We calculated Vp/Vs ratios for each station using the original catalog origin times, and the recalculated origin times from P-only and P- and S- phase relocation corrections that are output with improved spatial locations in hypoDD. We calculated $\mathrm{Vp} / \mathrm{Vs}$ ratios for before and after the first $\mathrm{M}=4.4$ in addition to the overall mean. If the $95 \%$ confidence bounds do not overlap, then the change from before and after is significant. The $95 \%$ confidence intervals are calculated using equation 4.5.1, where $\sigma$ is the standard deviation and $n$ is the number of earthquakes used in the calculation of the mean.

$$
\operatorname{mean}\left(\frac{V p}{V s}\right) \pm\left(\frac{\sigma}{\sqrt{n}} * 1.96\right)
$$

For calculation of $\mathrm{Vp} / \mathrm{Vs}$ ratio with the original catalog origin times, the $\mathrm{Vp} / \mathrm{Vs}$ ratios for most stations sit around this mean value with three notable exceptions: RITZ, TWVZ and TUVZ (Fig. 4. 47 A). Four stations show a significant change: KATZ, RITZ, TWVZ, and TUVZ. The direction of these changes is an increase at RITZ and TUVZ, and it is a decrease at KATZ and TWVZ. All four stations move closer to the average value after the $\mathrm{M}=4.4$. The $\mathrm{Vp} / \mathrm{Vs}$ calculations performed with relocated origin times (B and C) show considerably less variation and only a few minor changes between before and after the first $\mathrm{M}=4.4$ earthquake. The P-phase only and P and Sphase relocation origin times are similar with comparable errors. The Vp/Vs ratios using $\mathrm{P}$ and S-phase origin times are lower than those with P-phase only relocation origin times. These results indicate that there were not significant changes in $\mathrm{Vp} / \mathrm{Vs}$. 
The observations that RITZ, TUVZ, and TWVZ sit away from the average 1.70 value may be an artifact of fixed depth locations in the original catalogue.

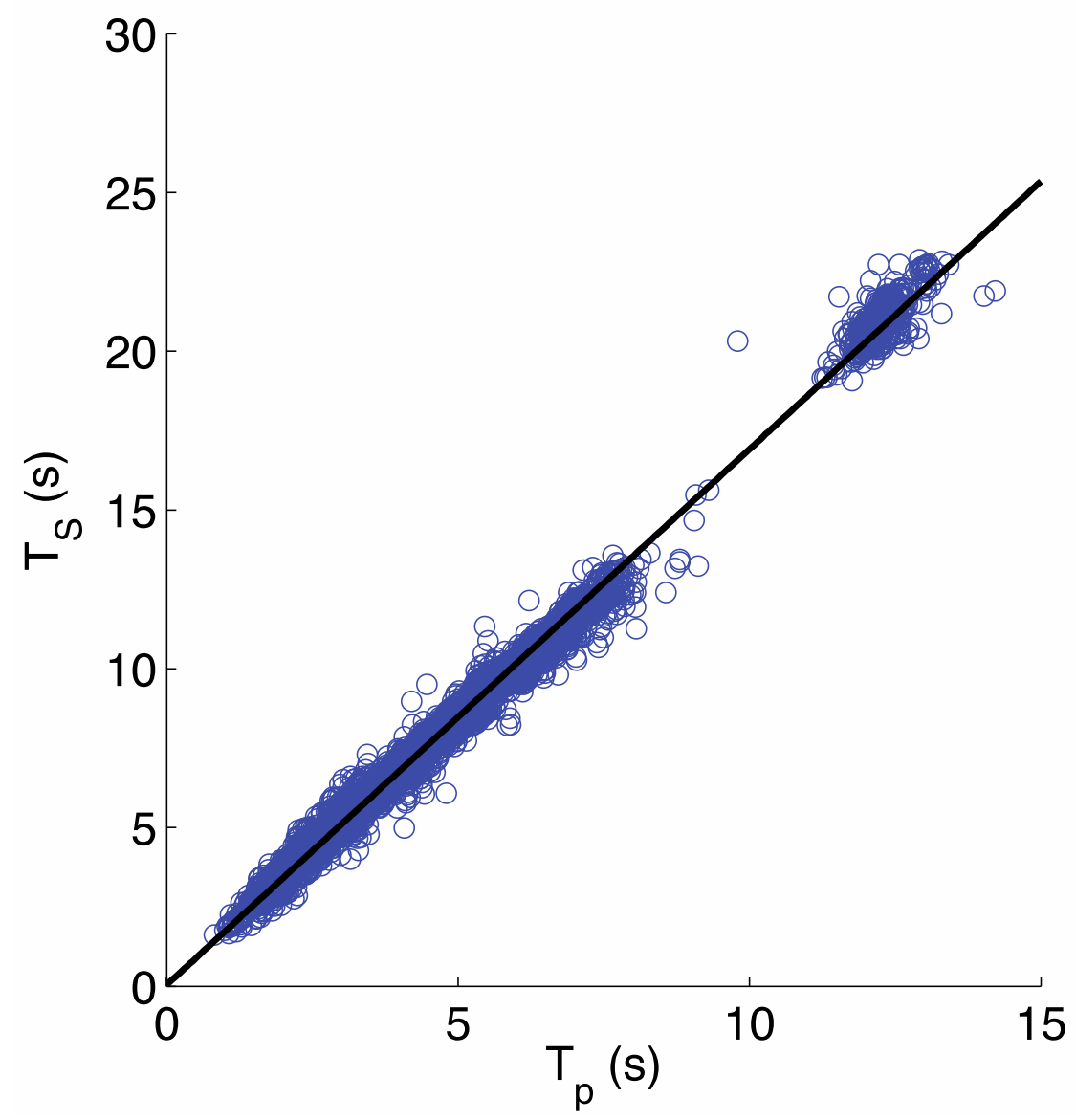

Figure 4.46 Plot of the arrival time of the P- vs the S- phases relative to the origin time for the set of earthquakes March $1^{\text {st }}$ to September $1^{\text {st }} 2009$ as measured on all twelve stations.

The average Vp/Vs velocity is 1.70 . The apparent gap in data is due to the lack of stations between the 11 closest stations, and the most distant station BKZ. 


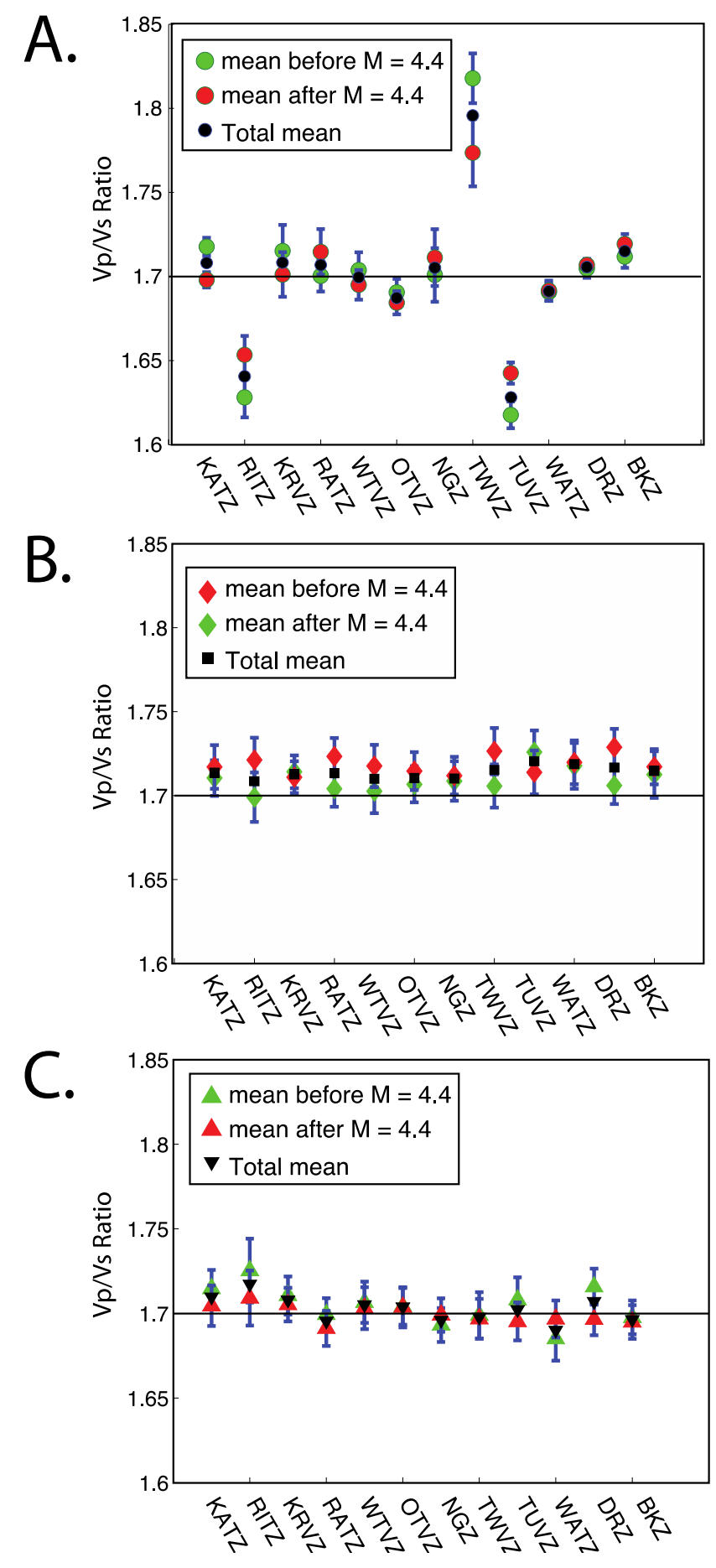

Figure 4.47 Mean Vp/Vs values by station for all Taupo earthquakes (black).

Green and Red symbols represent mean values before and after the first $\mathrm{M}=4.4$ earthquake. Error bars mark the bounds of the $95 \%$ confidence interval (see text for a full description). Before and after values that do not have overlapping confidence intervals represent significant changes (KATZ, RITZ, TWVZ, TUVZ). A) calculated with catalog origin times, B) calculated with P-phase only relocation origin times, and C) calculated with $\mathrm{P}$ and $\mathrm{S}$ phase relocation origin times. 


\subsubsection{Discussion}

The spatial separation of sequences 3 and 4 is very curious. Unraveling the underlying cause of this will help us understand more about what may have triggered these two swarms. We will focus first on swarm 4, the northernmost swarm that contains the two $\mathrm{M}=4.4$ earthquakes.

\subsubsection{Swarm 4, Evidence for fluid diffusion}

The observed migration of swarm 4 southwards with time suggests that fluids may play a role in the development of this swarm. A pocket of deeper events occurred early in swarm 4 (yellow events Fig 4.45). We plotted the depth with time for swarm 4 (Fig. 4.48 A) and found that the pocket of deep events shows a clear migration upwards prior to the first $M=4.4$. The migration of events suggests fluids or the presence of another physical movement within the seismic volume. All relocations, independent of phases used, show this upward migration (Fig. 4.49). To test for fluid diffusion we used the approach of Malagnini et. al. [2012] to calculate the diffusion curve for a 1-D pressure source. This requires the projection of the earthquake locations onto a single line (Fig. 4.50) and the calculation of the distance for each projected point and the potential source. We chose to use the first $M=4.4$ as the starting point for the distance calculation because the range of depths and the rate of earthquakes increases dramatically following this earthquake (Fig. 4.48 A). The azimuth of the projection was chosen to roughly match the orientation of mapped faults within the Tokaanu area (Fig. 4.41). Several diffusion rates were tested and we

plot the best fitting range, $35-45 \mathrm{~m}^{2} / \mathrm{s}$ in figure $4.48 \mathrm{~B}$. The diffusion curves appear to bound the locations reasonably well in the first 1-2 days of the swarm. The later part of the swarm, which is important for constraining the envelope of the diffusion curve, does not appear to have many events at the predicted maximum distances. This $\sim 5$ $\mathrm{km}$ distance approximately falls at the intersection of the diffusion front with the area 
previously ruptured by swarm 3 to the south. We infer that this area, having recently ruptured, was not available for pressure triggering. Similarly if there is a barrier that prevented swarm 3 from continuing northwards, the same barrier may very well act to stop swarm 4 from progressing southwards. The upward depth migration and subsequent fit to a diffusion curve strongly suggest that fluids played a role in swarm 4.

A.

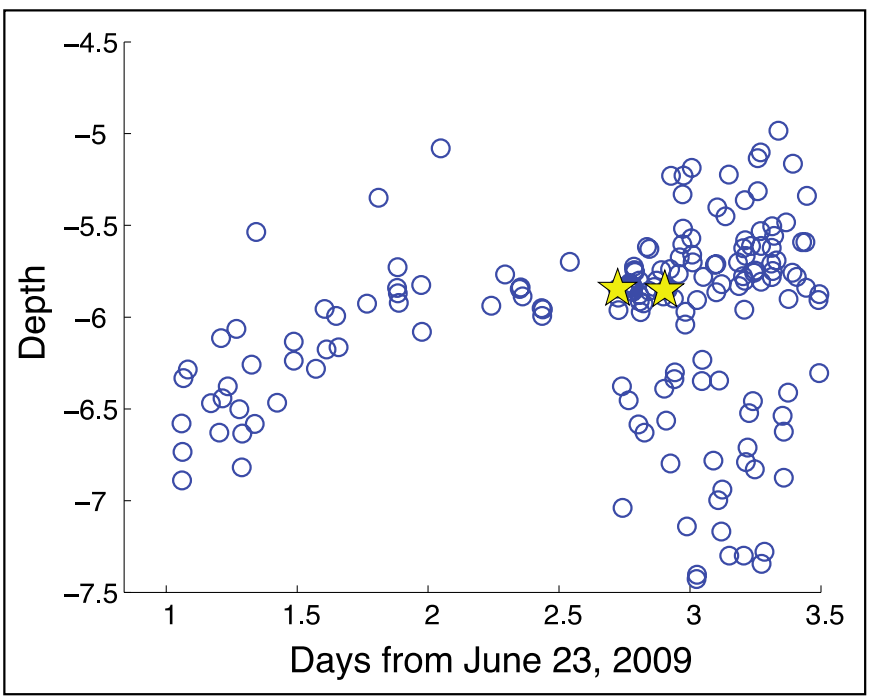

B.

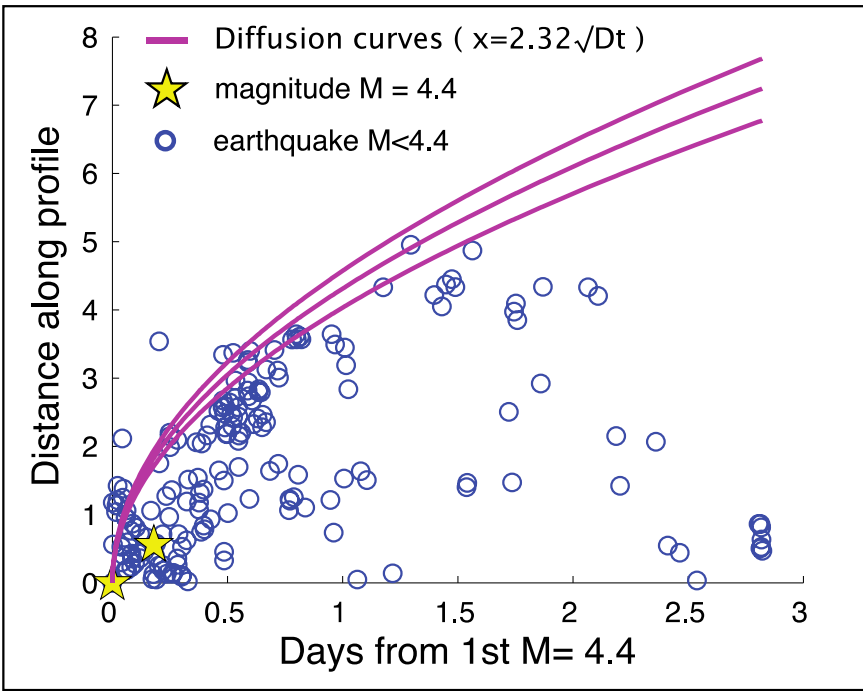

Figure 4.48 Depth and distance with time for Tokaanu Swarm 4.

A) Depth with time from June $23^{\text {rd }}$. Yellow starts represent the two $\mathrm{M}=4.4$ earthquakes. B). Earthquakes plotted with time from the first $\mathrm{M}=4.4$ earthquake and distance from that earthquake along the projection shown as a gray line in figure 4.50. The pink lines in $\mathrm{B}$ show several diffusion curves $(\mathrm{D}=35,40$, 45) that represent the maximum expected distance from a diffusion source given a particular diffusivity value D. 

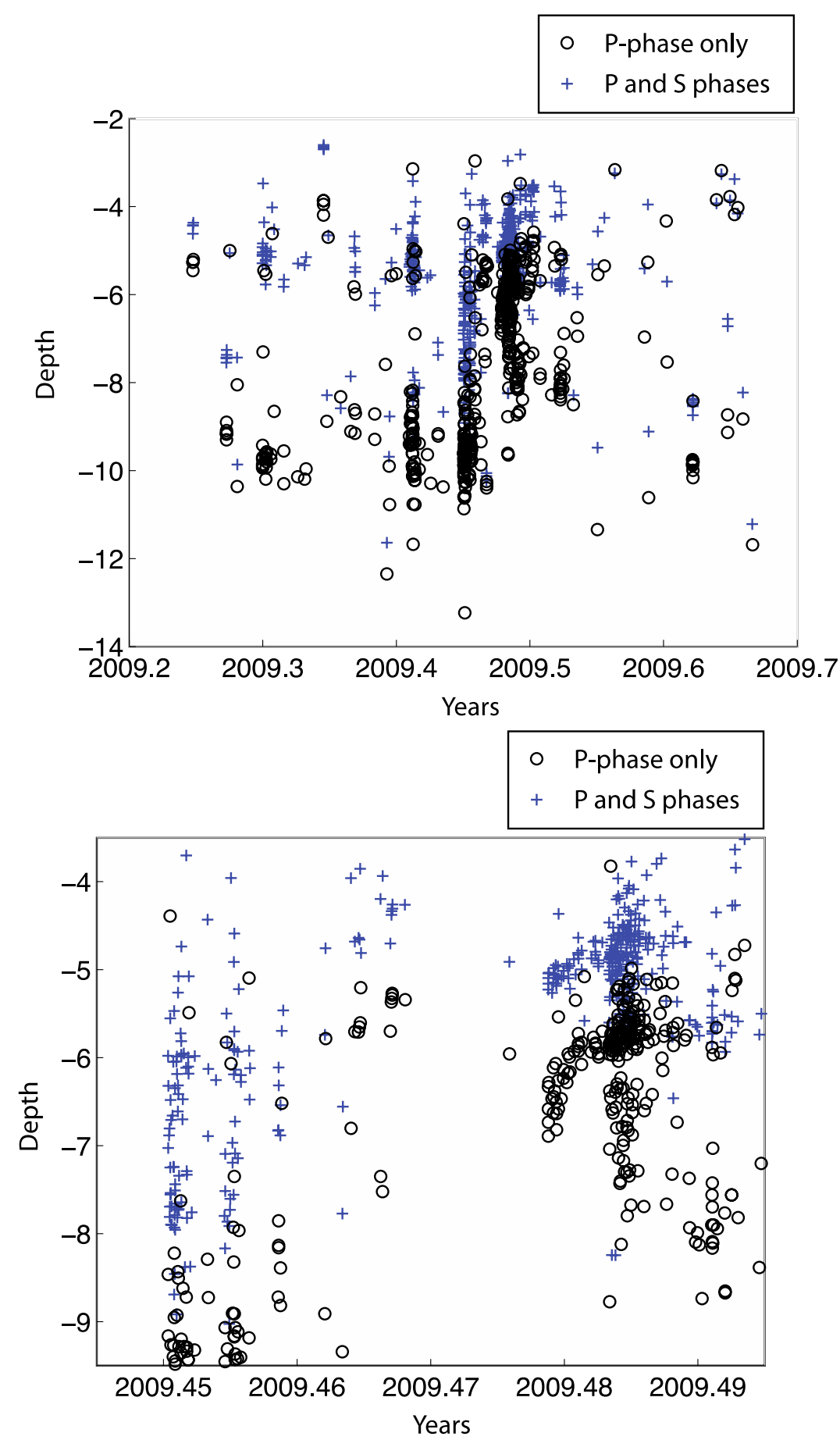

Figure 4.49 Depth with time comparisons of P-phase only, and P- and S- phase relocation results.

Top: whole time period, Bottom: time period from swarm 3 to the beginning of swarm 4.

\subsubsection{Spatial development and relationship to other observations}

Given that fluids were probably involved in swarm 4, it is reasonable to ask whether a migration of fluids from swarm 3 may have triggered swarm 4 . As discussed earlier however, despite the spatial proximity, swarm 4 starts on the 
northern side and then migrates south towards the location of swarm 3 (Fig. 4.41). The relocated depths also argue against direct triggering between the swarms because, while swarm 4 starts with events at deeper depths and migrates upwards, the deepest events of swarm 4 sit several kilometers above swarm 3 . Therefore we must investigate if a barrier of some kind exists that stopped earthquakes from swarm 3 , or fluids from migrating northwards.

The most obvious possibility is the Tokaanu-Waihi geothermal area boundary. Risk et. al. [2002] has summarized the available magneto-telluric (MT) and other geophysical and geochemical observations in this area. They suggest a model in which hydrothermal fluids rise nearly vertically under the high topography of Kakaramea and Tihia andesites and flow north towards the southern shore of Lake Taupo (see Fig. 4.50 for location of Kakaramea and Tihia). The apparent resistivity contours that outline the geothermal area appear to correlate well to the boundary between swarms 3 and 4 (Fig. 4.50). The observations of the distinct depth difference between swarms 3 and 4 also fit the concept of near vertical flow in this region associated with a flow boundary. There is also a tunnel from Lake Rotoaira and Tokaanu that was constructed in the 1970 s as part of a hydroelectric scheme and Risk et. al. [2002] note that in the tunnel there is change in lithology with the presence of hydrothermally altered clays about $1.8 \mathrm{~km}$ from the Lake Rotoaira end of the tunnel. We were unable to locate the original logs, but the boundary of this change as shown by Risk et. al. [2002] is shown on figure 4.50. If we extrapolate this boundary towards the peaks of Tihia and Kakaramea it also nicely separates the bulk of swarms 3 and 4 . We propose that if fluids were involved in swarm 3 that they were unable to flow across the boundary of the geothermal system. 


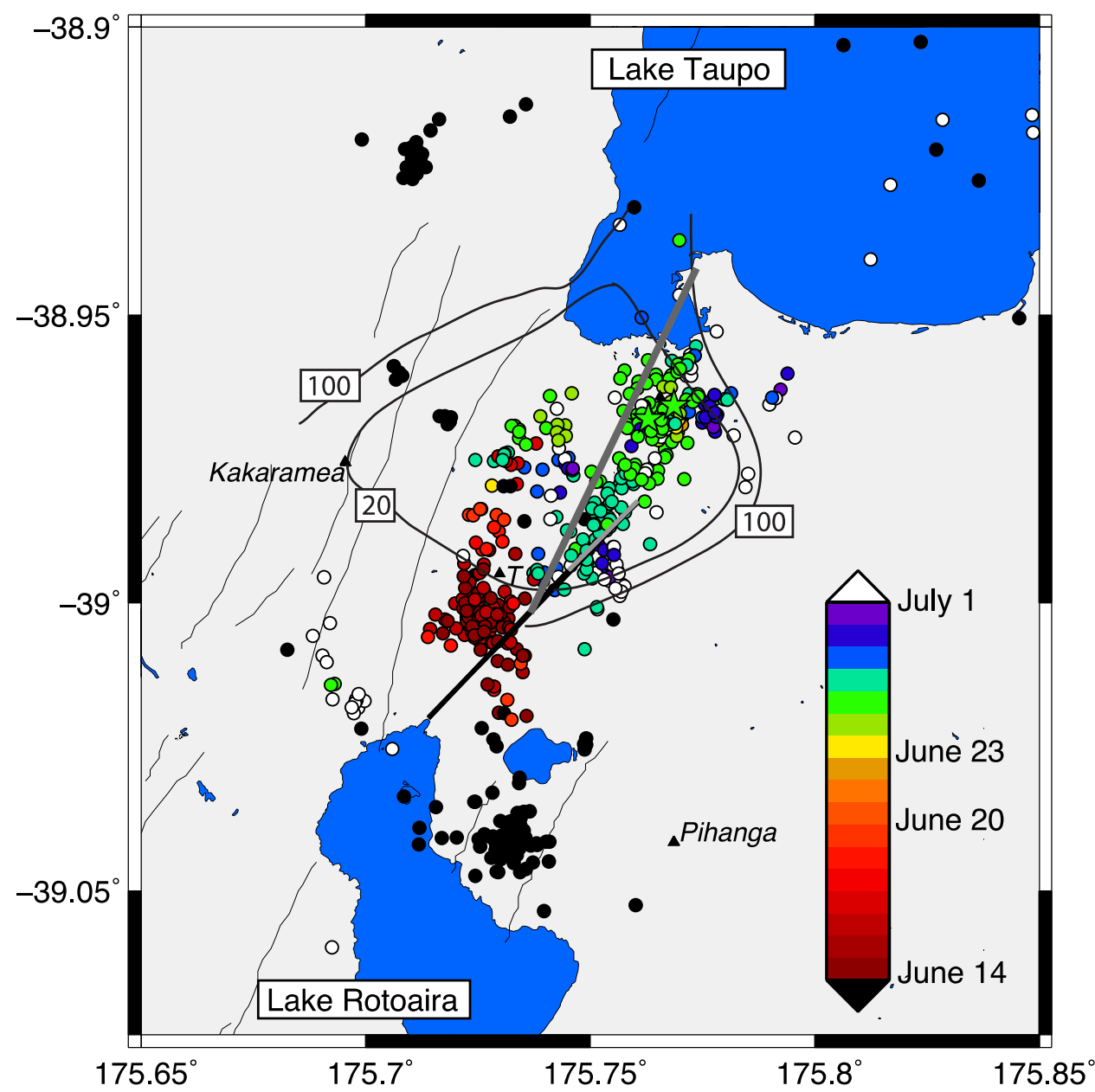

Figure 4.50 Earthquake relocations of all Taupo earthquakes using both $\mathrm{P}$ and $\mathrm{S}$ phases for relocation.

Earthquakes from June $14^{\text {th }}$-July $1^{\text {st }} 2009$ are colored with time. Black contours represent resistivity contours from Risk et al. [2002]. The thick gray line is the line used to project earthquakes for fluid diffusion testing (see figure 4.48). The line extending from the Northeast edge of Lake Rotoaira is the Tokaanu tunnel that was excavated in the 1970s for hydroelectric purposes. The change along the line from black to gray indicates the approximate location of a change in lithology, with gray representing the presence of hydrothermally altered clays (as shown in Risk et al. [2002] figure 2). The two stars mark the location of the two $M=4.4$ earthquakes. Three small andesitic cones are shown with black triangles (Tihia is abbreviated with T). 


\subsubsection{Possible physical triggers for the Tokaanu swarms}

Swarms 1-3 (table 4.5.1) seem to line up reasonably well north-south (Fig.

4.41, Fig 4.45). This suggests a moving triggering source at depth; however, their temporal pattern does not suggest any systematic movement in time. Because these swarms occur in one of the most active rhyolitic systems in the world we feel the need to briefly discuss the possibility of a magmatic source for the activity. Smith et. al. [2007] modeled leveling data and observed subsidence at Lake Taupo from 19861996 and proposed that a body of magma was intruded beneath the lake prior to 1983 (possibly around the time of the 1964-1965 swarm) and that dewatering of this magma body was associated with the observed swarms at the northern edge of the lake and in the center of the lake in 1983. They note that a swarm observed at the southern end of the lake, slightly west of the swarms considered here, was not associated with any changes in the lake leveling data. This fits with the suggestion of Otway and Sherburn [1994] that the deformation and swarms do not directly correlate, but are likely caused by the same underlying strain process. The swarms in 2009 discussed here are not significantly larger than the swarms observed during the period of subsidence. There is therefore no reason to conclude that intrusion of magma at shallow levels is necessary to produce the levels of seismicity recorded in 2009. We conclude that this set of swarms show no evidence that magma was intruded at shallow levels $(<12 \mathrm{~km})$. The overall observations do not allow us to rule out the possibility of a deeper magma intrusion. The occurrence of the four swarms relatively close in time that do not appear to trigger one another suggests something changed at depth. If a deep change did occur, the spatial manifestation of the shallow seismicity may be related to the location of existing fluids in the crust. 


\subsubsection{General implications for earthquake sequences}

To assess how these swarms relate to our general swarms we also plotted the inter-event time (rate-type) for swarm 4. The swarm has a strong acceleration prior to the first $\mathrm{M}=4.4$, another acceleration occurs after the first $\mathrm{M}=4.4$, and finally decays following the second $\mathrm{M}=4.4$ earthquake (Fig. 4.51). The accelerating rate corresponds to observed upward migration of earthquakes (Fig. 4.48 A). The link between accelerating rate and vertical migration may indicate a general model for such L-shaped sequences: that increased fluid pressure migrates and continues to pressurize until a more porous area is encountered, or faulting can release and diffuse the pressure.

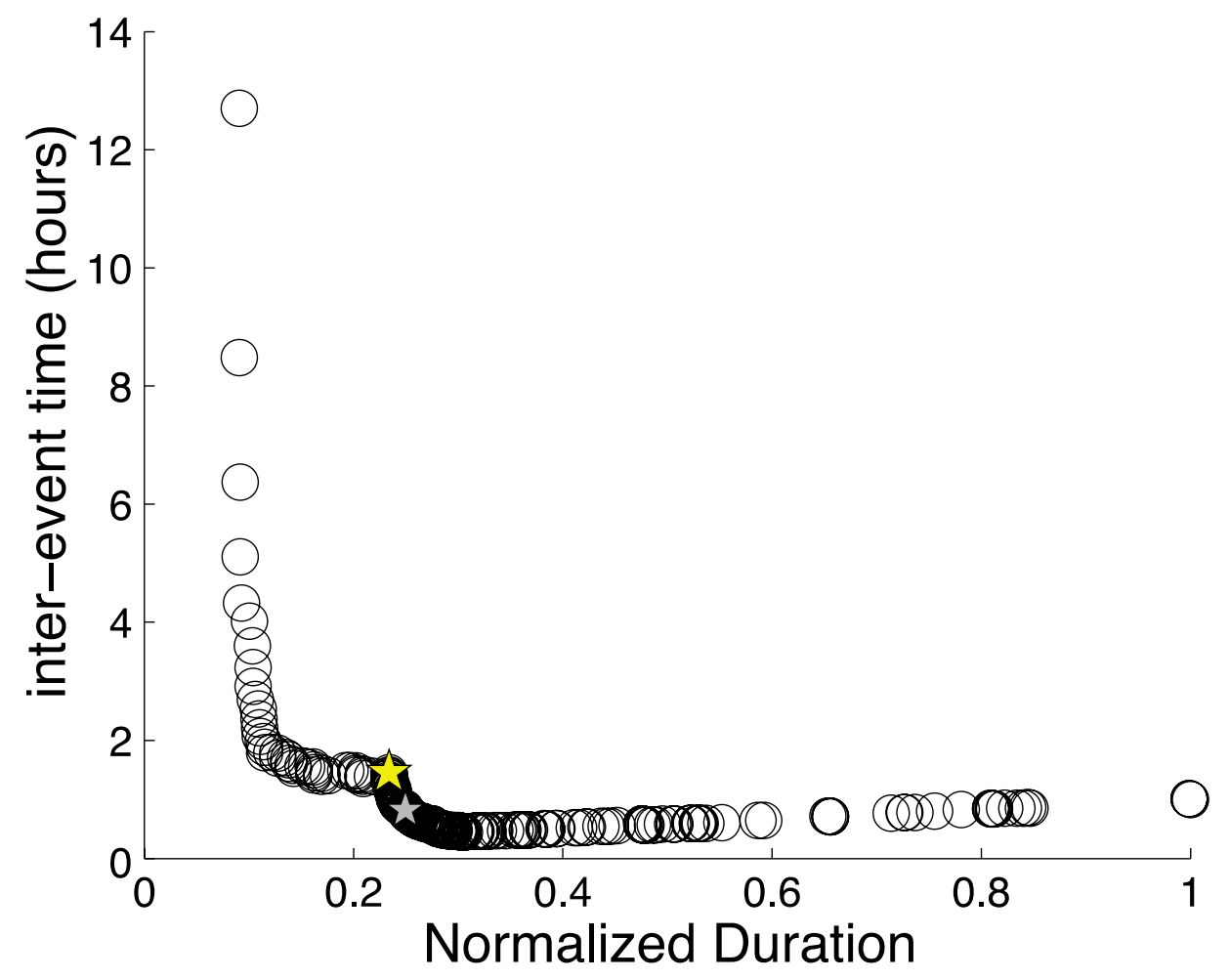

Figure 4.51 Average inter-event time versus normalized duration of swarm 4.

The Yellow star represents the first $\mathrm{M}=4.4$ and the gray star represents the second $\mathrm{M}$ $=4.4$. Note decreasing inter-event times indicate accelerating seismicity rates. 


\subsubsection{Conclusions}

Spatio-temporal evidence shows that fluids drove swarm 4 that started on June $23^{\text {rd }}$ and likely triggered the two $\mathrm{M}=4.4$ earthquakes that occurred during the swarm. The increased activity over the broad area around the southern end of Lake Taupo over these few months without clear migration or triggering between sequences may indicate that a deeper process or change occurred below the seismogenic zone triggering pockets of seismic activity over a widespread area that may be controlled by the location of crustal fluids. The relocated earthquake depths agree well with earlier geophysical observations that suggested that at shallow depths $(<10 \mathrm{~km})$ the Tokannu-Waihi system is separate from geothermal activity further south. The boundary of this system seems to act as a barrier to fluid flow and pressure increase. The average inter-event time plot for the largest swarm (4) has a pattern common to many other swarms and the accelerating rate is connected to an upward migration in depth. The link between a physical process and the rate may help us to interpret other earthquake swarms. 


\section{Triggering}

\subsection{Investigation of triggered sequences in the CVR}

Distal triggering of earthquakes by large earthquakes has, to our knowledge, not been hitherto demonstrated or observed in the CVR or in New Zealand. Recent observations in the Central Southern Alps (Boese $2012 \mathrm{PhD}$ thesis, hereafter referred to as Boese [2012]) suggest that triggering occurs in the central Southern Alps. That work has not been published, and it will be discussed in section 5.2. There have been a few studies of other potential triggers, including slow slip [Reyners and Bannister, 2007; Robinson, 2003; Wallace et al., 2012] but we will discuss those separately in section 5.3. Prior to the Boese [2012] study, the only known triggering observation by large global earthquakes in New Zealand was an observation of triggered tremor following the Maule Chile $\mathrm{M}_{\mathrm{W}}=8.8$ earthquake in 2010 [Fry et al., 2011]. The recent observations by Fry [2011] and Boese [2012] indicate that the lack of observations may be due to a lack of investigation, and a high magnitude of completeness in the New Zealand earthquake catalog. We thought it was important to try to quantify what effect, if any, large earthquakes have on the timing of earthquake sequence occurrence in the CVR. The sequence catalog produced by the CURATE method provides a new opportunity to undertake a statistical investigation of triggering because we have taken out most of the isolated background earthquakes.

\subsubsection{Trigger Test Parameters}

We began by compiling a list of global earthquakes from the USGS catalog of $M_{\mathrm{W}} \geq 7$ from January1993 through June 2007 to match the time period of our sequence catalog. We also made a list of earthquakes in New Zealand (NZ) $\mathrm{M}_{\mathrm{L}} \geq 6.0$ for the same time period using the GeoNet catalog. The magnitude threshold was lower for NZ events, as their proximity might be more important than their size in 
influencing their ability to exert stress triggering (e.g. [Stark and Davis, 1996]). This gave us 207 global earthquakes ( $73 \mathrm{M} \geq 7.5)$ and 43 New Zealand earthquakes as potential triggering events. Note that we did not limit distance of the global or regional catalogs and so there is overlap between the two lists. Eight earthquakes appear in both lists of large earthquakes. Three of those eight earthquakes have $\mathrm{M}_{\mathrm{L}}$ less than 7 in the GeoNet catalog. Complete versions of both lists can be found in the appendix (A2).

The number of potential triggers has a large impact on the accuracy of our current calculations. This is because factors such as directivity [Eberhart-Phillips et al., 2003; Gomberg, 2001], wave frequency and period [Brodsky and Prejean, 2005] can all influence the 'ability' of a large earthquake to trigger distant earthquakes. These quantities are all potentially site specific (e.g. [Gomberg, 2001; Prejean et al., 2004]). Thus, with more information on the specific triggering thresholds and physical mechanisms we may be able to discard large earthquakes that lack the necessary triggering characteristics and further refine the probabilities. Because there are no published observations of triggering in New Zealand we do not have criteria to help us exclude unlikely triggers. Distance is one possible parameter we could limit, but although current earthquake triggering observations have been at distances of $\leq$ $4000 \mathrm{~km}$ (e.g. [Eberhart-Phillips et al., 2003]), the observations of triggered tremor at $\geq 9000 \mathrm{~km}$ [Fry et al., 2011] suggest that earthquake triggering at greater distances may be possible under certain circumstances. As we are unable to exclude large earthquakes that do not have the power to trigger, the calculations result in minimum likelihoods.

Waveform triggered earthquakes can be $\mathrm{M}>3$, but are more commonly $\mathrm{M} \leq$ 1.5 [Hill et al., 1993; Prejean et al., 2004]. Our sequence catalog for the CVR has a 
Mc value of 2.45, which is somewhat high compared to previous global triggering observations. To account for the possibility that small earthquakes below the $\mathrm{M}_{\mathrm{c}}$ start soon after the passage of surface waves, and events above $M_{c}$ follow later we used a range of 1-7 days to look for triggered sequences.

\subsubsection{Likelihood calculation}

In addition to identifying sequences that begin around the time of large earthquakes, we also need to estimate how likely it is to observe a given number of earthquakes within a specified time window around the occurrence of large global earthquakes. To calculate this probability, we ran a code that generated 10,000 lists of random times, or simulations, within the catalogue dates (1993-June 2007). Each of these lists of random times has the same number of dates as the lists of potential triggers (207, and 43 events respectively for the global and NZ lists). Then we tested how many of the observed CVR sequences fall within time windows of 1-7 days of these random times. This allowed us to calculate an empirical cumulative distribution function of the number of our sequence start dates that fell within the tested time window of one of the randomly generated times. The empirical distribution function gives the probability that any number of CVR sequences will randomly fall within a given number of days after a large earthquake. Because the number of sequences is discrete there is variation between different runs of the code even though we are generating a large number of simulations. The difference between the runs is most prominent in the bins that contain the most sequences and highest likelihoods. Bins on the edges of the likelihood distribution tend to show less variation between runs as fewer sequences fall into those bins.

The variation in time window strongly influences the calculated probability. The lower, 1-day, limit covers $3 \%$ of the total days in the catalog (163 sequences $\geq 4$ 
earthquakes / 5297 days). The 7-day time window covers nearly twenty-two percent of the total days for sequences $\geq 4$ earthquakes.

\subsubsection{Triggering observations}

The numbers of sequences found that fall within various time windows of large global and New Zealand earthquakes are given in Table 5.1 along with the percent of the random list that have that number of sequences or less (percentile). For example, at a time window of 3 -days and a minimum sequence size of 8 earthquakes, the global $\mathrm{M} \geq 7.0$ show $22.3 \%$ of the 10,000 simulations have at least 8 sequences that align with the random times. We calculated these numbers for time windows of $[1,3,5$, and 7$]$ and for minimum sequence sizes of $[4,8,10$, and 20$]$ earthquakes. For some time windows there are no sequences of 8-9 earthquakes that occur within the window of one of the large earthquakes and so the number of sequences is the same for the 8 and 10 earthquake sequence size thresholds. However, the probabilities for these sequence sizes still change due to the number of sequences of $8-9$ earthquakes that do not fall within the time window of a large earthquake changing the probability of observing that number of sequences randomly. 
Table 5-1 Results of Likelihood Tests Looking for Potential Sequence Triggering. Tested triggers include 207 global earthquakes $M \geq 7.0,73$ global earthquakes $M \geq$ 7.5, and 43 New Zealand earthquakes $M \geq 6.0$. Along with the number of sequences that fall within the time frame, the likelihood that this many sequences would be observed randomly is also given. We consider anything with a likelihood $<10 \%\left(90^{\text {th }}\right.$ percentile) to be significant. Bold entries indicate the parameters that have the highest number of potentially triggered sequences for the global and New Zealand lists.

\begin{tabular}{|c|c|c|c|c|c|c|c|c|c|c|}
\hline \multirow[b]{2}{*}{ CVR } & \multirow{2}{*}{$\begin{array}{c}\text { time } \\
\text { window } \\
\text { (days) }\end{array}$} & \multirow[t]{2}{*}{ catalog } & \multicolumn{2}{|c|}{$\begin{array}{c}\text { Minimum seq } \\
\text { size: } 4\end{array}$} & \multicolumn{2}{|c|}{$\begin{array}{c}\text { Minimum seq } \\
\text { size: } 8\end{array}$} & \multicolumn{2}{|c|}{$\begin{array}{c}\text { Minimum seq } \\
\text { size: } 10\end{array}$} & \multicolumn{2}{|c|}{$\begin{array}{c}\text { Minimum seq } \\
\text { size: } 20\end{array}$} \\
\hline & & & $\#$ & Likelihood & $\#$ & Likelihood & $\#$ & Likelihood & $\#$ & Likelihood \\
\hline & & G 7.0 & 5 & $58.6 \%$ & 1 & $65.4 \%$ & 1 & $42.9 \%$ & 0 & $100 \%$ \\
\hline & & G 7.5 & 2 & $37.1 \%$ & 1 & $18.9 \%$ & 1 & $10.6 \%$ & 0 & $100 \%$ \\
\hline & & NZ 6.0 & 1 & $36.6 \%$ & 0 & $100 \%$ & 0 & $100 \%$ & 0 & $100 \%$ \\
\hline & & G 7.0 & 20 & $31.3 \%$ & 8 & $22.3 \%$ & 8 & $3.7 \%$ & 3 & $15.5 \%$ \\
\hline & & G 7.5 & 5 & $63.0 \%$ & 3 & $21.1 \%$ & 3 & $6.9 \%$ & 1 & $16.9 \%$ \\
\hline & & NZ 6.0 & 6 & $9.2 \%$ & 3 & $5.3 \%$ & 3 & $1.3 \%$ & 0 & $100 \%$ \\
\hline & & G 7.0 & 35 & $19.5 \%$ & 13 & $23.6 \%$ & 12 & $4.2 \%$ & 4 & $26.7 \%$ \\
\hline & & G 7.5 & 12 & $29.8 \%$ & 6 & $9.9 \%$ & 6 & $1.7 \%$ & 2 & $12.1 \%$ \\
\hline & & NZ 6.0 & 11 & $3.2 \%$ & 4 & $8.4 \%$ & 4 & $2.1 \%$ & 0 & $100 \%$ \\
\hline & & G 7.0 & 41 & $59.8 \%$ & 14 & $60.1 \%$ & 13 & $16.7 \%$ & 4 & $53.6 \%$ \\
\hline & & G 7.5 & 15 & $45.1 \%$ & 7 & $18.9 \%$ & 7 & $3.2 \%$ & 2 & $24.5 \%$ \\
\hline & & NZ 6.0 & 15 & $2.2 \%$ & 6 & $4.5 \%$ & 5 & $2.3 \%$ & 0 & $100 \%$ \\
\hline
\end{tabular}

\subsubsection{Global earthquakes}

The magnitudes of earthquakes will be a factor in their ability to trigger distant swarms, therefore we expected that in the global list $M \geq 7.5$ might return higher significance levels than the $M \geq 7.0$ list. Sequences of at least 20 earthquakes do not show any significant triggering results, indicating that the occurrence of those sequences is not influenced by the timing of external large earthquakes. For a time window of one day there are no significant $(<10 \%$ likelihood) triggering observations for either magnitude range of large global earthquakes. Despite the lack of significance we do see the expected increase in probability between $M \geq 7.0$ and 7.5. Because of the possible fluctuation in likelihood between different runs of the 10,000 simulations, it is possible that the global $\mathrm{M} \geq 7.5$ at one day time window and a 
sequence size of 10 should be considered significant as it is close to the threshold. The three-day time window shows significant triggering potential for the global $\mathrm{M} \geq$ 7.0 at a sequence size of $\geq 10$ earthquakes. While the triggering is significant for the larger global $M \geq 7.5$ sequences, it is not larger than for $M \geq 7.0$ (greater likelihood is less significant). The triggering significance is similar for the five-day time window, but with the larger global events now being more significant than $M \geq 7.0$. For the seven day time window only the $M \geq 7.5$ earthquakes have significance. The $M \geq 7.0$ earthquakes have the most significant results at a minimum sequence size of ten earthquakes for the three day time window. The maximum possible number of triggered sequences is twelve sequences (Table 5.1: time-window of five days and minimum sequences size of ten earthquakes). That is $7 \%$ of the total number of observed sequences (163). It is also possible that some of those twelve sequences are randomly aligned. The probabilities for this time window and sequence size limit are shown in Table 5.2. Low numbers of sequences occur with a high likelihood. For example, three or more sequences align with a list of 207 random times in $\sim 93.7 \%$ of the 10,000 simulations. While the observed twelve sequences are enough to pass the significance threshold, ten sequences would not be enough as 10 or more sequences are observed in $\sim 13.6 \%$ of simulations (Table 5.2 ). So some of the twelve potentially triggered sequences may not represent real triggering. Therefore the $7 \%$ of potentially triggered sequences is a maximum percentage.

\subsubsection{New Zealand earthquakes}

Like the global earthquakes, large New Zealand earthquakes do not show significant triggering for the smallest time window of one-day or for the largest minimum sequences size of twenty earthquakes (Table 5.1). There is significant triggering for sequences sizes of 10 or less and time windows between 3-7 days. 
These results are much more consistent than the global earthquake triggering. The highest percent of triggering in the CVR by large New Zealand earthquakes is a possible 15 sequences, or $9 \%$ of the total number of sequences. As discussed above with the global earthquakes, $9 \%$ is a maximum percentage as some sequences may still be randomly aligned (Table 5.2). The next section examines the parameters of the potential triggering earthquakes (those that are followed closely in time by a CVR sequence) to see if they have properties that are different from the overall population of large earthquakes. 
Table 5-2 Likelihood of sequences randomly aligning with a list of times the length of the potential triggers for the global and New Zealand lists that yield the largest number of potentially triggered sequences (Table 5.1).

Bold lines indicate the maximum number of observed sequences at these day and sequence limits.

\begin{tabular}{|c|c|c|c|}
\hline \multicolumn{2}{|c|}{$\begin{array}{l}\text { Global Earthquakes 5-day, } \\
\text { minimum earthquakes } 10\end{array}$} & \multicolumn{2}{|c|}{$\begin{array}{l}\text { NZ Earthquakes 7-day, } \\
\text { minimum earthquakes } 4\end{array}$} \\
\hline $\begin{array}{c}\text { \# seqs } \\
\text { randomly align }\end{array}$ & Likelihood & $\begin{array}{c}\text { \# seqs } \\
\text { randomly align }\end{array}$ & Likelihood \\
\hline 0 & $100.0 \%$ & 0 & $100.0 \%$ \\
\hline 1 & $99.4 \%$ & 1 & $99.8 \%$ \\
\hline 2 & $97.9 \%$ & 2 & $99.3 \%$ \\
\hline 3 & $93.7 \%$ & 3 & $98.0 \%$ \\
\hline 4 & $86.7 \%$ & 4 & $94.5 \%$ \\
\hline 5 & $75.4 \%$ & 5 & $88.4 \%$ \\
\hline 6 & $61.6 \%$ & 6 & $79.3 \%$ \\
\hline 7 & $46.9 \%$ & 7 & $67.7 \%$ \\
\hline 8 & $33.5 \%$ & 8 & $54.7 \%$ \\
\hline 9 & $22.3 \%$ & 9 & $40.7 \%$ \\
\hline 10 & $13.6 \%$ & 10 & $28.9 \%$ \\
\hline 11 & $7.8 \%$ & 11 & $19.1 \%$ \\
\hline 12 & $4.2 \%$ & 12 & $11.9 \%$ \\
\hline 13 & $2.2 \%$ & 13 & $7.1 \%$ \\
\hline 14 & $1.0 \%$ & 14 & $4.0 \%$ \\
\hline 15 & $0.4 \%$ & 15 & $2.2 \%$ \\
\hline 16 & $0.2 \%$ & 16 & $0.9 \%$ \\
\hline 17 & $0.0 \%$ & 17 & $0.4 \%$ \\
\hline 18 & $0.0 \%$ & 18 & $0.2 \%$ \\
\hline \multirow[t]{4}{*}{19} & $0.0 \%$ & 19 & $0.1 \%$ \\
\hline & & 20 & $0.0 \%$ \\
\hline & & 21 & $0.0 \%$ \\
\hline & & 22 & $0.0 \%$ \\
\hline
\end{tabular}

\subsubsection{Triggering with earthquake parameters}

The total number of sequences potentially triggered by both global and NZ large earthquakes is twenty-four unique sequences (Some sequences fall within a time window of both a large NZ and global earthquake.). That means that up to $15 \%$ of CVR sequences could be influenced by large distant earthquakes. For each sequence that falls within any of the four time windows we mark the corresponding large earthquake as a potential trigger. In this section we examine the distance, 
backazimuth, and depth of the potential triggers. If the triggering of sequences is real, we might expect the potential triggers identified by our simple time window investigation to vary from the overall distribution of large earthquakes. Figure 5.1 shows a map of all of the large global earthquakes used in the triggering test. The potential triggers come from a large range of backazimuths and distances. The New Zealand large earthquakes show a similar lack of trend (Figure 5.2) with potential triggers coming from all backazimuths, depths, and distances.

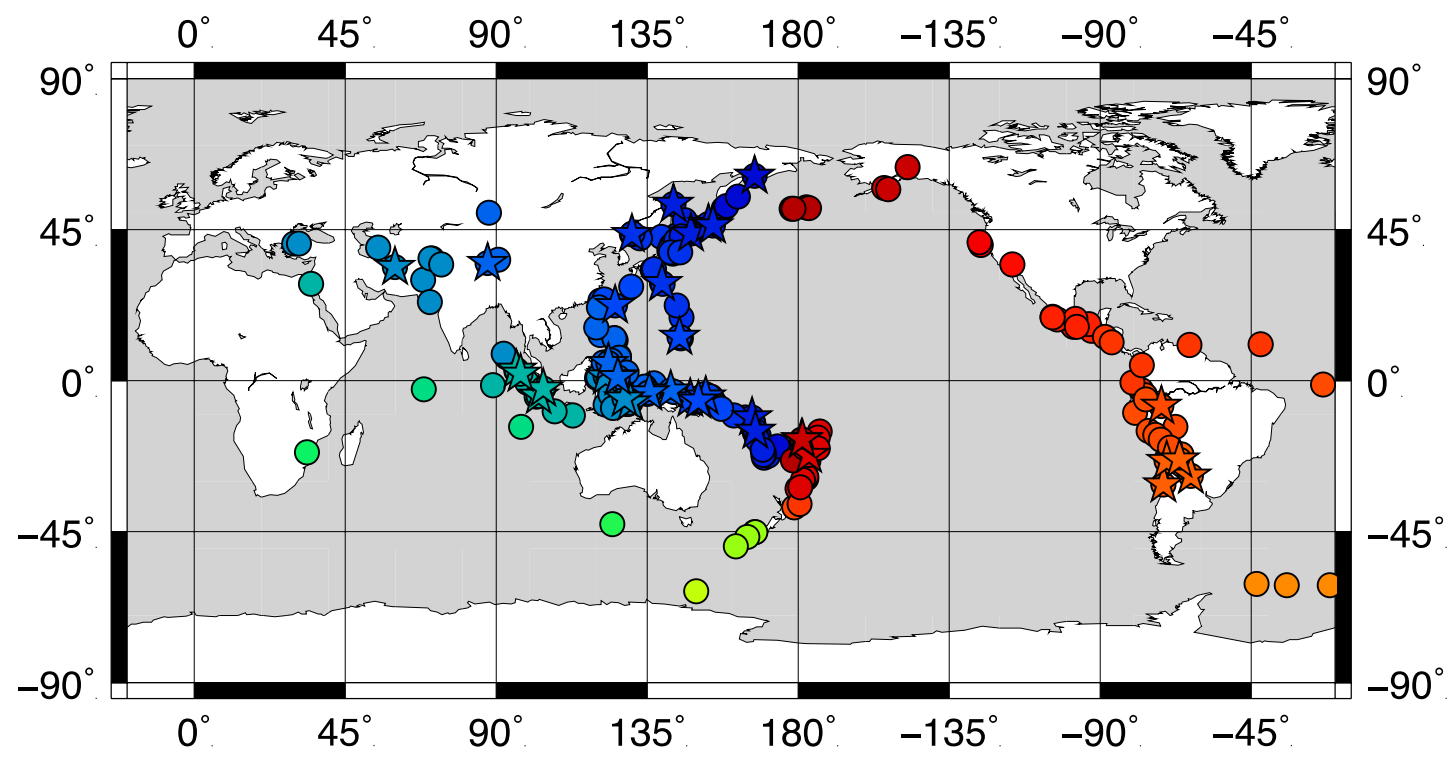

Figure 5.1 Map of large global earthquakes from the USGS catalog $(M \geq 7.0)$ between January1993 and July 2007.

Color indicates backazimuth from the center of the CVR, and stars indicate large earthquakes that occur 1-7 days before one of the CVR sequences (potential triggers). See the text for further explanation. 
A.

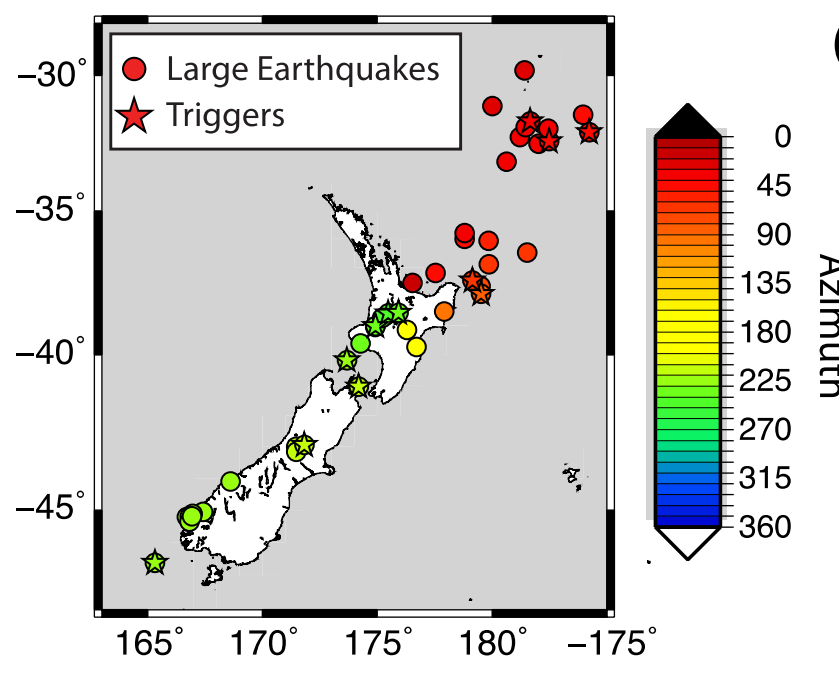

B.

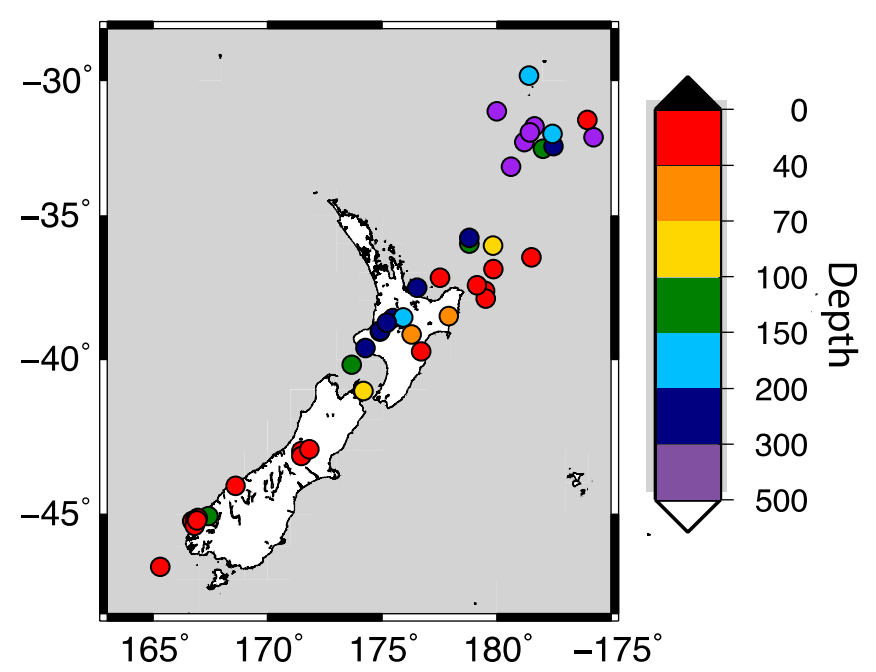

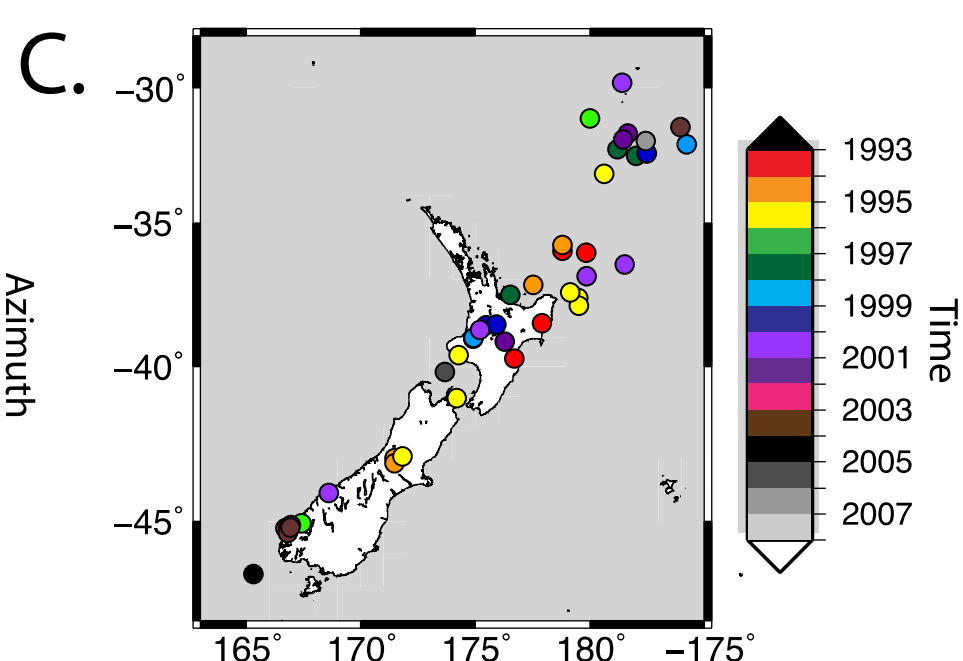

בְ'

Figure 5.2 Map of large New Zealand earthquakes $(M \geq 6.0)$ from January 1993 to July 2007 .

Color indicates backazimuth from the center of the CVR, and stars indicate large earthquakes that fall close enough in time that they may be considered potential triggers. B) Colors represent the depth of earthquakes. C) Colors represent the year in which the earthquake occurred. 
The distribution of these parameters is plotted in more detail in Figure 5.3. In general all of the distributions are similar. The global potential triggers seem to be skewed towards larger distances (5.3 A), the opposite of what we expect for real triggering. The potential triggers of sequences with at least 10 earthquakes are slightly less skewed, but have values beyond $10000 \mathrm{~km}$. The distribution of backazimuths for global potential triggers is different for small and large sequences, again suggesting some of the potential triggering is not real. Depth distributions show a similar percentage of shallow earthquakes, but more than $20 \%$ of potential triggers at $\geq 400 \mathrm{~km}$ depth.

The New Zealand potential triggers show a larger percentage of smaller distances (5.3 D), but still have $40 \%$ at greater than $500 \mathrm{~km}$ distance. The backazimuth of potential New Zealand triggers with small sequences (5.3 E) show a similar distribution to the overall earthquakes. The larger sequence triggers have more triggers from between 200-250 degrees, but this is not robust since there are only five sequences in that size range. 

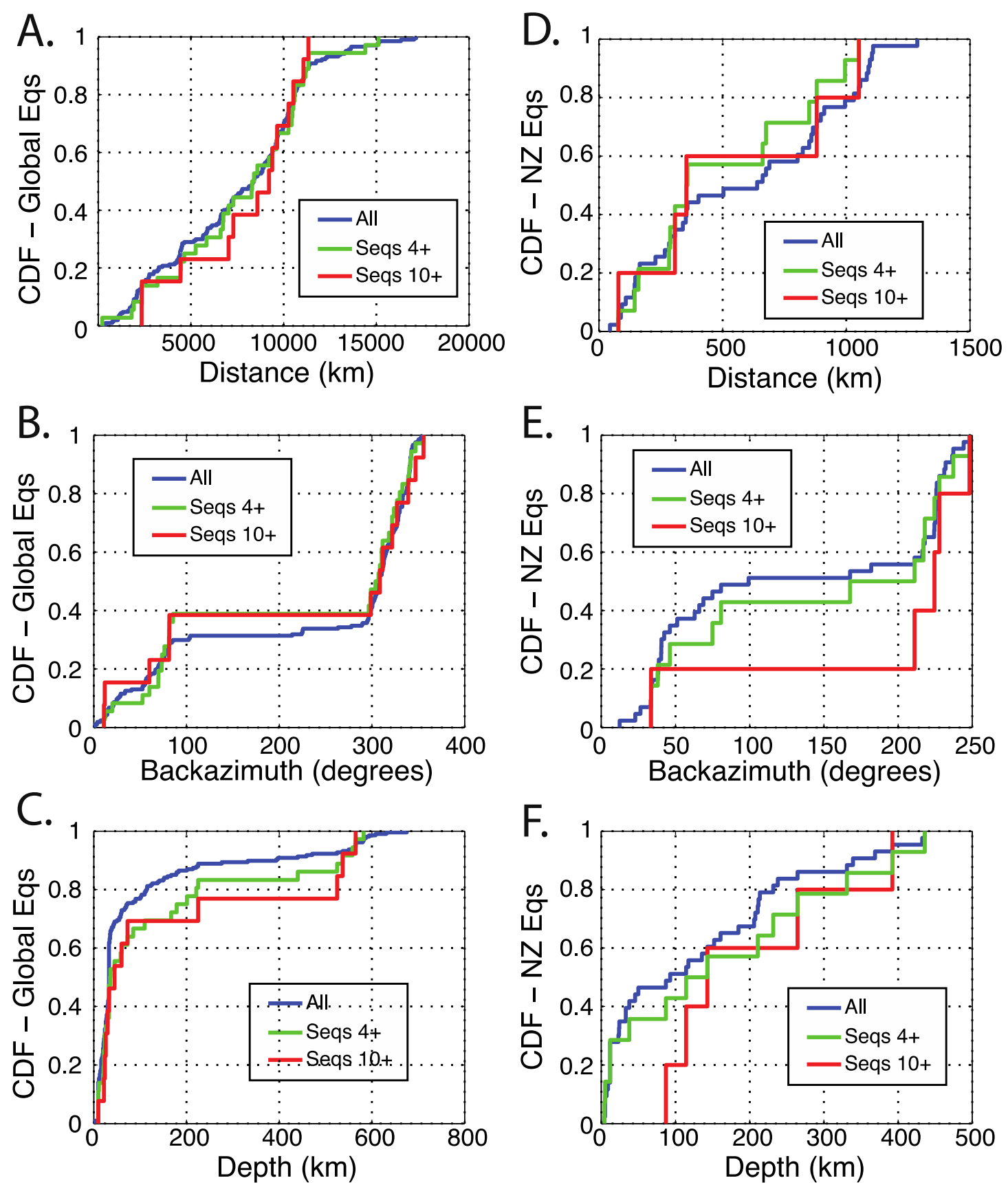

Figure 5.3 Cumulative Distribution Functions (CDF) of parameters for large earthquakes including potentially triggering events.

All plots show the population of all large earthquakes (blue) and potential triggers based on sequence size (green and red). A-C, are parameters from large global earthquakes $(M \geq 7.0)$ with A) Distance, B) Backazimuth, and C) Depth. D-F show CDF plots for large New Zealand earthquakes $(M \geq 6.0)$. 


\subsubsection{Discussion of triggering observations}

Up to $15 \%$ of sequences in the CVR may be triggered by either large global or NZ earthquakes. The percentage is likely to be substantially lower than that as some sequences will align randomly with the time windows around large earthquakes, but waveform level investigations of triggering are needed to define triggering thresholds and likely parameters of triggering earthquakes.

A one-day time window is insufficient to detect triggering at $\mathrm{Mc}=2.45$. Sequences of $\geq 20$ earthquakes are unlikely to be triggered, although we cannot rule out the possibility that large earthquakes may extend the duration of sequences already underway. While significant levels of triggering are observed for the global and NZ catalogues, the similarity between the parameter distributions for all earthquakes and the potential triggers suggests that the apparent triggering may not be real.

Prejean et al. [2004] notes that out of the hundreds of earthquakes identified as triggered in the Western US following the 2002 Denali Fault earthquakes, only 20 of the earthquakes in the first 30 minutes were located by routine processing. Thus waveform level investigation is needed to test whether the identified sequences are triggered. If they are found to be real instances of triggering, the identification of potentially triggered sequences will be another powerful application of the CURATE method that may lead to a better understanding of when and where triggering occurs. 


\subsection{SAMBA (sequences)}

The investigation here is my contribution to a study of swarm activity in a small area in the Central Southern Alps with fellow PhD student Carolin Boese (now graduated). I will use two references to her work. The first is a paper published about initial results and observations from the Southern Alps Micro-earthquake Borehole Array (SAMBA) installed for her project [Boese et al., 2012]. The other reference is to her parts of the work in this study that are presented in her thesis [Boese, 2012]. The work presented here is being developed into a publication and as such some of the text is necessarily joint work.

Boese [2012] observed temporal clustering of earthquakes in time and a high rate of earthquakes following the $2009 \mathrm{Mw} 7.8$ Dusky Sound earthquake, which occurred approximately $350 \mathrm{~km}$ to the south of the study area [Beavan et al., 2010]. An increase in earthquake rates was also observed by Boese [2012] after the $2010 \mathrm{Mw}$ 7.1Darfield earthquake, $175 \mathrm{~km}$ due east of the center of the SAMBA array [Gledhill et al., 2011; Quigley et al., 2012]. To try to identify distinct clusters Boese [2012] performed cross-correlation of waveforms. She found that few events have high cross-correlation coefficients $(\geq 0.8)$ and most events have values between 0.5 and 0.75. The sequences do not stand out from the background seismicity in terms of particularly high cross-correlation coefficients [Boese, 2012]. In order to better identify sequences I applied the CURATE method to this dataset and this allowed for an opportunity to better quantify the probability that the increased rate of earthquakes following the large regional earthquakes was random.

The collaborative study presented here sets out to identify individual sequences, establish the likelihood that sequences were triggered by regional earthquakes, and to compare triggered sequences to those that occur normally. For further information on 
peak-ground velocity and acceleration (PGV/PGA) and an investigation of possible physical mechanisms for the delayed triggering see Boese [2012]. My contributions to this study were application of the CURATE technique, the evaluation of probabilities, fitting Omori's law, contributing to references, and a general discussion of swarms and sequences.

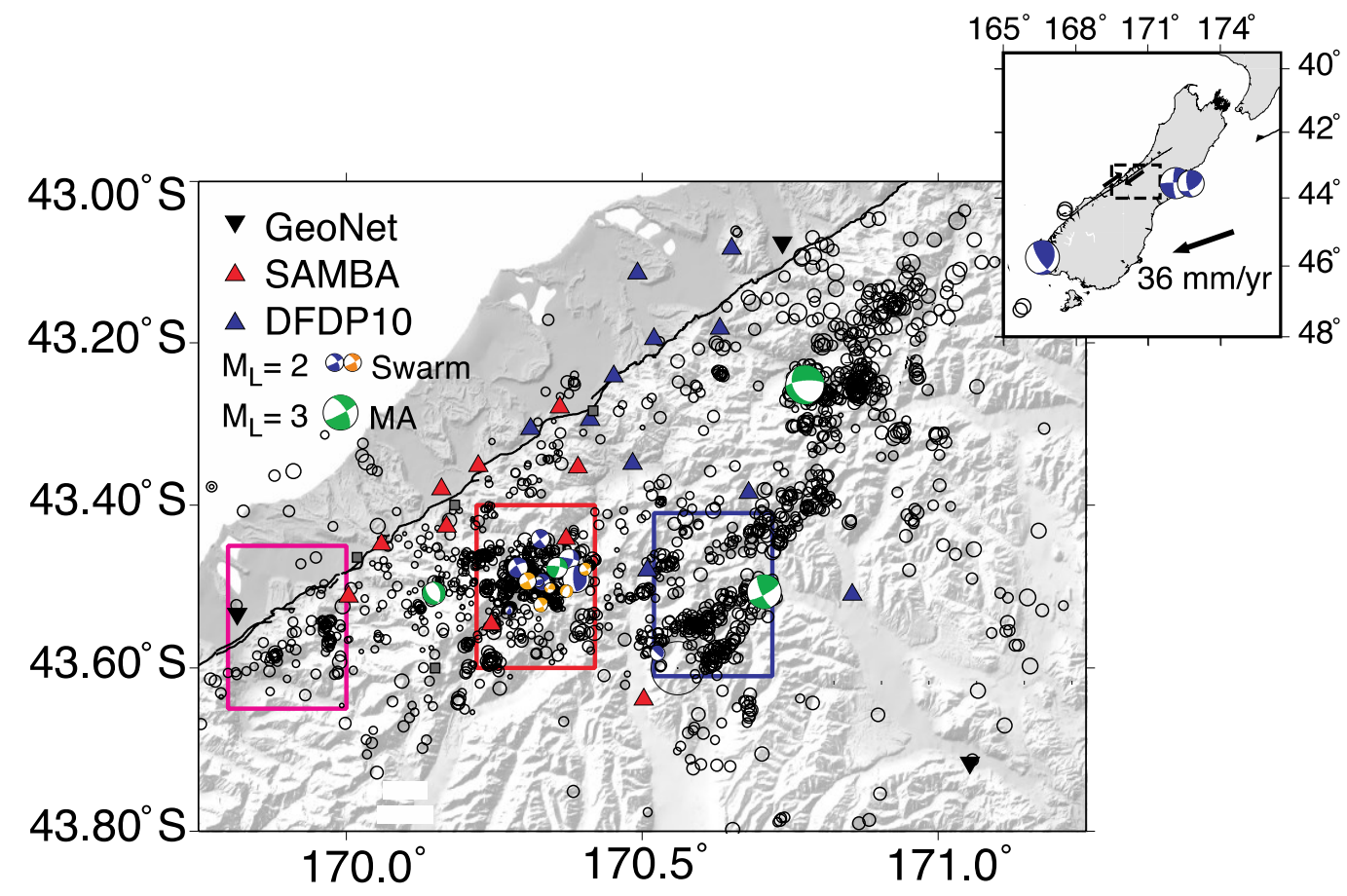

Figure 5.4 Location map for the SAMBA deployment.

Triangles show station locations from the permanent GeoNet (black) network, the SAMBA (red), and Deep Fault Drilling Project 2010 (DFDP10) (blue) temporary deployments. See Boese [2012] for further detail on when the deployments were operating. The three colored boxes show areas of interest described in the text, and with colors that match figure 5.8. Pink shows the Fox region, Red the most active area in the center of the SAMBA network, which also encompasses Mt. Cook, and blue the aftershock region of the Godley Valley earthquake (1984). The top inset shows the setting of the study area in the central South Island and focal mechanisms of large earthquakes: the 2009 MW 7.8 Dusky Sound earthquake approximately 350 km southwest and the 2010 MW 7.1 Darfield (Canterbury) and 2011 MW 6.3 Christchurch earthquakes $\sim 180 \mathrm{~km}$ east of the study area. Figure modified from Boese [2012]. 


\subsubsection{Sequence determination}

\subsubsection{CURATE application}

We ran the CURATE algorithm with this catalog as cross correlation of waveforms was inadequate to define sequences. Because the focus of this study was on waveform families, the CURATE algorithm was applied in a different way to the previous chapters. For this study we tested a number of distance and day rules to get the maximum number of similar waveform earthquakes, and we did not focus on obtaining a Poisson declustered catalog. Our best fitting parameters were a distance rule of $2.5 \mathrm{~km}$ and a day rule of 3 days.

We compared all events linked through cross-correlation with those events listed in the CURATE sequence catalogue. Those events chosen by visual inspection that clearly belong to one family form the events of the sequence catalogue. In other words, a sequence is identified based on seismicity rate-changes but the individual events in a sequence are selected on the basis of their waveform similarity. The final sequence catalogue is listed in Table 5.3. All sequences identified by the CURATE had at least one waveform family associated with them. The 'non-wff' column in Table 5.3 shows how many earthquakes were identified as part of a sequence by the CURATE method that were not part of the associated waveform family. Note that the waveform families are not limited by magnitude and so the families often contain more events than are observed in the CURATE sequences $(\mathrm{Mc} \geq 0.5$ and $1.0-$ see next section). 
Table 5-3 Sequence Catalog for the SAMBA and DFDP10 arrays (central Alpine Fault).

For delayed-triggered swarms the number in parenthesis after the start date shows the number of hours after the distant triggering earthquake. The "non-wff" label is the number of earthquakes identified as part of a CURATE sequence that are not part of the corresponding waveform-family. For Cross-Correlations the before and after columns report the number of waveform family members that occur before and after the duration of the identified CURATE sequence. The duration reported in the last column is given in days.

\begin{tabular}{|c|c|c|c|c|c|c|c|c|c|c|c|}
\hline \multicolumn{12}{|c|}{ Mainshock-aftershock sequences } \\
\hline \multirow[t]{2}{*}{ Start date } & \multicolumn{2}{|c|}{ Location } & \multicolumn{3}{|c|}{ CURATE } & \multicolumn{4}{|c|}{ Cross-Correlation } & \multirow[b]{2}{*}{ Mmax } & \multirow[b]{2}{*}{ Duration } \\
\hline & Latitude & Longitude & $M \geq 0.5$ & $M \geq 1.0$ & non-wff & total & before & after & $M \geq 0.5$ & & \\
\hline 12.03 .2009 & -43.494 & 170.321 & 9 & 4 & 0 & 16 & 2 & 0 & 14 & 2.4 & 4.5 \\
\hline 14.08.2009 & -43.254 & 170.778 & 10 & 9 & 0 & 15 & 1 & 3 & 15 & 4.2 & 2.1 \\
\hline 09.11 .2009 & -43.512 & 170.700 & 10 & 10 & 0 & 22 & 2 & 2 & 20 & 3.7 & 9.3 \\
\hline 28.08 .2010 & -43.508 & 170.152 & 12 & 6 & 0 & 11 & 0 & 0 & 10 & 2.6 & 2.7 \\
\hline \multicolumn{12}{|c|}{ Background swarms } \\
\hline 24.05 .2009 & -43.481 & 170.362 & 18 & 7 & 0 & 19 & 0 & 0 & 19 & 2.6 & $<1$ \\
\hline 26.07.2009 & -43.487 & 170.283 & 8 & 0 & 4 & 10 & 2 & 2 & 8 & 2 & 1.8 \\
\hline 29.07.2009 & -43.528 & 170.125 & 5 & 4 & 0 & 30 & 0 & 1 & 17 & 2.2 & 157 \\
\hline 12.09 .2009 & -43.442 & 170.331 & 6 & 5 & 0 & 17 & 2 & 6 & 13 & 2 & 37.3 \\
\hline 22.09 .2009 & -43.525 & 170.275 & 8 & 0 & 0 & 28 & 0 & 1 & 27 & 1.6 & 30 \\
\hline 16.12.2009 & -43.496 & 170.326 & 16 & 4 & 0 & 24 & 0 & 0 & 20 & 1.9 & 1.3 \\
\hline 29.01 .2010 & -43.493 & 170.323 & 9 & 8 & 1 & 11 & 2 & 0 & 11 & 1.1 & $<1$ \\
\hline 30.03 .2010 & -43.497 & 170.382 & 7 & 7 & 0 & 13 & 2 & 0 & 13 & 2.8 & 11.4 \\
\hline 29.08 .2010 & -43.571 & 170.501 & 9 & 0 & 2 & 21 & 0 & 0 & 19 & 2 & 9.7 \\
\hline \multicolumn{12}{|c|}{ Delayed-triggered swarms } \\
\hline 17.07.2009(8) & -34.493 & 170.303 & 17 & 11 & 1 & 35 & 0 & 3 & 22 & 2.8 & 3.8 \\
\hline 16.07.2009 (20) & -43.472 & 170.400 & - & - & - & 17 & 0 & 5 & 9 & 1.4 & $<1$ \\
\hline 18.07.2009 (70.6) & -43.522 & 170.330 & 30 & 11 & 15 & 47 & 2 & 2 & 21 & 1.8 & 7.9 \\
\hline $03.09 .2010(6.4)$ & -43.492 & 170.369 & 21 & 8 & 9 & 17 & 0 & 0 & 14 & 1.5 & 1.6 \\
\hline 04.09 .2010 (7.3) & -43.462 & 170.260 & 6 & 0 & 2 & 11 & 0 & 0 & 9 & 1.3 & 1.6 \\
\hline
\end{tabular}

\subsubsection{Sequence Identification}

During the search for sequences, we apply two magnitudes of completeness

thresholds: $\mathrm{Mc}=1$, the cut-off magnitude for the whole study area (Fig. 5.5 b), and Mc $=0.5$, the cut-off magnitude within the center of the SAMBA network (Fig. 5.4, red rectangle) where the majority of the sequences occur. Similar to the process in section 4.1, sequences are classified as mainshock-aftershock (MS-AS) sequences if the largest event occurs at the beginning of the sequence ( $\leq 20 \%$ of the events) and have a magnitude difference between the two largest events (Mdiff) of at least 0.5.

Sequences that do not fit both these criteria are considered swarms. Like our earlier catalogs, most of the sequences in this study with small Mdiff values have their 
largest magnitude event late in the sequence. This makes the sequences relatively insensitive to the exact MS-AS definition. The percent of events is smaller than our value of $30 \%$ used in section 4.1 . Using the first $30 \%$ of events affects just a single sequence, but we felt that the smaller Mc value warranted a tighter constraint (20\%) on the percent of events.

In total, we obtained 18 sequences comprising $\geq 10$ events of similar waveforms (Table 5.3). These sequences account for $15.3 \%$ of the total number of recorded earthquakes above the cut-off magnitude $(\mathrm{Mc}=1.0)$. We exclude from our analysis five sequences containing fewer than ten similar events because there may be more of these smaller sequences that have not been identified due to detection issues. These smaller sequences occur throughout the study area. Only four of the eighteen sequences are MS-AS sequences. All but four of the sequences (three MS-AS sequences and one swarm), occur in the center of the SAMBA network (Fig. 5.4 red rectangle), an area of $10 \times 12 \mathrm{~km}^{2}$. The only swarm outside the center of the SAMBA network occurs in the aftershock zone of the 1984 Godley Valley earthquake (Anderson and Webb, 1994). The MS-AS sequences have on average larger Mmax $\left(M_{L}\right.$ 2.4-4.2) than the largest event in each earthquake swarm $\left(M_{L} 1.1-2.8\right)$, similar to the findings for other regions in section 4.1. 

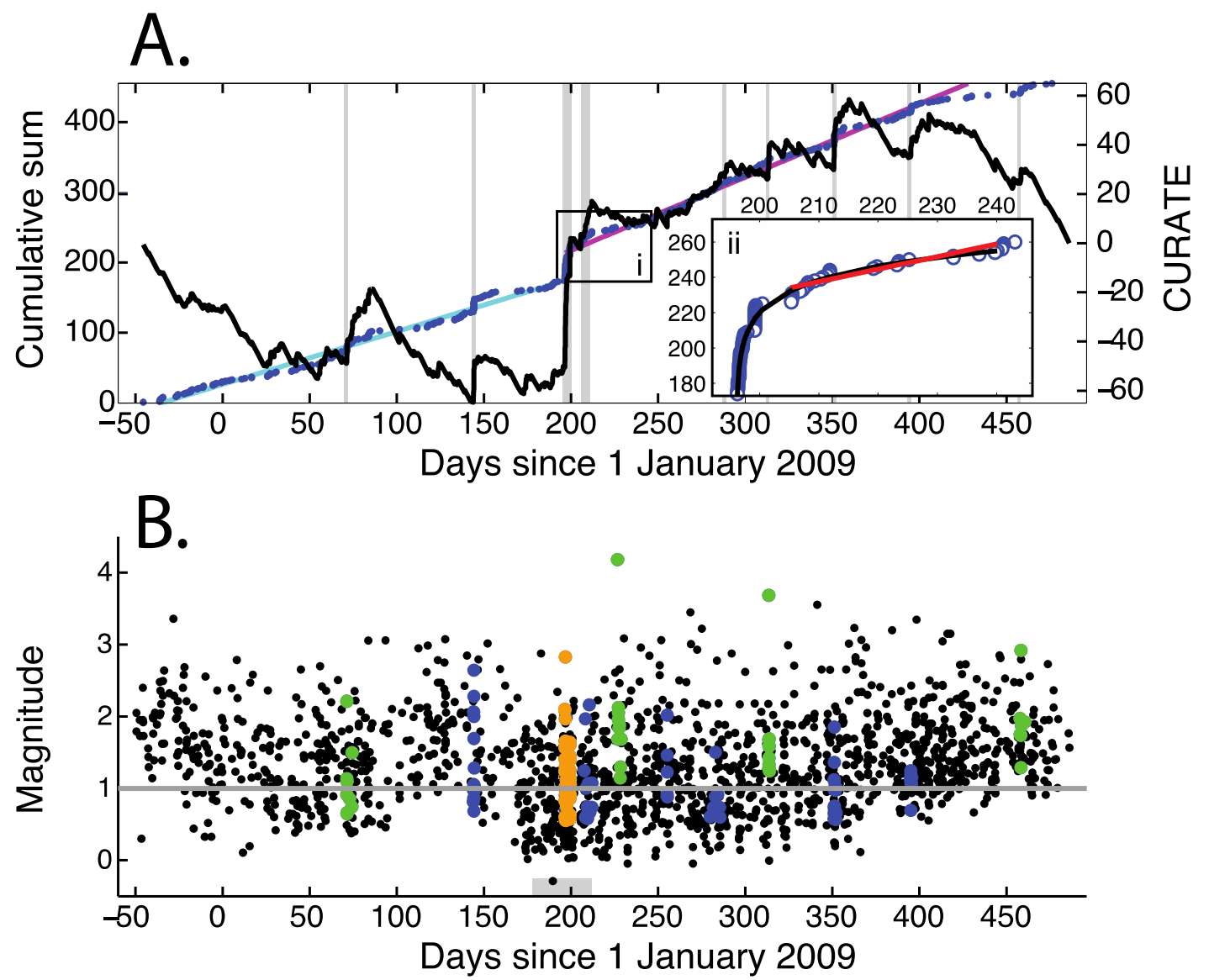

Figure 5.5 Seismicity with time details for the earthquakes in the SAMBA network. (A) Cumulative sum (blue) and CURATE (black) of earthquakes recorded on the SAMBA array between November 2008 and April 2010 for $\mathrm{Mc}=0.55$. Vertical grey lines mark times of the sequences listed in Table 5.3. The black box labeled ' $i$ ' defines the region shown at a larger scale in inset 'ii', which shows the cumulative sum of events after the Dusky Sound earthquake with a fit of an Omori's law (black line) and a constant linear rate obtained from regression (red line). Within a period of 45 days the Omori law fits the data better than any linear fit. (B) Magnitudes of earthquakes recorded in the study area versus time. Sequences of events that were identified in (A) are colored with background swarms in blue, delayed-triggered swarms in orange and mainshocks in green. Note that some of the mainshockaftershock sequences are not within the SAMBA network and are therefore not shown in (A). The horizontal line marks the cut-off magnitude of $\mathrm{Mc}=1$. The grey box indicates the period when events were identified on spectrograms of station WHYM.

\subsubsection{Probability Calculations}

Within the first twenty-four hours after the Dusky Sound earthquake, 146 micro-earthquakes occurred in the central Southern Alps region, the highest number 
of events per day recorded since the installation of the SAMBA array. The triggered seismicity commenced with the passage of the surface-wave trains and continued for approximately five days. It comprised three delayed-triggered swarms (orange in Fig. 5.4) in the center of the SAMBA array. The first and most energetic delayed-triggered swarm (ML $\leq 2.8)$ occurred eight hours after the passage of the surface waves of the Dusky Sound earthquake. This swarm continued for several days, with a second burst occurring 4 days later in the same location. The second swarm started 20 hours after the Dusky Sound earthquake, $8 \mathrm{~km}$ northeast of the first swarm. A third swarm started 70.6 hours after the passage of the surface waves at a distance of $3.7 \mathrm{~km} \mathrm{SE}$ and 7.7 $\mathrm{km} \mathrm{SW}$ of the first and second swarms, respectively.

Elevated seismicity rates in the study area were also observed after the $\mathrm{Mw}$ 7.1Darfield earthquake, $175 \mathrm{~km}$ due east of the center of the SAMBA [Gledhill et al., 2011; Quigley et al., 2012]. The triggered events were not as well recorded as following the Dusky Sound earthquake (only 53.4\% of the events could be located), because three out of ten SAMBA stations were not operating at the time following a particularly harsh and snowy southern hemisphere winter. As after the Dusky Sound event, the triggered seismicity commenced with the arrival of the surface waves and continued for two days. Two delayed-triggered swarms occurred 6.4 and 7.25 hours after the passage of the surface waves in the area where delayed-triggered swarms occurred after the Dusky Sound earthquake.

To show that the sequences following large earthquakes are likely to have been triggered, we calculate the probability of recording more than a given number of sequences in a short period of time. For this calculation, we only use sequences recorded inside the center of the SAMBA network with at least six earthquakes above the cut-off magnitude $\mathrm{Mc}=0.5$ (11 sequences). The average time between these 11 
sequences is 37 days. Note that this is the actual average time between sequences, rather than the total time divided by the number of sequences, which would give a longer mean time as it includes time intervals at the beginning and end of the catalogue in which sequences were not observed. The probability of observing a given number of sequences $\mathrm{n}$ then becomes,

$$
\mathrm{P}(n)=x^{n} / n ! \exp (-x)
$$

where $x$ is the time window of observation over $n$ ! the mean time between sequences (5/37). The Poisson probability of observing a number $\geq n$ is then

$$
\mathrm{P}(n)=1-[\mathrm{P}(0)+\mathrm{P}(1)+\ldots \mathrm{P}(\mathrm{n}-1)]
$$

Thus, the Poisson probability of observing at least one sequence in any fiveday window (1- $\mathrm{P}[0]$ )is $12.7 \%$. The probability of observing two swarms or more within five days after a large earthquake, but unrelated to it, is $0.8 \%$. Therefore, this analysis suggests that there is only a small chance of observing several background swarms by coincidence.

We now differentiate earthquake swarms as either background swarms, which occur randomly in time, or delayed-triggered swarms that occur several hours to days following a large earthquake and are indirectly caused by the surface waves of this event.

Given that the probabilities for observing several sequences by chance is small and that we observed delayed-triggered swarms repeatedly after large earthquakes, this suggests that earthquake triggering is a common phenomenon in the Southern Alps. 


\subsubsection{Fitting Omori's law}

Another observation relating to the number of earthquakes recorded by the SAMBA array after the Dusky Sound earthquake was a clear decay with time (5.5A). The decay looks like typical Omori's law. Husen [2004] and Brodsky [2006] both show that after other large earthquakes, there was an overall decay of triggered activity that could be fit with Omori's law decay.

We used the relative variance between a linear fit (constant rate) and the Omori decay model to test the goodness of fit. We cannot use traditional cumulative number tests like Kolmogorov-Smirinov (KS test) test because the model is made from the data and is not an independent distribution. The other standard way of testing for a decay, used by both Husen [2004] and Brodsky [2006], is a linear fit of $n(t)$ in logspace. This requires some binning of earthquakes in time. We did not think that we had a sufficient number of data to do that type of fitting.

We compared an Omori fit with several linear rate fits. Using the same maximum likelihood method described in section 4.3, we obtained a best fitting cvalue of 0.35 days, and a corresponding $K$-value of 14.4 earthquakes/day $(K=1 / \ln$ $(1+\operatorname{tmax} / c))$. To test the fit, we compare the sum of the residuals for the Omori's law decay with the residuals from linear fits. The residuals of the Omori and linear models are compared from five days to the end time of the simulation, and over a range of simulation times from 20-70 days. Linear fits are calculated using MATLAB robustfit for pre-Dusky Sound slope (0.75), post-Dusky Sound slope (1.02), and over the time period of residual comparison (slope $=0.71$ for time period up to 45 days).

The linear fit over the specific time period of interest (varying 20-70 days) always had a better fit than either the slope from before or after the DS earthquake. 
Fig 5.6 shows the cumulative residuals and fits for three different time ranges. The linear fit has a better fit at very short time periods (clearly shorter than the observed decay) and the Omori model fits better than all three linear models until 45 days after the Fiordland earthquake. On the forty-fifth day another sequence occurs, after which the residuals of the best linear fit are lower than the Omori residuals. This shows that delayed-triggered earthquakes are 'caused' by an underlying process that exhibits decay. Despite the good fit of the observed decay, the spatial evolution of the earthquakes occurs in distinct clustering that is not often observed in aftershock sequences.

A.

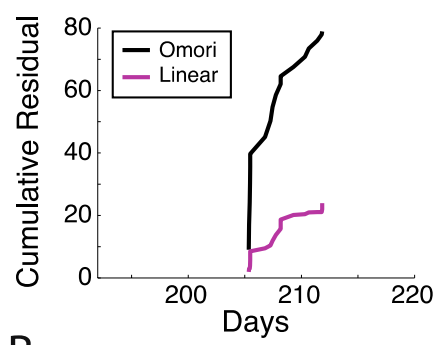

B.

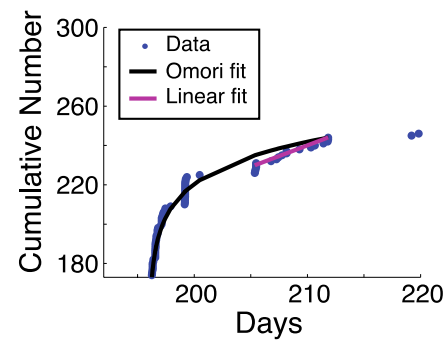

C.

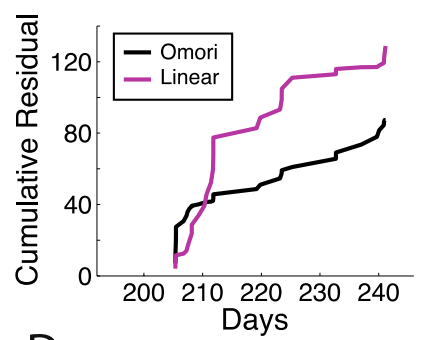

D.

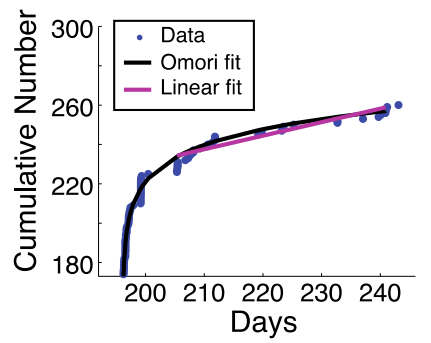

E.

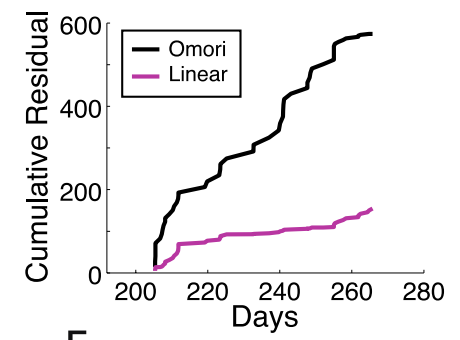

F.

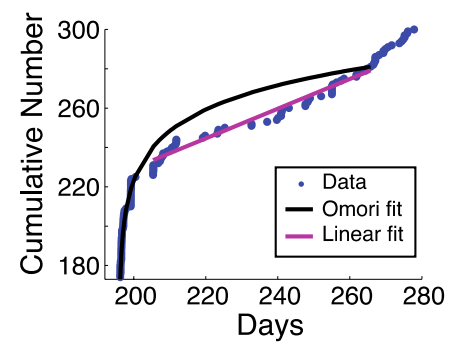

Figure 5.6 Earthquake rate fitting for the SAMBA catalog following the Dusky Sound earthquake.

Comparison of an Omori's law fit (black) and a linear best fit (magenta) to the earthquakes in the SAMBA catalogue following the Dusky Sound earthquake (blue dots). The days are marked as in Fig. 5.5. All of linear fits and the residual comparison start from five days after the Dusky Sound earthquake, although a hiatus in earthquake occurrence means the start of the fit is pushed to nearly 10 days. The top and bottom panels are pairs of figures for fits and cumulative residuals that end AB) 20 days, C-D) 45 days (best residual improvement for Omori's law, E-F) 70 days after the Dusky Sound earthquake. Note another hiatus in events roughly from day 211-219 shortens the overall length of the '20-day' fit. 


\subsubsection{Inter-event times}

The delayed-triggered swarms do not differ significantly from the background swarms in terms of their magnitude and depth distributions, focal mechanisms or inter-event time patterns except that there are more swarms within a shorter time span immediately following the Dusky Sound and Darfield earthquakes [Boese, 2012].

The inter-event time patterns obtained for MS-AS sequences should be a continuous decay. To demonstrate this, we calculated synthetic average inter-event time for an Omori's law decay with parameters $\mathrm{p}=1, \mathrm{c}=0.5 \mathrm{t}$, and $\mathrm{K}=10 \mathrm{n} / \mathrm{t}$ (Fig. 5.7A). The Omori's decay shows a steady increase in the average inter-event time with the cvalue controlling the intercept and contributing to the slope, and the K-value having the largest influence on the slope (Section 4.4.5.1). For sequences that we have identified as mainshock-aftershock type, we do observe an average inter-event time that is dominated by a decaying-rate (Fig $5.7 \mathrm{~B}, \mathrm{I})$. The observed swarms, however, all show a sharp decrease in the inter-event times mostly at the beginning of the sequence (Fig 5.7B, II-III). 
A. Synthetic average inter-event time I. Mainshock-Aftershock (Omori's law)

B. Observed average inter-event times
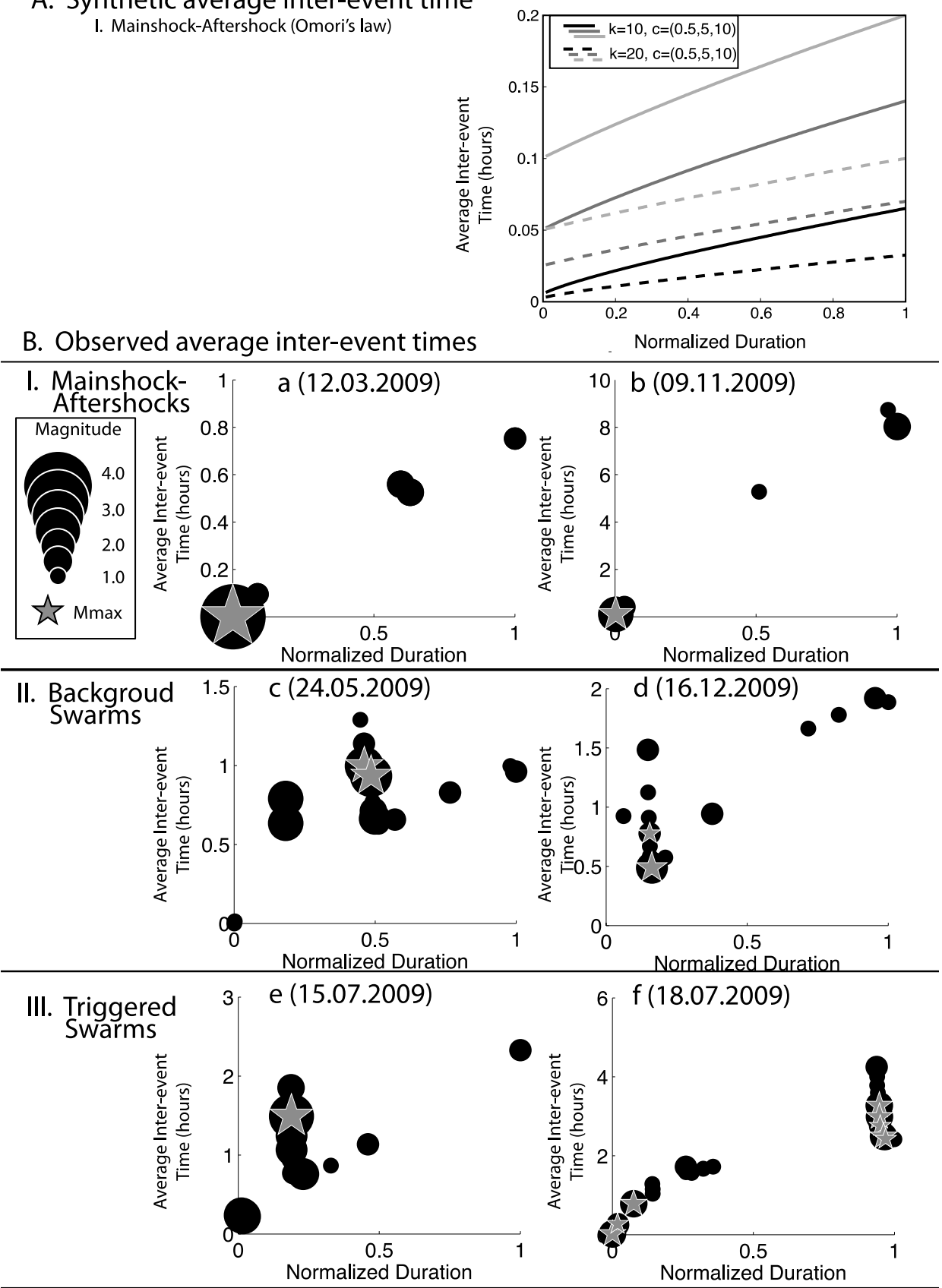

Figure 5.7 Synthetic and Observed average inter-event times for sequences in the SAMBA network.

A) Synthetic average inter-event times versus normalized duration calculated for a modified Omori-law with different parameters. The c-values are reported in hours and correspond to fractions of the duration of .002, .2, .4. The range of values is intended to show the influence of the c-value and not necessarily anticipated values. B) observed average inter-event time versus normalized duration in the central Southern Alps for mainshock-aftershock sequences (I), background swarms (II), and delayed-triggered swarms (III). The symbol size represents the magnitude of the events. All events with magnitudes within 0.5 units of Mmax are marked by stars. 


\subsubsection{Triggering investigation in the long term catalogue}

Although the swarms analyzed in this study occur predominantly in the center of the SAMBA network (Fig. 5.4, red rectangle), two large swarms known as the Mt. Cook and Fox swarms previously occurred outside this central area, but within the general SAMBA study area [Leitner et al., 2001; O'Keefe, 2008]. To extend the search for sequences and potential triggered events, we analyzed the seismicity in the GeoNet catalogue between 1993 and 2008 for different subregions throughout the study area. Note that a station upgrade of the GeoNet stations was undertaken in 2003 so apparent changes in the catalogue around this time could be an artifact of the network upgrade.

We compared this long-term seismicity record to the PGA values recorded at Haast (approximately $85 \mathrm{~km}$ southwest of the array) for large earthquakes similar to the Dusky Sound and Darfield earthquakes (Fig 5.8). There is no obvious indication of remotely-triggered seismicity commencing shortly after a large earthquake. However, the triggered swarms recorded by the SAMBA network in 2009 and 2010 are not listed in the GeoNet catalogue because of their small magnitudes. Therefore, as suggested in section 5.1, the cut-off magnitude of the GeoNet catalogue of Mc 2.6 [Petersen et al., 2011] is probably insufficient to establish whether triggering occurred previously in this area. 

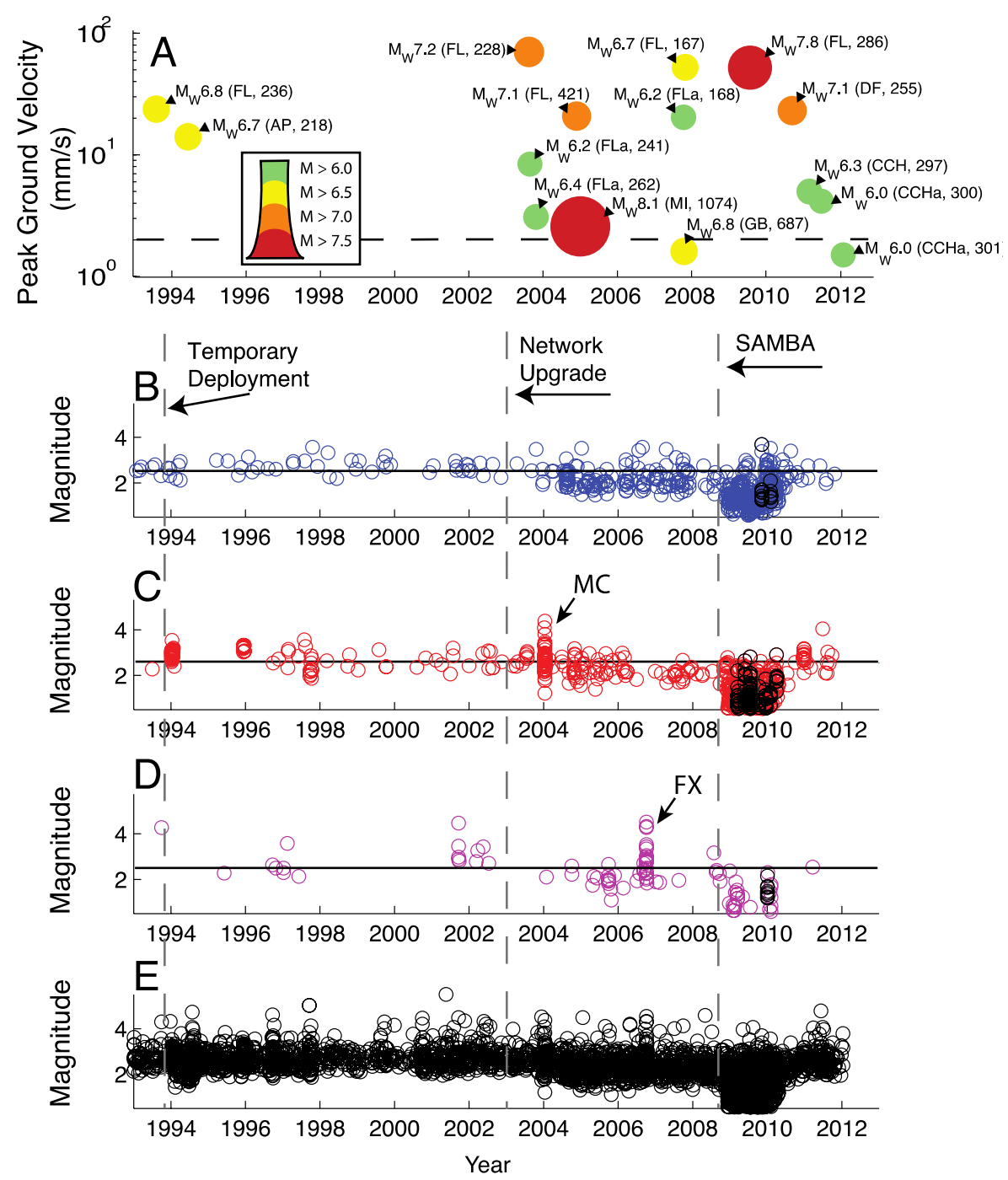

Figure 5.8 Search for triggered sequences in the central Southern Alps region after large earthquakes.

(A) Peak ground velocities (PGV) of major New Zealand earthquakes from Fiordland (FL), Arthur's Pass (AP), Macquarie Island (MI), Gisborne (GB), Darfield (DF) and Christchurch $(\mathrm{CCH})$ and their aftershocks recorded at Haast ca. $85 \mathrm{~km}$ southeast of the centre of the SAMBA array. The distance (in $\mathrm{km}$ ) of these events from the station at Haast is given in the brackets. The horizontal dashed line indicates the threshold proposed by Brodsky and Prejean [2005] above which triggering is independent of the waves' amplitude, duration and energy-density but dependent on the frequency of the waves. (B-D) Seismicity versus time for the subregions as shown in Fig. 5.4. Black circles indicate sequences detected in this study, horizontal solid lines show the Mc of the GeoNet catalog. Three vertical dashed lines mark a temporary seismometer deployment, an upgrade in the GeoNet catalog, and the installation of the SAMBA array. Annotated arrows show the Mt. Cook (MC) and Fox swarms (FX) and changes in the station network. The daily seismicity rate is $0.86(\mathrm{~B}), 1.66(\mathrm{C})$ and 0.45 (D) events/30 days/1000 km2 for the GeoNet catalogue (Mc 2.6) between 1993-2007. (E) Seismicity versus time for the whole study area. 


\subsubsection{Discussion}

The detection of low-magnitude earthquake swarms strongly depends on the local station network and its detection threshold [Frankel et al., 1980]. With the dense station networks in the central Southern Alps, we have recorded 18 sequences with event magnitudes $-0.5 \leq \mathrm{M}_{\mathrm{L}} \leq 4.2$ between November 2008 and September 2010. Of these, five swarms were remotely triggered and occurred with several hours delay after the arrival of the surface wave of the triggering event. The delayedtriggered swarms occur in the same area where background swarms occur frequently, consistent with observations elsewhere (e.g. [Hill et al., 1993]). The delayed-triggered swarms are similar to the background swarms in terms of waveform similarity, duration, inter-event time patterns, magnitude and depth distribution but they consist of more events.

Because dynamic triggering occurred twice within two years in the center of the SAMBA array, we conclude that earthquake triggering is a common phenomenon in the Southern Alps. The lack of triggering observations in the long-term record is almost certainly an effect of the high Mc value in the GeoNet catalog. This suggests that New Zealand may experience more triggering than is currently recognized. The creation of a sequence catalog with the CURATE method allows a quick way to determine probabilities of occurrence that is not easy if performed on an earthquake level, because background rates and the number of earthquakes triggered by a particular event may be variable.

Similar waveform groups are thought to be similar due to similar mechanisms and extreme spatial proximity (e.g. [Nadeau et al., 1995]). Swarms often occur over large areas (tens of $\mathrm{km}^{2}$ ) and therefore contain either no similar waveform families, or many different waveform families. This apparent contradiction raises some questions 
of causality. Does a single instance of a physical mechanism trigger all earthquakes in a swarm? Or do multiple instances of the same or different physical mechanisms cause separate groups of earthquakes within a single sequence? We cannot answer that question definitively, but a similar study by [Parotidis et al., 2003] found that both a swarm at large, and individual bursts within it, all had evidence of fluid diffusion. The occurrence of multiple families, or additional non-family events in the observed swarms with a small distance rule $(2.5 \mathrm{~km})$ suggests that waveform families may show similarity, solely because of the place they occur rather than the process that produces them.

Brodsky [2006] states that all delayed triggered earthquakes (those after the passage of the surface waves) may be considered as aftershocks of events that were triggered during the passage of the surface waves. She suggests that earthquakes up to $\mathrm{M}=3$ may be hidden in the noisy period of aftershocks that accompany the passage of the surface waves at Long Valley and that the number of observed delayedtriggered earthquakes fits the expected abundance for the hidden events. The directtriggered earthquakes following the Dusky Sound event are small in number and magnitude [Boese, 2012]. In contrast the three delayed-triggered-swarms are larger than others recorded during the observation period. Although small magnitude earthquakes may have larger magnitude aftershocks, that is not often the case. Another important characteristic of aftershocks is that they occur in a scattered spatial pattern with little development in distance from the mainshock, or migration with time (e.g. [Scholz, 2002] p. 225).

Our observations are most similar to Husen et al. [2004], who found that in Yellowstone, earthquakes show very few direct waveform triggered earthquakes and show distinct spatial clustering that is not typical of aftershock sequences. Husen et 
al. [2004] does not explore the reason for the disconnect between the observed temporal decay and spatial development. The fit of our data to Omori's law implies decay, but the spatial development of the triggered earthquakes suggets that the decay is driven by a different process than that governing true aftershock decay. Boese [2012] suggested that one possible reason for the delay was the release of fluids from a source north of the triggered swarm locations. The modeled 2-D diffusion of fluids from a reservoir broken by the passage of the surface waves is one possible mechanism for the timing delayed triggered swarms.

The difference in the observed inter-event time patterns for MS-AS sequences and swarms (Ch. 4.4) suggest that the processes underlying these two types of sequences are distinct and different, although the decay portions (increasing interevent times) may be similar. Toda et al. [2002] similarly suggested that differences in increase in the stressing rate are responsible for the different earthquake rate changes observed during the Izu islands swarm in 2000. While MS-AS sequences result from a step in the stressing rate, the swarms may represent a gradual increase in the stressing rate. Several swarms show that the largest events occur in the transition from decreasing to increasing inter-event times (Ch. 4.4.5.2, Fig. 4.36; Fig 5.7). This may indicate that the stress release corresponding to the stressing rate increases before the start of a decay process. While most of the swarms we observed from the SAMBA array exhibit the increasing inter-event times at the beginning of the sequence, not all swarms follow this pattern as strictly. A continuum of types between swarms and MSAS sequences exists [Sherburn, 1992b; Vidale and Shearer, 2006] and, as discussed throughout this thesis, swarm sequences likely represent multiple physical processes. 
In order to establish whether the triggering significantly altered the periodicity of swarms, we need a longer history of swarm activity in this region. The SAMBA network is still recording, so a longer-term analysis will be possible.

\subsection{Investigation of potential triggering by slow slip events}

\subsubsection{Previous seismic and slow slip observations in New Zealand}

Slow slip events (SSEs) are another possible trigger mechanism. Slow slip is the phenomenon of slip on a fault (often a subduction zone plate interface) that occurs over days to years, much slower than typical earthquake slip. Slow slip has been observed along both faults (e.g. [Linde et al., 1996]) and subduction zones (e.g. [Dragert et al., 2001; Peterson and Christensen, 2009]). SSEs have been shown to trigger earthquakes close to the slip area [Delahaye et al., 2009; Linde et al., 1996; Ozawa et al., 2003; Segall et al., 2006]. The triggered earthquakes occur within or on the edges of the slip patches and are often inferred to be due to static stress changes or fluids rather than dynamic mechanisms attributed to long-range triggering. These earthquakes take up a small portion of the total seismic moment with the highest value reported at $\sim 1 \%$ [Wallace et al., 2012]. Investigating possible triggering of local earthquakes by slow slip is important for two main reasons. Firstly if earthquake sequences accompany local slow slip events, they may be able to give us clues about the mechanism of slow slip and lead to a better understanding of the links between slow slip and locking along the plate interface. The second is that if slow slip influences the timing of earthquake sequences, like long-range triggering, it may be important information for earthquake forecasting models.

Due to the limited time frame that continuous GPS (cGPS) has been operating in New Zealand and worldwide it is difficult to make inferences about the recurrence times or patterns of SSEs and what their relationship is to the larger earthquake cycle 
of the subduction zone. Wech and Creager [2011] suggest slow slip may be a manifestation of progressive loading from the down to up-dip limit of the subduction interface. SSEs have been recognized in New Zealand since 2002 when an SSE occurred on the Raukumara peninsula close to Gisborne [Beavan et al., 2003]. Beaven et al. [2007] noted that due to the short duration of some SSEs, campaign GPS utilized prior to that time was not frequent enough to isolate them. More recently continuous GPS (cGPS) measurements have been made, and those observations are a key to identifying sequences related to slow slip [Petersen et al., 2011]. Following the densification of the cGPS network, starting in 2002, another SSE occurred in 2004 at very similar location to the Gisborne 2002 SSE (e.g. [Beavan et al., 2007; Douglas et al., 2005]). Since then more than twenty documented SSEs have been reported in the North Island of New Zealand (e.g. [Wallace and Beavan, 2010; Wallace et al., 2012]). There are four major regions where SSEs are observed in the North Island: Manawatu, Kapiti, Gisborne, and Hawkes Bay (Fig. 5.9). They mainly occur on the boundary between the locked and slipping portions of the margin as modeled by Wallace et al. [2004]. However, observations in Wallace et al. [2012] have identified two SSEs occurring within the locked region, a Cape Turnagain SSE in 2011 and a smaller event south of that in December 2009 that we refer to here as the 'Riversdale' SSE. These observations raise questions about the mechanisms of SSEs and the definition and manifestation of a 'locked' plate interface.

Recent observations of tremor near the Alpine Fault by Wech et al. [2012] may also indicate that the Alpine Fault also experiences some degree of slow slip. If slip exists, it has not been constrained, and so we will focus in this section on the better-documented seismic and geodetic features in the North Island. 


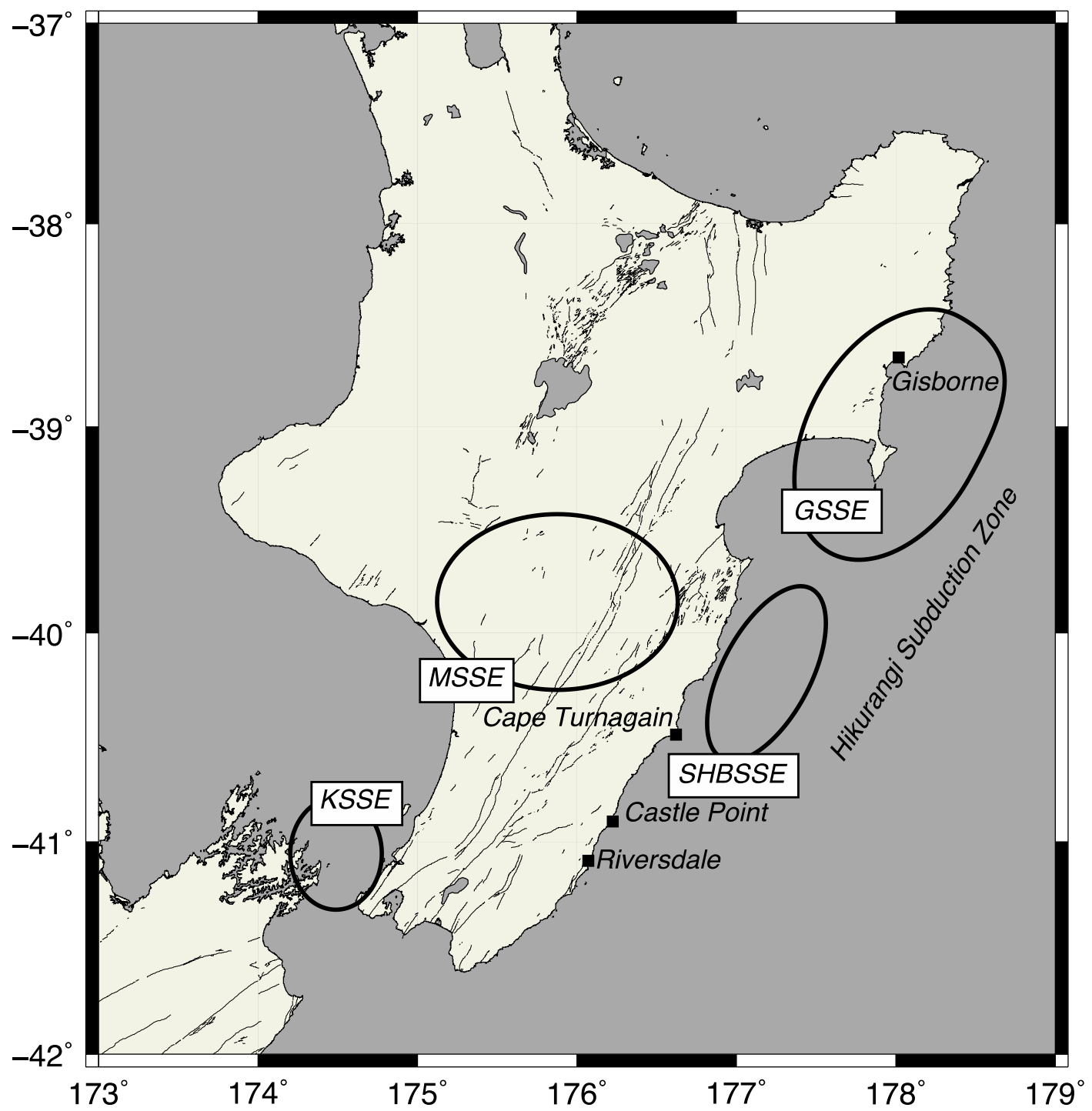

Figure 5.9 Map of main slow-slip regions and related locations.

Ellipses show the four main slow-slip regions: Gisborne (GSSE), Manawatu (MSSE), Southern Hawke's Bay (SHBSSE), and Kapiti (KSSE).

\subsubsection{Relationship to Seismicity (past observations)}

Low frequency tremor is probably the most common seismic observation accompanying slow slip (e.g. [Ide, 2012]). Despite attempts to find tremor along the Hikurangi margin, only earthquakes had been observed with SSEs in New Zealand until recently [Delahaye et al., 2009; Ide, 2012; Kim et al., 2011]. Seismicity has also been observed to accompany SSEs along the San Andreas Fault (e.g. [Linde et al., 1996]), Kilauea (e.g. [Brooks et al., 2006]), and the Boso Penninsula in Japan (e.g. 
[Ozawa et al., 2007]). In most cases the earthquakes are relatively small magnitudes

( $\leq 3.7$ ), but one SSE in the Boso Penninsula had an $\mathrm{M}_{\mathrm{L}}=5.3$ [Ozawa et al., 2007]. A recent study by Kim et al. [2011] was able to identify some tremor and seismicity associated with the 2010 Gisborne SSE. They concluded that both in the Hikurangi margin and the Boso Penninsula in Japan that thick layers of attenuating sediments may prevent the routine location of tremor observed in other subduction zones [Kim et al., 2011]. Several authors have shown probable links between an observed seismic sequence and a concurrent slow slip event in New Zealand. Reyners and Bannister [2007] document an earthquake sequence in Upper Hutt in 2004 and 2005 related to the Kapiti 2003-2004 slow slip event (Beavan et al. [2007]). They observe a swarm of normal faulting events in the subducting plate in April and May 2004 that contained four $M \geq 4.0$ earthquakes and numerous smaller ones. This swarm was followed by a $\mathrm{M}_{\mathrm{L}}=5.5$ earthquake and aftershocks in January 2005. All the events were relocated using waveform-based double difference techniques and show that the swarm took place on a plane about $1 \mathrm{~km}$ above the plane of the subsequent M 5.5 fault plane [Reyners and Bannister, 2007]. They argue that both these planes are in a region of increased Coloumb stress due to the occurrence of the Kapiti 2003-2004 slow slip event downdip on the interface. They also note that there may be frictional differences between the plate interface itself and within the top of the subducted plate as the Coloumb stress change should also have been favorable for triggering small thrust events on the interface even though none were observed.

However, such thrust events were observed by Delahaye [2009] in a search for tremor around the time of the Gisborne 2004 slow slip event. Their study searched a seven week time period of continuous broadband data spanning the 2004 Gisborne slow slip event. They found no tremor, but did find an apparent increased rate of 
microseismicity $(\mathrm{M}<2.0)$. They concluded that this seismicity is too small to be detected during current routine processing.

Robinson [2003] re-analyzed the Weber 1990 earthquake pair as potential evidence for slow slip. The Weber earthquakes $(\mathrm{M}=6.2$, and 6.4) occurred in Feb 19 1990 and May 131990 and subsequent $M=5.5$ earthquakes occurred in August 1990 and March 1992 [Robinson, 1994]. While there are not sufficient geodetic measurements over this time to look for slow slip, Robinson [2003] postulates that the activity may have been triggered by a SSE. A similar sequence was observed in 1942 [Robinson, 1994; 2003] further south along the margin, suggesting that this type of activity may occur regularly.

Recently, Wallace et. al 2012 showed that there was a series of earthquakes accompanying a slow slip event off Cape Turnagain in 2011. They point out other increases in seismicity in the catalog further back in time in 2006 and possibly 2001 and 2002, ([Wallace et al., 2012] figure 11). While the smaller Riversdale 2009 SSE overlaps with the Cape Turnagain 2011 SSE, Wallace et al. [2012] state that there is no seismicity associated with the Riversdale SSE.

Finally, the Weber 1990 sequence also seems similar to another series of earthquakes further south, off Cape Paliser, earlier in 1990 [Zhuang, 2000]. We do not know of any suggestions that the Cape Paliser earthquakes are associated with slow slip, but they also occur along and below the plate interface, similar to the Weber and Upper Hutt sequences [Zhuang, 2000]. SSEs have been documented to migrate along the margin over periods of months [Wallace and Beavan, 2010; Wallace et al., 2012]. Thus, the timing of this sequence within months of the Weber earthquakes also supports the possibility that these events may be related to slow slip. 
While slow slip has not yet been documented as far South as Cape Paliser, the recent observations of the Cape Turnagain 2011 and Riversdale 2009 SSEs within the locked zone suggest SSEs can occur within the locked region of the plate interface.

This is precisely the relationship we wish to investigate. Can the CURATE method identify sequences associated with SSEs using the catalog above Mc? Or, is the change in activity too small to be seen above Mc as suggested by Delahaye? First we address whether these observed earthquakes are truly clustered in time by creating a sequence catalog with our CURATE algorithm.

\subsubsection{Sequence Analysis}

Unfortunately, while recent periods of time have better GPS network and constraints, the GeoNet earthquake catalog has not been finalized. We have experienced drastic changes between preliminary and finalized version of the catalog so we only use finalized locations for our analysis. The recent GeoNet catalog is finalized for July 2008-June 2009, and from August 2009-September 2011 (inclusive). To deal with the patchy completeness, we use the observation in chapter 2 that potential sequences will be those that occur within a time less than or equal to ( $1 /$ mean-rate). To preserve the real mean rate when processing the data we calculate the average number of earthquakes that are expected for the month of July and put in evenly spaced synthetic earthquake times as placeholders through that month. The placeholder earthquakes are assigned locations and magnitudes randomly from the set of real earthquakes in the catalog. This allows us to run the CURATE method continuously from 2008 through 2011.

We also compared this mean-rate to the mean-rates calculated for the earlier earthquake catalog in these regions. As an example, the mean-rate reported for the 
Castle Point subsection of the Hikurangi margin from January 1993 through July 2007 was 388 earthquakes per year $\mathrm{M} \geq 2.45$, or 1.06 earthquakes per day. The overall rate of seismicity during 2008-2011 is higher (429/year, 1.18/day). This higher rate may indicate a broader change associated with the occurrence of SSEs. The seismic network upgrade could allow a larger number of low magnitude earthquakes to be located and these earthquakes could consequently inflate the rate. While additional low magnitude earthquakes may play a role in the increased rate, we note that all magnitudes appear to have a higher rate in the later time period. We have observed rate fluctuations in other regions and this increase is not unusual (Chapter 3).

\subsubsection{Related Sequences}

\subsubsection{Cape Turnagain and Riversdale}

To begin with we looked at whether we could see the 2011 Cape Turnagain seismicity described by Wallace et al. [2012]. This activity stands out as two separate sequences (Fig. 5.10) in the sequence catalog. The observed activity does indeed stand out from the background activity.

Having found the seismicity associated with the 2011 SSE we decided to also investigate the suggestion that there was no seismicity that accompanied the Riversdale 2009 SSE to the South [Wallace et al., 2012]. Despite the overlapping slip areas, Wallace et al. [2012] note that there is no seismicity associated with the northern edge of the 2009 slip Riversdale SSE. Our investigation also concludes that there is no seismicity in a comparable location to the Cape Turnagain 2011 SSE. However, we were able to identify seismicity along the southern edge of the Riversdale slip boundary (Fig. 5.10).

While it is not our goal here to directly investigate the mechanisms of SSEs we do note that these two recent SSEs occur in the locked portion of the subduction 
zone as modeled by Wallace and Beavan [2010]. The fact that the earthquake sequences associated with SSEs are also observed in zones with higher coupling coefficients implies that SSEs share a common mechanism in both partially and fully locked zones along the subduction margin. 

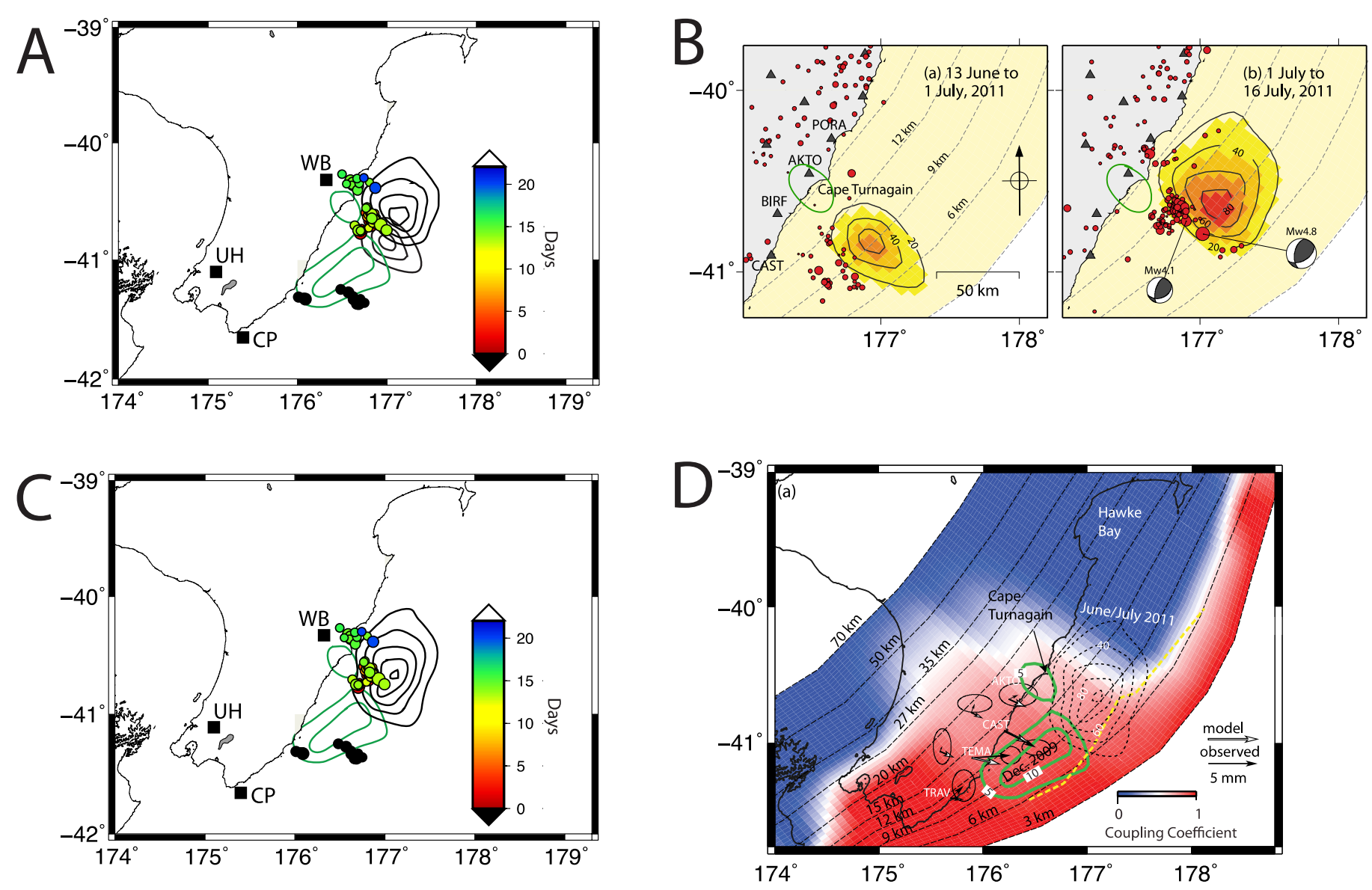

Figure 5.10 Maps of Slow Slip Events (SSE) and related seismicity in December 2009 and June and July 2011.

All slip contours are taken from Wallace et al. [2012]. A) shows the seismicity associated with the 2009 (black circles) and July 2011 SSE (colored in days from June 19). Black contours represent the slip observed in 2011 (B) and green contours represent the slip contours calculated for the December 2009 SSE. C) same as in A, but with the cumulative slip for the 2011 event (D). Colors are the same as in A. The locations of the Upper Hutt swarm (UH), Weber 1990 (WB), and Cape Paliser 1990 (CP) activity are shown as squares in A and C. B and D are figures 9 and 10 from Wallace et al. [2012] showing the slip contours. 


\subsubsection{Gisborne}

In the study of earthquake sequence decay (Section 4.3) we found an interesting rate development during a sequence in 2010. We looked at the sequence location, and decided to check if it correlates with slow slip (Fig. 5.11). It occurs along the edge of the documented 2010 Gisborne slow slip event [Wallace and Beavan, 2010] and in a similar location to the model for the earlier Gisborne slow slip events [Douglas et al., 2005]. This is one of the larger sequences in the catalog (62 earthquakes). It is the fifth largest sequence in the Hikurangi margin between 1993 and 2011 (exclusive of the last half of 2007 as data during that time were not finalized at the time of these analyses). Note that the last half of 2007 contains the largest magnitude earthquake in the Hikurangi margin over this time $\left(\mathrm{M}_{\mathrm{w}}=6.5\right)$.

Because we think that the March 2010 sequence was related to the concurrent SSE we decided to look for seismicity associated with the 2002 and 2004 SSE in the same location. As mentioned above, the seismicity related to the 2004 SSE was investigated previously by Delahaye [2009], who determined that the increased microseismicity was a feature that could not be detected through routine processing. In fact, our sequence catalogue contains two sequences during the same 2004 slip period. One sequence starts during the SSE October $26^{\text {th }}$, and the other begins at the end of the recorded slip on November $12^{\text {th }}$. In figure 5.11 we can see the spatial migration in the 2004 sequence noted by Delahaye et al. [2009]. While the routine processing was unable to detect earthquakes $\mathrm{M}<2.0$, the seismicity was energetic enough to be located by GeoNet and is easily detected using CURATE clustering analysis. This once again highlights the power of clustering techniques to identify subtle but significant changes in earthquake behavior over time. Moreover, the use of a sequence catalog allows us to put the seismicity associated with the 2004 SSE in a 
broader temporal context than was possible in the manual identification of events used by Delahaye et al. [2009] to detect smaller magnitudes. 

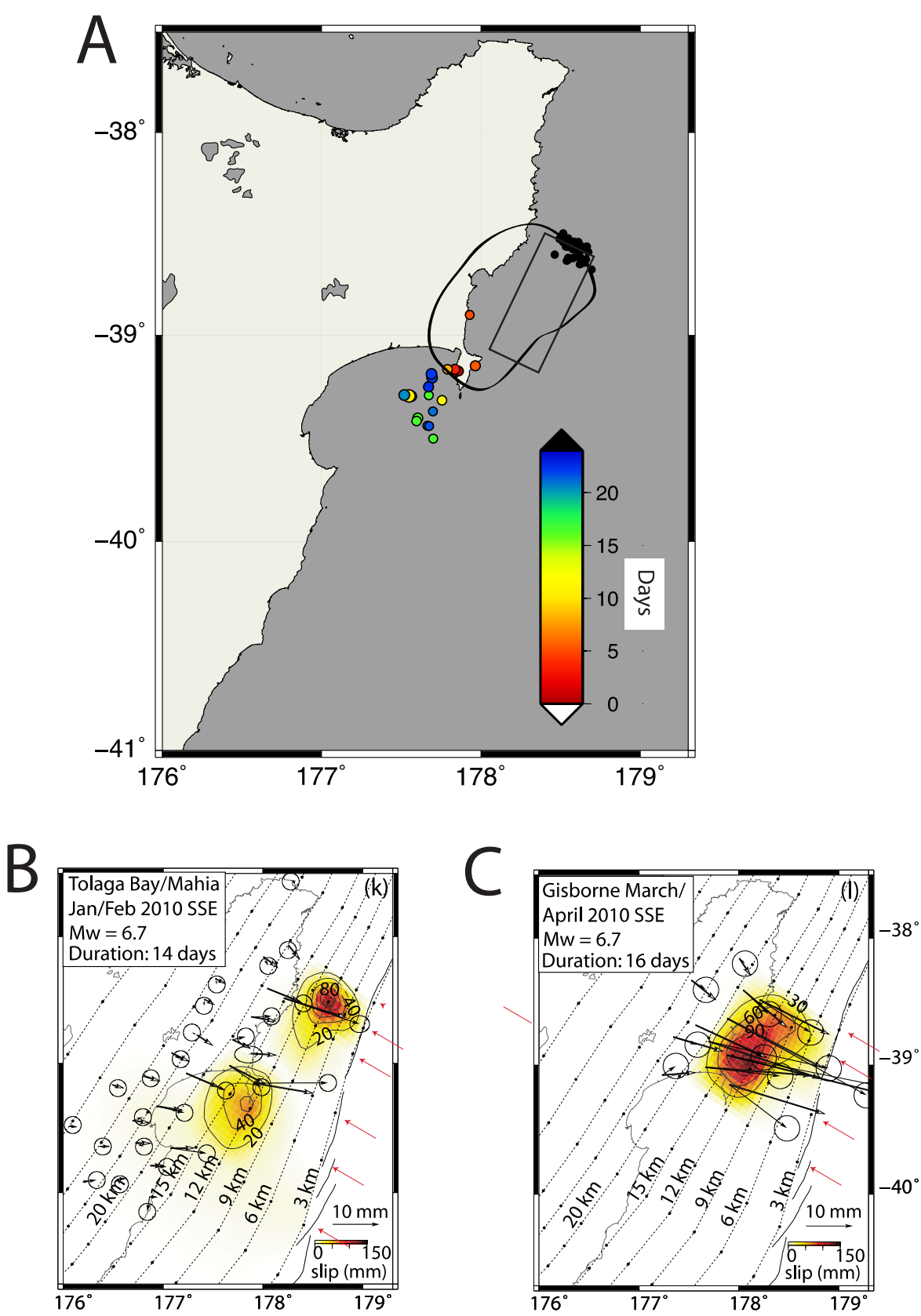

Figure 5.11 Seismicity and slip contours associated with Gisborne 2004 and 2010 SSEs.

Seismicity in A) black circles are events accompanying slip in 2010 and colored circles representing those associated with 2004 SSE (colored by days from October 26, 2004). Black rectangle is the slip patch calculated by Douglas et. al. [2005] for the October 2002 Gisborne SEE and the black loop is the $20 \mathrm{~mm}$ slip contour calculated for the Gisborne March-April 2010 SSE (shown in C). B) and C) taken from Wallace et al. 2012. B) Slip in the same area preceding the March slip shown in C) Numbered contours show depth to the subducted plate. 


\subsubsection{Weber}

Following the Darfield Mw 7.1 earthquake on September $4^{\text {th }}, 2010$ a number of $M \geq 4$ and 5 earthquakes occurred along the NE coast of the South Island and throughout the North Island. With heightened attention following the Darfield earthquake a number of questions were raised as to whether these earthquakes were triggered by the Darfield earthquake and whether they could represent an increased probability of large earthquakes on the subduction zone. Wallace et al. [2012] noted the temporal relationship between the Darfield earthquake and an acceleration of slip in the 2010 Manawatu slow slip event. This recent inference prompted us to reexamine these events. The biggest cluster includes two $\mathrm{M}_{\mathrm{L}} \sim 5.2$ and two additional $M_{L} \sim 4.2$ over four days from September $6^{\text {th }}$ thru September $9^{\text {th }}$. These events occur between the locations of the Weber 1990 earthquakes and the triggered Cape Turnagain 2011 sequence (orange events in Fig 5.12). Robinson [2003] modeled the stress change that would result from aseismic slip downdip of the Weber earthquakes, and concluded that such slip would be enough to load and to trigger the Weber earthquakes. Tremor has been identified associated with the earlier Manawatu SSE in 2004-2005 [Ide, 2012]. We note that the tremor identified by Ide [2012] in those locations is also observed during times when no SSE is occurring. Those locations are distributed to the north of the slip and are further away from the $30 \mathrm{~mm}$ slip contour than the tremor observed by Kim et al. [2011] associated with the Gisborne 2010 SSE [Ide, 2012]. Figure 5.12 compares the slip from the 2004-2005 and 2010 Manawatu events with the location of the $M \sim 5$ earthquakes in 2010 . We note that the locations of the $M \sim 5$ earthquakes in 2010 near Cape Turnagain are no further away (SE) than the tremor locations of Ide [2012] are to the North- northwest. This indicates that a Manawatu SSE, in addition to a more local Cape Turnagain SSE, is 
capable of triggering earthquakes near Weber and thus further supports Robinson's [2003] conclusion that the Weber earthquakes were triggered by aseismic slip. 

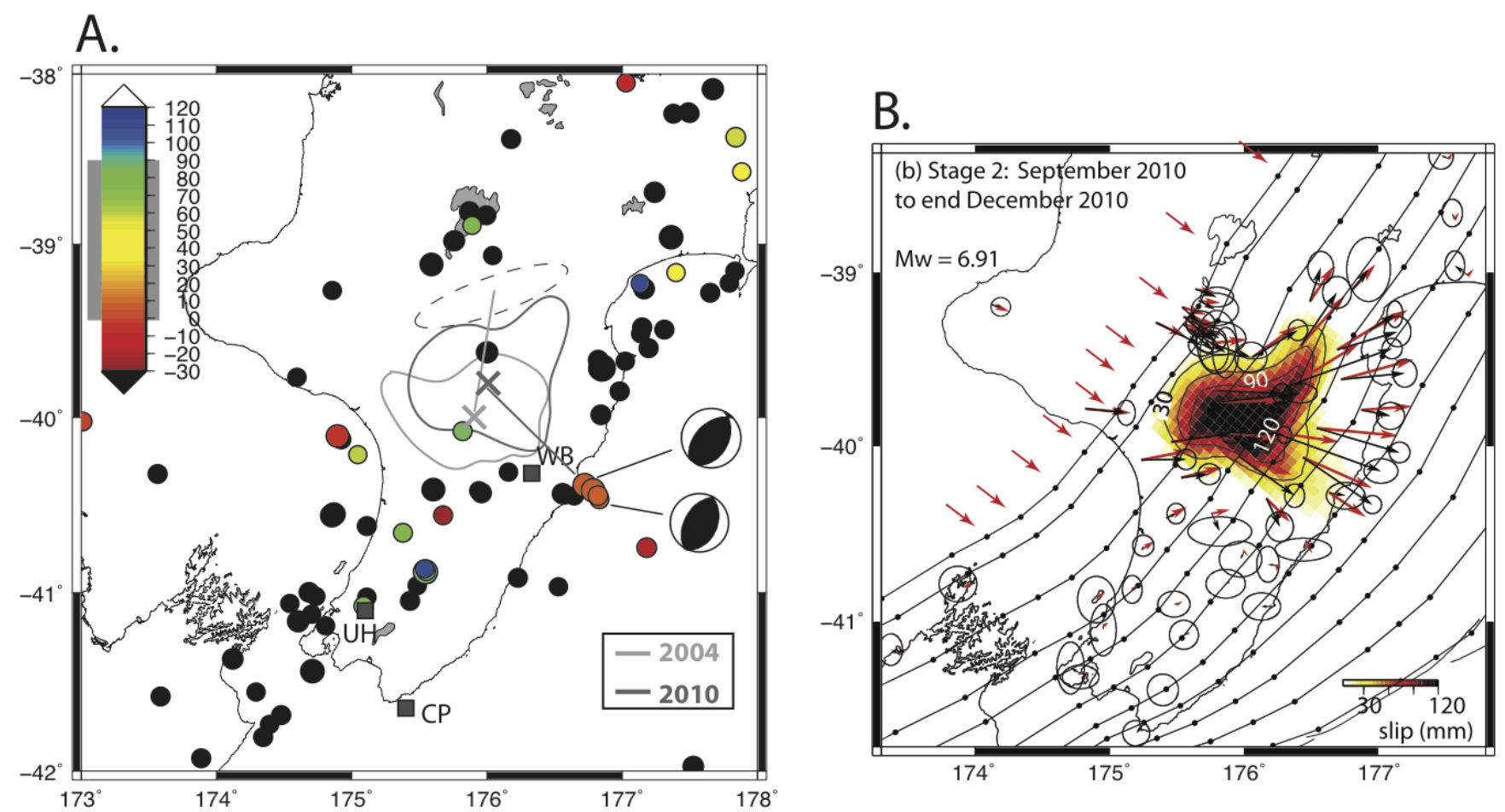

Figure 5.12 Plot of seismicity $M \geq 4.0$ from January 2008 through December 2010 .

A) The earthquakes are colored by time in days with $\mathrm{t}=0$ set to September $1^{\text {st }} 2010$ (earthquakes before September 2010 are all shown in black). The two gray contours show the $30 \mathrm{~mm}$ slip contour for the 2004 and 2010 events from Wallace et. al. [2006] and Wallace et al. [2012]. For each of those two SSEs an ' $x$ ' marks the center and lines of the same length point from the center to the center of the tremor (2004), and to the 2010 Weber earthquakes (2010). The dashed ellipse shows the approximate tremor region from Ide et al. 2012 associated with the 2004-2005 Manawatu event. Gray boxes indicate the Upper Hutt 2004 (UH), Cape Paliser 1990 (CP) and Weber 1990 (WB) sites as shown in figure 5.8. The CMT solutions determined by GeoNet are plotted for the two $\mathrm{M}_{\mathrm{L}} \geq 5.0$ earthquakes in the Cape Turnagain 2010 sequence. The gray box behind the color scale in A represents the time of slip in B) from Wallace et al. 2012, calculations of slip for stage 2 of 3 during the Manawatu SSE 2010 

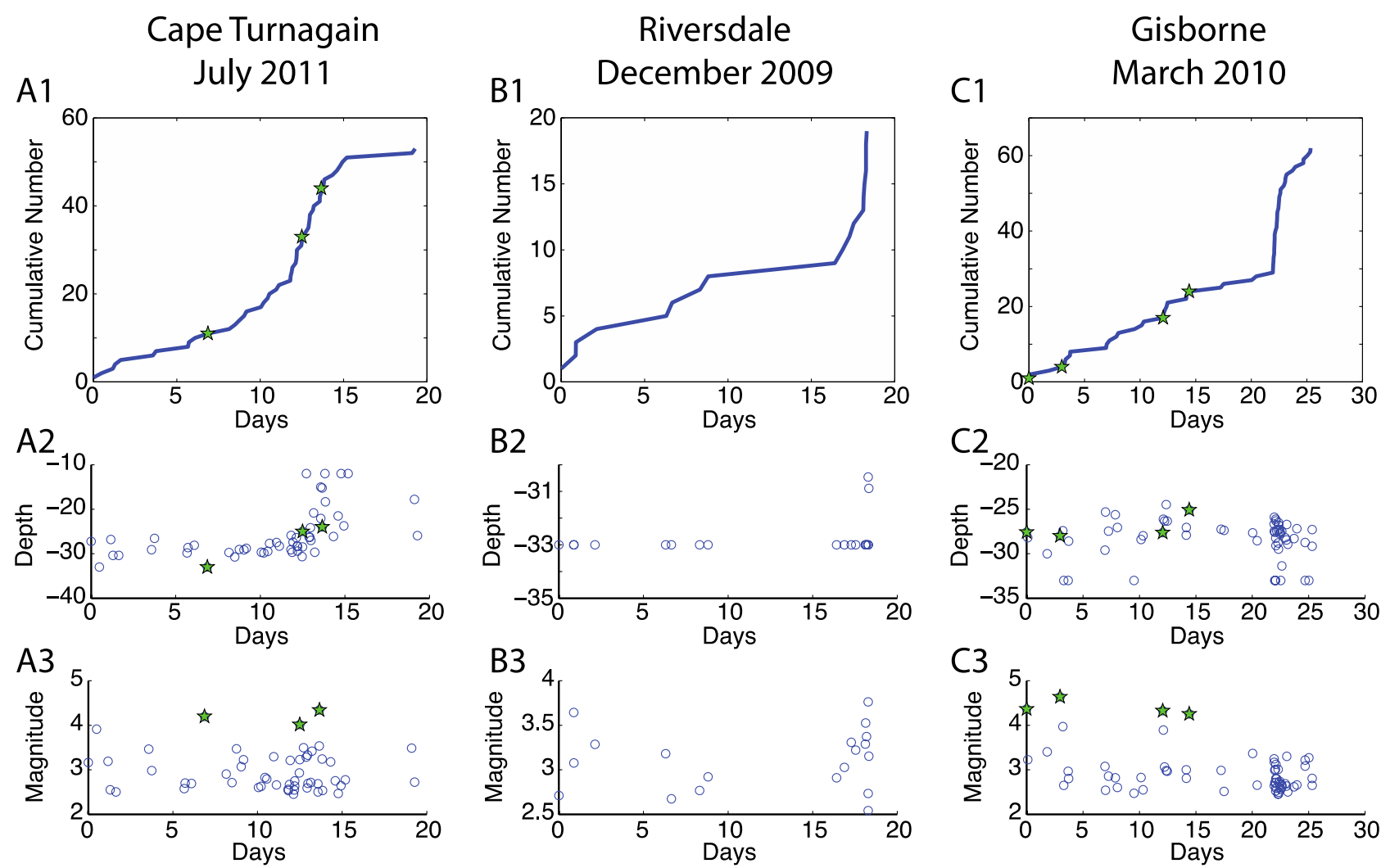

Figure 5.13 Rate with time for three sequences associated with slow slip.

Rows are rate (1), depth (2), and magnitude (3) are given for each of the three sequences. A) Cape Turnagain July 2011, B) Riversdale

December 2009 and C) Gisborne March 2010. Green stars mark the timing of earthquakes with magnitude $\geq 4.0$; note there are no events at this magnitude in the Riversdale 2009 sequence. 


\subsubsection{Discussion: Possibilities for seismic detection of slow slip events}

In chapter 3 we showed that the Hikurangi seismicity rate is more constant in time than other regions. All three aftershock-dominated regions have swarms that contain thousands of earthquakes. Both the CVR and the Hikurangi region have maximum sequences sizes of hundreds of earthquakes. The CVR is also more prone to clustering than the Hikurangi region. The sequences with at least ten earthquakes, analyzed in Chapter 4 , account for $38.5 \%$ of the total seismicity $\mathrm{M} \geq 2.5$ in the CVR, and only $12.5 \%$ of the total seismicity along the Hikurangi margin. Thus, we know that seismicity associated with SSE is not the dominant feature of observed seismicity along the margin. However, if seismicity associated with recorded SSEs have unique characteristics compared to other earthquake sequences we may be able to infer past SSEs prior to cGPS observations. In figure 5.13 we plot the cumulative number, magnitude with time, and depth with time of three of the triggered sequences. The depths are all poorly constrained and S-wave picks and relocations would be necessary to better constrain their relationship to the plate interface. The catalogue depths do hint that these earthquakes occur on the interface and this suggests that understanding slow slip will help us understand loading and release on the subduction interface. All three of the sequences (Fig. 5.13) have a similar rate development in time, with a steady increase followed by a sharp increase that is not related to the timing of the largest earthquakes (green stars). This suggests that the sequences may have a common temporal evolution (rate pattern), but three observations are not enough to confirm this. The Cape Turnagain (Fig. 5.13 A) swarms occur entirely within (June 19 - July 7) the published slip period (June 13 - July 16). Exact dates are not published for the Riversdale (2009) and Gisborne (2010) SSEs so it is difficult to relate the exact timing of the rate increase to accelerations in slip. 
While the overall percentage of clustered seismicity is low, the three sequences shown in figure 5.13 make up $25 \%$ of the total earthquakes in sequences of $\geq 10$ earthquakes. It is hard to calculate probabilities for SSEs because the potential trigger lasts anywhere from weeks to months with slip rates and spatial extent varying with time. For this reason New Zealand studies of seismicity related to slow slip have focused on Coloumb modeling of the entire slow slip event, and the simple association that earthquake occur concurrently with SSEs. With the recent development of more detailed spatio-temporal slip models (e.g. [Bartlow et al., 2011]), more detailed probability analysis may be possible in the near future. Thus, for now, we simply compare the amount of seismicity that occurs during periods of slow slip to the time over which that slip occurs. For the more recent earthquake catalog we analyzed the period July 2008 through September 2011 (not including June 2009), for a total of 38 months ( $\sim 1140$ days). We approximate the total duration of SSEs during that same time, not including the 2010 Manawatu SSE, using the durations given in Wallace and Beavan. [2010], and estimates from information in Wallace et al. [2012] and get approximately 147 days (August 2008, 12 days; December 2009, 30 days; February 2010, 14 days; March 2010, 16 days; June 2011, 30 days; August 2011, 30 days; September 2011, 15 days). This means that there is approximately twice as much clustered seismicity as we would expect if the seismicity occurred uniformly in time. Although it is not a definitive probability, it further suggests that these sequences are related to the timing of the SSEs.

Despite the Gisborne 2010 SSE occupying the same location as the earlier events in 2002 and 2004, sequences at those times are not similar. There are sequences and microseismicity [Delahaye et al., 2009] in 2004 on the southern end of the slip patch near the Mahia peninsula, but there do not appear to be events on the 
northern end in the location of the 2010 triggered sequence (Fig. 5.11). The 2002 SSE predates the upgrade of the GeoNet network around 2003 [Petersen et al., 2011] and may not have enough resolution to identify similar sequences, although $M>4$ earthquakes, which occurred in the 2010 sequence should have been captured by the 2002 network if they occurred. Another complication may be that between the 2004 and 2010 SSEs there was a $\mathrm{M}_{\mathrm{w}}=6.5$ earthquake within this slip patch. It could be that the $\mathrm{M}_{\mathrm{w}}=6.5$ changed the interface properties thus focusing triggering along the northern, instead of the southern (2004) side of the slow-slip patch.

The seismicity associated with the Riversdale 2009 SSE has an unusual location, and very few earthquakes were seen in this location at other times in the catalog (Fig. 5.10). The SSE itself is smaller than the Cape Turnagain (2011) SSE, and no $\mathrm{M}_{\mathrm{W}}$ measurement is given for the Riversdale (2009) event by Wallace [2012]. The Riversdale (2009) slip contours have a range of 5-10 mm in contrast to the Cape Turnagain (2011) slip from 20-100 mm [Wallace et al., 2012]. Regardless of the mechanism for SSE producing mircroseismicity, it is not surprising to have a smaller number of triggered events from a smaller SSE. While Wallace et al. [2012] were not able to identify a sequence on the northern edge of this slip, the sequence on the southern edge of the Riversdale slip emphasizes that we need to observe more of these sequences before we can anticipate the type and location of SSE triggered sequences.

We have shown that sequence catalogs are a powerful tool for identifying anomalous seismicity (Chapter 2). While Delahaye et al. [2009] concluded that the observed microseismicity was a phenomenon that could not be seen through routine processing we have identified two sequences at the same time as the 2004 Gisborne slow slip in the Mahia Peninsula area. This shows the power that clustering algorithms and sequence catalogs have in analyzing the data. Clustering techniques 
are extremely useful tools for identifying sequences. Sequence catalogues can also be used to quantify and compare the size of sequences at different times.

\subsubsection{Conclusions}

The few examples of earthquake sequences in this section show very clearly that slow slip events do influence the timing and distribution of seismicity along the Hikurangi margin. Sequences are not a significant percentage of the total seismicity rate in the Hikurangi margin. However, SSE related sequences account for $25 \%$ of the earthquakes observed in sequences of ten or more earthquakes in the Hikurangi sequence catalogues. This percentage is nearly twice what we'd expect randomly, and means there is a need for further investigation into the relationship and nature of triggered seismicity in order to fully understand the timing of sequences along this margin, and to understand how that may relate to the larger hazard of large subduction earthquakes.

The sequences outlined in this section also show that triggering is not entirely systematic. Slip may trigger earthquakes at one time, and then not do so for a comparable SSE at another time, or slip may trigger earthquakes in a different location. The variability in triggering behavior suggests the state of the interface, and local conditions also play a role in controlling whether triggered earthquakes occur. Both the triggering and its variability pose challenges for creating realistic earthquake forecasts. Clustering techniques offer a convenient way to identify and quantify these triggered sequences and their variability with time. Our identification of two swarms (Gisborne 2004 and Riversdale 2009) previously thought to not be identifiable in the GeoNet catalog shows the power of clustering techniques to resolve changes in rate even when clustering is not the dominant feature of an earthquake catalogue. More observations are necessary to confirm whether earthquake sequences triggered by 
SSEs have unique characteristics that may allow them to be identified without concurrent cGPS observations of the slip. The coupling coefficient of the plate does not appear to impact the ability of SSEs to trigger seismicity. Further investigation of the relationship between slow slip and seismicity will lead to a better understanding of plate coupling and the process of loading the subduction interface. 


\section{Discussion/Conclusions}

\subsection{How can we best identify sequences in an earthquake catalogue that} contains both MS-AS and swarm types?

We have developed a new clustering method, CURATE, which uses rate as the primary identification for sequence occurrence (Ch. 2). Rate increase is the main commonality between all sequence types, and the use of rate therefore ensures identification of all sequence types. The use of rate allows minimal physical assumptions and allows for physical sequence processes to be established later through other analyses.

The two secondary selection parameters, distance and day rules, can be varied over a larger range of values than other clustering methods without affecting the stability of the sequences (Ch. 2.3.3, Tbl. 2-4). The limiting rate at which earthquakes must occur in order to be considered as part of a sequence may be varied (mean/median/minimum), but similar distance rule values can be used for different geographic regions and earthquake catalogs, leading to more consistent and direct comparisons of sequences and their behavior (Ch. 3.2.2). The declustered catalogs and individual sequences do change with variations in selection parameters, but most of the differences occur in small sequences around the minimum number limit. Large sequences are relatively stable to changes in selection parameters. Poisson testing of the declustered catalog can be used to ensure that adequate selection parameters are applied (Ch. 2.5.2, Ch. 3).

The CURATE method also produces larger declustered catalogs for similar dispersion values (closer to a Poisson value) compared to other declustering techniques (Ch. 3.1.1). These larger declustered catalogs may be useful in identifying 
broad-scale changes in sequence occurrence and earthquake rates with time $(\mathrm{Ch}$. 3.3.1, Ch. 3.6).

6.2 What kinds of sequences occur in the CVR, and how do they compare to other regions of New Zealand and sequences in Southern California?

We have defined mainshock-aftershock sequences as any sequence that has a largest magnitude $\geq 0.5$ magnitude units larger than the second largest earthquake and in which the largest earthquake occurs within the first $30 \%$ of earthquakes (Ch. 4.2.2). The classification of sequences in this manner is most sensitive to the magnitude separation required between the two largest earthquakes. There is not one best definition, and we think that the one used here is sufficient in that throughout the analyses that the population of sequences meeting these criteria does behave differently to other sequences.

Over $90 \%$ of sequences in the CVR ( $\geq 10$ earthquakes) are swarms. While the CVR has the highest observed percentage of swarms of the regions we studied, we found that swarm type sequences dominate sequence catalogs at $\mathrm{Mc}<3$, accounting for $\sim 70 \%$ of sequences with at least 10 earthquakes in all regions. Vidale and Shearer [2006] found that only $20 \%(14 / 71)$ of their identified small sequences could be identified as mainshock-aftershock sequences. We need to understand swarm sequences to advance earthquake forecasting and other seismicity and hazard modelling (Ch. 4.2-4.4)

The two subduction zones in New Zealand behave differently from each other, with the Hikurangi margin containing more swarms than Fiordland region (Ch. 4). Although Fiordland is also a subduction zone, it has approximately the same swarm percentages as the Alpine Fault regions. We also analyzed data from Southern 
California and found that it has an intermediate percentage of swarms; more swarms than South Island and not as many swarms as North Island.

Both mainshock-aftershock and swarm sequences have an increased probability of Mmax occurring in the first $10 \%$ of the sequence by duration $(\mathrm{Ch}$. 4.2.3). For MS-AS this probability is $>60 \%$ whereas it is only around $15 \%$ for swarms. Both sequence types also have a relatively linear cumulative distribution of Mmax following the first $10 \%$ of the duration, indicating that the largest magnitude can occur at any later point in the sequence with almost uniform probability.

\subsection{How much decay is observed in swarm sequences and can decay, where observed, be explained by Omori's law?}

We found that all sequence types contain a large amount of decay through their duration. By comparing the residuals of individual sequences from Omori's law and linear fits we found that $89 \%$ of MS-AS, and $55 \%$ of swarms can be fit by an Omori's law over some amount of their duration (Ch. 4.3). Although many swarms pass our linear comparison test, in general, Omori's law gives less improvement over a linear fit for swarms than it does in MS-AS sequences. For some tests the relative improvement for MS-AS over swarm sequences is nearly double. These analyses can be used to give us an idea of how well we can expect ETAS type forecasts to perform when modelling catalogs that contain swarms.

\subsection{Are there patterns in sequence occurrence, and does sequence occurrence relate to broader tectonic and subduction processes?}

\subsection{1: Identified Patterns: Omori and others}

In addition to measuring the amount of decay, and comparing sequence decay to Omori's law, we have also been able to identify three non-Omori patterns in the rate of earthquakes with time ("rate types"). While previous studies have suggested 
the possibility that patterns exist in non-MS-AS sequences, a limited number of analyzed sequences has generally limited specific pattern identification (e.g.

[Sherburn, 1992b]). The use of sequence catalogs that include swarm sequences has allowed us to identify and quantify some of these patterns (Ch. 4.4).

We identified a Decay based rate type, as well as three additional patterns which we term: L-shaped, Steady, and Accelerating (Ch. 4.4). Not all sequences fit into these types, but sequence in the other category have intermediate values and may either result from inability to identify a rate-type, multiple instances of one or more rate types, or from a mixture of processes. As could be expected, most MS-AS fall into the decay rate-type (Ch. 4.4.3). The fact that we have identified multiple nondecay dominated patterns supports the idea that there are multiple physical processes that drive swarm sequences.

From several examples including, Haroharo, Tokaanu, and the Southern Alps, we think that $L$-shaped sequences may be associated with fluid injection or flow. Observed Vp/Vs changes at Haroharo, and a clear fluid diffusion signal at Tokaanu both support this idea (Ch. 4.4.5.3, Ch. 4.5.4.4).

The sequences associated with SSE in the Hikurangi margin (Ch. 5.3) do not fit any of the three new rate patterns, although they have similar rate development to one another. Thus, there could be more patterns to be discovered. Likewise analyzing the other rate-type in more detail may reveal sequences that contain multiple instances of one of the rate-patterns identified here. Complex small scale variations in average inter-event times currently prevent the use of a quantitative algorithm to identify these rate-patterns (Ch. 4.4.2), but this is likely to become possible as we understand and discover more about the patterns. 


\subsubsection{Are sequences related to broader tectonic and subduction processes?}

We have also identified broad scale temporal changes, on the order of several years, in the pattern of sequence occurrence (Ch. 3.2, 3.3, 3.6). This observation is most pronounced in Lake Taupo and Coso Geothermal (SCA) region. Changes are also seen in three other North Island subsets and the CVR-wide catalog (Ch. 3.4, 3.6.2). The widespread nature of these behavior changes implies a change in the deeper subduction zone or along a large portion of the subduction margin.

Broad changes are also seen in the Southern California earthquake catalogs (Ch. 3.2, 3.6). Some of these rate changes have been previously identified by other authors, including a decrease in years prior to Lander's 1992 earthquake [Wyss and Wiemer, 2000]. Our analysis shows that these rate changes may have been broader than previously reported. Two more recent periods of steady increase have also been identified in the southern California dataset.

Conservative declustering methods (e.g. Gardner and Knopoff 1974) may not be sensitive enough to detect these rate variations (Ch. 3.2.3). The timing of sequences does relate to broader tectonic processes and the investigation of shallow seismicity will continue to be a fruitful tool to investigate tectonic processes and hazards.

\subsection{Can statistics using complete catalogues identify potential triggering?}

A purely statistically analysis of earthquake triggering from global and regional New Zealand large earthquakes did return significant results of triggering (Ch. 5.1). The parameters of the potentially triggering large earthquakes however, did not differ significantly from the population of all large earthquakes and so we 
consider the search for triggering in the CVR to be inconclusive. While we do not think that this statistical study can definitively identify triggered sequences, we suggest that it could be a useful way to narrow down a list of large earthquakes for a waveform triggering study.

Through the application of the CURATE method to microseismicity in the Southern Alps of New Zealand we found evidence of triggered sequences, including delayed triggering, following two large New Zealand earthquakes (Ch. 5.2). The area where these triggered sequence occurred is one where swarm occurrence is common and a longer investigation is needed to see whether the repeat time of sequences was affected (clock advance) by the triggering.

\subsection{Does triggering occur in New Zealand? If so, what are its causes?}

We have not identified specific triggering in the CVR, but we have observed times of differing sequence behavior. These changes in behavior occur over periods of years, and may have larger implications for earthquake forecasting and hazard analysis.

We have identified triggered swarms in the Southern Alps (Ch. 5.2), and sequences associated with slow slip events (SSEs) along Hikurangi margin (Ch. 5.3). Using sequence catalogs we were able to identify two sequences associated with SSEs that were previously thought to be unidentifiable within the GeoNet earthquake catalog. Within the small set of SSE associated sequences there is variability, especially spatially, of the occurrence of seismicity even when similar areas slip at other times. The variability suggests that the state of the plate interface, fluid availability, or other factors may also play a role in the occurrence of triggered earthquakes. We also note that the observation of earthquakes accompanying slow 
slip in both the partially and fully locked portions (as modeled by Wallace and Beavan [2010]) provides a preliminary suggestion that the mechanism of slow slip is the same in both zones (Ch. 5.3.4.1).

A set of $M>5$ earthquakes near the location of Weber $1990 M>6$

earthquakes has been identified during the Manawatu 2010 SSE (Ch. 5.3.4.3). The occurrence of these earthquakes during the SSE provides further evidence that the Weber earthquakes were triggered by slow slip [Robinson, 2003]. Moreover the distance between the bulk of the slip and the $M>5$ earthquakes also suggests that SSE have a long range over which stress changes can act as potential triggers. We also suggest that recent observations of SSEs migrating along the margin may also implicate slow slip events in the 1990 Cape Paliser earthquake activity as well (Ch. 5.3.2).

\subsection{Conclusions: So, what can complete catalogues at high Mc tell us about earthquake swarms and their timing?}

Complete earthquake catalogs have the power to reveal much about individual sequences as well as broad tectonic processes. Creating sequence catalogs allows us to undertake quantitative analyses of various sequence parameters. Swarms account for $\sim 70 \%$ of sequences of $\geq 10$ earthquakes in all regions at $\mathrm{Mc}<3.0$ studied here. The large percentage of identified swarm sequences emphasizes the need for the study of swarms and for quantitative means by which to detect and define them. The new CURATE method is ideal for the identification of a variety of sequence types.

Our study of the dispersion of declustered catalogs with variation in Mc also provides support to recent ideas that sequences with smaller maximum magnitudes may behave differently than sequences with larger Mmax values. While it is possible 
that smaller magnitude sequence catalogs behave differently because they include more swarm sequences, which typically have smaller Mmax, we think that it is also possible that the rate changes and other behaviors are simply damped in catalogs with larger Mc values because there are fewer earthquakes. If this is true, then continued study of the behavior at small magnitudes may be necessary to further our understanding of earthquake behavior.

Statistically, we are not able to identify triggered sequences using analysis of the earthquake catalog alone as there are too many unknowns. However, sequence catalog analysis helps to identify sequences that can then be compared to times of potential triggers. This has worked to identify triggered swarms in the Southern Alps and in the Hikurangi region where we have been able to identify earthquake swarms associated with slow slip events. The sequences associated with slow slip events in the Hikurangi margin account for nearly $25 \%$ of all clustered seismicity (sequences $\geq$ 20 earthquakes). This is not obvious without the use of sequence catalogs and clustering techniques as only $12.5 \%$ of seismicity along that margin occurs in sequences of that size.

The use of sequence catalogs reaffirms the usefulness of sequence catalogs in quantitatively assessing earthquake sequences. The potential correlations of sequence parameters and the rate patterns identified here are another useful step forward in understanding earthquake swarms, as well as improving both earthquake forecasting and hazard analysis. 


\section{Suggestions for Future Work:}

We hope that the research presented here will serve as a solid starting point for a number of future studies. Outlined below are the topics we think may be most fruitful.

-Quantitative identification of rate-type patterns would allow for better incorporation into earthquake forecast models and could be used to test specific physical processes models. We may also be able to refine the other rate type and investigate the possibility of multiple patterns in a single sequence. The analysis of more earthquake datasets would also be useful for further testing and refining the ratetype patterns and other conclusions about sequence behavior made during this study.

-New spatio-temporal models of slow slip (e.g. Bartlow et. al. [2011]) could be used to constrain the slip amount and timing in order to further analyze the triggering probabilities associated with SSEs.

-The sequence type makeup could be used to define confidence bound on pure ETAS models that assume all sequences obey a magnitude dependent Omori's law branching model. The regional sequence type information may also be useful in limiting the amount of parameter fitting and obtaining more accurate estimates of the ETAS efficiency parameter alpha, which is typically underestimated in swarm regions.

-Similarly swarm information could be fully incorporated into an earthquake forecasting model that would only predict a certain percent of sequences to follow an ETAS-type model.

-The use of new information about sequence types and rate patterns should also be updated into a format that could be used for hazard analysis in real-time. 


\section{Bibliography}

Abercrombie, R. E., T. H. Webb, R. Robinson, P. J. McGinty, J. J. Mori, and R. J. Beavan (2000), The enigma of the Arthur's Pass, New Zealand, earthquake 1. Reconciling a variety of data for an unusual earthquake sequence, Journal of Geophysical Research-Solid Earth, 105(B7).

Bartlow, N. M., S. i. Miyazaki, A. M. Bradley, and P. Segall (2011), Space-time correlation of slip and tremor during the 2009 Cascadia slow slip event, Geophysical Research Letters, $38(18)$.

Bath, M. (1965), Lateral Inhomogeneities of Upper Mantle, Tectonophysics, 2(6).

Beanland, S., K. R. Berryman, and G. H. Blick (1989), Geological Investigations of the 1987 Edgecumbe Earthquake, New-Zealand, New Zealand Journal of Geology and Geophysics, $32(1)$.

Beanland, S., and J. Haines (1998), The kinematics of active deformation in the North Island, New Zealand, determined from geological strain rates, New Zealand Journal of Geology and Geophysics, 41(4).

Beavan, J., L. Wallace, and T. Hurst (2003), Observation of an aseismic deformation episode above the northern Hikurangi subduction zone, New Zealand, paper presented at EGS-AGUEUG Joint Assembly.

Beavan, J., L. Wallace, H. Fletcher, and A. Douglas (2007), Slow slip events on the Hikurangi subduction interface, New Zealand, Iag Symp, 130.

Beavan, J., S. Samsonov, P. Denys, R. Sutherland, N. Palmer, and M. Denham (2010), Oblique slip on the Puysegur subduction interface in the 2009 July M-W 7.8 Dusky Sound earthquake from GPS and InSAR observations: implications for the tectonics of southwestern New Zealand, Geophysical Journal International, 183(3).

Benoit, J. P., and S. R. McNutt (1996), Global volcanic earthquake swarm database 19791989, in USGS Open-File Report 96-69, edited, p. 334.

Bhattacharyya, J., S. Gross, J. Lees, and M. Hastings (1999), Recent earthquake sequences at Coso: Evidence for conjugate faulting and stress loading near a geothermal field, Bulletin of the Seismological Society of America, 89(3).

Bhattacharyya, J., and J. M. Lees (2002), Seismicity and seismic stress in the Coso Range, Coso geothermal field, and Indian Wells Valley region, southeast - central California, Mem. Geol. Soc. Am, 195.

Bibby, H. M., T. G. Caldwell, F. J. Davey, and T. H. Webb (1995), Geophysical Evidence on the Structure of the Taupo Volcanic Zone and Its Hydrothermal Circulation, Journal of Volcanology and Geothermal Research, 68(1-3).

Boese, C. M. (2012), Microseismicity in the central Southern Alps, Westland, New Zealand: a thesis submitted to the Victoria University of Wellington in fulfilment of the requirements for the degree of Doctor of Philosophy in Geophysics, 47.

Boese, C. M., J. Townend, E. Smith, and T. Stern (2012), Microseismicity and stress in the vicinity of the Alpine Fault, central Southern Alps, New Zealand, Journal of Geophysical Research-Solid Earth, 117. 
Brodsky, E. E., and S. G. Prejean (2005), New constraints on mechanisms of remotely triggered seismicity at Long Valley Caldera, Journal of Geophysical Research-Solid Earth, $110(\mathrm{~B} 4)$.

Brodsky, E. E. (2006), Long-range triggered earthquakes that continue after the wave train passes, Geophysical Research Letters, 33(15).

Brooks, B. A., J. H. Foster, M. Bevis, L. N. Frazer, C. J. Wolfe, and M. Behn (2006), Periodic slow earthquakes on the flank of Kilauea volcano, Hawai'i, Earth and Planetary Science Letters, 246(3-4).

Bryan, C. J., and S. Sherburn (1999), Seismicity associated with the 1995-1996 eruptions of Ruapehu volcano, New Zealand: narrative and insights into physical processes, Journal of Volcanology and Geothermal Research, 90(1-2).

Bryan, C. J., S. Sherburn, H. M. Bibby, S. C. Bannister, and A. W. Hurst (1999), Shallow seismicity of the central Taupo Volcanic Zone, New Zealand: its distribution and nature, New Zealand Journal of Geology and Geophysics, 42(4).

Cao, A. M., and S. S. Gao (2002), Temporal variation of seismic b-values beneath northeastern Japan island arc, Geophysical Research Letters, 29(15).

Christophersen, A., and E. G. C. Smith (2008), Foreshock rates from aftershock abundance, Bulletin of the Seismological Society of America, 98(5).

Console, R., A. M. Lombardi, M. Murru, and D. Rhoades (2003), Bath's law and the selfsimilarity of earthquakes, Journal of Geophysical Research-Solid Earth, 108(B2).

Davis, S. D., and C. Frohlich (1991), Single-Link Cluster-Analysis, Synthetic Earthquake Catalogs, and Aftershock Identification, Geophysical Journal International, 104(2).

Delahaye, E. J., J. Townend, M. E. Reyners, and G. Rogers (2009), Microseismicity but no tremor accompanying slow slip in the Hikurangi subduction zone, New Zealand, Earth and Planetary Science Letters, 277(1-2).

Dewey, J. W., G. Choy, B. Presgrave, S. Sipkin, A. C. Tarr, H. Benz, P. Earle, and D. Wald (2007), Seismicity associated with the Sumatra-Andaman Islands earthquake of 26 December 2004, Bulletin of the Seismological Society of America, 97(1).

Dixon, W. J., and F. J. Massey (1968), Introduction to statistical analysis, 3d ed., vii, 638 p. pp., McGraw-Hill, New York,.

Douglas, A., J. Beavan, L. Wallace, and J. Townend (2005), Slow slip on the northern Hikurangi subduction interface, New Zealand, Geophysical Research Letters, 32(16).

Dragert, H., K. L. Wang, and T. S. James (2001), A silent slip event on the deeper Cascadia subduction interface, Science, 292(5521).

Eberhart-Phillips, D., P. J. Haeussler, J. T. Freymueller, A. D. Frankel, C. M. Rubin, P. Craw, N. A. Ratchkovski, G. Anderson, G. A. Carver, A. J. Crone, T. E. Dawson, H. Fletcher, R. Hansen, E. L. Harp, R. A. Harris, D. P. Hill, S. Hreinsdottir, R. W. Jibson, L. M. Jones, R. Kayen, D. K. Keefer, C. F. Larsen, S. C. Moran, S. F. Personius, G. Plafker, B. Sherrod, K. Sieh, N. Sitar, and W. K. Wallace (2003), The 2002 Denali fault earthquake, Alaska: A large magnitude, slip-partitioned event, Science, 300(5622). 
Eiby, G. A. (1966), Earthquake Swarms and Volcanism in New Zealand, Bulletin volcanologique, 29.

Enescu, B., J. Mori, M. Miyazawa, and Y. Kano (2009), Omori-Utsu Law c-Values Associated with Recent Moderate Earthquakes in Japan, Bulletin of the Seismological Society of America, 99(2A).

Evison, F. (1982), Generalized Precursory Swarm Hypothesis, Journal of Physics of the Earth, 30(2).

Evison, F. F. (1977), Precursory Seismic Sequences in New-Zealand, New Zealand Journal of Geology and Geophysics, 20(1).

Evison, F. F., and D. A. Rhoades (1993), The Precursory Earthquake Swarm in New-Zealand - Hypothesis Tests, New Zealand Journal of Geology and Geophysics, 36(1).

Evison, F. F., and D. A. Rhoades (1997), The precursory earthquake swarm in New Zealand: hypothesis tests II, New Zealand Journal of Geology and Geophysics, 40(4).

Evison, F. F., and D. A. Rhoades (1999a), The precursory earthquake swarm in Japan: hypothesis test, Earth Planets and Space, 51(12).

Evison, F. F., and D. A. Rhoades (1999b), The precursory earthquake swarm and the inferred precursory quarm, New Zealand Journal of Geology and Geophysics, 42(2).

Felzer, K. R., T. W. Becker, R. E. Abercrombie, G. Ekstrom, and J. R. Rice (2002),

Triggering of the 1999 M-W 7.1 Hector Mine earthquake by aftershocks of the 1992 M-W 7.3 Landers earthquake, Journal of Geophysical Research-Solid Earth, 107(B9).

Felzer, K. R., R. E. Abercrombie, and G. Ekstrom (2004), A common origin for aftershocks, foreshocks, and multiplets, Bulletin of the Seismological Society of America, 94(1).

Fisher, M. A., N. A. Ruppert, R. A. White, F. H. Wilson, D. Comer, R. W. Sliter, and F. L. Wong (2009), A distal earthquake cluster concurrent with the 2006 explosive eruption of Augustine Volcano, Alaska, Tectonophysics, 469(1-4).

Frankel, A., W. R. Mccann, and A. J. Murphy (1980), Observations from a Seismic Network in the Virgin Islands Region - Tectonic Structures and Earthquake Swarms, Journal of Geophysical Research, 85(Nb5).

Frohlich, C., and S. D. Davis (1990), Single-Link Cluster-Analysis as a Method to Evaluate Spatial and Temporal Properties of Earthquake Catalogs, Geophysical Journal International, $100(1)$.

Frohlich, C., and S. D. Davis (1993), Teleseismic B-Values - or, Much Ado About 1.0, Journal of Geophysical Research-Solid Earth, 98(B1).

Fry, B., K. Chao, S. Bannister, Z. Peng, and L. Wallace (2011), Deep tremor in New Zealand triggered by the 2010 Mw8.8 Chile earthquake, Geophysical Research Letters, 38.

Gardner, J. K., and L. Knopoff (1974), Sequence of Earthquakes in Southern-California, with Aftershocks Removed, Poissonian, Bulletin of the Seismological Society of America, 64(15). 
Garrick, R. A., and S. J. Gibowicz (1983), Continuous Swarm-Like Seismicity - the Wanganui, New-Zealand, Earthquakes, Geophysical Journal of the Royal Astronomical Society, 75(2).

Gibowicz, S. J. (1973a), Variation of Frequency-Magnitude Relationship during Taupo Earthquake Swarm of 1964-65, New Zealand Journal of Geology and Geophysics, 16(1).

Gibowicz, S. J. (1973b), Variation of Frequency-Magnitude Relation during Earthquake Sequences in New-Zealand, Bulletin of the Seismological Society of America, 63(2).

Gledhill, K., J. Ristau, M. Reyners, B. Fry, and C. Holden (2011), The Darfield (Canterbury, New Zealand) M-w 7.1 Earthquake of September 2010: A Preliminary Seismological Report, Seismological Research Letters, 82(3).

Gomberg, J. (2001), The failure of earthquake failure models, Journal of Geophysical Research-Solid Earth, 106(B8).

Gomberg, J., P. A. Reasenberg, P. Bodin, and R. A. Harris (2001), Earthquake triggering by seismic waves following the Landers and Hector Mine earthquakes, Nature, 411(6836).

Gutenberg, R. a. R., C.F. (1944), Frequency of earthquakes in California, Bulletin of the Seismological Society of America, 34.

Hainzl, S., and T. Fischer (2002a), Indications for a successively triggered rupture growth underlying the 2000 earthquake swarm in Vogtland/NW Bohemia, Journal of Geophysical Research-Solid Earth, 107(B12).

Hainzl, S., and T. Fischer (2002b), Indications for a successively triggered rupture growth underlying the 2000 earthquake swarm in Vogtland/NW Bohemia, Journal of Geophysical Research-Solid Earth, 107(B12), 2338, doi:10.1029/2002jb001865.

Hainzl, S. (2004), Seismicity patterns of earthquake swarms due to fluid intrusion and stress triggering, Geophysical Journal International, 159(3).

Hainzl, S., and Y. Ogata (2005), Detecting fluid signals in seismicity data through statistical earthquake modeling, Journal of Geophysical Research-Solid Earth, 110(B5).

Hauksson, E., W. Z. Yang, and P. M. Shearer (2012), Waveform Relocated Earthquake Catalog for Southern California (1981 to June 2011), Bulletin of the Seismological Society of America, 102(5).

Hayes, G., M. Reyners, and G. Stuart (2004), The Waiouru, New Zealand, earthquake swarm: Persistent mid crustal activity near an active volcano, Geophysical Research Letters, 31(19).

Helmstetter, A., and D. Sornette (2003), Bath's law derived from the Gutenberg-Richter law and from aftershock properties, Geophysical Research Letters, 30(20).

Hill, D. P. (1977), Model for Earthquake Swarms, Journal of Geophysical Research, 82(8).

Hill, D. P., P. A. Reasenberg, A. Michael, W. J. Arabaz, G. Beroza, D. Brumbaugh, J. N. Brune, R. Castro, S. Davis, D. Depolo, W. L. Ellsworth, J. Gomberg, S. Harmsen, L. House, S. M. Jackson, M. J. S. Johnston, L. Jones, R. Keller, S. Malone, L. Munguia, S. Nava, J. C. Pechmann, A. Sanford, R. W. Simpson, R. B. Smith, M. Stark, M. Stickney, A. Vidal, S. Walter, V. Wong, and J. Zollweg (1993), Seismicity Remotely Triggered by the Magnitude 7.3 Landers, California, Earthquake, Science, 260(5114). 
Houghton, B. F., C. J. N. Wilson, M. O. Mcwilliams, M. A. Lanphere, S. D. Weaver, R. M. Briggs, and M. S. Pringle (1995), Chronology and Dynamics of a Large Silicic Magmatic System - Central Taupo Volcanic Zone, New-Zealand, Geology, 23(1).

Hurst, A. W., and P. J. McGinty (1999), Earthquake swarms to the west of Mt Ruapehu preceding its 1995 eruption, Journal of Volcanology and Geothermal Research, 90(1-2).

Hurst, T., S. Bannister, R. Robinson, and B. Scott (2008), Characteristics of three recent earthquake sequences in the Taupo Volcanic Zone, New Zealand, Tectonophysics, 452(1-4).

Husen, S., R. Taylor, R. B. Smith, and H. Healser (2004), Changes in geyser eruption behavior and remotely triggered seismicity in Yellowstone National Park produced by the 2002 M 7.9 Denali fault earthquake, Alaska, Geology, 32(6).

Husen, S., and J. L. Hardebeck (2010), Earthquake location accuracy, Community Online Resource for Statistical Seismicity Analysis.

Hutton, K., J. Woessner, and E. Hauksson (2010), Earthquake Monitoring in Southern California for Seventy-Seven Years (1932-2008), Bulletin of the Seismological Society of America, 100(2).

Ide, S. (2012), Variety and spatial heterogeneity of tectonic tremor worldwide, Journal of Geophysical Research-Solid Earth, 117.

Ishimoto, M. a. I., K. (1939), Observations of earthquakes registered with the microseismograph constructed recently, Bulletin of the Earthquake Research InstituteUniversity of Tokyo, 17.

Jackson, D. D. (1996), The Case for Huge Earthquakes, Seismological Research Letters, $67(1)$.

Jackson, D. D., and Y. Y. Kagan (1999), Testable Earthquake Forecasts for 1999, Seismological Research Letters, 70(4).

Jacobs, K. M., and S. R. McNutt (2006), Update of the Global Volcanic Earthquake Swarm Database: Search for forecasting possibilities, in Cities on Volcanoes, edited, Quito, Ecuador.

Jacobs, K. M., E. G. C. Smith, M. K. Savage, and J. C. Zhuang (2013), Cumulative rate analysis (CURATE): A clustering algorithm for swarm dominated catalogs, Journal of Geophysical Research-Solid Earth, 118(2).

Jolly, A. D., J. Neuberg, P. Jousset, and S. Sherburn (2012), A new source process for evolving repetitious earthquakes at Ngauruhoe volcano, New Zealand, Journal of Volcanology and Geothermal Research, 215.

Kagan, Y. Y. (1991), Seismic Moment Distribution, Geophysical Journal International, 106(1).

Kagan, Y. Y., and D. D. Jackson (2000), Probabilistic forecasting of earthquakes, Geophysical Journal International, 143(2).

Kagan, Y. Y. (2004), Short-term properties of earthquake catalogs and models of earthquake source, Bulletin of the Seismological Society of America, 94(4). 
Kagan, Y. Y., and H. Houston (2005), Relation between mainshock rupture process and Omori's law for aftershock moment release rate, Geophysical Journal International, 163(3).

Kanamori, H., and D. L. Anderson (1975), Theoretical Basis of Some Empirical Relations in Seismology, Bulletin of the Seismological Society of America, 65(5).

Kanamori, H. (1981), The Nature of Seismicity Patterns Before Large Earthquakes, in Earthquake prediction : an international review, edited by D. W. Simpson, et al., pp. viii, 680 p., American Geophysical Union, Washington, D.C.

Keats, B. S., J. H. Johnson, and M. K. Savage (2011), The Erua earthquake cluster and seismic anisotropy in the Ruapehu region, New Zealand, Geophysical Research Letters, 38.

Kilgour, G., V. Manville, F. Della Pasqua, A. Graettinger, K. A. Hodgson, and G. E. Jolly (2010), The 25 September 2007 eruption of Mount Ruapehu, New Zealand: Directed ballistics, surtseyan jets, and ice-slurry lahars, Journal of Volcanology and Geothermal Research, 191(1-2).

Kim, M. J., S. Y. Schwartz, and S. Bannister (2011), Non-volcanic tremor associated with the March 2010 Gisborne slow slip event at the Hikurangi subduction margin, New Zealand, Geophysical Research Letters, 38.

Leitner, B., D. Eberhart-Phillips, H. Anderson, and J. L. Nabelek (2001), A focused look at the Alpine fault, New Zealand: Seismicity, focal mechanisms, and stress observations, Journal of Geophysical Research-Solid Earth, 106(B2).

Lin, G. Q., P. M. Shearer, and E. Hauksson (2007), Applying a three-dimensional velocity model, waveform cross correlation, and cluster analysis to locate southern California seismicity from 1981 to 2005, Journal of Geophysical Research-Solid Earth, 112(B12).

Linde, A. T., M. T. Gladwin, M. J. S. Johnston, R. L. Gwyther, and R. G. Bilham (1996), A slow earthquake sequence on the San Andreas fault, Nature, 383(6595).

Llenos, A. L., J. J. McGuire, and Y. Ogata (2009), Modeling seismic swarms triggered by aseismic transients, Earth and Planetary Science Letters, 281(1-2).

Lohman, R. B., and J. J. McGuire (2007), Earthquake swarms driven by aseismic creep in the Salton Trough, California, Journal of Geophysical Research-Solid Earth, 112(B4).

Lombardi, A. M., W. Marzocchi, and J. Selva (2006), Exploring the evolution of a volcanic seismic swarm: The case of the 2000 Izu Islands swarm, Geophysical Research Letters, 33(7).

Lombardi, A. M., M. Cocco, and W. Marzocchi (2010), On the Increase of Background Seismicity Rate during the 1997-1998 Umbria-Marche, Central Italy, Sequence: Apparent Variation or Fluid-Driven Triggering?, Bulletin of the Seismological Society of America, $100(3)$.

Luen, B., and P. B. Stark (2012), Poisson tests of declustered catalogues, Geophysical Journal International, 189(1).

Malagnini, L., F. P. Lucente, P. De Gori, A. Akinci, and I. Munafo (2012), Control of pore fluid pressure diffusion on fault failure mode: Insights from the 2009 L'Aquila seismic sequence, Journal of Geophysical Research-Solid Earth, 117. 
McCaffrey, R., L. M. Wallace, and J. Beavan (2008), Slow slip and frictional transition at low temperature at the Hikurangi subduction zone, Nat Geosci, 1(5).

McNutt, S. R. (2005), Volcanic seismology, Annual Review of Earth and Planetary Sciences, 33.

Mogi, K. (1962), Magnitude-Frequency relation for elastic shocks accompanying fractures of various materials and some related problems in earthquakes (2nd paper), Bulletin of the Earthquake Research Institue, 40.

Mogi, K. (1963), Some Discussions on Aftershocks, Foreshocks and Earthquake Swarms, the Fracture of a Semi-infinite body Caused by an Inner Stress Origin and Its Realtion to the Earthquake Phenomena (Third Paper). Bulletin of the Earthquake Research InstituteUniversity of Tokyo, 41.

Moran, S. C., J. A. Power, S. D. Stihler, J. J. Sanchez, and J. Caplan-Auerbach (2004), Earthquake triggering at Alaskan volcanoes following the 3 November 2002 Denali fault earthquake, Bulletin of the Seismological Society of America, 94(6).

Mouslopoulou, V., and D. T. Hristopulos (2011), Patterns of tectonic fault interactions captured through geostatistical analysis of microearthquakes, Journal of Geophysical Research-Solid Earth, 116.

Nadeau, R. M., W. Foxall, and T. V. Mcevilly (1995), Clustering and Periodic Recurrence of Microearthquakes on the San-Andreas Fault at Parkfield, California, Science, 267(5197).

Nakagawa, M., K. Wada, T. Thordarson, C. P. Wood, and J. A. Gamble (1999), Petrologic investigations of the 1995 and 1996 eruptions of Ruapehu volcano, New Zealand: formation of discrete and small magma pockets and their intermittent discharge, Bulletin of Volcanology, 61(1-2).

Nur, A. (1972), Dilatancy, Pore Fluids, and Premonitory Variations of Ts-Tp Travel Times, Bulletin of the Seismological Society of America, 62(5).

O'Keefe, B. C. (2008), Microseismicity of the central Alpine Fault region, New Zealand. .

Ogata, Y. (1988), Statistical Models for Earthquake Occurrences and Residual Analysis for Point Processes, J Am Stat Assoc, 83(401).

Ogata, Y. (1998), Space-time point-process models for earthquake occurrences, Annals of the Institute of Statistical Mathematics, 50(2).

Okutani, T., and S. Ide (2011), Statistic analysis of swarm activities around the Boso Peninsula, Japan: Slow slip events beneath Tokyo Bay?, Earth Planets and Space, 63(5).

Otway, P. M., and S. Sherburn (1994), Vertical Deformation and Shallow Seismicity around Lake Taupo, New-Zealand, 1985-90, New Zealand Journal of Geology and Geophysics, $37(2)$.

Ozawa, S., S. Miyazaki, Y. Hatanaka, T. Imakiire, M. Kaidzu, and M. Murakami (2003), Characteristic silent earthquakes in the eastern part of the Boso peninsula, Central Japan, Geophysical Research Letters, 30(6).

Ozawa, S., H. Suito, and M. Tobita (2007), Occurrence of quasi-periodic slow-slip off the east coast of the Boso peninsula, Central Japan, Earth Planets and Space, 59(12). 
Page, E. S. (1954), Continuous Inspection Schemes, Biometrika, 41(1-2).

Paige, C. C., and M. A. Saunders (1982), Lsqr - an Algorithm for Sparse Linear-Equations and Sparse Least-Squares, Acm T Math Software, 8(1).

Parotidis, M., E. Rothert, and S. A. Shapiro (2003), Pore-pressure diffusion: A possible triggering mechanism for the earthquake swarms 2000 in Vogtland/NW-Bohemia, central Europe, Geophysical Research Letters, 30(20).

Petersen, T., K. Gledhill, M. Chadwick, N. H. Gale, and J. Ristau (2011), The New Zealand National Seismograph Network, Seismological Research Letters, 82(1).

Peterson, C. L., and D. H. Christensen (2009), Possible relationship between nonvolcanic tremor and the 1998-2001 slow slip event, south central Alaska, Journal of Geophysical Research-Solid Earth, 114.

Prejean, S. G., D. P. Hill, E. E. Brodsky, S. E. Hough, M. J. S. Johnston, S. D. Malone, D. H. Oppenheimer, A. M. Pitt, and K. B. Richards-Dinger (2004), Remotely triggered seismicity on the United States west coast following the M-W 7.9 Denali fault earthquake, Bulletin of the Seismological Society of America, 94(6).

Quigley, M., R. Van Dissen, N. Litchfield, P. Villamor, B. Duffy, D. Barrell, K. Furlong, T. Stahl, E. Bilderback, and D. Noble (2012), Surface rupture during the $2010 \mathrm{M}-\mathrm{w}$ 7.1 Darfield (Canterbury) earthquake: Implications for fault rupture dynamics and seismic-hazard analysis, Geology, 40(1).

Reasenberg, P. (1985), 2nd-Order Moment of Central California Seismicity, 1969-1982, Journal of Geophysical Research-Solid Earth and Planets, 90(Nb7).

Reyners, M. (1980), A Micro-Earthquake Study of the Plate Boundary, North-Island, NewZealand, Geophysical Journal of the Royal Astronomical Society, 63(1).

Reyners, M., and S. Bannister (2007), Earthquakes triggered by slow slip at the plate interface in the Hikurangi subduction zone, New Zealand, Geophysical Research Letters, 34(14).

Richardson, W. P. (1989), The Matata Earthquake of 1977 May 31 - a Recent Event near Edgecumbe, Bay of Plenty, New-Zealand, New Zealand Journal of Geology and Geophysics, $32(1)$.

Risk, G. F., H. M. Bibby, C. J. Bromley, T. G. Caldwell, and S. L. Bennie (2002), Appraisal of the Tokaanu-Waihi geothermal field and its relationship with the Tongariro geothermal field, New Zealand, Geothermics, 31(1).

Robinson, R. (1979), Variation of Energy-Release, Rate of Occurrence and B-Value of Earthquakes in the Main Seismic Region, New Zealand, Physics of the Earth and Planetary Interiors, $18(3)$.

Robinson, R. (1994), Shallow Subduction Tectonics and Fault Interaction - the Weber, NewZealand, Earthquake Sequence of 1990-1992, Journal of Geophysical Research-Solid Earth, 99(B5).

Robinson, R. (2003), Fault interactions and subduction tectonics: a re-examination of the Weber, New Zealand, earthquake sequence of 1990, Geophysical Journal International, 154(3). 
Roman, D. C., and K. V. Cashman (2006), The origin of volcano-tectonic earthquake swarms, Geology, 34(6).

Rydelek, P. A., and I. S. Sacks (1989), Testing the Completeness of Earthquake Catalogs and the Hypothesis of Self-Similarity, Nature, 337(6204).

Savage, M. K., and S. H. Rupp (2000), Foreshock probabilities in New Zealand, New Zealand Journal of Geology and Geophysics, 43(3).

Scholz, C. H. (1968), Frequency-Magnetude Relation of Microfracturing in Rock and Its Relation to Earthquakes, Bulletin of the Seismological Society of America, 58(1).

Scholz, C. H. (2002), The Mechanics of Earthquakes and Faulting, 2nd ed., 469 pp., Cambridge Universtiy Press, Cambridge.

Segall, P., E. K. Desmarais, D. Shelly, A. Miklius, and P. Cervelli (2006), Earthquakes triggered by silent slip events on Kilauea volcano, Hawaii, Nature, 442(7098).

Shearer, P. M. (2012), Self-similar earthquake triggering, Bath's law, and foreshock/aftershock magnitudes: Simulations, theory, and results for southern California, Journal of Geophysical Research-Solid Earth, 117.

Sherburn, S. (1992a), Seismicity of the Lake Taupo region, New Zealand, 1985-90, New Zealand Journal of Geology and Geophysics, 35(3).

Sherburn, S. (1992b), Characteristics of Earthquake Sequences in the Central Volcanic Region, New-Zealand, New Zealand Journal of Geology and Geophysics, 35(1).

Sherburn, S., S. Bannister, and H. Bibby (2003), Seismic velocity structure of the central Taupo Volcanic Zone, New Zealand, from local earthquake tomography, Journal of Volcanology and Geothermal Research, 122(1-2).

Sherburn, S., and I. A. Nairn (2004), Modelling geophysical precursors to the prehistoric c. AD1305 Kaharoa rhyolite eruption of Tarawera volcano, New Zealand, Nat Hazards, 32(1).

Smith, E. G. C., and T. H. Webb (1986), The seismicity and related deformation of the Central Volcanic Region, North Island, New Zealand. , Royal Soceity of New Zealand bulletin, 23.

Smith, E. G. C., and C. M. M. Oppenheimer (1989), The Edgecumbe Earthquake Sequence 1987 February 21 to March 18, New Zealand Journal of Geology and Geophysics, 32(1).

Smith, E. G. C., T. D. Williams, and D. J. Darby (2007), Principal component analysis and modeling of the subsidence of the shoreline of Lake Taupo, New Zealand, 1983-1999:

Evidence for dewatering of a magmatic intrusion?, Journal of Geophysical Research-Solid Earth, 112(B8).

Stark, M. A., and S. D. Davis (1996), Remotely triggered microearthquakes at The Geysers geothermal field, California, Geophysical Research Letters, 23(9).

Stern, T. A., W. R. Stratford, and M. L. Salmon (2006), Subduction evolution and mantle dynamics at a continental margin: Central North Island, New Zealand, Reviews of Geophysics, 44(4). 
Tam, D. (2009), A Theoretical Analysis of Cumulative Sum Slope (CUSUM-Slope) Statistic for Detecting Signal Onset (begin) and Offset (end) Trends from Background Noise Level, The Open Statistics and Probability Journal, 1.

Toda, S., R. S. Stein, and T. Sagiya (2002), Evidence from the AD 2000 Izu islands earthquake swarm that stressing rate governs seismicity, Nature, 419(6902).

Toda, S., R. S. Stein, and J. Lin (2011), Widespread seismicity excitation throughout central Japan following the $2011 \mathrm{M}=9.0$ Tohoku earthquake and its interpretation by Coulomb stress transfer, Geophysical Research Letters, 38.

Tormann, T., M. K. Savage, E. G. C. Smith, M. W. Stirling, and S. Wiemer (2008), Time-, distance-, and magnitude-dependent foreshock probability model for New Zealand, Bulletin of the Seismological Society of America, 98(5).

Umakoshi, K., H. Shimizu, and N. Matsuwo (2001), Volcano-tectonic seismicity at Unzen volcano, Japan, 1985-1999, Journal of Volcanology and Geothermal Research, 112.

Utsu, T., Y. Ogata, and R. S. Matsuura (1995), The Centenary of the Omori Formula for a Decay Law of Aftershock Activity, Journal of Physics of the Earth, 43(1).

van Stiphout, T., J. Zhuang, and D. Marsan (2012), Seismicity Declustering, Community Online Resource for Statistical Seismicity Analysis.

Vere-Jones, D. (1970), Stochastic Models for Earthquake Occurrence, Journal of the Royal Statistical Society. Series B (Methodological), 32(1).

Vere-Jones, D., and J. Zhuang (2008), Distribution of the largest event in the critical epidemic-type aftershock-sequence model, Physical Review E, 78(4).

Vidale, J. E., and P. M. Shearer (2006), A survey of 71 earthquake bursts across southern California: Exploring the role of pore fluid pressure fluctuations and aseismic slip as drivers, Journal of Geophysical Research-Solid Earth, 111(B5).

Villamor, P., and K. Berryman (2001), A late Quaternary extension rate in the Taupo Volcanic Zone, New Zealand, derived from fault slip data, New Zealand Journal of Geology and Geophysics, 44(2).

Villamor, P., and K. R. Berryman (2006), Evolution of the southern termination of the Taupo Rift, New Zealand, New Zealand Journal of Geology and Geophysics, 49(1).

Wadati, K. (1933), On the travel time of earthquake waves, Geophysics Magazine, 7.

Waldhauser, F., and W. L. Ellsworth (2000), A double-difference earthquake location algorithm: Method and application to the northern Hayward fault, California, Bulletin of the Seismological Society of America, 90(6).

Wallace, L. M., J. Beavan, R. McCaffrey, and D. Darby (2004), Subduction zone coupling and tectonic block rotations in the North Island, New Zealand, Journal of Geophysical Research-Solid Earth, 109(B12).

Wallace, L. M., and J. Beavan (2010), Diverse slow slip behavior at the Hikurangi subduction margin, New Zealand, Journal of Geophysical Research-Solid Earth, 115. 
Wallace, L. M., J. Beavan, S. Bannister, and C. Williams (2012), Simultaneous long-term and short-term slow slip events at the Hikurangi subduction margin, New Zealand: Implications for processes that control slow slip event occurrence, duration, and migration, J. Geophys. Res., 117(B11).

Wang, Q., D. D. Jackson, and J. C. Zhuang (2010a), Missing links in earthquake clustering models, Geophysical Research Letters, 37.

Wang, Q., D. D. Jackson, and J. C. Zhuang (2010b), Are spontaneous earthquakes stationary in California?, Journal of Geophysical Research-Solid Earth, 115.

Warren, N. W., and G. V. Latham (1970), An Experimental Study of Thermally Induced Microfracturing and Its Relation to Volcanic Seismicity, Journal of Geophysical Research, $75(23)$.

Webb, T. H., B. G. Ferris, and J. S. Harris (1986), The Lake Taupo, New-Zealand, Earthquake Swarms of 1983, New Zealand Journal of Geology and Geophysics, 29(4).

Wech, A. G., and K. C. Creager (2011), A continuum of stress, strength and slip in the Cascadia subduction zone, Nat Geosci, 4(9).

Wech, A. G., C. M. Boese, T. A. Stern, and J. Townend (2012), Tectonic tremor and deep slow slip on the Alpine Fault, Geophysical Research Letters, 39.

West, M., J. J. Sanchez, and S. R. McNutt (2005), Periodically triggered seismicity at Mount Wrangell, Alaska, after the Sumatra earthquake, Science, 308(5725).

Wiemer, A. (2001), A Software Package to Analyze Seismicity: ZMAP, Seismological Research Letters, 72.

Wiemer, S., and M. Wyss (1994), Seismic Quiescence before the Landers $(\mathrm{M}=7.5)$ and Big Bear (M=6.5) 1992 Earthquakes, Bulletin of the Seismological Society of America, 84(3).

Wiemer, S., and M. Wyss (2000), Minimum magnitude of completeness in earthquake catalogs: Examples from Alaska, the western United States, and Japan, Bulletin of the Seismological Society of America, 90(4).

Williams, D. (2001), Weighing the odds : a course in probability and statistics, xvii, $547 \mathrm{p}$. pp., Cambridge University Press, Cambridge ; New York.

Wilson, C. J. N., B. F. Houghton, M. O. Mcwilliams, M. A. Lanphere, S. D. Weaver, and R. M. Briggs (1995), Volcanic and Structural Evolution of Taupo Volcanic Zone, New-Zealand a Review, Journal of Volcanology and Geothermal Research, 68(1-3).

Woessner, J., and S. Wiemer (2005), Assessing the quality of earthquake catalogues: Estimating the magnitude of completeness and its uncertainty, Bulletin of the Seismological Society of America, 95(2).

Wyss, M. (1973), Towards a Physical Understanding of Earthquake Frequency Distribution, Geophysical Journal of the Royal Astronomical Society, 31(4).

Wyss, M., and S. Wiemer (2000), Change in the probability for earthquakes in southern California due to the Landers magnitude 7.3 earthquake, Science, 290(5495). 
Yamashita, T. (1999), Pore creation due to fault slip in a fluid-permeated fault zone and its effect on seismicity: Generation mechanism of earthquake swarm, Pure and Applied Geophysics, 155(2-4).

Zechar, J. D., J. L. Hardebeck, A. J. Michael, M. Naylor, S. Steacy, S. Wiemer, J. C. Zhuang, and C. W. Grp (2011), Community Online Resource for Statistical Seismicity Analysis, Seismological Research Letters, 82(5).

Zhuang, J., Y. Ogata, and D. Vere-Jones (2002), Stochastic declustering of space-time earthquake occurrences, J Am Stat Assoc, 97(458).

Zhuang, J., A. Christophersen, M. K. Savage, D. Vere-Jones, Y. Ogata, and D. D. Jackson (2008), Differences between spontaneous and triggered earthquakes: Their influences on foreshock probabilities, Journal of Geophysical Research-Solid Earth, 113(B11).

Zhuang, J. C. (2000), Statistical modelling of seismicity patterns before and after the 1990 Oct 5 Cape Palliser earthquake, New Zealand, New Zealand Journal of Geology and Geophysics, 43(3).

Zhuang, J. C., Y. Ogata, and D. Vere-Jones (2004), Analyzing earthquake clustering features by using stochastic reconstruction, Journal of Geophysical Research-Solid Earth, 109(B5). 


\section{Appendix A: CURATE user's manual}

General notes: We use the term 'sequence' to refer to a spatio-temporally clustered set of earthquakes. Sequences are of both mainshock-aftershock and swarm types. The initial identification of sequences in this code does not differentiate between sequence types. However later processing steps do determine which sequences fit the MS-AS definition given in Ch. 4.2. Below is a brief description of all the codes and their called function. The CURATE code uses pre-made codes to analyze the area. These are not distributed here, but the user is referred to a set of codes by Nima Moshtagh. The codes fit an ellipse around all data points and as a result the areas are maximum areas. These codes are listed under functions called (denoted by $*$ ), but are not separately explained in the function section.

\section{Necessary inputs:}

An earthquake catalog in MATLAB format is the only necessary initial input. The catalog is expected in the format:

[YYYY MM DD HR MIN SEC LAT LONG DEP MAG].

A bounding polygon is also required, but if one does not already exist, or if the user does not desire to use one the first script, polyCreate.m, will create one to the user's specifications.

Other inputs that are assigned as the codes are run include:

-Output file name for catalog/run (distance/day rules and Mcut appended to filename automatically)

-Desired Mcut (usually magnitude of completeness, Mc)

-Distance-rule range

-Day-rule range

\section{Boundary Checking:}

The codes included here do not perform any boundary checking of the input polygon. They could cut a sequence at the boundary. We have included our code we used to check the northern boundary in the CVR (newDist_test_northCat_2012pub.m), but due to the variety of potential input polygons we have not created a universal boundary checking code.

\section{Analysis using other programs:}

The CURATE codes output an additional format for use in ZMAP:

The earthquake catalog within the selected polygon and Mcut value are saved into an ascii file in zmap format (another MATLAB based program Wiemer et al.[Wiemer, 2001]). For analyzing the Mc value the whole catalog may be desirable, but many ZMAP functions have the option to use Mc as Mmin (this will be the case in the polygon cut earthquake catalog). Zmap also contains codes for running both Reasenberg and Gardner and Knopoff declustering. Both run fairly quickly and will give declustered catalogs that can be compared to the CURATE catalog. 
General MATLAB advice:

MATLAB has a relatively small memory. Figures take up lots of active memory so if things are running slowly, it may help to save and close figures when you are not using them. If you wish to close all figures (without saving), simply type 'close all' on the MATLAB command prompt. 'Clear all' deletes all variables from the workspace without saving. Figures can be saved into a variety of formats from MATLAB, but only the matlab format .fig will retain editing capabilities of the figure. It is useful to save a .fig version in addition to other saved formats for later editing if required. MATLAB has a great built in HELP function and the online resources for MATLAB are also quite good.

\section{Code Descriptions:}

Each script and function shows which direct functions are called, but sub functions are not listed.

Main scripts:

polyCreate.m :

\begin{tabular}{|l|l|l|l|}
\hline Purpose: & Inputs: & Outputs: & Functions \\
Called:
\end{tabular}


poly_subset.m :

\begin{tabular}{|l|l|l|l|}
\hline Purpose: & Inputs: & Outputs: & $\begin{array}{l}\text { Functions } \\
\text { Called: }\end{array}$ \\
\hline $\begin{array}{l}\text { Reads the information } \\
\text { from the file created by } \\
\text { polyCreate.m and } \\
\text { allows interactive } \\
\text { choices of several } \\
\text { parameters about initial } \\
\text { processing to } \\
\text { determine the }\end{array}$ & $\begin{array}{l}\text {-Eame for seq cat } \\
\text { CURATE and identify } \\
\text { Potential-sequences. }\end{array}$ & $\begin{array}{l}\text {-Potetntial } \\
\text { sequences }\end{array}$ & Decdays_fixlm.m \\
Curate_2010.m \\
\end{tabular}

grabSequenceData.m :

\begin{tabular}{|l|l|l|l|}
\hline Purpose: & Inputs: & Futputs: & Called: \\
\hline $\begin{array}{l}\text { Checks potential } \\
\text { sequences for } \\
\text { distance rule (input } \\
\text { in this step). }\end{array}$ & $\begin{array}{l}\text {-Pame for seq cat } \\
\text { Sequences }\end{array}$ & Proto-Sequences & Makeswarm.m \\
$\begin{array}{l}\text { Allows for a range } \\
\text { of distance rules to } \\
\text { be run at once. }\end{array}$ & $\begin{array}{l}\text {-Distance rule } \\
\text { range }\end{array}$ & $\begin{array}{l}\text { Files will save } \\
\text { separately for each } \\
\text { distance-rule. }\end{array}$ & Seq_cat.m \\
\hline
\end{tabular}


grab_sequence_m2012pub.m : Version associated with JGR 2012

\begin{tabular}{|l|l|l|l|}
\hline Purpose: & Inputs: & Outputs: & Functions \\
Called:
\end{tabular}

newDist_test_northCat_2012pub.m : Northern Boundary Testing (JGR 2012)

\begin{tabular}{|c|c|c|c|}
\hline Purpose: & Inputs: & Outputs: & $\begin{array}{l}\text { Functions } \\
\text { Called: }\end{array}$ \\
\hline $\begin{array}{l}\text { Uses a predetermined } \\
\text { earthquake catalog } \\
\text { with boundaries } \\
\text { greater than the } \\
\text { current sequence } \\
\text { catalog polygon to } \\
\text { check for truncation } \\
\text { of sequences at the } \\
\text { northern boundary. } \\
\text { Concatenates } \\
\text { earthquakes beyond } \\
\text { the boundary into } \\
\text { existing sequences, } \\
\text { but does not allow for } \\
\text { the creation of new } \\
\text { sequences. }\end{array}$ & $\begin{array}{l}\text {-Day and distance } \\
\text { rule ranges and } \\
\text { increments } \\
\text {-Initial Sequences } \\
\text {-Eq catalog with } \\
\text { obunds larger } \\
\text { than the limiting } \\
\text { polygon of the } \\
\text { sequence catalog } \\
\text { (set manually). }\end{array}$ & $\begin{array}{l}\text { Initial-Sequences (will } \\
\text { receive one more check in } \\
\text { Catalogue_paramTest.m } \\
\text { bevofre becoming } \\
\text { finalized sequences). } \\
\text { Files will save separately } \\
\text { for each distance-day rule } \\
\text { combination. }\end{array}$ & $\mathrm{N} / \mathrm{A}$ \\
\hline
\end{tabular}


Catalogue_paramTest_2012.m: Version associated with JGR 2012

\begin{tabular}{|l|l|l|l|}
\hline Purpose: & Inputs: & Functions \\
Called:
\end{tabular}

grab_sequence_m.m : Version that does not include boundary checking.

\begin{tabular}{|c|c|c|c|}
\hline Purpose: & Inputs: & Outputs: & $\begin{array}{l}\text { Functions } \\
\text { Called: }\end{array}$ \\
\hline $\begin{array}{l}\text { Uses the day-rule to } \\
\text { concatenate Proto- } \\
\text { Sequences if they are } \\
\text { close enough in time. } \\
\text { Unlike JGR version, } \\
\text { this version does not } \\
\text { have boundary checking } \\
\text { and so takes on the } \\
\text { functions of } \\
\text { Catalogue_paramTest.m }\end{array}$ & $\begin{array}{l}\text {-Day rule range } \\
\text { and increment } \\
\text {-Proto } \\
\text { Sequences }\end{array}$ & $\begin{array}{l}\text { Sequences } \\
\text { Basic initial } \\
\text { analysis } \\
\text { Files will save } \\
\text { separately for each } \\
\text { distance-day rule } \\
\text { combination. }\end{array}$ & $\begin{array}{l}\text { Grab_seq_rerun.m } \\
\text { Multi_sw_cat.m } \\
\text { Seq_PostProcess.m }\end{array}$ \\
\hline
\end{tabular}




\section{Functions:}

The following tables describe the purpose of functions called by the codes above:

Decdays_fixlm.m:

\begin{tabular}{|l|l|l|l|}
\hline Purpose: & Inputs: & Outputs: & $\begin{array}{l}\text { Functions } \\
\text { Called: }\end{array}$ \\
\hline $\begin{array}{l}\text { Convert earthquake } \\
\text { catalog times into } \\
\text { decimal years. } \\
\text { (Written by Euan } \\
\text { Smith) }\end{array}$ & $\begin{array}{l}\text {-Eq catalog [YYYY } \\
\text { MMD HR MIN }\end{array}$ & $\begin{array}{l}\text { List of decimal times } \\
\text { for all earthquakes }\end{array}$ & N/A \\
\hline
\end{tabular}

Curate_2010.m:

\begin{tabular}{|l|l|l|l|}
\hline Purpose: & Inputs: & Outputs: & Functions \\
Called:
\end{tabular}

make_swarm.m :

\begin{tabular}{|c|c|c|c|}
\hline Purpose: & Inputs: & Outputs: & $\begin{array}{l}\text { Functions } \\
\text { Called: }\end{array}$ \\
\hline $\begin{array}{l}\text { Uses the day-rule to } \\
\text { concatenate Proto- } \\
\text { Sequences if they are } \\
\text { close enough in time. }\end{array}$ & $\begin{array}{l}\text {-name of polydata } \\
\text { file } \\
\text {-cell size for spatial } \\
\text { gridding (optional) }\end{array}$ & $\begin{array}{l}\text {-Proto seq list } \\
\text {-eq list related to seq } \\
\text { list } \\
\text {-bounding polygon } \\
\text {-mean rate } \\
\text {-spatial grid indexing }\end{array}$ & findswarm.m \\
\hline
\end{tabular}


findswarm.m :

\begin{tabular}{|l|l|l|l|}
\hline Purpose: & Inputs: & Outputs: & Functions \\
& & & Called: \\
\hline $\begin{array}{l}\text { Determines the } \\
\text { CURATE }\end{array}$ & $\begin{array}{l}\text {-List of earthquake } \\
\text { times }\end{array}$ & -Proto seq list & N/A \\
& & -mean rate \\
& & \\
\hline
\end{tabular}

\section{findswarmMod.m :}

\begin{tabular}{|c|c|c|c|}
\hline Purpose: & Inputs: & Outputs: & $\begin{array}{l}\text { Functions } \\
\text { Called: }\end{array}$ \\
\hline $\begin{array}{l}\text { Same as findswarm.m } \\
\text { but takes a mean rate. } \\
\text { Used for searching the } \\
\text { earthquakes rejected } \\
\text { from sequences for } \\
\text { additional sequences. }\end{array}$ & $\begin{array}{l}\text {-List of earthquake } \\
\text { times } \\
\text {-mean rate }\end{array}$ & $\begin{array}{l}\text {-Proto seq list } \\
\text {-mean rate } \\
\text {-CUARTE through time }\end{array}$ & N/A \\
\hline
\end{tabular}

\section{Cut_seq_byDistWWc.m :}

\begin{tabular}{|l|l|l|l|}
\hline Purpose: & Inputs: & Outputs: & Functions \\
Called:
\end{tabular}


Grab_seq_rerun.m :

\begin{tabular}{|c|c|c|c|}
\hline Purpose: & Inputs: & Outputs: & $\begin{array}{l}\text { Functions } \\
\text { Called: }\end{array}$ \\
\hline $\begin{array}{l}\text { Create new set of } \\
\text { general sequence } \\
\text { parameters after } \\
\text { distance limitation or } \\
\text { sequence } \\
\text { concatenation with } \\
\text { day-rule. }\end{array}$ & $\begin{array}{l}\text {-seq list } \\
\text {-eq locations } \\
\text {-distance limit } \\
\text {-indexing column }\end{array}$ & $\begin{array}{l}\text {-updated seq list } \\
\text {-eq list in new seq order } \\
\text {-updated general seq } \\
\text { parameters including a } \\
\text { mark of which eqs are } \\
\text { further from the new } \\
\text { median location than } \\
\text { the distance limit }\end{array}$ & N/A \\
\hline
\end{tabular}

checkEdge.m :

\begin{tabular}{|l|l|l|l|}
\hline Purpose: & Inputs: & Outputs: & Functions \\
& & & Called: \\
\hline $\begin{array}{l}\text { Checks for a cookie } \\
\text { cutter effect at the } \\
\text { edge of sequences. }\end{array}$ & -seq list & -eq locations \\
-distance limit & -eq list in new seq order \\
-indexing column & -number of sequences & \\
& & & \\
& & & \\
\hline
\end{tabular}


Seq_cat.m :

\begin{tabular}{|c|c|c|c|}
\hline Purpose: & Inputs: & Outputs: & $\begin{array}{l}\text { Functions } \\
\text { Called: }\end{array}$ \\
\hline $\begin{array}{l}\text { Put the sequence list } \\
\text { back together again } \\
\text { after processing small } \\
\text { pieces of it for } \\
\text { additional distance } \\
\text { testing etc. }\end{array}$ & $\begin{array}{l}\text {-Main seq list } \\
\text {-Main eq locations } \\
\text {-Main gen seq params } \\
\text {-Sub seq list } \\
\text {-Sub eq locations } \\
\text {-Sub gen seq params }\end{array}$ & $\begin{array}{l}\text {-concatenated seq list } \\
\text {-updated eq list in } \\
\text { new seq order } \\
\text {-updated general seq } \\
\text { param list }\end{array}$ & N/A \\
\hline
\end{tabular}

Multi_sw_cat.m:

\begin{tabular}{|l|l|l|l|}
\hline Purpose: & Inputs: & Outputs: & Functions \\
Called:
\end{tabular}


newDist_seqcatSrch.m:

\begin{tabular}{|c|c|c|c|}
\hline Purpose: & Inputs: & Outputs: & $\begin{array}{l}\text { Functions } \\
\text { Called: }\end{array}$ \\
\hline $\begin{array}{l}\text { Checks the time } \\
\text { relationship of } \\
\text { sequences. }\end{array}$ & $\begin{array}{l}\text {-seq list } \\
\text {-general seq info } \\
\text {-distance rule } \\
\text {-day rule }\end{array}$ & $\begin{array}{l}\text {-A variety of variable } \\
\text { defining related } \\
\text { sequences }\end{array}$ & $\mathrm{N} / \mathrm{A}$ \\
\hline
\end{tabular}

aftshk_srt.m :

\begin{tabular}{|l|l|l|l|}
\hline Purpose: & Inputs: & Outputs: & Functions \\
\hline $\begin{array}{l}\text { Gathers Mmax and } \\
\text { relevant magnitude } \\
\text { information. Stores } \\
\text { indexing for later use. } \\
\begin{array}{l}\text { Defines sequences as } \\
\text { MS-AS or swarms. }\end{array}\end{array}$ & $\begin{array}{l}\text {-seq list } \\
\text {-general seq details } \\
\text {-minimum number } \\
\text { of earthquakes a } \\
\text { sequence must } \\
\text { contain (Nmin) }\end{array}$ & $\begin{array}{l}\text {-Magnitude (Mmax) } \\
\text { information and } \\
\text { indexing } \\
\text { separately }\end{array}$ & N/A \\
& & & \\
\hline
\end{tabular}


Ellipse_boundGrid.m :

\begin{tabular}{|c|c|c|c|}
\hline Purpose: & Inputs: & Outputs: & $\begin{array}{l}\text { Functions } \\
\text { Called: }\end{array}$ \\
\hline $\begin{array}{l}\text { Calculate area of } \\
\text { sequences. Uses a } \\
\text { best fit ellipse and a } \\
\text { gridded seismicity } \\
\text { approach. }\end{array}$ & $\begin{array}{l}\text {-sequence list } \\
\text {-eq locations } \\
\text {-number of seqs } \\
\text { to process } \\
\text {-Mmax info } \\
\text {-general seq } \\
\text { details } \\
\text {-latitiude } \\
\text { correction }\end{array}$ & $\begin{array}{l}\text {-Parameters } \\
\text { containing several } \\
\text { area calculations } \\
\text {-List of points } \\
\text { defining best fit } \\
\text { ellipses for each } \\
\text { sequence }\end{array}$ & $\begin{array}{l}\text { MinVolEllipse.m* } \\
\text { Ellipse_plot.m* }\end{array}$ \\
\hline
\end{tabular}

sizeSwCrnr.m :

\begin{tabular}{|l|l|l|l|}
\hline Purpose: & Inputs: & Outputs: & Functions \\
& Called: \\
\hline $\begin{array}{l}\text { Pulls relevant } \\
\text { sequence data for } \\
\text { sequences limited by a } \\
\text { minimum number of } \\
\text { earthquakes (Nmin). }\end{array}$ & $\begin{array}{l}\text {-list of sequences } \\
\text {-General sequence } \\
\text { parameters } \\
\text { (SwarmCorners) }\end{array}$ & $\begin{array}{l}\text { Size limited general } \\
\text { sequence parameters }\end{array}$ & N/A \\
& & & \\
\hline
\end{tabular}


SeqPostProcess.m :

\begin{tabular}{|c|c|c|c|}
\hline Purpose: & Inputs: & Outputs: & $\begin{array}{l}\text { Functions } \\
\text { Called: }\end{array}$ \\
\hline $\begin{array}{l}\text { Basic Analysis of } \\
\text { final sequence } \\
\text { parameters. } \\
\text { Includes area } \\
\text { calculation. }\end{array}$ & $\begin{array}{l}\text {-list of sequences } \\
\text {-General sequence } \\
\text { parameters } \\
\text { (SwarmCorners) }\end{array}$ & $\begin{array}{l}\text { Size limited general } \\
\text { sequence parameters }\end{array}$ & \\
\hline
\end{tabular}




\section{Appendix B: HypoDD inputs}

This Appendix contains the parameters that were varied through the hypoDD relocations shown in chapter 4.5. No cross-correlations were performed and so, where inputs were required for cross-correlation parameters values were set to -9 the flag to not use data in hypoDD. These parameters are not shown. The maximum distance between the cluster centroid and stations (DIST) was set to $100 \mathrm{~km}$ for all runs.

Table B.1 Input arameters for phase2dt (hypoDD preparation)

\begin{tabular}{|c|c|c|}
\hline Parameter & Description & Value \\
\hline MINWGHT & Minimum pick weight (0-1) & 0 \\
\hline MAXDIST & $\begin{array}{l}\text { Maximum distance (in } \mathrm{km} \text { ) between event } \\
\text { pair and station }\end{array}$ & 150 \\
\hline MAXSEP & $\begin{array}{l}\text { Maximum hypocentral separation between } \\
\text { event pairs in } \mathrm{km} \text {. }\end{array}$ & 10 \\
\hline MAXNGH & Maximum number of neighbors per event. & 10 \\
\hline MINLNK & $\begin{array}{l}\text { Minimum number of links required to } \\
\text { define a neighbor. }\end{array}$ & 6 \\
\hline MINOBS & Minimum number of links per pair saved & 4 \\
\hline MAXOBS & $\begin{array}{l}\text { Maximum number of links per pair saved } \\
\text { (ordered by distance from event pair). }\end{array}$ & 20 \\
\hline
\end{tabular}


Table B.2 Weighting and iteration information for several hypoDD relocation runs. The only weighting changed between P-only and runs with $\mathrm{P}$ and $\mathrm{S}$ phases is the S-phase weighting. Values of -9 indicate

that data or parameter wasn't used.

\begin{tabular}{|c|c|c|c|c|c|c|}
\hline & NITER & WTCTP & WTCTS & WRCT & WDCT & DAMP \\
\hline & $\begin{array}{l}\text { Numbe } \\
\text { r of } \\
\text { iteratio } \\
\text { ns }\end{array}$ & $\begin{array}{l}\text { Weighting } \\
\text { of Catalog } \\
\text { P-phase } \\
\text { picks }\end{array}$ & $\begin{array}{l}\text { Weighting } \\
\text { of Catalog } \\
\text { S-phase } \\
\text { picks }\end{array}$ & $\begin{array}{l}\text { Cutoff threshold for } \\
\text { outliers (values } \geq 1= \\
\text { factor to multiply } \\
\text { standard deviation). }\end{array}$ & $\begin{array}{l}\text { Maximum event } \\
\text { separation } \\
\text { distance (in km) } \\
\text { for catalog data }\end{array}$ & $\begin{array}{c}\text { Damping } \\
\text { (LSQR } \\
\text { only) }\end{array}$ \\
\hline P-only & 5 & 1 & -9 & -9 & -9 & 50 \\
\hline P-only & 5 & 1 & -9 & -9 & 6 & 50 \\
\hline P-only & 5 & 0.5 & -9 & 6 & 4 & 47 \\
\hline P-only & 10 & 0.1 & -9 & 4 & 4 & 47 \\
\hline$P$ and $S-1$ & & & 0.5 & & & \\
\hline $\mathrm{P}$ and $\mathrm{S}-1$ & & & 0.5 & & & \\
\hline $\mathrm{P}$ and $\mathrm{S}-1$ & & & 0.1 & & & \\
\hline $\mathrm{P}$ and $\mathrm{S}-1$ & & & 0.01 & & & \\
\hline$P$ and $S-2$ & & & 0.5 & & & \\
\hline $\mathrm{P}$ and $\mathrm{S}-2$ & & & 0.1 & & & \\
\hline$P$ and $S-2$ & & & 0.01 & & & \\
\hline$P$ and $S-2$ & & & 0 & & & \\
\hline
\end{tabular}




\section{Appendix C: Earthquakes used in triggering analyses}

Table C.1 List of large global earthquakes tested as potential triggers in Chapter 5.1.

\begin{tabular}{cccccccccc}
\hline Year & Month & Day & Hour & Min & Sec & Lat & Long & Mag & Depth \\
\hline 1993 & 1 & 15 & 11 & 6 & 5.95 & 43.3 & 143.69 & 7.6 & 102 \\
1993 & 3 & 6 & 3 & 5 & 49.87 & -10.97 & 164.18 & 7.1 & 20 \\
1993 & 5 & 11 & 18 & 26 & 51.32 & 7.22 & 126.57 & 7 & 58 \\
1993 & 5 & 24 & 23 & 51 & 28.24 & -22.67 & -66.54 & 7 & 221 \\
1993 & 6 & 8 & 13 & 3 & 36.48 & 51.22 & 157.83 & 7.5 & 70 \\
1993 & 7 & 12 & 13 & 17 & 11.96 & 42.85 & 139.2 & 7.7 & 16 \\
1993 & 8 & 8 & 8 & 34 & 24.93 & 12.98 & 144.8 & 7.8 & 59 \\
1993 & 8 & 9 & 12 & 42 & 48.19 & 36.38 & 70.87 & 7 & 214 \\
1993 & 8 & 10 & 0 & 51 & 53.25 & -45.28 & 166.93 & 7 & 28 \\
1993 & 9 & 10 & 19 & 12 & 54.62 & 14.72 & -92.64 & 7.2 & 34 \\
1993 & 11 & 13 & 1 & 18 & 4.18 & 51.93 & 158.65 & 7 & 34 \\
1993 & 12 & 29 & 7 & 48 & 14.2 & -20.23 & 169.79 & 7 & 33 \\
1994 & 1 & 21 & 2 & 24 & 29.96 & 1.01 & 127.73 & 7 & 19 \\
1994 & 2 & 12 & 17 & 58 & 23.99 & -20.55 & 169.36 & 7 & 27 \\
1994 & 3 & 9 & 23 & 28 & 6.78 & -18.04 & -178.41 & 7.6 & 562 \\
1994 & 3 & 14 & 4 & 30 & 15.75 & -1.28 & -23.57 & 7 & 10 \\
1994 & 6 & 2 & 18 & 17 & 34.02 & -10.48 & 112.83 & 7.8 & 18 \\
1994 & 6 & 9 & 0 & 33 & 16.23 & -13.84 & -67.55 & 8.2 & 631 \\
1994 & 7 & 13 & 2 & 35 & 56.02 & -16.62 & 167.52 & 7.2 & 33 \\
1994 & 7 & 21 & 18 & 36 & 31.74 & 42.34 & 132.87 & 7.3 & 471 \\
1994 & 9 & 1 & 15 & 15 & 53.08 & 40.4 & -125.68 & 7.1 & 10 \\
1994 & 10 & 4 & 13 & 22 & 55.84 & 43.77 & 147.32 & 8.3 & 14 \\
1994 & 10 & 9 & 7 & 55 & 39.58 & 43.9 & 147.92 & 7.3 & 33 \\
1994 & 11 & 14 & 19 & 15 & 30.66 & 13.52 & 121.07 & 7.1 & 31 \\
1994 & 12 & 28 & 12 & 19 & 23.03 & 40.53 & 143.42 & 7.8 & 26 \\
1995 & 1 & 6 & 22 & 37 & 34.32 & 40.25 & 142.18 & 7 & 26 \\
1995 & 2 & 5 & 22 & 51 & 5.14 & -37.76 & 178.75 & 7.1 & 21 \\
1995 & 4 & 7 & 22 & 6 & 56.89 & -15.2 & -173.53 & 7.4 & 21 \\
1995 & 4 & 21 & 0 & 34 & 46.09 & 12.06 & 125.58 & 7.2 & 20 \\
1995 & 5 & 5 & 3 & 53 & 45.05 & 12.63 & 125.3 & 7.1 & 16 \\
1995 & 5 & 16 & 20 & 12 & 44.22 & -23.01 & 169.9 & 7.7 & 20 \\
1995 & 5 & 27 & 13 & 3 & 52.65 & 52.63 & 142.83 & 7.1 & 11 \\
1995 & 7 & 3 & 19 & 50 & 50.62 & -29.21 & -177.59 & 7.2 & 35 \\
1995 & 7 & 30 & 5 & 11 & 23.63 & -23.34 & -70.29 & 8 & 45 \\
1995 & 8 & 16 & 10 & 27 & 28.63 & -5.8 & 154.18 & 7.7 & 30 \\
1995 & 8 & 16 & 23 & 10 & 23.98 & -5.77 & 154.35 & 7.2 & 33 \\
1995 & 8 & 23 & 7 & 6 & 2.76 & 18.86 & 145.22 & 7.1 & 594 \\
1995 & 9 & 14 & 14 & 4 & 31.43 & 16.78 & -98.6 & 7.4 & 23
\end{tabular}


Table C.1 (global triggering investigation) continued

\begin{tabular}{|c|c|c|c|c|c|c|c|c|c|}
\hline Year & Month & Day & Hour & Min & $\mathrm{Sec}$ & Lat & Long & Mag & Depth \\
\hline 1995 & 10 & 3 & 1 & 51 & 23.9 & -2.75 & -77.88 & 7 & 24 \\
\hline 1995 & 10 & 9 & 15 & 35 & 53.91 & 19.06 & -104.21 & 8 & 33 \\
\hline 1995 & 10 & 18 & 10 & 37 & 26.38 & 27.93 & 130.18 & 7.1 & 28 \\
\hline 1995 & 10 & 21 & 2 & 38 & 57.12 & 16.84 & -93.47 & 7.2 & 159 \\
\hline 1995 & 11 & 22 & 4 & 15 & 11.94 & 28.83 & 34.8 & 7.2 & 10 \\
\hline 1995 & 12 & 3 & 18 & 1 & 8.99 & 44.66 & 149.3 & 7.9 & 33 \\
\hline 1995 & 12 & 25 & 4 & 43 & 24.46 & -6.9 & 129.15 & 7.1 & 141 \\
\hline 1996 & 1 & 1 & 8 & 5 & 10.83 & 0.73 & 119.93 & 7.9 & 24 \\
\hline 1996 & 2 & 7 & 21 & 36 & 46.3 & 45.32 & 149.89 & 7.2 & 42 \\
\hline 1996 & 2 & 17 & 5 & 59 & 30.55 & -0.89 & 136.95 & 8.2 & 33 \\
\hline 1996 & 2 & 21 & 12 & 51 & 1.3 & -9.59 & -79.59 & 7.5 & 10 \\
\hline 1996 & 2 & 25 & 3 & 8 & 15.87 & 15.98 & -98.07 & 7.1 & 21 \\
\hline 1996 & 4 & 16 & 0 & 30 & 54.67 & -24.06 & -177.04 & 7.2 & 110 \\
\hline 1996 & 4 & 29 & 14 & 40 & 41.09 & -6.52 & 155 & 7.2 & 44 \\
\hline 1996 & 6 & 2 & 2 & 52 & 9.55 & 10.8 & -42.25 & 7 & 10 \\
\hline 1996 & 6 & 10 & 4 & 3 & 35.48 & 51.56 & -177.63 & 7.9 & 33 \\
\hline 1996 & 6 & 10 & 15 & 24 & 56 & 51.48 & -176.85 & 7.3 & 26 \\
\hline 1996 & 6 & 11 & 18 & 22 & 55.73 & 12.61 & 125.15 & 7.1 & 33 \\
\hline 1996 & 6 & 17 & 11 & 22 & 18.54 & -7.14 & 122.59 & 7.9 & 587 \\
\hline 1996 & 7 & 22 & 14 & 19 & 35.77 & 1 & 120.45 & 7 & 33 \\
\hline 1996 & 8 & 5 & 22 & 38 & 22.09 & -20.69 & -178.31 & 7.4 & 550 \\
\hline 1996 & 11 & 12 & 16 & 59 & 44.03 & -14.99 & -75.68 & 7.7 & 33 \\
\hline 1997 & 1 & 11 & 20 & 28 & 26.02 & 18.22 & -102.76 & 7.2 & 33 \\
\hline 1997 & 1 & 23 & 2 & 15 & 22.97 & -22 & -65.72 & 7.1 & 276 \\
\hline 1997 & 2 & 27 & 21 & 8 & 2.36 & 29.98 & 68.21 & 7.1 & 33 \\
\hline 1997 & 4 & 21 & 12 & 2 & 26.43 & -12.58 & 166.68 & 7.7 & 33 \\
\hline 1997 & 5 & 10 & 7 & 57 & 29.72 & 33.83 & 59.81 & 7.3 & 10 \\
\hline 1997 & 5 & 25 & 23 & 22 & 33.19 & -32.12 & 179.79 & 7.1 & 332 \\
\hline 1997 & 7 & 9 & 19 & 24 & 13.17 & 10.6 & -63.49 & 7 & 19 \\
\hline 1997 & 9 & 20 & 16 & 11 & 32.15 & -28.68 & -177.62 & 7 & 30 \\
\hline 1997 & 10 & 14 & 9 & 53 & 18.15 & -22.1 & -176.77 & 7.8 & 167 \\
\hline 1997 & 10 & 15 & 1 & 3 & 33.46 & -30.93 & -71.22 & 7.1 & 58 \\
\hline 1997 & 10 & 28 & 6 & 15 & 17.33 & -4.37 & -76.68 & 7.2 & 112 \\
\hline 1997 & 11 & 8 & 10 & 2 & 52.61 & 35.07 & 87.32 & 7.5 & 33 \\
\hline 1997 & 11 & 15 & 18 & 59 & 24.3 & -15.15 & 167.38 & 7 & 123 \\
\hline 1997 & 11 & 25 & 12 & 14 & 33.63 & 1.24 & 122.54 & 7 & 24 \\
\hline 1997 & 12 & 5 & 11 & 26 & 54.69 & 54.84 & 162.04 & 7.8 & 33 \\
\hline 1997 & 12 & 22 & 2 & 5 & 50.08 & -5.49 & 147.87 & 7.2 & 179 \\
\hline 1998 & 1 & 4 & 6 & 11 & 58.97 & -22.3 & 170.91 & 7.5 & 100 \\
\hline 1998 & 1 & 30 & 12 & 16 & 8.69 & -23.91 & -70.21 & 7.1 & 42 \\
\hline 1998 & 3 & 25 & 3 & 12 & 25.07 & -62.88 & 149.53 & 8.1 & 10 \\
\hline 1998 & 3 & 29 & 19 & 48 & 16.21 & -17.55 & -179.09 & 7.2 & 537 \\
\hline 1998 & 4 & 1 & 17 & 56 & 23.36 & -0.54 & 99.26 & 7 & 55 \\
\hline 1998 & 5 & 3 & 23 & 30 & 21.91 & 22.31 & 125.31 & 7.5 & 33 \\
\hline
\end{tabular}


Table C.1 (global triggering investigation) continued

\begin{tabular}{|c|c|c|c|c|c|c|c|c|c|}
\hline Year & Month & Day & Hour & Min & $\mathrm{Sec}$ & Lat & Long & Mag & Depth \\
\hline 1998 & 7 & 16 & 11 & 56 & 36.42 & -11.04 & 166.16 & 7 & 110 \\
\hline 1998 & 7 & 17 & 8 & 49 & 13.28 & -2.96 & 141.93 & 7 & 10 \\
\hline 1998 & 8 & 4 & 18 & 59 & 20.1 & -0.59 & -80.39 & 7.2 & 33 \\
\hline 1998 & 8 & 20 & 6 & 40 & 55.82 & 28.93 & 139.33 & 7.1 & 440 \\
\hline 1998 & 11 & 9 & 5 & 38 & 44.22 & -6.92 & 128.95 & 7 & 33 \\
\hline 1998 & 11 & 29 & 14 & 10 & 31.96 & -2.07 & 124.89 & 7.7 & 33 \\
\hline 1999 & 1 & 19 & 3 & 35 & 33.84 & -4.6 & 153.24 & 7 & 114 \\
\hline 1999 & 2 & 6 & 21 & 47 & 59.47 & -12.85 & 166.7 & 7.3 & 90 \\
\hline 1999 & 3 & 4 & 8 & 52 & 1.9 & 5.4 & 121.94 & 7.1 & 33 \\
\hline 1999 & 4 & 5 & 11 & 8 & 4 & -5.59 & 149.57 & 7.4 & 150 \\
\hline 1999 & 4 & 8 & 13 & 10 & 34.08 & 43.61 & 130.35 & 7.1 & 565 \\
\hline 1999 & 5 & 10 & 20 & 33 & 2.09 & -5.16 & 150.88 & 7.1 & 138 \\
\hline 1999 & 5 & 16 & 0 & 51 & 20.46 & -4.75 & 152.49 & 7.1 & 73 \\
\hline 1999 & 6 & 15 & 20 & 42 & 5.93 & 18.39 & -97.44 & 7 & 70 \\
\hline 1999 & 8 & 17 & 0 & 1 & 39.13 & 40.75 & 29.86 & 7.6 & 17 \\
\hline 1999 & 9 & 20 & 17 & 47 & 18.49 & 23.77 & 120.98 & 7.7 & 33 \\
\hline 1999 & 9 & 30 & 16 & 31 & 15.69 & 16.06 & -96.93 & 7.5 & 60 \\
\hline 1999 & 10 & 16 & 9 & 46 & 44.13 & 34.59 & -116.27 & 7.2 & 0 \\
\hline 1999 & 11 & 12 & 16 & 57 & 19.55 & 40.76 & 31.16 & 7.2 & 10 \\
\hline 1999 & 11 & 15 & 5 & 42 & 43.22 & -1.34 & 88.98 & 7 & 10 \\
\hline 1999 & 11 & 19 & 13 & 56 & 46.45 & -6.35 & 148.76 & 7 & 33 \\
\hline 1999 & 11 & 26 & 13 & 21 & 15.57 & -16.42 & 168.21 & 7.5 & 33 \\
\hline 1999 & 12 & 6 & 23 & 12 & 33.92 & 57.41 & -154.49 & 7 & 66 \\
\hline 1999 & 12 & 11 & 18 & 3 & 36.45 & 15.77 & 119.74 & 7.3 & 33 \\
\hline 2000 & 1 & 8 & 16 & 47 & 20.58 & -16.92 & -174.25 & 7.2 & 183 \\
\hline 2000 & 2 & 25 & 1 & 43 & 58.64 & -19.53 & 173.82 & 7.1 & 33 \\
\hline 2000 & 3 & 28 & 11 & 0 & 22.51 & 22.34 & 143.73 & 7.6 & 126 \\
\hline 2000 & 4 & 23 & 9 & 27 & 23.32 & -28.31 & -62.99 & 7 & 608 \\
\hline 2000 & 5 & 4 & 4 & 21 & 16.21 & -1.11 & 123.57 & 7.6 & 26 \\
\hline 2000 & 5 & 12 & 18 & 43 & 18.12 & -23.55 & -66.45 & 7.2 & 225 \\
\hline 2000 & 6 & 4 & 16 & 28 & 26.17 & -4.72 & 102.09 & 7.9 & 33 \\
\hline 2000 & 6 & 18 & 14 & 44 & 13.31 & -13.8 & 97.45 & 7.9 & 10 \\
\hline 2000 & 8 & 6 & 7 & 27 & 12.9 & 28.86 & 139.56 & 7.4 & 394 \\
\hline 2000 & 10 & 4 & 16 & 58 & 44.31 & -15.42 & 166.91 & 7 & 23 \\
\hline 2000 & 10 & 29 & 8 & 37 & 8.77 & -4.77 & 153.95 & 7 & 50 \\
\hline 2000 & 11 & 16 & 4 & 54 & 56.74 & -3.98 & 152.17 & 8 & 33 \\
\hline 2000 & 11 & 16 & 7 & 42 & 16.93 & -5.23 & 153.1 & 7.8 & 30 \\
\hline 2000 & 11 & 17 & 21 & 1 & 56.49 & -5.5 & 151.78 & 7.8 & 33 \\
\hline 2000 & 12 & 6 & 17 & 11 & 6.4 & 39.57 & 54.8 & 7 & 30 \\
\hline 2001 & 1 & 1 & 6 & 57 & 4.17 & 6.9 & 126.58 & 7.5 & 33 \\
\hline 2001 & 1 & 9 & 16 & 49 & 28 & -14.93 & 167.17 & 7.1 & 103 \\
\hline 2001 & 1 & 10 & 16 & 2 & 44.23 & 57.08 & -153.21 & 7 & 33 \\
\hline 2001 & 1 & 13 & 17 & 33 & 32.38 & 13.05 & -88.66 & 7.7 & 60 \\
\hline 2001 & 1 & 26 & 3 & 16 & 40.5 & 23.42 & 70.23 & 7.7 & 16 \\
\hline
\end{tabular}


Table C.1 (global triggering investigation) continued

\begin{tabular}{|c|c|c|c|c|c|c|c|c|c|}
\hline Year & Month & Day & Hour & Min & $\mathrm{Sec}$ & Lat & Long & Mag & Depth \\
\hline 2001 & 2 & 13 & 19 & 28 & 30.26 & -4.68 & 102.56 & 7.4 & 36 \\
\hline 2001 & 2 & 24 & 7 & 23 & 48.73 & 1.27 & 126.25 & 7.1 & 35 \\
\hline 2001 & 6 & 3 & 2 & 41 & 57.16 & -29.67 & -178.63 & 7.2 & 178 \\
\hline 2001 & 6 & 23 & 20 & 33 & 14.13 & -16.26 & -73.64 & 8.4 & 33 \\
\hline 2001 & 7 & 7 & 9 & 38 & 43.52 & -17.54 & -72.08 & 7.6 & 33 \\
\hline 2001 & 8 & 21 & 6 & 52 & 6.25 & -36.81 & -179.57 & 7.1 & 33 \\
\hline 2001 & 10 & 12 & 15 & 2 & 16.84 & 12.69 & 144.98 & 7 & 37 \\
\hline 2001 & 10 & 19 & 3 & 28 & 44.46 & -4.1 & 123.91 & 7.5 & 33 \\
\hline 2001 & 10 & 31 & 9 & 10 & 20 & -5.91 & 150.2 & 7 & 33 \\
\hline 2001 & 11 & 14 & 9 & 26 & 10.01 & 35.95 & 90.54 & 7.8 & 10 \\
\hline 2001 & 12 & 12 & 14 & 2 & 35.04 & -42.81 & 124.69 & 7.1 & 10 \\
\hline 2002 & 1 & 2 & 17 & 22 & 48.76 & -17.6 & 167.86 & 7.2 & 21 \\
\hline 2002 & 3 & 3 & 12 & 8 & 19.74 & 36.5 & 70.48 & 7.4 & 225 \\
\hline 2002 & 3 & 5 & 21 & 16 & 9.13 & 6.03 & 124.25 & 7.5 & 31 \\
\hline 2002 & 3 & 31 & 6 & 52 & 50.49 & 24.28 & 122.18 & 7.1 & 32 \\
\hline 2002 & 4 & 26 & 16 & 6 & 7 & 13.09 & 144.62 & 7.1 & 85 \\
\hline 2002 & 6 & 28 & 17 & 19 & 30.27 & 43.75 & 130.67 & 7.3 & 566 \\
\hline 2002 & 8 & 19 & 11 & 1 & 1.19 & -21.7 & -179.51 & 7.7 & 580 \\
\hline 2002 & 8 & 19 & 11 & 8 & 24.31 & -23.88 & 178.49 & 7.7 & 675 \\
\hline 2002 & 9 & 8 & 18 & 44 & 23.71 & -3.3 & 142.95 & 7.6 & 13 \\
\hline 2002 & 10 & 10 & 10 & 50 & 20.57 & -1.76 & 134.3 & 7.6 & 10 \\
\hline 2002 & 11 & 2 & 1 & 26 & 10.7 & 2.82 & 96.08 & 7.4 & 30 \\
\hline 2002 & 11 & 3 & 22 & 12 & 41 & 63.52 & -147.44 & 7.9 & 4 \\
\hline 2002 & 11 & 17 & 4 & 53 & 53.54 & 47.82 & 146.21 & 7.3 & 459 \\
\hline 2003 & 1 & 20 & 8 & 43 & 6.07 & -10.49 & 160.77 & 7.3 & 33 \\
\hline 2003 & 1 & 22 & 2 & 6 & 34.61 & 18.77 & -104.1 & 7.6 & 24 \\
\hline 2003 & 3 & 17 & 16 & 36 & 17.31 & 51.27 & 177.98 & 7.1 & 33 \\
\hline 2003 & 5 & 26 & 9 & 24 & 33.4 & 38.85 & 141.57 & 7 & 68 \\
\hline 2003 & 5 & 26 & 19 & 23 & 27.94 & 2.35 & 128.85 & 7 & 31 \\
\hline 2003 & 6 & 20 & 6 & 19 & 38.91 & -7.61 & -71.72 & 7.1 & 558 \\
\hline 2003 & 7 & 15 & 20 & 27 & 50.53 & -2.6 & 68.38 & 7.6 & 10 \\
\hline 2003 & 8 & 4 & 4 & 37 & 20.13 & -60.53 & -43.41 & 7.6 & 10 \\
\hline 2003 & 8 & 21 & 12 & 12 & 49.79 & -45.1 & 167.14 & 7.2 & 28 \\
\hline 2003 & 9 & 25 & 19 & 50 & 6.36 & 41.81 & 143.91 & 8.3 & 27 \\
\hline 2003 & 9 & 25 & 21 & 8 & 0.03 & 41.77 & 143.59 & 7.4 & 33 \\
\hline 2003 & 9 & 27 & 11 & 33 & 25.08 & 50.04 & 87.81 & 7.3 & 16 \\
\hline 2003 & 10 & 31 & 1 & 6 & 28.28 & 37.81 & 142.62 & 7 & 10 \\
\hline 2003 & 11 & 17 & 6 & 43 & 6.8 & 51.15 & 178.65 & 7.8 & 33 \\
\hline 2003 & 12 & 27 & 16 & 0 & 59.45 & -22.01 & 169.77 & 7.3 & 10 \\
\hline 2004 & 1 & 3 & 16 & 23 & 21.02 & -22.25 & 169.68 & 7.1 & 22 \\
\hline 2004 & 2 & 5 & 21 & 5 & 2.84 & -3.62 & 135.54 & 7 & 16 \\
\hline 2004 & 2 & 7 & 2 & 42 & 35.21 & -4 & 135.02 & 7.3 & 10 \\
\hline 2004 & 7 & 15 & 4 & 27 & 14.73 & -17.66 & -178.76 & 7.1 & 565 \\
\hline 2004 & 7 & 25 & 14 & 35 & 19.06 & -2.43 & 103.98 & 7.3 & 582 \\
\hline
\end{tabular}


Table C.1 (global triggering investigation) continued

\begin{tabular}{cccccccccc}
\hline Year & Month & Day & Hour & Min & Sec & Lat & Long & Mag & Depth \\
\hline 2004 & 9 & 5 & 10 & 7 & 7.82 & 33.07 & 136.62 & 7.2 & 14 \\
2004 & 9 & 5 & 14 & 57 & 18.61 & 33.18 & 137.07 & 7.4 & 10 \\
2004 & 10 & 9 & 21 & 26 & 53.69 & 11.42 & -86.67 & 7 & 35 \\
2004 & 11 & 11 & 21 & 26 & 41.15 & -8.15 & 124.87 & 7.5 & 10 \\
2004 & 11 & 15 & 9 & 6 & 56.56 & 4.7 & -77.51 & 7.2 & 15 \\
2004 & 11 & 22 & 20 & 26 & 23.9 & -46.68 & 164.72 & 7.1 & 10 \\
2004 & 11 & 26 & 2 & 25 & 3.31 & -3.61 & 135.4 & 7.1 & 10 \\
2004 & 11 & 28 & 18 & 32 & 14.13 & 43.01 & 145.12 & 7 & 39 \\
2004 & 12 & 23 & 14 & 59 & 4.41 & -49.31 & 161.35 & 8.1 & 10 \\
2004 & 12 & 26 & 0 & 58 & 53.45 & 3.3 & 95.98 & 9 & 30 \\
2004 & 12 & 26 & 4 & 21 & 29.81 & 6.91 & 92.96 & 7.2 & 39 \\
2005 & 2 & 5 & 12 & 23 & 18.94 & 5.29 & 123.34 & 7.1 & 525 \\
2005 & 3 & 2 & 10 & 42 & 12.23 & -6.53 & 129.93 & 7.1 & 201 \\
2005 & 3 & 28 & 16 & 9 & 36.53 & 2.09 & 97.11 & 8.6 & 30 \\
2005 & 6 & 13 & 22 & 44 & 33.9 & -19.99 & -69.2 & 7.8 & 115 \\
2005 & 6 & 15 & 2 & 50 & 54.19 & 41.29 & -125.95 & 7.2 & 16 \\
2005 & 7 & 24 & 15 & 42 & 6.21 & 7.92 & 92.19 & 7.2 & 16 \\
2005 & 8 & 16 & 2 & 46 & 28.4 & 38.28 & 142.04 & 7.2 & 36 \\
2005 & 9 & 9 & 7 & 26 & 43.73 & -4.54 & 153.47 & 7.6 & 90 \\
2005 & 9 & 26 & 1 & 55 & 37.67 & -5.68 & -76.4 & 7.5 & 115 \\
2005 & 10 & 8 & 3 & 50 & 40.8 & 34.54 & 73.59 & 7.6 & 26 \\
2005 & 11 & 14 & 21 & 38 & 51.42 & 38.11 & 144.9 & 7 & 11 \\
2006 & 1 & 2 & 6 & 10 & 49.76 & -60.96 & -21.61 & 7.4 & 13 \\
2006 & 1 & 2 & 22 & 13 & 40.49 & -19.93 & -178.18 & 7.2 & 582 \\
2006 & 1 & 27 & 16 & 58 & 53.67 & -5.47 & 128.13 & 7.6 & 397 \\
2006 & 2 & 22 & 22 & 19 & 7.8 & -21.32 & 33.58 & 7 & 11 \\
2006 & 4 & 20 & 23 & 25 & 2.15 & 60.95 & 167.09 & 7.6 & 22 \\
2006 & 5 & 3 & 15 & 26 & 40.29 & -20.19 & -174.12 & 8 & 55 \\
2006 & 5 & 16 & 10 & 39 & 23.34 & -31.81 & -179.31 & 7.4 & 152 \\
2006 & 7 & 17 & 8 & 19 & 26.68 & -9.28 & 107.42 & 7.7 & 20 \\
2006 & 8 & 20 & 3 & 41 & 48.04 & -61.03 & -34.37 & 7 & 13 \\
2006 & 11 & 15 & 11 & 14 & 13.57 & 46.59 & 153.27 & 8.3 & 10 \\
2006 & 12 & 26 & 12 & 26 & 21.14 & 21.8 & 120.55 & 7.1 & 10 \\
2007 & 1 & 13 & 4 & 23 & 21.16 & 46.24 & 154.52 & 8.1 & 10 \\
2007 & 1 & 21 & 11 & 27 & 45.06 & 1.07 & 126.28 & 7.5 & 22 \\
2007 & 3 & 25 & 0 & 40 & 1.61 & -20.62 & 169.36 & 7.1 & 34 \\
2007 & 4 & 1 & 20 & 39 & 58.71 & -8.47 & 157.04 & 8.1 & 24
\end{tabular}


Table C.2 List of large New Zealand earthquakes tested as potential triggers in Chapter 5.1.

\begin{tabular}{|c|c|c|c|c|c|c|c|c|c|}
\hline Year & Month & Day & Hour & Min & $\mathrm{Sec}$ & Lat & Long & Mag & Depth \\
\hline 1993 & 4 & 11 & 6 & 59 & 49.65 & -39.73 & 176.71 & 6.1 & 37.84 \\
\hline 1993 & 6 & 2 & 20 & 42 & 53.53 & -36.06 & 179.85 & 6.1 & 92.96 \\
\hline 1993 & 8 & 10 & 0 & 51 & 51.61 & -45.21 & 166.71 & 6.7 & 5.00 \\
\hline 1993 & 8 & 10 & 9 & 46 & 39.93 & -38.53 & 177.91 & 6.3 & 45.53 \\
\hline 1993 & 8 & 13 & 11 & 2 & 22.98 & -36.00 & 178.80 & 6.5 & 117.38 \\
\hline 1994 & 6 & 18 & 3 & 25 & 14.61 & -43.01 & 171.48 & 6.7 & 4.27 \\
\hline 1994 & 6 & 19 & 13 & 43 & 51.74 & -43.17 & 171.47 & 6.1 & 5.00 \\
\hline 1994 & 12 & 4 & 22 & 5 & 57.83 & -35.79 & 178.80 & 6.1 & 210.99 \\
\hline 1994 & 12 & 15 & 11 & 20 & 19.56 & -37.20 & 177.53 & 6.0 & 12.00 \\
\hline 1995 & 2 & 5 & 22 & 51 & 2.30 & -37.65 & 179.49 & 7.0 & 12.00 \\
\hline 1995 & 2 & 10 & 1 & 44 & 56.34 & -37.92 & 51 & 6.6 & 12.00 \\
\hline 1995 & 2 & 13 & 0 & 11 & 43.99 & -37.46 & 179.14 & 6.2 & 12.00 \\
\hline 1995 & 3 & 22 & 19 & 43 & 31.05 & -41.05 & 174.18 & 6.4 & 87.08 \\
\hline 1995 & 6 & 3 & 20 & 58 & 59.19 & -33.21 & -179.37 & 6.7 & 432.49 \\
\hline 1995 & 9 & 19 & 22 & 52 & 24.73 & -39.61 & 174.27 & 6.6 & 205.89 \\
\hline 1995 & 11 & 24 & 6 & 18 & 57.88 & -42.95 & 171.82 & 6.3 & 7.26 \\
\hline 1996 & 2 & 28 & 14 & 23 & 10.89 & -45.06 & 167.41 & 6.2 & 114.37 \\
\hline 1996 & 9 & 25 & 10 & 35 & 17.72 & -39.05 & & .3 & 213.78 \\
\hline 1996 & 11 & 5 & 9 & 41 & 34.43 & -31.16 & 180.00 & 7.3 & 369.00 \\
\hline 1997 & 5 & 3 & 16 & 46 & 1.84 & -32.55 & -177.98 & 7.1 & 135.49 \\
\hline 1997 & 5 & 25 & 23 & 22 & 30.59 & -32.31 & -17 & 7.9 & 339.38 \\
\hline 1997 & 5 & 27 & 3 & 14 & 1.68 & -37.55 & $17 €$ & 6.1 & 212.02 \\
\hline 1998 & 4 & 20 & 23 & 34 & 18.11 & -39.02 & 174.92 & 6.8 & 231.78 \\
\hline 1998 & 7 & 9 & 14 & 45 & 37.12 & -32.13 & -175.77 & 7.2 & 330.96 \\
\hline 1999 & 4 & 20 & 19 & 4 & 4.55 & -32.46 & -177.51 & 7.0 & 207.63 \\
\hline 1999 & 5 & 18 & 9 & 19 & 35.69 & -38.59 & 175.48 & 6.5 & 264.45 \\
\hline 1999 & 10 & 25 & 20 & 31 & 42.51 & -38.57 & 175.91 & 7.0 & 160.81 \\
\hline 2000 & 5 & 8 & 21 & 35 & 40.97 & -31.72 & -178.35 & 7.2 & 392.40 \\
\hline 2000 & 8 & 8 & 10 & 31 & 19.55 & -39.16 & 176.28 & 6.2 & 49.98 \\
\hline 2000 & 8 & 15 & 4 & 30 & 4.99 & -31.94 & -178.55 & 7.6 & 436.17 \\
\hline 2000 & 11 & 1 & 10 & 35 & 55.78 & -45.12 & 166.95 & 6.2 & 9.00 \\
\hline 2001 & 5 & 24 & 18 & 7 & 57.88 & -38.75 & 175.20 & 6.5 & 237.88 \\
\hline 2001 & 6 & 3 & 2 & 41 & 57.44 & -29.81 & -178.58 & 7.3 & 185.00 \\
\hline 2001 & 8 & 21 & 6 & 51 & 57.71 & -36.49 & -178.48 & 7.1 & 33.00 \\
\hline 2001 & 10 & 21 & 0 & 29 & 20.43 & -36.89 & 179.86 & 6.3 & 12.00 \\
\hline 2001 & 12 & 7 & 19 & 27 & 35.04 & -44.11 & 168.61 & 6.2 & 5.00 \\
\hline 2003 & 5 & 4 & 20 & 8 & 43.82 & -31.48 & -176.05 & 6.4 & 33.00 \\
\hline 2003 & 8 & 21 & 12 & 12 & 49.87 & -45.19 & 166.83 & 7.0 & 24.35 \\
\hline 2003 & 8 & 21 & 14 & 12 & 27.57 & -45.36 & 166.82 & 6.1 & 23.83 \\
\hline 2003 & 9 & 4 & 8 & 40 & 44.08 & -45.19 & 166.93 & 6.0 & 23.13 \\
\hline 2004 & 11 & 22 & 20 & 26 & 32.09 & -46.61 & 165.32 & 7.1 & 12.00 \\
\hline 2005 & 3 & 13 & 15 & 8 & 13.14 & -40.18 & 173.67 & 6.4 & 142.94 \\
\hline 2006 & 5 & 16 & 10 & 39 & 14.17 & -32.00 & -177.56 & 7.5 & 152.00 \\
\hline
\end{tabular}

